Viscothermal

Wave Propagation

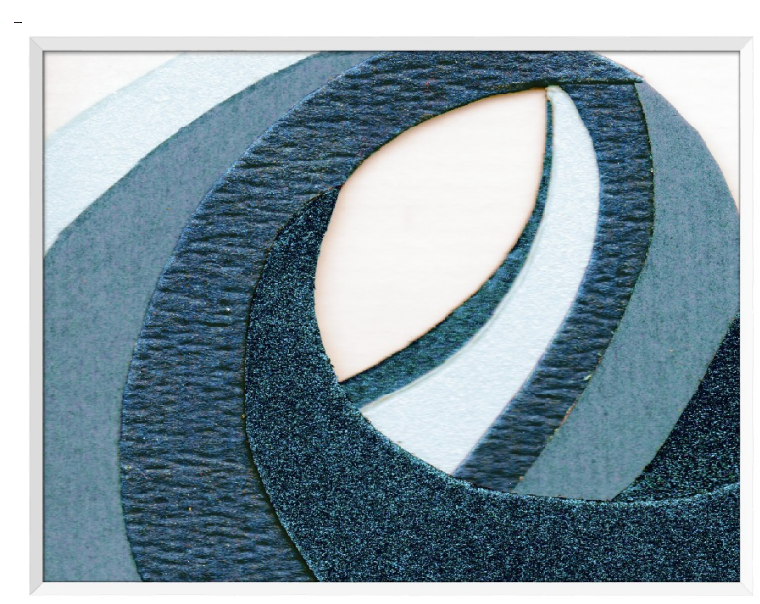

Marten Nijhof 

VISCOTHERMAL WAVE PROPAGATION

Marten Nijhof 
De promotiecommissie is als volgt samengesteld:

Voorzitter en secretaris:

Prof.dr. F. Eising

Universiteit Twente

Promotor:

Prof.dr.ir. A. de Boer

Universiteit Twente

Assistent-promotor:

Dr.ir. Y.H. Wijnant

Leden:

Prof.dr.ir. H.W.M. Hoeijmakers

Universiteit Twente

Prof.dr. S. Luding

Universiteit Twente

Universiteit Twente

Prof.dr. J.P. Coyette

Dr. M.A. Bochev

Katholieke Universiteit Leuven, België

Dr.ir. W.M. Beltman

Universiteit Twente

Intel Corporation, USA

This research was performed in the framework of an INTEL research grand, contract number 11336.

Viscothermal Wave Propagation

Nijhof, Marten Jozef Johannes

$\mathrm{PhD}$ thesis, University of Twente, Enschede, The Netherlands

November 2010

ISBN 978-90-365-3119-1

DOI 10.3990/1.9789036531191

Subject headings: acoustics, vibrations, viscothermal wave propagation

Copyright $\odot 2010$ by M.J.J. Nijhof, Enschede, The Netherlands

Printed by Ipskamp drukkers b.v., Enschede, The Netherlands

Cover created by M.J. Nijhof, ProudMary. 


\section{VISCOTHERMAL WAVE PROPAGATION}

\section{PROEFSCHRIFT}

ter verkrijging van

de graad van doctor aan de Universiteit Twente, op gezag van de rector magnificus, prof. dr. H. Brinksma, volgens besluit van het College voor Promoties in het openbaar te verdedigen op vrijdag 10 december 2010 om 13.15 uur

door

Marten Jozef Johannes Nijhof

geboren op 8 november 1979

te Borne 
Dit concept proefschrift is goedgekeurd door de promotor

Prof.dr.ir. A. de Boer

en de assistent-promotor

Dr.ir. Y.H. Wijnant 


\section{Summary}

In engineering practice, the simplest, most efficient model that yields the desired level of accuracy is usually the model of choice. This is particulary true if an optimization process is involved, in which case the choice of the underlying models is often a trade-off between efficiency and accuracy. It is therefore important to know not only how efficient a model is, but also how accurate.

In this work, the accuracy, efficiency and range of applicability of various (approximate) models for viscothermal wave propagation are investigated in a general setting. Models for viscothermal wave propagation describe the wave behavior of fluids including viscous and thermal effects. Cases where viscothermal effects are significant generally involve small fluid domains, low frequencies, or fluid systems near resonance. Examples of practical applications of these models are, for instance, describing the behavior of in-ear hearing aids, MEMS devices, microphones, inkjet printheads and muffler systems involving acoustic resonators.

Amongst the various models for viscothermal wave propagation that are considered, a prominent role is taken by the family of approximate models known as Low Reduced Frequency (or LRF) models. These are the most efficient approximate models available and they have been used extensively to model a wide variety of problems involving viscothermal wave propagation. Nevertheless, LRF models are only available for a limited number of geometries and can become inaccurate under certain conditions.

A second family of models that is considered consists of exact solutions to the equations describing viscothermal wave propagation. These models, which are less efficient than the LRF models, provide reference solutions which can be used to determine the accuracy of the LRF models. A drawback of the exact models is that they are only available for a small number of geometries. Therefore a third family of models is considered, which is based on a newly developed Finite Element (or FE) approximation of the equations for viscothermal wave propagation. The main attraction of these FE models is that they can be used to model arbitrary geometries and boundary conditions. A drawback is that obtaining a solution requires much more computing power than needed for the LRF or exact models. The 
numerical stability and convergence properties of the developed FE methods are investigated to ensure that they can yield reference solutions of a desired accuracy for cases where an exact solution is not available.

Using these three families of models, a number of parameter studies are carried out that yield detailed information on accuracy of the highly efficient LRF models for a range of geometries and boundary conditions. The gathered data provides a means of estimating the accuracy of simple coupled LRF models a priori.

Besides the investigation into the accuracy of LRF models, two engineering applications where viscothermal wave propagation takes a prominent role are described. The first application involves the passive silencing of a speed controlled cooling fan in a personal computer with acoustic resonators. The dimensions of the resonator system, which exhibits viscothermal wave propagation, is optimized for broadband noise reduction. The underlying meta model is built from LRF models and includes a newly developed model describing the radiating and scattering properties of the rotating fan. A demonstrator setup was designed and built based on the outcome of optimization. The measurement results obtained with this setup indicate that broadband noise reduction is achieved.

The second application involves the optimization of a resonator system for sound absorbtion. Again, the underlying meta model consists of LRF models. The predicted performance in terms of absorption for a previously obtained optimization result is determined with an LRF model, the newly developed FE model and by direct measurement. Based on the measurement results, it is concluded that the accuracy of the FE model offers a significant improvement over that of the LRF model.

Note, that the family of LRF models and exact solutions are described in a very general setting and are extended to include geometries for which solutions were previously unavailable. In addition, a hybrid FE/analytical method is developed which offers an efficient alternative for expensive full 3D FE calculations for a number of geometry classes. Together with the numerically stable FE methods, that can be used for arbitrary geometries and boundary conditions, these models expand the engineering toolkit for modeling viscothermal wave propagation. 


\section{Samenvatting}

In het algemeen geven ingenieurs de voorkeur aan modellen die zo simpel en efficiënt mogelijk zijn en tegelijkertijd de vereiste nauwkeurigheid bieden. Deze voorkeur komt nog sterker tot uiting wanneer een model gebruikt wordt binnen een optimalisatie proces. De modelkeuze berust dan vaak op een trade-off tussen efficiency en nauwkeurigheid. Het is daarom belangrijk om naast kennis over de efficiency van een model ook een goede voorspelling te hebben over de nauwkeurigheid ervan.

In dit onderzoek worden in een brede context de efficiëntie en nauwkeurigheid van diverse (benaderings)modellen voor visco-thermische golfvoortplanting onderzocht. Dergelijke modellen beschrijven het golfgedrag van gassen en vloeistoffen inclusief de effecten van viscositeit en warmteoverdracht. Deze zogenaamde visco-thermische effecten zijn van belang wanneer kleine vloeistof/gas volumes of lage frequenties worden beschouwd en bij het modeleren van systemen die (bijna) in resonantie zijn. Praktische toepassing van deze modellen zijn bijvoorbeeld: het beschrijven van het gedrag van hoorapparaten, MEMS structuren, microfoons, inktjet printkoppen en geluidsreductie met akoestische resonatoren.

De zogenaamde Low Reduced Frequency (LRF) modellen hebben een prominente plaats in de rij modellen voor visco-thermische golfvoortplanting die in dit werk beschouwd worden. Deze groep van modellen is zeer efficiënt en wordt veelvuldig toegepast om een breed scala aan problemen te modeleren die verband houden met visco-thermische golfvoortplanting. Echter, LRF modellen zijn slechts voor een beperkt aantal geometrieën beschikbaar en kunnen onnauwkeurig worden onder bepaalde omstandigheden.

Een tweede groep modellen die beschouwd wordt zijn exacte oplossingen van de vergelijkingen die visco-thermische golfvoortplanting beschrijven. Deze modellen, die minder efficiënt zijn dan LRF modellen, kunnen als referentiemodel gebruikt worden om de nauwkeurigheid van LRF modellen vast te stellen. Een nadeel van deze 'exacte modellen' is dat ze slechts beschikbaar zijn voor een zeer beperkt aantal geometrieën.

De derde groep modellen die beschouwd wordt zijn zogenaamde Eindige Ele- 
viii

menten (EE) benaderingen van de vergelijkingen voor visco-thermische golfvoortplanting. Een groot voordeel van deze modellen is dat ze gebruikt kunnen worden voor het modeleren van willekeurige geometrieën en randvoorwaarden. Een groot nadeel is dat het verkrijgen van een EE oplossing een veelvoud van de rekenkracht vereist in verhouding tot de rekenkracht die nodig is voor het verkrijgen van een LRF of exacte oplossing. De numerieke stabiliteit en het convergentie gedrag van de ontwikkelde EE methodes zijn grondig onderzocht om zeker te stellen dat EE modellen een (referentie) oplossing met een gewenste nauwkeurigheid kunnen bieden voor gevallen waar een exacte oplossing niet beschikbaar is.

Met gebruik van de modellen uit deze drie groepen zijn er diverse parameterstudies uitgevoerd die informatie verschaffen over de nauwkeurigheid van diverse gekoppelde LRF modellen. De verzamelde gegevens maken het mogelijk om de nauwkeurigheid van simpele gekoppelde LRF modellen te voorspellen.

Naast een onderzoek naar de nauwkeurigheid van LRF modellen zijn er ook twee toepassingen van visco-thermischemodellen beschreven. De eerste toepassing is het optimaliseren van een systeem voor passieve geluidsreductie van een snelheidsgeregelde computerventilator met akoestische resonatoren. Visco-thermische effecten hebben een grote invloed op de optimale afmetingen van het resonatorsysteem dat gericht is op breedbandige geluidsreductie. Het onderliggende metamodel is samengesteld uit gekoppelde LRF modellen en een nieuw ventilatormodel dat de geluidsafstraling en weerkaatsing van de ventilator beschrijft. Op basis van een optimalisatie met dit model is een resonatorsysteem ontworpen en gebouwd. De metingen die uitgevoerd zijn met dit systeem laten zien dat er inderdaad sprake is van breedbandige geluidsreductie.

De tweede applicatie is het ontwerp van een resonatorsysteem voor passieve geluidsabsorptie van starre wanden. Opnieuw is het onderliggende metamodel opgebouwd uit gekoppelde LRF modellen. De gerealiseerde geluidsabsorptie van een geoptimaliseerd ontwerp is bepaald met het gekoppeld LRF model, de ontwikkelde EE modellen en door een directe meting met een experimentele opstelling. Gebaseerd op de meetresultaten kan de conclusie getrokken worden dat de voorspellingen op basis van EE modellen significant nauwkeuriger zijn dan die op basis van het gebruikte LRF model.

Verder, is de groep van LRF modellen uitgebreid met modellen voor geometrieën waar voorheen geen oplossing voor beschikbaar was. Daarnaast is een hybride EE/analytische methode ontwikkeld voor een aantal klasses van geometrieën, die een efficiënt alternatief is voor volledige 3D EE berekeningen. Samen met de numeriek stabiele EE methodes, die toegepast kunnen worden op willekeurige geometrieën en randvoorwaarden, vergroten deze modellen de inhoud van de 'gereedschapskist' voor het beschrijven van visco-thermische golfvoortplanting. 


\section{Contents}

Summary $\quad$ v

Samenvatting vii

Contents $\quad$ ix

1 Introduction 1

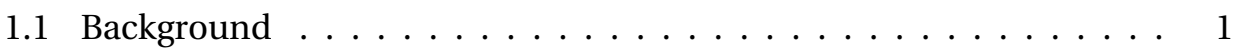

1.2 Viscothermal wave propagation . . . . . . . . . . . . 1

1.3 Applications of viscothermal models . . . . . . . . . . . . . . . 3

1.4 Models for viscothermal wave propagation . . . . . . . . . . . 4

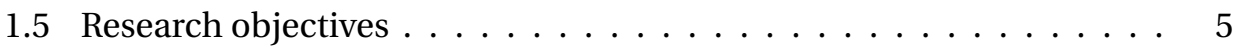

1.6 Waveguide models . . . . . . . . . . . . . . . 6

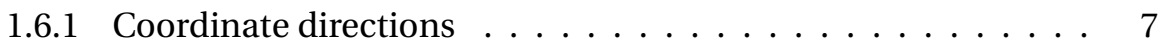

1.7 Outline ........................ 7

2 Viscothermal wave propagation 9

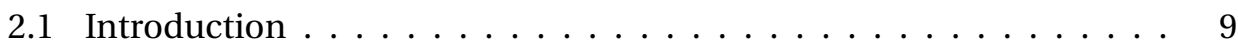

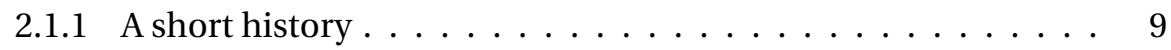

2.2 Basic equations . . . . . . . . . . . . . . . 14

2.2 .1 Equations of state . . . . . . . . . . . . . . 14

2.2.2 Energy equation for an ideal gas . . . . . . . . . . . . . 15

2.3 Linearized governing equations $\ldots \ldots \ldots \ldots$

2.3.1 Total derivative . . . . . . . . . . . . . . 16

2.3.2 Small density, pressure and temperature variations . . . . . . 17

2.3 .3 Viscous dissipation . . . . . . . . . . . . . . . . . . . . 18

2.3 .4 Governing equations . . . . . . . . . . . . . . . . . . . . . . . 18

2.4 Fourier transformation . . . . . . . . . . . . . . . 18

2.4.1 Effects of an imposed steady state solution . . . . . . . . . . 19

2.5 Reduced models . . . . . . . . . . . . . . . . . 21 
2.5.1 Inviscid adiabatic wave propagation . . . . . . . . . . 21

2.5 .2 Linear elasticity . . . . . . . . . . . . . . . . . 22

2.5 .3 Stokes flow . . . . . . . . . . . . . . . . . . 24

2.6 Dimensionless equations and groups . . . . . . . . . . . 25

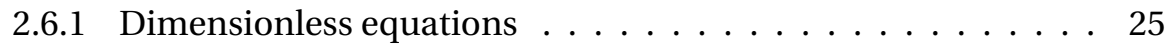

2.6.2 Dimensionless wave numbers . . . . . . . . . . . . . . . 26

2.6 .3 Characteristic length . . . . . . . . . . . . . . . . . . . . . . . . . 27

2.6 .4 Dimensionless groups . . . . . . . . . . . . 27

2.7 Boundary conditions . . . . . . . . . . . . . . . 29

2.7 .1 Velocity boundary conditions . . . . . . . . . . 30

2.7.2 Temperature boundary conditions . . . . . . . . . . . 31

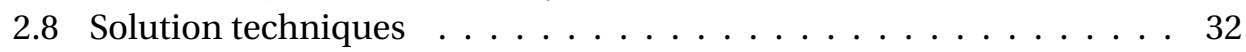

2.8.1 (A) BEM/FEM boundary layer approximation . . . . . . . . 32

2.8 .2 (B) (C) (D) LRF models . . . . . . . . . . . . . . . . . . . . . . . . . . . . . . . 35

2.8 .3 (E) SLNS models . . . . . . . . . . . . . . . . . . . . . . . . . . . . . . . . . . . 37

$2.8 .4(\mathrm{~F})(\mathrm{G})$ Kirchhoff models . . . . . . . . . . . . . . . . . . . . . . . . . . 38

2.8 .5 (H) BEM/FEM full LNSF models . . . . . . . . . . . . . . . . . . . . . . . . . . . . . . . . . . . . .

2.9 Summary .............................. 42

3 Full linearized Navier-Stokes models $\quad \mathbf{4 3}$

3.1 Exact Kirchhoff solutions . . . . . . . . . . . . . . . . . . . . 44

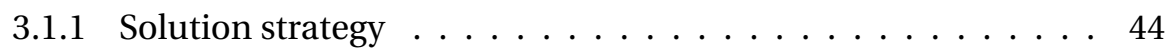

3.2 General form of exact solutions . . . . . . . . . . . . . . . 45

3.2 .1 Reduced form . . . . . . . . . . . . . . . . . . . 45

3.2 .2 Fourth order equation . . . . . . . . . . . . . . 47

3.2 .3 Vector Helmholtz equation ............... 48

3.2 .4 Boundary conditions . . . . . . . . . . . . . 49

3.2.5 Pilot vector and surface normal coincide . . . . . . . . . . 50

3.2.6 Symmetry in flat waveguides . . . . . . . . . . . . . . 52

3.2.7 Pilot vector and surface normal do not coincide . . . . . . . . 53

3.2 .8 Solving the system . . . . . . . . . . . . . . . . . . 55

3.3 Examples of exact solutions . . . . . . . . . . . . . . 58

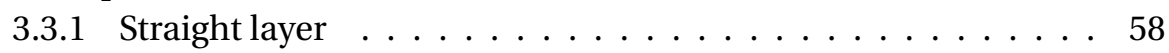

3.3.2 Curved layers, spherical layers, tubes, and annular tubes . . . 60

3.4 Kirchhoff-FEM solutions . . . . . . . . . . . . . . . 60

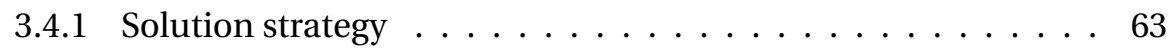

3.4.2 Non-orthogonal coordinate systems . . . . . . . . . . . . . 65

3.4.3 Examples of Kirchhoff-FEM solutions . . . . . . . . . . . . . 66

3.5 LRF and inviscid adiabatic approximation . . . . . . . . . . . . . . 72

3.5.1 Wave numbers .................... 72 


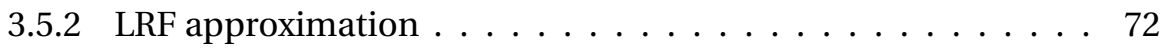

3.5.3 Inviscid adiabatic approximation $\ldots \ldots \ldots \ldots \ldots \ldots$

3.6 Mode Matching . . . . . . . . . . . . . . . . . . . 75

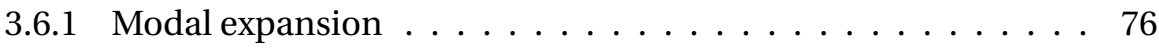

3.6 .2 Boundary conditions . . . . . . . . . . . . . . . 77

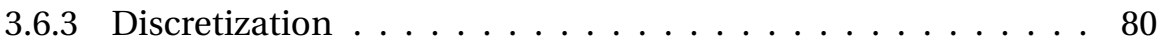

3.7 Summary and discussion $\ldots \ldots \ldots \ldots \ldots \ldots$

4 Low reduced frequency models $\quad 87$

4.1 General Solution . . . . . . . . . . . . . . . . . 88

4.1 .1 Splitting operators $\ldots \ldots \ldots \ldots \ldots$

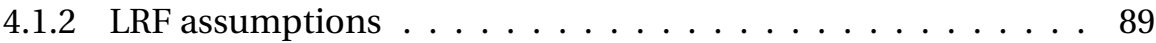

4.1 .3 LRF equations . . . . . . . . . . . . . . . . . 91

4.1.4 Solutions for velocity, temperature and density . . . . . . . . 92

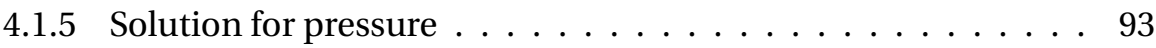

4.2 Geometry specific LRF solutions . . . . . . . . . . . . . . . . 97

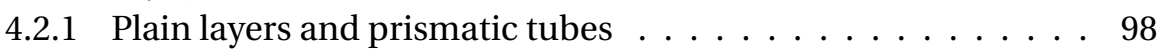

4.2.2 Curved tubes and cylindrical layers . . . . . . . . . . . . 104

4.2.3 Straight tubes and plain layers with non-constant cross section 112

4.3 Acoustic two-port networks . . . . . . . . . . . . . . . 118

4.3.1 General two-port description . . . . . . . . . . . . . 119

4.3 .2 Prismatic tubes . . . . . . . . . . . . . . . . . 120

4.3 .3 Circular layers . . . . . . . . . . . . . . . . . . . . 121

4.3.4 Arbitrary 1D waveguides . . . . . . . . . . . . . . . . 122

4.3.5 Coupling two-ports . . . . . . . . . . . . . . . . . . . . 124

4.3.6 Length corrections . . . . . . . . . . . . . . . . . . 125

4.4 Summary and discussion $\ldots \ldots \ldots \ldots \ldots$

5 Viscothermal acoustic FE models 129

5.1 Viscothermal acoustic problems . . . . . . . . . . . . . 130

5.1.1 Velocity, temperature and pressure/density based FE methods 131

5.1.2 Velocity- and temperature-based FE methods . . . . . . . . . 133

5.1.3 Velocity-based FE methods . . . . . . . . . . . . . . . . 134

5.1.4 FE spaces, convergence, approximability, and stability . . . . 135

5.2 Stability of FE methods for the LNSF equations . . . . . . . . . . 139

5.2.1 Existence and uniqueness results for saddle point problems . 141

5.2.2 Coercivity and semi-positive definiteness . . . . . . . . 143

5.2 .3 Alternative weak form . . . . . . . . . . . . . . 145

5.2.4 Existence and uniqueness for the LNSF equations $\ldots \ldots \ldots 146$

5.2 .5 FE formulation . . . . . . . . . . . . . . . . . 149 
5.2.6 Error bounds, optimal convergence and the inf-sup condition 151

5.3 The numerical inf-sup test . . . . . . . . . . . . . . . . . . 159

5.3.1 Matrix vector form of the LNSF equations . . . . . . . . . . . 159

5.3.2 The inf-sup condition and the singular value decomposition . 160

5.3 .3 The inf-sup test . . . . . . . . . . . . . . . . . 162

5.3 .4 Influence of $\mathrm{B}_{v}$ and $\mathrm{B}_{T} \ldots \ldots \ldots \ldots \ldots$

5.3 .5 Stable FE methods . . . . . . . . . . . . . . . . 165

5.4 Stability and convergence results . . . . . . . . . . . . . . . . . . . . . . . . . . . . .

5.4 .1 Test setup . . . . . . . . . . . . . . . 167

5.4 .2 Element types . . . . . . . . . . . . . . . . 170

5.4.3 Example: stable vs. unstable LNSF element . . . . . . . . . . . 174

5.4 .4 Numerical inf-sup test . . . . . . . . . . . . . . . . . 178

5.4 .5 Convergence results . . . . . . . . . . . . . . . 182

5.4 .6 Example of convergence results . . . . . . . . . . . . 183

5.4 .7 Velocity-temperature based elements . . . . . . . . . . . . 185

5.5 Conclusions . . . . . . . . . . . . . . . . 190

6 Performance 193

6.1 LRF assumptions and relative accuracy . . . . . . . . . . . . . . . . . . . . . 197

6.1 .1 Relative accuracy of LRF models . . . . . . . . . . . . . . 197

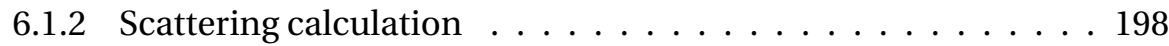

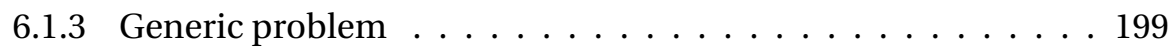

6.1 .4 Relative errors . . . . . . . . . . . . . . . . . . 204

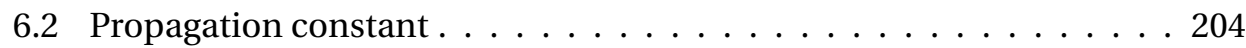

6.2.1 Isothermal no-slip boundary conditions . . . . . . . . . . 205

6.2.2 Adiabatic no-slip boundary conditions . . . . . . . . . . . 207

6.2 .3 Error in complex amplitude . . . . . . . . . . . . . . . 208

6.3 Bifurcation of waveguides . . . . . . . . . . . . . 210

6.4 Change in thermal boundary conditions . . . . . . . . . . . . 213

6.5 Change in cross-sectional geometry . . . . . . . . . . . . 216

6.6 Change in curvature . . . . . . . . . . . . . . . 221

6.7 Scattering calculation using mode matching . . . . . . . . . . . . . 226

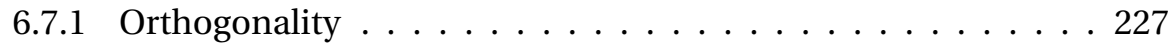

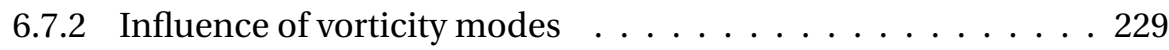

6.8 Conclusions . . . . . . . . . . . . . . . . . . . 232

7 Engineering applications $\mathbf{2 3 5}$

7.1 Passive reduction of fan noise . . . . . . . . . . . . . 235

7.1.1 Influence of source impedance and termination impedance . 236

7.1.2 Wave propagation in the presence of mean flow . . . . . . . . 238 
7.1.3 Fan model . . . . . . . . . . . . . . . . . . . . . . . . 238

7.1.4 Determining the scattering matrix and source strength vector 241

7.1.5 Measured source characteristics . . . . . . . . . . . . . 244

7.1 .6 Experimental validation . . . . . . . . . . . . . . . 248

7.1 .7 Resonator design . . . . . . . . . . . . . . . . . 250

7.1 .8 Results . . . . . . . . . . . . . . . . . . . 252

7.2 Sound absorption of aircraft cabin noise $\ldots \ldots \ldots \ldots$

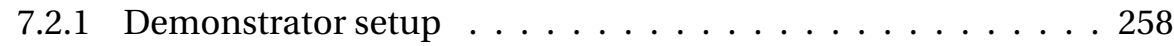

7.2 .2 LRF, FE, and FE-BLA model . . . . . . . . . . . . . 260

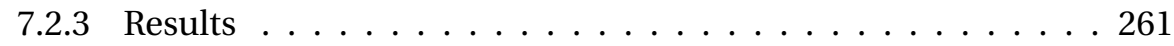

8 Conclusions and discussion $\quad 265$

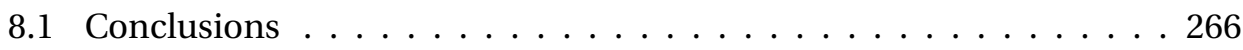

8.2 Discussion . . . . . . . . . . . . . . . . 267

$\begin{array}{ll}\text { Nomenclature } & 271\end{array}$

A Derivation of the basic equations 275

A.1 Continuum hypothesis . . . . . . . . . . . . . . . . 275

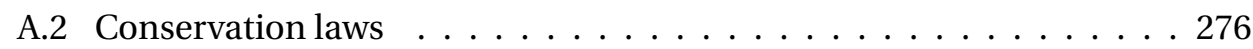

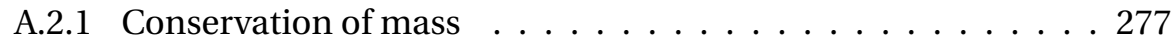

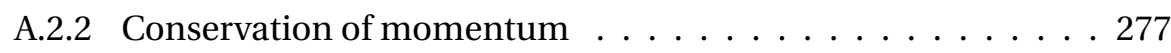

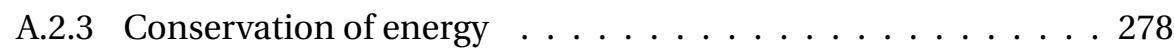

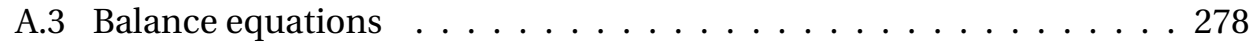

A.4 Constitutive Relations . . . . . . . . . . . . . . . . . . . . . . 279

A.4.1 Constitutive relations for Navier-Stokes-Fourier fluids . . . . 280

A.4.2 Material parameters in the literature . . . . . . . . . . 281

A.5 Governing equations for Navier-Stokes-Fourier fluids . . . . . . . 282

$\begin{array}{ll}\text { B Properties of air } & 283\end{array}$

C LNSF equations for arbitrary fluids $\quad 285$

$\begin{array}{ll}\text { D Coordinate systems } & 287\end{array}$

E Geometry specific LRF solutions $\quad 289$

E.1 Analytical solutions . . . . . . . . . . . . . . . . . . . . . . . . 289

E.2 Semi-analytical solutions . . . . . . . . . . . . . . . . . . . 292

E.3 Example curved tube of rectangular cross section . . . . . . . . . 294 
F 1D waveguides of non-constant cross section 295

F.1 One-parameter waves . . . . . . . . . . . . . . . 295

F.2 Numerical solutions for tapered 1D waveguides . . . . . . . . . . . . . . . . . . . . . . . . . . . . . .

F.3 Boundary layer approximation . . . . . . . . . . . . . . . 297

G Non-orthogonality of mode shapes $\quad 299$

H Mathematical backgrounds 301

H.1 Null spaces, image and closed range theorem . . . . . . . . . . . . 301

H.2 Requirements for existence and uniqueness for sesquilinear forms . . 302

$\begin{array}{ll}\text { I Convergence results } & 305\end{array}$

J FE boundary layer approximation $\quad 309$

J.1 FEM Helmholtz equation for pressure . . . . . . . . . . . . . . . . . . . . . . . . . . . . . . . . . . . . . .

J.2 Boundary layer theory . . . . . . . . . . . . . . . 310

K LRF performance $\quad \mathbf{3 1 3}$

K.1 No-slip isothermal/adiabatic boundary conditions . . . . . . . . . . 313

K.2 Changes in thermal boundary conditions . . . . . . . . . . . . . . 314

$\begin{array}{lll}\text { L Resonator design } & \mathbf{3 1 7}\end{array}$

$\begin{array}{lr}\text { Bibliography } & \mathbf{3 1 9}\end{array}$

$\begin{array}{lr}\text { Nawoord } & 329\end{array}$ 


\section{Chapter 1}

\section{Introduction}

\subsection{Background}

Acoustics is an important aspect of the design of many everyday objects. For some products, the importance lies in achieving good sound quality, for instance in the case of hearing aids design, or speaker design. For other objects, the importance lies in controlling the level of noise that is produced, for instance in the design of computers, household appliances, airplanes, or cars. Over the years, acoustic properties have become an important selling point for an increasing number of consumer products. The demands of consumers provide engineers with the challenge of incorporating acoustic aspects in the design of their products.

As with any engineering aspects, an efficient design process that involves acoustic aspects implies the use of models that predict the acoustic properties. Preferably, such models are time efficient, accurate, and applicable for all possible choices of the design parameters. In practice, the model that is selected from a set of applicable models often offers a trade-off between efficiency and accuracy. This is especially true when extensive optimization is involved and time efficiency is critical. In order to select the most suitable model, a priori information about the efficiency and accuracy of a model is valuable.

The present study focusses on the accuracy, efficiency and applicability of models for viscothermal wave propagation, a form of acoustic wave propagation that takes viscous and thermal effects into account.

\subsection{Viscothermal wave propagation}

Viscothermal wave propagation can be seen both as a special case of fluid dynamics and a generalization of acoustic wave propagation. To arrive at a physically and 
mathematically sound description, a logical starting point is the set of so-called Linearized Full Navier-Stokes-Fourier equations (or LNSF equations), which are the linearized form of the equations describing fluid dynamics. This approach is taken in chapter 2 . On the other hand, to explain the physics behind the viscous and thermal effects on wave propagation, a more convenient starting point is perhaps acoustic wave propagation.

\section{Acoustic wave propagation}

Acoustic wave propagation is a description of material behavior due to (harmonic) excitation in terms of pressure fluctuations. These fluctuations take the form of pressure waves which are accompanied by fluctuations in fluid velocity, density and temperature. As an acoustic wave travels through a medium, a conversion of energy occurs repeatedly between kinetic energy (in the form of fluid velocity) and potential energy (in the form of increased pressure and temperature). The fluctuations in velocity, density and temperature can all be efficiently described in terms of pressure fluctuations. However, such a description of the fluid behavior does not account for shearing forces in the fluid or heat flows due to thermal conduction. This corresponds with approximating the fluid as inviscid (implying the fluid does not resist shearing) and approximating wave propagation as an adiabatic process (implying temperature gradients do not result in heat flows). These approximations lead to the term inviscid adiabatic acoustics, which is used in this thesis to indicate 'classical' or 'standard' acoustics (thus underlining the distinction with viscothermal acoustics).

\section{Effects of viscosity}

In many cases involving wave propagation, the fluid domain under consideration is bounded. Close to fluid boundaries, the inviscid and adiabatic approximation can become inaccurate. The effects of adding viscosity to the description of the fluid behavior is easiest understood by considering a wave traveling parallel to a fluid boundary. If the boundary is fixed in space, the velocity of the fluid at the boundary is zero. At the same time a certain distance away from the boundary, the fluid is speeding up and down due to the forces of a passing pressure wave. This difference in fluid velocity causes shearing of the fluid. For viscid fluids, viscous forces will oppose the shearing motion, thus slowing down and damping out the acoustic wave while dissipating a part of the wave energy as heat. As the distance to the boundary increases, the velocity gradients become smaller and with it the viscous forces. At a certain distance away from the boundary, the forces are negligible implying that viscous effects are confined to a boundary layer. 


\section{Effects of heat flow}

If the boundary has a fixed temperature a similar account can be given of the influence of thermal effects. The fluid experiences temperature fluctuations due to the passing pressure wave. The temperature difference between boundary and fluid will induce heat flows in directions normal to the boundary. As a result, the magnitude of the temperature variations is zero at the boundary, and increases with distance from the boundary. The temperature gradient and heat flow decrease until, at a certain distance from the boundary, they become negligible thus confining thermal effects to a boundary layer. Close to the boundary, energy is no longer buffered as heat as the pressure rises and falls. Wave propagation can thus be characterized as an isothermal process at the boundary and as an adiabatic process outside the thermal boundary layer. This behavior affects the relation between fluctuations in density and pressure, which governs the speed of the pressure wave.

\subsection{Applications of viscothermal models}

The combined effects of viscosity and thermal conduction slow down and damp out acoustic waves. The question arises when these effects are significant, and when they can be neglected. Naturally, the influence of viscothermal effects is expected to be pronounced if the volume enclosed in the boundary layers takes up a significant part of the total volume of a fluid domain. Due to miniaturization trends in many fields of technological development, these conditions are met in an increasing number of applications. Examples of practical applications where viscothermal models are important due to the size of the domain relative to the viscous and thermal boundary layers include:

- Wave propagation in in-ear hearing aids [1]

- The behavior of MEMS devices with thin fluid layers $[2,3,4]$

- The behavior of microphones, consisting of a membrane backed by a thin air layer $[5,6,7,8]$

- The behavior of inkjet print heads, including the wave propagation in narrow ink channels $[9,10]$

- The behavior of plates and double wall panels with a thin fluid layer for reduction of sound transmission [11, 12, 13, 14, 15, 16, 17]

- Modeling wave propagation in the cochlea [18] 
- Modeling wave propagation in the vocal tract, trachea and lungs [19]

Note that in many cases where the boundary-layer thicknesses are small compared to the dimensions of the domain, neglecting viscothermal effects leads to a very accurate and efficient description of the fluid behavior. Nevertheless, a second class of problems where the viscothermal effects can become significant, even if the boundary-layer thicknesses are relatively small, is in systems near resonance. At resonance, the viscothermal effects prevent the amplitude of acoustic waves becoming infinite upon excitation of the fluid. These damping effects are also strong for frequencies near resonance. In addition, the effects of damping also cause the resonance frequency to shift somewhat. So for frequencies near resonances of a system, viscothermal effects should be accounted for in order to obtain a good estimate of the wave amplitudes and resonance frequencies. Practical examples where these aspects are of importance are, for instance;

- Passive noise reduction with resonators in the case of reflecting or transmitting surfaces $[19,20]$

- Passive noise reduction in duct systems using reactive mufflers [19, 21, 22, 23]

The use of viscothermal models for optimization of resonator designs is illustrated by the applications presented in chapter 7 .

\subsection{Models for viscothermal wave propagation}

An overview of various viscothermal models is given at the end of chapter 2 . Some models are based on the full set of LNSF equations, while others are based on an approximation of the equations. The models vary from being purely analytical to purely numerical. These two aspects have a profound influence on their accuracy, efficiency, and applicability, where applicability refers to the classes of geometries that can be modeled. The different models have their own advantages and disadvantages, and may or may not be useful depending on the specifics of the problem. Two type of models that play a central role in this thesis are shortly introduced here.

\section{Low reduced frequency models}

Low Reduced Frequency models (LRF models) are based on an approximation of the full set of equations describing viscothermal wave propagation. The approximation is based on the notion that for certain combinations of fluid domain geom- 
etry and coordinate systems, it is possible to distinguish between coordinate directions in which waves propagate and coordinate directions in which waves do not propagate. Due to this approach, the geometries that can be modeled are limited to a class of basic shapes ${ }^{1}$. More complex geometries can be modeled by coupling multiple LRF models, however, the direction of wave propagation in the coupled system must be known a priori. LRF models for certain simple basic shapes only involve analytical solutions and are extremely time-efficient. The accuracy of such LRF models is known to be very good under most practical conditions ${ }^{2}$. For more complex (coupled) geometries, computational costs rise and the level of accuracy is not known a priori. Nevertheless, LRF models are the most efficient viscothermal models for a given geometry. The obvious appeal of using LRF models is the combination of efficiency and (in most cases) accuracy.

\section{Finite Element models}

An alternative to the approximate (often analytical) LRF models is the use of Finite Element models (FE models) based on the full set of LNSF equations. The use of FE models is not limited to any class of geometries, and if proven to be numerically stable, a solution of any desired accuracy can be obtained, provided enough computing power is available. A drawback of FE methods for the full set of LNSF equations is the huge amount of computing power required to evaluate them. Due to the required computing power, studies involving FE methods for the full set of equations are novelties. Due to the ongoing increase in memory, cpu-power and solver efficiency, the attention of researchers has gradually being drawn to such FE models over the last few years. Literature on such models is scarce, however $[24,25,26,27]$. The numerical stability of FE methods for viscothermal wave propagation has not been investigated previously.

\subsection{Research objectives}

With hindsight, it can be assessed that research objectives of this study are:

- Extend the toolkit of viscothermal models to include numerically stable FE methods for arbitrary geometries.

- Extend the knowledge on the accuracy of coupled LRF models using such FE models as reference.

\footnotetext{
${ }^{1}$ Uncoupled LRF models can only describe wave propagation in tubes and layers that are bounded by the coordinate planes of certain suitable coordinate systems.

${ }^{2}$ Inaccuracies are observed for very high frequencies and very small structures.
} 
- Extend the toolkit of approximate viscothermal models for efficient and accurate modeling of a wider set of problems.

The appeal of having a numerically stable FE model that is based on the full set of LNSF equations and is applicable to arbitrary geometries is that it can serve as a reference model to determine the accuracy of more efficient approximate models. For instance, assessing the accuracy of the promising new approximate model, recently proposed by Kampinga [1], would have been difficult without it. The FE methods presented in chapter 5 should therefore be seen as an enrichment of the toolkit of available models, and not as a replacement of successful approximate models. Their primary function is that of a benchmarking tool. They also serve as a convenient back-up in case all other models should fail to provide the desired accuracy.

In light of the second objective, it can be said that the accuracy of coupled LRF models was not systematically investigated previously. In almost all instance, the accuracy is assessed by validating individual cases experimentally ${ }^{3}$. In most cases, excellent agreement between measurement and LRF results is obtained. However, the application in chapter 7 demonstrates that this conclusion cannot be drawn automatically for LRF models of more complex geometries. In the present work a parameter study is carried out to investigate the accuracy of coupled LRF models by comparison with FE models based on the full set of LNSF equations. By doing this, the observed errors are known to be caused by using an approximate model and are not polluted by other modeling errors or measurement errors.

\subsection{Waveguide models}

In most cases where viscothermal effects become important, the fluid domain is finite, i.e., it is completely enclosed by boundaries. Waves traveling towards a boundary that represents, for instance, an enclosing structure will be (largely) reflected. When a large part of the boundary will not allow waves to pass freely, the directions in which waves will propagate are restricted by the geometry of the domain, i.e., the waves are 'guided' by the geometry of the enclosing boundaries. Therefore, fluid filled domains that are mostly enclosed by reflecting boundaries are referred to as waveguides. The non-reflecting sections are the waveguide entrances/openings. A priori knowledge on the directions of wave propagation can often be used to arrive at efficient approximate models. LRF models are an example of efficient waveguide models.

\footnotetext{
${ }^{3}$ An exception is the recent work of Kampinga [1] who compares LRF results with results obtained using the FE methods described in chapter 5 .
} 


\subsubsection{Coordinate directions}

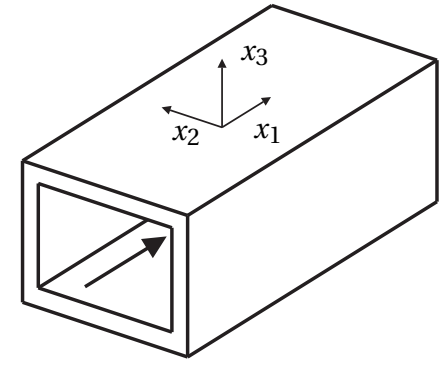

$c d$-coordinates: $\mathbf{x}_{c d}=x_{2}, x_{3}$

$p d$-coordinates: $x_{p d}=x_{1}$

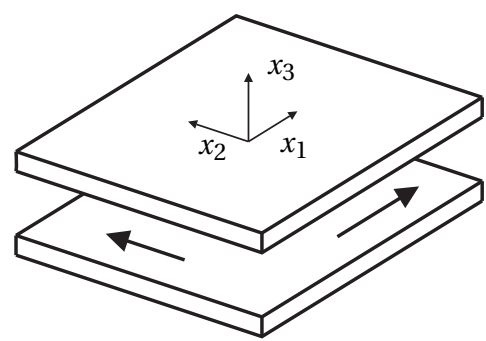

$c d$-coordinates: $x_{c d}=x_{3}$

pd-coordinates: $\mathbf{x}_{p d}=x_{1}, x_{2}$

Figure 1.1: Distribution of coordinates in propagation direction ( $p d$-coordinates), and coordinates in cross section ( $c d$-coordinates) for 1D and 2D waveguides.

The derivation of the LRF models presented in chapter 4 and the exact models in chapter 3 require the choice of a suitable coordinate system. These systems are chosen in such a way that a distinction can be made between two sets of coordinates; The first set consists of all coordinates that coincide with the propagational directions (or $p d$-directions), the directions in which wave propagation occurs. The second set consists of all coordinates that coincide with the crosssectional directions (or $c d$-directions), the coordinates in which the cross section (or thickness) of the waveguide can be described. From this point on, these sets of coordinates are referred to as $p d$-coordinates and $c d$-coordinates, respectively. Accordingly, velocities in $p d$ - and $c d$-directions are indicated as $p d$-velocities and $c d$-velocities, respectively. Note that the terms $p d / c d$-coordinates, $p d / c d$-directions and $p d / c d$-velocities can indicate a single coordinate, coordinate direction or velocity despite the plural form that is being used. An example of these conventions for a prismatic rectangular tube and a plane square layer is given in figure 1.1. Waveguides with one $p d$-direction are called 1D waveguides, waveguides with two $p d$-directions are called 2D waveguides. Note that a layer geometry for which wave propagation in only one $p d$-direction is assumed is also called a $1 \mathrm{D}$ waveguide (or 1D layer).

\subsection{Outline}

The equations governing viscothermal wave propagation are introduced in chapter 2. A small literature overview on their inception and the different solution pro- 
cedures that were developed over time is presented. The chapter finishes with an overview of the different solution strategies and their merits and drawbacks.

In chapter 3, two general solution strategies to arrive at solutions for the full set of LNSF equations are presented. The first strategy leads to models for 2D waveguides. The second strategy leads to a new type of model for $1 \mathrm{D}$ waveguides, adding to the toolkit of viscothermal models. The derivation includes an overview of the boundary conditions and geometries for which a closed form solution is available. The different models yield an infinite number of solutions, or so-called modes. It is demonstrated that LRF models approximate the first (acoustic) mode.

A general solution strategy to arrive at LRF approximations of the full set of LNSF equations is presented in chapter 4 . The effect of different boundary conditions and geometries on the form of the solution is discussed. Two newly developed LRF models for curved and tapered waveguides are presented. Lastly, techniques for coupling multiple LRF models are described in the context of acoustic two-port networks.

In chapter 5 various FE methods for the LNSF equations are developed. Both primal and mixed methods are considered. The numerical stability of mixed FE methods for the LNSF equations are studied in a general setting. Based on the results of the numerical inf-sup test and convergence studies, three families of numerically stable FE methods are identified. Case studies for the unstable primal method are also presented.

The performance of coupled LRF models is investigated in chapter 6 . The results of parameter studies on the accuracy of LRF models that include a change in boundary conditions, cross section or curvature are presented. The dependency of some of the encountered errors on the different model parameters is studied further using a model based on mode-matching.

Chapter 7 describes two applications of the presented results. First, the design of a side resonator system of an in-duct axial fan is presented. An acoustic twoport model for the fan is derived and the results of an experimental verification are presented. The layout of the two-port LRF model used for optimization is described and the performance of the optimized design is investigated. Thereafter, the optimization of a resonator design for acoustic noise reduction in aircraft cabins using LRF models is studied. The results of the optimized LRF model and a reference FE model are compared with results from experimental measurements.

Lastly, the main conclusions of this study are summarized in chapter 8. 


\section{Chapter 2}

\section{Viscothermal wave propagation}

\subsection{Introduction}

In this chapter the LNSF equations, which govern linearized viscothermal wave propagation in ideal (or perfect) gases, are introduced. First, a historic overview is given of the development of acoustic models including viscous and thermal effects. The basic equations, consisting of the conservation laws for mass, momentum and energy, are stated. They are supplemented by constitutive equations describing material behavior of ideal gases. Linearization is necessary to obtain a model that allows straightforward transformation to the frequency domain. Special attention is paid to the assumptions and considerations involving linearization of the governing equations. Stokes flow and inviscid isentropic wave propagation (or 'classical' acoustic wave propagation), both special cases of viscothermal wave propagation, are discussed in section 2.5.1. In the final section, five solution strategies to solve the LNSF equations are discussed. The usefulness of the different models is put in perspective by evaluating their limitations, accuracy and computational efficiency.

\subsubsection{A short history}

In this section a short history of the developments in the field of viscothermal wave propagation is presented. The overview is not complete, but is intended to highlight the breakthroughs that were made by different scientists. The developments in numerical methods for modeling viscothermal wave propagation are given extra attention. 
Acoustic wave equation The history of viscothermal wave propagation is rooted in that of the wave theory of sound. The first significant contributions to the study of wave propagation in fluids were made by Newton who related the speed of sound in air to the pressure and density under the assumptions of isothermal compression [28, 29]. This relation, an equation of state, was complemented by the equations of motion for a compressible fluid, resulting in a wave equation for sound. These developments were mainly due to the work on inviscid flow by Euler, d'Alembert and Lagrange [28].

Effects of heat and viscosity in free waves In this classic model of acoustic wave propagation in a compressible fluid, a constant ratio between pressure and density is assumed, which agrees with an isothermal process. As an exception, Lagrange considered the relation between pressure and density to be governed by a polytropic law instead. Laplace was among the first to recognize that for gases with constant heat capacity, a polytropic law agrees with an adiabatic process [28, 29]. Navier added frictional resistance terms to the equations of motion for fluids, and later Stokes [30] introduced the coefficient of viscosity and presented the momentum equations in their currently accepted form.

Effects of heat and viscosity in bounded waves The models of Laplace and Stokes concerned the propagation of free waves. The effect of viscosity on sound in a constrained body of fluid was studied by Helmholtz, who obtained an approximate expression for the phase velocity of a harmonic disturbance in a liquid-filled tube [29]. An investigation by Kirchhoff [31] showed that for gas-filled tubes, losses due to heat conductivity were of the same order of magnitude as the losses due to viscous dissipation. He included the energy equation to account for thermal boundary effects, derived a dispersion equation from the resulting set of equations (being the LNSF equations), and solved it for cases in which viscous and thermal effects are confined to small boundary layers (wide tubes). In his monumental work, the concept of separating the pressure, temperature and velocity fields into an acoustic, entropic (thermal) and vortical (viscous) contribution was already presented. It was Rayleigh [29] who used Kirchhoff's equations to determine the solution for cases in which the viscous and thermal effects are significant throughout the domain (narrow tubes).

Kirchhoff's dispersion equation using boundary admittance In 1948 Cremer [32] introduced the concept of the acoustic boundary layer. In this approach viscothermal effects are assumed to take place only in small regions near the boundaries, while wave propagation in the rest of the domain is considered inviscid and 
adiabatic. This leads to an expression for the so-called apparent admittance, an impedance-like boundary condition that accounts for the thermal and acoustic effects in the boundary layer. Beatty [33] used this concept to obtain an analytical approximation for the higher order modes of viscothermal wave propagation in waveguides.

Low Reduced Frequency approximations Although Kirchhoff and Rayleigh had already obtained an approximate solution to Kirchhoff's viscothermal wave propagation model in the mid 18 hundreds, it was not until 1949 that Zwikker and Kosten [34] presented a model that could be used for tubes of arbitrary width (ranging from narrow to wide). In this approximate model, the propagation constant is governed by a density term (mass), which is influenced by viscous effects but not by thermal effects, and the fluids compression modulus (spring) for which the opposite holds true. Zwikker and Kosten demonstrated that their model converges to the limiting cases presented by Kirchhoff and Rayleigh.

Tijdeman [35] compared the results of the model of Zwikker and Kosten, which he called a Low Reduced Frequency ${ }^{1}$ (LRF) model, with a numerical solution to Kirchhoff's dispersion equation. He put the different approximate models for viscothermal wave propagation available at the time in perspective. All models were rewritten in a convenient dimensionless form and he demonstrated that only the LRF model could be used for the entire range of narrow to wide tubes. Two decades later, Beltman $[5,10,36]$ compared the available models for layer geometries using a similar dimensionless notation (see section 2.6). He demonstrated that the relatively simple LRF models, which are computationally very efficient, are highly accurate for most fluids under standard atmospheric conditions under the same assumptions required for continuum mechanics. For some cases, it can be shown that LRF models approximate the exact solution for the lowest acoustic mode obtained by solving the corresponding Kirchhoff model (see section 3.5.2).

Beltman et al. [37] also presented an acoustic Finite Element (FE) for layers based on the LRF model. In this formulation, the velocity and temperature profiles across the thickness of the layer are solved analytically from the LRF equations while the equation for propagation behavior is discretized using a finite element method. In the work of Cummings [38] the LRF equations governing the velocity and temperature profiles are discretized, leaving a one dimensional wave equation to be solved analytically. Astley and Cummings [39] extended this model to account for mean flow.

\footnotetext{
${ }^{1}$ In some sources the model is referred to as the Zwikker-Kosten-Daniels model.
} 
Numerical solutions to Kirchhoff's dispersion equation In 1965, Shields et al. [40] presented an iterative method to solve Kirchhoff's dispersion equation without making additional assumptions concerning viscothermal effects. Building on this concept, Scarton [41] and Scarton and Rouleau [42] used numerical techniques to find the roots of Kirchhoff's dispersion equation. They obtained 'exact' solutions to the linearized Navier-Stokes equations for higher order acoustic modes, excluding thermal boundary effects. They also demonstrated the existence of a second series of modes that are dominated by viscous effects (vortical or vorticity modes) and indicated that, when thermal effects are included, a third series of modes can be found (entropic or thermal modes). Bruneau et al. [43] and Liang and Scarton [44] independently extended the method to include thermal effects.

Numerical solution using acoustic boundary layer approximation Dokumaci [45] formulated a Boundary Element Model (BEM) based on a fourth order equation for pressure (equivalent to Kirchhoff's equation for temperature). The equation is transformed into its integral form and the resulting higher order derivatives of pressure are rewritten as lower order derivatives of temperature and the vorticity vector (the curl of the velocity vector) using the original equations. Subsequently, a boundary layer approximation is used to obtain an expression for the normal heat flux and vorticity vector in terms of pressure, temperature and normal and tangential velocity. The resulting equations allow a straightforward application of temperature and tangential velocity boundary conditions yielding an integral equation in terms of pressure and normal velocity only. Due to the boundary layer approximation, the use of the model is limited to cases where the boundary layers are small compared to the dimension of the domain and the boundary can be considered locally flat. A similar, but simpler model was developed independently by Karra and Ben Tahar [46, 8]. In this work, the (normal component of the) rotational (solenoidal) part of the velocity field is neglected when applying the boundary conditions. As a consequence, the resulting model does not include viscous effects.

Bossart et al. [47] proposed a hybrid numerical and analytical method that can be used with 'classical' (inviscid and adiabatic) acoustic Finite Element Models (FEM) or BEM. The influence of the viscous and thermal effects in the boundary layers on the inviscid adiabatic part of the domain is represented by an admittancelike boundary condition which follows from boundary layer theory. Note that the calculated impedance corresponds with locations where the transition between boundary layer and bulk occurs. Since these locations depend on the boundary layer thickness and thus frequency, the calculated impedances are prescribed at the domain boundary instead. This additional approximation causes only small 
errors if viscous and thermal boundary layers are small compared to the dimensions of the domain, i.e., the boundary layers are well separated, but the errors become significant for larger boundary layers. In addition, the boundary layer approximations only hold when the boundaries can be considered locally flat.

Numerical solution to the full LNSF equations A FE model of the full LNSF equations was developed independently by Malinen et al. [24], Joly [48], Christensen et al. [26], and Nijhof et al. [25] and Kampinga et al. [27] (see chapter 5). The formulations that are used by the different authors are similar (and in some cases equivalent), however, the actual equations and variables that are used vary.

Cutanda et al. [49, 50] presented a BE model for viscothermal wave propagation based on the full LNSF equations. In these models, the wave equations for the acoustic, thermal and vorticity contributions to the pressure, temperature and velocity fields are discretized independently. Subsequently, the temperature, velocity and pressure are expressed in terms of the system matrices obtained by discretizing the independent fields. These expressions are inserted in the original equations to eliminate the thermal and tangent velocity boundary conditions. The approach ultimately yields a single system that relates the normal surface velocity to acoustic pressure. The total pressure and velocity field are obtained as a postprocessing step.

These FE and BE models are all suitable to model arbitrary geometries independent of the size of the boundary layers and the curvature of the boundary. However, at present, Cutanda's BE models are limited to axisymmetrical geometries.

SLNS models Recently, Kampinga et al. [1] proposed a method that bridges the gap between LRF models and FE models based on the full LNSF equations. The resulting models are called Sequential Linearized Navier Stokes models (or SLNS models). The adopted solution strategy is equivalent to that for deriving an LRF model. The main difference is that the independent equations describing temperature and velocity are no longer solved for a cross section, but for the entire geometry using FEM. This means that there is no longer a need to associate the directions of wave propagation with specific coordinate axes, which restricts the use of (semi-)analytic LRF models to relatively simple geometries. Solving SLNS models requires more computational effort than solving analytical (or mixed analytical/numerical) LRF models. Compared to solving the full LNSF equations numerically, the SLNS models are more efficient since they involve solving only three independent scalar equations instead of solving a system of three to five coupled equations. 


\subsection{Basic equations}

The equations governing viscothermal wave propagation are the fundamental conservation principles for mass, momentum and energy combined with constitutive laws which represent material specific behavior. For convenience, a concise derivation including the necessary assumptions is included in appendix A. The resulting equations are

$$
\begin{aligned}
\frac{D \rho}{D t}+\rho \nabla \cdot \mathbf{v} & =0 \\
\rho \frac{D \mathbf{v}}{D t} & =\nabla \cdot \sigma+\rho \mathbf{b} \\
\rho \frac{D e}{D t} & =-p \nabla \cdot \mathbf{v}+\Phi-\nabla \cdot \mathbf{q}+\rho r_{e}
\end{aligned}
$$

with $\rho$ the density, $\mathbf{v}$ the velocity vector, $p$ the pressure, $T$ the temperature, $e$ the specific internal energy, $\mathbf{b}$ the specific body force vector, and $r_{e}$ the rate of specific energy supply. The stress tensor $\sigma$, the viscous dissipation function $\Phi$ and the heat flux vector $\mathbf{q}$ are respectively defined as:

$$
\begin{aligned}
& \sigma=-p \mathbf{I}+\tau \\
& \Phi=\tau:(\nabla \mathbf{v})^{T} \\
& \mathbf{q}=-\kappa \nabla T
\end{aligned}
$$

with $\kappa$ the coefficient of thermal conductivity and the viscous stress tensor $\tau$ defined as:

$$
\tau=\lambda(\nabla \cdot \mathbf{v}) \mathbf{I}+\mu\left[\nabla \mathbf{v}+(\nabla \mathbf{v})^{T}\right]
$$

where $\mu$ is the coefficient of shear viscosity, $\lambda$ is the second coefficient of viscosity and $\nabla$ is the gradient operator ${ }^{2}$. The total derivative with respect to time, denoted as $D / D t$ (which is also known as material derivative), is defined as the sum of the partial derivative with respect to time and the convective derivative;

$$
\frac{D}{D t} \equiv \frac{\partial}{\partial t}+(\mathbf{v} \cdot \nabla)
$$

\subsubsection{Equations of state}

In order to complete the description of the behavior of any fluid, additional constitutive relations are needed. These so-called equations of state only involve the

\footnotetext{
${ }^{2}$ Note that the term $(\nabla \mathbf{v})^{T}$ can alternatively be defined as $(\nabla \mathbf{v})^{T}=\mathbf{v} \overleftarrow{\nabla}$, where $\overleftarrow{\nabla}$ is the so-called post gradient.
} 
state variables $\rho, T, p, e$ or $s$ (the latter representing specific entropy). The equations of state for an ideal gas, that will be used throughout this thesis, are stated below. Note that the presented models and solution strategies are also applicable to fluids which are described by equations of state other than those for an ideal gas. The use of equations of state for arbitrary fluids is commented upon in appendix C.

The constitutive relations used in the material description of ideal gases are the so-called thermal and caloric equation of state. The thermal equation of state for a classical ideal or perfect gas is given by the ideal gas law.

$$
p=\rho R_{0} T
$$

with $R_{0}$ the specific gas constant. For ideal gases the specific internal energy $e$ depends on the temperature only; the caloric equation of state for an ideal gas yields

$$
\mathrm{d} e=C_{V} \mathrm{~d} T
$$

where $d$ indicates a differential change. For an ideal gas, the specific heat at constant volume $C_{V}$ and the specific heat at constant pressure $C_{p}$, are related by

$$
R_{0}=C_{p}-C_{V}
$$

For many gasses that are compliant with the equations of state for an ideal gas, the specific heats can be considered a function of the temperature only. Moreover, for small temperature ranges $C_{V}$ and $C_{p}$ can be taken to be constant. Dry air is considered an ideal or perfect gas mixture under standard atmospheric conditions. The properties of air, the medium that is considered throughout this thesis, are listed in appendix B.

The viscosities and thermal conductivity of a perfect gas are typically taken to be a function of temperature only. For arbitrary fluids, these material properties may also depend on pressure. In case a perfect gas experiences only small temperature variations (or an arbitrary fluid governed by equation (2.1) experiences only small temperature and pressure variations), the values of the viscosities and thermal conductivity can be taken as constants.

\subsubsection{Energy equation for an ideal gas}

Substituting the caloric equation of state (2.8) and the Fourier law (2.4) in equation (2.1c) yields the energy equation for an ideal gas ${ }^{3}$ :

$$
\rho C_{V} \frac{D T}{D t}=-p \nabla \cdot \mathbf{v}+\Phi+\nabla \cdot(\kappa \nabla T)+\rho r_{e}
$$

\footnotetext{
${ }^{3}$ Note that $C_{\nu}$ and $C_{p}$ are taken to be constants.
} 
Rewriting $T$ in the left-hand side as a function of $p$ and $\rho$ using the thermal equation of state (2.7) and equation (2.9) yields

$$
\rho C_{p} \frac{D T}{D t}=\frac{D p}{D t}-\frac{p}{\rho} \frac{D \rho}{D t}-p \nabla \cdot \mathbf{v}+\Phi+\nabla \cdot(\kappa \nabla T)+\rho r_{e}
$$

By applying the equation for conservation of mass (equation (2.1a)) to the second right-hand side term, it is observed that the second and third right-hand side term cancel each other. So, the above equation reduces to

$$
\rho C_{p} \frac{D T}{D t}=\frac{D p}{D t}+\Phi+\nabla \cdot(\kappa \nabla T)+\rho r_{e}
$$

Note that this form of the energy balance is also known as the enthalpy equation.

\subsection{Linearized governing equations}

The different nonlinear terms found in the governing equations prohibit straightforward transformation to the frequency domain. The set of equations that is being linearized consists of the equation for conservation of mass (2.1a), conservation of momentum (2.1b), conservation of energy for an ideal gas (2.12) and the thermal equation of state for an ideal gas (2.7). The different assumptions that are necessary to linearize this set of equations are stated in the next subsections. For an extensive overview of the literature on nonlinear phenomena in acoustics see $[51,52,53]$.

\subsubsection{Total derivative}

The second term of the total derivative, known as the convective derivative (see equation (2.6)), is a cause of nonlinearity which is found in all balance equations. In order to linearize the total derivative, it is assumed that variation of a variable due to motion of the fluid (the convective derivative) is small compared to all other variations (the partial derivative with respect to time). The mathematical representation of this assumption is:

$$
\left|\frac{\partial}{\partial t}\right| \gg|\mathbf{v} \cdot \nabla|
$$

Under this assumption, the total derivatives in the different equations can be replaced by partial time derivatives. It can be shown a priori that for a (single) progressive plane acoustic wave, equation (2.13) holds if the velocity remains small compared to the acoustic speed of sound $\left(|\mathbf{v}| \ll c_{0}\right)$. In many acoustic studies, 
this velocity criteria is used to identify the significance of nonlinear effects. However, in the vicinity of localized sources or in the case of enclosed fluids close to resonance, contribution of the nonlinear terms can be significant while $|\mathbf{v}| \ll c_{0}$ [28]. It thus seems advisable to verify criterion (2.13) a posteriori to determine if the results of the linearized system are internally consistent with the linearization. Note that even if the linear model is internally consistent, the nonlinear solution may still differ significantly from the result of the linearized system. Examples of internally consistent linear acoustic models that prove to produce inaccurate or erroneous results are presented in reference [53, 54, 55, 56].

Despite what the examples found in these references suggest, the errors made by linearization are usually not significant as long as the amplitudes of the considered waves are not too large. This is true in both inviscid adiabatic acoustic wave propagation and viscothermal wave propagation. In the remainder of the text, criterion 2.13 is assumed to hold and the convective derivative is neglected. This implies that phenomena such as vortex shedding and acoustic streaming are not described by the developed models. Note that in the derivation presented below, criteria (2.13) is assumed to hold for both the unsteady and steady part of the velocity. This implies that changes in mass, momentum and energy due to convection by a steady velocity field are also assumed to be small compared to all other changes. The effects of adding a steady velocity field that does not satisfy (2.13) is discussed in section 2.4.1.

\subsubsection{Small density, pressure and temperature variations}

In order to linearize equations (2.1a), (2.1b) and (2.12) it is assumed that density variations are small compared to the average density. In order to linearize equation (2.7) the temperature and pressure variations have to be small compared to their average value as well. These assumptions can be mathematically represented by the following statements

$$
\begin{array}{lll}
\rho=\rho_{0}+\bar{\rho}(\mathbf{x}, t), & \text { with } & \bar{\rho}(\mathbf{x}, t) \ll \rho_{0} \\
T=T_{0}+\bar{T}(\mathbf{x}, t), & \text { with } & \bar{T}(\mathbf{x}, t) \ll T_{0} \\
p=p_{0}+\bar{p}(\mathbf{x}, t), & \text { with } & \bar{p}(\mathbf{x}, t) \ll p_{0}
\end{array}
$$

in which $\rho_{0}, T_{0}$ and $p_{0}$ are constant average values (in time and space) for density, temperature and pressure respectively, and $\bar{\rho}, \bar{T}$ and $\bar{p}$ are all small variations in time and space of these variables.

It was already mentioned in section 2.2.1 that for sufficiently small temperature (and pressure) variations the coefficients of specific heat, viscosity and thermal conductivity can all be taken as constants. The amplitude of the temperature 
(and pressure) variations for which the linear model predicts results of reasonable accuracy is dependent on the rate at which these material properties change with temperature (and pressure) for a given fluid and state. For certain fluids, these properties are known to vary significantly; for instance, if the fluid is in a state close to phase change. In such cases, the requirements in (2.14) may not be stringent enough to justify taking these material properties as constant.

\subsubsection{Viscous dissipation}

As a last step in the linearization process, it is assumed that energetic losses due to viscous dissipation can be neglected; the viscous dissipation function, $\Phi$ is removed from equation (2.12). Under these assumptions viscous losses do not contribute to an increase in internal energy (in the case of adiabatic boundary conditions) or an additional heat flow over the boundary (in the case of isothermal boundary conditions). Hence, the system does not conserve all energy due to this linearization. However, viscous effects are taken into account when the conservation of momentum is concerned. In other words; in the linear system, viscous effects 'slow down' and 'damp out' propagating waves, however, they do not contribute to the energy balance.

\subsubsection{Governing equations}

After all assumptions described above are taken into account, the linearized governing equations for the behavior of an ideal gas are found to be

$$
\begin{aligned}
\frac{\partial \bar{\rho}}{\partial t}+\rho_{0} \nabla \cdot \mathbf{v} & =0 \\
\rho_{0} \frac{\partial \mathbf{v}}{\partial t} & =-\nabla \bar{p}+(\lambda+\mu) \nabla(\nabla \cdot \mathbf{v})+\mu \Delta \mathbf{v}+\rho_{0} \mathbf{b} \\
\rho_{0} C_{p} \frac{\partial \bar{T}}{\partial t} & =\frac{\partial \bar{p}}{\partial t}+\kappa \Delta \bar{T}+\rho_{0} r_{e} \\
\frac{\bar{p}}{p_{0}} & =\frac{\bar{\rho}}{\rho_{0}}+\frac{\bar{T}}{T_{0}}
\end{aligned}
$$

\subsection{Fourier transformation}

The linearized system of equations in (2.15) is now transformed to the frequency domain. The independent variables $\bar{\rho}, \bar{p}, \bar{T}$ and $\mathbf{v}$ and external loads $\mathbf{b}$ and $r_{e}$ are now all a function of the angular frequency $\omega$. By transforming the system of 
equations to the frequency domain, the time derivatives are replaced by $i \omega$ and the following set of equations is obtained.

$$
\begin{aligned}
i \omega \bar{\rho}+\rho_{0} \nabla \cdot \mathbf{v} & =0 \\
i \omega \rho_{0} \mathbf{v} & =-\nabla \bar{p}+(\lambda+\mu) \nabla(\nabla \cdot \mathbf{v})+\mu \Delta \mathbf{v}+\rho_{0} \mathbf{b} \\
i \omega \rho_{0} C_{p} \bar{T} & =i \omega \bar{p}+\kappa \Delta \bar{T}+\rho_{0} r_{e} \\
\frac{\bar{p}}{p_{0}} & =\frac{\bar{\rho}}{\rho_{0}}+\frac{\bar{T}}{T_{0}}
\end{aligned}
$$

with $i=\sqrt{-1}$ the imaginary unit. Next, the system is solved in the frequency domain and the solutions can be transformed back to the time domain with the equations in (2.17) and (2.18) if necessary. In most cases studied in practice, the boundary conditions that are applied to the system can be represented or approximated by a discrete frequency spectrum. If boundary conditions are applied only at discrete frequencies, it suffices to sum the solutions found for these individual frequencies to find the total response of the system. In other words, for linear systems with boundary conditions at discrete frequencies it suffices to solve the system per frequency. If the different variables and external loads are real-valued functions in the time domain, back transformation of the solution to the time domain for a specific value of $\omega$ is defined as

$$
\begin{aligned}
& \bar{\rho}(t)=\Re\left[\bar{\rho}(\omega) e^{i \omega t}\right], \quad \bar{p}(t)=\Re\left[\bar{p}(\omega) e^{i \omega t}\right], \quad \bar{T}(t)=\Re\left[\bar{T}(\omega) e^{i \omega t}\right] \\
& \mathbf{v}(t)=\Re\left[\mathbf{v}(\omega) e^{i \omega t}\right], \quad \mathbf{b}(t)=\Re\left[\mathbf{b}(\omega) e^{i \omega t}\right], \quad r_{e}(t)=\Re\left[r_{e}(\omega) e^{i \omega t}\right]
\end{aligned}
$$

If boundary conditions are a continuous function of frequency, an inverse Fourier transformation is appropriate. In the remainder of this thesis, $\bar{\rho}, \bar{p}, \bar{T}, \mathbf{v}, \mathbf{b}$ and $r_{e}$ are taken to be functions of the angular frequency $\omega$.

\subsubsection{Effects of an imposed steady state solution}

If the nonlinear terms in the governing equations are not removed prior to Fourier transformation, convolution terms arise in the equations for the frequency domain, making it impossible to solve the system per frequency. However, an order of magnitude analysis of the convolution terms reveals that the governing linearized equations can be modified to account for large ${ }^{4}$ spatial gradients of the different variables describing the time invariant state of the fluid, still allowing to solve the system per frequency.

\footnotetext{
${ }^{4}$ The steady state 'perturbations' may be larger than the acoustic perturbation and may involve significant nonlinear terms.
} 
First, the effect of a steady velocity field on the convective terms is considered. In section 2.3.1 it is assumed that variation of a variable due to motion of the fluid is small compared to all other variations. If this restriction is dropped for the null frequency, the effects of a steady velocity field of arbitrary magnitude can be included. The velocity can be written as the sum of a steady (zero frequency) field $\mathbf{v}_{0}$ and unsteady field $\overline{\mathbf{v}}$

$$
\mathbf{v}=\mathbf{v}_{0}+\overline{\mathbf{v}}
$$

The convolution of the resulting convective terms in equations (2.1) will yield additional terms compared to the linearized set in equations (2.16) that account for the convection of mass, momentum and energy due to the steady state velocity field. Under the aforementioned assumption that the variation of a variable due to motion of the fluid is small compared to all other variations except at the null frequency, the influence of the acoustic disturbances on the steady velocity field is negligible, i.e., the steady velocity field can be determined independently. Subsequently, it can be used to account for convection of mass, momentum and energy due to the steady velocity field in the equations describing the acoustic (unsteady) field. The LNSF equations in the frequency domain for a steady velocity field including the convective terms are

$$
\begin{aligned}
i \omega \bar{\rho}+\mathbf{v}_{0} \cdot \nabla \rho+\rho_{0} \nabla \cdot \overline{\mathbf{v}} & =0 \\
\rho_{0}\left(i \omega \overline{\mathbf{v}}+\mathbf{v}_{0} \cdot \nabla \overline{\mathbf{v}}+\overline{\mathbf{v}} \cdot \nabla \mathbf{v}_{0}\right) & =-\nabla \bar{p}+(\lambda+\mu) \nabla(\nabla \cdot \overline{\mathbf{v}})+\mu \Delta \overline{\mathbf{v}}+\rho_{0} \mathbf{b} \\
\rho_{0}\left(i \omega C_{p} \bar{T}+\mathbf{v}_{0} \cdot \nabla \bar{T}\right) & =i \omega \bar{p}+\mathbf{v}_{0} \cdot \nabla p+\kappa \Delta \bar{T}+\rho_{0} r_{e} \\
\frac{\bar{p}}{p_{0}} & =\frac{\bar{\rho}}{\rho_{0}}+\frac{\bar{T}}{T_{0}}
\end{aligned}
$$

Casting these equations in a weak form to obtain an FE description as described in chapter 5 can be seen as an extension of the work of Peat [57]. He adopted a similar approach to obtain a finite element description of inviscid adiabatic acoustics wave propagation including the effects of a steady inviscid mean flow of low Mach number. The approach presented in section 3.4 using equations (2.20) can be seen as a generalization of the work of Astley and Cummings [39]. They presented a waveguide description of viscothermal wave propagation in tubes subjected to a steady mean flow that was based on a reduced (LRF-like) form of the full NSF equations. As a result of the simplifications made by Astley and Cummings, higher order modes are not predicted correctly. Using the full set of equations yields an FE model that also yields valid higher order modes.

In a similar way, the governing linearized equations can by modified to account for a steady density, pressure or temperature field that varies with the spatial coordinates. The effects of a combined axial mean flow and temperature gra- 
dient on the wave propagation in a cylindrical duct was presented independently by Peat and Kirbya [58] and Dokumaci [59]. The appropriate assumptions for the acoustic (unsteady) density, pressure and temperature is that variations must remain small compared to the local steady state values. Since the steady state values for density, pressure and temperature can now vary significantly throughout the medium, the different material parameters may become dependent on the spatial coordinates, resulting in additional terms in the linearized model. In [58] these additional terms are taken into account and it is shown that the influence of steady state temperature gradients on the coefficient of viscosity should not be neglected.

\subsection{Reduced models}

In some cases, additional assumptions can be made that can reduce the full set of LNSF equations to other canonical fluid dynamics models. In this section, three reduced models that are of importance in this thesis are given. The models under consideration describe linear elasticity, Stokes flow, and adiabatic inviscid wave propagation which is usually referred to as acoustic wave propagation.

\subsubsection{Inviscid adiabatic wave propagation}

Inviscid adiabatic/acoustic wave propagation is usually modeled with a simplified form of the equations in (2.1). To arrive at this simplified form, the following assumptions are introduced: viscous forces are assumed to be small with respect to inertial forces, i.e., the flow is considered to be inviscid. Changes in internal energy caused by thermal conduction are assumed to be small with respect to changes in internal energy due to expansion, i.e., the process is considered to be adiabatic ${ }^{5}$. In addition, it is assumed that there are no body forces present and the system is not subjected to volumetric heating. These assumptions can be expressed mathematically as

$$
\mu=0, \quad \lambda=0, \quad \kappa=0, \quad \mathbf{b}=0, \quad r_{e}=0 \quad(2.21 \mathrm{a}, \mathrm{b}, \mathrm{c}, \mathrm{d}, \mathrm{e})
$$

Under these assumptions, the equations are known as the Euler equations and describe reversible adiabatic processes only (such processes are also isentropic). The assumption of inviscid adiabatic behavior is only valid for free waves. If the thickness of the viscous and/or thermal boundary layer is not small compared to the

\footnotetext{
${ }^{5}$ Assuming the opposite (a relatively large heat flow due to conduction) results in an isothermal idealization of sound propagation. If for instance air or water is considered, neither is exactly true. However, for standard air and water conditions the adiabatic assumption is appropriate for frequencies considerably smaller than $10^{9} \mathrm{~Hz}$ and $2 \cdot 10^{12} \mathrm{~Hz}$, respectively. The adiabatic assumption is better for lower frequencies [28].
} 
dimension of the fluid bulk, viscous and/or thermal effects can contribute considerably to the behavior of the fluid. In other words, a viscothermal model is appropriate for wave propagation in fluid-filled geometries involving features of comparable size to the viscous or thermal boundary layers.

After linearization as described in section 2.4 the equations of conservation of mass and momentum are of the form of equation (2.15a) and (2.15b), respectively, without the terms involving viscosity and body forces. In order to eliminate density from this system, the thermal equation of state (2.7) and the energy equation (2.12) (with $\kappa=0$ and $r_{e}=0$ ) are combined, yielding the relation

$$
\frac{D p}{D t}=\gamma R_{0} T \frac{D \rho}{D t}=c^{2} \frac{D \rho}{D t}
$$

with $\gamma$ the ratio of specific heats defined as $\gamma=C_{p} / C_{V}$, and $c$ the (adiabatic) speed of sound, defined for ideal gases as $c=\sqrt{\gamma R_{0} T}$. Combining the linearized form of equation (2.22) with the linear equation for conservation of mass differentiated with respect to time and the divergence of the linear equation for conservation of momentum yields the so-called wave equation. After transformation to the frequency domain as described in 2.4 the so-called Helmholtz equation is obtained.

$$
\Delta \bar{p}+k^{2} \bar{p}=0
$$

where $k$ the wave number defined as $k=\omega / c_{0}$ with $c_{0}$ the adiabatic speed of sound at temperature $T_{0}$. An expression for velocity in terms of pressure is obtained by rewriting equation (2.16b) as

$$
\mathbf{v}=\frac{i}{\omega \rho_{0}} \nabla \bar{p}
$$

\subsubsection{Linear elasticity}

For small frequencies, the term representing contributions due to inertia can be neglected in the LNSF equations. In that case, the form of the momentum equation for the LNSF equations is equivalent to that of the equation for static deformation of linear elastic materials. In addition, for a large frequency range, (an approximation of) the stress tensor for the LNSF equations is analogous to the stress tensor for linear elastic materials which can be denoted as

$$
\sigma=\frac{1}{2} K \nabla \cdot \mathbf{u}+G\left(\nabla \mathbf{u}+(\nabla \mathbf{u})^{T}-\frac{1}{3} \nabla \cdot \mathbf{u}\right)
$$

where $\mathbf{u}$ is the displacement, and $K$ and $G$ are the bulk and shear modulus respectively. An equivalent notation using Lamé's first constant, which is defined as $\lambda_{L}=K-2 / 3 G$, reads

$$
\sigma=\frac{1}{2} \lambda_{L} \nabla \cdot \mathbf{u}+G\left(\nabla \mathbf{u}+(\nabla \mathbf{u})^{T}\right)
$$




\section{Similarity with viscothermal wave propagation}

Outside the region that is significantly affected by viscous and thermal boundary layers, wave propagation in fluids can often be adequately described by 'classical' acoustic wave propagation. It was already mentioned above that, for many fluids, acoustic wave propagation is an adiabatic process by approximation at most frequencies. This implies that outside the boundary layers, the energy equation and the thermal equation of state can be approximated by the relation between the gradient of pressure and density in equation (2.22). Note that if $c$ is corrected for the thermal effects of the boundary, equation (2.22) also offers a good approximation of the relation between pressure and density inside the thermal boundary layer. For instance, for isothermal boundary conditions, $c$ will vary from the adiabatic speed of sound outside the thermal boundary layer to the isothermal speed of sound at the boundary.

Substitution of this relation in the equation for conservation of mass and subsequently substituting the result in the expression for the stress tensor in (2.2) yields - after neglecting the term involving bulk viscosity:

$$
\sigma=-i \frac{c^{2} \rho_{0}}{\omega} \nabla \cdot \mathbf{v}+\mu\left(\nabla \mathbf{v}+(\nabla \mathbf{v})^{T}\right)
$$

Even though the variables differ, and the constants involving the various material parameters and frequency are different, the form of the above equation and equation (2.26) is identical. This resemblance is important in understanding why fluids in which wave propagation occurs seem to behave as nearly incompressible materials for a large frequency range.

\section{Nearly incompressible behavior}

Linear elastic materials described by equation (2.25) or (2.26) become nearly incompressible under the conditions $K \gg G$ or $\lambda_{L} \gg G$. These conditions imply that deforming the bulk of the material takes much more effort than shearing it. In the limiting case that $\lambda_{L}$ or $K$ becomes infinite, the material resists bulk deformation completely, and the material is said to be incompressible. For the LNSF equations, the analogue condition for near incompressibility is

$$
\frac{c^{2} \rho_{0}}{\omega} \gg \mu
$$

i.e., if this condition is satisfied, the fluid will behave as a nearly incompressible fluid when it undergoes wave propagation. To be precise, the fluid resists changes in deformation, not the deformation itself ${ }^{6}$. It is clear that the condition will be

\footnotetext{
${ }^{6}$ The definition of a fluid entails that it does not resist static deformation.
} 
satisfied for smaller values ${ }^{7}$ of $\omega$. To get an indication of the frequency range where nearly incompressible behavior is to be expected, the inequality is rewritten as

$$
\omega \ll \frac{c^{2} \rho_{0}}{\mu}
$$

This implies that for wave propagation in air at standard atmospheric conditions, the behavior of the fluid will be nearly incompressible if the frequency $f \ll 1.2$ $\mathrm{GHz}$ in case of adiabatic wave propagation, and $f \ll 870 \mathrm{MHz}$ in case of isothermal wave propagation. This implies that in most practical cases, the behavior of fluid undergoing wave propagation can be called nearly incompressible. This is an important notion, since (near) incompressibility can have a negative influence on the numerical properties of algebraic systems of equations that are obtained by employing FE methods to solve the differential equations describing the problem. Based on the similarities between the momentum equation for the LNSF equation and the equation for static deformation of linear elastic materials, similar numerical problems are expected when FE methods are used to solve the LNSF equations. These issues will be addressed thoroughly in chapter 5 .

\subsubsection{Stokes flow}

It is important to realize that the case of a fully incompressible linear elastic material leads to the same equations as the Stokes equations. Therefore, it should come as no surprise that the LNSF equations, which closely resemble the equations for linear elastic materials, reduce to the Stokes equations as $\omega$ vanishes.

As mentioned above, for very low frequencies, inertial forces become small compared to viscous forces and can be neglected (this corresponds with a low Reynolds number). In addition, wave propagation becomes a nearly incompressible process, and thermal effects can be represented by assuming a linear relation between pressure and density, thus eliminating temperature as a variable. Moreover, in the limit of zero frequency, the full set of LNSF equations in (2.16) reduces to two decoupled sets of equations: A Poisson equation for temperature, and the Stokes equations for velocity and pressure. The Stokes equation (including body forces) is given by

$$
\begin{aligned}
\nabla \cdot \mathbf{v} & =0 \\
\mu \Delta \mathbf{v} & =\nabla p-\rho_{0} \mathbf{b}
\end{aligned}
$$

\footnotetext{
${ }^{7}$ Note that a bulk deformation at zero frequency implies a permanent state of expanding or delating. In this limit, the LNSF description of the fluid predicts full incompressibility, which would require an infinite amount of effort to achieve bulk deformation, thus preventing such nonphysical behavior.
} 
Numerical methods to solve this set of equations have been studied intensively in the literature (see for instance [60,61,62] for an overview). Numerical issues similar to those observed for FE methods for the equations of linear elasticity are also encountered for FE methods for the Stokes equations. The methods that are used to assess the numerical stability of the FE models for the full set of equations proposed in chapter 5 are an extension of the methods that assess the stability of FE models for the equations describing Stokes flow and linear elastic materials presented in [61].

\subsection{Dimensionless equations and groups}

The system defined by equations (2.16) can be written in dimensionless form by introducing dimensionless variables. Subsequently, rewriting the equations using dimensionless operators allows a description in terms of five dimensionless groups which characterize viscothermal wave propagation. This dimensionless form allows an order of magnitude analysis of the different terms in the equation that leads to the LRF approximation presented in chapter 4. Using dimensionless variables provides a proper scaling of the different equations prior to the FE discretization scheme proposed in chapter 5 .

\subsubsection{Dimensionless equations}

To arrive at the dimensionless form of equations (2.16), the density, velocity, temperature and pressure are written in dimensionless form using

$$
\tilde{\rho}=\frac{\bar{\rho}}{\rho_{0}}, \quad \tilde{p}=\frac{\bar{p}}{p_{0}}, \quad \tilde{T}=\frac{\bar{T}}{T_{0}}, \quad \tilde{\mathbf{v}}=\frac{\mathbf{v}}{c_{0}} \quad \text { (2.31a,b,c,d) }
$$

Subsequently, the continuity equation is divided by $\omega \rho_{0}$, the momentum equation is divided by $\omega \rho_{0} c_{0}$, the energy equation is divided by $\omega p_{0} C_{p} / R_{0}$. Omitting body forces and volumetric energy supply yields the following dimensionless equations for viscothermal wave propagation

$$
\begin{aligned}
i \tilde{\rho}+\frac{1}{k} \nabla \cdot \tilde{\mathbf{v}} & =0 \\
i \tilde{\mathbf{v}} & =-\frac{1}{\gamma k} \nabla \tilde{p}+\frac{1}{s^{2}}(\xi+1) \nabla(\nabla \cdot \tilde{\mathbf{v}})+\frac{1}{s^{2}} \Delta \tilde{\mathbf{v}} \\
i \tilde{T} & =i\left[\frac{\gamma-1}{\gamma}\right] \tilde{p}+\frac{1}{s_{t}^{2}} \Delta \tilde{T} \\
\tilde{p} & =\tilde{\rho}+\tilde{T}
\end{aligned}
$$


with $k$ the wave number, $s$ the shear wave number, $s_{t}$ the thermal wave number, $\gamma$ the ratio of specific heats and $\xi$ the viscosity ratio defined, respectively, as

$$
k=\frac{\omega}{c_{0}}, \quad s=\sqrt{\frac{\rho_{0} \omega}{\mu}}, \quad s_{t}=\sqrt{\frac{\rho_{0} \omega C_{p}}{\kappa}}, \quad \gamma=\frac{C_{p}}{C_{v}}, \quad \xi=\frac{\lambda}{\mu} \quad(2.33 \mathrm{a}, \mathrm{b}, \mathrm{c}, \mathrm{d}, \mathrm{e})
$$

Note that while equations (2.32) are in dimensionless form (as are the ratio of specific heat and the viscosity ratio), the above wave numbers are still dimensional.

\subsubsection{Dimensionless wave numbers}

When doing an order of magnitude analysis or a parameter study, it is often convenient to switch to dimensionless wave numbers. This is achieved by scaling the wave lengths corresponding to the different wave numbers with an appropriate length scale. To this aim, the so-called characteristic length $l$ is introduced. This length scale characterizes waveguide geometry in directions perpendicular to the directions that allow wave propagation. When wave propagation in waveguides is considered, $l$ characterizes the length scale of the cross section of a waveguide. The appropriate characteristic length for different example geometries are defined in section 2.6.3. The resulting dimensionless wave numbers are defined as

$$
\tilde{k}=l k=\frac{\omega l}{c_{0}}, \quad \tilde{s}=l s=l \sqrt{\frac{\rho_{0} \omega}{\mu}}, \quad \tilde{s}_{t}=l s_{t}=l \sqrt{\frac{\rho_{0} \omega C_{p}}{\kappa}} \quad(2.34 \mathrm{a}, \mathrm{b}, \mathrm{c})
$$

where the indicates that the wave number is in its dimensionless form. If the dimensional wave numbers in equation (2.32) are replaced by their dimensionless counterparts, the gradient and Laplace operators have to be replaced by the following dimensionless operators

$$
\tilde{\nabla}=l \nabla, \quad \tilde{\Delta}=l^{2} \Delta
$$

In the work of Beltman, the wave numbers and operators are all used in their dimensionless form. He also introduces dimensionless coordinates requiring the gradient and Laplace operators to be redefined for different waveguide geometries. The notation in dimensionless groups, dimensionless operators and dimensionless coordinates offers useful insights into the order of magnitude of the different terms if the scaling of coordinates is applied properly. However, the use of dimensionless operators and dimensionless coordinates is somewhat cumbersome in most numerical settings and not even always meaningful. For instance, if the FE approximation of the equations presented in chapter 5 is regarded, a meaningful 
scaling of the different coordinates is out of reach for arbitrary geometries. Therefore equation (2.32) will only be used with dimensional wave numbers, operators and coordinates in this thesis. However, the different parameter studies that are presented make use of dimensionless wave numbers, which can easily be derived from their dimensional counterparts with equations (2.34). For convenience, the viscous and thermal dimensionless wave numbers are addressed as viscous and thermal wave numbers, respectively, throughout the rest of this thesis.

\subsubsection{Characteristic length}

In this work the characteristic length is taken to equal twice the so-called hydraulic radius, $r_{h}$

$$
l=2 r_{h}=\frac{2 S}{P}
$$

with $S$ and $P$ the surface area and length of the perimeter of the waveguide cross section, respectively. The characteristic length is given for a few cross sections of simple geometric shape in figure 2.1.
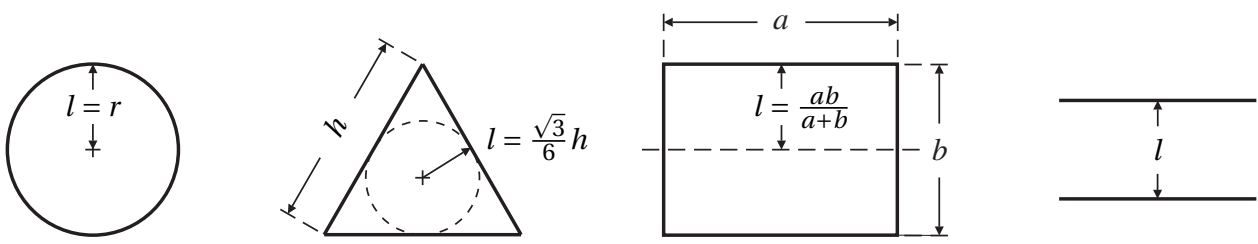

Figure 2.1: Characteristic length, $l$, for a number of simple geometric cross sectional shapes.

Note that in many publications that involve the definition of a shear wave number (for instance [10, 19,36]), the chosen (or implied) characteristic length for a layer/slit is half its width, while the characteristic length of the other geometries is as defined here. This 'inconsistent' choice of the characteristic length hinders a straightforward comparison of the results for different waveguide cross sections with that of a layer/slit.

\subsubsection{Dimensionless groups}

Viscothermal wave propagation can be characterized by the three dimensionless wave numbers and the ratio of heats and viscosities introduced above. The impact of these five dimensionless groups on the behavior of an ideal gas is described below. 
The dimensionless acoustic wave number, $\tilde{k}$, is the ratio between the characteristic length of the geometry and the acoustic wavelength of a free wave. For $\tilde{k} \ll 1$ all propagating acoustic waves in straight tubular waveguides will be planar by approximation.

The shear wave number, $\tilde{s}$, is the square root of an unsteady Reynolds number; it is a measure for both the ratio between inertial and viscous forces and the ratio between the characteristic length and the viscous boundary layer thickness. If wave propagation in waveguides is considered, a high shear wave number $(\tilde{s} \gg 1)$ indicates that the viscous boundary layer is small compared to the length scales of a waveguide cross section, i.e., inertial forces dominate. For low shear wave numbers $(\tilde{s} \ll 1)$ the boundary layer is large, and viscous effects are dominant. The quotient $\tilde{k} / \tilde{s}$, which is known as Stokes number [63], is a measure for the ratio between the viscous boundary layer thickness and the wavelength of a free wave

$$
\frac{\tilde{k}}{\tilde{s}}=\frac{k}{s}=\sqrt{\frac{\omega \mu}{\rho_{0} c_{0}^{2}}}
$$

Notice that this ratio is independent of the characteristic length, so $\tilde{k} / \tilde{s}$ and $k / s$ are interchangeble. For small values $(\tilde{k} / \tilde{s} \ll 1)$ the free wavelength is large compared to the viscous boundary layer thickness. For most gases and liquids, the ratio is small in the frequency and dimensional ranges that do not conflict with the assumptions of continuum mechanics [36]. For free waves, $\tilde{k} / \tilde{s} \ll 1$ leads to the assumption of inviscid wave propagation.

The thermal wave number, $\tilde{s}_{t}$, is the square root of an unsteady Péclet number for thermal diffusion and can be written as the product of the shear wave number and the square root of the Prandtl ${ }^{8}$ number $\tilde{s}_{t}=\tilde{s} \sqrt{\operatorname{Pr}}$. It is a measure for the ratio between heat energy that is stored and heat energy that is conducted and also quantifies the ratio between the characteristic length and the thermal boundary layer thickness. For $\operatorname{Pr} \approx 1$ the viscous and thermal boundary layer are of the same order. This is the case for many gases including air. For liquids, values of the Prandtl number range from very small values (e.g., $0.01<\operatorname{Pr}<0.05$ for liquid metals) to very high values (e.g., $100<\operatorname{Pr}<40.000$ for engine oil). Analogous to the shear wave number, $\tilde{k} / \tilde{s}_{t}$ is a measure for the ratio between the thermal boundary layer thickness and the wave length of a free wave

$$
\frac{\tilde{k}}{\tilde{s}_{t}}=\frac{k}{s_{t}}=\sqrt{\frac{\omega \kappa}{\rho_{0} c_{0}^{2} C_{p}}}
$$

Notice that the ratio is independent of the characteristic length. For small values of $\tilde{k} / \tilde{s}_{t}$ the free wavelength is large compared to the thermal boundary layer thick-

\footnotetext{
${ }^{8}$ The Prandtl number is defined as $\operatorname{Pr}=\frac{\mu C_{p}}{\kappa}$.
} 
ness. For free waves, this leads to the assumption of adiabatic wave propagation. The adiabatic index, $\gamma$, is defined as the ratio of specific heats. When an acoustic wave passes through a medium, the product of pressure and volume for a constant mass is not constant; the acoustic wave performs positive and negative work on the medium and as a result adds and retracts heat from it. The adiabatic index reflects the ratio between heat changes due to variations in density (proportional to $C_{p}$ ) and heat changes due to variations in pressure (proportional to $-C_{v}$ ). For many ideal gas mixtures (including air) under standard atmospheric conditions $\gamma \sim 1.4$.

The viscosity ratio, $\xi$, is defined as the ratio between the second and first coefficient of viscosity ( $\lambda$ and $\mu$, respectively). The second coefficient of viscosity can also be related to the bulk viscosity coefficient $\eta$ and the first coefficient of viscosity by equation (A.11). The value of $\mu$ determines the resistance of a material against the rate of shear deformation, while the value of $\eta$ is related to the resistance of a material against the rate of contraction/dilatation. For $\eta=0$, the resistance a material has against bulk deformation is not dependent on the rate at which the deformation takes place. In the audible frequency range, $\eta=0$ often yields satisfying results for air if the maximum length scale of the domain under consideration is in the order of a few wavelengths. In this thesis, $\eta=0$ is assumed, thus $\xi=-2 / 3$ (this is known as Stokes hypothesis). For a discussion on the value of $\lambda$ and $\eta$ in the literature see section A.4.2.

Notice that $\gamma$, Pr and $\xi$ are dependent on material properties only, while $\tilde{s}, \tilde{s}_{t}$ and $\tilde{k}$ are also dependent on the characteristic length and frequency.

\subsection{Boundary conditions}

In order to find a solution to physical problems described by the system of equations in (2.16) or (2.32) the equations need to be accompanied by appropriate boundary conditions. In many cases, the velocity and temperature boundary conditions prescribed at the surfaces of the waveguides can be expressed as a form of an (inhomogeneous) Robin boundary condition;

$$
\left(\alpha_{\psi}+\beta_{\psi} \frac{\partial}{\partial n}\right) \psi=f_{\psi}, \quad \text { where } \quad \psi=\left\{v_{1}, v_{2}, v_{3}, T\right\}
$$

with $\frac{\partial}{\partial n}$ the derivative in the direction normal to the waveguide surface, $\alpha_{\psi}, \beta_{\psi}$ and $f_{\psi}$ functions of the different coordinates that describe the boundary conditions for the different velocity components $v_{1}, v_{2}$ and $v_{3}$ and the temperature T. The types of boundary conditions that are enforced for different values of these coefficients are listed in table 2.1. The physical interpretation of the different types of boundary conditions is discussed below. 


\begin{tabular}{cccc}
\hline & $\alpha_{j} \neq 0, \beta_{j}=0$ & $\alpha_{j}=0, \beta_{j} \neq 0$ & $\alpha_{j} \neq 0, \beta_{j} \neq 0$ \\
\hline \multirow{2}{*}{$f_{j}=0$} & Homogeneous & Homogeneous & Homogeneous \\
& Dirichlet & Neumann & Robin (Impedance) \\
\multirow{2}{*}{$f_{j} \neq 0$} & Inhomogeneous & Inhomogeneous & Inhomogeneous \\
& Dirichlet & Neumann & Robin (Jump) \\
\hline
\end{tabular}

Table 2.1: Boundary conditions, with $j=\left\{v_{1}, v_{2}, v_{3}, T\right\}$.

The homogeneous part of the boundary conditions is represented by the linear operator $L$

$$
L=\left(\alpha+\beta \frac{\partial}{\partial n}\right)
$$

Applying a boundary condition of the form in equation (2.39) to a function $\psi$ at boundary $\mathbf{x}=\partial S$ can now conveniently be denoted as

$$
\left.L \psi\right|_{\mathbf{x}=\partial S}=f_{\psi}
$$

When boundary equations are applied to a system of equations, $L$ can be written as a matrix where each of its elements are linear operators. For instance, consider a system with three velocity variables $v_{1}, v_{2}$ and $v_{3}$ and one temperature variable $T$, with a boundary perpendicular to the direction of $v_{1}$ at $\mathbf{x}=\partial S$. Prescribing a normal velocity $v_{b}$, zero traction in both tangential directions, and a heat flux $q$ at the boundary can be conveniently denoted as

$$
\left.[L]\left\{\begin{array}{c}
v_{1} \\
v_{2} \\
v_{3} \\
T
\end{array}\right\}\right|_{\mathbf{x}=\partial S}=\left\{\begin{array}{c}
v_{b} \\
0 \\
0 \\
q
\end{array}\right\}, \quad \text { with } \quad L=\left[\begin{array}{cccc}
1 & 0 & 0 & 0 \\
\frac{\partial}{\partial x_{2}} & \frac{\partial}{\partial x_{1}} & 0 & 0 \\
\frac{\partial}{\partial x_{3}} & 0 & \frac{\partial}{\partial x_{1}} & 0 \\
0 & 0 & 0 & 1
\end{array}\right]
$$

\subsubsection{Velocity boundary conditions}

Assume inhomogeneous Dirichlet boundary conditions for the different velocity components with $f_{v_{i}}$ the velocity of the waveguide surface in each coordinate direction. This corresponds with continuity of velocity at the fluid-waveguide interface, i.e., assuming a no-slip condition and matching the normal velocities of the waveguide and fluid. In this way, a full acousto-elastic coupling is realized. In many cases, the structure that is connected to the fluid is assumed to be rigid in tangential direction, which leads to a constant velocity $f_{v_{i}}$ over the waveguide surface in tangential directions. LRF models including tangential motion of rigid 
waveguides were presented by Hannink [20]. If there is no need to model structural motion in a certain direction, the velocity of the fluid is taken to equal zero at the fluid wall interface, i.e., $f_{v_{i}}=0$ which corresponds with a homogeneous Dirichlet boundary condition.

For rarefied gases, the continuum hypothesis is (slightly) violated at the fluid wall interface. In such cases the no-slip assumption is not valid. In the literature, models for rarefied gases are presented that include a correction for a jump in tangential velocity at the wall to circumvent this issue $[64,65,66]$. A simple model results if the velocity jump (defined as the velocity at the wall minus the velocity of the waveguide surface) is assumed to be proportional to the normal derivative of the velocity near the wall. In that case $\alpha_{v_{i}}=1, f_{v_{i}}$ equals the tangential velocity of the waveguide and $\beta_{v_{i}}$ is a constant related to the tangential momentum accommodation coefficient ${ }^{9}$.

A second alternative to the no-slip assumption, is assuming zero tangential force at the waveguide surfaces. This is achieved by taking the gradients of the tangential velocity normal to the wall zero, i.e., a homogeneous Neumann boundary condition is applied. As a result, the boundary does not induce viscous effects. Assuming zero tangential force (and zero normal velocity) is appropriate for symmetric boundary conditions (see [20]).

\subsubsection{Temperature boundary conditions}

Assuming a Dirichlet boundary condition for temperature with $f_{T}$ equal to the temperature of the waveguide, corresponds with continuity of temperature over the fluid wall interface. In this way, a full coupling with the thermal model of the waveguide surfaces can be established. In many cases it suffices to assume isothermal conditions at the gas wall interface (i.e., $f_{T}=0$ ) and a thermal model of the waveguide surface is not required. This approximation is appropriate when the so-called thermal inertia ${ }^{10}$ of the wall, defined as the product of volumetric heat capacity and thermal conductivity, exceeds the thermal inertia of the fluid significantly.

As mentioned above, the continuum hypothesis is (slightly) violated at the fluid gas interface for rarefied gases. In such cases, models that include a correction for a jump in temperature are more appropriate than assuming continuity of temperature $[64,65,66]$. Analogous to a velocity jump, the temperature jump is assumed to be proportional to the normal derivative of the temperature near the

\footnotetext{
${ }^{9}$ See [65] for appropriate expressions for the tangential momentum accommodation coefficient and thermal accommodation coefficient.

${ }^{10}$ Thermal inertia is the tendency of a material to resist changes in temperature.
} 
wall, i.e., $\alpha_{T}=1, f_{T}$ equals the temperature of the waveguide surface and $\beta_{T}$ is a constant related to the thermal accommodation coefficient ${ }^{9}$.

An alternative to the assumption of isothermal walls, is assuming an adiabatic process at the waveguide surface. This is achieved by taking the heat flux normal to the wall to be zero, i.e., a Neumann boundary condition is applied for temperature and $\alpha_{T}=0, \beta_{T}=1$ and $f_{T}=0$. As a result, wave propagation is not thermally influenced by the boundary. Prescribing a zero heat flux is appropriate in case a symmetry boundary condition is modeled (see [20]).

\subsection{Solution techniques}

In section 2.1.1 a historic overview was presented of the different viscothermal acoustic models that were developed based on the LNSF equations given in equation 2.16. The merits and limitations of the five prominent models are briefly discussed in this section. The different models that are evaluated are characterized by two main properties: Firstly, they are either based on the full set of equations or on a simplified (or reduced) form. Secondly, they involve either analytic solution techniques or only numerical solution techniques. A schematic overview of the different models according to these criteria is given in figure 2.2. A short description of the geometries for which each model is suited is included in the overview. The relative computational efficiency of each model for results of similar accuracy is indicated for a virtual test case. The case under consideration is a geometry consisting of coupled waveguides for arbitrary parameter settings. In addition, the best relative accuracy for a worst-case scenario is given. In this case, the virtual test case is a geometry consisting of coupled waveguides evaluated for parameter settings that are disadvantageous for accuracy for the model under consideration.

\subsection{1 (A) BEM/FEM boundary layer approximation}

If the viscous and thermal boundary layers remain small compared to the dimensions of the fluid domain, a boundary layer approximation can be used to describe viscothermal effects (see [28, 32]). Examples are given by Bossart et al. [47] and Dokumaci [45]. In the work of Bossart et al., the boundary layer approximation is used to give an expression for the normal acoustic velocity in terms of acoustic pressure at the point of transition from boundary layer to bulk under the assumptions of isothermal, stationary boundaries. This expression (which depends on the local pressure gradient) is rewritten in the form of an impedance boundary condition. This approach allows the use of (standard) FE and BE models for inviscid adiabatic wave propagation, while the viscothermal effects are represented by an 


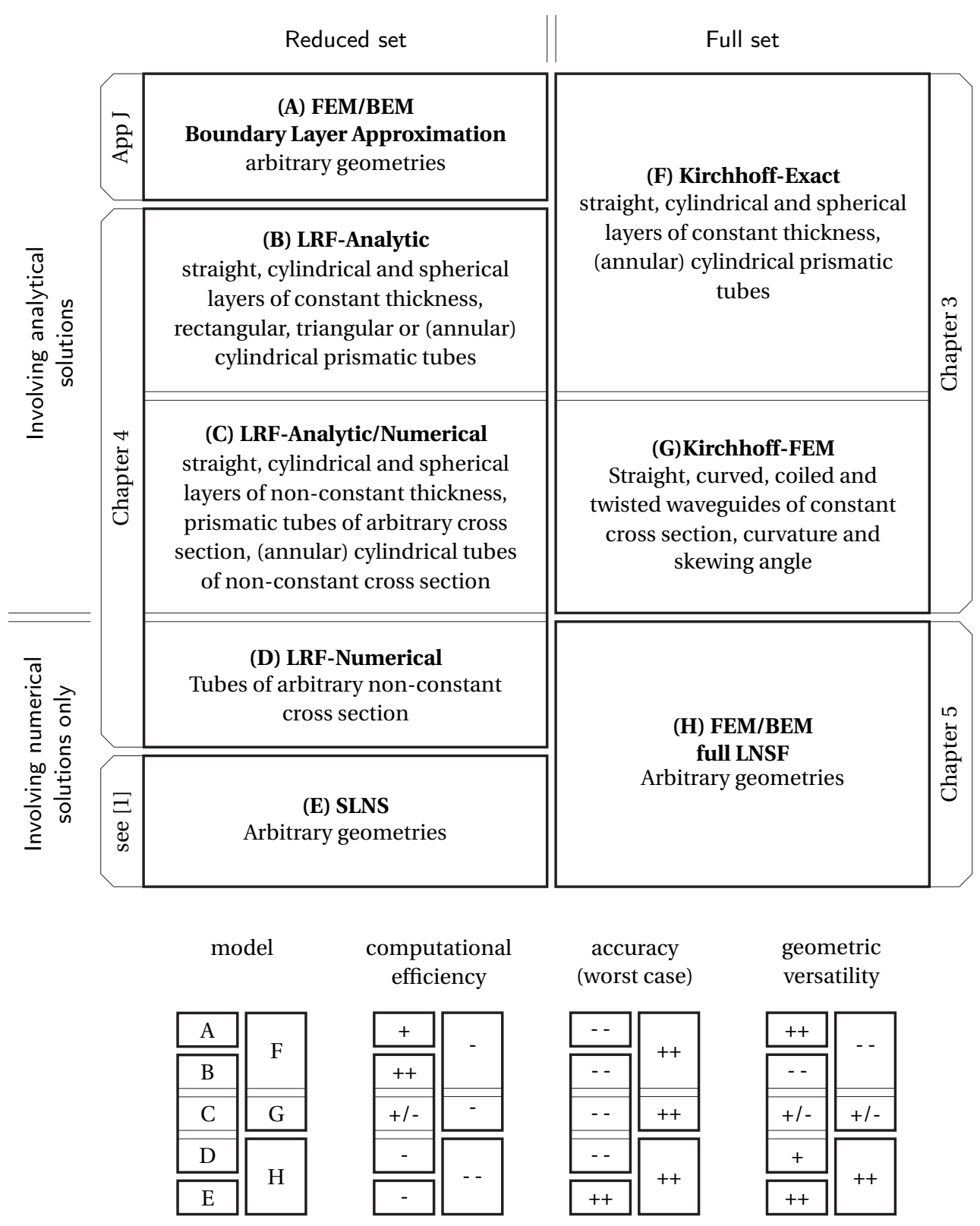

Figure 2.2: Overview of five prominent viscothermal acoustic models and geometries to which they can be applied. The relative computational efficiency for results of similar accuracy, the best accuracy obtained for coupled waveguides and disadvantageous parameters, and the geometric versatility of different viscothermal acoustic models are indicated. 
admittance-like boundary condition.

In the work of Dokumaci, the terms involving the normal heat flux and the vorticity vector (curl of the velocity vector) are written in terms of pressure, temperature and velocity on the boundary by applying boundary layer theory. The resulting equations allow a straightforward application of temperature and tangential velocity boundary conditions, yielding an integral equation in terms of pressure and normal velocity, which can be discretized with standard BE techniques.

Limitations and efficiency The FEM/BEM boundary layer approximation is far more efficient compared to solving the full LNSF equations with FEM/BEM. In the case of FEM, the method involves solving a system depending on a single variable instead of solving a system with three to five coupled variables. In addition, there is no need to refine the mesh near boundaries to accurately describe the boundary layer, since all boundary layer effects are accounted for in the boundary conditions. This is an additional computational advantage of using the FEM boundary layer approximation compared to solving the full set of equations using FEM. In the case of the BEM boundary layer approximation, two influence matrices are constructed and one is inverted, while solving the full LNSF equations with FEM/BEM involves constructing six influence matrices and inverting four.

The different assumptions and approximations inherent to these models can cause significant errors depending on the thermal and viscous boundary layer thickness. The assumptions necessary for boundary layer approximations only hold if the thermal and viscous wavelengths are small compared to the dimensions of the domain (the boundary layers must be well separated). In addition, it is required that the boundaries must be locally flat, or more specifically; the boundary layer thicknesses must remain small compared to the radius of curvature of the boundary.

In the work of Bossart et al. [47], additional assumptions are made. The impedance is calculated at the locations where the transition between boundary layer and bulk occurs. However, since these locations depend on the boundary layer thickness and thus frequency, they do not automatically coincide with element boundaries of the FE mesh in use. Therefore, the calculated impedances are prescribed at the domain boundary instead. As a result, the model yields accurate results only if the viscous and thermal boundary layers are significantly smaller than the (local) dimensions of the domain. Note that the required difference between boundary layer thickness and domain dimension is larger than that required for the method presented by Dokumaci [45] (which only requires well separated boundaries). 


\subsection{2 (B) (C)(D) LRF models}

The most prominent reduced (analytical) models for viscothermal wave propagation were put in perspective by Tijdeman [35] and Beltman [36]. Their reviews of the different models showed that LRF models are highly accurate and most efficient to describe viscothermal wave propagation in isothermal, rigid waveguides of simple geometry structure for most fluids under standard atmospheric conditions. Depending on the geometry that is being modeled, LRF models involve pure analytic solutions, pure numerical solutions, or a mixture of both. The nature of the solution influences the computational efficiency of the LRF model compared to other models. The limitations and efficiency of LRF models is commented on below for different types of geometries. A general technique to derive LRF models for different geometries is presented in chapter 4 .

(B) LRF-Analytic models The use of analytical LRF models is limited to layers and tubes of simple geometric shape. In the literature (semi) analytical LRF models can be found for square, circular, annular or triangular prismatic tubes [20, 66, $67,68,69]$ and rectangular, circular and elliptical air layers [10, 22, 25, 36]. An LRF model for wave propagation in a curved air layer is given in section 4.2.2. The individual models require relatively little computational effort. More complex geometries can be modeled by coupling the analytical expressions for these simple geometries (see section 4.3). However, wave propagation in coupled models is not accurately described in the transition zones between sub models which can lead to significant errors. This is due to the fact that only the first acoustic (propagating) mode is represented, while contributions of higher order acoustic, thermal and viscous (evanescent) modes are not represented. These modes can also become important when applying boundary conditions at the end of short tubes and layers. In most cases, these so-called inlet effects, which are mainly due to omission of the higher order acoustic modes can be represented accurately by applying a length correction to the waveguide with the smallest cross sectional dimensions. Obtaining length corrections for a coupling between (multiple) arbitrary waveguides is not trivial and deriving suitable analytical expressions is only feasible in special cases. Expressions for length corrections are available for a limited number of waveguide geometries for the inviscid adiabatic case [70]. In chapter 6 it is demonstrated that these length corrections give satisfying results in the viscothermal case as well.

(C) LRF-Analytic/Numerical models Tube geometries with arbitrary cross sectional shape can be modeled by employing numerical techniques to obtain the temperature and velocity profiles over the waveguide cross section (see for in- 
stance [39]). The equation for pressure, describing behavior in propagation direction, can be solved analytically. In contrast, for straight layers of arbitrary shape the equations for pressure have to be solved numerically (for instance with FE [37] or Trefftz [21] method), while the equations describing the temperature and velocity profiles across the layer can be solved analytically. Naturally, obtaining the solution to the full set of equations in these ways requires more computational effort than solving a purely analytical LRF model. However, these techniques are still far more efficient than solving the full set of equations.

(D) LRF-Numerical models An LRF model of tubes and layers with non-constant cross section can also be derived (see section 4.2.3). Waveguide models for nonconstant cross sections lead to a differential equation for pressure with non-constant coefficients, adding to the complexity of the model. In these cases, an analytical solution of the equation for pressure is in general not available. For tubes with simple cross sections, and layer geometries of arbitrary shape, the added computational effort is low; the velocity and temperature profiles for the different cross sections/layer thicknesses are determined analytically, while the equation for pressure has to be solved numerically. However, for tubes of arbitrary, and non-constant cross section the velocity and temperature profiles have to be determined with numerical techniques for each cross section that is required in the (numerical) procedure used to solve the equation for pressure. These models also suffer from loss of accuracy due to geometric spreading if changes in cross sectional profiles are not sufficiently small compared to the acoustic wavelength. Note that there are only two type of models with a non-constant cross section that do not suffer from errors due to geometric spreading [71]. The first exception is the model for a tapered layer in cylindrical coordinates presented in section 4.2 .3 , the second exception is a model for a conical geometry (of constant flare) described in spherical or conical coordinates.

The problem of geometric spreading is closely related to that of coupling different LRF solutions. The inaccuracies result from the fact that the propagation directions described by the LRF model do not correspond (locally) with the true propagation directions. If changes in cross sectional profiles are small compared to the acoustic wavelength, inaccuracies due to geometrical spreading are small. In such cases, changes in cross section can often be represented accurately with a finite number of coupled waveguides of constant cross section. For more abrupt changes in waveguide cross sections the errors due to geometric spreading become significant (see section 6.7). 
LRF models for arbitrary geometries In the derivation of all LRF models, the directions of propagation are assumed to be known a priori. This makes the method highly appropriate for most waveguide geometries. However, for larger (open) volumes or volumes of more complex geometry, the directions of wave propagation are not always known a priori and a suitable LRF model might not be obtainable. In such cases, using an LRF-FE model (see section 2.8.3) or an FE/BE model based on boundary layer approximation (see section 2.8.1 and appendix J) might be more suitable.

\subsection{3 (E) SLNS models}

Recently, a very promising alternative to above-mentioned implementation of the boundary layer approximation for FEM has been proposed by Kampinga [1]. The underlying assumptions and solution strategy are similar to those used in deriving LRF models. First, viscous forces due to changes in bulk are neglected (which is known to be a reasonable assumption for $k / s \ll 1$ ). Now, both the momentum and energy equation are in the form of an inhomogeneous (vector) Helmholtz equation. Secondly, variations in pressure are assumed not to contribute to heat exchange, and viscous forces due to variation in the pressure gradient are assumed not to contribute to momentum exchange (even though the pressure and pressure gradient themselves are the driving force behind heat and momentum exchange, respectively). The right-hand side of the energy and momentum equation is substituted by a unit driving force, and the equations are solved independently for homogeneous boundary conditions. The resulting solutions describe the temperature and velocities for a unit pressure and unit pressure gradients, respectively. Note that if Cartesian coordinates are used, the vector Helmholtz equation for conservation of momentum yields three identical scalar equations. Identical boundary conditions are imposed on all equations ${ }^{12}$, so a single scalar equation has to be solved. The solutions for the velocities are adjusted to account for the fact that viscous forces do not occur for pressure gradients directed normal to the bound$\operatorname{ary}^{13}$.

Next, the temperature and corrected velocity solutions for a unit driving force are multiplied with the pressure and the pressure gradients to obtain an approximation of the temperature and velocity as a function of pressure and pressure gradient respectively. The approximations for velocity and temperature (in terms of the pressure gradient and pressure, respectively) that are thus obtained are in-

\footnotetext{
${ }^{12}$ Boundary conditions in tangential directions are assumed identical and are imposed on all equations.

${ }^{13}$ Velocities normal to the boundary are directly correlated to the pressure gradient in the direction of the boundary, just as for inviscid acoustics.
} 
serted into the continuity equation which yields a Helmholtz-like equation ${ }^{14}$. The coefficients found in the pressure and the Laplacian-like term account for thermal and viscous effects, respectively. The contributions of inhomogeneous boundary conditions serve as right-hand side (driving terms). Solving this third scalar equation yields the pressure field from which velocity and temperature can be reconstructed if necessary.

Limitations and efficiency Note that the LRF solutions involve similar coefficients accounting for viscothermal effects, and in many ways, SLNS models can be thought of as LRF models where all equations are solved with FEM. One of the advantages of this method is that only three independent scalar equations have to be solved (in the case of homogeneous traction or temperature boundary condition). This makes the method more efficient than solving the full discretized system (which involves solving a system of three to five coupled equations), and slightly less efficient than using the boundary layer approximation (which involves solving only a single scalar equation on a simpler mesh). The only additional approximation errors that are introduced are due to neglecting viscous forces due to compression/dilatation and variations in the pressure gradient, and neglecting transport of heat due to pressure variations (assumptions that are the cornerstone of 'classical' inviscid adiabatic acoustics). These errors are usually of no significance ${ }^{15}$ and can be reduced, if necessary, by adjusting the propagation constant to account for these effects in the bulk of the fluid when calculating the (acoustic) pressure (see for instance $[28,72]$ ). The SLNS models do not suffer from the limitations mentioned for the FEM/BEM boundary layer approximation described above and can be applied to arbitrary geometries. A drawback of the model is that it can only be used in combination with homogeneous boundary conditions for temperature, and velocities tangential to the boundary (normal velocities can be prescribed). Thermal excitation, or excitation due to a shearing motion of the boundary can thus not be modeled. These limitations also prevent a straightforward coupling between LRF-FEM models and thermal models of structures that share a boundary. It also prevents a full coupling between LRF-FEM models and vibrating structures that share a boundary, since tangential motion cannot be coupled.

\subsection{4 (F)(G) Kirchhoff models}

\footnotetext{
${ }^{14}$ Taking the divergence of the approximated velocity yields and extra term which is neglected. Note that the independent terms of the pressure Laplacian are multiplied with different coefficients.

${ }^{15}$ Errors due to neglecting heat conduction become significant in air and water well above the the audible frequency range $[28,72]$.
} 
(F) Kirchhoff-Exact An 'exact' solution to the full LNSF equations can be obtained by solving Kirchhoff's dispersion equation. Numerical solutions to the dispersion equation are presented by various authors [35, 40, 41, 42, 44]. Deriving the dispersion equation requires the analytical solution of a vector Helmholtz equation, which is available for six orthogonal coordinate systems only (see section 3.2 .3 or $[73,74])$. All geometries that can be modeled are bound by the coordinate surfaces ${ }^{16}$ of these systems, limiting the use of this method to simple geometric shapes (while other considerations concerning the boundary conditions, limit the number of suitable geometries even further). The method yields a countable infinite number of modes, both propagating and evanescent in nature. Higher order modes damp out progressively with increasing order.

(G) Kirchhoff-FEM For straight, curved, coiled and twisted waveguides having a constant (arbitrary) cross section, constant skewing angle, and constant boundary conditions, an efficient approximate solution to the full LNSF equations can be obtained. Under these conditions, the variables are separable in contributions depending on the $p d$ - and $c d$-coordinates only (see section 3.4). The solution depending on the $p d$-coordinate is assumed to be an exponential function (with an unknown propagation constant). Substituting this solution in the original equations yields a system with terms independent and dependent on the propagation constant and its square. Subsequently, FEM techniques can be used to rewrite the system as a discrete $2 \mathrm{D}$ nonlinear eigenvalue problem. The resulting eigenvalues and mode shapes correspond to the propagation constant and velocity, pressure and temperature profiles of the different modes.

Coupled waveguides More complex geometries can be modeled by combining the solutions found for the different (lower order) modes of multiple waveguide geometries. By demanding continuity of velocity, force, temperature and heat flux, the different modes can be matched in such a way that conservation of mass, momentum and energy are preserved at the interface. This technique is demonstrated in section 6.7 (the technique is applied to a reduced version of Kirchhoff's dispersion equation in $[75,76])$. It is demonstrated that the effects of geometrical spreading are incorporated in the full LNSF solution for waveguides that are coupled in this way. So, in contrast to LRF models, there is no need for length corrections. A general technique to solve the full LNSF equations using a dispersion equation is presented in chapter 3 for a number of geometries.

\footnotetext{
${ }^{16} \mathrm{~A}$ coordinate surface is defined as a surface for which one coordinate is constant.
} 
Limitations and efficiency For certain simple geometries, the LRF models are an approximation of the first propagation mode found by solving the dispersion equation (see section 3.5.2 or [77]). The LRF approximation is very good for most fluids under conditions where the assumptions for continuum mechanics hold. Finding the roots of the dispersion equation with numerical methods is far more complex and computationally intensive than evaluating the (partially) analytical LRF equations. In addition, the number of geometries for which an exact Kirchhoff model is available is very limited compared to the number of available LRF models. The Kirchhoff-FEM approach allows more geometrical freedom since waveguides with cross sections of arbitrary shape can be modeled. Applicability of the Kirchhoff solution in a design or optimization process is limited if only the lowest acoustic mode is of interest. In that case the use of efficient and more versatile LRF models is almost always preferable. However, if higher order modes are also important, LRF models cannot be used and the Kirchhoff approaches are an efficient alternative to using FE/BE models based on the full set of equations.

\subsection{5 (H) BEM/FEM full LNSF models}

The full set of LNSF equations can be discretized using standard techniques to form a mixed FE description. Joly [48] uses a reduced form of the equations involving only velocity and temperature degrees of freedom. Malinen et al. [24] argue that using an additional degree of freedom is necessary. He presented an order of magnitude analysis which shows that the reduced form can lead to locking effects. To eliminate locking effects, he used a formulation that involves a density like auxiliary variable besides velocity and temperature. Alternatively, Nijhof et al. [25] and Kampinga et al. [27] used a formulation ${ }^{17}$ with velocity, temperature and pressure as degrees of freedom, which is also free of locking ${ }^{18}$. Cheng et al. [78] eliminate the energy equation and temperature variable by using an equation of state that relates pressure directly to density (thus the effects of a thermal boundary layer are not taken into account). They also recognize the issue of locking and use a mixed formulation with pressure and displacements as degrees of freedom. Note that the approaches of Joly, Nijhof et al., Kampinga et al. and Cheng et al. lead to a FE scheme in which all essential and natural boundary conditions are physically meaningful. In chapter 5 it is demonstrated that locking effects mentioned by Malinen et al. and Cheng et al. can become significant for parameter values that are within the scope of acoustics in air. This makes the mixed formulations

\footnotetext{
${ }^{17}$ Kampinga et al. use the dimensional form of the equations while Nijhof et al. use a dimensionless form.

${ }^{18}$ In the work of Christensen et al. [26], it is not explicitly mentioned which degrees of freedom are discretized to obtain the presented results.
} 
attractive since they are robust, i.e., they are stable over the entire range of parameters. Descriptions of the different FE models for viscothermal wave propagation are given in chapter 5 .

To obtain a BEM formulation of the full system, the equations are rewritten to form five independent Helmholtz equations that are coupled through the boundary conditions. Application of the boundary conditions for tangential velocity and analytical manipulation allows the two equations for tangential velocity to be eliminated. The three remaining (inhomogeneous) Helmholtz equations are discretized using standard BEM techniques. The resulting influence matrices are combined with the original equations and after application of the temperature and normal velocity boundary conditions an equation for normal velocity in terms of pressure on the boundary is obtained (see [49]).

Limitations and efficiency Both FE and BE representations can be used to describe viscothermal wave propagation in arbitrary geometries. No assumptions are made in addition to those needed for linearization, and the accuracy of both models is limited only by the discretization errors inherent to BEM and FEM. Solving the full LNSF equations with BEM or FEM is computationally intensive compared to solving the Helmholtz equation for inviscid adiabatic fluids when using a FEM/BEM boundary layer approximation. The number of variables in a viscothermal FE model are three to five ${ }^{19}$ times that of a FE model of the Helmholtz equation for inviscid adiabatic fluids. In addition, the bandwidth of the system matrix is also roughly four times larger for a viscothermal FE model adding to the required computational efforts. The viscothermal BE model based on the full LNSF equations that is proposed in [49] involves construction and inversion of several fully populated influence matrices. The computational efforts required for solving the full LNSF equations with BEM are more than three times higher than for solving a BEM boundary layer approximation model. Therefore, it is concluded that BEM/FEM full LNSF models should only be used in cases where the much more efficient LRF models, FEM/BEM boundary layer models or discrete LRF models cannot be used due to geometric complexity, or in cases where the approximate models are expected to be incapable of producing results of the desired accuracy due to the required additional assumptions.

\footnotetext{
${ }^{19}$ The number of degrees of freedom depends on the problem's dimension and if the system of equations is reduced prior to descritization (see chapter 5).
} 


\subsection{Summary}

A short history of the work on viscothermal wave propagation that can be found in the literature was presented. Over the last two decades, focus of developments has gradually shifted from analytical methods and approximate models towards numerical methods and full LNSF models. This trend is driven, firstly, by the desire to develop techniques that are applicable to arbitrary geometries and boundary conditions, and secondly, by the continuing growth in computational power readily available to scientists and engineers.

The equations governing linearized viscothermal wave propagation in an ideal gas were introduced. The different assumptions and requirements necessary for linearization were outlined. The coefficients in the equations can be adjusted to accommodate the use of the linearized equations of state of arbitrary fluids. Note that the requirements necessary for the linearization to be meaningful can differ from those for an ideal gas.

In the last section, five prominent viscothermal models were introduced. Two of these methods are based on the full LNSF equations, while three offer solutions to a reduced set of equations. The solution techniques used in the different methods vary from purely analytical to purely numerical. Both characteristics impact the computational effort, accuracy and limitations to the geometric shapes that can be described. Methods that are purely numerical generally require more computational effort, while (partially) analytical methods usually restrict the geometries for which a solution exists to a limited set. Solving a reduced system generally requires less computational power, however, neglecting certain terms can have a negative influence on accuracy. In addition, the required assumptions needed for the approximations can also reduce the types of geometries for which a solution exists. A schematic overview of the different methods outlining their merits and disadvantages was presented in figure 2.2. 


\section{Chapter 3}

\section{Full linearized Navier-Stokes models}

A common and efficient way to model wave propagation in waveguides of constant cross section with homogeneous and constant boundary conditions, is a description in terms of modes. These 'modes' are waveforms that satisfy the governing equations and the boundary conditions at the waveguide surface for a given frequency. A description of wave propagation in terms of modes can be found in many areas of research, such as acoustics, optics and electronics. In the case of viscothermal acoustic wave propagation a complete description of the propagation behavior in waveguides is obtained by taking a linear combination of the countable infinite number of modes that satisfy equation (2.32) such that the boundary conditions at the waveguide surface are met.

The first objective of this chapter is to present two general solution strategies to find propagation constants and corresponding mode shapes for different waveguide geometries. The first solution strategy is presented in section 3.1. Expressions for the mode shapes obtained with this strategy are known analytically for a limited number of waveguide geometries. The implementation of the strategy leading to the different solutions for these waveguide geometries can be found in the subsequent sections (3.2 through 3.3). The second solution strategy is presented in section 3.4. The resulting mode shapes are determined by numerical approximations and the strategy is applicable to waveguides of arbitrary cross section.

The second objective of this chapter is to demonstrate that the first mode can generally be approximated very well by the LRF solutions presented in chapter 4 and that the viscothermal acoustic modes reduce to the inviscid adiabatic modes for high shear and thermal wave numbers. The derivations leading to these ap- 
proximations are given in section 3.5.

The third objective is to introduce a technique that uses multiple higher order modes to reconstruct the velocity and temperature fields at waveguide interfaces. This technique, which is known as mode matching, is presented for viscothermal wave propagation in section 3.6.

\subsection{Exact Kirchhoff solutions}

The solution strategy to obtain modes for which analytical expressions exist is presented in this section. Analytical expressions for mode shapes are only known to exist for modes in waveguides that are bounded by the coordinate surfaces of certain orthogonal coordinate systems. Solutions of this type are henceforward called exact solutions, since the expressions that describe the mode shapes are known in closed form and are obtained from the linearized system without additional assumptions. Note, however, that the propagation constants are obtained by solving a transcendental equation with numerical techniques.

A large part of the theory concerning these 'exact' solutions was already described in Kirchhoff's monumental paper [31] from 1868. Therefore, the models that are derived in this chapter are henceforward called Kirchhoff models. In Kirchhoff's original work only cylindrical prismatic tubes were considered. The more general derivation of the exact solutions given in this chapter follows the work of Bruneau et al. [43] to a large extent. The notation is based on the work of Beltman [10, 36] who presented the work of Bruneau et al. in the dimensionless form that was introduced in section 2.6. Close attention is paid to the limitations put on the waveguide geometry by requiring analytical expressions for the mode shapes. It is made plausible, that in the case of constant waveguide cross section and boundary conditions, analytical expressions are only obtainable for three types of geometries (see section 3.2.4). The different types include curved waveguides of constant curvature for which the solutions (for viscothermal wave propagation) are given here for the first time.

\subsubsection{Solution strategy}

To find the different modes, the number of unknowns and equations in the system of governing equations is reduced by eliminating the pressure and density degrees of freedom through analytical manipulation (see section 3.2.1). The resulting equations for velocity and temperature can be solved independently (see sections 3.2.2 and 3.2.3) yielding a number of independent solutions that are a function of the dimensionless propagation constant $\Gamma$. In their general form, the solutions can 
be written as

$$
\begin{aligned}
\tilde{v}_{i} & =W_{1} \Psi_{v_{i}}^{(1)}(\Gamma)+W_{2} \Psi_{v_{i}}^{(2)}(\Gamma)+W_{3} \Psi_{v_{i}}^{(3)}(\Gamma)+W_{4} \Psi_{v_{i}}^{(4)}(\Gamma) \\
\tilde{T} & =W_{1} \Psi_{T}^{(1)}(\Gamma)+W_{2} \Psi_{T}^{(2)}(\Gamma)
\end{aligned}
$$

With $\Psi_{v_{i}}^{(j)}$ and $\Psi_{T}^{(j)}$ functions of the different independent solutions for the velocity components $\tilde{v}_{i}$ and temperature, respectively, and $W_{j}$ participation factors. Although the equations for temperature and velocity could be solved independently, the different independent solutions are coupled through the boundary conditions. This means that the participation factors $W_{j}$ of the different solutions which are determined by application of boundary conditions (see section 3.2.4) have to be obtained simultaneously. Application of (homogeneous) temperature and velocity boundary conditions on the waveguide surface results in a system of equations of the form

$$
[\Psi(\Gamma)]\{\mathbf{W}\}=0
$$

where the elements of the matrix $\Psi(\Gamma)$ are functions of the independent solutions for velocity and temperature evaluated on the waveguide surface (which are dependent on the propagation constant $\Gamma$ ), and $\mathbf{W}$ is a vector of participation factors. The right-hand side of the equation is zero since homogeneous boundary conditions are considered. What remains is to determine values for $\Gamma$ for which a solution to equation (3.2) exists. This system of equations will only have a nontrivial solution (non-zero participation factors) for values of $\Gamma$ that make the matrix $\psi(\Gamma)$ singular. Values for $\Gamma$ that yield a solution can therefore be obtained by minimizing the determinant, smallest eigenvalue or smallest singular value of the matrix $\psi(\Gamma)$. It turns out that the values found for $\Gamma$ correspond with three infinite series of modes, describing the different types of waveforms that can occur in the waveguide. The modes are acoustic, vortical and thermal in nature.

\subsection{General form of exact solutions}

\subsubsection{Reduced form}

In order to arrive at the general form of the solution, the system of dimensionless equations (2.32) is written in a reduced form. As a first step, the velocity vector $\mathbf{v}$ is split into a rotational and solenoidal part

$$
\tilde{\mathbf{v}}=\tilde{\mathbf{v}}_{\nu}+\tilde{\mathbf{v}}_{l}
$$

The rotational (or transverse) part, $\tilde{\mathbf{v}}_{v}$, accounts for velocity due to shearing which is related to viscous effects. The solenoidal (or longitudinal) part, $\tilde{\mathbf{v}}_{l}$, accounts for 
velocity due to compression and dilatation which is mainly related to acoustic and thermal effects. The rotational and solenoidal parts are defined as the curl of a vector potential A and the gradient of a scalar potential $\psi$ respectively

$$
\tilde{\mathbf{v}}_{v} \equiv \nabla \times \mathbf{A}, \quad \tilde{\mathbf{v}}_{l} \equiv \nabla \psi
$$

this definition immediately leads to

$$
\nabla \cdot \tilde{\mathbf{v}}_{l}=0, \quad \nabla \times \tilde{\mathbf{v}}_{l}=0
$$

Note that by this definition, both parts are coupled only through the boundary conditions. The expression for $\tilde{\mathbf{v}}$ in equation (3.3) is inserted in equation (2.32b). Subsequently, the equation is rewritten with the vector identity

$$
\Delta\left(\tilde{\mathbf{v}}_{l}+\tilde{\mathbf{v}}_{v}\right)=\nabla\left(\nabla \cdot \tilde{\mathbf{v}}_{l}\right)+\nabla \times \nabla \times \tilde{\mathbf{v}}_{v}
$$

The resulting equation is split into a rotational and longitudinal part by taking its curl and divergence. Next, equation (3.3) and the equation of state (2.32d) are substituted in the equation for conservation of mass, and the following system is obtained

$$
\begin{aligned}
\frac{1}{k} \nabla \cdot \tilde{\mathbf{v}}_{l} & =i \tilde{T}-i \tilde{p} \\
i \tilde{\mathbf{v}}_{l} & =-\frac{1}{\gamma k} \nabla \tilde{p}+\frac{1}{s^{2}}(\xi+2) \Delta \tilde{\mathbf{v}}_{l} \\
i \tilde{\mathbf{v}}_{v} & =\frac{1}{s^{2}} \Delta \tilde{\mathbf{v}}_{v} \\
i \tilde{T} & =i\left[\frac{\gamma-1}{\gamma}\right] \tilde{p}+\frac{1}{s_{t}^{2}} \Delta \tilde{T}
\end{aligned}
$$

The divergence of equation (3.7b) is substituted in equation (3.7a). Subsequently, the Laplacian of equation (3.7a) and identities (3.6) and (3.5) can be used to eliminate $\tilde{\mathbf{v}}_{l}$. Next, equation (3.7d) and its Laplacian are used to eliminate $\tilde{p}$ from the resulting equation. After multiplying through $k^{2}$, the following fourth order equation for temperature is obtained

$$
\frac{i}{s_{t}^{2}}\left[1+\frac{i \gamma k^{2}}{s^{2}}(\xi+2)\right] \Delta \Delta \tilde{T}+\left[1+i k^{2}\left[\frac{1}{s^{2}}(\xi+2)+\frac{\gamma}{s_{t}^{2}}\right]\right] \Delta \tilde{T}+k^{2} \tilde{T}=0
$$

Note that this equation also holds for $\tilde{\mathbf{v}}_{l}$ and $\tilde{p}$. The original system is now in the desired form which consists of a single fourth order equation for either $\tilde{T}, \tilde{\mathbf{v}}_{l}$ or $\tilde{p}$ (equation (3.8)) and a vector Helmholtz equation for $\tilde{\mathbf{v}}_{v}$ (equation (3.7c)). The 
equations are only coupled through the velocity boundary conditions. A derivation of the general solutions to the individual equations is given in the next two sections. Note that the solution to both equations is written in terms of solutions to the scalar Helmholtz equation. See appendix D for an overview of general solutions to the scalar Helmholtz equations in a number of orthogonal coordinate systems.

\subsubsection{Fourth order equation}

Equation (3.8) can be seen as a second order polynomial as function of the Laplace operator. After factorization the right-hand side takes the form of the product of two Helmholtz equations

$$
\left[\Delta+k_{a}^{2}\right]\left[\Delta+k_{h}^{2}\right] \tilde{T}=0
$$

where $k_{a}$ and $k_{h}$ are the roots of the polynomial defined as

$$
k_{a}^{2}=\frac{C_{2}-\sqrt{C_{2}^{2}-4 C_{1} k^{2}}}{2 C_{1}}, \quad k_{h}^{2}=\frac{C_{2}+\sqrt{C_{2}^{2}-4 C_{1} k^{2}}}{2 C_{1}}
$$

with

$$
C_{1}=\frac{i}{s_{t}^{2}}\left[1+\frac{i \gamma k^{2}}{s^{2}}(\xi+2)\right], \quad C_{2}=\left[1+i k^{2}\left[\frac{1}{s^{2}}(\xi+2)+\frac{\gamma}{s_{t}^{2}}\right]\right]
$$

The solution for temperature consists of a linear combination of the solutions of the Helmholtz equation for the Helmholtz operators in equation (3.9).

$$
T=W_{1} \psi_{a}+W_{2} \psi_{h}
$$

with $\psi_{a}$ and $\psi_{h}$ the solutions to the individual Helmholtz equations ${ }^{1}$.

$$
\left[\Delta+k_{a}^{2}\right] \psi_{a}=0, \quad\left[\Delta+k_{h}^{2}\right] \psi_{h}=0
$$

and $W_{1}$ and $W_{2}$ are participation factors that have to be determined from the boundary conditions. Equations (3.13) are combined with equation (3.7d) to obtain an expression for $p$ in terms of $\psi_{a}, \psi_{h}, W_{1}$ and $W_{2}$

$$
\tilde{p}=\frac{\gamma}{\gamma-1}\left[W_{1}\left[1-\frac{i k_{a}^{2}}{s_{t}^{2}}\right] \psi_{a}+W_{2}\left[1-\frac{i k_{h}^{2}}{s_{t}^{2}}\right] \psi_{h}\right]
$$

\footnotetext{
${ }^{1}$ See appendix D for general solutions to the scalar Helmholtz equation.
} 
This expression for $p$ is substituted in equation (3.7b). Combining the result with equations (3.13) yields an expression for $\tilde{\mathbf{v}}_{l}$ in terms of $\psi_{a}, \psi_{h}, W_{1}$ and $W_{2}$

$$
\tilde{\mathbf{v}}_{l}=\alpha_{a} W_{1} \nabla \psi_{a}+\alpha_{h} W_{2} \nabla \psi_{h}
$$

where

$$
\alpha_{a}=\frac{i}{k \gamma} \frac{\gamma}{\gamma-1}\left[\frac{1-\frac{i k_{a}^{2}}{s_{t}^{2}}}{1-\frac{i k_{a}^{2}}{s^{2}}(\xi+2)}\right], \quad \alpha_{h}=\frac{i}{k \gamma} \frac{\gamma}{\gamma-1}\left[\frac{1-\frac{i k_{h}^{2}}{s_{t}^{2}}}{1-\frac{i k_{h}^{2}}{s^{2}}(\xi+2)}\right]
$$

\subsubsection{Vector Helmholtz equation}

Analytical solutions to the vector Helmholtz equation in (3.7c) can be obtained by separation of variables for certain coordinate systems. For six of the eleven coordinate systems in which the scalar Helmholtz equation is separable, solutions to the vector Helmholtz equation in (3.7c) are given by Morse and Feshbach [74].

$$
\mathbf{L}=\nabla \psi_{L}, \quad \mathbf{M}=\nabla \times\left(\mathbf{e}_{p} w \psi_{M}\right), \quad \mathbf{N}=\nabla \times \nabla \times\left(\mathbf{e}_{p} w \psi_{N}\right)
$$

with $w$ a coordinate-system dependent scaling factor, $\mathbf{e}_{p}$ a so-called pilot vector and $\psi_{L}, \psi_{N}$ and $\psi_{M}$ so-called generating functions. These functions are solutions to the corresponding scalar Helmholtz equation ${ }^{1}$.

$$
\left[\Delta+k_{v}^{2}\right] \psi_{m}=0, \quad \text { where } \quad k_{v}^{2}=-i s^{2}, \quad \text { and } \quad m=\{L, M, N\}
$$

For Cartesian, and circular-, elliptical- and parabolic-cylindrical coordinate systems $w=1$, while for spherical and conical coordinate systems $w=r$ (where $r$ is the radial coordinate). For other coordinate systems a solution of the vector Helmholtz equation of the presented form is not available. The pilot vector $\mathbf{e}_{p}$ is a unit vector pointing in the direction of a coordinate with scaling factor equal to unity: for all cylindrical coordinate systems the vector points in axial direction, for spherical and conical coordinates it points in radial direction. Note that $\mathbf{L}$ represents the longitudinal part of the general solution while $\mathbf{M}$ and $\mathbf{N}$ represent the transversal parts. By taking the divergence of the longitudinal part and considering equations (3.5) and (3.18) it follows that the longitudinal part does not contribute to the total solution. The general solution to the vector Helmholz equation in (3.7c) for these coordinate systems is thus a linear combination of the transversal solutions given above

$$
\tilde{\mathbf{v}}_{\nu}=W_{3} \nabla \times\left(\mathbf{e}_{p} w \psi_{M}\right)+W_{4} \nabla \times \nabla \times\left(\mathbf{e}_{p} w \psi_{N}\right)
$$


Consider a coordinate system $\left(x_{1}, x_{2}, x_{3}\right)$ that yields a solution of this form with the pilot vector pointing in the direction of the $x_{3}$ coordinate. For such systems, the corresponding scaling factors are such that expressions for the curl and double curl operator can be simplified considerably ${ }^{2}$

$$
\begin{aligned}
\nabla \times\left(\mathbf{e}_{p} w \psi_{M}\right) & =\nabla_{1}\left(w \psi_{M}\right) \mathbf{e}_{1}+\nabla_{2}\left(w \psi_{M}\right) \mathbf{e}_{2} \\
\nabla \times \nabla \times\left(\mathbf{e}_{p} w \psi_{N}\right) & =\nabla\left(\nabla_{3}\left(w \psi_{N}\right)\right)-\Delta\left(w \psi_{N}\right) \mathbf{e}_{3}
\end{aligned}
$$

where $\mathbf{e}_{1}$ and $\mathbf{e}_{2}$ are unit vectors corresponding to the first two coordinates and the operators $\nabla_{1}, \nabla_{2}$ and $\nabla_{3}$ are the components of the gradient for the different coordinate directions defined as

$$
\nabla_{i}=\frac{1}{h_{i}} \frac{\partial}{\partial x_{i}}, \quad \text { where } \quad i=\{1,2,3\}
$$

with $h_{i}$ the shape factors of the coordinate system under consideration (see appendix D).

\subsubsection{Boundary conditions}

The last step in solving the full set of viscothermal equations is finding sets of participation factors and propagation factors for which the boundary conditions are met. In general, boundary conditions will not be available for the individual acoustic, thermal and vortical components that make up temperature and velocity. Recall that the acoustic and thermal part of the temperature field are related according to equation (3.12). The total velocity vector is constructed by substitution of (3.15) and (3.19) in equation (3.3). With the curl and double curl as defined in (3.20) and the gradient components as defined in (3.21), the expressions for temperature and velocity can be cast into the following form

$$
\left\{\begin{array}{c}
\tilde{v}_{1} \\
\tilde{v}_{2} \\
\tilde{\mathbf{v}}_{3} \\
\tilde{T}
\end{array}\right\}=\left[\begin{array}{cccc}
\alpha_{a} \nabla_{1} \psi_{a} & \alpha_{h} \nabla_{1} \psi_{h} & \nabla_{2}\left(w \psi_{M}\right) & \nabla_{1} \nabla_{3}\left(w \psi_{N}\right) \\
\alpha_{a} \nabla_{2} \psi_{a} & \alpha_{h} \nabla_{2} \psi_{h} & \nabla_{1}\left(w \psi_{M}\right) & \nabla_{2} \nabla_{3}\left(w \psi_{N}\right) \\
\alpha_{a} \nabla_{3} \psi_{a} & \alpha_{h} \nabla_{3} \psi_{h} & 0 & {\left[\nabla_{3} \nabla_{3}+k_{v}^{2}\right]\left(w \psi_{N}\right)} \\
\psi_{a} & \psi_{h} & 0 & 0
\end{array}\right]\left\{\begin{array}{l}
W_{1} \\
W_{2} \\
W_{3} \\
W_{4}
\end{array}\right\}
$$

The coordinates are ordered so that the pilot vector (see section 3.2.3) points in the direction of the third coordinate. Note that there are an uncountable infinite number of solutions available for $\psi_{a}, \psi_{h}, \psi_{M}$ and $\psi_{N}$ that can be combined to satisfy

\footnotetext{
${ }^{2}$ For such systems the scaling factor $h_{3}=1$ and the quotient $h_{1} / h_{2}$ is independent of $x_{3}$. These properties do not only lead to the simplification but are a prerequisite for separability of the vector Helmholtz equation [74].
} 
the boundary conditions. For arbitrary geometries and boundary conditions, determining the contribution of the independent solutions involves the non-trivial task of reducing the uncountable infinite set to a countable infinite subset that forms a basis capable of describing all possible solutions. Subsequently, the subset has to be truncated to obtain a system of finite size. Application of boundary conditions (by using the method of weight residuals, or point collocation) yields a system of equations (which is in general ill-conditioned) that can be solved numerically. This non-trivial and computational intensive process, which is known as Trefftz method [79, 80], only yields an approximate solution involving truncation and numerical errors [81].

Fortunately, for a number of simple geometries having constant homogeneous boundary conditions, there exist exact solutions that only use a single set of $\psi_{a}$, $\psi_{h}, \psi_{M}$ and $\psi_{N}$. Note that solutions exist for both bounded and unbounded domains. Solutions in bounded domains concern wave propagation through waveguides and internal cavities, while solutions in unbounded domains concern scattering problems (such as a planar wave impinging on a flat surface) [43]. In this thesis only solutions for bounded domains are considered. The objective is to find non-trivial solutions for the (coordinate independent) constants $\mathbf{W}$ that satisfy the system in (3.22) over the complete waveguide surface. Careful inspection of the coordinate systems for which a solution of the form in equation (3.22) exists reveals that for homogeneous boundary conditions that are constant over the waveguide surface such an exact solution is available for only three types of geometries; straight layers, spherical layers (a complete sphere being a special case) and cylindrical layers (tube and annular tube geometries being a special cases of a cylindrical layer). All these geometries have the form of a 2D waveguide that is bounded by coordinate surfaces. Consequently, on the waveguide surface, a distinction can be made between coordinates that are normal to and tangential to the waveguide surface. In the remainder of this chapter, coordinates in $p d$-direction are referred to as tangential coordinates, while coordinates in $c d$-direction are referred to as normal coordinates.

The different waveguide geometries considered in this chapter are divided into two groups. The distinction between both groups is based on the alignment of the normal and tangential coordinates with the pilot vector that is used to generate the solutions to the vector Helmholtz equation (3.19). The two groups are evaluated in the next section.

\subsubsection{Pilot vector and surface normal coincide}

Consider a 2D waveguide with its surface normal coinciding with the pilot vector (see figure 3.1 for examples). In order to satisfy the boundary conditions every- 


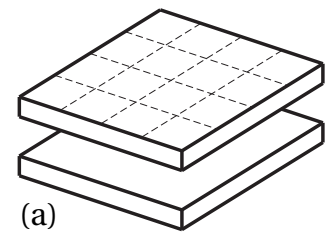

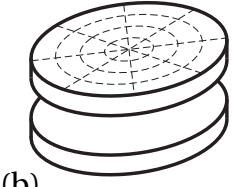

(b)

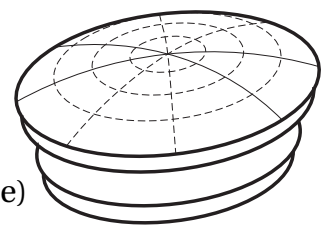

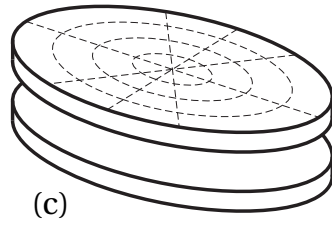

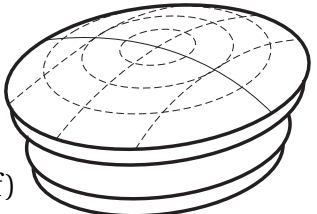

Figure 3.1: 2D waveguide geometries with their surface normal coinciding with the pilot vector in coordinate systems that allow an exact solution: Cartesian (a), circular-cylindrical (b), elliptic-cylindrical (c), parabolic-cylindrical (d), spherical (e), and conical (f).

where on the waveguide surface, the different matrix elements in equation (3.22) must all vary with the same rate of change for the tangential coordinates. For the solutions corresponding to the coordinate systems under consideration, this holds true only if the participation factor $W_{3}$ equals zero and the propagation constant for the tangential direction is taken equal for $\psi_{a}, \psi_{h}$ and $\psi_{N}$. In that case, the dependence of the components on the tangential coordinates is identical in each equation. Division of the equations by these common expressions makes the system dependent on the normal coordinate only. The first two equations in (3.22) are now linear dependent and one can be removed from the system. The velocity component corresponding to the equation that is removed can always be reconstructed with equation (3.22) after the participation factors are determined. The solutions $\psi_{a}, \psi_{h}$ and $\psi_{N}$ are expanded as a linear combination of two solutions to the corresponding scalar Helmholtz equation. After application of the boundary conditions and rearranging the participation factors the following expression is obtained

$$
\left[\begin{array}{ll}
\mathbf{P}_{1}\left(k_{t}, s_{1}\right) & \mathbf{P}_{2}\left(k_{t}, s_{1}\right) \\
\mathbf{P}_{1}\left(k_{t}, s_{2}\right) & \mathbf{P}_{2}\left(k_{t}, s_{2}\right)
\end{array}\right]\left\{\begin{array}{l}
\mathbf{W}_{1} \\
\mathbf{W}_{2}
\end{array}\right\}=\left[\mathbf{P}\left(k_{t}\right)\right]\{\mathbf{W}\}=0
$$

where $s_{1}$ and $s_{2}$ are the values of the normal coordinate on the waveguide surface, and $k_{t}^{2}=k_{1}^{2}+k_{2}^{2}$, the sum of the propagation constants for the tangential direc- 
tions ${ }^{3}$. The sub-matrices $\mathbf{P}_{1}$ and $\mathbf{P}_{2}$ are of the form

$$
\mathbf{P}_{i}\left(k_{t}, s_{i}\right)=\left.[\mathbf{L}]\left[\begin{array}{ccc}
\alpha_{a} \psi_{a i} & \alpha_{h} \psi_{h i} & \nabla_{3}\left(w \psi_{v i}\right) \\
\alpha_{a} \nabla_{3} \psi_{a i} & \alpha_{h} \nabla_{3} \psi_{h i} & {\left[\nabla_{3} \nabla_{3}+k_{v}^{2}\right]\left(w \psi_{v i}\right)} \\
\psi_{a i} & \psi_{h i} & 0
\end{array}\right]\right|_{x_{3}=s_{i}}
$$

with $\psi_{a i}, \psi_{h i}$ and $\psi_{v i}$ the part of the individual solutions that make up $\psi_{a}, \psi_{h}$ and $\psi_{\nu}$ in normal direction. The wave numbers used in these solutions are

$$
k_{a}^{x_{3}}=\sqrt{k_{a}^{2}-k_{t}^{2}}, \quad k_{h}^{x_{3}}=\sqrt{k_{h}^{2}-k_{t}^{2}}, \quad k_{v}^{x_{3}}=\sqrt{k_{v}^{2}-k_{t}^{2}} \quad(3.25 \mathrm{a}, \mathrm{b}, \mathrm{c})
$$

The operator $\mathbf{L}$ representing the boundary conditions is a matrix which elements are linear operators acting on the equations (as described in section 2.7). The subvectors $\mathbf{W}_{1}$ and $\mathbf{W}_{2}$ with participation factors are defined as

$$
\mathbf{W}_{1}=\left\{\begin{array}{l}
W_{11} \\
W_{21} \\
W_{41}
\end{array}\right\}, \quad \mathbf{W}_{2}=\left\{\begin{array}{l}
W_{12} \\
W_{22} \\
W_{42}
\end{array}\right\}
$$

This form can be obtained for all coordinate systems under consideration as long as the pilot vector is normal to the waveguide surface. Once solved, the equation yields a countable infinite number of modes. Incidentally, the solution is identical for Cartesian, circular-, elliptic- and parabolic-cylindrical coordinates for a given $k_{t}$ since the solution in axial direction is identical in all cases. In fact, the calculated values of $k_{t}$ and the corresponding velocity and temperature profiles are exact solutions for a flat 2D waveguide in any cylindrical coordinate system. Note that the propagation constant $k_{t}$ is related to the dimensionless propagation constant $\Gamma$, by $k_{t}=k \Gamma$. The propagation constant $k \Gamma$ in the finite element formulation for viscothermal wave propagation in flat layers proposed by Beltman et al. [37] can be substituted by solutions for $k_{t}$ to obtain a model for flat layers of arbitrary shape. In that case, errors due to the LRF approximation are avoided and the FE solution will converge to the exact solution. Since the solution in r-direction is identical for spherical and conical coordinates, their solutions are also identical for a given $k_{t}$ and inner and outer radii of the waveguide surface.

\subsubsection{Symmetry in flat waveguides}

For flat 2D waveguide, the components of the different solutions to the Helmholtz equations $\psi_{a} i, \psi_{h} i$, and $\psi_{\nu} i$ can be split into a symmetric and mirror-symmetric

\footnotetext{
${ }^{3}$ Examples of the expression for $k_{t}$ are: $k_{t}^{2}=k_{x}^{2}+k_{y}^{2}$ for Cartesian coordinates, $k_{t}^{2}=k_{\theta}^{2}+k_{z}^{2}$ for circular-cylindrical coordinates, and $k_{t}^{2}=k^{2}-k_{r}^{2}=l(l-1)$ for spherical and conical coordinates, where $l$ is the degree of the spherical harmonic and ellipsoidal harmonic, respectively.
} 
part by rearranging the participation factors. After such a separation, equation (3.23) consists of two uncoupled sets of equations. For instance, application of no-slip, isothermal boundary conditions results in two individual systems of the form

$$
[\mathbf{L}]\left[\begin{array}{ccc}
\alpha_{a} \psi_{a 1} & \alpha_{h} \psi_{h 1} & i k_{v 3}\left(\psi_{v 1}\right) \\
i \alpha_{a} k_{a 3} \psi_{a 2} & i \alpha_{h} k_{h 3} \psi_{h 2} & {\left[k_{v}^{2}-k_{v 3}^{2}\right]\left(\psi_{v 2}\right)} \\
\psi_{a 1} & \psi_{h 1} & 0
\end{array}\right]\left\{\begin{array}{l}
W_{1} \\
W_{2} \\
W_{4}
\end{array}\right\}=0
$$

where

$$
\begin{aligned}
& \left.\begin{array}{l}
\psi_{m 1}=\cos \left(k_{m 3} x_{3}\right) \\
\psi_{m 2}=\sin \left(k_{m 3} x_{3}\right)
\end{array}\right\} \quad \text { for symmetrical modes, } \quad m=\{a, h, v\} \\
& \left.\begin{array}{l}
\psi_{m 1}=\sin \left(k_{m 3} x_{3}\right) \\
\psi_{m 2}=\cos \left(k_{m 3} x_{3}\right)
\end{array}\right\} \quad \text { for mirror-symmetrical modes, } \quad m=\{a, h, v\}
\end{aligned}
$$

The individual systems yield symmetrical and mirror-symmetrical modes, respectively. Similar separations can be made for other symmetrical boundary conditions. If boundary conditions are asymmetrical or if the waveguide is described by spherical or conical coordinates the full set of equations in (3.23) has to be solved.

\subsubsection{Pilot vector and surface normal do not coincide}

Consider a 2D waveguide with surfaces that have a tangential coordinate coinciding with the pilot vector (see figure 3.2 for examples in circular-cylindrical coordinates). The coordinates are arranged such that the $x_{1}$ coordinate is normal to the
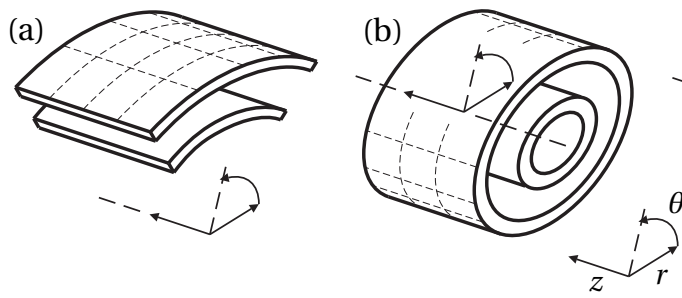

(c)

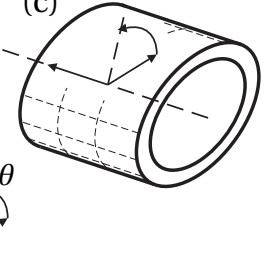

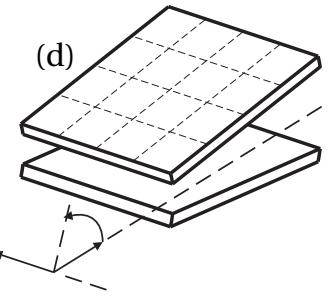

Figure 3.2: Two 2D Waveguide geometries in circular cylindrical coordinates with $x_{1}$ in the direction of the surface normal, $x_{2}$ and $x_{3}$ the propagation directions, and $x_{3}$ coinciding with the pilot vector: $\left(x_{1}, x_{2}, x_{3}\right)=(r, \theta, z)(\mathrm{a})(\mathrm{b})(\mathrm{c})$, and $\left(x_{1}, x_{2}, x_{3}\right)=(\theta, r, z)(\mathrm{d})$.

waveguide surface (the $x_{3}$ coordinate is in the direction of the pilot vector). The general form developed in equation (3.23) is in general not valid in case the pilot 
vector is tangential to the waveguide surface. However, if

$$
\nabla_{2} \psi_{M}=\nabla_{2} \psi_{N}=m \psi_{N}, \quad \nabla_{3} \psi_{N}=n \psi_{N}
$$

where $m$ and $n$ are coefficients that are independent of the tangential coordinates, all terms in the system vary identically with the tangential coordinates. Imposing these conditions to the solutions of the different coordinate systems reveals that this is only possible for Cartesian coordinates (see figure 3.1(a)), and cylindrical coordinates with the $\theta$-and $z$-coordinates tangential to the waveguide service (see figure 3.2(a)(b)(c)). The system is made independent of the tangential coordinates by dividing through the common term. Next, the solutions $\psi_{a}, \psi_{h}$ and $\psi_{N}$ are expanded as a linear combination of two solutions to the corresponding scalar Helmholtz equation. After application of the boundary conditions and rearranging the participation factors the following expression is obtained

$$
\left[\begin{array}{ll}
\mathbf{P}_{1}\left(k_{t}, s_{1}\right) & \mathbf{P}_{2}\left(k_{t}, s_{1}\right) \\
\mathbf{P}_{1}\left(k_{t}, s_{2}\right) & \mathbf{P}_{2}\left(k_{t}, s_{2}\right)
\end{array}\right]\left\{\begin{array}{l}
\mathbf{W}_{1} \\
\mathbf{W}_{2}
\end{array}\right\}=\left[\mathbf{P}\left(k_{t}\right)\right]\{\mathbf{W}\}=0
$$

The sub-matrices $P_{1}$ and $P_{2}$ are both of the form

$$
\mathbf{P}_{i}\left(k_{t}, s_{i}\right)=\left.[\mathbf{L}]\left[\begin{array}{cccc}
\alpha_{a} \nabla_{1} \psi_{a i} & \alpha_{h} \nabla_{1} \psi_{h i} & m \psi_{N i} & n \nabla_{1}\left(\psi_{N i}\right) \\
\alpha_{a} m \psi_{a i} & \alpha_{h} m \psi_{h i} & \nabla_{1} \psi_{N i} & m n \psi_{N i} \\
\alpha_{a} n \psi_{a i} & \alpha_{h} n \psi_{h i} & 0 & {\left[n^{2}+k_{v}^{2}\right]\left(\psi_{N i}\right)} \\
\psi_{a i} & \psi_{h i} & 0 & 0
\end{array}\right]\right|_{x_{1}=s_{i}}
$$

with $\psi_{a i}, \psi_{h i}$ and $\psi_{N i}$ the individual solutions that make up $\psi_{a}, \psi_{h}$ and $\psi_{N}$. The subvectors $\mathbf{W}_{1}$ and $\mathbf{W}_{2}$ with participation factors are defined as

$$
\mathbf{W}_{1}=\left\{\begin{array}{l}
W_{11} \\
W_{21} \\
W_{31} \\
W_{41}
\end{array}\right\}, \quad \mathbf{W}_{2}=\left\{\begin{array}{l}
W_{12} \\
W_{22} \\
W_{32} \\
W_{42}
\end{array}\right\}
$$

For a 2D waveguide described by Cartesian coordinates this system will yield identical propagation constants and mode shapes as the system in (3.23). In cylindrical coordinates the solution $\psi_{a i}, \psi_{h i}$ and $\psi_{N i}$ take the form

$$
\left.\begin{array}{l}
\psi_{m 1}=C_{m 1}^{r} J_{k_{m}^{\theta}}\left(k_{m}^{r}\right) \\
\psi_{m 2}=C_{m 2}^{r} Y_{k_{m}^{\theta}}\left(k_{m}^{r}\right)
\end{array}\right\}, \quad \text { with } \quad m=\{a, h, v\}
$$

A countable infinite number of modes can be obtained for instance by excluding wave propagation in axial direction. In that case, solving equation (3.31) involves 
evaluation of Bessel functions of complex order and argument. The equation also describes wave propagation in an annular tube if the curved waveguide completes a full circle (see figure 3.2(b)). In that case, the cyclic boundary conditions in $\theta$ direction are only satisfied for integer (real) values of the propagation constant in that direction. Again, a countable infinite number of modes can be obtained. To describe a tube that is not annular, continuity of the velocity and temperature derivatives are enforced at the center of the tube (see figure 3.2(c)). As a result, solutions involving Bessel functions of the second kind do not contribute $\left(\mathbf{W}_{2}=0\right)$ and the corresponding equations can be removed from the system.

\subsubsection{Solving the system}

As mentioned above, equation (3.23) and (3.31) are satisfied only when the vector of participation factors $\mathbf{W}$ is an eigenvector (or right-hand singular vector) of the matrix $\mathbf{P}$ corresponding to an eigenvalue (or singular value) of zero. Finding solutions that satisfy the boundary conditions thus reduces to finding the values of the propagation constants that minimize either the smallest eigenvalue, the smallest singular value or the determinant of the matrix $\mathbf{P}$ in an absolute sense. These three matrix properties can be used as an objective function in any optimization algorithm for unconstrained problems to obtain appropriate values for the propagation constants.

\section{The method of eigenvalleys}

If both tangential propagation constants are varied, their real and imaginary parts span a four dimensional search space in which the objective function is zero on a countable infinite number of surfaces. If one of the propagation constants in tangential directions is fixed (for instance by assuming the lack of wave propagation in a certain direction), the objective function is a 2D response surface spanned by the real and imaginary part of the free propagation constant. The absolute value of the objective function reaches zero at the bottom of a countable infinite number of valleys. An example of the locations in the complex plane of the dimensionless propagation constant for different modes is given in figure 3.3 for a straight layer. The objective function, being the absolute value of the determinant of the matrix $\mathbf{P}$, is plotted on a log scale. The points where this function has a minimum are indicated by black spots.

A detailed description of the location of the propagation constant of different modes in the complex plane, and the dependency of these locations on different parameters (such as viscosity coefficient and frequency) is given by Scarton [41, 42]. He named this approach the method of eigenvalleys, and applied it to 

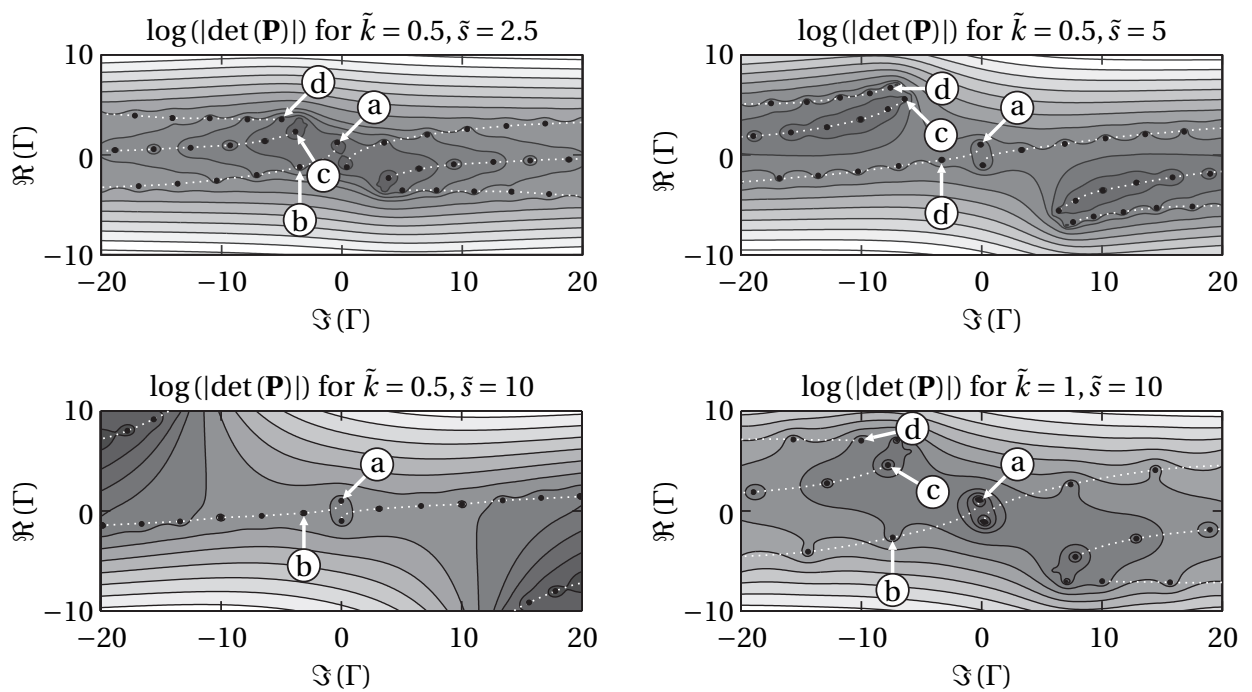

Figure 3.3: Location of the propagation constants for different values of $\tilde{k}$ and $\tilde{s}$ in the complex plane for a 1D layer: first acoustic mode (a), second acoustic mode (b), first thermal mode (c), and first vorticity mode (d). Propagation constants corresponding to the same type of mode are connected by white dotted lines.
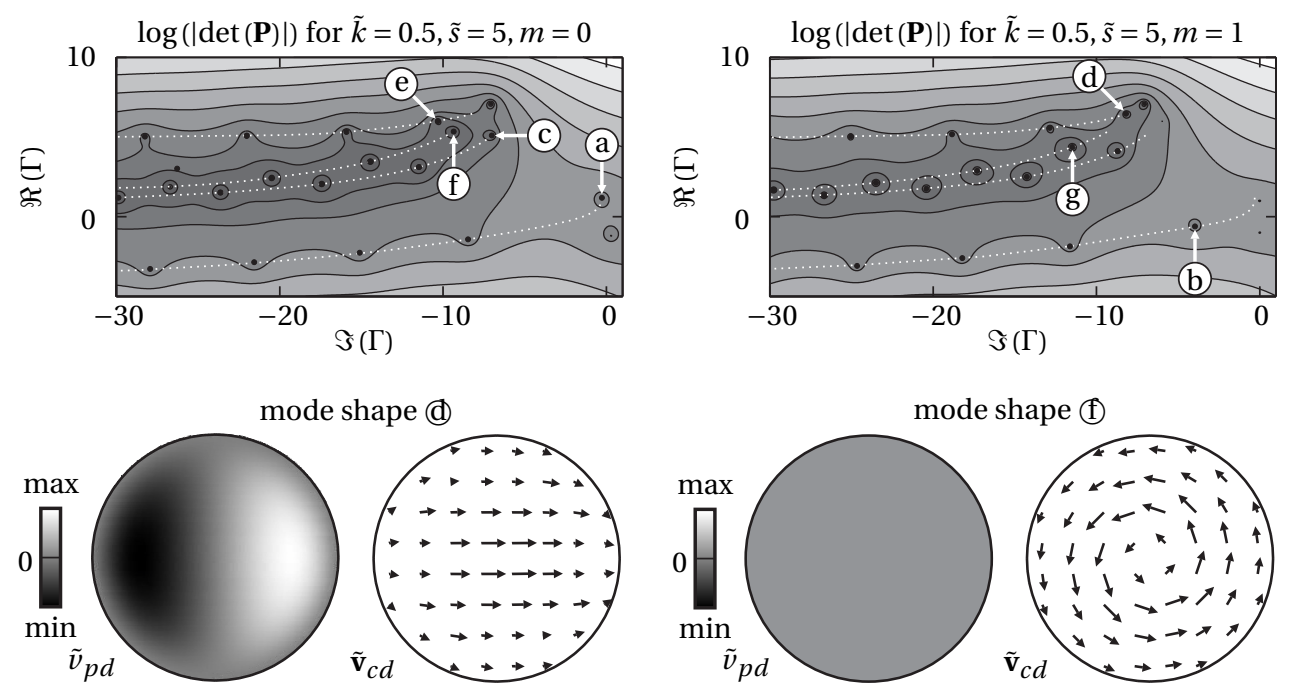

Figure 3.4: Location of propagation constants in the complex plane for a cylindrical tube: first acoustic mode (a), second (b), first thermal mode (C), first $p d$-vorticity mode (d), second $p d$-vorticity mode (e), first $c d$-vorticity mode (f), and second $c d$-vorticity mode (g). 
isothermal viscous wave propagation in cylindrical prismatic tubes. Scarton only considered axisymmetrical solutions. In that case (and in the case of layers) two infinite sets of modes can be found: acoustic modes and vorticity modes. For viscothermal wave propagation, temperature is an additional variable and a third infinite set of modes is obtained, which are known as thermal modes. For layers, an example of the location of the propagation constant of the different modes in the complex plane was given in figure 3.3, while examples of the different mode shapes are given in the next section. Note that two minima in the value of $\log (\mid \operatorname{det}((\mathbf{P}) \mid)$ are observed at exactly $\sqrt{i} s_{t} / k$ and $\sqrt{i} s / k$. These minima can be related to the thermal and vorticity modes by extending the white dotted lines connecting the modes of these types, however, they do not correspond to actual modes (since none of the eigenvalues of the matrix $\mathbf{P}$ at these locations equals zero).

If asymmetric modes in cylindrical tubes are also considered, velocity in circumferential direction is added as a fourth variable, and a fourth infinite series of modes emerges. In addition, solutions can be found for all integer values of the radial wave number, i.e., each integer radial wave number yields four infinite series of modes. An example of the location of the propagation constant of the four different types of modes is given in figure 3.4 for two values of the radial wave number $m$. The modes corresponding to the additional fourth series are a second type of vorticity modes. These modes are characterized by high $c d$-velocities compared to the $p d$-velocities, while the axisymmetrical vorticity modes involve $p d$ and $c d$-velocities of comparable magnitude. These different types are henceforward called $c d$-and $p d$-vorticity modes, respectively. The distinction between the two types of vorticity modes is most clear for lower order modes. As an example, the mode shapes of the first $c d$ - and $p d$-vorticity mode are given in figure 3.4. The $p d$-vorticity mode can be seen as a shear wave; if the fluid in the center of the tube is harmonically forced from left to right at a certain cross section, a shear wave that rapidly decays in the axial direction of the tube will result. The $c d$-vorticity mode can be seen as a torsional shear wave; if the fluid in the center of the tube is harmonically twisted around the tube axis, a shear wave that spirals around the tube axis results. Note that the solution technique for waveguides of arbitrary 2D cross section that is presented in the next section will also yield both types of vorticity modes.

\section{Scaling}

Proper scaling of the matrix $\mathbf{P}$ is important to avoid numerical inaccuracies when calculating the matrix property that is being minimized. Scaling also affects the accuracy of the resulting eigenvectors (or singular vectors) $W_{1}$ and $W_{2}$ that are used as participation factors in the process of reconstructing the sound field in 
the waveguide. The optimal scaling depends on the matrix property that is being minimized. The following scaling operation is proposed when the determinant is the objective function

$$
\left[\mathbf{P}_{o}\right]=[\mathbf{Q}][\mathbf{P}][\mathbf{R}]
$$

where $\mathbf{P}_{o}$ is the matrix used to construct the objective function, and $\mathbf{Q}$ and $\mathbf{R}$ are diagonal matrices with their diagonal elements defined as

$$
Q_{i i}=\frac{1}{\left|\sum_{j} P_{i j}\right|}, \quad R_{j j}=\frac{1}{\left|\sum_{i} P_{i j}\right|}
$$

When the smallest singular value or smallest eigenvalue is minimized, the scaling matrix $\mathbf{Q}$ is the identity matrix while $\mathbf{R}$ is as defined above. Note that the scaling affects the shape of the response surface. In gradient-based searches, it influences which minimum is found for a given starting vector thus influencing the required accuracy of the initial guess. In some cases, numerical inaccuracies can be reduced by splitting the matrix $\mathbf{P}$ into parts yielding symmetrical and mirrorsymmetrical modes prior to minimization. In that case, it is also feasible to find a solution for the participation factors using two of the equations in (3.27), and subsequently determining the propagation constant by minimizing the residual of the remaining equation. This technique can be particulary useful when scaling of the matrix $\mathbf{P}$ does not yield the required accuracy.

\subsection{Examples of exact solutions}

In this section the first few mode shapes found by solving equation (3.23) or (3.31) for different geometries are presented. For each example, the waveguide walls are fixed and isothermal, so the operator $L$ containing the boundary conditions is a unit tensor. For the tube example, the boundary condition on the axis of symmetry reduces the system to include only Bessel functions of the first order $\left(\mathbf{W}_{2}=0\right)$. All mode shapes are normalized with respect to the magnitude and phase of pressure profile integrated over the waveguide cross section. For each waveguide, the profiles are plotted as a function of the arc length of the dotted lines indicated in figure 3.5. Note that the length of the dotted line does not coincide with the characteristic length in the case of the circular cylindrical tube (figure 3.5b), while it does for the other waveguides.

\subsubsection{Straight layer}

As a first example, a flat 2D waveguide in Cartesian coordinates is considered. The surface normal is in $z$-direction and wave propagation in y-direction is excluded, so $k_{t}=k_{x}$. Figure 3.6 shows profiles of the velocity in propagation and 

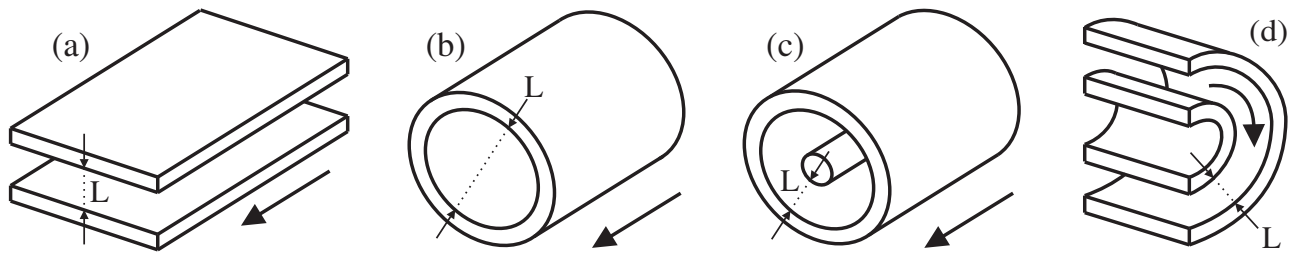

$\longrightarrow$ propagation direction

Figure 3.5: Various waveguides which mode shapes are plotted in figure 3.6, 3.7, 3.8, 3.9, and 3.10. The mode shapes are plotted as a function of the arc length of the dotted lines indicated here in the waveguide cross sections.

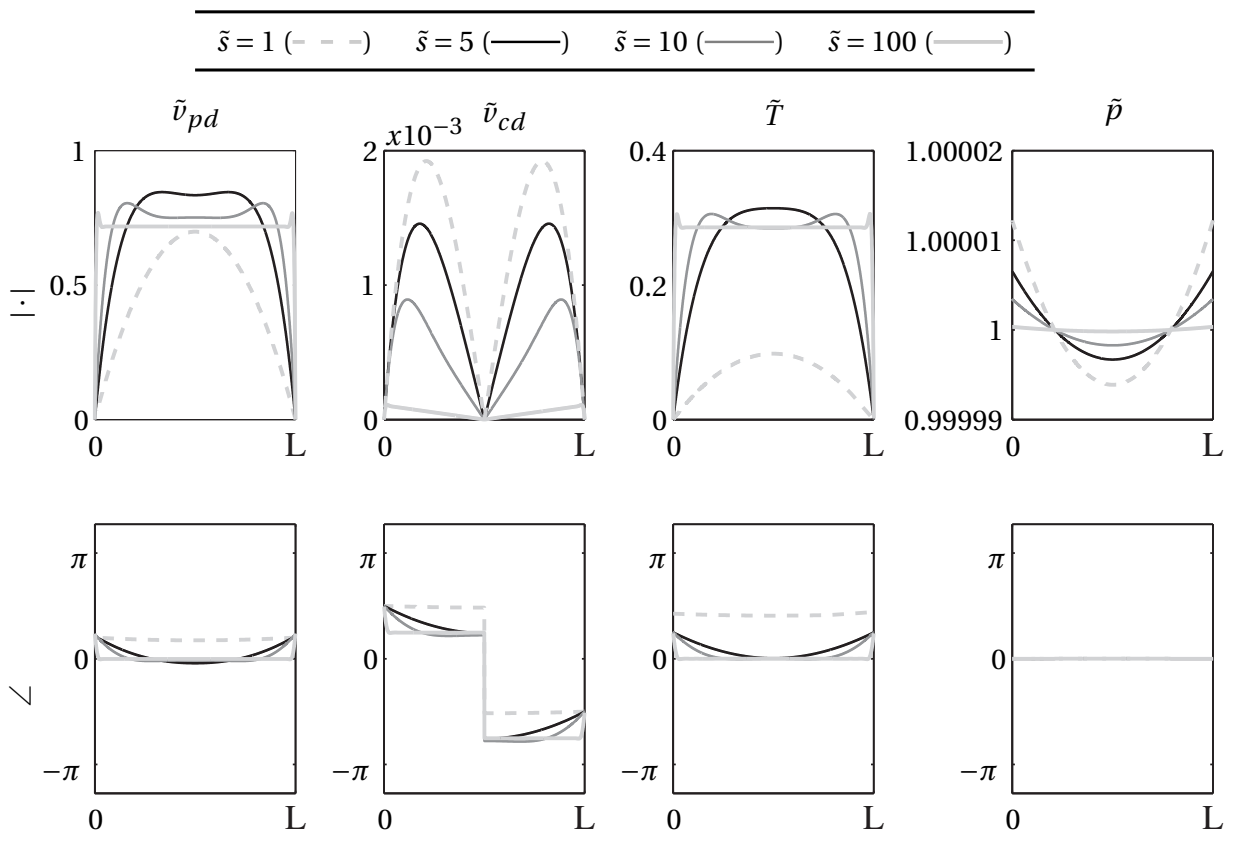

Figure 3.6: Velocity, temperature and pressure profiles for the first acoustic mode of a flat 2D waveguide for different shear wave numbers and a reduced frequency $\tilde{k}=0.01$.

normal direction, the temperature and the pressure of the first mode for $\tilde{k}=0.01$ and $\tilde{s}=1,5,10,100$. In figures $3.7,3.8$ and 3.9 the velocity, temperature and pressure profiles of the first three acoustic, vorticity and thermal modes are plotted. All modes are evaluated for $\tilde{k}=0.01$ and $\tilde{s}=10$. These plots can be useful when inspecting the orthogonality of the different mode shapes. Non-orthogonality of the different mode shapes can become important when using the mode-matching 
method which is described in section 3.6 and is applied to model various problems in chapter 6.

\subsubsection{Curved layers, spherical layers, tubes, and annular tubes}

In the second example the velocity and temperature profiles for the first acoustic mode are plotted for a straight layer, a curved layer, a spherical layer, a tube, and an annular tube. For all cases $\tilde{k}=0.01$ and $\tilde{s}=10$. The ratio between outer and inner radius for the annular and curved layer is a factor of 100 . The plots illustrate the similarities of viscothermal wave propagation in straight layers, tubes and annular tubes, and the substantial difference of viscothermal wave propagation in these geometries compared to that in curved layers. The differences in the propagation constant for the first mode for these geometries and the dependency of these differences on the shear wave number are commented on in section 4.2. Curved waveguides of arbitrary cross-sectional geometry are considered in the next section.

\subsection{Kirchhoff-FEM solutions}

The second solution strategy to obtain viscothermal acoustic waveguide modes for tubes of arbitrary cross section is presented here for the first time. It involves solving an FE model based on a discretization of equation (2.32) similar to that proposed in chapter 5 . Solutions of this type are approximate in nature; the expressions for both the mode shape and propagation constant are not known analytically and approximated by employing numerical techniques.

Note that the proposed method is an extension to the work of Astley and Cummings [39], who employed it to a simplified form of the Navier-Stokes equations (that accounted for an imposed steady flow). The assumptions proposed by Astley and Cummings that are necessary for the simplifications are identical to those required to obtain the LRF models presented in chapter 4 . Their model can thus be seen as an LRF model that accounts for mean flow. The method requires assuming a constant pressure over the waveguide cross section. An assumption that does not hold true for the higher order (acoustic) modes. Consequently, the higher order modes obtained with the model in [39] are no solutions to the Navier-Stokes equations. In contrast, no additional assumptions are required for the technique described below, which makes it suitable to obtain all viscothermal acoustic modes in straight, curved, coiled and twisted waveguides of arbitrary cross section (having constant curvature and cross section). The effects of a steady state mean flow as described in [39] can be easily incorporated in the new model if desired. 

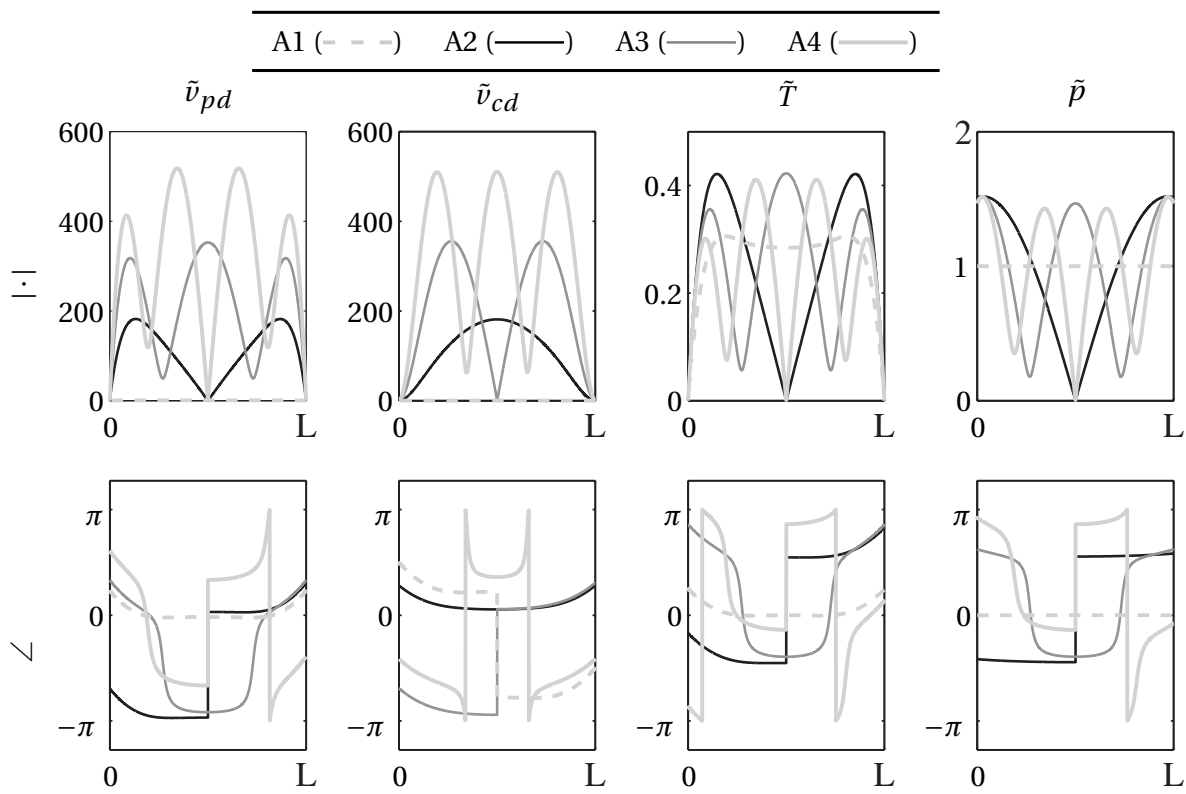

Figure 3.7: Velocity, temperature and pressure profiles for the first four acoustic modes (A1-A4) of a flat 2D waveguide for $\tilde{k}=0.01$ and $\tilde{s}=10$.
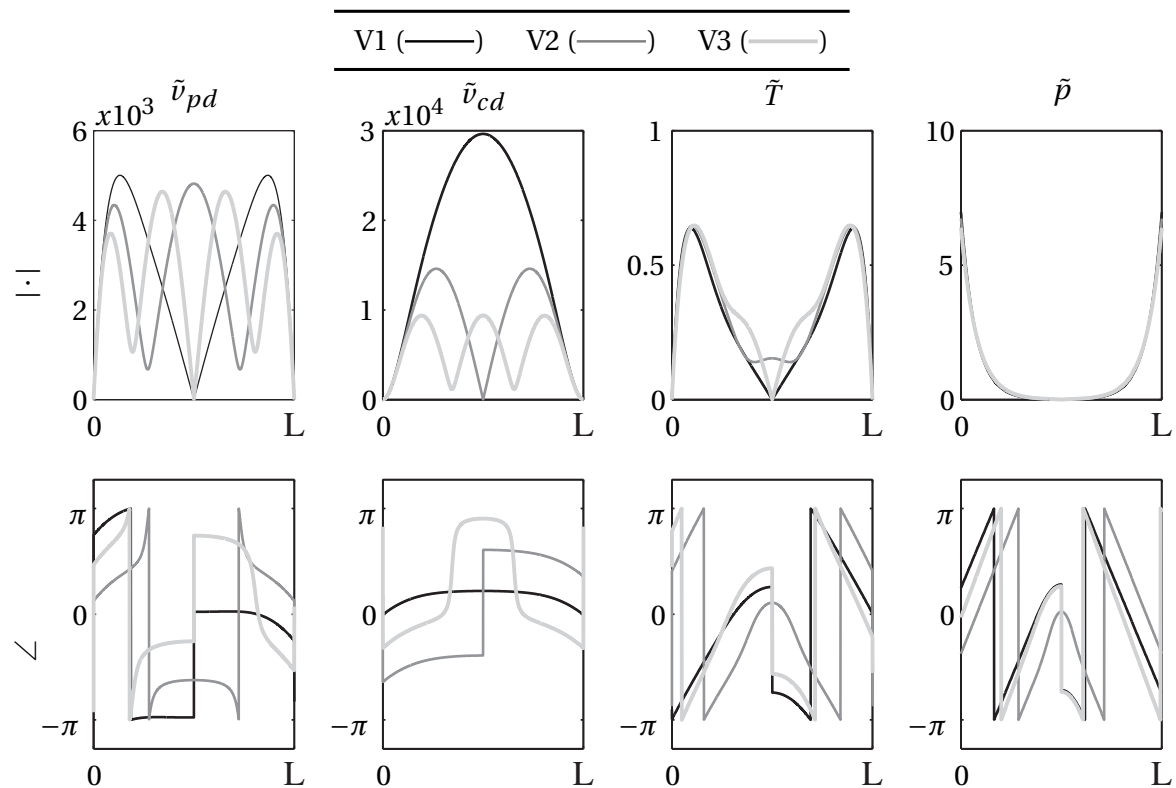

Figure 3.8: Velocity, temperature and pressure profiles for the first three vorticity modes (V1-V3) of a flat 2D waveguide for $\tilde{k}=0.01$ and $\tilde{s}=10$. 

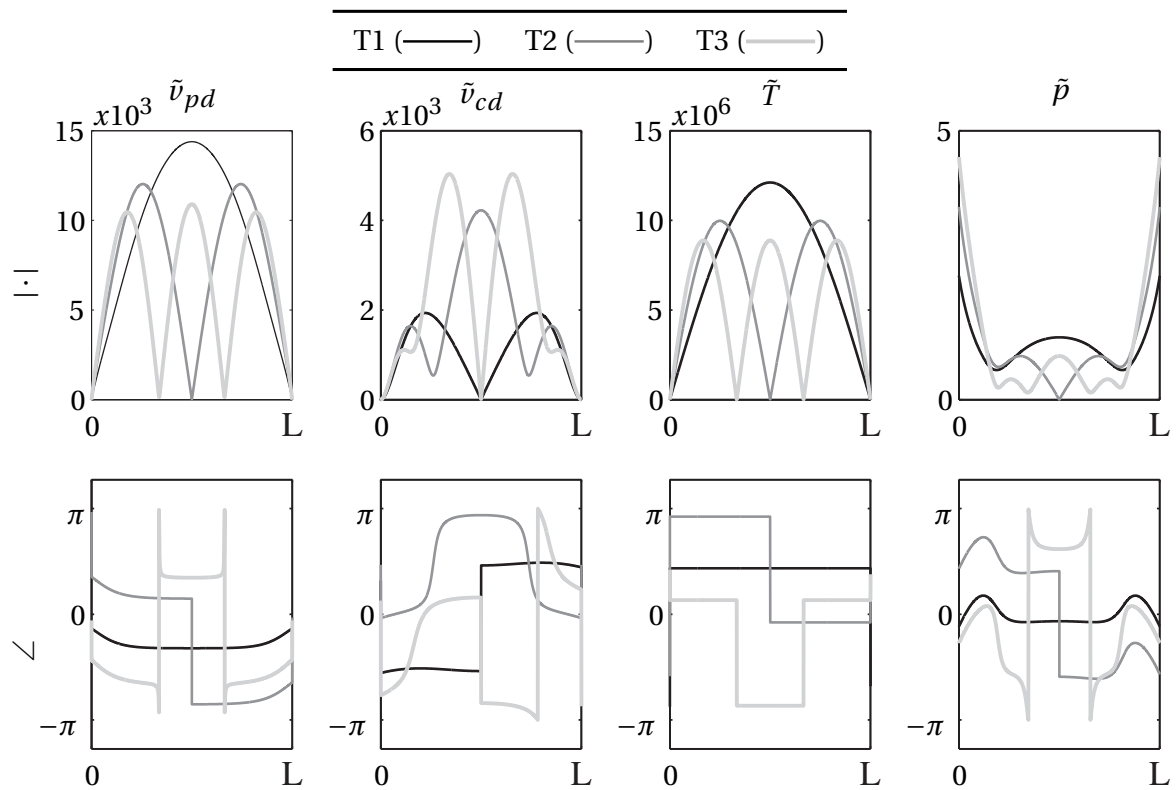

Figure 3.9: Velocity, temperature and pressure profiles for the first three thermal modes (T1-T3) of a flat 2D waveguide for $\tilde{k}=0.01$ and $\tilde{s}=10$.

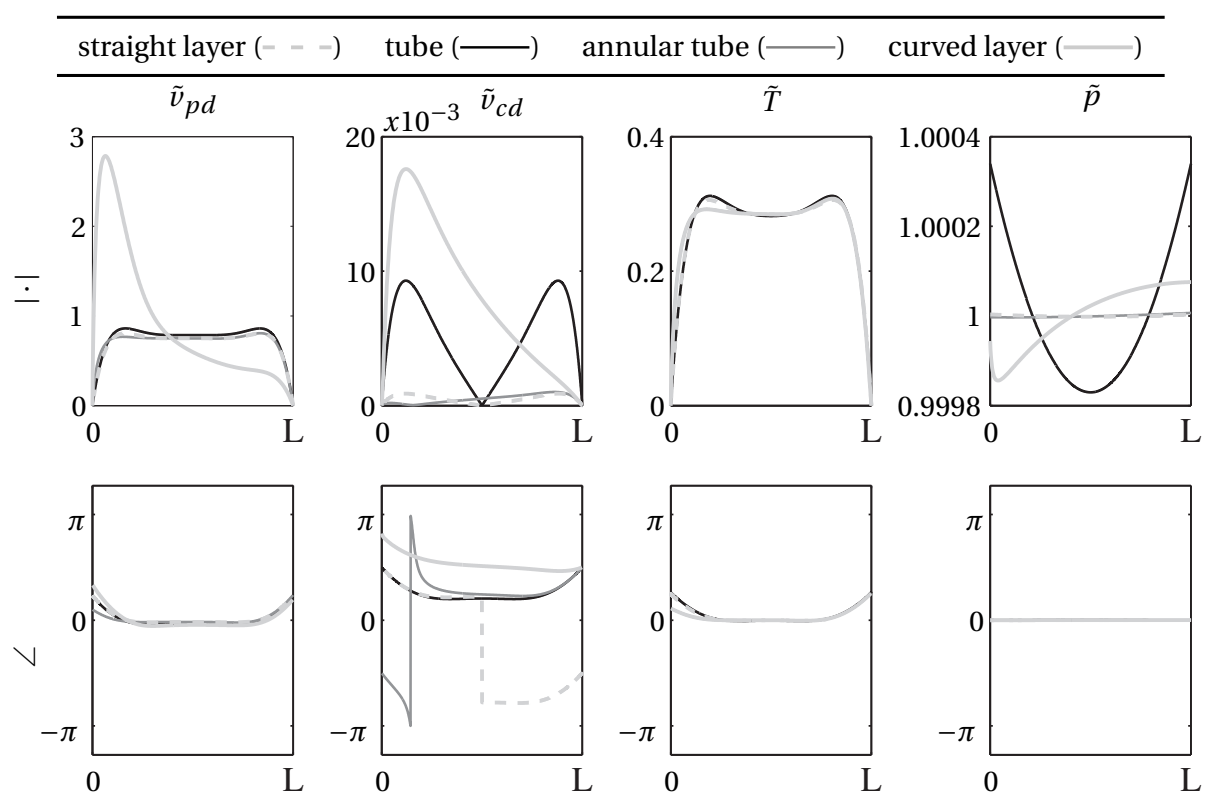

Figure 3.10: Velocity, temperature and pressure profiles for the first acoustic mode of different types of waveguides for $\tilde{k}=0.01$ and $\tilde{s}=10$. (For tubes, $L$ is the diameter.) 


\subsubsection{Solution strategy}

The strategy is presented here for $1 \mathrm{D}$ waveguides only, but can be easily modified to obtain numerical approximations of the solutions for layers that were presented in the previous sections. The method only applies to waveguides of constant cross sections and the boundary conditions on the waveguide surface are again assumed to be homogeneous and constant. An additional requirement is that the curvature and skewing angle between the plane of the cross section and the propagation constant are also constant. Under these conditions, the coefficients preceding the different derivatives in the gradient and Laplace operator do not depend on the $p d$-coordinates. This means the variables are separable in $p d$-coordinates and $c d$-coordinates. In order to satisfy constant boundary conditions over the entire waveguide surface, the $p d$-dependent part must be equal for velocity, temperature and pressure. Solving the $p d$-dependent part yields a solution in terms of exponential functions. Consequently, the expression for velocity, temperature and pressure for modes propagating/attenuating in the positive $p d$ direction can be cast into the following form

$$
\mathbf{v}=\mathbf{v}\left(\mathbf{x}_{c d}\right) e^{-i k \Gamma x_{p d}}, \quad T=T\left(\mathbf{x}_{c d}\right) e^{-i k \Gamma x_{p d}}, \quad p=p\left(\mathbf{x}_{c d}\right) e^{-i k \Gamma x_{p d}} \quad(3.37 \mathrm{a}, \mathrm{b}, \mathrm{c})
$$

Solutions of this form correspond to mode shapes in a waveguide that extends infinitely in positive and negative $p d$-direction. Two examples of waveguide geometries that meet these requirements are straight waveguides described in Cartesian coordinates (see figure 3.11(a)) and curved waveguides described in circular cylindrical coordinates (see figure 3.11(b)).

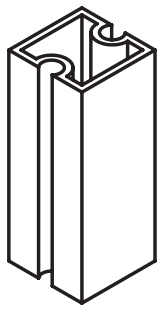

(a)

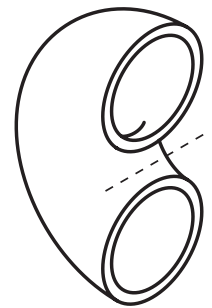

(b)

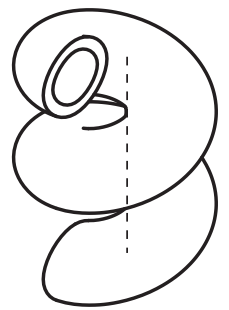

(c)

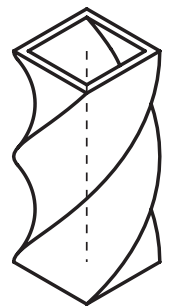

(d)

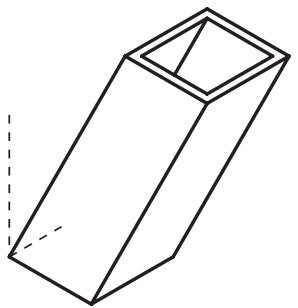

(e)

Figure 3.11: Waveguide geometries for which approximation solutions of the modes can be obtained: Straight tubes (a), curved tubes (b), coiled tubes (c), twisted tubes (d) and skewed tubes (e).

Equations (2.32) are brought into their weak form as described in chapter 5. The expressions for velocity, temperature and pressure in equation (3.37) are substituted and the $c d$-dependent part of the shape and weighting functions are ap- 
proximated by a discrete function space (while the $p d$-dependent parts are given by $\left.e^{i k \Gamma x_{p d}}\right)$. This results in an algebraic system of the form

$$
[K] \mathbf{u}=\mathbf{f}, \quad \text { with } \quad \mathbf{u}=\left\{\hat{\mathbf{v}}_{1}, \hat{\mathbf{v}}_{2}, \hat{\mathbf{v}}_{3}, \hat{\mathbf{T}}, \hat{\mathbf{p}}\right\}^{T}
$$

where the elements of the vector $\mathbf{u}$ are vectors $\hat{\mathbf{v}}_{1}, \hat{\mathbf{v}}_{2}, \hat{\mathbf{v}}_{3}, \hat{\mathbf{T}}$ and $\hat{\mathbf{p}}$ representing the degrees of freedom of the velocity, temperature and pressure fields, and the elements of vector $\mathbf{f}$ represent the natural boundary condition (and body forces) corresponding to each degree of freedom. Care must be taken when applying boundary conditions, since the solution in $p d$-direction is already explicitly assumed. The boundary conditions that are enforced on the waveguide cross section must match with the solution for the $p d$-direction. A mismatch introduces terms that act as body forces. To circumvent this issue, the divergence of the stress tensor and the gradient of temperature multiplied with the corresponding weighting functions are separated in a contribution in $p d$ - and $c d$-direction prior to using Greens theorem. The divergence is rewritten as

$$
\nabla \cdot \mathbf{A}=\nabla \cdot \mathbf{A}_{p d}+\nabla \cdot \mathbf{A}_{c d}, \quad \text { where } \quad \mathbf{A}=\{\sigma \cdot \mathbf{w}, q \nabla T\}
$$

where $\mathbf{w}$ and $q$ are the weighting functions for velocity and temperature respectively. For traction and thermal boundary conditions the $p d$-dependent part of $\mathbf{A}$ is given, respectively, by

$$
A_{p d}=\sigma_{p d} \cdot \mathbf{w} \quad A_{p d}=q \nabla_{p d} T
$$

where $\sigma_{p d}$ and $\nabla_{p d} T$ represent the part of the stress tensor and heat flux vector that corresponds with a force vector on, and heat flux through a surface perpendicular to the $p d$-coordinates, respectively. Terms resulting from the $p d$ components of $\mathbf{A}$ are retained in the right-hand side of the weak equations. The other terms are rewritten as boundary integrals by applying Greens theorem and moved to the left-hand side to serve as natural boundary conditions. Using this approach, the natural boundary conditions thus only enforce tangential forces, normal forces and heat fluxes resulting from gradients in stress and temperature that are perpendicular to the $p d$-direction ${ }^{4}$. Application of homogeneous boundary conditions on the waveguide surfaces now leads to an algebraic system of the form

$$
[M] \mathbf{u}=0, \quad \text { with } \quad M_{i j}=\int \frac{f\left(x_{c d}\right)}{|J|} d x_{c d} \int\left(e^{-i k \Gamma x_{p d}}\right)^{2} d x_{p d}
$$

\footnotetext{
${ }^{4}$ In the case of non-orthogonal coordinate systems (see section 3.4.2) these gradients are not necessarily gradients in $c d$-direction.
} 
where the function $f$ is an expression in terms of the $c d$-dependent part of shape and weighting functions and their first-order derivatives, and $J$ is the Jacobian matrix for the 3D coordinate system under consideration. The integral involving the $p d$-dependent terms will only affect the magnitude of the mode shapes $\mathbf{u}$, which is not of interest here, and can be taken to be equal to unity. The terms in matrix $[K]$ can be grouped by occurrence of $\Gamma$ and $\Gamma^{2}$ that result from the terms with a derivative in $p d$-direction. After regrouping, the system has the form of a quadratic eigenvalue problem

$$
\left(\Gamma^{2}[A]+\Gamma[B]+[C]\right)\{\mathbf{u}\}=0
$$

where $\Gamma$ is the propagation constant corresponding to the mode shape described by the eigenvector $u$. Several methods and algorithms are available to solve equations of the form in (3.42) (see [82] for an overview). A common approach is to rewrite the quadratic eigenvalue problem as an equivalent generalized eigenvalue problem

$$
\Gamma\left[\begin{array}{cc}
I & 0 \\
0 & -A
\end{array}\right]\{q\}=\left[\begin{array}{ll}
0 & I \\
C & B
\end{array}\right]\{q\}, \quad \text { with } \quad q=\left\{\begin{array}{c}
\mathbf{u} \\
\Gamma \mathbf{u}
\end{array}\right\}
$$

which can be solved using standard techniques. This system yields $2 n$ independent eigenvalues and eigenvectors for system matrices $[A],[B]$ and $[C]$ of size $n \times n$. The complex eigenvalues are found in pairs with opposite signs having identical eigenvectors. These solution pairs correspond to waves of identical shape traveling in the positive and negative propagation direction. Note that if the terms accounting for main flow are introduced, the symmetry of these pairs is lost and the solutions for upstream and downstream traveling waves will not be identical [39]. In section 3.4.3 this is demonstrated at the hand of an example.

\subsubsection{Non-orthogonal coordinate systems}

In contrast to the exact solutions described in sections 3.1 through 3.3 , no assumptions were made concerning the orthogonality of the coordinate system in the approximate method presented above. As long as the coefficients of the different derivatives in the gradient and Laplace operator do not depend on the $p d$ coordinates, the equations are separable in $p d$-coordinates and $c d$-coordinates, and the method can be successfully applied by straightforward substitution of the appropriate expressions for the gradient and divergence operator. Three examples of waveguides in non-orthogonal coordinate systems that meet the requirements stated above are coiled or twisted waveguides in helical coordinates (see figure 3.11(c) and 3.11(d)) and skewed waveguides in oblique coordinates (see figure 3.11(e)). Note that different definitions of helical coordinate systems can be found in the literature. An overview of a number of helical coordinate systems and 
references to studies involving their application to the (nonlinear) Navier Stokes equations for (non-Newtonian) viscothermal fluids are presented in [83]. Applying the proposed method to helical waveguides can be seen as an extension of the work of Campos and Serrao [84]. He presented an analysis of inviscid adiabatic wave propagation in terms of modes in a coiled waveguide described in helical coordinates.

A consequence of the requirement that all geometric features of the waveguide (such as cross section, curvature and skewing) are constant with the $p d$-coordinates is that these geometries can slide along the $p d$-coordinates within their bounding surfaces. Helical geometries are the only type of geometries that possess this property besides straight and rotation symmetric waveguides ${ }^{5}$. This suggests that the type of geometries depicted in figure 3.11 are the only types that allow for an approximate solution of the presented form.

\subsubsection{Examples of Kirchhoff-FEM solutions}

For a waveguide with the arbitrary cross-sectional geometry shown in figure 3.12(a), the differences in mode shapes between a straight and curved waveguide, and a straight waveguide with and without flow are illustrated in figure 3.13 .

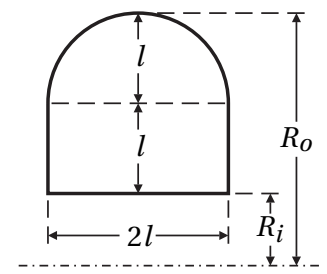

(a)

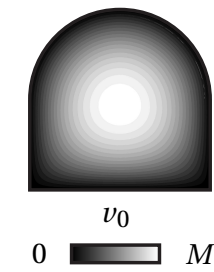

(b)

Figure 3.12: Waveguide geometry and characteristic dimensions (a). Velocity profile of the mean flow (b).

The values for the dimensionless wave numbers for all calculations are $\tilde{s}=10$ and $\tilde{k}=0.01$. The flow profile describing $\nu_{0}$ over the cross section of the waveguide for the case with mean flow is obtained (under the assumption of laminar flow) by solving the following Poisson equation for $\nu_{0}$ (in dimensional and dimensionless quantities, respectively);

$$
\mu \Delta v_{0}=\nabla_{p d} p_{0} \quad \frac{1}{s^{2}} \Delta \tilde{\mathbf{v}}_{0}=\frac{1}{k \gamma} \nabla_{p d} \tilde{p}_{0}
$$

\footnotetext{
${ }^{5}$ The equations for modes of helical waveguides include those for straight waveguides and curved waveguides as special cases. For the inviscid adiabatic acoustical case see [83, 84].
} 
where $\nabla_{p d} p_{0}$ and $\nabla_{p d} \tilde{p}_{0}$ are the dimensional and dimensionless pressure gradient in $p d$-direction of the solution at zero frequency. The pressure gradient that is used for the calculation of the results in figure 3.13 is such, that the maximum resulting velocity of the flow is Mach 0.025 (i.e., $\left.\max \left(\tilde{\mathbf{v}}_{0}\right)=0.025\right)$. The resulting velocity profile of the flow is given in figure 3.12(b). The dimensions of the cross section in terms of the characteristic length $l$ and the location of the axis of rotational symmetry with respect to the cross section for curved waveguides are sketched in figure 3.12(a). For the results in figure 3.13 the ratio between the most outer and inner part of the boundary, defined by $R_{0}$ and $R_{i}$, is 11 . The magnitude of all modes is normalized so that the maximum of the $p d$-velocity equals unity. The phase of the solution is normalized on the angle of $p$ integrated over the cross section. The two arrow plots given for each mode differ 90 degrees in phase, where the coloring indicates $\tilde{v}_{p d}$ at that phase.

From the results in figure 3.13, it is clear that the sound waves in a curved tube 'cut the corner', in the sense that the velocity is higher close to the inner boundary, This behavior was already observed for the first acoustic mode in a curved layer in figure 3.10. The effects of viscosity on this feature of wave propagation in curved tubes is elaborated on in section 4.2.2. Figure 3.13 suggests that the behavior also applies to higher order modes, since the velocity is higher closer to the inner boundary of the curved waveguide. However, the propagation constant for these modes, given in table 3.1, suggests differently.

\begin{tabular}{cccc}
\hline$\frac{R_{0}}{R_{i}}$ & $\Gamma_{A 1}$ & $\Gamma_{A 2}$ & $\Gamma_{A 3}$ \\
\hline 1 & $1.1036-0.1144 i$ & $-11.81-160.0 i$ & $-12.25-164.0 i$ \\
1.1 & $1.0984-0.1141 i$ & $-11.77-158.1 i$ & $-12.25-163.7 i$ \\
1.5 & $1.0777-0.1133 i$ & $-11.74-150.3 i$ & $-12.38-161.1 i$ \\
2 & $1.0538-0.1132 i$ & $-11.85-141.3 i$ & $-12.65-157.0 i$ \\
3 & $1.0151-0.1144 i$ & $-12.27-127.4 i$ & $-13.32-148.9 i$ \\
6 & $0.9456-0.1215 i$ & $-13.67-104.7 i$ & $-15.29-132.5 i$ \\
11 & $0.8935-0.1334 i$ & $-15.63-89.26 i$ & $-17.96-199.3 i$ \\
21 & $0.8547-0.1500 i$ & $-18.29-78.22 i$ & $-21.59-108.5 i$ \\
\hline
\end{tabular}

Table 3.1: Propagation constant for the first three acoustic modes in a curved waveguide (A1-A3) for $\tilde{s}=10$ and $\tilde{k}=0.01$. The propagation constant is given for several values of curvature of the waveguide.

The table shows that the real part of the propagation constant for the first mode decreases with increasing curvature, while the imaginary part increases. This suggests that the wave is traveling faster and is more damped by traveling 


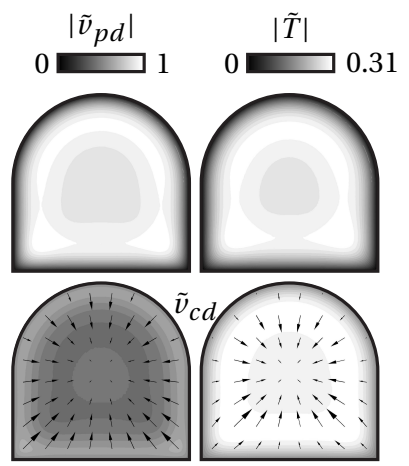

(a) Al, straight, no flow

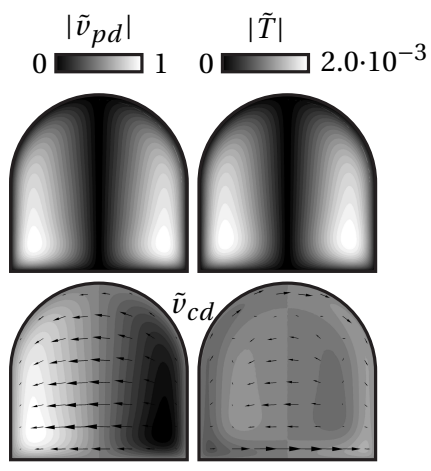

(d) A2, straight, no flow

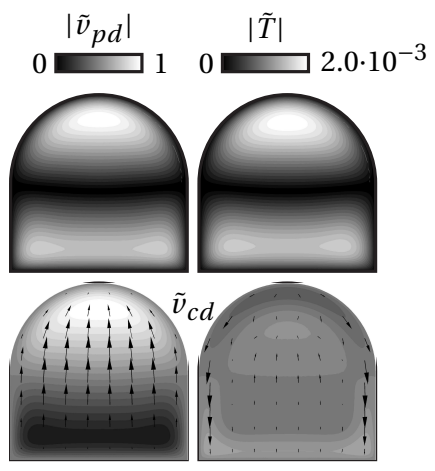

(g) A3, straight, no flow

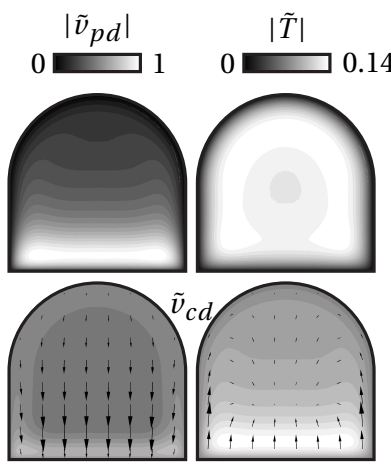

(b) A1, curved, no flow

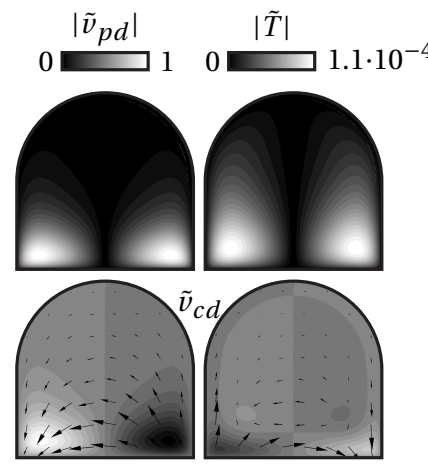

(e) A2, curved, no flow

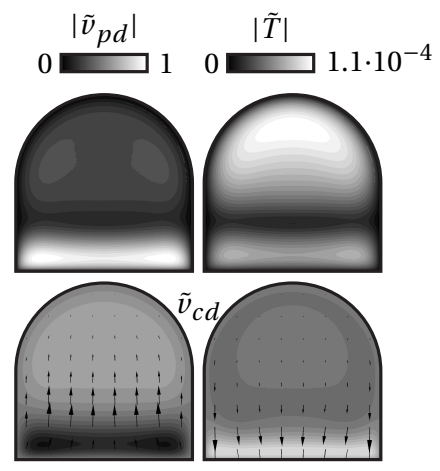

(h) A3, curved, no flow

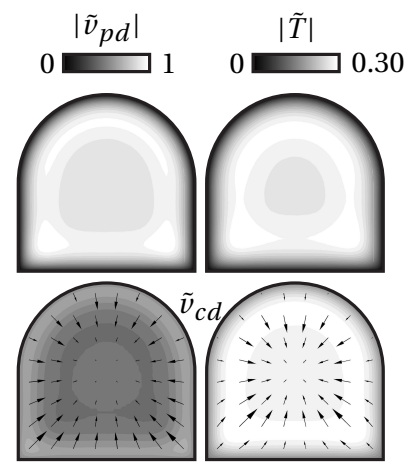

(c) A1, straight, flow

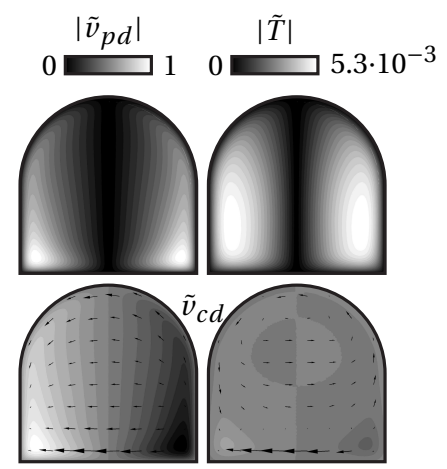

(f) A2, straight, flow

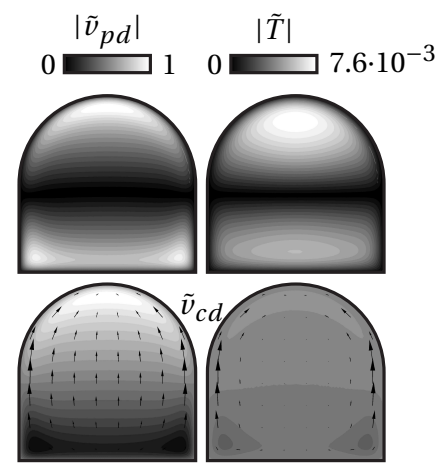

(i) A3, straight, flow

Figure 3.13: Velocity and temperature fields for the first three acoustic modes (A1A3) over the cross section of a straight and curved waveguide with and without a mean (Stokes) flow. Arrow plots for $\tilde{\mathbf{v}}_{c d}$ differ 90 degrees in phase (coloring represents $\tilde{v}_{p d}$ ). 
through tighter corners, which concurs with the premise based on the velocity data that the first acoustic mode 'cuts the corner'. For the higher order acoustic modes, the propagation constants behave opposingly different. The real part increases with curvature, while the imaginary part decreases. This suggests that the corresponding waves travel slower and damp out less as the waveguides curvature increases, which does not concur with 'cutting the corner'. The temperature profiles for the higher order modes in the curved waveguide differ significantly from those of the straight waveguide, while differences in temperature profile are relatively small for the first acoustic mode. This might explain the difference in the effect of waveguide curvature on propagation speed between the first and higher order modes. Note that if a straight and a curved waveguide of identical cross section are coupled both the difference in velocity profile and propagation constant of the different modes will affect how a propagating wave impinging on the junction will be scattered. A study on the errors that are made by neglecting these differences is presented in section 6.6.

It is already mentioned above that the symmetry between backward and forward waves is broken if a mean flow is present. The velocity profiles given in figure 3.13 for the waveguide with a mean flow correspond to higher order acoustic waves traveling downstream, i.e., in the same direction of the flow. The differences between the velocity profiles of the upstream and downstream traveling modes is illustrated by figure 3.14. All modes are normalized on the magnitude and the phase of $\tilde{p}$. The arrow plots for $\tilde{\mathbf{v}}_{c d}$ are all taken at the same phase, with the coloring indicating $\tilde{v}_{p d}$ at that phase.

The presented results are valid for $\tilde{s}=10, \tilde{k}=0.01$ and a Mach number of $M=0.1$ for the maximum velocity of the mean flow. The corresponding modes for the case without flow are depicted once more for ease of comparison. Note that the flow seems to push the velocity peaks in the velocity profiles slightly outward compared to the case without flow. The rippling in the profiles of the upstream traveling modes are only observed for higher order modes. This unexpected feature of the upstream modes appears not to be an artifact of the FE method that is used; it is mesh independent and is observed for all the (stable) element types described in chapter 5. The phenomena is dependent on the Mach number of the mean flow, however at $M=0$, the ripple-free solution of a waveguide without flow is obtained. As the Mach number increases the wavelength of the ripples decreases while the amplitude of the oscillations increases. Note that in many cases, mode shapes of lower order modes are smooth while higher order modes become increasingly oscillatory. In that light, the highly oscillatory ripples in the mode shape of the second and third mode seem somewhat strange. This apparent paradox can be explained by the fact that if the order of a mode is determined 

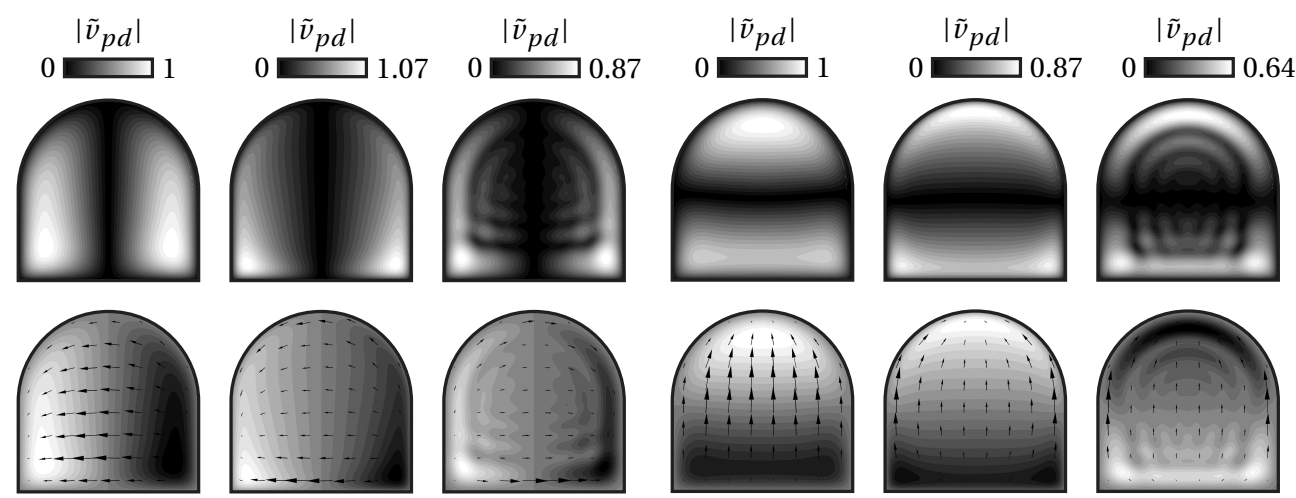

$\max \left|\tilde{\mathbf{v}}_{c d}\right|=0.97 \max \left|\tilde{\mathbf{v}}_{c d}\right|=0.86 \max \left|\tilde{\mathbf{v}}_{c d}\right|=0.70$

$\max \left|\tilde{\mathbf{v}}_{c d}\right|=0.81 \max \left|\tilde{\mathbf{v}}_{c d}\right|=0.61 \max \left|\tilde{\mathbf{v}}_{c d}\right|=0.49$

(a) A2, from left to right: no mean flow, downstream mode, upstream mode

(b) A3, from left to right: no mean flow, downstream mode, upstream mode

Figure 3.14: Velocity fields for the second and third acoustic modes (A2 and A3) over the cross section of a straight waveguide with and without a mean (Stokes) flow. Both downstream and upstream modes are presented.

by the distance of its propagation constant to the origin in the complex plane, the different modes shift in order as the Mach number increases. The labels 'second acoustic mode' and 'first acoustic mode' are based on the situation where $M=0$, but the propagation constant of many modes is closer to the origin as $M$ increases. The rippling phenomenon is expected to be a physical result from the interaction between the flow and the higher order acoustic waves, but is not further investigated here. The influence of the flow on the propagation constant of the different modes is illustrated by the results in table 3.2 .

Not surprisingly, these results show that the waves corresponding to the first acoustic mode traveling upstream are speeded up by the flow while waves traveling downstream are slowed down. The downstream traveling waves are damped less as the Mach number grows, while the upstream traveling waves are damped more with increasing Mach number. The higher order modes are damped less as the Mach number grows independent of the direction of the wave. Note that the direction of higher order modes is determined by the direction in which the amplitude of the wave attenuates. Using this definition, there are up- and downstream traveling waves for all considered wave numbers. In the work of Astley and Cummings [39], who presented an approximating model based on the LRF assumptions, it is mentioned that there are only downstream traveling waves above a certain Mach number well below unity. Note, however, that they are referring to the direction in which wave tops are traveling and not to the direction in which waves 


\begin{tabular}{cccc}
\hline$M$ & $\Gamma_{A 1+}$ & $\Gamma_{A 2+}$ & $\Gamma_{A 3+}$ \\
\hline 0 & $1.1036-0.1144 i$ & $-11.81-160.0 i$ & $-12.25-164.0 i$ \\
0.001 & $1.1029-0.1143 i$ & $-17.40-158.7 i$ & $-18.01-162.5 i$ \\
0.01 & $1.0970-0.1129 i$ & $-33.64-134.8 i$ & $-34.12-137.9 i$ \\
0.02 & $1.0905-0.1177 i$ & $-33.47-121.9 i$ & $-33.63-124.9 i$ \\
0.05 & $1.0712-0.1067 i$ & $-29.59-107.2 i$ & $-29.23-110.3 i$ \\
0.1 & $1.0400-0.0995 i$ & $-25.82-98.60 i$ & $-25.05-102.0 i$ \\
0.2 & $0.9811-0.0869 i$ & $-22.37-91.88 i$ & $-21.17-95.77 i$ \\
\hline$M$ & $\Gamma_{A 1-}$ & $\Gamma_{A 2-}$ & $\Gamma_{A 3-}$ \\
\hline 0 & $-1.1036+0.1144 i$ & $11.81+160.0 i$ & $12.25+164.0 i$ \\
0.001 & $-1.1042+0.1146 i$ & $5.975+159.8 i$ & $6.210+163.7 i$ \\
0.01 & $-1.1102+0.1161 i$ & $-14.04+137.1 i$ & $-13.87+140.4 i$ \\
0.02 & $-1.1168+0.1113 i$ & $-14.77+123.9 i$ & $-14.26+127.1 i$ \\
0.05 & $-1.1371+0.1229 i$ & $-11.80+109.1 i$ & $-10.87+112.7 i$ \\
0.1 & $-1.1719+0.1320 i$ & $-8.862+100.7 i$ & $-7.765+104.9 i$ \\
0.2 & $-1.2454+0.1525 i$ & $-6.319+94.25 i$ & $-5.237+99.04 i$ \\
\hline
\end{tabular}

Table 3.2: Propagation constant for the first three acoustic modes traveling downstream (A1+ through $\mathrm{A} 3+)$ and upstream (A1- through A3-) in a straight waveguide with Stokes flow for $\tilde{s}=10$ and $\tilde{k}=0.01$. The propagation constant is given for several values of the Mach number $M$ of the maximum velocity of the flow.

are attenuating. Taking this difference in definition into account, a similar phenomenon is observed here. However, the current study predicts that the wave tops of all higher order modes travel upstream above $M=0.01$ and not downstream as suggested by Astley and Cummings.

It must also be noted that for the current value of the shear wave number, the effects of viscosity on the convection of the first acoustic mode are significant. The wave speed predicted by the propagation constant in table 3.2 would not be obtained by correcting the wave speed for a waveguide without flow by assuming a plug flow ${ }^{6}$ of equivalent speed (for instance the average or maximum speed of the Stokes flow) and simply adding or subtracting it to the wave speed for the case without flow.

\footnotetext{
${ }^{6}$ Plug flows have a constant velocity profile.
} 


\subsection{LRF and inviscid adiabatic approximation}

In many cases, the first mode is most important to characterize the sound field in a waveguide. Under the assumptions that pressure variations in thickness directions are small and $k / s \ll 1$, an approximation of the first acoustic mode shape and corresponding propagation constant can be made. In this section, it is demonstrated that this approximation is equal to the expressions of the LRF model in section 3.5.2 in the case of $1 \mathrm{D}$ straight layers and prismatic cylindrical tubes. In addition, it is demonstrated that the viscothermal acoustic modes reduce to the inviscid adiabatic acoustic modes in the case of high shear and thermal wave number.

\subsubsection{Wave numbers}

Keeping in mind that $s_{t}=s \sqrt{\mathrm{Pr}}$, the wave numbers $k_{a}$ and $k_{h}$ as defined in equation (3.10) can be seen as functions of $k^{2} / s^{2}$. For most gases and liquids at atmospheric conditions this dimensionless ratio is small. This notion leads to a simplification of the expressions for the acoustic and thermal wave number. By taking the first order Taylor expansion of the wave numbers in equation (3.10) around $k^{2} / s^{2}=0$ the following approximations for the wave numbers are obtained for small values of $k^{2} / s^{2}$

$$
k_{a}^{2} \approx k^{2}, \quad k_{h}^{2} \approx-i s_{t}^{2}-(\gamma-1)\left[i s_{t}^{2}(\xi+2)+i s^{2}\right] \frac{k^{2}}{s^{2}}
$$

In the limit $k^{2} / s^{2} \downarrow 0$, the viscous and thermal boundary layers become infinitely small compared to the acoustic wavelength and the expressions for the wave numbers $k_{a}$ and $k_{h}$ become

$$
k_{a}^{2} \approx k^{2}, \quad k_{h}^{2} \approx-i s_{t}^{2}
$$

This analysis demonstrates that the wave number $k_{a}$ is closely related to the acoustic wave number $k$, while the wave number $k_{h}$ is closely related to the thermal wave number $s_{t}$ (see equation (2.33) for a definition of $k$ and $s_{t}$ ).

\subsubsection{LRF approximation}

A derivation of an approximate expression for the propagation constant of the first mode of a straight layer and circular prismatic tube with fixed isothermal walls is given below ${ }^{7}$. The expressions will turn out to be identical to the corresponding LRF models presented in the next chapter. For fixed isothermal walls $\alpha_{i}=1, \beta_{i}=0$

\footnotetext{
${ }^{7} \mathrm{~A}$ very concise version of the derivation can be found in [77].
} 
in equation (3.32). Assume $k_{2}=0$, so there is only propagation in one direction (the axial direction for tubes). Equating the determinant of $\mathbf{P}$ corresponding to the symmetric modes for no-slip, adiabatic boundary conditions (see (3.27)) to zero yields the following dispersion equation for both tubes and layers after rearranging the different terms

$$
\left(1-\frac{\alpha_{a}}{\alpha_{h}}\right) \frac{k_{3}^{2}}{k_{v}^{2}+k_{3}^{2}} \Psi_{v}+\Psi_{a}-\frac{\alpha_{h}}{\alpha_{a}} \Psi_{h}=0
$$

with

$$
\left.\begin{array}{ll}
\Psi_{m}=k_{m 1} \tan \left(k_{m 1} x_{1}\right) & \text { for layers } \\
\Psi_{m}=k_{m 1} \frac{J_{1}\left(k_{m 1} x_{1}\right)}{J_{0}\left(k_{m 1} x_{1}\right)} & \text { for tubes }
\end{array}\right\} \quad \text { with } m=\{a, h, v\}
$$

where

$$
k_{a 1}=\sqrt{k_{a}^{2}-k_{3}^{2}}, \quad k_{h 1}=\sqrt{k_{h}^{2}-k_{3}^{2}}, \quad k_{v 1}=\sqrt{k_{v}^{2}-k_{3}^{2}}
$$

and $x_{1}$ is the $c d$-coordinates at the boundary (half the layer thickness or the tube radius). From here on, it is assumed that $\sqrt{\operatorname{Pr}} \gtrsim 1$. Since $s_{t}=s \sqrt{\operatorname{Pr}}$, small values of $k^{2} / s^{2}$ imply small values of $k^{2} / s_{t}^{2}$, i.e., for small values of $k^{\prime} s^{2}$ the viscous and thermal boundary layers are small compared to the free wavelength. If the boundary layers are also considered small compared to the wavelength of bounded waves, then $k_{3}^{2} / s^{2}$ and $k_{3}^{2} / s_{t}^{2}$ must be small as well. Approximating $k_{h 1}$ and $k_{v 1}$ given in (3.49b) and (3.49c), respectively, by assuming $k_{3}^{2} / s_{t}^{2} \ll 1$ and $k_{3}^{2} / s^{2} \ll 1$ and using the approximation for $k_{h}$ for small values of $k^{2} / s^{2}$ given in (3.46b) leads to

$$
k_{h 1} \approx s_{t} i \sqrt{i}, \quad k_{\nu 1} \approx s i \sqrt{i}
$$

Using the relation $s_{t}=s \sqrt{\operatorname{Pr}}$ allows both $\alpha_{h}$ and $\alpha_{a}$ (equation (3.16)) to be written in terms of $k^{2} / s^{2}$. A first order Taylor expansion of $\alpha_{h} / \alpha_{a}$ around $k^{2} / s^{2}=0$ demonstrates that

$$
\frac{\alpha_{h}}{\alpha_{a}} \approx-(\gamma-1) \frac{k^{2}}{i s_{t}^{2}}
$$

for small values of $k^{2} / s^{2}$. Using this approximation, the definition $k_{v}=-i s^{2}$ (see equation (3.18)) and the fact that $k^{2} / s^{2} \ll 1$ implies $k^{2} / s_{t}^{2} \ll 1$ and $k_{3}^{2} / s^{2} \ll 1$ the dispersion equation in (3.47) can be approximated by

$$
-\frac{k_{3}^{2}}{i s^{2}} \Psi_{\nu}+\Psi_{a}+(\gamma-1) \frac{k^{2}}{i s_{t}^{2}} \Psi_{h}=0
$$


In addition, it is assumed that pressure variations in directions other than the propagation directions are small (an assumption also required to derive the LRF models). If equation (3.14) is considered for small values of $k^{2} / s^{2}$, the assumption that the pressure is nearly constant in non-propagation directions implies that the value of $k_{a 1} x_{1}$ must be small. The first order Taylor expansion of $\Psi_{a}$ in the case of layers and tubes around $k_{a 1} x_{1}=0$ demonstrates that

$$
k_{a 1} \tan \left(k_{a 1} x_{1}\right) \approx k_{a 1}^{2} x_{1}, \quad \quad k_{a 1} \frac{J_{1}\left(k_{a 1} x_{1}\right)}{J_{0}\left(k_{a 1} x_{1}\right)} \approx \frac{1}{2} k_{a 1}^{2} x_{1}
$$

for small values of $k_{a 1} x_{1}$. These results are substituted in equation (3.47) together with the expression for $k_{a 1}$ given in (3.49a), the approximations for $k_{v 1}, k_{h 1}$ and $\alpha_{h} / \alpha_{a}$ given in (3.50a), (3.50b), and (3.51), respectively. After rearranging the different terms extensively ${ }^{8}$, the following approximation for $k_{3} / k^{2}$ is obtained for layers and tubes, respectively

$$
\begin{aligned}
\frac{k_{3}^{2}}{k^{2}} \approx \frac{\gamma-(\gamma-1)\left[1-\frac{\tan \left(s_{t} i \sqrt{i} x_{1}\right)}{s_{t} i \sqrt{i} x_{1}}\right]}{1-\frac{\tan \left(s i \sqrt{i} x_{1}\right)}{s i \sqrt{i} x_{1}}} \\
\frac{k_{3}^{2}}{k^{2}} \approx \frac{\gamma-(\gamma-1) \frac{J_{2}\left(s_{t} \sqrt{i} x_{1}\right)}{J_{0}\left(s_{t} \sqrt{i} x_{1}\right)}}{\frac{J_{2}\left(s \sqrt{i} x_{1}\right)}{J_{0}\left(s \sqrt{i} x_{1}\right)}}
\end{aligned}
$$

These expressions for $k_{3}^{2} / k^{2}$ are identical to the LRF expression for the propagation constants $\Gamma$ for layers and tubes in equation (4.24).

\subsubsection{Inviscid adiabatic approximation}

For the inviscid adiabatic case, the shear wave number and thermal wave number become infinitely large. If equation (3.14) is evaluated for the limit $k^{2} / s^{2} \downarrow 0$ and is combined with equations (3.13), the Helmholtz equation for acoustic pressure with wave number $k$ is obtained (while the solution for temperature decouples). If equation (3.14) is combined with equation (3.15) for the same limiting case, Eulers equation is obtained. So, in the limit $k^{2} / s^{2} \downarrow 0$, the full system of equations reduces to the simplified inviscid adiabatic case as is to be expected.

\footnotetext{
${ }^{8}$ In the case of tubes, the following recurrence relation for Bessel functions is used: $J_{m}(x)=\frac{x}{2 m}\left[J_{m+1}(x)+J_{m-1}(x)\right]$
} 


\subsection{Mode Matching}

The modes obtained with the methods presented in this chapter apply to wave propagation in infinite waveguides. Without further assumptions, such solutions are mainly of academic interest, since realistic boundary conditions at the waveguide entrances will in general not match the velocity and temperature profiles of a specific mode. A common assumption that allows the use of waveguide modes is that of a relatively long waveguide. Under the assumption that the waveguide length is large compared to the dimensions of the cross section and the additional assumption that $k<1$, only the first acoustic mode will propagate while the higher order modes attenuate quickly. As a consequence, the contribution of the higher order modes to the velocity, temperature and pressure fields is only significant at short distances from the waveguide entrances. The propagation behavior of the waveguide is thus governed by the first acoustic mode; the so-called inlet effects of the higher order modes are significant close to the waveguide entrances only. However, to accurately describe realistic boundary conditions, the influence of higher order modes can become important. Examples include the boundary conditions at the interface of two waveguides of different cross section (see chapter 6). A technique, known as mode matching, that combines multiple higher order modes to obtain a more accurate description of the boundary conditions at the entrances of $1 \mathrm{D}$ waveguides is described for viscothermal wave propagation in this section. The focus is on boundary conditions needed to model scattering phenomena that occur at waveguide discontinuities.

Mechel $[75,76]$ recently presented a mode-matching method for viscothermal wave propagation. In contrast to the method presented here, Mechel's description of viscothermal wave propagation is not based on the full model. Before introducing the mode-matching scheme, he simplifies the governing set of equations by eliminating the temperature variable. The effects of the thermal boundary layer are accounted for by using an adjusted acoustic wave number for free field wave propagation. The adjusted wave number accounts for heat flow over the waveguide boundaries, thus preserving the non-adiabatic character of viscothermal wave propagation. As a result of the simplification, the method does not include thermal modes. In addition, Mechel disregards the vorticity modes and only uses acoustic modes ${ }^{9}$ to establish a coupling between different waveguides. The more general method presented here uses all modal types. In chapter 6 it is concluded that disregarding thermal modes will only cause minor inaccuracies, while disregarding vorticity modes can prevent the mode-matching method from converging to the physically correct solution for smaller wave numbers $(\tilde{s}<10)$.

\footnotetext{
${ }^{9}$ Mechel addresses the acoustic modes as Carcino modes.
} 


\subsubsection{Modal expansion}

To keep matters simple, the technique is presented here for straight waveguide sections (see figure 3.15) although it applies to curved, twisted and coiled waveguides as well. As a first step, the unknown velocity and temperature fields in each waveguide section are expressed as a combination of four infinite series of modes.

$$
f=\sum_{i=1}^{\infty} \sum_{j}\left[A_{i j+} f_{i j} e^{-i k_{i j} x}+A_{i j-} f_{i j} e^{i k_{i j} x}\right]
$$

where $f$ represents the different fields (a velocity component or temperature) and $j$ represents the different type of modes ( $a=$ acoustic, $t=$ thermal, $v_{1}=p d$-vorticity and $v_{2}=c d$-vorticity), i.e.

$$
f=\left\{\tilde{v}_{p d}, \tilde{v}_{c d 1}, \tilde{v}_{c d 2}, \tilde{T}\right\}, \quad \text { and } \quad j=\{a, v 1, v 2, t\}
$$

The functions $f_{i j}$ are the different dimensionless velocity and temperature profiles corresponding to mode $i$ and mode type $j, k_{i j}$ is the corresponding propagation constant, and $A_{i j+}$ and $A_{i j-}$ are the participation factors for the modes traveling/attenuating in positive and negative $x$-direction, respectively ${ }^{10}$. In the case of geometries that only allow velocities and temperature gradients in a single direction (such as two 1D layers - see figure 3.15(b) - or axisymmetrical circular geometries) the velocity and temperature fields are essentially 2D. Consequently, the velocities in one of the $c d$-direction can be omitted and $c d$-vorticity modes are not present, i.e.,

$$
f=\left\{\tilde{v}_{p d}, \tilde{v}_{c d}, \tilde{T}\right\}, \quad \text { and } \quad j=\{a, v, t\}
$$

In the remainder of this section, the focus is on geometries of this type (while the method is easily extended to waveguides of arbitrary cross section having $3 \mathrm{D}$ velocity and temperature fields). To improve legibility of the different equations, the $p d$ - and $c d$-velocities are denoted as

$$
\tilde{u}=\tilde{v}_{p d}=\tilde{v}_{c d}
$$

in the remainder of this section.

\footnotetext{
${ }^{10}$ The 'direction' of a wave is determined by the direction in which it attenuates, not by the sign of its phase speed. The phase speed of the higher order (evanescent) acoustic modes can become negative due to viscothermal effects, causing the direction of attenuation and the direction in which wave tops travel to be opposite.
} 


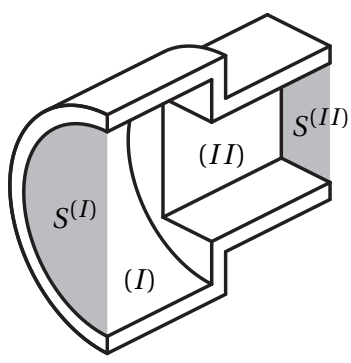

(a)

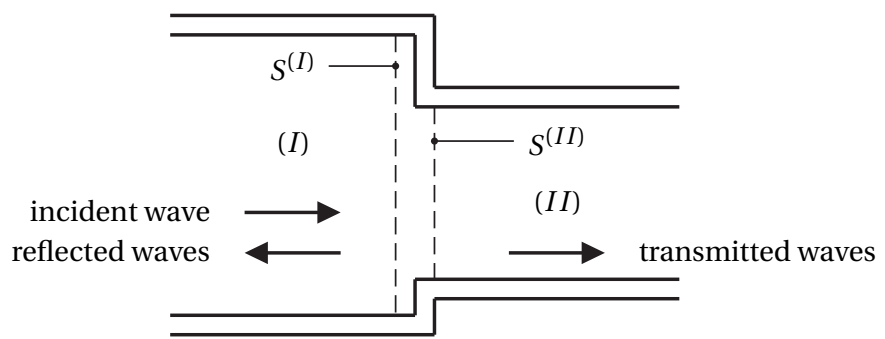

(b)

Figure 3.15: Schematic representation of scattering problems involving two waveguide sections resulting in a $3 \mathrm{D}$ and $2 \mathrm{D}$ mode-matching scheme: A coupled circular and square prismatic tube leading to a $3 \mathrm{D}$ model (a), and two coupled layers leading to a $2 \mathrm{D}$ model (b).

\subsubsection{Boundary conditions}

Two sets of velocity and temperature boundary conditions have to be prescribed at the waveguide entrances in order to determine the value of the participation factors and subsequently the resulting velocity and temperature fields of the waveguide. Naturally, instead of prescribing velocities and temperature, it is also possible to impose boundary values on their derivatives or combinations thereof. The different boundary conditions for velocity and temperature can be gathered conveniently in a matrix $L$ as described in section 2.7. Alternatively, the participation factors of waves traveling/attenuating in positive or negative $x$-direction can be prescribed directly, reducing the number of required boundary conditions.

In chapter 6, mode-matching is used to study scattering phenomena in waveguides. More precisely, scattering phenomena are investigated that occur when a propagating (incident) wave encounters a junction of two waveguides of different cross section (see figure 3.15(b)). The incident sound field (described by all waves traveling/attenuating in positive $x$-direction in section $I$ ) consists of the lowest acoustic mode only; the corresponding participation factor equals unity, while the participation factors of the other waves traveling/attenuating in positive $x$-direction equal zero. In the second waveguide section, no waves are assumed to be reflected from the unconnected entrance; all participation factors for waves traveling/attenuating in the negative $x$-direction equal zero. These "bound- 
ary conditions" can be summarized as

$$
\begin{array}{ll}
A_{0 a+}^{(I)}=1 & \\
A_{i j+}^{(I)}=0, & \forall i \in \mathbb{N}, j \in\{a, v, t\}: i, j \notin\{i=0 \wedge j=a\} \\
A_{i j-}^{(I I)}=0, & \forall i \in \mathbb{N}, j \in\{a, v, t\}
\end{array}
$$

The two boundary conditions that remain to be applied must realize the coupling between the two waveguide sections. If the medium in the waveguides is identical and if it is assumed that the medium is continuous at the interface, the boundary conditions at the interface must enforce conservation of energy and impedance (or alternatively admittance) at every point of the interface. For inviscid acoustics, these conditions are met if continuation of pressure and velocity are enforced at the interface; integrating the product of pressure and velocity over an infinitesimal area yields sound intensity at a point, while integrating the quotient yields acoustic impedance at a point. So, if pressure and velocity are continuous at each point, the product and quotient are identical for both waveguides and conservation of energy and impedance is satisfied at each point. In the case of viscothermal acoustics the conditions are met if continuity of velocity, temperature, stress vector (traction vector) and normal heat flux are enforced at the interface ${ }^{11}$. Enforcing continuity of these quantities is achieved by simply equating their values at the interface of two waveguide sections. In order to apply the boundary conditions involving the stress vector, it is convenient to introduce pressure as a fourth variable and expand it in terms of modes according to equation (3.56). Note that the expression for pressure is composed of the same functions used to express velocity and temperature (see equation (3.14)), so the modal behavior of the system and its solutions remain unchanged. Application of the continuity boundary conditions can now be simply stated as

$$
\left[L^{P}\right]\left\{\begin{array}{c}
\tilde{u}^{(I)} \\
\tilde{w}^{(I)} \\
\tilde{T}^{(I)} \\
\tilde{p}^{(I)}
\end{array}\right\}=\left[L^{P}\right]\left\{\begin{array}{c}
\tilde{u}^{(I I)} \\
\tilde{w}^{(I I)} \\
\tilde{T}^{(I I)} \\
\tilde{p}^{(I I)}
\end{array}\right\}, \quad\left[L^{D}\right]\left\{\begin{array}{c}
\tilde{u}^{(I)} \\
\tilde{w}^{(I)} \\
\tilde{T}^{(I)} \\
\tilde{p}^{(I)}
\end{array}\right\}=\left[L^{D}\right]\left\{\begin{array}{c}
\tilde{u}^{(I I)} \\
\tilde{w}^{(I I)} \\
\tilde{T}^{(I I)} \\
\tilde{p}^{(I I)}
\end{array}\right\}
$$

where the superscripts ${ }^{(I)}$ and ${ }^{(I I)}$ indicate the different waveguide sections, and

\footnotetext{
${ }^{11}$ Integrating the product of stress and velocity over an area, and integrating the normal heat flux over an area yields the energy flows due to work and heat conduction. Integrating the quotient of stress and velocity over an area yields mechanical impedance, while integrating the quotient of heat flux and temperature over an area yields thermal admittance.
} 
the operators $L^{P}$ and $L^{D}$ describing the boundary conditions are defined as

$$
L^{P}=\left[\begin{array}{llll}
1 & 0 & 0 & 0 \\
0 & 1 & 0 & 0 \\
0 & 0 & 1 & 0
\end{array}\right], \quad L^{D}=\left[\begin{array}{cccc}
\frac{1}{s^{2}}(\xi+2) \frac{\partial}{\partial x_{p d}} & \frac{1}{s^{2}} \frac{\partial}{\partial x_{c d}} & 0 & -\frac{1}{\gamma k} \\
\frac{1}{s^{2}} \frac{\partial}{\partial x_{c d}} & \frac{1}{s^{2}} \frac{\partial}{\partial x_{p d}} & 0 & 0 \\
0 & 0 & -\frac{1}{s_{t}^{2}} \frac{\partial}{\partial x_{p d}} & 0
\end{array}\right]
$$

The operator $L^{P}$ enforces boundary conditions on the primary variables, i.e., it enforces continuity of velocity and temperature. The operator $L^{D}$ enforces boundary conditions on the secondary or derived variables, i.e., it enforces continuity of stress and normal and tangential direction, and the normal heat flux. The first two rows of $L^{D}$ express the dimensionless stress vector in 2D Cartesian coordinates, and the last row expresses the dimensionless normal heat flux in Cartesian coordinates. The dimensionless stress vector $\tilde{\mathbf{f}}_{s}$ and the dimensionless normal heat flux $\tilde{q}_{n}$, are defined respectively as

$$
\tilde{\mathbf{f}}_{s}=\frac{1}{\omega \rho_{0} c_{0}} \tilde{\sigma} \cdot \mathbf{n}, \quad \quad \tilde{q}_{n}=\frac{R_{0}}{\omega p_{0} C_{p}} \mathbf{q} \cdot \mathbf{n}
$$

where $\tilde{\sigma}$ is the stress tensor as defined in equation (2.2), $\mathbf{q}$ is the heat flux vector as defined in equation (2.4)), and $\mathbf{n}$ is the unit vector in $p d$-direction.

Note that the continuity boundary conditions have to be satisfied only at the intersection, $S$, of the cross-sectional surfaces of the waveguides $\left(S=S^{(I)} \cap S^{(I I)}\right.$, see figure 3.15). Outside this area, the behavior of the walls that are perpendicular to the $p d$-direction must be prescribed. Using the operators $L^{D}$ and $L^{P}$, applying a prescribed velocity, temperature, stress vector, normal heat flux or acoustic/thermal impedances or admittances is straightforward. For the scattering problems investigated in chapter (6), the walls perpendicular to the $p d$-direction are assumed to be rigid and isothermal, and a no-slip condition is enforced. The boundary conditions at such walls can be written as

$$
\left[L^{P}\right]\left\{\begin{array}{l}
\tilde{u}^{(I)} \\
\tilde{w}^{(I)} \\
\tilde{T}^{(I)} \\
\tilde{p}^{(I)}
\end{array}\right\}=0
$$

An alternative way to prescribe these boundary conditions is to pad the different mode shapes of the waveguides with zeros outside the waveguides cross-sectional surfaces, and demand continuity of velocity and temperature over the union of the cross-sectional surfaces instead of the intersection ${ }^{12}$. This is the approach adopted below. An example of boundary conditions for nonrigid walls at the interface (for the inviscid acoustic case) is presented in [85].

\footnotetext{
${ }^{12}$ Continuity of the stress vector and normal heat flux is only demanded over the intersection of the cross-sectional surfaces.
} 


\subsubsection{Discretization}

The velocity, temperature and pressure in equation (3.59) and (3.62) are expanded in modes using equation (3.56). After enforcing a part of the boundary conditions by prescribing the corresponding participation factors as described in equations (3.58) the velocity, temperature and pressure in both sections can be written as

$$
\left\{\begin{array}{c}
\tilde{u}^{(I)} \\
\tilde{w}^{(I)} \\
\tilde{T}^{(I)} \\
\tilde{p}^{(I)}
\end{array}\right\}=\sum_{i=1}^{\infty}\left[M_{i}^{(I)}\right]\left\{A_{i-}^{(I)}\right\}+\left\{A_{0+}^{(I)}\right\}, \quad\left\{\begin{array}{l}
\tilde{u}^{(I I)} \\
\tilde{w}^{(I I)} \\
\tilde{T}^{(I I)} \\
\tilde{p}^{(I I)}
\end{array}\right\}=\sum_{i=1}^{\infty}\left[M_{i}^{(I I)}\right]\left\{A_{i+}^{(I I)}\right\}
$$

where the matrices $M_{i}^{(I)}$ and $M_{i}^{(I I)}$, and the vectors $A_{i-}^{(I)}, A_{0+}^{(I)}$ and $A_{i+}^{(I I)}$ are, respectively defined as

$$
\begin{gathered}
{\left[M_{i}^{(I)}\right]=\left[\begin{array}{lll}
u_{i q-}^{(I)} & u_{i \nu-}^{(I)} & u_{i t-}^{(I)} \\
w_{i a-}^{(I)} & w_{i \nu-}^{(I)} & w_{i t-}^{(I)} \\
T_{i q-}^{(I)} & T_{i \nu-}^{(I)} & T_{i t-}^{(I)} \\
p_{i a-}^{(I)} & p_{i v-}^{(I)} & p_{i t-}^{(I)}
\end{array}\right], \quad\left[M_{i}^{(I I)}\right]=\left[\begin{array}{lll}
u_{i q+}^{(I I)} & u_{i \nu+}^{(I I)} & u_{i t+}^{(I I)} \\
w_{i a+}^{(I I)} & w_{i \nu+}^{(I I)} & w_{i t+}^{(I I)} \\
T_{i q+}^{(I I)} & T_{i \nu+}^{(I I)} & T_{i t+}^{(I I)} \\
p_{i a+}^{(I I)} & p_{i v+}^{(I I)} & p_{i t+}^{(I)}
\end{array}\right]} \\
\left\{A_{i-}^{(I)}\right\}=\left\{\begin{array}{l}
A_{i a-}^{(I)} \\
A_{i \nu-}^{(I)} \\
A_{i t-}^{(I)}
\end{array}\right\}, \quad\left\{A_{0+}^{(I)}\right\}=\left\{\begin{array}{c}
A_{0 a+}^{(I)} \\
0 \\
0
\end{array}\right\}, \quad\left\{A_{i+}^{(I I)}\right\}=\left\{\begin{array}{l}
A_{i a+}^{(I I)} \\
A_{i j+}^{(I I)} \\
A_{i t+}^{(I I)}
\end{array}\right\}
\end{gathered}
$$

Note that the complex amplitudes of all waves were chosen equal to their participation factor at $x=0$ in order to arrive at these expressions (i.e., the amplitude of the incident acoustic wave at $x=0$ equals $A_{0 a^{+}}^{(I)}$ ). Using these expressions, the six equations in $(3.59 \mathrm{a}, \mathrm{b})$ and three equations in (3.62) can be written in terms of the six infinite series of unknown participation factors in (3.65a) and (3.65c) as

$$
\sum_{i=1}^{\infty}\left[\begin{array}{cc}
L^{P} M_{i}^{(I)} & -L^{P} M_{i}^{(I I)} \\
L^{D} M_{i}^{(I)} & -L^{D} M_{i}^{(I I)}
\end{array}\right]\left\{\begin{array}{c}
A_{i-}^{(I)} \\
A_{i+}^{(I I)}
\end{array}\right\}=-\left[\begin{array}{c}
L^{P} M_{0}^{(I)} \\
L^{D} M_{0}^{(I)}
\end{array}\right]\left\{A_{0+}^{(I)}\right\}
$$

where the top equation is expected to hold over the intersection of both waveguide surfaces, while the bottom equation is expected to hold only over their union. There are different techniques available to obtain an approximation of the values of the unknown participation factors. Two techniques that are encountered in the literature for the inviscid adiabatic case, being Galerkin mode-matching and point collocation, are discussed shortly. For a short discussion on the merits and drawbacks of both techniques for the inviscid adiabatic case see for instance [86, 87]. A summary of these discussions and the usefulness of both the methods in the viscothermal case are also given below. 


\section{Analytical or Galerkin mode matching}

The first technique is known alternatively as analytical mode matching or Galerkin mode matching. It involves multiplying each of the equations with a weighting function and integrating over the cross section of the waveguide. Using the Galerkin approach, the resulting equations are expected to hold (in an integrated sense) for all possible weighting functions in the solution space. The functions in this space are expanded in terms of a suitable (infinite) base. Successively, the infinite series in equations (3.56), and the infinite series resulting from expanding the space of weighting functions are both truncated. The maximum number of modes and the maximum number of weighting functions that are taken into account are $n$ and $m$, respectively, and the following algebraic system (of finite size) is obtained

$$
[K]\left\{\begin{array}{c}
A_{0-}^{(I)} \\
A_{0+}^{(I)} \\
\vdots \\
A_{n-}^{(I)} \\
A_{n+}^{(I)}
\end{array}\right\}=\left[\begin{array}{c}
\int_{S} L_{P} M_{0}^{(I)} W_{0}^{P} \\
\int_{U} L_{D} M_{0}^{(I)} W_{0}^{D} \\
\vdots \\
\int_{S} L_{P} M_{0}^{(I)} W_{m}^{P} \\
\int_{U} L_{D} M_{0}^{(I)} W_{m}^{D}
\end{array}\right]\left\{A_{0}^{(I)}\right\}
$$

where the matrix $K$ is defined as

$$
[K]=\left[\begin{array}{ccccc}
\int_{S} L^{P} M_{0}^{(I)} W_{0}^{P} & \int_{S} L^{P} M_{0}^{(I I)} W_{0}^{P} & \cdots & \int_{S} L^{P} M_{n}^{(I)} W_{0}^{P} & \int_{S} L^{P} M_{n}^{(I I)} W_{0}^{P} \\
\int_{U} L^{D} M_{0}^{(I)} W_{0}^{D} & \int_{U} L^{D} M_{0}^{(I I)} W_{0}^{D} & \cdots & \int_{U} L^{D} M_{n}^{(I)} W_{0}^{D} & \int_{U} L^{D} M_{n}^{(I I)} W_{j}^{D} \\
\vdots & & & & \\
\int_{S} L^{P} M_{0}^{(I)} W_{m}^{P} & \int_{S} L^{P} M_{0}^{(I I)} W_{m}^{P} & \cdots & \int_{S} L^{P} M_{n}^{(I)} W_{m}^{P} & \int_{S} L^{P} M_{n}^{(I I)} W_{j}^{P} \\
\int_{U} L^{D} M_{0}^{(I)} W_{m}^{D} & \int_{U} L^{D} M_{0}^{(I I)} W_{m}^{D} & \cdots & \int_{U} L^{D} M_{n}^{(I)} W_{m}^{D} & \int_{U} L^{D} M_{n}^{(I I)} W_{j}^{D}
\end{array}\right]
$$

where $S$ and $U$ are the intersection and union of the cross-sectional surfaces of the waveguides $S^{(I)}$ and $S^{(I I)}$ (see figure $3.15(\mathrm{~b})$ ), respectively, and $W_{j}^{P}$ and $W_{j}^{D}$ are diagonal matrices of size $3 \times 3$. The diagonal elements of these matrices are weighting functions, allowing the individual boundary conditions defined by $L^{P}$ and $L^{D}$ to be weighted differently.

\section{Point collocation}

A second approach to obtain an approximate solution to equation (3.66) is known as point collocation. Instead of enforcing the boundary conditions in a weighted sense over the entire boundary, the boundary conditions are enforced at specific points on the boundary. This results in an algebraic system of the form in equation 
(3.67), however, the matrix $K$ is now defined as

$$
[K]=\left[\begin{array}{ccccc}
\left.L^{P} M_{0}^{(I)}\right|_{x_{c d}=S_{0}} & \left.L^{P} M_{0}^{(I I)}\right|_{x_{c d}=S_{0}} & \cdots & \left.L^{P} M_{n}^{(I)}\right|_{x_{c d}=S_{0}} & \left.L^{P} M_{n}^{(I I)}\right|_{x_{c d}=S_{0}} \\
L^{D} M_{0}^{(I)} \mid x_{c d}=U_{0} & \left.L^{D} M_{0}^{(I I)}\right|_{c d}=U_{0} & \cdots & \left.L^{D} M_{n}^{(I)}\right|_{x_{c d}=U_{0}} & \left.L^{D} M_{n}^{(I I)}\right|_{c d}=U_{0} \\
\vdots & & & & \vdots \\
\left.L^{P} M_{0}^{(I)}\right|_{x_{c d}=S_{m}} & \left.L^{P} M_{0}^{(I I)}\right|_{x_{c d}=S_{m}} & \cdots & \left.L^{P} M_{n}^{(I)}\right|_{x_{c d}=S_{m}} & \left.L^{P} M_{n}^{(I I)}\right|_{x_{c d}=S_{m}} \\
\left.L^{D} M_{0}^{(I)}\right|_{x_{c d}=U_{m}} & \left.L^{D} M_{0}^{(I)}\right|_{x_{c d}=U_{m}} & \cdots & \left.L^{D} M_{n}^{(I)}\right|_{x_{c d}=U_{m}} & \left.L^{D} M_{n}^{(I I)}\right|_{x_{c d}=U_{m}}
\end{array}\right]
$$

where the elements of $S_{j}$ and $U_{j}$ are the coordinates of the locations where the boundary conditions are enforced on the union and intersection of the cross-sectional surfaces, respectively. Note that point collocation can be seen as a form of analytical mode-matching. In that setting, the weighting functions in the analytical mode-matching scheme are Dirac functions that are non-zero at the evaluation points, which form an orthogonal incomplete basis for the boundary conditions that are to be prescribed. The location of the evaluation points influences the condition of the resulting matrix. To avoid a tedious search for the best location with respect to accuracy of the resulting solution, an overdetermined system of equations can be formulated by taking into account more evaluation points than modes. An approximate solution to such a system can be found by using a least squares algorithm.

\section{Accuracy and convergence}

The basis in which the space of weighting functions is expanded must be chosen with care, since this choice influences the accuracy of the calculated participation factors. The best accuracy that can be achieved is, however, limited by the shape functions (the mode shapes in (3.64)), which are determined by the problem at hand. This can be understood by realizing that mode-matching methods are in essence Trefftz methods; a finite number of exact solutions to the system of differential equations is combined to meet the boundary conditions at the interface. Note that in order to guarantee convergence of the Trefftz approximation to the solution of the boundary value problem, the finite set of exact solutions must be a subset of a so-called T-complete set. For the Helmholtz equation, such sets are available for different coordinate systems, making the Trefftz method applicable to solve inviscid adiabatic acoustic problems. However, for the set of equations describing viscothermal acoustics, the T-completeness of the corresponding set of eigenfunctions has not been proven. In this thesis, it is simply assumed that the eigenfunctions form a T-complete set, and in chapter 6 it is demonstrated that the method presented here indeed converges to a reference FE solution for the cases considered (however, this does not necessarily imply that eigenfunctions form a T-complete set). 
As is generally the case with Trefftz methods [81], the problem of finding the participation factors in equation (3.67) involves solving a system of equations that becomes ill-conditioned as more modes and weighting functions are taken into account. The condition of the system of equations is affected by the orthogonality of the mode shapes and the choice of the weighting function. Note that in the context of mode-matching the combination of the velocity and temperature profiles and the profiles describing the stress vector and heat flux define the mode shape of each mode. If the mode shapes that are used to construct the system of equations in (3.67) are not orthogonal, arbitrary linear combinations of the mode shapes can become almost linearly dependent as more modes are added to the system. It is observed that for practical choices of the weighting functions (see below) non-orthogonality of the mode-shapes causes the system to become increasingly ill-conditioned, i.e., the system becomes (almost) rank deficient from a numerical point of view. An ill-conditioned system leads to serious numerical inaccuracies in the calculated participation factors, rendering the resulting solution inaccurate or even useless. This drawback of Trefftz methods limits the number of higher order modes that can be taken into account and thus the accuracy that ultimately can be attained. At the same time, non-orthogonality of the shape functions requires taking into account a relatively high number of modes since the participation factors of higher order modes influence the participation factors of lower order modes. Three causes for non-orthogonality of the shape functions that have a negative effect on the condition of the system of equations are discussed in appendix G. Note that it is the combination of shape functions (determined by the problem at hand) and weighting functions that determines the condition of the system. A short discussion on the choice of weighting functions is given below.

\section{Weighting functions}

Unfortunate combinations of weighting and shape functions can cause the algebraic system resulting from a Galerkin mode-matching scheme to become ill-conditioned. In that case, small changes in the coefficients of the system matrix or right-hand side vector, for instance due to numerical inaccuracies, cause changes in the solution of much greater order. In other words, ill-conditioning limits the expected accuracy of the solution obtained by solving an algebraic system of equations numerically. Obviously, it is desirable that the combination of weighting and shape functions leads to a well-conditioned system implying the solution is relatively insensitive to numerical inaccuracies. However, finding a set of weighting functions that makes such a fortunate combination is not a trivial task if the set of shape functions does not form an orthogonal set.

The shape functions are governed by the problem at hand, and determine 
which sets of weighting functions lead to ill-conditioned systems, and which sets lead to well-conditioned systems. Unfortunately, a priori information on the relation between weighting functions and the condition of the resulting system is not available. It was found that using the mode shapes as weighting functions (e.g., using the Bubnov-Galerkin approach) generally worsens ill-conditioning due to strong non-orthogonality. Similar conclusions were drawn by Kirby [86] and Mechel [76] (both, however, do not explicitly mention the Trefftz method, and only Mechel reports that the system of equations becomes ill-conditioned by taking more modes into account).

Kirby reports that the Bubnov-Galerkin approach (using the shape functions as weighting functions) works well for the inviscid adiabatic case if the problem describing the individual waveguide sections are Sturm-Liouville. In that case, the velocity and temperature profiles form orthogonal sets, and the resulting mode shapes are only slightly non-orthogonal (see appendix G), so inaccurate results are only to be expected when many higher order modes are taken into account. However, if the velocity and pressure profiles are non-orthogonal, for instance due to dissipative walls or the presence of a mean flow, the mode shapes become strongly non-orthogonal. Kirby states that in these cases using the Bubnov-Galerkin approach does not guarantee that the approximate solution obtained with modematching converges to the physical solution. He concludes that using a pointcollocation scheme instead of analytical mode-matching remedies the problems due to non-orthogonality.

Mechel, who investigated analytical mode-matching for the viscothermal case, recognizes that the mode shapes of the acoustic modes can become less orthogonal due to the effects of viscosity. Only acoustic modes are used in his approach, and the boundary conditions at the interface only enforce continuity of pressure and normal velocity. The shape functions are thus a combination of the pressure and velocity profiles. Mechel experiments with different weighting functions, but for the most part uses a combination of two sets of weighting functions. The first set weights the velocity part of the shape function with the velocity profiles, while the pressure part is multiplied by zero. The second set weights the pressure part of the shape function with potential functions, while the velocity part of the shape function is multiplied by zero. Note that the potential functions are equal to the pressure profiles of the different modes if forces due to bulk deformation are neglected. The weighting functions are thus not identical to the shape functions, nevertheless, they are constructed from almost similar profiles. Mechel reports that the condition of the resulting algebraic system can become ill-conditioned, but insists that taking more modes into account improves the accuracy of the solution, although convergence is not proven. 
A parameter study was carried out to determine which set of weighting functions provides accurate results for various values of the acoustic and shear wave number and various discontinuities in cross section. The approaches that were tested included the Bubnov-Galerkin approach, point collocation, using a base of sine and cosine functions, and an extension of the method proposed by Mechel that incorporates vorticity modes. The result was compared with reference solutions obtained with the FE approach described in chapter 5. It was found that point collocation (using more evaluation points than modes) yields satisfactory results in all cases, and often performs best with respect to the obtained accuracy of the first propagating mode and the errors in the velocity profiles at the interface. The results obtained using mode-matching presented in section 6.7 are all obtained by using point collocation. In all these cases, convergence is not yet halted due to ill-conditioning of the system.

\section{Edge condition}

Note that in the inviscid adiabatic case, the ratio between the number of modes that is taken into account in each waveguide section must be chosen so that the so-called edge condition is satisfied [85, 88]. If this condition is not met, an energy flow of infinite magnitude can occur at sharp edges in the approximate solution, which prevents the mode-matching approximation from converging to the correct solution. This problem is related to the magnitude of the fluid velocity at sharp edges which also tends to infinity for the inviscid adiabatic case. In the viscothermal case with a no-slip condition at the boundaries, the fluid velocity is zero at the entire boundary, including the sharp edges found at discontinuities in cross section. It can therefore be assumed that the edge condition is always satisfied independent of the ratio between the number of modes that is taken into account in the different waveguide sections. A parameter study that was carried out supported this conclusion (The ratio between the number of modes that is taken into account in each waveguide influences the accuracy, but does not effect the convergence behavior). The results obtained using mode-matching that are presented in this thesis are all obtained by taking the same number of modes into account in each waveguide section.

\subsection{Summary and discussion}

Two solution strategies to obtain solutions to the full set of LNSF equations in terms of modes were presented. The first strategy yields mode shapes that can be expressed in closed form, while the corresponding propagation constants are 
obtained by finding the roots of a transcendental equation with numerical techniques. The second strategy leads to a discrete eigenvalue problem yielding numerical approximations of the mode shapes and propagation constants (being the eigenvectors and eigenvalues, respectively).

For each strategy it is made plausible that the geometries for which solutions are presented in this chapter are the only type of waveguide geometries for which solutions of the presented form can be obtained. The waveguide geometries for the first strategy are geometries with two pd-directions and include a straight layer, a cylindrical layer ${ }^{13}$ and a spherical layer. The waveguide geometries for the second strategy are 1D waveguide geometries and include straight, skewed, curved, coiled and twisted tubes of arbitrary cross section.

It was demonstrated that the LRF solutions for straight layers and cylindrical prismatic tubes that are presented in the next chapter are in fact approximations of the first acoustic modes found by solving the full set of LNSF equations. The assumptions used in this derivation are identical to those that are required to obtain the LRF solutions.

Finally, the concept of mode matching was discussed. Using this technique, waveguides of different geometry can be coupled by demanding continuity of velocity, temperature, stress and normal heat flux at the waveguide interfaces. These quantities are represented in terms of modal contributions for the individual waveguides. The infinite series of modes are truncated and the equations are solved approximately using the Galerkin approach or point collocation. It is shown that the method classifies as a Trefftz method, which generally involves solving a system of equations that becomes increasingly ill-conditioned as the number of independent exact solutions (e.g., the number of modes) that is taken into account is increased. It is explained that the condition of the system of equations is affected by the method used to weight different equations in the discrete model. Different methods of discretization were investigated, and it was found that a point collocation scheme, using more evaluation points than the number of accounted modes, yields satisfactory results in all cases, and often performs best with respect to the accuracy of the solution.

\footnotetext{
${ }^{13}$ Cylindrical prismatic (annular) tubes can be thought of as special cases of a cylindrical layer.
} 


\section{Chapter 4}

\section{Low reduced frequency models}

In this chapter, so-called Low Reduced Frequency models (LRF models) are introduced in a general setting. The presented LRF models are characterized by the feature that they are based on a simplified form of the full set of LNSF equations, and the fact that the accuracy of these approximate models is dependent on the (reduced) frequency. In the literature, LRF models are alternatively known as ZwikkerKosten-Daniels models, or (in many cases) are not endowed with a specific name. Derivations of (semi-) analytical LRF models can be found for rectangular, circular and elliptically shaped plane layers and straight prismatic tubes of rectangular, triangular, circular (closed and annular) cross section [10, 20, 21, 22, 36, 66]. In the present study, the semi-analytical LRF equations are also derived for elliptical (closed and annular) straight prismatic tubes and tapered and curved tube and layer geometries. In addition, the general framework for LRF models is extended to include tangential velocity boundary conditions and temperature boundary conditions that may vary throughout the waveguide.

In section 2.6, the LNSF equations were presented in dimensionless form. Applying assumptions concerning the different dimensionless parameters and boundary conditions to the dimensionless LNSF equations leads to a set of simplified equations which form the basis for the different LRF models. This set of equations can be partially solved to obtain a general solution for different LRF models. In the first section of this chapter, this general framework for LRF models is presented, and the general form of a solution of LRF models is derived. Subsequently, LRF models and their solutions for specific geometries are given in section 4.2. The next section describes techniques for coupling multiple LRF models which make it possible to describe problems involving more complex geometries. 


\subsection{General Solution}

\subsubsection{Splitting operators}

To facilitate an easy comparison of the order of magnitude of the different terms in the system a new, more compact notation is introduced. It is convenient to split terms involving gradients in pressure, heat flux, volume and viscous stresses into their contribution in $c d$-directions and $p d$-directions. For terms involving the gradient operator, such a separation is straightforward. The gradient operator is written as the sum of two individual operators

$$
\nabla=\nabla_{p d}+\nabla_{c d}
$$

with $\nabla_{p d}$ and $\nabla_{c d}$ the individual contribution of the gradient in $p d$ - and $c d$-directions respectively. Such a straightforward separation is, however, not always possible for terms involving the divergence operator, the Laplacian, and the vector Laplacian. To illustrate this, the gradient operator and velocity vector $\mathbf{v}$ are written in terms of their vector components as

$$
\begin{aligned}
\nabla & =\mathbf{e}_{1} \frac{1}{h_{1}} \frac{\partial}{\partial x_{1}}+\mathbf{e}_{2} \frac{1}{h_{2}} \frac{\partial}{\partial x_{2}}+\mathbf{e}_{3} \frac{1}{h_{3}} \frac{\partial}{\partial x_{3}} \\
\mathbf{v} & =\mathbf{e}_{1} v_{1}+\mathbf{e}_{2} v_{2}+\mathbf{e}_{3} v_{3}
\end{aligned}
$$

where $\mathbf{e}_{i}$ is the unit vector in the direction of the $i$-coordinate and $h_{i}$ the shape factors corresponding to the metric of the coordinate system that is used (see appendix $\mathrm{D}$ for a definition of the shape factor). The direction of the unit vector determines whether a term is labeled as a contribution in $p d$-or $c d$-direction. Using this notation, the divergence of a vector $\mathbf{v}$ and the Laplace operator can now be written in terms of vector components as (see for instance [89])

$$
\begin{aligned}
\nabla \cdot \mathbf{v} & =\sum_{i=1}^{3} \sum_{j=1}^{3}\left[\mathbf{e}_{i} \cdot \mathbf{e}_{j} \frac{1}{h_{i}} \frac{\partial v_{j}}{\partial x_{i}}+\mathbf{e}_{i} \cdot \frac{\partial \mathbf{e}_{j}}{\partial x_{i}} \frac{v_{j}}{h_{i}}\right] \\
\Delta & =\sum_{i=1}^{3} \sum_{j=1}^{3}\left[\mathbf{e}_{i} \cdot \mathbf{e}_{j} \frac{1}{h_{i}} \frac{\partial}{\partial x_{i}}\left(\frac{1}{h_{j}} \frac{\partial}{\partial x_{j}}\right)+\mathbf{e}_{i} \cdot \frac{\partial \mathbf{e}_{j}}{\partial x_{i}} \frac{1}{h_{i} h_{j}} \frac{\partial}{\partial x_{i}}\right]
\end{aligned}
$$

For orthogonal coordinate systems, the first term in each series will only yield contributions for $i=j$. These terms can be attributed to either the $p d$ - or $c d$-part of the operator. The second term in each series can introduce contributions for $i \neq j$. If $x_{i}$ and $x_{j}$ are not both $p d$-coordinates or $c d$-coordinates, these terms cannot be labeled solely as $p d$ - or $c d$-contributions. In the remainder of this chapter, 
the divergence, the Laplacian and the vector Laplacian are separated as follows in contributions in $p d$ and $c d$-directions:

$$
\begin{aligned}
\nabla \cdot \mathbf{v} & =\nabla \cdot\left(\mathbf{v}_{p d}+\mathbf{v}_{c d}\right) \\
\Delta p & =\nabla \cdot\left(\nabla_{p d} p+\nabla_{c d} p\right) \\
\Delta \mathbf{v} & =\left(\Delta_{p d}+\Delta_{c d}+\Delta_{p c d}\right) \mathbf{v}
\end{aligned}
$$

with $\mathbf{v}_{p d}$ and $\mathbf{v}_{c d}$ velocity components in $p d$ - and $c d$-direction, respectively, $\Delta_{p d}$ and $\Delta_{c d}$ representing contributions only due to gradients in $p d$-and $c d$-direction, respectively, and $\Delta_{p c d}$ representing contributions due to gradients in $p d$ - and $c d$ directions. If the waveguide geometry is such that the axis describing the $p d$ directions define a plain layer or straight line, the expressions $\nabla_{p d} \cdot \mathbf{v}_{c d}, \nabla_{c d} \cdot \mathbf{v}_{p d}$, $\Delta_{p d} \mathbf{v}_{c d}, \Delta_{p d} \mathbf{v}_{c d}, \Delta_{p c d} \mathbf{v}$ and $\Delta_{p c d} T$ equal zero. For other waveguide geometries (for instance curved or tapered tubes and layers) these cross terms can become non-zero. In order to arrive at the LRF formulation given below, it may be necessary to neglect some of the cross terms even though their contribution can be significant. In the case of curved layers and tubes, this can lead to inaccuracies in the LRF model for small radii (see section 4.2.2). To arrive at a convenient notation, the additional cross terms that are taken into account are included in the expressions retained in the LRF model. As a result, these terms may be different from the definitions given above. In such cases the operators are explicitly redefined depending on the case at hand.

\subsubsection{LRF assumptions}

The LRF model is a simplified form of the dimensionless equations in (2.32). The aim is to rewrite the system in such a way that equations governing behavior in $c d$-direction decouple from the equation describing behavior in $p d$-direction. In the literature, different formulations can be found of the assumptions that are necessary for this simplification in addition to the assumptions made for linearization in section 2.3. The following assumptions are made either explicitly or implicitly in most derivations

- Pressure gradients are much larger in $p d$-directions than in $c d$-directions

- The magnitude of the $c d$-velocities are small compared to that of the $p d$ velocities

- Temperature gradients are much larger in $c d$-directions than in $p d$-directions

- Velocity gradients of $p d$-velocities are much larger in $c d$-directions than in $p d$-directions 
These assumptions also form the basis of acoustic boundary layer theory (see [32, 28]). They can be expressed mathematically as:

$$
\nabla_{c d} \tilde{p}=0, \quad\left|\tilde{\mathbf{v}}_{c d}\right| \ll\left|\tilde{\mathbf{v}}_{p d}\right|, \quad\left|\nabla_{p d} \tilde{T}\right| \ll\left|\nabla_{c d} \tilde{T}\right|, \quad\left|\nabla_{p d} \tilde{\mathbf{v}}_{p d}\right| \ll\left|\nabla_{c d} \tilde{\mathbf{v}}_{p d}\right| \quad(4.9 \mathrm{a}, \mathrm{b}, \mathrm{c}, \mathrm{d})
$$

It must be verified a posteriori if these assumptions are internally consistent with the resulting models ${ }^{1}$. Validity of the assumptions does not only depend on the material parameters and frequency of the propagating waves, but also on the geometry and boundary conditions that are prescribed. For straight tubes and plain layers of constant cross section having boundary conditions that do not vary with the $p d$-coordinates, it can be verified that these assumptions are valid if the following criteria are met:

- The viscous and thermal boundary layers are small compared to the acoustic wavelength

- The characteristic length is small compared to the acoustic wavelength

- The characteristic length is small compared to the length of the waveguide

The first two criteria can be expressed mathematically in terms of dimensionless wave numbers as

$$
\frac{\tilde{k}}{\tilde{s}} \ll 1, \quad \frac{\tilde{k}}{\tilde{s}_{t}} \ll 1, \quad \tilde{k} \ll 1
$$

Notice that these criteria are entirely described by the length scale, the frequency and the material parameters and can be verified a priori.

\section{Implications}

A posteriori inspection of the order of magnitude of the different terms reveals which terms can be removed from the set of equations. For long, straight waveguides with constant boundary conditions, the criterion in (4.10a) justifies removing terms describing viscosity induced impulse changes due to velocity gradients in $p d$-direction from the full set of equations. If the bulk viscosity is of the same order as the first coefficient of viscosity or smaller, terms related to bulk deformation can also be removed. The criterion in (4.10b) justifies removing the terms describing heat flow in the propagation direction. The criterion in (4.10c) implies a constant pressure of the waveguide cross section (assuming the criterion in (4.10a)

\footnotetext{
${ }^{1}$ Note that even if the assumptions hold and the resulting models are internally consistent, solutions to the full set of equations may exist that do not satisfy the assumptions.
} 
is also satisfied). Note that for an unbounded (free) wave, the criterion in (4.10a) coincides with the assumption of inviscid wave propagation, while the criterion in (4.10b) coincides with the assumption of adiabatic wave propagation.

Recall that the shear and thermal wave number only differ by a factor $\sqrt{\operatorname{Pr}}$. For air under standard conditions $\sqrt{P r} \sim 0.85$ and the range of thermal wave numbers for which the criterion in (4.10b) is met includes the range of shear wave numbers that satisfy the criterion in (4.10a). Conforming to the literature, the focus is on the criterion in (4.10a) in the parameter studies found throughout this thesis.

Under the above conditions, LRF models approximate the first acoustic mode found by solving Kirchhoff's dispersion equation very well. However, boundary conditions at the waveguide openings can also excite the thermal, vorticity and higher order acoustic modes. Due to their complex wave numbers, the thermal and vorticity modes will be evanescent in nature. If in addition $\tilde{k} \ll 1$, higher order acoustic modes that are excited by the boundary conditions at the waveguide openings will attenuate rapidly as well. In other words: if the length of the waveguide is long compared to the viscous and thermal boundary layer thickness and the characteristic length, neglecting the modes that cause these so-called inlet effects will only have a significant influence on the accuracy of the approximation very close to the waveguide entrances. Nevertheless, these influences can be of importance if multiple waveguide models are coupled (see section 4.3 for a two-port network description of multiple LRF models). Since the higher order evanescent modes contribute to the solution at the interfaces significantly, their influence on interaction between propagating waves in the different waveguide sections can become important. As a result, the amplitudes of the propagating waves as predicted by a coupled LRF model can become inaccurate due to the omission of the influence of higher order modes at the interface (see section 6.7).

\subsubsection{LRF equations}

For straight or plain waveguides with constant boundary conditions, applying the criteria in equation (4.9) to the equations in (2.32) yields a system in which the order of magnitude of the different terms can be observed. Neglecting the different terms that are expected to be small yields the following simplified system of 
equations after rewriting

$$
\begin{aligned}
i k \tilde{\rho}+\nabla \cdot \tilde{\mathbf{v}}_{p d}+\nabla \cdot \tilde{\mathbf{v}}_{c d} & =0 \\
i \tilde{\mathbf{v}}_{p d}-\frac{1}{s^{2}} \Delta_{c d} \tilde{\mathbf{v}}_{p d} & =-\frac{1}{\gamma k} \nabla_{p d} \tilde{p} \\
0 & =-\frac{1}{\gamma k} \nabla_{c d} \tilde{p} \\
i \tilde{T}-\frac{1}{s_{t}^{2}} \nabla \cdot \nabla_{c d} \tilde{T} & =i\left[\frac{\gamma-1}{\gamma}\right] \tilde{p} \\
\tilde{p} & =\tilde{\rho}+\tilde{T}
\end{aligned}
$$

For curved waveguides or in case the boundary conditions or waveguide cross section vary with the $p d$-coordinates, the contribution of the neglected terms can become significant. In such cases, the LRF approximation can be less accurate (see chapter 6).

\subsubsection{Solutions for velocity, temperature and density}

For prismatic waveguides and plain layers, equation (4.11b) and (4.11d) are Helmholtz equations with an inhomogeneous term that varies linearly with the pressure gradient and pressure, respectively. For other geometries, additional terms may arise in equation (4.11b) (see section 4.1.1). Solutions for specific geometries are presented in section 4.2. In all cases, the general solution is composed of the particular solution of the inhomogeneous equations satisfying homogeneous Robin boundary conditions and the solution of the homogeneous equations satisfying inhomogeneous Robin boundary conditions (equation (2.39)). Depending on the geometry of the waveguide, the equations can be solved analytically, by series expansion or with numerical techniques. Once the equations are solved, the resulting expressions for velocity and temperature can be written in the following form

$$
\begin{aligned}
\tilde{T} & =\left[\frac{\gamma-1}{\gamma}\right] C\left(s_{t}\right) \tilde{p}+C_{b}\left(s_{t}\right) \\
\tilde{\mathbf{v}}_{p d} & =\frac{i}{\gamma k}[\mathbf{A}(s)] \nabla_{p d} \tilde{p}+\mathbf{A}_{b}(s)
\end{aligned}
$$

with $\mathbf{A}$ and $\mathbf{A}_{b}$ (vector) functions describing the shape of the velocity profile (or profiles in the case of multiple $p d$-directions), and $C$ and $C_{b}$ functions describing the shape of the temperature profile over the waveguide cross section.

Note that $\mathbf{A}_{b}$ is a vector valued function. The elements corresponding to the $p d$-directions describe velocity profiles, while the elements corresponding to the 
$c d$-directions are zero. The term $\mathbf{A}$ is a diagonal matrix with scalar functions on the diagonal that describe velocity profiles in the $p d$-directions, while the diagonal elements corresponding to $c d$-directions are zero. It is stressed that the term $\mathbf{A}$ is a matrix (not a tensor). This distinction becomes important when the divergence of the velocity is taken for coordinate systems that are not Cartesian. The product $[\mathbf{A}] \nabla_{p d} \tilde{p}$ is defined as

$$
[\mathbf{A}] \nabla_{p d} \tilde{p}=\sum_{i=1}^{3} \mathbf{e}_{i} A_{i i} \nabla_{i} \tilde{p}
$$

Note that for layer geometries having equal curvature in both $p d$-directions and $p d$-velocity boundary conditions that are identical for the $p d$-directions, the velocity profiles are identical for both $p d$-directions. In that case (and for 1D waveguides), the diagonal matrix $\mathbf{A}$ can be replaced by an equivalent scalar function. The term involving $\mathbf{A}$ is the particular solution to the inhomogeneous equation (4.11b) satisfying homogeneous boundary conditions, while the term $\mathbf{A}_{b}$ represents the solution to the homogeneous part of equation (4.11b) that satisfies the inhomogeneous boundary conditions. The latter represents the contribution due to a prescribed $p d$-velocity or force in $p d$-direction (or a combination). In the case of homogeneous boundary conditions (e.g., zero $p d$-velocity, zero force in $p d$-direction or a prescribed impedance/admittance) the function $\mathbf{A}_{b}$ equals zero.

In a similar way, the functions $C$ and $C_{b}$ describe the thermal profile, with $C$ the contribution due to the pressure term and $C_{b}$ the contribution due to a prescribed temperature or heat flux (or a combination). In appendix E expressions for these functions are given for no-slip/isothermal boundary conditions for a number of geometries. Note that $\mathbf{A}, \mathbf{A}_{b}, C$ and $C_{b}$ can be a function of the propagation coordinates.

The expression for temperature is substituted in equation (4.11e), yielding the density in terms of pressure

$$
\tilde{\rho}=\left[1-\left[\frac{\gamma-1}{\gamma}\right] C\left(s_{t}\right)\right] \tilde{p}-C_{b}\left(s_{t}\right)
$$

\subsubsection{Solution for pressure}

The expression for density and $p d$-velocity and the divergence of the $c d$-velocity are integrated over the $c d$-coordinates and divided by the cross-sectional surface $S$. The averaged values are substituted in equation (4.11a), yielding an equation in terms of the averaged pressure. The divergence theorem is used to rewrite the term involving the $c d$-velocity as a source term. The general equation for pressure now takes the form

$$
\frac{n}{\gamma E} \nabla \cdot\left(E[\mathbf{B}] \nabla_{p d} \tilde{p}\right)+k^{2} \tilde{p}=k n\left[i \Re+i \nabla \cdot \mathbf{B}_{b}+k D_{b}\right]
$$


with

$$
\begin{aligned}
n\left(s_{t}\right) & =\left[1-\left[\frac{\gamma-1}{\gamma}\right] D\left(s_{t}\right)\right]^{-1} \\
D\left(s_{t}\right) & =\frac{1}{S} \int_{S} C\left(s_{t}\right) d S \\
D_{b}\left(s_{t}\right) & =\frac{1}{S} \int_{S} C_{b}\left(s_{t}\right) d S \\
\Re & =\frac{1}{S} \int_{\partial S} \tilde{\mathbf{v}} \cdot \mathbf{e}_{n} d \partial S
\end{aligned}
$$

where $S$ is the cross-sectional area, $\partial S$ is the boundary of $S$ and $\mathbf{e}_{n}$ is the outward normal on $\partial S$. The term $\mathbf{B}_{b}$ is a vector where the elements corresponding to the $c d$-directions are zero, and the term $\mathbf{B}$ is a diagonal matrix where the elements corresponding to the $c d$-directions are zero. The elements of $\mathbf{B}$ and $\mathbf{B}_{b}$ are defined as

$$
\begin{aligned}
& B_{i i}(s)=\frac{1}{S} \int_{S} A_{i i}(s) d S \\
& B_{b i}(s)=\frac{1}{S} \int_{S} A_{b i}(s) d S
\end{aligned}
$$

The definition of the term $E$ that is related to changes in the cross-sectional surface area $S$, and the term $\Re$ that is related to motion of the waveguide surface in $c d$ directions is given below.

\section{Non-constant cross section}

If the waveguide cross section varies with the $p d$-coordinates, equation (4.16) also includes contributions due to changes in cross-sectional geometry. The function $E$ represents changes of the cross-sectional surface area $S$ due to changes of the cross section with respect to the $c d$-coordinates (as a function of the $p d$-coordinates). The definition of $E$ thus depends on the shape of the waveguide, the coordinate system that is being used and the choice of $p d$-coordinates and $c d$-coordinates. If the cross section does not change with respect to the $c d$-coordinates, $E$ is constant and can be eliminated by taking it through the divergence operator. For example, $E$ will be constant for a prismatic tube section or a plain layer section described in Cartesian coordinates (see figure 4.1(a)), a tapered layer section of constant opening angle described in cylindrical coordinates (see figure 4.1(b)), or a conical tube 


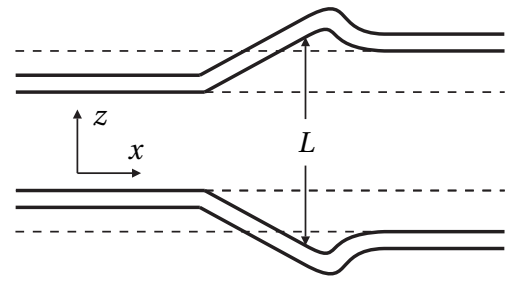

(a) $E=S=L$

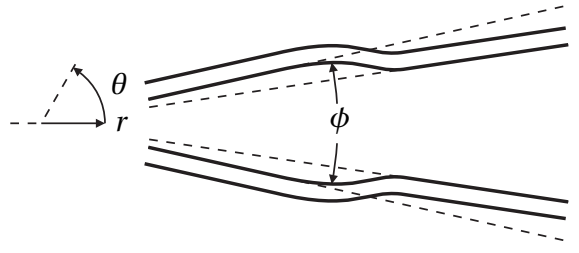

(b) $S=r \phi$ and $E=\frac{S}{r}=\phi$

Figure 4.1: Definition of $E$ for a 1D layer in Cartesian coordinates (a), and cylindrical coordinates (b). Lines of constant $E$ are indicated by (- - -) .

section of constant opening angle described in spherical or conical coordinates. In these cases, contributions to equation (4.16) due to changes in cross-sectional surface area are already included in the divergence operator. An example of the definition of $E$ for different coordinate systems is given in table 4.1.

\begin{tabular}{lcc}
\hline coordinate system & $\begin{array}{c}p d \text {-coordinates/ } \\
c d \text {-coordinates }\end{array}$ & E \\
\hline cylindrical systems $^{2}$ & $x_{p d}=z \quad$ or $\quad x_{c d}=z$ & $S$ \\
circular cylindrical & $x_{p d}=r \quad$ or $\quad x_{p d}=r, z$ & $\frac{S}{r}$ \\
spherical coordinates & $x_{p d}=r$ & $\frac{S}{r^{2}}$ \\
\hline
\end{tabular}

Table 4.1: Definition of $E$ for different coordinate systems and choices of the $p d$-coordinates and $c d$-coordinates.

\section{Inhomogeneous terms}

The inhomogeneous terms or source terms in equation (4.16) account for the inhomogeneous part of the velocity and temperature boundary conditions. The term involving $\Re$, also referred to as the squeeze term, accounts for the pressure changes due to a squeezing motion of the wall. The terms involving $\mathbf{B}_{b}$ and $D_{b}$ account for tangential motion of the boundary and expansion/rarefaction due to heating/cooling of the boundary, respectively. If homogeneous boundary conditions are applied (for instance: zero normal/tangential velocity or force, and isothermal or adiabatic walls) the contribution of the terms involving $\Re, \mathbf{B}_{b}$ and $D_{b}$ are zero. 
Expressions for these terms are easily derived from $\mathbf{B}$ and $D$ (see appendix E).

Equation (4.16) is the general equation describing pressure in waveguides for LRF models. For certain types of geometries and boundary conditions the equation reduces to a simplified form. Two specific cases are considered below.

\section{Case A}

Consider a waveguide of constant cross section and curvature, with the homogeneous part of the $p d$-velocity boundary conditions independent of the $p d$-coordinates. Under these conditions, the expressions for $E$ and $\mathbf{B}$ are constant with the $p d$-coordinates. The term $\mathbf{B}$ can be written as a scalar in the case of a $1 \mathrm{D}$ waveguide, and in the case of a 2D waveguide if the curvature and homogeneous part of the $p d$-velocity boundary conditions are equal in $p d$-directions. For these cases, equation (4.16) can be written as

$$
\Delta_{p d} \tilde{p}+k^{2} \Gamma^{2} \tilde{p}=k n \Gamma^{2}\left[i \Re+i \nabla \cdot \mathbf{B}_{b}\left(\mathbf{x}_{p d}\right)+k D_{b}\left(\mathbf{x}_{p d}\right)\right]
$$

with

$$
\Gamma\left(\mathbf{x}_{p d}\right)=\sqrt{\frac{\gamma}{n\left(s_{t}, \mathbf{x}_{p d}\right) \mathbf{B}(s)}}
$$

Note that in this formulation, $D, \mathbf{B}_{b}, D_{b}$ and $\Gamma$ may be dependent on the $p d$ coordinates. Under these conditions, equation (4.23) is in the form of an (inhomogeneous) Helmholtz equation which also describes inviscid adiabatic wave propagation (equation (2.23)). In the LRF model, the wave number $\tilde{k}$ is multiplied by the so-called propagation constant, $\Gamma$, to account for the viscous and thermal influence of the boundaries. The propagation constant is dependent on the shear wave numbers $\tilde{s}$ and $\tilde{s}_{t}$, but independent of the reduced frequency $\tilde{k}$. It is governed by the polytropic constant $n\left(s_{t}\right)$ which accounts for thermal influences of the boundary, and the function $B(s)$ which accounts for viscous influences of the boundary. The real part of $\Gamma$ accounts for the difference in phase speed with respect to free waves due to viscothermal effects ( $\Gamma=1$ in the absence of viscothermal effects). The imaginary part of $\Gamma$ accounts for the attenuation of sound waves due to viscothermal effects. A typical curve for the real and imaginary part of $\Gamma$ as a function of the shear wave number $\tilde{s}$ is given in figure 4.2 for a circular cylindrical tube and a plain layer.

\section{Case B}

Consider a layer of constant thickness and curvature, with the homogeneous part of the temperature boundary conditions independent of the $p d$-coordinates $(E$ 

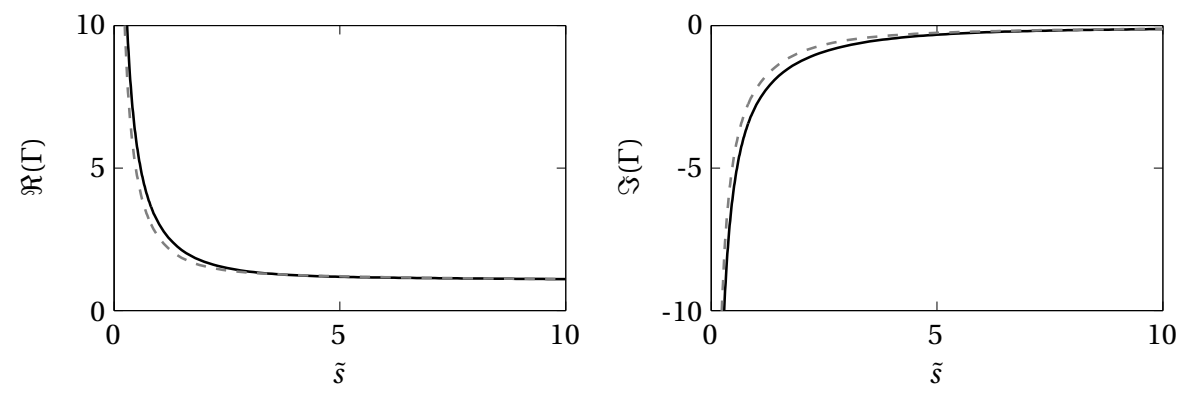

Figure 4.2: Real and imaginary part of the propagation constant $\Gamma$ as a function of the shear wave number $\tilde{s}$ for a plain layer (_- ) , and a cylindrical prismatic tube ( - - -) .

and $D$ are constant). If the layer has a curvature that is different for both $p d$ directions or if the homogeneous part of the $p d$-velocity boundary conditions are different for both $p d$-directions, the velocity profile is different for both $p d$ directions. As a result, the term $\mathbf{B}$ is a diagonal matrix, where the non-zero diagonal elements describe the averaged velocity for the individual $p d$-directions. Under these conditions equation (4.16) can be written as

$$
\nabla \cdot\left([\mathbf{K}] \nabla_{p d} \tilde{p}\right)+k^{2} \tilde{p}=k n\left[i \Re+i \nabla \cdot \mathbf{B}_{b}+k D_{b}\right]
$$

where $\mathbf{K}$ is a diagonal matrix with elements corresponding to the $c d$-direction equaling zero and the elements corresponding to the $p d$-directions defined as

$$
K_{i i}=\frac{1}{\Gamma_{i}}
$$

with $\Gamma_{i}$ the propagation constant (as defined in equation (4.24)) calculated for the values of $B_{i i}$ for the individual $p d$-directions. This equation can be thought of as an anisotropic Helmholtz equation in which the wave number for each direction is scaled with a different factor that accounts for viscothermal effects. As an example, the difference in propagation constants for axial and circumferential direction for wave propagation in a cylindrical prismatic air layer is given as a function of the shear wave number in figure 4.3. The LRF models used to calculate these results are presented in the next section.

\subsection{Geometry specific LRF solutions}

In this section, an overview is given of LRF models for specific geometries. The different subsections cover straight prismatic waveguides, curved prismatic waveguides and straight non prismatic waveguides, respectively. Solution techniques 


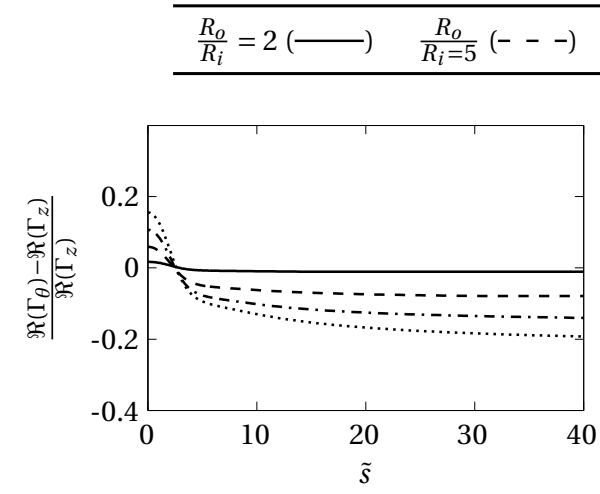

$\frac{R_{o}}{R_{i}}=10(-\cdot-\cdot) \quad \frac{R_{o}}{R_{i}}=20(\cdots \cdots)$

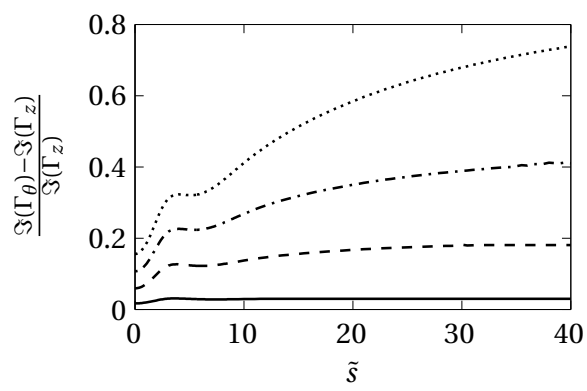

Figure 4.3: Relative difference (in real and imaginary part) between the propagation constant in axial and circumferential direction of a cylindrical prismatic air layer for various values of the ratios between the inner and outer radius of the boundary, denoted by $R_{i}$ and $R_{0}$, respectively.

(and solutions) are given for velocity profiles $A$ and $C$ (necessary to obtain the propagation constants $\Gamma$ ) and the homogeneous part of equation (4.16). Independent from waveguide geometry, the equations for $p d$-velocity $((4.11 b))$ and temperature $((4.11 \mathrm{~d}))$ can be written in the following form

$$
\left(\Delta_{c d}-i \lambda^{2}\right) f=g
$$

where $f, \lambda$ and $g$ are given in table 4.2. For plain layers and prismatic tubes, equation (4.27) is in the form of an inhomogeneous Helmholtz equation. For other geometries, the operator $\Delta_{c d}$ can include additional terms (see section 4.1.1).

\begin{tabular}{cccc}
\hline variable & $f$ & $\lambda$ & $g$ \\
\hline$p d$-velocity & $\tilde{\mathbf{v}}_{p d}$ & $s$ & $\frac{s^{2}}{\gamma k} \nabla_{p d} \tilde{p}$ \\
temperature & $\tilde{T}$ & $s_{t}$ & $-i s^{2}\left[\frac{\gamma-1}{\gamma}\right] \tilde{p}$ \\
\hline
\end{tabular}

Table 4.2: Variables, wave numbers and inhomogeneous terms for equation (4.27).

\subsubsection{Plain layers and prismatic tubes}

For plain layers and prismatic tubes, the operator $\Delta_{c d}$ involves derivatives in $c d$ direction only, and the homogeneous part $g$ is dependent on the $p d$-direction 
only. For homogeneous boundary conditions, this allows the solution for $f$ to be written as

$$
f=\left(1-f_{b}\right) g
$$

where $f_{b}$ is a solution the boundary value problem defined by the following homogeneous equation and boundary conditions

$$
\left(\Delta_{c d}-i \lambda^{2}\right) f_{b}=0, \quad \text { satisfying }\left.\quad f_{b}\right|_{\mathbf{x}_{c d}=\partial S}=1
$$

with $\partial S$ the boundary of the waveguide cross section.

The expression $\left(1-f_{b}\right)$ equals the functions $A$ and $C$ for $p d$-velocity and temperature, respectively. Applying inhomogeneous boundary conditions to the general solution of equation (4.29) yields $\mathbf{A}_{b}$ and $C_{b}$. The simplified equation for pressure given in equation (4.23) can be used for prismatic tubes. This equation also applies to layers in case the homogeneous part of the $p d$-velocity boundary conditions are equal for both $p d$-directions.

\section{Prismatic tubes}

For prismatic tubes, equation (4.23), which describes the propagation behavior, is in the form of a 1D Helmholtz equation. In case the inhomogeneous terms vanish (zero normal velocity, and homogeneous $p d$-velocity and temperature boundary conditions), the general solution for the averaged value over the waveguide cross section of pressure and velocity in $p d$-direction is

$$
\begin{aligned}
\tilde{p}(x) & =p^{(-)} e^{i k \Gamma x}+p^{(+)} e^{-i k \Gamma x} \\
\tilde{v}_{x}(x) & =\frac{1}{\Gamma n}\left[p^{(-)} e^{i k \Gamma x}-p^{(+)} e^{-i k \Gamma x}\right]
\end{aligned}
$$

where $p^{(-)}$and $p^{(+)}$are the complex amplitudes of the wave traveling in negative and positive $x$-directions at location $x=0$, respectively. The propagation constant $\Gamma$ can be obtained with equation (4.24). For straight prismatic tubes, equation (4.27) is the form of a 2D inhomogeneous scalar Helmholtz equation. If the boundary conditions are constant over the perimeter of the waveguide cross section and the waveguide surfaces are described by coordinate lines of a coordinate system in which the scalar Helmholtz equation is separable, analytical or semi-analytical solutions are available.

Overviews of the different analytical solutions found in the literature are given for instance by Kozlov and Fedorov [66] and Stinson [67]. The former presented solutions in the case of Robin (jump) boundary conditions for velocity and temperature (which include the solutions for Dirichlet boundary conditions presented 
in [67] as a special case). Hannink [20] presented the solution for the annular cylindrical case for Dirichlet and Neumann boundary conditions (which is not included in the aforementioned overviews). As a quick reference the known analytical solutions in the case of no-slip/isothermal boundary conditions are given in appendix E.

A general method to obtain the velocity and temperature profiles by expanding $f_{b}$ in a series of eigenmodes of the $\Delta_{c d}$ operator is presented in appendix E. For certain simple geometries, the eigenmodes of the Helmholtz equation are known analytically, resulting in efficient semi-analytical LRF models. Examples of this technique are reported in the literature for a prismatic tube with a rectangular cross section $[66,68,69]$. The method was also used to obtain the results for prismatic tubes with (closed and annular) elliptical cross sections (figures 4.5(e) and $4.5(\mathrm{~g})$ ). In the next subsection, the technique is used to obtain the velocity profile for curved layers and rectangular curved tubes of constant curvature and cross section. For waveguide geometries of more complex shape, numerical techniques such as FEM are best suited to obtain solutions to equation (4.27).

\section{Plain layers}

For plain layers, equation (4.27) yields two identical inhomogeneous Helmholtz equations for the $p d$-velocities and a third inhomogeneous Helmholtz equation for the temperature. The solution for arbitrary Robin boundary conditions is known analytically ${ }^{3}$. Equation (4.23) describing the propagation behavior has the form of a 2D inhomogeneous Helmholtz equation. For certain simple geometries and boundary conditions a solution can be obtained by separation of variables. Using separation of variables for arbitrary geometries and boundary conditions leads to the Trefftz method (for an example see [21]). A more general approach based on FE methods, which is suitable for layers of arbitrary shape is presented in [37]. An overview of the literature on viscothermal wave propagation in plain layers is given in references [10, 36].

\section{Propagation constant}

To calculate the propagation constants, the integrals of the velocity and temperature profiles are needed. The shape of these profiles is influenced by the shear wave number and thus by the choice of the characteristic length. If the definition of the characteristic length in equation (2.36) is used, the magnitude of the integrated velocity and temperature profile depends only weakly on the shear and

\footnotetext{
${ }^{3}$ See [66], or appendix E for the case with no-slip/isothermal boundary conditions.
} 
thermal wave number for higher values of these wave numbers $\left(s, s_{t}>10\right)$. Depending on the wave numbers and desired accuracy the integrals of the profiles can be approximated by the values found for wave propagation in a plain layer for which analytical expressions are available. In figure 4.4 the relative difference in propagation constant of a plain layer compared to that of the different crosssectional geometries given in figure 4.5 is plotted for a range of shear wave numbers.

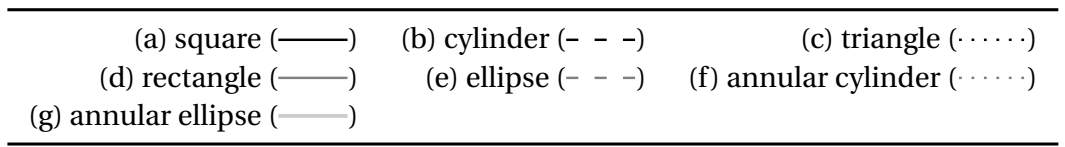

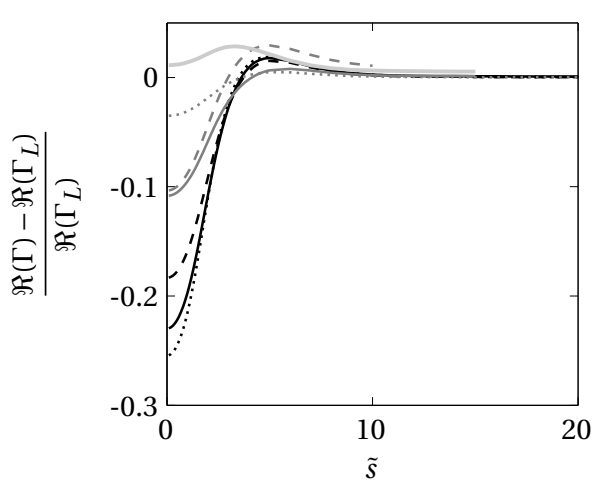

(a) Relative differences in real part

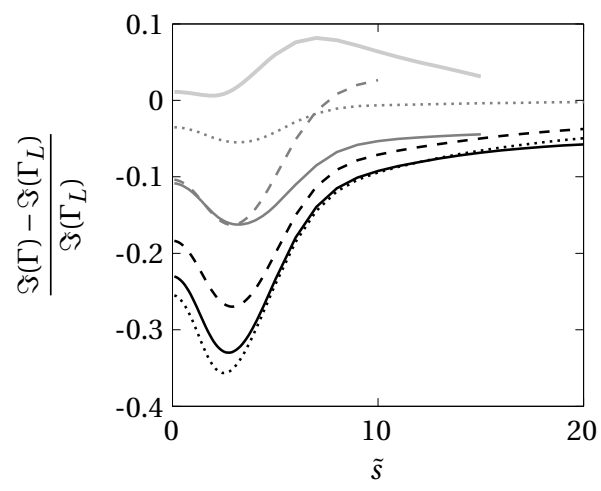

(b) Relative differences in imaginary part

Figure 4.4: Difference in propagation constant of a layer, $\Gamma_{L}$, compared with $\Gamma$ for the different cross-sectional geometries in figure 4.5 .

From figure 4.4 it is clear that the value of the propagation constant for the different geometries converges to that of a layer for higher shear wave numbers ${ }^{4}$. For lower shear wave numbers, the difference in propagation constant can be quite substantial. The differences can be explained by looking at the velocity profiles depicted in figure 4.5. For higher shear wave numbers a distinct boundary layer is present in all geometries. In that case, the magnitude of the viscous forces $F_{v}$ is proportional to the length of the wetted perimeter $P$ and the size of the viscous boundary layer $l_{v}$, while the magnitude of the inertial forces $F_{i}$ is proportional to

\footnotetext{
${ }^{4}$ The relative difference in the imaginary part of the propagation constant does not converge to zero for geometries with semi-analytical solutions (figure 4.5(d)(e) and (g)) because a limited number of eigenfunctions is taken into account. The corresponding curves are discontinued for higher shear wave numbers.
} 

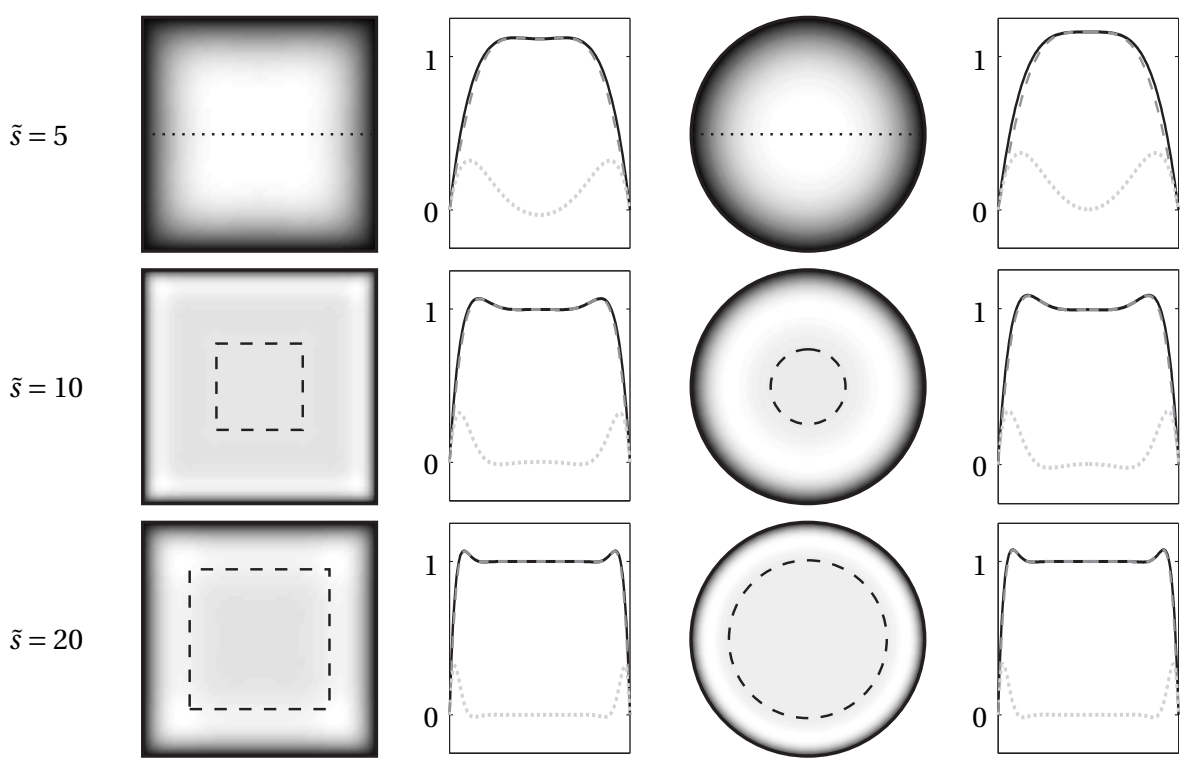

(a) square cross section

(b) cylindrical cross section
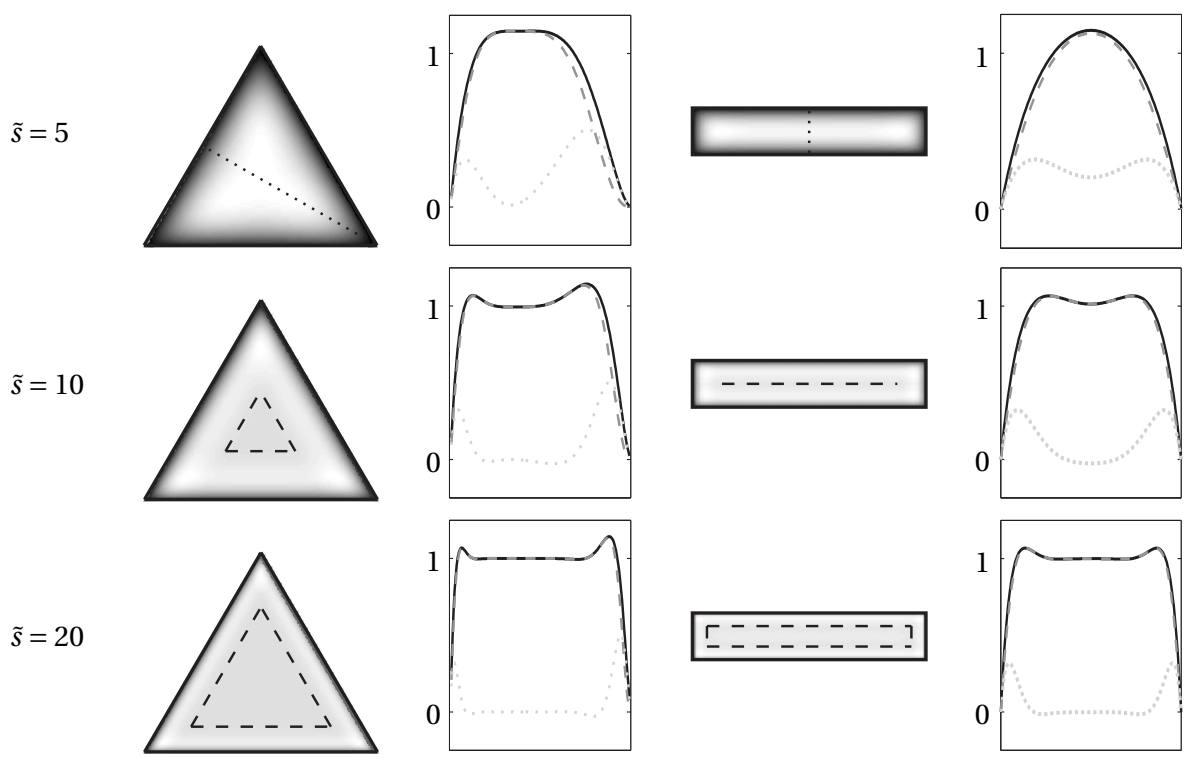

(c) triangular cross section

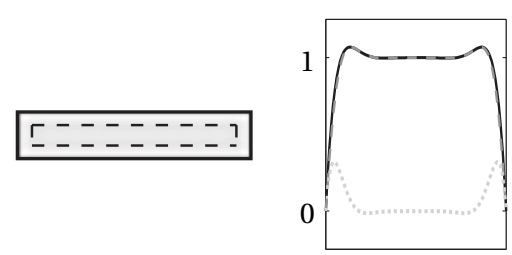

(d) rectangular cross section

Figure 4.5: Velocity profiles for different cross sections; absolute value $(-)$, real value $(---)$, and imaginary value $(. . .$.$) of the velocity are plotted along the dotted$ line $(\cdots \cdots)$. The shape of the viscous boundary layer is indicated by $(---)$. 

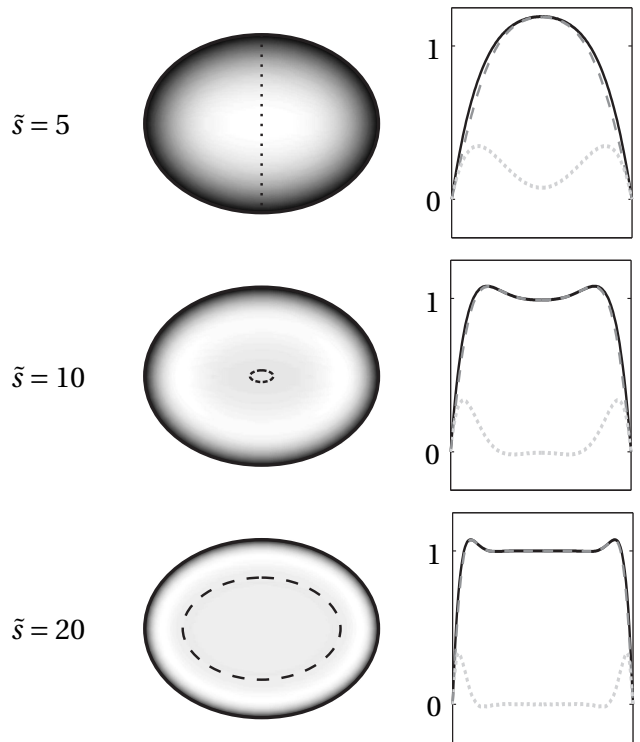

(e) elliptic cross section
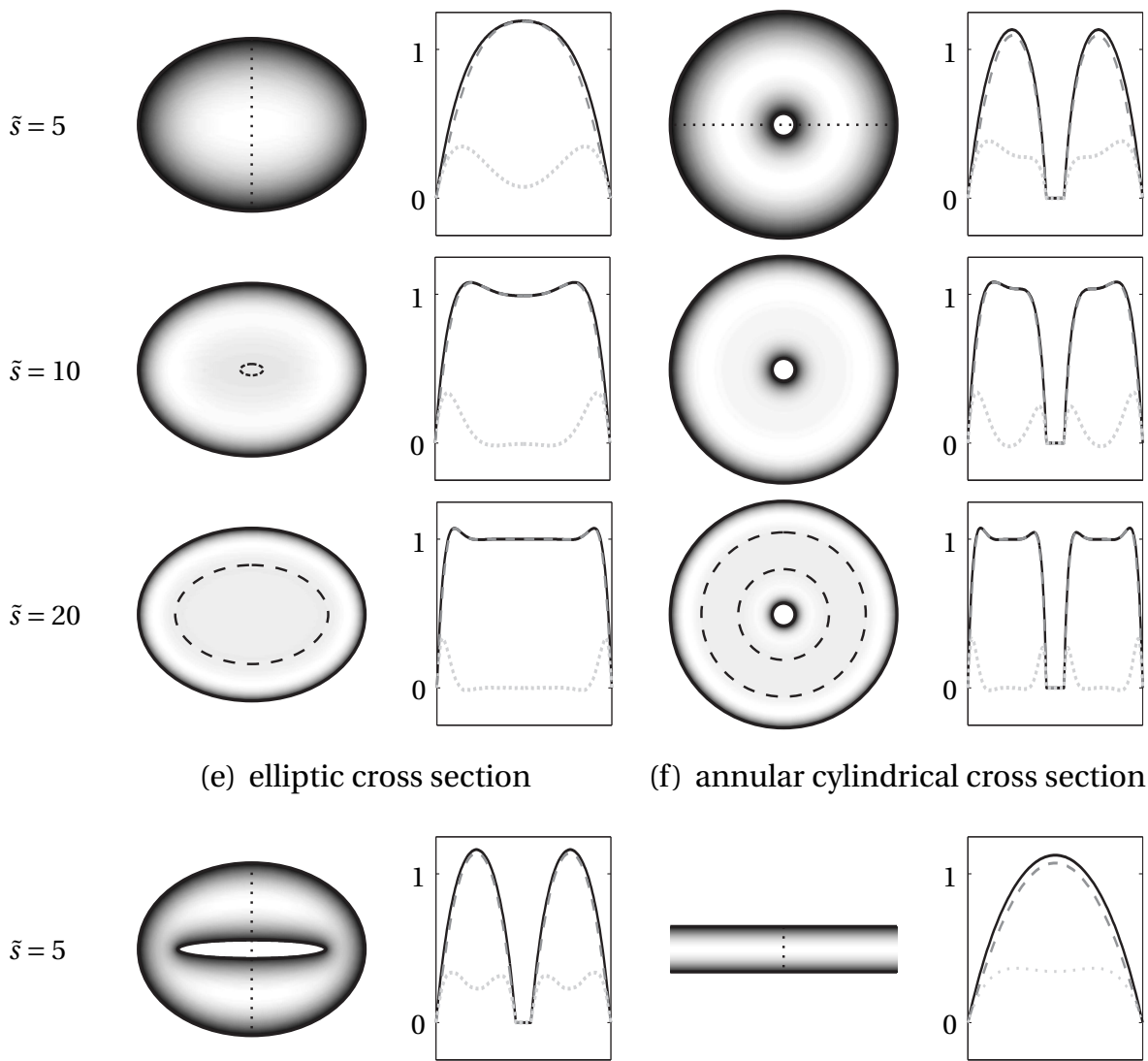

(f) annular cylindrical cross section
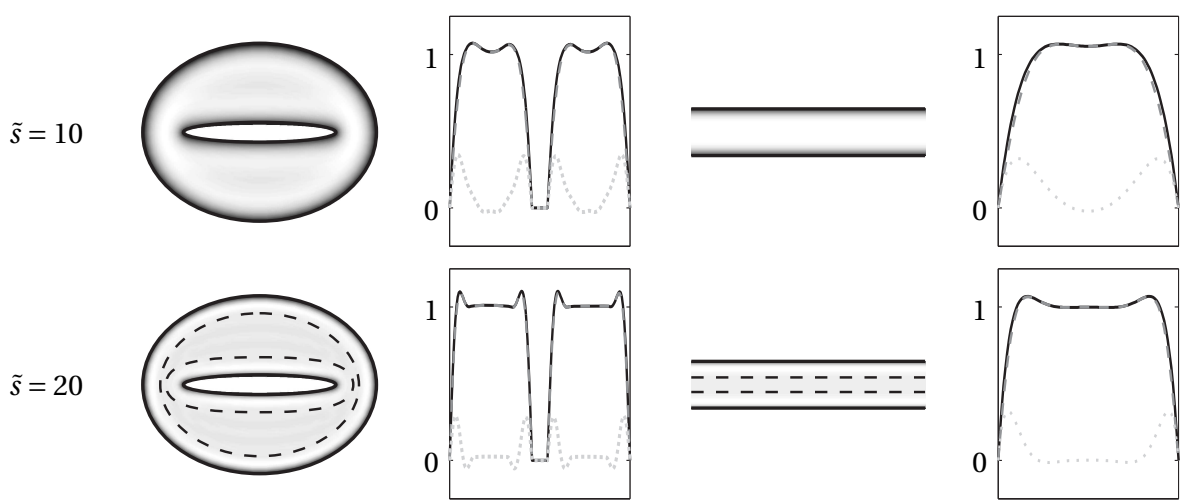

(g) annular elliptic cross section

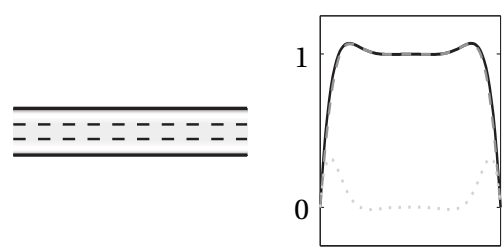

(h) layer

Figure 4.5: continuation Velocity profiles for different cross sections; absolute value $(-)$, real value $(---)$, and imaginary value $(. . .$.$) of the velocity are plotted along$ the dotted line $(\cdots \cdots)$. The shape of the viscous boundary layer is indicated by $(---)$. 
the cross-sectional surface area $S$ :

$$
F_{v} \sim P l_{v}, \quad F_{i} \sim S
$$

Recall that the shear wave number is a measure for both the ratio between inertial and viscous forces and the ratio between the characteristic length and the viscous boundary layer. Combining these relations leads to

$$
s=\frac{l}{l_{v}}=\frac{F_{i}}{F_{v}} \sim \frac{S}{P l v} \quad \Rightarrow \quad l \sim \frac{S}{P}
$$

Since the hydraulic radius is defined as the ratio between cross-sectional surface area and wetted perimeter, it is a natural choice for the characteristic length for higher shear wave numbers. However, as the shear wave number decreases, the size of the viscous boundary layer is of the same order or bigger than the dimensions of the cross section. Viscous forces and inertial forces are no longer proportional to the wetted perimeter and cross-sectional surface area. As a result the simple relation between the hydraulic radius and shear wave number ceases to be meaningful. This is easily understood by comparing the velocity profiles of the cylindrical and annular cylindrical cross section in figure 4.5. The small inner core has very little influence on the hydraulic radius, so the wetted perimeter and cross-sectional surface area are nearly identical. For large shear wave numbers, the additional viscous forces introduced by the core are negligible and the propagation constant for both geometries differs only slightly. As the shear wave number decreases, the size of the cross-sectional area in the viscous boundary layer almost doubles as a result of the core resulting in a considerable difference in propagation constant. A similar analysis can be carried out for the thermal wave number. In that case the heat energy that is stored is proportional to the crosssectional surface, while the heat energy that is conducted is proportional to the wetted perimeter and the size of the thermal boundary layer. The velocity profiles in figure 4.5 are equal to the temperature profiles for $s_{t}=s /$ Pr. From figure 4.4 it is clear that the propagational behavior is not solely determined by the shear and thermal wave number; the shape of the cross-sectional area affects the speed and attenuation of acoustic waves significantly and is therefore an important design parameter for waveguides.

\subsubsection{Curved tubes and cylindrical layers}

A semi-analytical LRF model for wave propagation in curved tubes and cylindrical layers of constant curvature and cross section is presented here for the first time. When describing wave propagation in curved tubes, it is convenient to introduce a 


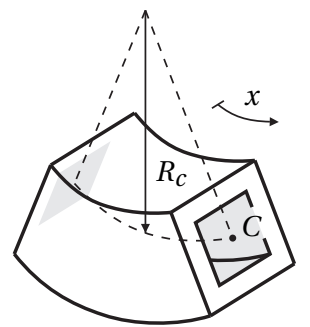

(a)

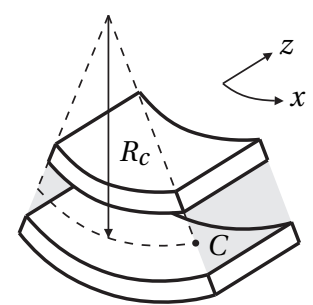

(b)

Figure 4.6: Definition of $R_{c}$ : Distance from the centroid $C$ of the cross section of a curved tube (a), or a cylindrical layer (b) to the center of curvature.

coordinate along the path of propagation. A propagation constant corresponding to such a coordinate allows a direct comparison with the propagation constant of a straight waveguide. To this aim, the distance traveled along a curve through the centroid $C$ of the waveguide cross section is defined as

$$
x=\theta R_{c}
$$

where $R_{c}$ is the distance of the centroid of the cross section to the origin (see figure 4.6). For a curved tube and a cylindrical layer the expressions for pressure (for homogeneous boundary conditions) are of the following form, respectively

$$
\begin{gathered}
\tilde{p}=p^{(-)} e^{i k \Gamma x}+p^{(+)} e^{-i k \Gamma x} \\
\tilde{p}=\left(p^{(x-)} e^{i k_{x} \Gamma_{x} x}+p^{(x+)} e^{-i k_{x} \Gamma_{x} x}\right)\left(p^{(z-)} e^{i k_{z} \Gamma_{z} z}+p^{(z+)} e^{-i k_{z} \Gamma_{z} z}\right)
\end{gathered}
$$

It follows from the derivation given below that the propagation constants $\Gamma_{x}$ and $\Gamma_{z}$ are not identical. Using $x$ as a $p d$-coordinate instead of $\theta$ allows a direct comparison of the propagation constants $\Gamma$ and $\Gamma_{x}$ with those obtained for straight tubes (as was done in figure 4.6). Note that in the derivation of the velocity and temperature profiles, a description in terms of $\theta$ is more intuitive, and is thus adopted below.

\section{Equation for velocity}

Cylindrical layers and curved (square) tubes are easiest described in cylindrical coordinates. First, equation (4.27) is considered using the $\theta$-component of the normal vector Laplacian instead of the LRF operator $\Delta_{c d}$. This allows an inspection of the terms that are neglected under the LRF assumptions. For curved tubes, equation (4.27) takes the following form for the $p d$-velocity:

$$
\frac{\partial^{2} \tilde{v}_{\theta}}{\partial r^{2}}+\frac{1}{r^{2}} \frac{\partial^{2} \tilde{v}_{\theta}}{\partial \theta^{2}}+\frac{\partial^{2} \tilde{v}_{\theta}}{\partial z^{2}}+\frac{1}{r} \frac{\partial \tilde{v}_{\theta}}{\partial r}+\frac{2}{r^{2}} \frac{\partial \tilde{v}_{r}}{\partial \theta}-\frac{\tilde{v}_{\theta}}{r^{2}}-i s^{2} \tilde{v}_{\theta}=\frac{s^{2}}{\gamma k} \frac{1}{r} \frac{\partial \tilde{p}}{\partial \theta}
$$


For cylindrical layers a second equation, describing the (dimensionless) impulse balance in $z$-direction, is required (involving the $z$-component of the normal vector Laplacian)

$$
\frac{\partial^{2} \tilde{v}_{z}}{\partial r^{2}}+\frac{\partial^{2} \tilde{v}_{z}}{\partial \theta^{2}}+\frac{\partial^{2} \tilde{v}_{z}}{\partial z^{2}}+\frac{1}{r} \frac{\partial \tilde{v}_{z}}{\partial r}-i s^{2} \tilde{v}_{z}=\frac{s^{2}}{\gamma k} \frac{\partial \tilde{p}}{\partial z}
$$

Upon applying the LRF assumptions, only the terms involving $p d$-velocities are retained while terms involving derivatives in $c d$-direction are removed from the above equation. It is demonstrated in section 6.6 that these simplifications can lead to small, but significant errors for small tube/layer radii. The $\Delta_{c d}$ operator in equation (4.27) is redefined by dropping the different terms. For curved tubes, the following equation is obtained for $\tilde{v}_{\theta}$

$$
\left(\Delta_{c d}-i s^{2}\right) \tilde{v}_{\theta}=\frac{s^{2}}{\gamma k} \frac{1}{r} \frac{\partial \tilde{p}}{\partial \theta}, \quad \text { where } \quad \Delta_{c d}=\frac{\partial^{2}}{\partial r^{2}}+\frac{\partial^{2}}{\partial z^{2}}+\frac{1}{r} \frac{\partial}{\partial r}-\frac{1}{r^{2}}
$$

for cylindrical layers, the following equations, which are only coupled through the inhomogeneous term, are obtained for $\tilde{v}_{\theta}$ and $\tilde{v}_{z}$

$$
\begin{aligned}
& \left(\Delta_{c d}-i s^{2}\right) \tilde{v}_{\theta}=\frac{s^{2}}{\gamma k} \frac{1}{r} \frac{\partial \tilde{p}}{\partial \theta}, \quad \text { where } \quad \Delta_{c d}=\frac{\partial^{2}}{\partial r^{2}}+\frac{1}{r} \frac{\partial}{\partial r}-\frac{1}{r^{2}} \\
& \left(\Delta_{c d}-i s^{2}\right) \tilde{v}_{z}=\frac{s^{2}}{\gamma k} \frac{\partial \tilde{p}}{\partial z}, \quad \text { where } \quad \Delta_{c d}=\frac{\partial^{2}}{\partial r^{2}}+\frac{1}{r} \frac{\partial}{\partial r}
\end{aligned}
$$

Using the assumption that $p$ only depends on the $p d$-coordinates, separation of variables allows the solution for $\tilde{v}_{\theta}$ to be written as

$$
\tilde{v}_{\theta}=\tilde{v}_{\theta}\left(\mathbf{x}_{c d}\right) \tilde{v}_{\theta}\left(\mathbf{x}_{p d}\right)
$$

where the $c d$-dependent part and the $p d$-dependent part are solutions of

$$
\begin{gathered}
\left(\Delta_{c d}-i s^{2}\right) \tilde{v}_{\theta}\left(\mathbf{x}_{c d}\right)=\frac{s^{2}}{\gamma k} \frac{1}{r} \\
\tilde{v}_{\theta}\left(\mathbf{x}_{p d}\right)=\frac{\partial \tilde{p}}{\partial \theta}
\end{gathered}
$$

where $\Delta_{c d}$ is as defined in equations (4.39) and (4.40) for curved tube and cylindrical layer geometries, respectively. When equation (4.43) is compared with the corresponding equation for a prismatic tube or plain layer, it is observed, firstly, that the operator $\Delta_{c d}$ yields additional terms, and secondly, that the inhomogeneous part of the equation is no longer a constant but depends on the $c d$-coordinates. As a result, there are differences in the velocity profiles for tubes with identical cross sections but different curvature, and differences in the velocity profiles for the different $p d$-directions in a cylindrical layer. 


\section{Solving equations for temperature and velocity}

An efficient semi-analytical solution for $\tilde{v}_{\theta}$ is found by decomposing it in the eigenvalues of the $\Delta_{c d}$ operator implicitly defined by equation (4.39) or (4.40), and substituting these in the above equations. The technique is elaborated upon in appendix E.

For cylindrical layers, there is also a velocity profile in $z$-direction. The profile is obtained by solving equation 4.41, describing the (dimensionless) impulse balance in $z$-direction. Note that the equation is identical to the corresponding equation for annular cylindrical prismatic tubes. This is to be expected as the solution for an annular cylindrical prismatic tube can be considered a special case of wave propagation in a cylindrical layer (by assuming wave propagation in $z$-direction only). The expressions for the velocity profile in $\theta$-direction and $z$-direction expressed in terms of $\tilde{v}_{\theta}$ and $f_{b}$ is given, respectively, by

$$
A_{\theta}=r \tilde{v}_{\theta}(r, z), \quad A_{z}=1-f_{b}
$$

where $f_{b}$ is defined by equation (4.29).

The equation describing the (dimensionless) energy balance is also identical to the corresponding equation for annular cylindrical prismatic tubes. The solution for the temperature profile is $C=1-f_{b}$, where $f_{b}$ is again obtained using equation (4.29).

\section{Curved vs straight tubes}
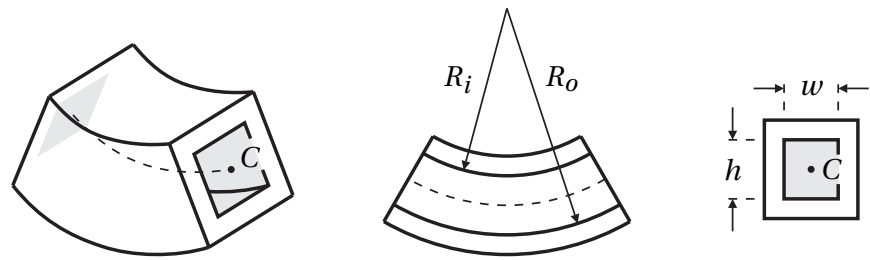

Figure 4.7: Dimensions of a curved tube.

As a first approximation, a curved square tube or layer can be modeled as a prismatic tube or plain layer of equivalent length. The length is measured along the curve passing through the centroid $C$ of the waveguide cross section. To get an impression of the accuracy of such an approximation, the propagation constant of a curved waveguide having a square cross section (see figure 4.7) is compared to that of a straight waveguide having an identical cross section. 


\section{Inviscid adiabatic case}

Inviscid adiabatic wave propagation in curved waveguides was studied previously by Rostafinski [90, 91]. He demonstrates that the pressure and velocity profiles for the different acoustic modes in curved waveguides are frequency dependent (in contrast to the frequency independent profiles for straight waveguides). Consequently, the propagation constant used in equation (4.35), which equals unity for a straight waveguide in the inviscid adiabatic case, is also frequency dependent for a curved waveguide. In figure 4.8 , the propagation constant is plotted as a function of $\tilde{k}$ for different values of $R_{o} / R_{i}$, where $R_{i}$ and $R_{o}$ represent the inner and outer radius of the cross section (see figure 4.7). The different graphs are based on the data presented in [90]. In [91] it is demonstrated that for low frequencies

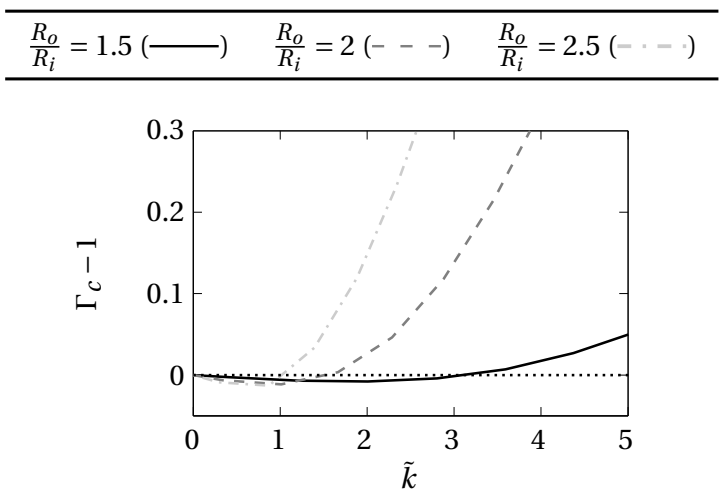

Figure 4.8: Relative difference in wave number in a curved and straight waveguide of equivalent length for various ratios of the inner and outer radius (data obtained from [90]).

$(\tilde{k}<1)$ the pressure is approximately constant over the waveguide cross section for the first acoustic mode. Consequently, equation (2.24) implies that the magnitude of the $p d$-velocity is inverse proportional to the radius (see [90]). So, for low frequencies, the $p d$-velocity increases as the distance to the center of curvature of the waveguide decreases. Put in other words, the acoustic wave is 'cutting the corner'. This is supported by the plots in figure 4.8 , which indicate that for $\tilde{k}<1$, the propagation constant in $p d$-direction in a curved waveguide is smaller than unity (being the propagation constant of a straight waveguide). The plots imply that for $\tilde{k}<1$, the phase speed in a curved waveguide becomes lower as the waveguide is curved tighter.

As the frequency increases so that $\tilde{k}>1$, the pressure is no longer constant for the first mode. Instead, the pressure profile has lower values at the inner radius 
and higher values at the outer radius (see [91]). The difference between highest and lowest pressure increases with increasing values of $\tilde{k}$ and with increasing values of $R_{o} / R_{i}$. Equation (2.24) implies that such non-constant pressure profiles counter the effect of 'cutting the corner'. Indeed, in figure 4.8 it can be seen, that for tight corners $\left(R_{o} / R_{i} \ll 1\right)$ and high frequencies $\tilde{k}>1$ the propagation constant of a curved waveguide is higher than unity. The figure implies that for $\tilde{k}>1$, the phase speed in curved waveguides becomes higher as the waveguide is curved tighter. The focus in the remainder of this section is on the case where $\tilde{k}<1$ and the pressure is approximately constant over the waveguide cross section for the first acoustic mode.

\section{Viscothermal case}

In figure 4.9 the relative differences (for the real and imaginary part) between the propagation constant of a curved and straight waveguide are plotted as a function of the ratio $R_{i} / R_{o}$. The differences are plotted for various shear wave numbers and different aspect ratios of the cross section (see figure 4.7).

These differences, which indicate the error of the straight waveguide approximation, can be quite substantial for both the real part of the propagation constant, governing the phase speed, and the imaginary part, governing the attenuation. A positive difference in the real part implies a lower phase speed, while a positive difference in the imaginary part implies more attenuation in the curved waveguide.

The error in the real part of the propagation constant becomes more negative as viscothermal effects become less significant (higher shear wave numbers). For $\tilde{s} \gg 1$, the real part of the propagation constant is negative for all values of $R_{i} / R_{0}$. This corresponds with the fact that the propagation constant for a curved tube is smaller than unity for the inviscid adiabatic case as was pointed out above. In figure 4.10 the velocity profiles for viscothermal wave propagation in a square curved tube are given for various values of the shear wave number. These plots indicate that the velocity is higher close to the center of curvature which is also in line with the results for the inviscid adiabatic case ${ }^{5}$. Note that this effect of 'cutting the corner' reduces with increasing viscous effects.

A second effect of acoustic waves 'cutting the corner' is that attenuation due to viscothermal losses is slightly smaller due to the fact that the effective distance a sound wave travels is shorter (the areas with higher velocities/displacements have a shorter path). This is supported by the fact that the imaginary part of the propagation constant is negative given the aspect ratio $w / h$ is small and becomes more negative with increasing viscous effects (see the line $w=h / 4$ ). For larger values

\footnotetext{
${ }^{5}$ Note that for wave propagation in a cylindrical layer in axial direction the velocity is lower near the center (see figure 4.5(f)).
} 


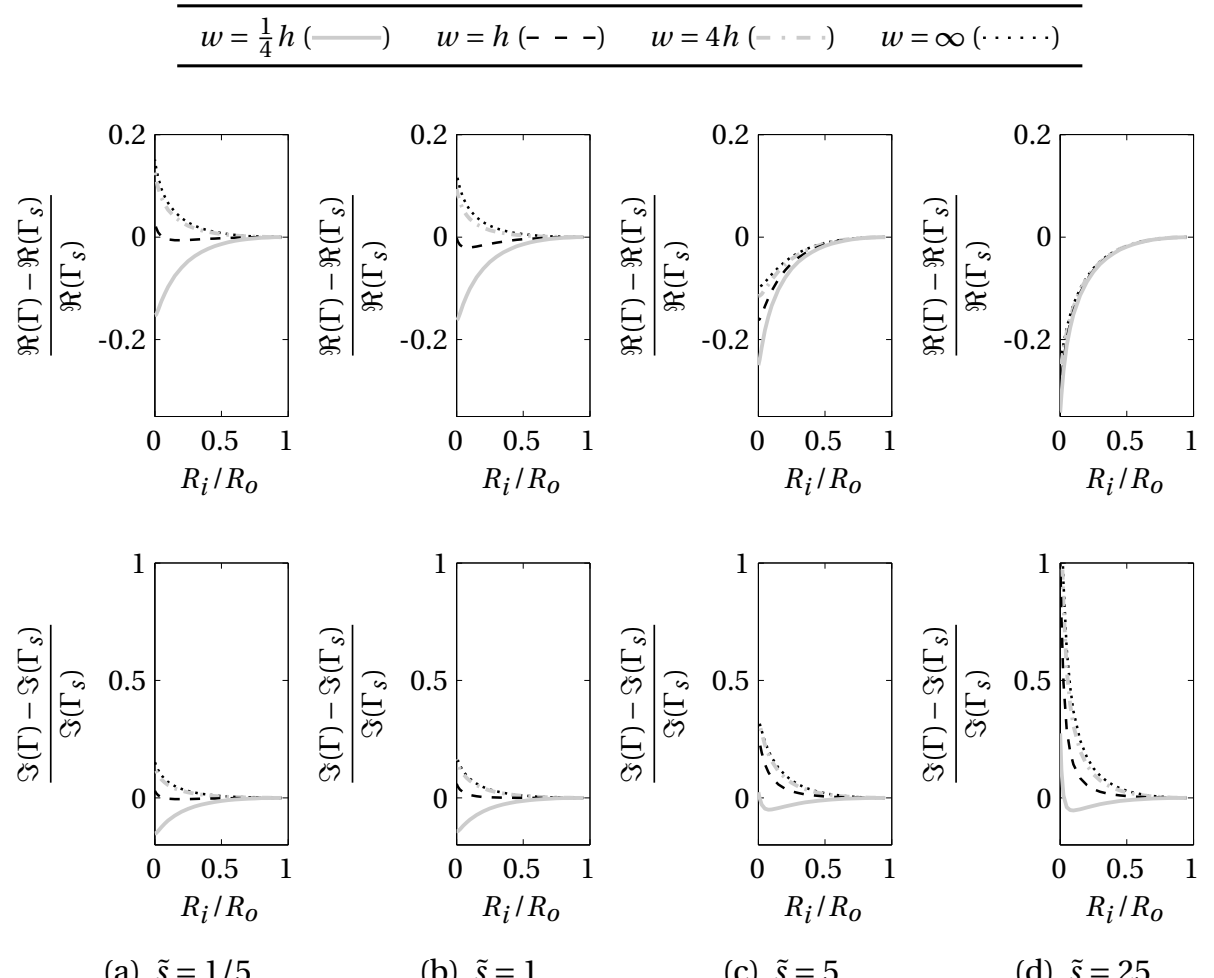

Figure 4.9: Relative difference in real and imaginary part of the propagation constant, $\Gamma$, for a curved tube/layer of rectangular cross section with respect to the propagation constant, $\Gamma_{S}$ for a straight tube of identical cross section. The difference is considered for various aspect ratios and various shear wave numbers (plots (a) through (d)).

of $w / h$ this effect of 'cutting the corner' is overshadowed by other effects (as described below).

An important factor influencing the impact of viscous effects is the aspect ratio of the cross section $w / h$. The aspect ratio influences the factor $B$ (in equation (4.24)), describing the averaged fluid velocity for a unit pressure gradient in $x$-direction (at $r=R_{c}$ ). The value of $B$ for a curved waveguide can differ substantially to that of a straight waveguide. As an example, table 4.3 lists the value of $B$ for the velocity profiles depicted in 4.10 . Note that for larger aspect ratio, additional viscous effects can completely cancel the increase in phase speed and decrease in attenuation due to cutting the corner.

The influence of the aspect ratio can be explained as follows. For small values of $w / h$, the waveguide boundaries perpendicular to the axis of curvature are relatively long. For $w / h \ll 1$ a flat disc perpendicular to the axis of curvature is 

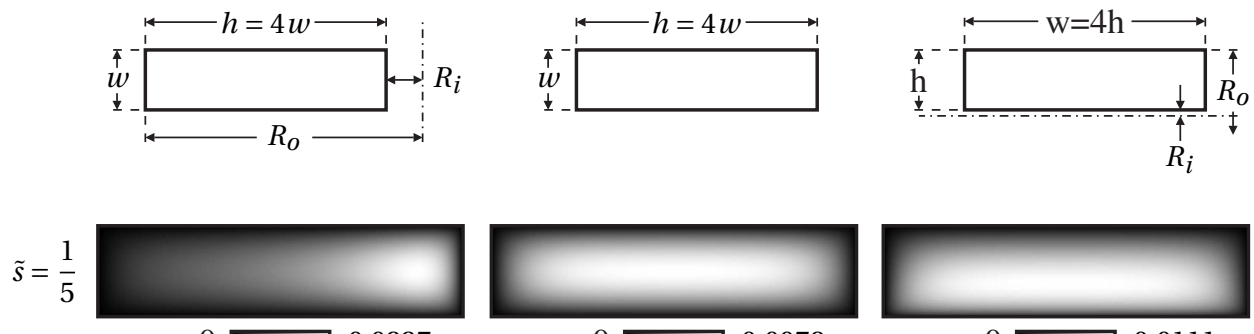

$0 \longleftarrow 0.0078$

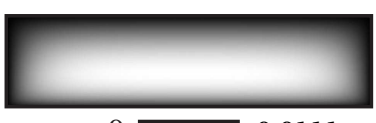

$0 \rightleftharpoons 0.0111$

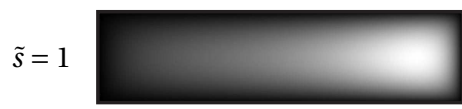

$0 \rightleftharpoons 0.589$

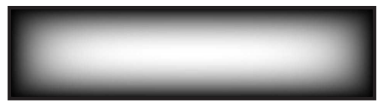

0

0.192

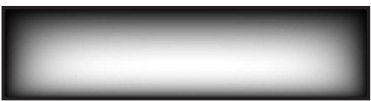

$0 \longmapsto 0.274$

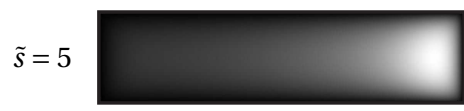

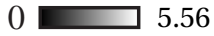

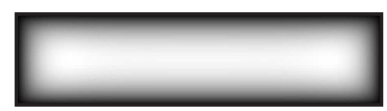

$0 \Longleftarrow 1.19$

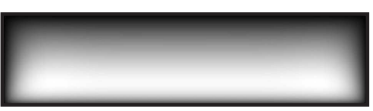

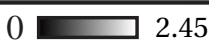

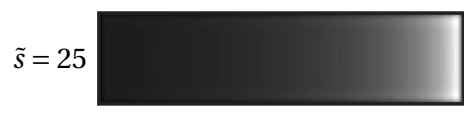

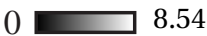

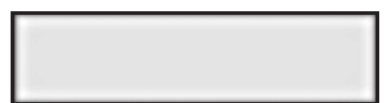

$0 \stackrel{\square}{\square} 1.12$

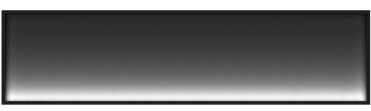

$0 \rightleftharpoons 52$

(a) Curved tube $(w=h / 4)$

(b) Straight tube $(w=h / 4)$

(c) Curved tube $(w=4 h)$

Figure 4.10: Velocity profiles $|A|$ for curved and straight tubes of rectangular cross section $\left(R_{i} / R_{o}=1 / 10\right)$.

obtained. The boundaries that are perpendicular to the axis of curvature are relatively long and dominate the viscous effects. Consequently, viscous effects due to velocity gradients in radial directions are small. This implies that the effects of viscosity on the phase speed and attenuation are similar for wave propagation in circumferential direction in a flat disc and wave propagation in a flat layer. Consequently, the error in propagation constant in figure 4.9 is only slightly dependent on the shear wave number for $w / h \ll 1$. For intermediate and large values of the aspect ratio, viscous effects are significantly influenced by velocity gradients in radial directions. Viscous effects are significantly larger in such waveguides due to the fact that the pressure gradient in propagation direction is not constant with the radial coordinate, leading to bigger gradients in the $p d$-velocity. As a result of the additional viscous losses in curved waveguides, the differences in the propagation constant in figure 4.9 depend heavily on the shear wave number for $w / h>1 / 4$.

Note that the shape of the temperature profile and its average value $D$ is only weakly dependent on the curvature (although the dependency strengthens as the 
shear wave number decreases). The averaged values for $D$ corresponding to the cases proposed in figure 4.9 are also given in table 4.3.

\begin{tabular}{ccccc}
\hline & $\tilde{s}$ & $\begin{array}{c}\text { curved tube }(w=h / 4) \\
\text { figure } 4.10(\mathrm{a})\end{array}$ & $\begin{array}{c}\text { straight tube } \\
\text { figure } 4.10(\mathrm{~b})\end{array}$ & $\begin{array}{c}\text { curved tube }(w=4 h) \\
\text { figure } 4.10(\mathrm{c})\end{array}$ \\
\hline \multirow{3}{*}{$B \quad 1 / 5$} & $0.0000+0.0053 i$ & $0.0000+0.0043 i$ & $0.0000+0.0039 i$ \\
& 1 & $0.0173+0.1307 i$ & $0.0150+0.1064 i$ & $0.0105+0.0965 i$ \\
& 5 & $0.9243+0.3742 i$ & $0.7125+0.2536 i$ & $0.7733+0.3832 i$ \\
& 25 & $1.2937+0.0878 i$ & $0.9336+0.0542 i$ & $1.2432+0.1243 i$ \\
\hline & $1 / 5$ & $0.0000+0.0031 i$ & $0.0000+0.0031 i$ & $0.0000+0.0035 i$ \\
& 1 & $0.0077+0.0767 i$ & $0.0079+0.0777 i$ & $0.0098+0.0869 i$ \\
& 5 & $0.6540+0.3006 i$ & $0.6572+0.2982 i$ & $0.6885+0.2794 i$ \\
& 25 & $0.9234+0.0641 i$ & $0.9230+0.0630 i$ & $0.9241+0.0597 i$ \\
\hline
\end{tabular}

Table 4.3: Average values, $B$, corresponding to the velocity profiles $A$ given in figure 4.10 , and averaged values $D$ of the corresponding temperature profile $C$.

\subsubsection{Straight tubes and plain layers with non-constant cross section}

For non-constant waveguide cross sections the function $\mathbf{B}, D$ and (in some cases) $E$ depend on the $p d$-coordinates. As a result the most general equation for pressure given in equation (4.16) must be used. The first left-hand side term of the equation can be split into several contributions

$$
\frac{n}{\gamma E} \nabla \cdot\left(E \mathbf{B} \nabla_{p d} \tilde{p}\right)=\frac{n}{\gamma}\left[\frac{(\nabla E)}{E}+\nabla\right] \cdot\left(\mathbf{B} \nabla_{p d} \tilde{p}\right)
$$

The first term between the square brackets accounts for changes in cross-sectional surface area due to changes in cross section with respect to the $c d$-coordinates (i.e., area changes that are not accounted for by taking the divergence). This term vanishes for waveguides that have a constant cross section with respect to the $c d$ coordinates such as straight layers/tubes, tapered layers/square tubes and conical tubes described in a Cartesian, circular cylindrical and spherical coordinate system, respectively. In the case of a single $p d$-direction, the second right-hand side term in equation (4.46) can be written as

$$
\nabla \cdot\left(\mathbf{B} \nabla_{p d} \tilde{p}\right)=\nabla \mathbf{B} \cdot \nabla_{p d} \tilde{p}+\mathbf{B} \nabla \cdot \nabla_{p d} \tilde{p}
$$

where the first term accounts for changes in the averaged velocity, and the second term accounts for pressure gradients and changes in cross-sectional surface area for a cross section that is constant with respect to the $c d$-coordinates. For multiple 
$p d$-directions, the term $\mathbf{B}$ is a diagonal matrix (not a tensor). The second righthand side term in equation (4.46) can be written as a series

$$
\begin{array}{r}
\nabla \cdot\left(\mathbf{B} \nabla_{p d} \tilde{p}\right)=\sum_{i=1}^{3} \sum_{j=p d}\left[\mathbf{e}_{i} \cdot \mathbf{e}_{j}\left(\nabla_{i} B_{j j}\right) \nabla_{j} \tilde{p}+\mathbf{e}_{i} \cdot \mathbf{e}_{j} B_{j j}\left(\nabla_{i} \nabla_{j} \tilde{p}\right)+\right. \\
\left.\left(\mathbf{e}_{i} \cdot \nabla_{i} \mathbf{e}_{j}\right) B_{j j} \nabla_{i} \nabla_{j} \tilde{p}\right]
\end{array}
$$

The first term accounts for changes in the averaged velocity. The second and third terms in the series are related to pressure gradients, and changes in cross-sectional surface area for a cross section that is constant with respect to the $c d$-coordinates, respectively.

\section{Horn equation}

For 1D waveguides, equation (4.16) represents the viscothermal equivalent of the so-called horn equation. This can easily be seen by considering equation (4.16) for the inviscid adiabatic case with rigid waveguide walls $\left(\mathbf{B}=D=1\right.$ and $\Re=\mathbf{B}_{b}=$ $D_{b}=0$ ), which reduces it to the horn equation:

$$
\frac{1}{E} \nabla \cdot E \nabla_{p d} \tilde{p}+k^{2} \tilde{p}=0
$$

The horn equation and its solutions have been studied intensively for the inviscid adiabatic case, and different 'families' of analytical solutions are available for a limited number of axisymmetrical geometries (for an overview, see for instance [92]). Approximations including mean flow can be found for instance in [93, 94]. Note that the horn equation only accounts for $p d$-velocities, while it does not involve $c d$-velocities. Similarly, equation (4.16) only accounts for the impulse balance in $p d$-direction, while it does not involve the impulse balance in $c d$-direction. A short discussion on the effects of neglecting the balance equation for the $c d$ direction is given in F.1.

\section{LRF approximation}

In the case of a tapered 1D layer (having a constant opening angle), the velocity and temperature profiles $A$ and $C$, and their averaged values $B$ and $D$ are found by solving equation 4.27 in circular cylindrical coordinates. Assuming no-slip velocity boundary conditions, the expressions for $A$ and $B$ are given by

$$
A=1-\frac{\cos (\sqrt{-i} s r \alpha)}{\cos (\sqrt{-i}(s r \alpha / 2))}, \quad B=1-\frac{\tan (\sqrt{-i}(s r \alpha / 2))}{\sqrt{-i}(s r \alpha / 2)}
$$


where $\alpha$ is the opening angle of the tapered layer. For isothermal temperature boundary conditions, the expressions for $C$ and $D$ are obtained with these equations by substituting $s$ with $s_{t}$. Due to the dependency of the coefficients $B$ and $D$ (and therefore the propagation constant) on the $p d$-coordinate, finding a closed form solution to equation 4.16 is not trivial in the viscothermal case.

An efficient alternative is to approximate the waveguide geometry with a series of coupled prismatic waveguides. Each waveguide is represented by a twoport description using a mobility matrix approach (see section 4.3). The solutions of the individual waveguides are coupled by demanding continuity of the averaged pressure and volume flow at the interfaces. This approach is very efficient especially in case the waveguide includes prismatic sections. The merits of two other attractive numerical techniques that are encountered in the literature are discussed shortly in appendix F.2. One of these techniques, which is based on a so-called step-integration-algorithm is used below as a reference solution to investigate the accuracy of the two-port approximation. A third alternative to solve equation (4.16) is the use of a boundary layer approximation as described in appendix F.3.

\section{Convergence of an 1D LRF two-port model}

$$
\begin{gathered}
\frac{r_{2}-r_{1}}{L}=1 \\
\frac{r_{2}}{r_{1}}=2
\end{gathered}
$$

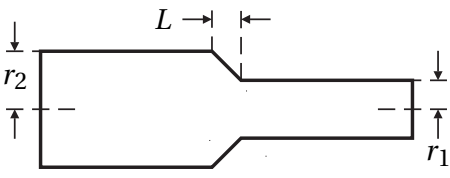

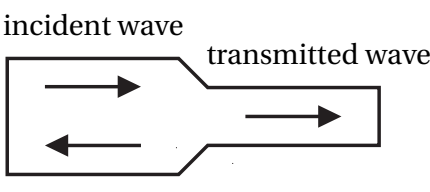

reflected wave

Figure 4.11: Waveguide of non-constant cross section.

To get an indication of the rate of convergence of an LRF model based on a two-port approximation, the two-port description is applied to the layer geometry depicted in figure 4.11 using different numbers of two-ports. The results are compared with the results obtained with a step-integration-algorithm for a (very) small fixed step ${ }^{6}$. The number of layers of constant thickness that are used in the two-port model to describe the tapered section is varied. A unit normal velocity is assumed as the boundary condition at the the entrance with the larger cross section. The boundary condition at the smaller cross section is an impedance of

\footnotetext{
${ }^{6}$ The step-integration-method used is the well known Runge-Kutta $(4,5)$ algorithm. The fixed step size equals $L / 5000$.
} 
an outgoing wave. For the step-integration model an equivalent set of boundary conditions is prescribed; a unit velocity and impedance of an outgoing wave are prescribed at the smaller cross section. An 1D two-port LRF model (as proposed in section 4.3.2) describing the straight layer sections at both waveguide ends is used to calculate the magnitude of the reflected and transmitted wave at the entrances of the conical section (based on the pressure and pressure gradients found at the waveguide entrances). The results of both models are scaled so the amplitude of the incident wave equals unity. The difference in reflected and transmitted wave (absolute value and phase angle) between the step-integration-method and the two-port network for $1,10,100$ and 1000 waveguide sections are plotted in figure 4.12 for $\tilde{s}=10$ and $\tilde{s}=100$, and a fixed ratio of $k / s=1 /(4 \pi)$.

The figure demonstrates that the propagation constant found for the coupled LRF layers converges to that found with the step-integration-method. A parameter study that was carried out showed that the two models appear to converge for
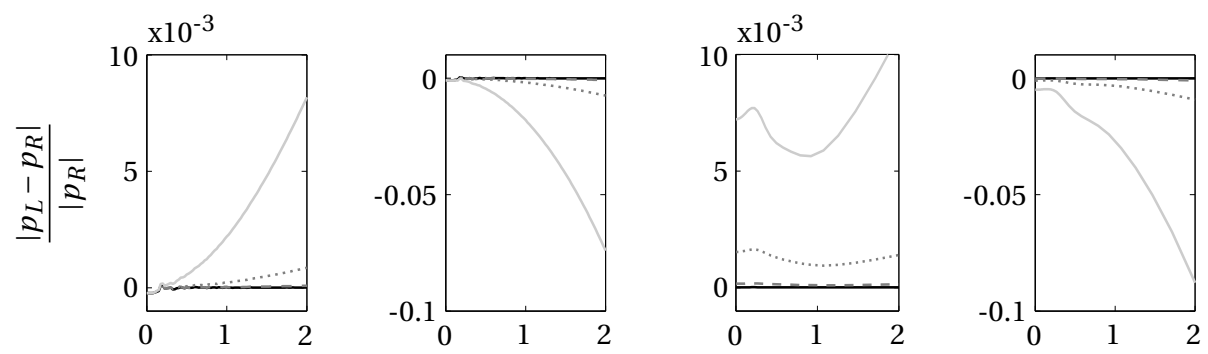

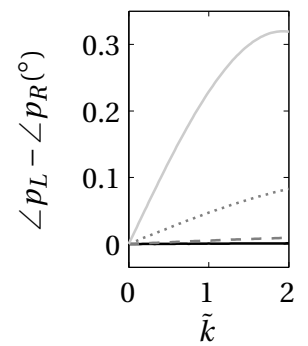

(a) Transmitted wave, $\tilde{s}=100$

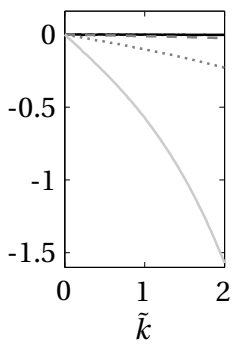

(b) Reflected wave, $\tilde{s}=100$

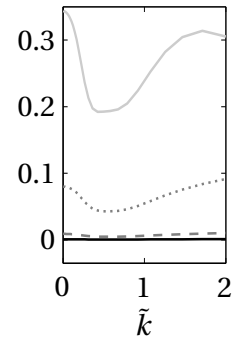

(c) Transmitted wave, $\tilde{s}=10$

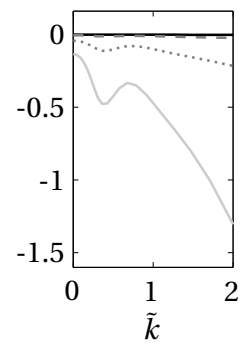

(d) Reflected wave, $\tilde{s}=10$

Figure 4.12: Difference in the reflected and transmitted waves (see figure 4.11) between solution of the step-integration-method approximation $\left(p_{R}\right)$ and $N$ coupled LRF layers $\left(p_{L}\right)$ for $k / s=1 /(4 \pi)$. 
arbitrary changes in cross section ${ }^{7}$. The rate of convergence, however, depends on $\tilde{s}, \tilde{k}$, and the rate of change of the cross section, but is only weakly dependent on the ratio $k / s$ for $k / s<1$. In chapter 6 , the effects of viscosity on the accuracy of LRF two-port models for waveguides of non-constant cross section are investigated.

\section{Tapered waveguides}

In the case of a conical or tapered waveguide, the waveguide surfaces can be described conveniently by the coordinate surfaces of a spherical or circular cylindrical coordinates system, respectively. In appendix F.1 it is explained that for these combinations of waveguide and coordinate system, a so-called one-parameter approximation allows the surfaces of constant pressure to be modeled exactly for the inviscid adiabatic case, while for the viscothermal case only errors due to the LRF approximation remain (apart from discretization errors). So, at first glance it seems attractive to model conical parts of a waveguide geometry in cylindrical coordinates. However, the use of models in circular cylindrical and spherical coordinates is limited. As an example, consider the layer geometry including a tapered section shown in figure 4.13(a), 4.13(b) and 4.13(c). Again an impedance condition is prescribed at the small waveguide opening while a pressure wave of unit amplitude is inciding at the other opening. The layer is filled with air and $k=0.1$ and $s=10$ (the characteristic length is the thickness of the thick part of the waveguide). Isobars resulting from FEM calculations are given in 4.13(a). Note that at small distances from the tapered section, the isobars are already straight by approximation. This is as expected, since for the given values of $\tilde{k}$ and $\tilde{s}$ all higher order modes have a fairly large decay rate.

The geometry can also be considered as an assembly of two straight waveguide sections and one tapered section. The boundary conditions at the inlet of each section is a prescribed pressure, while the boundary condition at the outlet corresponds to the impedance of straight layer with its cross-sectional surface equal to that of the outlet. The pressure fields of the different subsections found with FEM are given in figure 4.13(d). The isobars of the subsections match up well with those of the corresponding LRF models in Cartesian and circular cylindrical coordinates presented in figure 4.13(f). The results of a 1D LRF approximation in Cartesian coordinates only is given in figure 4.13(b) and 4.13(e). From figure 4.13(b) and 4.13(c) it is clear that the LRF approximations fail to accurately predict the pressure field in the conical section when the entire geometry is considered.

Figure 4.13(c) demonstrates the drawback of modeling the LRF approximation of the conic section in cylindrical coordinates; there is no common boundary over

\footnotetext{
${ }^{7}$ In the case of discontinuities, the step-integration-method must be used in succession on the different continuous subsections.
} 


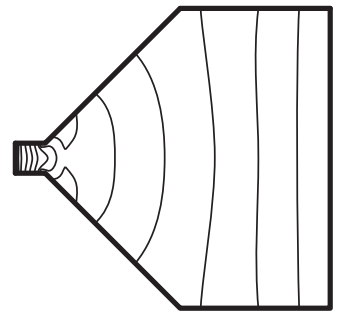

(a) FEM

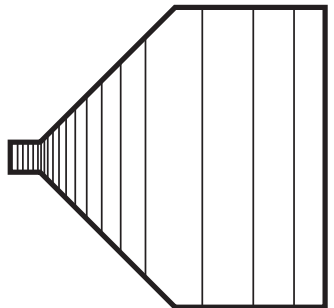

(b) LRF (Cartesian coordinates)

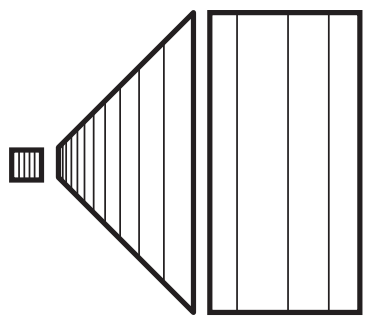

(e) LRF (Cartesian coordinates) subsections

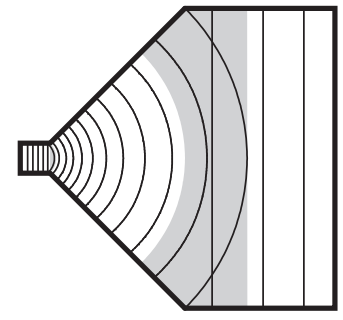

(c) LRF (cylindrical coordinates)

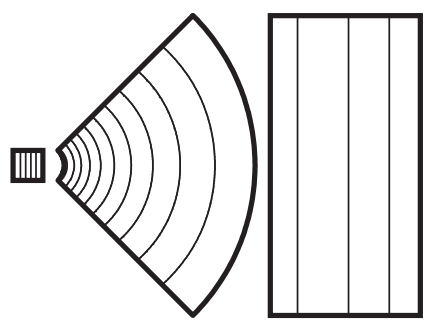

(f) LRF (cylindrical coordinates) subsections

Figure 4.13: Isobars $|p|$ in a 1D layer section of non-constant thickness for different models at $\tilde{k}=0.01$ and $\tilde{s}=10$. The gray areas indicate where the pressure fields predicted by the sub models are ambiguous.

which the pressure in both models is constant, and it is not clear how the conical and straight LRF model should be coupled at the interface to obtain the most accurate results. Note, the LRF approximation will always be (locally) inaccurate since the inlet effects at the interfaces of the waveguide sections are not modeled. Nevertheless, when two tapered waveguides are coupled that only differ slightly in opening angle, the errors that are induced by incompatibility in geometry and neglecting inlet effects at the interface both remain small.

It is concluded that the use of LRF models in circular cylindrical and spherical coordinates is thus limited to pure tapered or conical geometries, or geometries for which the rate of change of the cross section varies gradually. Additionally, in the latter case, using a two-port network of prismatic waveguides yields results of comparable accuracy and efficiency. 


\subsection{Acoustic two-port networks}

To obtain an accurate LRF model, the directions of wave propagation in a waveguide should correspond with the coordinate directions of a coordinate system. This implies that the complexity of a waveguide geometry determines whether a (simple) LRF solution is feasible. If the propagation directions of an arbitrary waveguide are not known a priori or vary with frequency, finding an appropriate coordinate system to derive an LRF model is not trivial. In such cases solving the full set of equations using numerical techniques is more appealing (see chapter $5)$.

Fortunately, in many cases, a good estimation of the propagation directions can be made even for relatively complex waveguide geometries. Finding a single coordinate system with coordinate directions that correspond with the propagation directions in a complex geometry at every point is well nigh impossible, however. In the case of complex waveguide geometries, it is therefore more appropriate to use a so-called lumped-parameter model. In this model, the total geometry is divided into multiple waveguides, each having their own LRF model. The behavior of the independent LRF models can be described by modeling them as acoustic two-ports. Accordingly, a geometry represented by multiple coupled two-port models is called a two-port network. An example of a two-port network for three coupled tubes is given in figure 4.14.

In the two-port network, the pressure and velocity are no longer represented as fields varying with position throughout the entire geometry. Instead, the be-

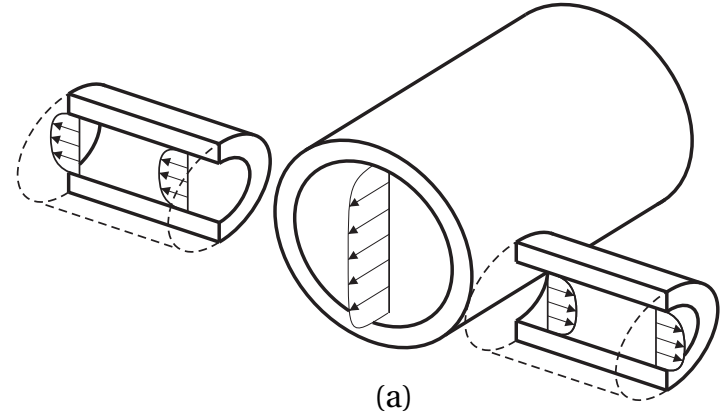

velocity/temperature profile
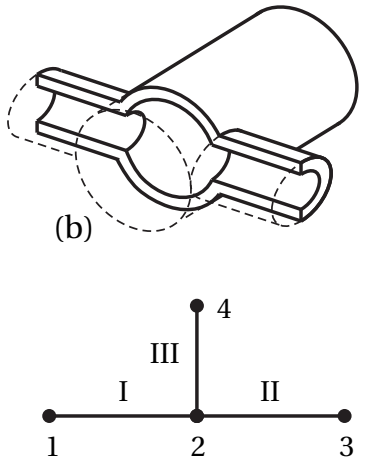

(c)

Figure 4.14: Two-port network of three coupled tubes: Individual waveguides (a), total geometry (b) and a representation in elements and nodes (c). 
havior of the two-port network is characterized by state variables describing the state of the individual two-ports. Below, the state variables that are used are introduced and the general form of a two-port description in terms of a mobility matrix is given. In the subsequent subsections, the mobility matrix for LRF models is derived for prismatic tubes, circular layers and 1D waveguides of arbitrary geometry. Note that two-port models are alternatively called four-pole models in older literature.

\subsubsection{General two-port description}

A variety of two-port models can be found in the literature $[95,96]$. The models can be categorized based on the choice of input and output state variables that are used. An overview of the three most prominent approaches used in acoustics is given in figure 4.15 .

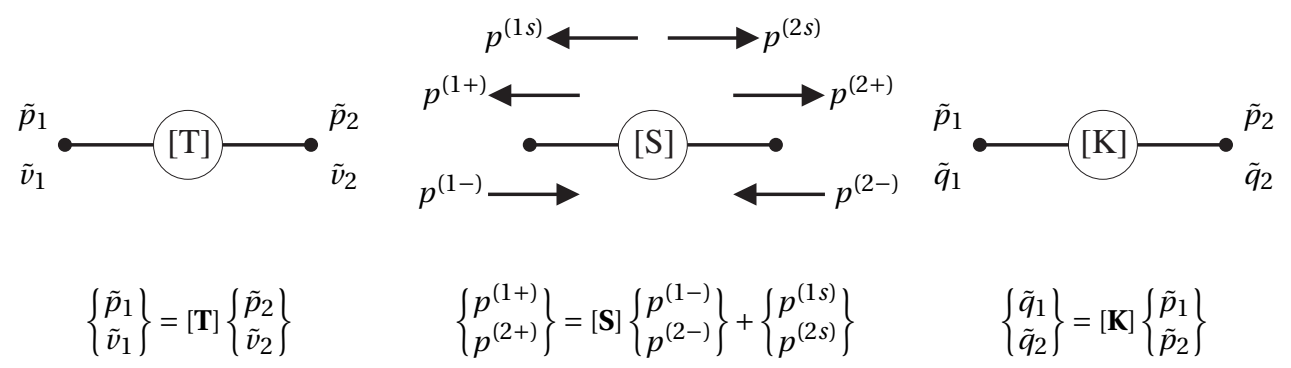

(a)

(b)

(c)

Figure 4.15: Schematic overview of different two-port approaches: transfer matrix [T] approach (a), scattering matrix $[\mathbf{S}]$ approach (b), and mobility matrix $[\mathbf{K}]$ approach (c).

Most commonly, the averaged pressure $\tilde{p}_{2}$ and averaged velocity $\tilde{v}_{2}$ at one port are taken as input state variables yielding the averaged pressure $\tilde{p}_{1}$ and averaged velocity $\tilde{v}_{1}$ at the other port as output state variables [97] (see figure 4.15(a). This so-called transfer-matrix approach is less suited to model two-port networks with multiple branches or two-port networks including loops [19, 95]. Glav et al. [95] and Eversman [98] presented an alternative method that uses the complex amplitudes of incident waves at both ports, $p^{(1-)}$ and $p^{(2-)}$, and the complex amplitude of the source waves (representing internal sources) at both ports, $p^{(1 s)}$ and $p^{(2 s)}$, as input state variables. The complex amplitude of the outgoing waves at both ports, $p^{(1+)}$ and $p^{(2+)}$, serve as output state variables (see figure 4.15(b)). This so-called 
scattering-matrix approach ${ }^{8}$ reflects the waveguide nature of the problem and is well suited for arbitrary two-port networks. A third approach, presented by Frid [99], uses the averaged pressures at both ports, $\tilde{p}_{1}$ and $\tilde{p}_{2}$, as input variables yielding the total volume flows at both ports, $\tilde{q}_{1}$ and $\tilde{q}_{2}$, as output variables (see figure 4.15(c)). This so-called mobility-matrix approach ${ }^{8}$ is adopted in this thesis. It is suited to model arbitrary two-port networks and is the most efficient approach in terms of the required number of state variables ${ }^{9}$ [95]. Using this approach, the general form of a two-port description for arbitrary waveguides is

$$
\left\{\begin{array}{c}
\tilde{q}_{1} \\
\tilde{q}_{2}
\end{array}\right\}=[\mathbf{K}]\left\{\begin{array}{c}
\tilde{p}_{1} \\
\tilde{p}_{2}
\end{array}\right\}
$$

where $\mathbf{K}$ is the so-called mobility matrix or admittance matrix.

An In-house software tool for two-port modeling based on the mobility-matrix approach was previously developed at the University of Twente [19]. It is extended to include two-port LRF models for circular air layers and curved air layers. In addition, the formulation used in the scattering-matrix approach is rewritten to obtain the mobility matrix for waveguides of arbitrary shape, based on a (measured) scattering matrix (that relates the incident and outgoing waves). In section 7.1.3 it is shown how source waves can also be incorporated in the mobility-matrix approach.

\subsubsection{Prismatic tubes}

The solution for pressure for prismatic tubes and curved tubes of constant cross section that were presented in the previous sections all take the following form for homogeneous $p d$-velocity and temperature boundary conditions that are constant in $p d$-direction

$$
\tilde{p}=p^{(-)} e^{i k \Gamma x}+p^{(+)} e^{-i k \Gamma x}
$$

where $p^{(+)}$and $p^{(-)}$are the complex amplitudes at $x=0$ of the waves traveling in positive and negative $x$-direction, respectively (see figure 4.16(a) and 4.16(b)).

The velocity in propagation direction as a function of the pressure is given in equation (4.13). Substituting the above expression for pressure, integrating over the waveguide cross section, and rewriting the result with equations (4.24) and

\footnotetext{
${ }^{8}$ Confusingly, in [19] the scattering matrix approach is called recursive formulation, while the mobility matrix approach is called transfer matrix formulation.

${ }^{9}$ Compared to the mobility-matrix approach, the transfer-matrix approach requires an equal number of state variables; depending on the problem, the scattering-matrix approach requires equal or more state variables.
} 


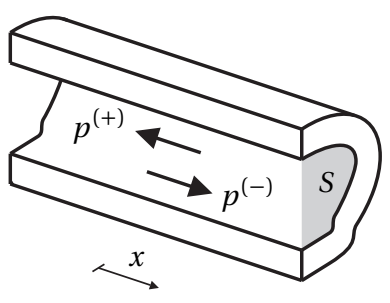

(a)

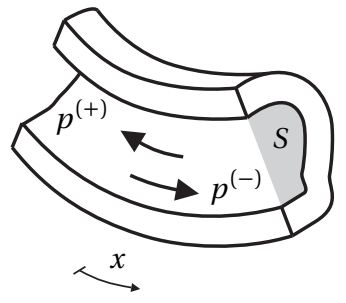

(b)

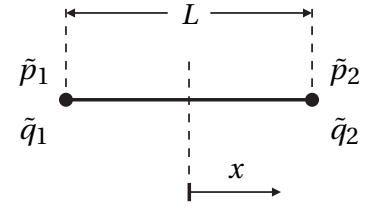

(c)

Figure 4.16: Prismatic tube of arbitrary cross section (a) and curved tube of arbitrary constant cross section (b) and a schematic representation of the corresponding two-port LRF model (c).

(4.18) yields the following expression for the volume flow $\tilde{q}$ through a waveguide cross section with cross-sectional surface area $S$

$$
\tilde{q}=-\frac{S}{\Gamma n}\left[p^{(-)} e^{i k \Gamma x}-p^{(+)} e^{-i k \Gamma x}\right]
$$

For a waveguide section of length $L$ the pressures $\tilde{p}_{1}$ and $\tilde{p}_{2}$, and volume flows $\tilde{q}_{1}$ and $\tilde{q}_{2}$ at the waveguide entrances can be expressed as a function of the complex amplitudes at $x=0$

$$
\begin{aligned}
\left\{\begin{array}{l}
\tilde{p}_{1} \\
\tilde{p}_{2}
\end{array}\right\}=[\mathbf{V}]\left\{\begin{array}{l}
p^{(-)} \\
p^{(+)}
\end{array}\right\}, \quad \text { with } \quad[\mathbf{V}]=\left[\begin{array}{cc}
e^{i k \Gamma L / 2} & e^{-i k \Gamma L / 2} \\
e^{-i k \Gamma L / 2} & e^{i k \Gamma L / 2}
\end{array}\right] \\
\left\{\begin{array}{l}
\tilde{q}_{1} \\
\tilde{q}_{2}
\end{array}\right\}=[\mathbf{W}]\left\{\begin{array}{l}
p^{(-)} \\
p^{(+)}
\end{array}\right\}, \quad \text { with } \quad[\mathbf{W}]=\frac{S}{\Gamma n}\left[\begin{array}{ll}
-e^{i k \Gamma L / 2} & e^{-i k \Gamma L / 2} \\
e^{-i k \Gamma L / 2} & -e^{i k \Gamma L / 2}
\end{array}\right]
\end{aligned}
$$

Equation (4.54) can be rewritten to yield the complex amplitudes $p^{(-)}$and $p^{(+)}$ in terms of the pressures $\tilde{p}_{1}$ and $\tilde{p}_{2}$. Substitution of the result in equation (4.55) leads to a relation between volume flows and pressures in the form presented in equation (4.51)

$$
\left\{\begin{array}{l}
\tilde{q}_{1} \\
\tilde{q}_{2}
\end{array}\right\}=[\mathbf{K}]\left\{\begin{array}{c}
\tilde{p}_{1} \\
\tilde{p}_{2}
\end{array}\right\}, \quad \text { with } \quad[\mathbf{K}]=[\mathbf{W}][\mathbf{V}]^{-1}
$$

\subsubsection{Circular layers}

In the case of circular layer waveguides (see figure 4.17), the solution for axisymmetrical boundary conditions is of the form

$$
\tilde{p}=p^{(-)} H_{0}^{(1)}(k \Gamma r)+p^{(+)} H_{0}^{(2)}(-k \Gamma r)
$$




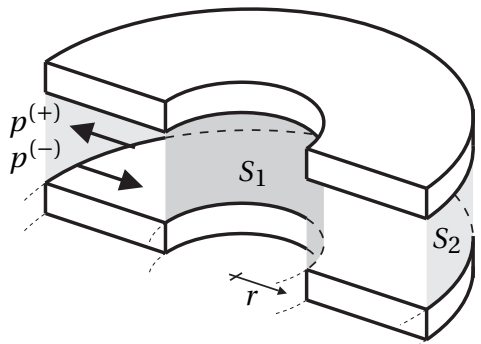

(a)

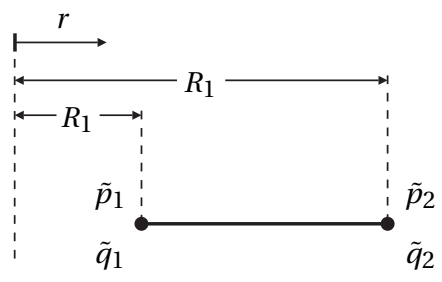

(b)

Figure 4.17: Circular layer waveguide (a) and a schematic representation of the corresponding two-port LRF model (b).

where $H_{n}^{(1)}$ and $H_{n}^{(2)}$ are $\mathrm{n}^{\text {th }}$-order Hankel functions of the first and second kind, respectively. Applying the same strategy as for prismatic tubes to obtain an expression for volume flows in terms of pressure yields

$$
\tilde{q}=\frac{S}{\Gamma n}\left[p^{(-)} H_{1}^{(1)}(k \Gamma r)-p^{(+)} H_{1}^{(2)}(-k \Gamma r)\right]
$$

Again, the complex amplitudes $p^{(-)}$and $p^{(+)}$are expressed in terms of the pressures at the location of the two-ports. Substituting the result in the above equation for $r=R_{1}$ and $r=R_{2}$ yields an expression for the (inward) volume flows at the ports of the form presented in (4.56), except the matrices $\mathbf{V}$ and $\mathbf{W}$ are now defined as

$$
\begin{aligned}
{[\mathbf{V}] } & =\left[\begin{array}{cc}
H_{0}^{(1)}\left(k \Gamma R_{1}\right) & H_{0}^{(2)}\left(-k \Gamma R_{1}\right) \\
H_{0}^{(1)}\left(k \Gamma R_{2}\right) & H_{0}^{(2)}\left(-k \Gamma R_{2}\right)
\end{array}\right] \\
{[\mathbf{W}] } & =\frac{1}{\Gamma n}\left[\begin{array}{cc}
H_{1}^{(1)}\left(k \Gamma R_{1}\right) S_{1} & -H_{1}^{(2)}\left(-k \Gamma R_{1}\right) S_{1} \\
-H_{1}^{(1)}\left(k \Gamma R_{2}\right) S_{2} & H_{1}^{(2)}\left(-k \Gamma R_{2}\right) S_{2}
\end{array}\right]
\end{aligned}
$$

where $S_{1}$ and $S_{2}$ the cross-sectional surface areas of the corresponding ports, with $l$ the thickness of the waveguide.

\subsubsection{Arbitrary 1D waveguides}

In this section wave propagation in a waveguide of arbitrary shape that does not include sources is considered (see figure 4.18). The acoustic fields are assumed to be approximately $1 \mathrm{D}$ at the waveguide entrances in correspondence with the LRF assumption $\tilde{k} \ll 1$. Note that this assumption does not apply to the waveguide interior allowing waveguides of arbitrary interior size having arbitrary sound fields 

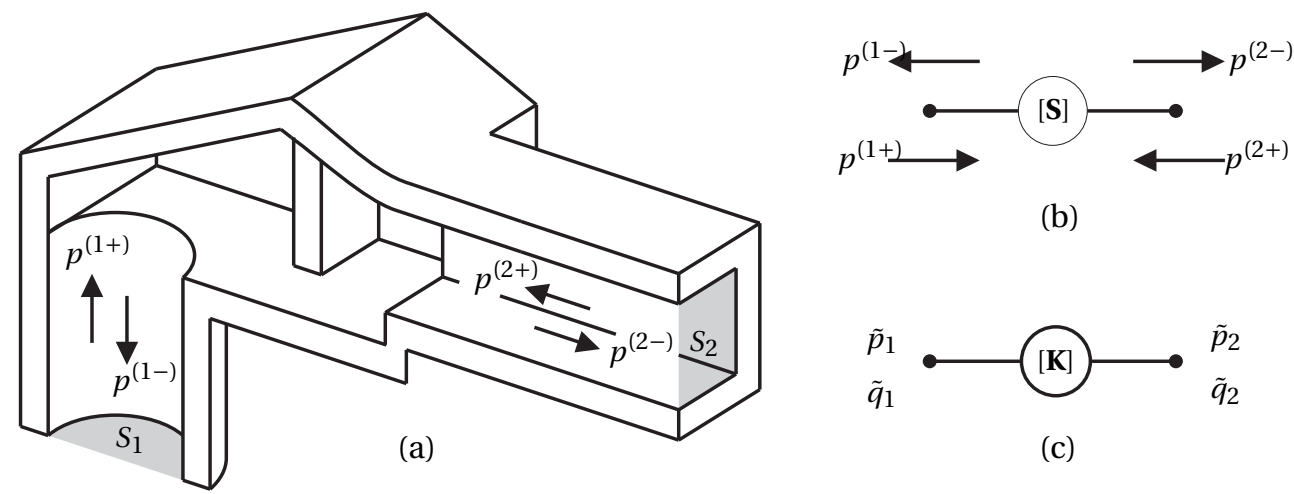

(b)

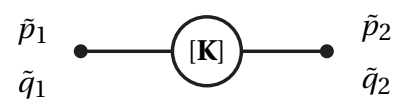

(c)

Figure 4.18: Waveguide of arbitrary shape including an internal boundary (a) and two schematic representations of the corresponding two-port LRF model: scattering-matrix approach (b), and mobility-matrix approach (c).

to be modeled. The interior can also include elements impeding, reflecting or attenuating the sound waves such as fan blades, resonators or acoustic lining and may involve changes in media (for instance in the form of a foam barrier).

Under these assumptions, the complex amplitude of the outgoing waves, $p^{(1-)}$ and $p^{(2-)}$, is completely determined by the complex amplitude of the incident waves $p^{(1+)}$ and $p^{(2+)}$ (see figure 4.18(a) and 4.18(b)). The so-called scattering matrix $\mathbf{S}$ determines how the complex amplitudes of the incident waves and outgoing waves are related;

$$
\left\{\begin{array}{l}
p^{(1-)} \\
p^{(2-)}
\end{array}\right\}=[\mathbf{S}]\left\{\begin{array}{l}
p^{(1+)} \\
p^{(2+)}
\end{array}\right\}
$$

The off-diagonal terms of $\mathbf{S}$ represent how well incident waves are transmitted to the other outlet, while the diagonal terms represent how well incident waves are reflected back to the corresponding inlet. For straight prismatic tubes (or circular air layers) incident waves are not reflected, leading to a scattering matrix with all zero diagonal terms. For an induct fan, waves are both reflected and transmitted leading to a fully populated scattering matrix (see, section 7.1.3). In the case of complex waveguide shapes or complex boundary conditions, analytical expressions for the scattering-matrix elements may not be available. Alternatively, these coefficients can be extracted from more detailed FE/BE models or obtained from measurement (see, section 7.1.4).

Equation (4.61) can be rewritten so it expresses the complex amplitudes of the incident and outgoing wave at one port in terms of the complex amplitudes of the 
waves at the other port

$$
\left\{\begin{array}{l}
p^{(1+)} \\
p^{(1-)}
\end{array}\right\}=[\mathbf{R}]\left\{\begin{array}{l}
p^{(2+)} \\
p^{(2-)}
\end{array}\right\}, \quad \text { with } \quad[\mathbf{R}]=\frac{1}{S_{21}}\left[\begin{array}{cc}
-S_{22} & 1 \\
S_{21} S_{12}-S_{11} S_{22} & S_{11}
\end{array}\right]
$$

Equations (4.52) and (4.53) are used to express the dimensionless pressure $p_{1}$ and $p_{2}$ and volume flows $q_{1}$ and $q_{2}$ in terms of the complex amplitudes of the plain waves traveling through both ports

$$
\begin{array}{ll}
\tilde{p}_{1}=p^{(1+)}+p^{(1-)}, & \tilde{p}_{2}=p^{(2+)}+p^{(2-)} \\
\tilde{q}_{1}=\frac{S_{1}}{\Gamma_{1} n}\left(-p^{(1+)}+p^{(1-)}\right), & \tilde{q}_{2}=\frac{S_{2}}{\Gamma_{2} n}\left(p^{(2+)}-p^{(2-)}\right)
\end{array}
$$

where $S_{1}$ and $S_{2}$ are the cross-sectional surface areas and $k \Gamma_{1}$ and $k \Gamma_{2}$ are the propagation constants for the plain waves at the different ports. The expressions for $p_{1}$ and $q_{1}$ can also be written in terms of $p^{(2+)}$ and $p^{(2-)}$ by invoking equation (4.62) yielding the following systems

$$
\begin{aligned}
& \left\{\begin{array}{l}
p_{1} \\
p_{2}
\end{array}\right\}=[\mathbf{V}]\left\{\begin{array}{l}
p^{(2+)} \\
p^{(2-)}
\end{array}\right\}, \quad \text { with } \quad[\mathbf{V}]=\left[\begin{array}{cc}
\left\{\begin{array}{cc}
1 & 1
\end{array}\right\}[\mathbf{R}] \\
\{1 & 1
\end{array}\right\} \\
& \left.\left\{\begin{array}{l}
q_{1} \\
q_{2}
\end{array}\right\}=[\mathbf{W}]\left\{\begin{array}{l}
p^{(2+)} \\
p^{(2-)}
\end{array}\right\}, \quad \text { with } \quad[\mathbf{W}]=\left[\begin{array}{cc}
\left\{-\frac{S_{1}}{\Gamma_{1} n}\right. & \left.\frac{S_{1}}{\Gamma_{1} n}\right\}[\mathbf{R}] \\
\left\{\frac{S_{2}}{\Gamma_{2} n}\right. & -\frac{S_{2}}{\Gamma_{2} n}
\end{array}\right\}\right]
\end{aligned}
$$

Again, the volume flows $q_{1}$ and $q_{2}$ are positive for inward velocities. Equation (4.65) is rewritten to give the complex amplitudes of the backward and forward traveling waves at port 2 in terms of the pressures at both ports. Substitution of the result in (4.66) yields an expression of the desired form

$$
\left\{\begin{array}{c}
\tilde{q}_{1} \\
\tilde{q}_{2}
\end{array}\right\}=[\mathbf{K}]\left\{\begin{array}{c}
\tilde{p}_{1} \\
\tilde{p}_{2}
\end{array}\right\}, \quad \text { with } \quad[\mathbf{K}]=[W][V]^{-1}
$$

\subsubsection{Coupling two-ports}

The two-ports can be seen as acoustic FE elements with the LRF solution for wave propagation as shape functions. When compared to a 1D structural element, nodal pressures are equivalent with displacements, volume flows are equivalent with forces and the mobility or admittance matrix $\mathbf{K}$ is equivalent with the stiffness matrix. A system matrix for a geometry consisting of multiple two-ports is constructed using standard FE techniques; the nodes of the independent elements are coupled by demanding continuity of pressure and volume flow at the inlets of 
the waveguides. For example, the system matrix of three coupled tubes depicted in figure 4.19 is

$$
\left\{\begin{array}{l}
\tilde{q}_{1} \\
\tilde{q}_{2} \\
\tilde{q}_{3} \\
\tilde{q}_{4}
\end{array}\right\}=\left[\begin{array}{cccc}
K_{11}^{(I)} & K_{12}^{(I)} & 0 & 0 \\
K_{21}^{(I)} & K_{22}^{(I)}+K_{11}^{(I I)}+K_{11}^{(I I I)} & K_{12}^{(I I)} & K_{12}^{(I I I)} \\
0 & K_{21}^{(I I)} & K_{22}^{(I I)} & 0 \\
0 & K_{21}^{(I I)} & 0 & K_{22}^{(I I I)}
\end{array}\right]\left\{\begin{array}{l}
\tilde{p}_{1} \\
\tilde{p}_{2} \\
\tilde{p}_{3} \\
\tilde{p}_{4}
\end{array}\right\}
$$

where the elements of the (force) vector $\tilde{\mathbf{q}}$ are the inflows of volume at each node and the elements of the (displacement) vector $\tilde{\mathbf{p}}$ are the pressures at each node. Applying the boundary conditions by prescribing a pressure $\tilde{p}_{i}$, a volume flow $\tilde{q}_{i}$, or an impedance $\tilde{q}_{i} / \tilde{p}_{i}$ is done using standard FE techniques [19].

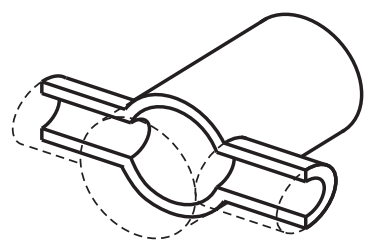

(a)

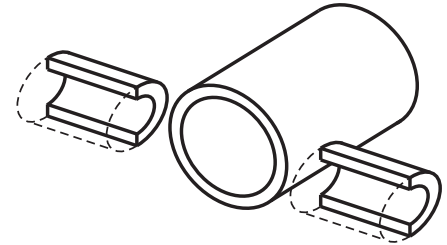

(b)

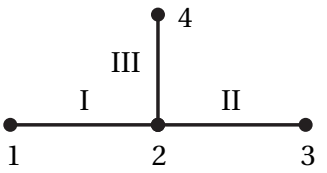

(c)

Figure 4.19: Structure of multiple coupled waveguides (a), individual waveguides (b), and a schematic representation of the corresponding two-port network (c). The system of equations describing the two-port network is given in equation (4.68).

\subsubsection{Length corrections}

The two-port models presented above are based on LRF solutions for waveguides of infinite length. In general, the velocity, temperature and pressure profiles of the different waveguide sections will not match at the interface if several two-ports are coupled (see figure 4.20). The actual velocity, temperature and pressure fields at discontinuities are therefore not accurately represented by two-port networks, which are based on continuity of pressure and fluid flow in an averaged sense only. For practical cases, the velocities in $c d$-direction can be of the same magnitude as velocities in the $p d$-directions, and the pressure field may vary substantially over the waveguide cross sections in the vicinity of a waveguide junction. Consequently, the LRF assumptions are violated locally and the accuracy of the velocity and pressure amplitudes predicted at the entrances of a two-port network can decrease considerably.

For the inviscid adiabatic acoustic case, similar effects occur when $\tilde{k} \sim 1$ or $\tilde{k}>1$. For a limited number of types of waveguide junctions, so-called length 


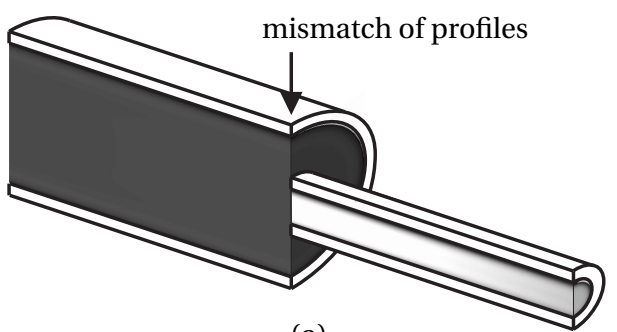

(a)

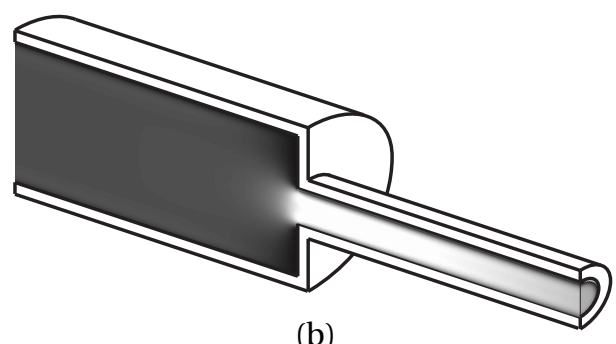

(b)

Figure 4.20: Mismatch in $p d$-velocity profiles for two coupled waveguides: Two-port LRF solution (a) and mode-matching/FEM solution (b).

corrections or end corrections are available, that compensate for the local behavior $[28,29,70,72]$. The corrections are aimed at compensating for the effects of higher order acoustic modes which are not captured by the two-port model. The literature ${ }^{10}$ suggests that if viscous effects are confined to boundary layers, these corrections are suitable for use with two-port LRF models as well. To the author's knowledge, no appropriate expressions are available in case viscous effects are significant throughout the bulk of the fluid in some part of the two-port network $(\tilde{s}<5)$. The results for a simple waveguide junction presented in chapter 6 suggest that although other phenomena (related to the omission of viscous modes) dominate the error if $\tilde{s}<5$, the errors remain relatively small for $k / s \ll 1$.

Note that for more complex junctions of waveguides, expressions for end corrections are in general not available and alternative techniques such as mode matching as described in section 6.7 or the various FEM techniques described in chapter 5 , appendix J or reference [1] are required to obtain a more accurate solution. An example of where the use of 'standard' end corrections does not lead to good accuracy is the application presented in section 7.2.

\subsection{Summary and discussion}

The general solutions strategy to obtain LRF models for different geometries was presented. The method was extended to include curved waveguides, waveguides of non-constant cross section and arbitrary homogeneous and non-homogeneous boundary conditions that may vary both over the boundary of the cross section

\footnotetext{
${ }^{10}$ Morse and Ingard [72] derive expressions for end-corrections based on mode-matching techniques using inviscid adiabatic modes. They propose to correct the different acoustic modes for viscothermal effects by applying an equivalent admittance based on boundary layer approximation which is valid for $(\tilde{s} \gg 1)$.
} 
and in the direction of wave propagation. The importance of the influence of the cross-sectional shape on the propagation constant for lower shear wave numbers was demonstrated $(\tilde{s}<10)$. It was shown that for waveguides of large curvature, LRF models for curved waveguides provide a far more accurate value for the propagation constant than using LRF models for straight waveguides of equivalent length ${ }^{11}$. Three numerical techniques to obtain a solution for the pressure were discussed for LRF models describing waveguides of varying cross section. The influence of the dimensionless acoustic and shear wave number on the rate of convergence of the most attractive one was briefly investigated. The concepts of acoustic two-ports and two-port networks were introduced. Three types of twoport descriptions were discussed: transfer-matrix approach, scattering-matrix approach and mobility-matrix approach. The two-port descriptions in terms of mobility matrices were found to be most attractive, and were given for straight and curved tubes/layers, circular cylindrical layers and waveguides having a known (arbitrary) scattering matrix.

\footnotetext{
${ }^{11}$ In section 6.6 it is shown that the effect of these large deviations on the complex amplitude of traveling waves (before and after a curved section) are nevertheless small if a waveguide with a 180 degree bend is considered.
} 


\section{Chapter 5}

\section{Viscothermal acoustic FE models}

In this chapter, different finite element approximations are derived for the linearized set of equations describing viscothermal wave propagation that was introduced in chapter 2. Prior to discretization, the set of equations can be reduced by eliminating the density, pressure and/or temperature variables. In the first section, three FE methods depending on different sets of variables are considered and their weak form is presented. The convergence behavior of the resulting FE approximations depends on the formulations of the problem, which is the main topic of this chapter. The concepts of optimal convergence, stability and robustness, which are important in the study of convergence, are introduced at the end of the first section.

In the second section, it is shown that the convergence behavior of FE methods for viscothermal acoustics is closely related to that of FE methods for the Stokes problem. The amount of literature on FE methods for the Stokes equations is vast (see for instance [60, 61, 100, 101, 102, 103]). A summary of some of the results for the Stokes equations that are interesting in light of the theory on the related LNSF equations is given at the beginning of the second section. Thereafter, the requirements for optimal convergence of FE methods for the LNSF equations are given, and the influence of both locking and spurious modes, two phenomena that can adversely influence the convergence behavior of the FE solution, is commented on.

In the last section, a numerical test for the stability of FE methods is introduced. The stability and convergence behavior of various promising FE methods for the LNSF equations are inspected with this numerical test. The majority of the inspected methods are based on FE methods which are known to be stable for the Stokes equations. Note that all FE results presented in this chapter were obtained with a dedicated FE package for MATLAB written by the author. The FE results 
found in other chapters of this thesis were obtained through implementation of the LNSF equations in the commercial FE package COMSOL. Implementation of the presented mixed methods in COMSOL was a joint effort by W.R. Kampinga $[1,27]$ and the author.

In the course of establishing the different FE approximations, certain abstract function spaces (known as Sobolev spaces) are used. These spaces are essential when proving the existence and uniqueness of solutions to the (continuous) problem and examining the convergence behavior of the proposed approximations. However, the theory concerning the definition and properties of these spaces is outside the scope of this thesis. For a precise definition and development of these function spaces (including an investigation into the properties which are important to establish a mathematical foundation for FE methods) see for instance [60, 100]. The different problems presented in this chapter and onwards are posed in arbitrary domains $\Omega$ of $\mathbb{R}^{n}$. It must be noted however, that some of the proofs in the mentioned references are derived for domains with sufficiently smooth boundary, leaving unanswered the question of their validity for domains with corners.

\subsection{Viscothermal acoustic problems}

The linearized equations for viscothermal wave propagation in the frequency domain given in equation (2.16) can be rewritten in many forms involving different numbers of variables. Three different types that are considered as a basis for a FE method are discussed below. The original equations are stated here once again in a more convenient form. The momentum equation is given in terms of the stress tensor and heat flux vector. All terms in the balance equations for momentum and energy except the body forces are moved to the left-hand side. The ideal gas law is rewritten to yield density in terms of pressure and temperature. The result is substituted in the equation for conservation of mass to eliminate density from the set of equations. After these modifications, the system of equations in (2.16) takes the form

$$
\begin{aligned}
i \omega \rho_{0} \mathbf{v}-\nabla \cdot \sigma & =\rho_{0} \mathbf{b} \\
i \omega \rho_{0} C_{p} T-i \omega p+\nabla \cdot \mathbf{q} & =\rho_{0} r_{e} \\
i \frac{\omega}{p_{0}} p-i \frac{\omega}{T_{0}} T+\nabla \cdot \mathbf{v} & =0
\end{aligned}
$$

where the stress tensor $\sigma$ and heat flux vector $\mathbf{q}$ are as defined in equation (2.2) and (2.4), respectively. They are stated here once more for convenience;

$$
\sigma=-p \mathbf{I}+\lambda(\nabla \cdot \mathbf{v}) \mathbf{I}+\mu\left(\nabla \mathbf{v}+(\nabla \mathbf{v})^{T}\right), \quad \mathbf{q}=-\kappa \nabla T
$$


Note that in this chapter the bars over $T$ and $p$, indicating that these quantities are small perturbations from a steady state, are dropped.

\subsubsection{Velocity, temperature and pressure/density based FE methods}

Equations (5.1) are the basis of the viscothermal velocity, temperature and pressurebased (v, $T, p$-based) FE method presented in Nijhof et al. [25] and Kampinga et al. [27]. The equations used by Nijhof et al. are scaled to yield a dimensionless form prior to discretization. Malinen et al. [24] use a similar (dimensionless) set of equations to construct their FE method. They remove the pressure variable from the set of equations as described in the next section and introduce an auxiliary (dimensionless) density-like variable $\phi$ that scales linearly with the divergence of velocity as

$$
\phi=\frac{i s^{2}-\gamma k^{2} \xi}{i s^{2} \gamma k}\left(\frac{p}{p_{0}}-\frac{T}{T_{0}}\right)=\frac{i s^{2}-\gamma k^{2} \xi}{s^{2} \gamma k \omega} \nabla \cdot \mathbf{v}
$$

Cheng et al. [78] also use a set of equations similar to equations (5.1) to obtain their FE method. However, they use an equation of state that relates pressure to density directly. This equation of state corresponds with the assumption of adiabatic $^{1}$ wave propagation and does not include temperature as a variable. Using this approach, the energy equation decouples from the other equations, and the effects of thermal boundary layers are not taken into account. In this thesis, the equations in (5.1) are used in their (unchanged) dimensional form.

\section{Weak form}

The equations are brought into a weak form in the usual way; the equations are multiplied by weighting functions (u, $S$ and $q$ ) and integrated over the domain $\Omega$. The divergence of the stress tensor and the Laplacian of the temperature (the divergence of the heat flux) are rewritten by successively applying the product rule and the divergence theorem, thus reducing the order of the differential equations involved. The resulting surface integrals serve as natural boundary conditions and are moved to the right-hand side. As a last step, the energy equation in the weak form is divided through $-T_{0}$ and the continuity equation is multiplied through -1 (thus arriving at a complex, symmetric formulation of the problem). The weak

\footnotetext{
${ }^{1}$ Isothermal wave propagation can also be modeled by using the isothermal speed of sound.
} 
form of the problem can now be stated as: find $(\mathbf{v}, T, p) \in X \times Y \times Z$ so that

$$
\begin{aligned}
i \omega \rho_{0}\langle\mathbf{v}, \mathbf{u}\rangle+\langle\sigma, \nabla \mathbf{u}\rangle & =\rho_{0}\langle\mathbf{b}, \mathbf{u}\rangle+\left\langle\mathbf{f}_{s}, \mathbf{u}\right\rangle_{\partial S} \\
-i \frac{\omega \rho_{0} C_{p}}{T_{0}}\langle T, S\rangle+i \frac{\omega}{T_{0}}\langle p, S\rangle+\frac{1}{T_{0}}\langle\mathbf{q}, \nabla S\rangle & =-\frac{\rho_{0}}{T_{0}}\left\langle r_{e}, S\right\rangle+\frac{1}{T_{0}}\left\langle q_{n}, S\right\rangle_{\partial S} \\
-i \frac{\omega}{p_{0}}\langle p, q\rangle+i \frac{\omega}{T_{0}}\langle T, q\rangle-\langle\nabla \cdot \mathbf{v}, q\rangle & =0
\end{aligned}
$$

for all $(\mathbf{u}, S, q) \in X \times Y \times Z$, where the sesquilinear forms on the left-hand side involving the stress tensor and the heat flux vector can be expanded as

$$
\begin{aligned}
& \langle\sigma, \nabla \mathbf{u}\rangle=-\langle p, \nabla \cdot \mathbf{u}\rangle+\langle\lambda \nabla \cdot \mathbf{v}, \nabla \cdot \mathbf{u}\rangle+\mu\left\langle\nabla \mathbf{v}+(\nabla \mathbf{v})^{T}, \nabla \mathbf{u}\right\rangle \\
& \langle\mathbf{q}, \nabla S\rangle=-\kappa\langle\nabla T, \nabla S\rangle
\end{aligned}
$$

The sesquilinear forms on the right-hand side involving the stress vector $\mathbf{f}_{s}$ and normal heat flux $q_{n}$ serve as natural boundary conditions. If desired they can be expressed in terms of the variables of the problem as follows

$$
\mathbf{f}_{s}=\sigma \cdot \mathbf{e}_{n}, \quad q_{n}=\mathbf{q} \cdot \mathbf{e}_{n}=-\kappa \nabla T \cdot \mathbf{e}_{n}
$$

with $\mathbf{e}_{n}$ the outward normal on the boundary surface $\partial S$ of the domain. The trial and test spaces $X, Y$ and $Z$ are defined as

$$
X=\left[H^{1}(\Omega)\right]^{n}, \quad Y=H^{1}(\Omega), \quad Z=L^{2}(\Omega)
$$

where $n$ is the dimensionality of the problem, and the abstract function spaces $L^{2}$ and $H^{1}$ are so-called Hilbert spaces (more precisely, they are Sobolev spaces of zeroth and first order having $L^{2}$ norms). For a definition and concise description of the properties of these function spaces, see for instance $[60,100]$. The inner products $\langle$,$\rangle (used in (5.4), (5.5) and (5.6)) are sesquilinear forms over the domain$ $\Omega$, defined for scalar-, vector- and second order tensor-valued functions, respectively, as

$$
\langle a, b\rangle=\int_{\Omega} a b^{H} d \Omega, \quad\langle\mathbf{a}, \mathbf{b}\rangle=\int_{\Omega} \mathbf{a} \cdot \mathbf{b}^{H} d \Omega, \quad\langle\alpha, \beta\rangle=\int_{\Omega} \alpha: \beta^{H} d \Omega \quad(5.9 \mathrm{a}, \mathrm{b}, \mathrm{c})
$$

where the superscript ${ }^{H}$ indicates complex conjugation. The inner products $\langle,\rangle_{\partial S}$ are similar sesquilinear forms where the integrals are taken over the boundary $\partial S$ of the domain $\Omega$ instead of over the domain itself.

Different types of boundary conditions can be enforced by placing restrictions on the trial and/or test spaces, and/or adjusting the natural boundary conditions 
(see, for instance, [104] for the restrictions/adjustments needed to model (non)homogeneous Dirichlet, Neumann, Robin and mixed boundary conditions in the case of the related Stokes equations). To keep the derivation general, no specific boundary conditions are assumed here.

Note that any solution of the strong form in equation (5.1) will also be a solution of the weak form in equation (5.4), and sufficiently smooth solutions $(\mathbf{v}, T, p)$ of the weak problem are also a solution of equations (5.1). Issues involving existence and uniqueness of weak solutions and their FE approximations are addressed in section 5.2 .

If an FE method for which the FE spaces for the trial and test functions are equal is used to approximate the system of equation in (5.4) (i.e., using a BubnovGalerkin method), the resulting system of equations is symmetric (but not Hermitian). This is a favorable property, since it can significantly reduce the amount of memory and time used by certain solvers during the solution process [27]. Note that the symmetry can be lost by application of certain boundary conditions or body forces that are dependent on the field variables.

\subsubsection{Velocity- and temperature-based FE methods}

Finite element models based on the weak form presented in the previous section approximate the velocity, temperature and pressure field. In light of the relation between degrees of freedom, calculation time and memory requirements, it seems advantageous to eliminate one or more variables prior to discretization to reduce the fields that need to be approximated, and thus the degrees of freedom in the system. In this section, the weak form of a set of equations from which the pressure is eliminated is described. Such a system still allows boundary conditions in terms of velocity, force, temperature and heat flux to be prescribed in a straightforward manner. Equation (5.1c) can be rewritten to yield pressure in terms of temperature and the divergence of velocity. Substitution of this expression in the equation (2.2) allows the stress tensor to be expressed in terms of velocity and temperature only

$$
\sigma=\left(-\frac{p_{0}}{T_{0}} T-i \frac{p_{0}}{\omega} \nabla \cdot \mathbf{v}+\lambda \nabla \cdot \mathbf{v}\right) I+\mu\left(\nabla \mathbf{v}+(\nabla \mathbf{v})^{T}\right)
$$

Substitution of (5.1c) in equation (5.1b) yields the energy equation in terms of temperature and velocity. The temperature-dependent terms of the resulting equation are rewritten using the relation $R_{0}=C_{p}-C_{\nu}$ to obtain:

$$
i \omega \rho_{0} C_{\nu} T+p_{0} \nabla \cdot \mathbf{v}-\kappa \Delta T=\rho_{0} r_{e}
$$

Although scaled differently, equation (5.1a) and equation (5.11) (with the stress tensor as defined in equation (5.10)) form the set of equations that is the basis 
of the viscothermal velocity and temperature-based (v, $T$-based) FE method proposed by Joly [48]. Another scaling that results in a dimensionless form of these equations is discussed by Malinen et al. [24]. They perform an order of magnitude analysis on the different dimensionless terms and conclude that straightforward discretization of equations can cause a so-called locking effect for low values of the dimensionless acoustic wave number $(\tilde{k} \downarrow 0)$ (or for very high values of the bulk modulus $\lambda \rightarrow \infty$ ). The cause of locking, which has an adverse effect on the convergence behavior, and possible remedies to counter these effects are investigated in section 5.2.

\section{Weak form}

The equations are brought into weak form as described in the previous section. The equation for energy is again divided through $-T_{0}$ so a symmetric system matrix is obtained if the Bubnov-Galerkin method is used. The weak form of the problem can now be stated as: find $(\mathbf{v}, T) \in X \times Y$ so that

$$
\begin{aligned}
& i \omega \rho_{0}\langle\mathbf{v}, \mathbf{u}\rangle+\langle\sigma, \nabla \mathbf{u}\rangle= \rho_{0}\langle\mathbf{b}, \mathbf{u}\rangle+\left\langle\mathbf{f}_{s}, \mathbf{u}\right\rangle_{\partial S} \\
&-i \frac{\omega \rho_{0} C_{v}}{T_{0}}\langle T, S\rangle-\frac{p_{0}}{T_{0}}\langle\nabla \cdot \mathbf{v}, S\rangle \\
&-\frac{\kappa}{T_{0}}\langle\nabla T, \nabla S\rangle=-\frac{\rho_{0}}{T_{0}}\left\langle r_{e}, S\right\rangle+\frac{1}{T_{0}}\left\langle q_{n}, S\right\rangle_{\partial S}
\end{aligned}
$$

for all (u, $S) \in X \times Y$, with the trial and test spaces $X$ and $Y$ as defined in equation (5.8a,b), and $\sigma$ as defined in (5.10).

\subsubsection{Velocity-based FE methods}

Analytical manipulation also allows equation (5.1) to be rewritten as a single fourth order vector equation with only velocity variables. This is done by taking the divergence of (5.1a) (with the stress tensor as defined in equation (5.10)) and substituting the result in equation (5.11). This result can be rewritten to give the temperature as a function of velocity only. Substituting this expression in equation (5.10) yields the stress tensor expressed in terms of velocity only.

$$
\begin{aligned}
\sigma=-i \frac{\gamma p_{0}}{\omega}(\nabla \cdot \mathbf{v}) \mathbf{I}+\lambda(\nabla \cdot \mathbf{v}) \mathbf{I}+\mu\left(\nabla \mathbf{v}+(\nabla \mathbf{v})^{T}\right) \\
+i \frac{\kappa(\gamma-1)}{\omega} \frac{T_{0}}{p_{0}}\left(-i \omega \rho_{0} \nabla \cdot \mathbf{v}-i \frac{p_{0}}{\omega} \Delta(\nabla \cdot \mathbf{v})+(\lambda+\mu) \Delta(\nabla \cdot \mathbf{v})\right. \\
\left.+\mu \nabla \cdot \Delta \mathbf{v}+\nabla \cdot \rho_{0} \mathbf{b}\right) \mathbf{I}+i \frac{p_{0}}{\omega T_{0} C_{v}} r_{e} \mathbf{I}
\end{aligned}
$$


Using this expression for the stress tensor, the resulting equation for conservation of momentum is a fourth order equation in terms of velocity only. Eliminating the temperature variable might seem advantageous from a computational point of view at first glance, however, the price to pay for eliminating the temperature variable is the requirement of inter-element continuity of higher order (weak) derivatives. The equations for the $(\mathbf{v}, T, p)$ and $(\mathbf{v}, T)$-based FE methods are second order and require $\mathbf{v}$ and $T$ to be elements of the space $H^{1}$ when considering the weak form (in $1 \mathrm{D}$ settings, this implies $C^{0}$ inter-element continuity is required). This is obviously not sufficient for the proposed fourth order equation which requires $\mathbf{v}$ and $T$ to be elements of the spaces $H^{2}$ when considering the weak form (in 1D settings this implies $C^{1}$ inter-element continuity). FE discretization schemes that meet this demand are available, however, constructing and implementing FE spaces in $H^{2}$ is a non-trivial task which is considered more cumbersome than implementing mixed methods (with FE spaces in $H^{1}$ and $L^{2}$ ). An additional disadvantage is that temperature-related boundary conditions cannot be prescribed in a straightforward way. Furthermore, the complications due to the locking phenomenon mentioned for the $(\mathbf{v}, T)$-based methods will also occur for the velocity-based elements. Considering these drawbacks, the development of a FE method based on a formulation in terms of velocity is not pursued here.

\subsubsection{FE spaces, convergence, approximability, and stability}

The weak forms of the different formulation presented above yield systems of infinite size which cannot be numerically evaluated by a computer. An approximate solution can be obtained, by evaluating the equations on a finite dimensional subspace of the function space governing the problem. FE methods can be seen as a general technique to build finite-dimension subspaces that allow the weak form of a continuous problem to be approximated. The approximate solutions of an FE method are dependent on both the weak form that is chosen to represent the original problem and the way the finite-dimensional subspaces are constructed. In this subsection, the construction of FE spaces and the concepts of convergence, approximability, optimal convergence/stability and robustness are discussed briefly. For a more rigorous introduction of FE methods and these concepts, see for instance $[62,100,104,105,106]$.

\section{FE spaces}

For the above weak forms, the subspaces $X_{h} \subset X, Y_{h} \subset Y$ and $Z_{h} \subset Z$ are the FE spaces in which the FE solutions $\tilde{\mathbf{v}}_{h}, \tilde{T}_{h}$ and $\tilde{p}_{h}$ are sought. For FE methods, these subspaces are constructed by subdividing the domain of the problem 
in non-overlapping, connected sub-domains called elements, forming a so-called $F E$-mesh. Subsequently, the spaces $X, Y$ and $Z$ on each sub-domain are approximated by a finite subspace of 'simple' functions, the so-called shape functions or trial functions, that meet certain matching conditions at the interface. The functions that make up these FE spaces are usually polynomial functions up to a certain order defined on a reference element which are mapped to the elements in the mesh. The spaces that govern the weak problem might be restricted to functions that have certain 'continuity' properties imposed on them and their (weak) derivatives up to a certain order. For instance, if a one dimensional domain is considered, the space $H^{1}$ only contains continuous functions. The matching conditions at the element boundaries ensure that these 'continuity' properties are preserved for spaces that are constructed by stitching the spaces defined on the individual elements together. If these properties are preserved, the embedding $X_{h} \subset X, Y_{h} \subset Y$ and $Z_{h} \subset Z$ is achieved and the FE method is called conforming. If the embedding is not achieved, the FE method is called non-conforming and the resulting approximations may be polluted by consistency errors. The FE methods considered in this thesis are all conforming. Furthermore, for all methods considered, the subspaces of the dual spaces (which contain the so-called weighting functions or test functions) are chosen equal to the subspaces for the shape/trial functions. This approach is known as the Bubnov-Galerkin method. A useful feature of mixed elements is that the shape functions defined on the elements may be chosen differently for each variable.

\section{Convergence}

Naturally, it is desirable that the accuracy of an FE approximation can be improved by manipulating the subspaces used in the approximation. The two most common methods to increase the accuracy of FE approximations are based on increasing the dimension of the FE spaces. The first method involves mesh refinement by increasing the number of elements in the mesh ( $h$-adaptivity), the second method involves increasing the order of the polynomial functions within each element ( $p$-adaptivity). A combination of both methods ( $h p$-adaptivity) and a third method based on restructuring the mesh without increasing the dimension of the FE spaces ( $r$-adaptivity) are also encountered in the literature. In this thesis, only the method of mesh refinement ( $h$-adaptivity) is considered. Usually, $h$ is taken as some measure for the size of the elements in a mesh (for instance, the averaged or largest value of the longest element boundary or the radius of the circumcircle/circumsphere passing through all vertices of the element). Consequently, $h$ is inversely proportional to the number of elements if a sequence of $\mathrm{FE}$ meshes is obtained by successively partitioning each element. Ideally, the FE solutions of such 
a sequence converge to the solution of the continuous problem, and the error of the FE approximation approaches zero as $h$ goes to zero, i.e.,

$$
\lim _{h \downarrow 0}\left(\left\|\mathbf{v}-\tilde{\mathbf{v}}_{h}\right\|_{X}+\left\|T-\tilde{T}_{h}\right\|_{Y}+\left\|p-\tilde{p}_{h}\right\|_{Z}\right)=0
$$

As mentioned previously, at zero frequency, the LNSF equation reduces to the Stokes equations, involving velocity and pressure, and the Poisson equation for temperature. For these two sets of uncoupled equations, the solution for pressure and temperature is only uniquely determined up to a constant pressure and temperature field. These non-unique parts of the solution can obviously not be expected to converge. To circumvent this, the norms on $T$ and $p$ can be replaced by so-called quotient norms which 'disregard' the non-unique parts of the solution. If $T$ and $p$ are elements of $Y$ and $Z$, and the non-unique parts $p_{0}$ and $T_{0}$ belong to the subspaces $Y_{0}$ and $Z_{0}$, the quotient norms on $T$ and $p$ are defined, respectively, as:

$$
\|T\|_{Y / Y_{0}}=\inf _{T_{0} \in Y_{0}}\left\|T-T_{0}\right\|_{Y} \quad\|p\|_{p / p_{0}}=\inf _{p_{0} \in X_{0}}\left\|p-p_{0}\right\|_{X}
$$

Substituting these norms in (5.14) yields a useful definition of convergence for solutions that are only uniquely determined up to elements in $Y_{0}$ and $X_{0}$. Quotient norms are used extensively in the expressions for the different error bounds presented in the next sections.

\section{Approximability}

If an FE approximation is expected to converge to the solution of the continuous problem, a necessary property for a discretization scheme is that it offers approximability. Approximability means that given a mesh family consisting of an infinite number of increasingly refined meshes, an arbitrary solution can be approximated with any desired accuracy by selecting a sufficiently refined mesh. A formal definition of approximability requires a definition of how well a solution can be approximated by the elements of a given FE space. Consider an abstract problem having a solution $v \in V$ and an FE space $V_{h}$. A suitable measure for how well $v$ can be approximated by the elements of $V_{h}$ is the magnitude of the approximation error $d\left(\nu, V_{h}\right)$, which is defined as

$$
d\left(\nu, V_{h}\right)=\inf _{v_{h} \in V_{h}}\left\|v-v_{h}\right\|_{V}
$$

The 'magnitude of the approximation error', simply called the 'approximation error' from here on, can also be interpreted as the orthogonal 'distance' between the 
exact solution $v$ and the solution space $V_{h}$. When considering $h$-adaptivity, a discretization scheme is now said to offer approximability if

$$
\lim _{h \downarrow 0} d\left(v, V_{h}\right)=0, \quad \forall v \in V
$$

This means that given an arbitrary element or 'solution' $v \in V$ and a family of subspaces $V_{h}$ corresponding to a sequence of decreasing $h$, the approximation error vanishes as the size of the elements goes to zero. Approximability is assumed for all discretization schemes that are presented in this thesis. Note that approximability depends on the family of spaces $V_{h}$ resulting from the FE method, but not on the particular problem under consideration. From the next sections, it will become clear that approximability is necessary, but not sufficient to obtain convergence of an FE solution to the exact solution. In that light, it is useful to introduce the concept of optimal convergence.

\section{Optimal convergence/stability}

Consider an abstract problem having a solution $v \in V$ and an FE method yielding a solution $\tilde{v}_{h} \in V_{h}$. The FE method is said to be stable or to have optimal convergence if

$$
\left\|v-\tilde{v}_{h}\right\|_{V} \leq C d\left(v, V_{h}\right)
$$

with $C$ constant (independent of $h$ ) and of finite size. This means that for a stable method, the magnitude of the FE error, being the difference between the FE solution and actual solution, is bounded asymptotically by the approximation error. In other words, the rate of convergence of the FE error is (at least) of the same order as the rate of convergence of the approximation error ${ }^{2}$ in the limit that $h \downarrow 0$. Note that if $C$ in the above inequality is not constant but increases with $h$, inequality (5.18) does not predict optimal convergence. Convergence at a lower order might still be guaranteed depending on how $C(h)$ varies with $h$. If $C(h)$ increases faster with $h$ than $d\left(v, V_{h}\right)$ decreases, the $\mathrm{FE}$ error is not bounded by the approximation error, and the FE method might not converge at all. The value of $C$ (and its dependence on $h$ ) which will later be brought into connection with the phenomena of locking and spurious modes, is thus important for the stability (and robustness) of an FE method. Note that optimal convergence is introduced here for a single variable. The definition is easily extended to the case of multiple variables by considering compound variables (for instance $v=(\mathbf{v}, p)$ or $v=(\mathbf{v}, T, p)$ ) as is done in the next section.

\footnotetext{
${ }^{2}$ See for instance [107] for the order of convergence of the approximation error in the case of approximations of Sobolev spaces by polynomial shape functions.
} 


\section{Robustness}

The stability of an FE method is influenced by many factors stemming from both the mathematical formulation used and the physical problem that is modeled. The function $C(h)$ is, of course, influenced by the choice of the weak formulation that is used to describe the problem. It also depends on the choice of subspaces, e.g. the element shape (triangle/tetraeder or quadrilateral/cube), the order of polynomial functions used in each element for each field, and on mesh properties (unstructured/structured, element quality, etc.). Other important factors influencing $C(h)$ are the physical parameters and boundary conditions that describe the problem. For instance for the viscothermal case, $C(h)$ (and thus the stability of the method) depends on the frequency.

The fact that $C(h)$ depends on mesh properties, boundary conditions, and physical parameters, implies that ad hoc stability results are not a very practical criterion for the selection of a suitable FE method. To that end, the concept of robustness is introduced; An FE method is called robust for a range of (physical) parameters if it is stable (has optimal convergence) for all meshes, boundary conditions and problems within that range of parameters ${ }^{3}$. In the next section, relatively concrete conditions for stability and robustness are provided, however, analytical verification of these conditions can be quite cumbersome. An alternative criterion based on numerical results is presented in section 5.3.

\subsection{Stability of FE methods for the LNSF equations}

It was mentioned above that approximability of an FE method is a necessary, but not sufficient condition for optimal convergence of FE errors. In this section, the additional requirements needed to guarantee optimal convergence of FE methods for the LNSF problem are investigated. As mentioned previously, the LNSF equations reduce to the Stokes equations for velocity and temperature and an uncoupled Poisson equation for temperature when the frequency reduces to zero. So, not surprisingly, the requirements needed to obtain stable FE methods for the LNSF equation are very similar to those needed for the Stokes equations (and the Poisson equation). The relation between the LNSF equations, the Stokes equations and the Poisson equations for temperature are sketched in figure 5.1 using the different elements in their respective solution spaces. Below, the case of the Stokes equations is considered briefly. Subsequently, an outline of the rest of this section is given.

\footnotetext{
${ }^{3} \mathrm{~A}$ similar but more precise definition of robustness is proposed in [108].
} 


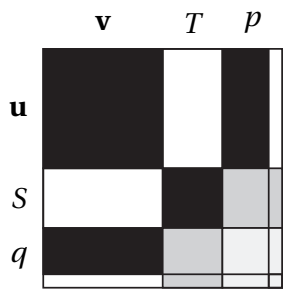

(a) $\operatorname{LNSF} \omega>0$

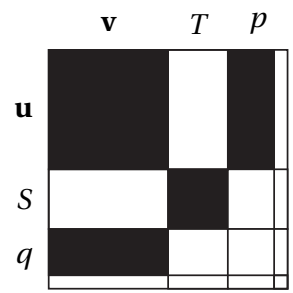

(b) $\operatorname{LNSF} \omega=0$

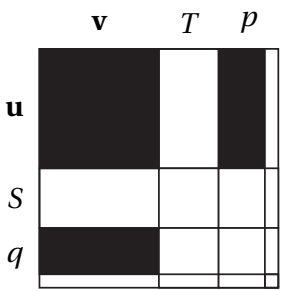

(c) Stokes

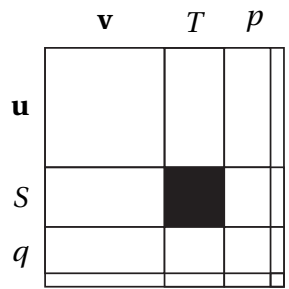

(d) Poisson

Figure 5.1: Abstract representation of the coupling between the different trial and test functions $\mathbf{v}, \mathbf{u} \in X, T, S \in Y$ and $p, q \in Z$ describing FE methods for the LNSF, the Stokes, and the Poisson equations. Black indicates the norm of the operator coupling the different elements is large, white indicates a zero norm (there is no direct coupling between elements) and gray indicates a small operator norm. Note that the strength of the coupling of the gray areas is frequency dependent $(\omega=0 \Longrightarrow$ white, $\omega>>0 \Longrightarrow$ dark).

\section{Stability of FE methods for the Stokes equations}

For the class of saddle point problems including the Stokes problem, it turns out that if the continuous problem has a unique ${ }^{4}$ solution and a conformal FE method yields a unique ${ }^{4}$ solution for all $h$ including the limit $h \downarrow 0$, the FE method has optimal convergence. Proof of the existence of unique (FE) solutions for this class of saddle point problems and the resulting error bounds for the FE solution which predict optimal convergence is given for instance by Brezzi and Fortin [61]. In the following subsections, it is demonstrated that sufficient requirement for optimal convergence of FE methods for the LNSF equation can also be stated in terms of the requirements for existence and uniqueness of a solution to both the continuous and FE problem.

It must be stressed that if the requirements for existence of a unique solution are met for a continuous problem, they are not necessarily met for arbitrary FE approximations of that problem. This implies that the requirements for existence and uniqueness of an FE solution must be verified for individual FE methods. For mixed FE formulations of the Stokes equations, it is observed that some FE methods having an unfortunate combination of subspaces for velocity and pressure, yield systems of equations that do not offer a (unique) FE solution for all $h$. Consequently, optimal convergence is not guaranteed for such FE methods. A sufficient condition for the existence of a unique solution for mixed FE methods independent of $h$ is the so-called inf-sup condition. If the inf-sup condition holds for an FE method, optimal convergence is guaranteed.

\footnotetext{
${ }^{4}$ The solution for pressure is unique only up to a well-defined subspace.
} 
Two particular conditions which lead to a system that fails to satisfy the infsup condition (and consequently does not yield a unique FE solution) are known as locking and spurious modes. If an FE method suffers from locking, existence of a unique solution is compromised in the limit $h \downarrow 0$ where an infinite number of elements are considered. If an FE method suffers from spurious modes, uniqueness of the solution is compromised for systems of finite size. If the inf-sup condition is met, the FE method suffers from neither, and optimal convergence is guaranteed, i.e., the method is said to be stable. For a formal introduction of the locking phenomena and spurious modes at the hand of the (somewhat simpler) Stokes equations see for instance [60,61].

\section{Outline}

The aim of this section is to extend the results for existence, uniqueness and stability, which are presented by Brezzi and Fortin [61] for a number of saddle point problems (including the Stokes equations), to a more general class that includes the LNSF equations describing viscothermal wave propagation. In the first subsection, the requirements for existence and uniqueness of a class of saddle point problems that includes the Stokes equations as a special case are considered. In the subsequent subsection it will be shown that the LNSF equations describing viscothermal wave propagation are of a similar form. This leads to the criteria for existence and uniqueness of solutions to the LNSF equations, which leads to the definition of an inf-sup condition for viscothermal wave propagation. The effects of locking and spurious modes on problems belonging to this broader class are elaborated on. In the next section, a numerical inf-sup test is presented, which can be used to determine the stability of FE methods for the LNSF equations, along with the results of this test for a few promising FE methods.

\subsubsection{Existence and uniqueness results for saddle point problems}

Brezzi and Fortin extended the results for existence and uniqueness for the Stokes problem (given by equation (2.30)) to the following general class of saddle point problems: let $X$ and $Z$ be Hilbert spaces, and $X^{\prime}$ and $Z^{\prime}$ their associated dual spaces. For a given $(\mathbf{f}, g) \in X^{\prime} \times Z^{\prime}$, find $(\mathbf{v}, p) \in X \times Z$ so that

$$
\begin{aligned}
& a(\mathbf{v}, \mathbf{u})+b(\mathbf{u}, p)=\langle\mathbf{f}, \mathbf{u}\rangle \\
& b(\mathbf{v}, q)-c(p, q)=\langle g, q\rangle
\end{aligned}
$$

for all $(\mathbf{u}, q)$ in $X^{\prime} \times Z^{\prime}$. The terms $a(),, b($,$) and c($, ) are continuous bilinear forms, having an associated linear operator $A: X \rightarrow X^{\prime}, B: X \rightarrow Z^{\prime}$, and $C: Z \rightarrow Z^{\prime}$, which 
can be defined, respectively, by

$$
\begin{aligned}
\langle A \mathbf{v}, \mathbf{u}\rangle & =a(\mathbf{v}, \mathbf{u}), & & \forall \mathbf{v}, \mathbf{u} \in X \\
\langle B \mathbf{v}, q\rangle=\left\langle\mathbf{v}, B^{T} q\right\rangle & =b(\mathbf{v}, q), & & \forall \mathbf{v} \in X, q \in Z \\
\langle C p, q\rangle & =c(p, q), & & \forall p, q \in Z
\end{aligned}
$$

The generalized saddle point problem in (5.19) can be restated in operator form as: for a given (f, $g) \in X \times Z$, find $(\mathbf{v}, p) \in X \times Z$ so that

$$
\left[\begin{array}{cc}
A & B^{T} \\
B & -C
\end{array}\right]\left\{\begin{array}{l}
\mathbf{v} \\
p
\end{array}\right\}=\left\{\begin{array}{l}
\mathbf{f} \\
g
\end{array}\right\}
$$

Note that the saddle point problem describing the Stokes equations (see equation (2.30)) is obtained by setting $C=0$. For this special case, the necessary requirements for existence and uniqueness of a solution are elaborated on in [61]. These results can be summarized as: for any (f, $g) \in X \times \operatorname{Im} B$, existence of a solution to the problems in (5.19) and (5.23) is guaranteed if

- $A$ is invertible on $\operatorname{ker} B$

- $\operatorname{Im} B$ is closed in $Z^{\prime}$

Furthermore, the solution for $\mathbf{v}$ is unique, and the solution for $p$ is unique up to an element of $\operatorname{ker} B^{T}$. The requirements for an operator to be closed, or invertible on a subspace, are given in appendix H. In [61], it is demonstrated that the following additional assumptions are sufficient for existence of a solution for the case $C \neq 0$ :

- $A$ is positive semi-definite

- $C=\lambda \bar{C}$, where $\lambda \geq 0$, and $\bar{C}$ is symmetric, positive semi-definite, and invertible on $\operatorname{ker} B^{T}$

The definition for positive semi-definiteness is given in (5.26). Under these conditions, $\mathbf{v}$ is uniquely determined in $X$. In addition, $C$ is invertible on $\operatorname{ker} B^{T}$ for $\lambda>0$, so the solution for $p$ is uniquely determined on $Z$ for $\lambda>0$. As mentioned above, if $\lambda=0$, the Stokes equations are obtained (with $C=0$ ). Since $\operatorname{Im} B$ is closed in $Z^{\prime}, B$ is invertible from $(\operatorname{ker} B)^{\perp}$ on $\left(\operatorname{ker} B^{T}\right)^{\perp}$, so $p$ is uniquely determined in $Z$ up to an element of $\operatorname{ker} B^{T}$ if $\omega=0$. This can be summarized as: $(\mathbf{v}, p)$ is uniquely determined in $X \times Z / M$ where the subspace $M$ is defined as

$$
M=\operatorname{ker} B^{T} \cap \operatorname{ker} C
$$


It must be stressed that the second set of requirements given above are sufficient but not (always) necessary. For instance, if $A$ is coercive on $X$, the requirement that $C$ is symmetric can be dropped. If $A$ and $C$ are both coercive, the requirements on $B$ can also be dropped (see [61]). A similar condition will be encountered in the case of the LNSF equations for non-zero frequencies. The definition for coerciveness is given below in equation (5.25).

\subsubsection{Coercivity and semi-positive definiteness}

A bilinear form $a($, ) and its associated operator $A$ are said to be coercive and positive semi-definite on a space $V$ if, respectively,

$$
\begin{aligned}
& \exists \alpha>0 \quad \text { such that } \frac{a(v, v)}{\|v\|_{V}^{2}}>\alpha, \quad \forall v \in V \\
& a(v, v) \geq 0, \quad \forall v \in V
\end{aligned}
$$

So, coercivity implies positive semi-definiteness, while the reverse is not true. Furthermore, coercivity of a continuous bilinear form on a space $V$, implies that the associated operator is invertible on all subspaces of $V$. The fact that invertibility is inherited on arbitrary subspaces is what makes coercivity such an attractive property in the development of stable FE methods. Note that on finite dimensional spaces the concept of coercivity coincides with that of positive definiteness.

It naturally follows that if the negative of a form is coercive, it is also negative semi-definite. For a bilinear form $a($, ) defined as a linear combination of two bilinear forms by

$$
a(u, v)=a_{1}(v, u)+\gamma a_{2}(v, u), \quad \forall v, u \in V
$$

the following statements hold for all $\gamma \geq 0$ :

- If $a_{1}($,$) and a_{2}($,$) are both positive semi-definite, a($, ) is also positive semidefinite

- If $a_{1}($,$) is coercive and a_{2}($,$) is positive semi-definite, a($,$) is also coercive$

These relations are useful when studying the coerciveness of compound forms that are encountered below in the reformulated weak form of the LNSF equations.

\section{Sesquilinear forms}

The theory presented above (as well as the theory for the Stokes equation) is based on bilinear forms which map real valued vector fields to the field of real numbers. However, the weak formulation of the LNSF equations presented in section (5.1) 
includes sesquilinear forms which map complex valued vector fields to the field of complex numbers. The definitions in (5.25), and (5.26) do not make sense for sesquilinear forms, since $a(v, v)$ can be a complex number. To be able to extend the presented theory to describe the LNSF equations, the definitions of coercivity and positive semi-definiteness have to be extended to the case of sesquilinear forms.

The definition of positive (semi-)definiteness can be extended to complex nonHermitian forms by requiring that the Hermitian part (see equation (5.28)) is positive (semi-)definite ${ }^{5}$. In this study, the definition of coercivity is extended to complex valued non-Hermitian forms/operators in a similar way. The extended definition is based on the fact that an operator $A$ associated with an arbitrary sesquilinear form $a$ (, ) can always be split into a Hermitian and skew-Hermitian part according to

$$
A=A_{h}+i A_{s}, \quad \text { where } \quad A_{h}=\frac{A+A^{H}}{2}, \quad A_{s}=\frac{A-A^{H}}{2 i}
$$

where $A_{h}$ denotes the Hermitian part and $i A_{s}$ denotes the skew-Hermitian part. Using this separation, the following holds for the associated forms $a_{h}\left(\right.$, ) and $a_{s}($, )

$$
\begin{array}{lll}
a_{h}(u, v)=\left[a_{h}(v, u)\right]^{H}, & a_{h}(\nu, v) \in \Re, & \forall u, v \in V \\
a_{s}(u, v)=\left[a_{s}(v, u)\right]^{H}, & a_{s}(\nu, v) \in \Re, & \forall u, v \in V
\end{array}
$$

i.e., the forms $a_{h}\left(\right.$, ) and $a_{s}\left(\right.$, ) (and their associated operators $A_{h}$ and $A_{s}$ ) are all Hermitian, and the expressions $a_{h}(v, v)$ and $a_{s}(v, v)$ map arbitrary elements $v \in V$ to the set of real numbers. This means that the definitions in (5.25) and (5.26) which do not make sense for an arbitrary (non-Hermitian) sesquilinear form $a($, ), do make sense for the forms $a_{h}\left(\right.$, ) and $a_{s}($, ).

Using the separation in equation (5.28), the definition of coercivity for arbitrary sesquilinear forms and their associated operators can be stated as: an arbitrary non-Hermitian sesquilinear form $a$ (,) (or operator $A$ ) is called coercive if its Hermitian part $a_{h}$ (,) (or $A_{h}$ ) is coercive. Using the above definition, if either $A_{h}$, $-A_{h}, A_{s}$ or $-A_{s}$ corresponding to an operator $A$ is coercive, $A,-A, i A$, or $-i A$ is coercive, respectively. Furthermore, it is obvious that if either $A_{h},-A_{h}, A_{s},-A_{s}$ is coercive, there is no element $v \neq 0 \in V$ that makes $a(v, v)=0$, which implies invertibility of $A$. So, using this definition of coercivity for sesquilinear forms, the fact that either $A,-A, i A$, or $-i A$ is coercive, implies that $A$ is invertible. More importantly, the property that coercivity of forms/operators is inherited on arbitrary

\footnotetext{
${ }^{5}$ Confusingly, in the literature the discussion of positive (semi-)definite operators is often restricted to only Hermitian operators, or symmetric operators in the case of real matrices.
} 
subspace remains true using this definition. This means that an operator $A$ (and its associated sesquilinear form $\mathrm{a}()$,$) for which either A,-A, i A$ or $-i A$ is coercive is invertible on arbitrary subspaces (such as for instance FE spaces). In other words, the characteristic that makes coercivity an attractive property of forms/operators in an FE setting is preserved by extending the definition to include sesquilinear forms as described above.

\subsubsection{Alternative weak form}

The weak form of the LNSF equations in (5.4), which describes viscothermal wave propagation, can be cast in a form that is similar to the equations in (5.23). The variable $\mathbf{v}$ is replaced by the compound variable $(\mathbf{v}, \bar{T})$, and the natural boundary condition $\mathbf{f}$ is replaced by the compound natural boundary condition $(\mathbf{f}, q)$, where $\mathrm{f}$ is the sum of the specific body force vector and the stress vector, and $q$ is the sum of the specific energy supply and the normal heat flux. The space $X$ is replaced by $X \times Y$ (as defined in equation (5.8)). The temperature trial and test functions and the right-hand side term $q$ in the weak form of the LNSF equations presented in (5.1.1) are scaled by the imaginary unit as follows.

$$
\bar{T}=-i T \quad \bar{S}=i S \quad \bar{q}=i q \quad(5.31 \mathrm{a}, \mathrm{b}, \mathrm{c})
$$

Note that the bar indicating that $\bar{T}, \bar{S}$ and $\bar{q}$ are scaled is dropped in the following. The problem in (5.4) can now be reformulated as: Given (f, $q) \in X^{\prime} \times Y^{\prime}$, find $(\mathbf{v}, T, p) \in X \times Y \times Z$ so that

$$
\left[\begin{array}{cc}
A & B^{H} \\
B & -C
\end{array}\right]\left\{\begin{array}{c}
(\mathbf{v}, T) \\
p
\end{array}\right\}=\left\{\begin{array}{c}
(\mathbf{f}, q) \\
0
\end{array}\right\}
$$

The operators $A, B$ and $C$ are defined by their associated sesquilinear forms $a($, ), $b($, ) and $c($, ) in the usual way

$$
\begin{aligned}
a((\mathbf{v}, T),(\mathbf{u}, S)) & =\langle A(\mathbf{v}, T),(\mathbf{u}, S)\rangle \\
b((\mathbf{v}, T), q) & =\langle B(\mathbf{v}, T), q\rangle \\
b((\mathbf{u}, S), p) & =\left\langle(\mathbf{u}, S), B^{H} p\right\rangle \\
c(p, q) & =\langle C p, q\rangle
\end{aligned}
$$


where the sesquilinear forms $a(),, b($,$) and c($,$) are defined as$

$$
\begin{aligned}
& a((\mathbf{v}, T),(\mathbf{u}, S))= i \omega \rho_{0}\langle\mathbf{v}, \mathbf{u}\rangle+\lambda\langle\nabla \cdot \mathbf{v}, \nabla \cdot \mathbf{u}\rangle+\mu\left\langle\nabla \mathbf{v}+(\nabla \mathbf{v})^{T}, \nabla \mathbf{u}\right\rangle \\
&+i \frac{\omega \rho_{0} C_{p}}{T_{0}}\langle T, S\rangle+\kappa \frac{1}{T_{0}}\langle\nabla T, \nabla S\rangle \\
& b((\mathbf{v}, T), q)=-\langle\nabla \cdot \mathbf{v}, q\rangle-\frac{\omega}{T_{0}}\langle T, q\rangle \\
& b((\mathbf{u}, S), p)=-\langle\nabla \cdot \mathbf{u}, p\rangle-\frac{\omega}{T_{0}}\langle S, p\rangle \\
& c(p, q)=i \frac{\omega}{p_{0}}\langle p, q\rangle
\end{aligned}
$$

Note that in this form the off-diagonal terms $B$ and $B^{H}$ are each other's complex conjugates. In addition, both the Hermitian and skew-Hermitian part of the diagonal terms also have the favorable property of being positive semi-definite, and in addition, for $\omega>0$, the operators $A$ and $-i C$ are both coercive (this is demonstrated below).

\subsubsection{Existence and uniqueness for the LNSF equations}

In the previous subsection, the weak formulation in equation (5.4) was brought in a form very similar to equation (5.23) presented in section 5.2.1 for which existence and uniqueness results were presented by Brezzi and Fortin [61]. The only differences are that the bilinear forms in equation (5.23) are replaced by (compound) sesquilinear forms in (5.32), and the off-diagonal terms in equation (5.32) are each other's complex conjugates instead of each other's transpose as is the case for the system in (5.23). The requirements for existence and uniqueness of solutions have to be altered slightly, but the proof remains virtually unchanged by these changes. In appendix H.2 the need for these changes is elaborated on.

\section{Requirements in the case of sesquilinear forms}

The key difference in the requirements for the existence of a unique solution to the equations in (5.23) and (5.32) can be stated as: the requirements that $A$ and $\bar{C}$ are both positive semi-definite, and $C$ is symmetric, are replaced by the requirement that both $A_{h}$ and $C_{h}$ are positive or negative semi-definite, and both $A_{s}$ and $C_{s}$ are positive or negative semi-definite ${ }^{6}$. The additional requirement that $C$ is sym-

\footnotetext{
${ }^{6}$ Note that if both $A_{h}$ and $C_{h}$ are negative definite, and/or both $A_{s}$ and $C_{s}$ are negative definite, a scaling of the test and/or trial functions for velocity, temperature and/or pressure with the imaginary unit, can be used to arrive at a form where $A_{h}, C_{h}, A_{s}$ and $C_{s}$ are all positive semi-definite. An example is the scaling of temperature and the corresponding weighting function in (5.31) which is used to cast the LNSF equations in the form in (5.32).
} 
metrical can be dropped; if $c($,$) is a bilinear form (as in the original problem), this$ condition is automatically satisfied under these new requirements. The requirement that if $C=\lambda \bar{C}$ with $\lambda \geq 0$ then $\operatorname{ker} \bar{C}$ must be invertible on $\operatorname{ker} B^{H}$ remains unaltered. Note that for the LNSF equations, $\lambda$ can be related to a parameter of the physical model, being the frequency, i.e., $\lambda=\omega$. Summarizing, sufficient requirements for the existence of a solution $(\nu, T, p)$ can be stated as:

- $A$ is invertible on $\operatorname{ker} B$

- $\operatorname{Im} B$ is closed on $Z^{\prime}$

- $C=\omega \bar{C}$, where $\omega \geq 0$, and $\bar{C}$ is invertible on $\operatorname{ker} B^{H}$

- $\alpha A$ and $\alpha C$ are both positive semi-definite for $\alpha=1 \vee-1$ and $\alpha=i \vee-i$

It follows that if $\alpha A$ and $\beta C$ are coercive, and $\beta A$ and $\alpha C$ are positive semi-definite for a certain $\alpha, \beta \in\{1,-1, i,-i\}$, all requirements on $A$ and $C$ are automatically satisfied ${ }^{7}$. In that case, the solution for $\mathbf{v}$ and $p$ can be obtained by solving

$$
\left[A+B^{H} C^{-1} B\right]\{(\mathbf{v}, T)\}=\{(\mathbf{f}, q)\}, \quad\left[C+B A^{-1} B^{H}\right]\{p\}=\{0\}
$$

It is clear that for a unique solution to these equations to exist, no additional requirements on $B$ (other than that $b($, ) is a bounded form) is required. Below, it is demonstrated that the LNSF equations meet the required conditions for this to be the case if $\omega>0$.

\section{Requirements in the case of the LNSF equations}

A useful property of inner products that are used in the definition of an inner product space is that they constitute a coercive form on the associated space by definition. This means that the inner product $\langle p, q\rangle$ in the definition of the sesquilinear form $c($,$) is coercive. The terms \left\langle\left(\nabla \mathbf{v}+(\nabla \mathbf{v})^{T}, \nabla \mathbf{u}\right\rangle\right.$ and $\langle\nabla T, \nabla S\rangle$ are also coercive for $X=\left[H_{0}^{1}\right]^{n}$ and $Y=H_{0}^{1}$, respectively (see for instance [61] and [104] ). The Hilbert space $H_{0}^{1}$ is the subspace of $H^{1}$ that includes all functions that are zero on the boundary of the domain. Note that this restricts the results of the presented analysis to problems with homogeneous Dirichlet boundary conditions for both velocity and temperature. Nevertheless, for many related problems (for instance described by the Stokes equations or the Poisson equation) an extension of the results to problems involving non/homogeneous Dirichlet, Neumann, mixed and/or Robin boundary conditions is possible (see [104] for various examples). It

\footnotetext{
${ }^{7}$ Note that if this holds for $\alpha=\beta=1$, the operators $A$ and $C$ are both coercive. This special case, with $a($, ) and $c($,) bilinear forms instead of sesquilinear forms, is considered in [61].
} 
is assumed here that such extensions are also possible for the case of the LNSF equations. Note that the numerical results presented in section 5.4 are based on problems with non-homogeneous boundary conditions.

The other inner products on $L^{2}$ in the definition of $a($, ) are all (at least) positive semi-definite by definition. This means that the terms involving the inner products are either Hermitian and positive semi-definite, or skew-Hermitian with their imaginary part being positive semi-definite. Using the fact that the sum of two positive semi-definite forms is also positive semi-definite (see section 5.2.2), it follows that $A_{h}, A_{s}, C_{h}$ and $C_{s}$ are all positive semi-definite (independent of the value of $\omega$ ). In addition, $A$ is coercive on $X \times Y$ and thus invertible on $\operatorname{ker} B$, and $-i \bar{C}$ is coercive on $Z$ and thus invertible on $\operatorname{ker} B^{H}$. The operator $B$ can be split into two operators according to

$$
[B]\{(\mathbf{v}, T)\}=\left[\begin{array}{ll}
B_{v} & B_{T}
\end{array}\right]\left\{\begin{array}{c}
\mathbf{v} \\
T
\end{array}\right\}=\left[\begin{array}{ll}
B_{v} & \omega \bar{B}_{T}
\end{array}\right]\left\{\begin{array}{c}
\mathbf{v} \\
T
\end{array}\right\}
$$

where $B_{v}$ represents the frequency independent right-hand side term in (5.34b), while $B_{T}$ represents the frequency dependent term. In [109] it is proven that operator $B_{v}$ is closed, while operator $B_{T}$ is trivially closed ${ }^{8}$. From the null spaces of the Hermitian of both operators, it can be inferred that operator $B$ is also closed. The properties of $B$ as $\omega$ approaches zero are somewhat special. Considering the null spaces of $B_{v}^{H}$ and $\bar{B}_{T}^{H}$ (see $[61,109]$ ), it can be inferred that for $\omega>0$, the dimension of $\operatorname{ker} B^{H}$ is zero, while for $\omega=0$ the dimension of $\operatorname{ker} B^{H}$ is one. This implies that as $\omega \downarrow 0$, the elements in $Z$ which are in $\operatorname{ker} B_{v}^{H}$, will transcend from not being an element in $\operatorname{ker} B^{H}$ to being an element of $\operatorname{ker} B^{H}$. If $\omega$ is taken infinitely close to zero, these elements are 'infinitely close' to being in $\operatorname{ker} B^{H}$. This corresponds with the fact that for $\omega>0$ the solution for $p$ is unique in $Z$, while for $\omega=0$, the solution is unique only up to an element of $\operatorname{ker} B^{H}$. This behavior can cause numerical issues for small values of $\omega$ as will be demonstrated in section 5.4.

Summarizing, all requirements for the existence of a solution are met. Moreover, the solution is unique for $\omega>0$ since $-i C$ is coercive and thus invertible on $\operatorname{ker} B^{H}$. For $\omega=0$ the solution for $\mathbf{v}$ and $T$ are unique in $X$ and $Y$, while the solution for $p$ is uniquely determined in $Z$ only up to an element of $\operatorname{ker} B$. The main result of this analysis can thus be concisely written as: for the continuous LNSF equations in their weak form having arbitrary right-hand side terms $\mathbf{f} \in X$ and $q \in Y$, the solution $(\mathbf{v}, T, p)$ is uniquely determined in $X \times Y \times Z / M$, where the space $M$ is defined as

$$
M=\operatorname{ker} B^{H} \cap \operatorname{ker} C
$$

\footnotetext{
${ }^{8}$ The linear operator $B_{T}$ is bounded over its entire domain, which implies $B_{T}$ is closed (see [104]).
} 


\subsubsection{FE formulation}

The conforming FE spaces $X_{h} \in X, Y_{h} \in Y$ and $Z_{h} \in Z$ are constructed according to section 5.1.4. The FE formulation corresponding to the continuous problem in (5.32) is obtained by simply replacing all functions in $X, Y$ and $Z$, with their FE counterparts in $X_{h}, Y_{h}$ and $Z_{h}$, respectively. Using these FE spaces, the discrete operators

$$
A_{h}:\left(X_{h} \times Y_{h}\right) \rightarrow\left(X_{h}^{\prime} \times Y_{h}^{\prime}\right), \quad B_{h}:\left(X_{h} \times Y_{h}\right) \rightarrow Z_{h}^{\prime}, \quad C_{h}: Z_{h} \rightarrow Z_{h}^{\prime} \quad(5.38 \mathrm{a}, \mathrm{b}, \mathrm{c})
$$

are defined in the same way as was done for the continuous problem. The FE formulation of the problem can be stated in operator form as: find $\left(\mathbf{v}_{h}, T_{h}, p_{h}\right)$ in $X_{h} \times Y_{h} \times Z_{h}$ so that

$$
\left[\begin{array}{ll}
A_{h} & B_{h}^{T} \\
B_{h} & C_{h}
\end{array}\right]\left\{\begin{array}{c}
\left(\mathbf{v}_{h}, T_{h}\right) \\
p_{h}
\end{array}\right\}=\left\{\begin{array}{c}
\left(\mathbf{f}_{h}, q_{h}\right) \\
0
\end{array}\right\}
$$

where the functionals $\mathbf{f}_{h}$ and $q_{h}$ satisfy

$$
\begin{array}{rr}
\left\langle\mathbf{f}_{h}, \mathbf{v}_{h}\right\rangle=\left\langle\mathbf{f}, \mathbf{v}_{h}\right\rangle, & \forall \mathbf{v}_{h} \in X_{h} \\
\left\langle q_{h}, S_{h}\right\rangle=\left\langle q, S_{h}\right\rangle, & \forall S_{h} \in Y_{h}
\end{array}
$$

\section{Existence and uniqueness of FE solutions}

The requirements for existence and uniqueness of $\mathrm{FE}$ solutions are equivalent to requirements for the continuous system described in the previous section. Since the properties of positive semi-definiteness and coerciveness are inherited on subspaces, the first, third and fourth requirement from the previous section are automatically satisfied on FE spaces obtained with arbitrary conformal FE methods. The requirement on $B$ is discussed below for the cases $\omega=0$ and $\omega>0$.

\section{Zero frequency $(\omega=0)$}

If $\omega=0$, the requirement that $\operatorname{Im} B_{h}$ is closed in $Z_{h}^{\prime}$ is needed for the existence of $\mathrm{FE}$ solutions. This condition is always satisfied for FE systems of finite size. However, despite the fact that all requirements for a solution to exist are met for systems of finite dimension, the convergence behavior of an FE method depends on the question whether $\operatorname{Im} B_{h}$ continues to be closed in $Z_{h}^{\prime}$ when the dimension of the systems grows to infinity. This question is closely related to the definition of the inf-sup condition and the locking phenomenon, which are introduced in section (5.2.6).

Furthermore, $p$ is only uniquely determined up to an element of $\operatorname{ker} B_{h}^{H}$. Note that $\operatorname{ker} B_{h}^{H}$ can be bigger than its continuous counterpart depending on the FE 
method that is considered. If this occurs, the system suffers from spurious pressure modes (see section (5.2.6)). The adverse influence on convergence of spurious modes depends on $\omega$. For $\omega=0$, spurious modes only affect the pressure solution and can often be filtered out with relative ease (as is the case for the Stokes equations).

\section{Non-zero frequency $(\omega>0)$}

For $\omega>0$, operator $-i C$ is coercive, and the requirement that $\operatorname{Im} B_{h}$ is closed in $Z_{h}^{\prime}$ for all $h$, can be dropped. So, a unique solution for $v_{h}, T_{h}$, and $p_{h}$ is not only guaranteed for the continuous problem, but also for FE approximations of the problem obtained with arbitrary conformal FE methods. Nevertheless, if $B_{h}$ is not 'well behaved' for a certain FE method, the FE approximation can be poor if $\omega$ is small. This happens for instance if operator $B_{h}$ is not closed in the limit that $h \downarrow 0$. In that case, the behavior of the LNSF equations is similar to that of the Stokes equations and the equations for linear elasticity for nearly incompressible materials. In addition, convergence behavior can be adversely influenced if $B_{h}$ suffers from spurious pressure modes. Below, these issues are discussed in more detail.

\section{Small non-zero frequency}

Besides the possibility that $B_{h}$ is not closed in the limit that $h$ goes to zero for a certain FE method, there is a second cause for ill-conditioning of numerical systems obtained by using mixed FE methods to solve the LNSF equations. In fact, the properties of operator $B$ in the continuous problem are such that mixed FE methods for the LNSF equations inherently yield ill-conditioned systems for small non-zero frequencies.

As mentioned above, the dimension of $\operatorname{ker} B^{H}$ changes from zero to one in the limit that $\omega$ goes to zero. This implies that as $\omega \downarrow 0$, the elements in $Z$ that are in $\operatorname{ker} B_{v}^{H}$ will transcend from not being an element in $\operatorname{ker} B^{H}$ to being an element of $\operatorname{ker} B^{H}$. At the same time operator $C$ will vanish as $\omega$ goes to zero, leaving the part of the solution associated with $\operatorname{ker} B_{v}^{H}$ undetermined. In other words, the system of equations transforms from an invertible to a non-invertible system. This transition is also observed when looking at the null space of the corresponding FE operators $B_{h}$ and $C_{h}$, and causes the numerical system of equations to become increasingly ill-conditioned as $\omega$ decreases. In the limit where $\omega=0$, the condition number of the matrix grows to infinity, i.e., the numerical system does not have an inverse and $p$ can only be uniquely determined up to an element of $B_{v h}$, the discrete operator associated with $\operatorname{ker} B_{v}^{H}$. It is thus expected that the FE error of the part of the solution related to $\operatorname{ker} B_{v h}^{H}$ grows unboundedly as $\omega$ vanishes. The 
question arises whether the ill-conditioning of the numerical system has a negative influence on parts of the solution other than that related to $\operatorname{ker} B_{v h}^{H}$. This question is partially answered in the next subsection. The impact of the properties of operator $B_{h}$ on numerical results is investigated further in section 5.2.6 and 5.3.

\subsubsection{Error bounds, optimal convergence and the inf-sup condition}

Above it was demonstrated that the requirements for the existence and uniqueness of a solution presented in section 5.2.4 are satisfied for both the continuous and FE version of the LNSF equations. It was also demonstrated that the LNSF equations belong to the class of equations given by (5.23) after extending it to sesquilinear forms. Brezzi and Fortin [61] presented an error bound for the FE problem corresponding to equation (5.23), which also bounds the FE error corresponding to the LNSF equations. For the LNSF equations the error bound takes the following form:

$$
\begin{aligned}
&\left\|\mathbf{v}-\tilde{\mathbf{v}}_{h}\right\|_{X}+\left\|T-\tilde{T}_{h}\right\|_{Y}+\left\|p-\tilde{p}_{h}\right\|_{Z / \operatorname{ker} B^{H}} \leq \\
& K\left(\|a\|,\|b\|,\|c\|, \frac{1}{\alpha_{0}}, \frac{1}{\beta_{0}}, \frac{\|\bar{c}\|}{\overline{\gamma_{0}}}\right)\left(d\left(\mathbf{v}, X_{h}\right)+d\left(T, Y_{h}\right)+d\left(p, Z_{h}\right)\right)
\end{aligned}
$$

where $(\mathbf{v}, T, p)$ and $\left(\tilde{\mathbf{v}}_{h}, \tilde{T}_{h}, \tilde{p}_{h}\right)$ are the solution to a continuous and FE problem, respectively, and $d\left(\mathbf{v}, X_{h}\right), d\left(T, Y_{h}\right)$, and $d\left(p, Z_{h}\right)$ are the distances between the solutions for $\mathbf{v}, T$, and $p$ and the best approximation of these solutions in the FE spaces $X_{h}, Y_{h}$ and $Z_{h}$, respectively. The expressions for $\alpha_{0}, \beta_{0}$ and $\bar{\gamma}_{0}$ are constants satisfying $\alpha_{h} \geq \alpha_{0}, \beta_{h} \geq \beta_{0}$, and $\bar{\gamma}_{h} \geq \bar{\gamma}_{0}$ for all $h$, where the terms $\alpha_{h}, \beta_{h}$ and $\bar{\gamma}_{h}$ are defined by the following inequalities, respectively:

$$
\begin{gathered}
\inf _{\left(\mathbf{v}_{h}, T_{h}\right) \in \operatorname{ker} B_{h}} \sup _{\left(\mathbf{u}_{h}, S_{h}\right) \in \operatorname{ker}} \frac{\left|a\left(\left(\mathbf{v}_{h}, T_{h}\right),\left(\mathbf{u}_{h}, S_{h}\right)\right)\right|}{\left\|\left(\mathbf{v}_{h}, T_{h}\right)\right\|_{X \times Y}\left\|\left(\mathbf{u}_{h}, S_{h}\right)\right\|_{X \times Y}} \geq \alpha_{h} \\
\inf _{q_{h} \in Z_{h}} \sup _{\left(\mathbf{v}_{h}, T_{h}\right) \in X \times Y} \frac{\left|b\left(\left(\mathbf{v}_{h}, T_{h}\right), q_{h}\right)\right|}{\left\|\left(\mathbf{v}_{h}, T_{h}\right)\right\|_{X \times Y}\left\|q_{h}\right\|_{Z / \operatorname{ker} B^{H}}} \geq \beta_{h} \\
\inf _{q_{h} \in\left(\operatorname{ker} B_{h}^{H}\right)^{\perp}} \sup _{p_{h} \in\left(\operatorname{ker} B_{h}^{H}\right)^{\perp}} \frac{\left|\bar{c}\left(q_{h}, p_{h}\right)\right|}{\left\|p_{h}\right\|_{Z}\left\|q_{h}\right\|_{Z}} \geq \bar{\gamma}_{h}
\end{gathered}
$$

Note that if these inequalities hold with $\alpha_{h}>0, \beta_{h}>0$ and $\bar{\gamma}_{h}>0$ it is guaranteed ${ }^{9}$ that $a\left(\right.$, ) is invertible on $\operatorname{ker} B_{h}, \operatorname{Im} B_{h}$ is closed on $Z_{h}^{\prime}$, and $\bar{c}($,$) is invertible on$ $\operatorname{ker} B_{h}^{H}$, respectively.

\footnotetext{
${ }^{9}$ Note that for $\operatorname{Im} B_{h}$ to be closed on $Z_{h}^{\prime}$, the requirement that inequality (5.44) holds with $\beta_{h}>$ 0 is actually too strict. A necessary and sufficient requirement is obtained if $\left\|q_{h}\right\|_{Z / \operatorname{ker} B^{H}}$ in the denominator of (5.44) is replaced by $\left\|q_{h}\right\|_{Z / \operatorname{ker} B_{h}^{H}}$. Enforcing inequality (5.44) without replacing the norm yields $\beta_{h}=0$ if spurious modes are present.
} 
In section 5.3, it will be shown that for $\omega$ the value of $\beta_{h}$ is related to the smallest singular value of $B_{h}$. It turns out that the smallest singular value of $B_{h}$ vanishes as $\omega$ goes to zero, which implies the value of $\beta_{h}$ will also vanish. This means that the error bound in (5.42) is dependent on $\omega$ through the term $\beta_{h}$.

\section{Optimal convergence and the inf-sup condition}

The error bound in (5.42) predicts optimal convergence if the value of $K$ is finite, and independent of $h$. Upon a closer look at the derivation presented in [61] of the error bound in (5.42), it is observed that the value of $K$ grows when the value of its arguments increase, but remains finite if its arguments are finite. The first three arguments are operator norms of bounded sesquilinear forms and thus finite by definition. This means that the error bound in (5.42) predicts optimal convergence if there exist $\alpha_{0}>0, \beta_{0}>0$ and $\overline{\gamma_{0}}>0$ so that $\alpha_{h} \geq \alpha_{0}, \beta_{h} \geq \beta_{0}$ and $\bar{\gamma}_{h} \geq \bar{\gamma}_{0}$ independent of $h$. Note that using these definitions, the requirements for stability of an FE method for the LNSF equations are also requirements for the existence and uniqueness of an FE solution for all $h$ (including the limit $h \downarrow 0$ ).

Due to the coercivity of $a$ (,) on $X$, and $\bar{c}($, ) on $Z$, these forms are invertible on all subspaces of $X$ and $Z$, respectively. This means conditions (5.43) and (5.45) are met with $\alpha_{h}>0$ and $\bar{\gamma}_{h}>0$ for all $h$, i.e., there exist $\alpha_{0}$ and $\bar{\gamma}_{0}$ so that $\alpha_{h} \geq \alpha_{0}>0$ and $\bar{\gamma}_{h} \geq \bar{\gamma}_{0}>0$ for all $h$. On the other hand, a non-zero value of $\beta_{0}$, bounding $\beta_{h}$ above zero, is not guaranteed automatically.

Even if $B_{h}$ is closed, small values of $\beta_{h}$ are obtained for small values of $\omega$. In the limit that $\omega$ goes to zero, $\beta_{h}$ vanishes, making $K$ infinite and causing the bound in (5.42) to explode. This behavior is due to the physical nature of the problem and cannot be controlled by the choice of the numerical approximation. On the other hand, if $B_{h}$ is not closed for all $h$, the value of $\beta_{h}$ can become infinitely small independent of the value of $\omega$. This behavior is dependent on the FE method that is used. It is important to realize that the condition that $\operatorname{Im} B$ is closed on $Z^{\prime}$ does not imply that $B_{h}$ is closed on $Z_{h}^{\prime}$ for all $h$. More specifically, for a system of finite dimension, the condition that $B_{h}$ is closed is automatically satisfied, however, it is not necessarily true that $\operatorname{Im} B_{h}$ is closed on $Z_{h}^{\prime}$ as $h \downarrow 0$ and the FE spaces become infinite dimensional. This means that $\beta_{h}$ in condition (5.44) is not automatically bounded above zero as $h \downarrow 0$. The requirement that there is a $\beta_{0}$, so that $\beta_{h} \geq \beta_{0}>0$ for all $h$ including the limit $h \downarrow 0$ is known alternatively as the inf-sup condition, the Babuška-Brezzi condition, or the LB or LBB criterion. In the present text it will be known as the inf-sup condition. This condition must be verified for individual FE methods; it does not follow from the fact that the existence of a solution to the continuous problem and FE problems of finite size is guaranteed.

Recall that $K$ will become unbounded if $\beta_{0} \downarrow 0$. This implies that based on the 
error bound in (5.42), loss of accuracy or a lack of convergence of the FE solution can be expected, if the inf-sup condition is not met. Nevertheless, even if $\beta_{h}$ is not bounded from below, convergence at a lower order might still be achieved depending on how rapidly $\beta_{h}, d\left(\mathbf{v}, X_{h}\right)$ and $d\left(p, Z_{h}\right)$ converge to zero. It must also be noted that the error bounds are upper bounds indicative of the limit behavior as $h$ decreases. This means that the actual rate of convergence may be higher (or lower outside of the asymptotic range where $h>0$ ). The alternative error bound derived below, shows that (depending on the frequency) the bound in (5.42) can indeed be too strict. Nevertheless, checking the inf-sup condition is an important step in determining the stability and robustness of FE methods for saddle point problems such as described by the LNSF equations, the Stokes equations and the equations for linear elasticity. Proving analytically that the condition is satisfied for a certain FE method is hard in most cases, and this will not be attempted here. Instead, in section 5.4, the numerical inf-sup test, which is presented in section 5.3, is used to verify whether certain FE methods that have been proven to be stable for the Stokes equations are also likely to be stable for the LNSF equations.

\section{Frequency independent error bound}

Above, it is concluded that the error bound in (5.42) is dependent on $\omega$ since $\beta_{0}$ is dependent on $\omega$. The function $K$ grows to infinity as $\omega$ goes to zero making the bound useless for small values of $\omega$. As mentioned previously, this is related to ill-conditioning of the system due to the fact that the solution is only uniquely determined up to an element of $\operatorname{ker} B_{v}^{H}$ in the limit that $\omega$ goes to zero. The question arises if it is possible to bound the part of the solution excluding $\operatorname{ker} B_{v}^{H}$ independent on $\omega$. It turns out that a bound on $\mathbf{v}_{h} \in X_{h}$ and $p_{h} \in Z_{h} / \operatorname{ker} B_{v}^{H}$ can be constructed by eliminating temperature from the system of equations. The operator $A$ can be split into a velocity and temperature dependent part according to

$$
A(\mathbf{v}, T)=\left[\begin{array}{cc}
A_{\nu} & 0 \\
0 & A_{T}
\end{array}\right]\left\{\begin{array}{l}
\mathbf{v} \\
T
\end{array}\right\}
$$

Since $A_{T}$ is coercive, its inverse exists, allowing $T$ to be eliminated. The system in (5.32) can thus be rewritten as

$$
\left[\begin{array}{cc}
A_{v} & B_{v}^{H} \\
B_{v} & -\omega \bar{D}
\end{array}\right]\left\{\begin{array}{c}
(\mathbf{v}) \\
p
\end{array}\right\}=\left\{\begin{array}{c}
\mathbf{f} \\
-B_{T} A_{T}^{-1} q
\end{array}\right\}
$$

where operator $D$ is given by

$$
D=C+B_{T} A_{T}^{-1} B_{T}^{H}, \quad \text { and } \quad D=\omega \bar{D}
$$


It follows from the positive semi-definiteness of $-i A_{T}$ and the coerciveness of $-i \bar{C}$, that operator $-i \bar{D}$ is also coercive. The resulting system is thus of the same form as that in (5.32), and satisfies all requirements for existence and uniqueness of a solution. However, in contrast to the operator $B$ in (5.32), the operator $B_{v}$ in (5.47) is not dependent on $\omega$ (which is also true for its FE counterpart $B_{v h}$ ). The error bound for the LNSF equations in (5.42) takes the following form for the reduced system in (5.47)

$$
\begin{aligned}
& \left\|\mathbf{v}-\tilde{\mathbf{v}}_{h}\right\|_{X}+\left\|p-\tilde{p}_{h}\right\|_{Z / \operatorname{ker} B^{H}} \leq \\
& K\left(\left\|a_{v}\right\|,\left\|b_{v}\right\|,\|d\|, \frac{1}{\alpha_{0}}, \frac{1}{\beta_{0}}, \frac{\|\bar{c}\|}{\overline{\gamma_{0}}}\right)\left(d\left(\mathbf{v}, X_{h}\right)+d\left(p, Z_{h}\right)\right)
\end{aligned}
$$

with $\alpha_{0}, \beta_{0}$ and $\bar{\gamma}_{0}$ satisfying $\alpha_{h} \geq \alpha_{0}, \beta_{h} \geq \beta_{0}$ and $\bar{\gamma}_{h} \geq \bar{\gamma}_{0}$ for all $h$, where $\alpha_{h}, \beta_{h}$, and $\bar{\gamma}_{h}$ are defined by

$$
\begin{gathered}
\inf _{\mathbf{v}_{h} \in \operatorname{ker} B_{v h}} \sup _{\mathbf{u}_{h} \in \operatorname{ker} B_{v h}} \frac{\left|a\left(\mathbf{v}_{h}, \mathbf{u}_{h}\right)\right|}{\left\|\mathbf{v}_{h}\right\|_{X} \|_{\mathbf{u}_{h} \|_{X}}} \geq \alpha_{h} \\
\inf _{q_{h} \in Z_{h}} \sup _{\mathbf{v}_{h} \in X} \frac{\left|b\left(\mathbf{v}_{h}, q_{h}\right)\right|}{\left\|\mathbf{v}_{h}\right\| X\left\|q_{h}\right\|_{Z / \operatorname{ker} B^{H}}} \geq \beta_{h} \\
\inf _{q_{h} \in\left(\operatorname{ker} B_{h}^{H}\right)^{\perp}} \sup _{p_{h} \in\left(\operatorname{ker} B_{h}^{H}\right)^{\perp}} \frac{\left|\bar{d}\left(q_{h}, p_{h}\right)\right|}{\left\|p_{h}\right\|_{Z}\left\|q_{h}\right\|_{Z}} \geq \bar{\gamma}_{h}
\end{gathered}
$$

Note that since $B_{v h}$ is not dependent on $\omega$, the term $\beta_{h}$ also does not depend on $\omega$. The function $K$ is thus not dependent on $\omega$ and (5.49) predicts optimal convergence for $\mathbf{v}$ and $p$ as long as $B_{v h}$ is closed as $h$ goes to zero ${ }^{10}$. Based on this error bound, it is expected that the accuracy of the solution for velocity and the part of the pressure in the orthogonal complement of $\operatorname{ker} B_{v h}^{H}$ do not suffer from the ill conditioning of the system as $\omega$ vanishes. This is indeed supported by the numerical results presented in section 5.4. The same conclusion cannot be drawn for the temperature solution, and it will be seen in section 5.4 that the error in the temperature solution will indeed grow as $\omega$ vanishes.

\section{Inf-sup independent error bound}

An important feature of the error bound in (5.42) is that the nonlinear function $K$ is uniformly bounded in $\omega$ for all $k<\omega<\infty$, with $k$ an arbitrary non-zero constant. The function $K$ in the error bound in (5.49) is inherently independent of $\omega$. This

\footnotetext{
${ }^{10}$ Operator $D$ (specifically the second term in the definition in (5.47)) does depend on $\omega$. Nevertheless, $\bar{\gamma}_{h}$ can be bounded above zero independent of $\omega$, so $K$ does not depend on $\omega$.
} 
implies that if the inf-sup condition is satisfied, these error bounds predict optimal convergence independent of the value of $\omega$ (in the aforementioned frequency range). However, if the inf-sup is not satisfied, the error bounds becomes useless and convergence is not predicted. On the other hand, if $\omega$ is bounded above zero, the existence of a unique FE solution for all $h$ including $h \downarrow 0$ is guaranteed independent of the properties of $B$, since $A$ and $-i C$ are both coercive (as was already mentioned above). This implies that the bounds in (5.42) and (5.49) might not always be sharp for $\omega>0$ if the inf-sup condition is not satisfied.

Using the same strategy as is adopted in [61] to obtain (5.42), a second error bound can be obtained by replacing all requirements for existence and uniqueness by assuming that $A$ and $-i C$ are coercive (which is true for $\omega>0$ ). Under this condition, the continuous and the FE problem have a unique solution, which leads to the following error bound:

$$
\begin{aligned}
\left\|\mathbf{v}-\tilde{\mathbf{v}}_{h}\right\|_{X}+\left\|T-\tilde{T}_{h}\right\|_{Y}+\left\|p-\tilde{p}_{h}\right\|_{Z} \leq \\
K\left(\|a\|,\|b\|,\|c\|, \frac{1}{\alpha_{0}}, \frac{1}{\gamma_{0}}\right)\left(d\left(\mathbf{v}, X_{h}\right)+d\left(T, Y_{h}\right)+d\left(p, Z_{h}\right)\right)
\end{aligned}
$$

with $\alpha_{0}$ and $\gamma_{0}$ constant independent of $h$ so that $\alpha_{h} \geq \alpha_{0}$ and $\gamma_{h} \geq \gamma_{0}$, where $\alpha_{0}$ is defined by (5.43), and $\gamma_{h}$ is defined by ${ }^{11}$

$$
\inf _{q_{h} \in\left(\operatorname{ker} B_{h}^{H}\right)^{\perp}} \sup _{p_{h} \in\left(\operatorname{ker} B_{h}^{H}\right)^{\perp}} \frac{\left|c\left(q_{h}, p_{h}\right)\right|}{\left\|p_{h}\right\|_{Z}\left\|q_{h}\right\|_{Z}} \geq \gamma_{h}
$$

Again, the value of $K$ grows when its arguments increase, but remains finite if its arguments are finite. The nonlinear function $K$, becomes unbounded if $\omega \downarrow 0$ since $\gamma_{h}$ and thus $\gamma_{0}$ is linearly dependent on $\omega$. In contrast to the first bound, the nonlinear function $K$ does not depend on $\beta_{0}$. This implies that optimal convergence is predicted irrespective of whether the inf-sup condition is violated or holds, however, the error bound explodes for $\omega \downarrow 0$.

\section{Locking}

Based on the behavior of $K$ in the error bounds in (5.42), (5.53), and (5.49) it is to be expected that conformal FE methods for the LNSF equations always exhibit optimal convergence (for $\mathbf{v}$ and $p$ ) in the case $0<\omega<\infty$. Moreover, if the inf-sup condition is satisfied, or if $\omega$ is not too small, one of the error bounds will generally provide a reasonable bound on the FE error in terms of the approximation error for all $h$. On the other hand, if the inf-sup condition is not met, the bounds in

\footnotetext{
${ }^{11}$ This is inequality (5.44) where $\bar{c}($,$) and \bar{\gamma}_{0}$ are substituted by $c\left(\right.$, ) and $\gamma_{h}$.
} 
(5.42) and (5.49) become useless, and the bound on the FE error predicted with (5.42) and (5.53) can be very high for systems having a relatively small number of elements if $\omega$ is small. The situation where the inf-sup condition is not satisfied is of particular interest since it causes the solution to 'lock' as $\omega$ vanishes ${ }^{12}$.

It is important to realize that the error is only bounded from above by (5.53), and the actual FE error as a function of $h$ can behave differently depending on the type of problem, model parameters, boundary conditions, FE method and FE mesh under consideration. On the one hand, the observed FE error can have the same order of magnitude as predicted by the bound and exhibit optimal convergence in the entire range of $h$. Alternatively, the observed FE error can be considerably lower than predicted with (5.53) for a system having relatively few elements, while convergence occurs at a lower rate in the corresponding range of $h$. In that case, optimal convergence is observed only when $h$ becomes 'small enough'. For larger values of $h$ (i.e. systems with relatively few elements), the solution does not seem to converge properly and appears to be 'locked'.

Babuška and Suri [108] give a description (and a proper definition) of this phenomena, which is known as locking. They state that "Locking involves the 'shifting' of the asymptotic range of the calculations and will eventually disappear when the level of discretization is increased enough, depending on the strength of the degeneracy", and they mention that an infeasible level of discretization may be required before convergence can be observed. Their very general description of the locking phenomenon offers a framework for studying the convergence behavior of FE methods applied to various problems including linear elasticity, plate bending, heat conduction through highly orthotropic materials, Stokes flow, and viscothermal wave propagation.

Note that this description of the locking phenomenon does not correspond with the definition that is given in $[61,110]$ in the context of the Stokes equations. According to this definition, an FE method is classified as locking free when the constant $K$ in the above error bounds remains finite (thus ensuring optimal convergence as $h \downarrow 0$ ). Using the definition of locking given by Babuška and Suri, an FE method is only classified as locking free when the actual observed convergence rate becomes optimal within a reasonable range of $h$ (and not just in the limit $h \downarrow 0$ ). For many problems, this corresponds with the situation that $K$ is not only finite, but also not too large. For the LNSF equations, this implies that the FE method must either satisfy the inf-sup condition, or the frequency must be 'sufficiently high'.

Babuška et al. also give a mathematically precise definition of robustness,

\footnotetext{
${ }^{12}$ For an introduction to the concept of locking within the framework of the Stokes equation, see for instance [61, 60, 106, 62].
} 
which is based on their definition of locking. The definition is similar to the definition given in 5.1.4, although it requires optimal convergence of FE methods within a reasonable range of $h$ and not just in the limit $h \downarrow 0$. Using this definition, FE methods that do not satisfy the inf-sup condition are not robust for arbitrary frequencies. In section 5.4 it is shown that methods which do not satisfy the inf-sup condition are not robust in the range of audible frequencies.

\section{Spurious modes $(\omega=0)$}

It was already mentioned that if $\omega=0$, the equations describing pressure and velocity reduce to the Stokes equations, and the continuous and FE solutions for pressure are only uniquely determined up to an element $\operatorname{ker} B^{H}$ and $\operatorname{ker} B_{h}^{H}$, respectively. Depending on the FE method that is considered, $\operatorname{ker} B_{h}^{H}$ can be bigger than its continuous counterpart $\operatorname{ker} B^{H}$, which only contains constant pressure functions. If this is the case, there are non-constant pressure functions that can be added to the solution for $p$ while still satisfying the set of discretized equations. Such non-constant pressure functions are known as spurious pressure modes, or spurious modes for short. They result from the fact that FE solutions only have to satisfy the continuous equations in a weighted sense and have no physical meaning. Recall that the operator $B_{h}$ can be split into a v and $T$ dependent part, denoted by $B_{v h}$ and $B_{T h}$, according to equation (5.36). The spurious modes can be related to operator $B_{v h}$, since $B_{T h}=\{0\}$ if $\omega=0$, implying $\operatorname{ker} B_{h}^{H}=\operatorname{ker} B_{v h}^{H}$.

By definition $B_{v h}^{H} p_{h}=0$ for all $p_{h} \in \operatorname{ker} B_{h}^{H}$, implying that spurious modes do not contribute to the discrete momentum equation. At the same time, using spurious modes to weight the outcome of the term $B_{v h} \mathbf{v}_{h}$ in the continuity equation yields zero for all elements $\mathbf{v}_{h} \in X$. So, for the Stokes equation and the LNSF equations with $\omega=0$, spurious modes can be removed from the discrete system without altering the solution of the remaining system. This can be done by performing a coordinate transformation so that each spurious mode is only coupled with a single degree of freedom and subsequent removal of those degrees of freedom (a task that is not trivial for large systems). It follows that spurious modes do not influence the accuracy of the FE solution for velocity or the part of the solution for pressure that is orthogonal to $\operatorname{ker} B_{h}^{H}$. Alternatively, a system still including spurious modes can be solved, and the influence of spurious modes can often be filtered out successfully yielding an accurate pressure solution [61]. It must be noted, however, that systems including spurious modes are non-invertible which limits the number of suitable solvers. 


\section{Spurious modes $(\omega>0)$}

Now consider the LNSF equations for the case $\omega>0$, so $C \neq 0$. Since $-i C$ is coercive for $\omega>0$ the contribution of the spurious modes to the pressure solution is uniquely determined for $\omega>0$, and the discrete system of equations is invertible. Depending on the discretization of $p$ and $T$, the elements of the subspace $\operatorname{ker} B_{v h}^{H}$ containing the spurious modes are coupled with the elements in its orthogonal complement $\left(\operatorname{ker} B_{v h}^{H}\right)^{\perp}$, and the FE space for temperature, respectively, through the operators $C_{h}$ and $B_{T h}$. The coupling with $B_{T h}$ and $C_{h}$, is not established only if the following conditions are met

$$
\begin{aligned}
c\left(p_{0}, q_{0}\right)=0, & \forall p_{0} \in \operatorname{ker} B_{v h}^{H}, q_{0} \in\left(\operatorname{ker} B_{v h}^{H}\right)^{\perp} \\
b_{T}(T, S)=0, & \forall T \in T_{s}, q_{0} \in\left(T_{S}\right)^{\perp}
\end{aligned}
$$

where $T_{S}$ is the subspace of $B_{T}$ that yields a non-zero value when weighted with elements of $\operatorname{ker} B_{h}^{H}$ :

$$
T_{s}=\left\{T \in Y \mid b_{T}\left(T, q_{0}\right) \neq 0, \quad \forall q_{0} \in \operatorname{ker} B_{v h}^{H}\right\}
$$

In general these conditions are not met, and the spurious modes are coupled with elements in $\left(\operatorname{ker} B_{v h}^{H}\right)^{\perp}$ and $Y$. Both $B_{T h}$ and $C_{h}$ are linearly dependent on $\omega$, implying the strength of the coupling is determined by the value of $\omega$. As mentioned above, for $\omega=0$, there is no coupling (equation (5.55) and (5.56) are met) and the spurious modes can be removed. However, for $\omega>0$, the spurious modes can in general not be isolated by a coordinate transformation and cannot be removed from the system. As previously mentioned, the LNSF equations are related to the equations for linear elasticity problems. It is known from the literature that large solution errors can occur for FE approximations of these equations, if spurious modes are present and non-zero displacements are prescribed as boundary conditions (see, for instance, [110]). Based on the analogy between the equations for linear elasticity and viscothermal wave propagation, it is to be expected that prescribing non-zero velocity (or temperature) boundary conditions can cause FE solutions for the LNSF equations to be polluted by spurious modes.

Note that the norm on $\|q\|$ in inequality (5.44), which defines $\beta_{0}$, does not exclude the influence of spurious modes. Consequently, $\beta_{0}=0$ if spurious modes are present, making $K$ in error bound (5.42) and (5.42) infinite. So, according to these bounds, optimal convergence will not occur if spurious modes are present. However, just as for the FE methods that do not satisfy the inf-sup condition, these error bounds are not always sharp for FE methods suffering from spurious modes. 


\subsection{The numerical inf-sup test}

The system of equations in (5.39) must first be brought into a suitable matrix/vector form, before numerical solution techniques can be applied. Such a form, which also provides the basis for the numerical inf-sup test, is presented in the first subsection. Next, the relation between the inf-sup condition and the generalized singular values of the discrete operator $B_{h}$ is discussed. Two decompositions that can be used two obtain these generalized singular values are introduced. In the next subsection, the numerical inf-sup test is defined, and the consequence of using the numerical inf-sup test compared to verifying the inf-sup condition analytically is commented on. The last subsection contains results of the numerical inf-sup test for a number of FE methods for the LNSF equations.

\subsubsection{Matrix vector form of the LNSF equations}

The spaces $X_{h}, Y_{h}$, and $Z_{h}$ are assumed to be of dimension $k, l$, and $m$. The elements of these spaces are written in terms of a sum of the contributions of the set of functions that form a basis for the spaces, i.e.,

$$
\mathbf{v}=\sum_{i=1}^{k} \alpha_{i} \mathbf{v}_{i}, \quad T=\sum_{i=1}^{l} \beta_{i} T_{i}, \quad \quad p=\sum_{i=1}^{m} \gamma_{i} p_{i}
$$

where $\mathbf{v}_{i}, T_{i}$, and $p_{i}$ are functions in the bases of $X_{h}, Y_{h}$, and $Z_{h}$ respectively, and $\alpha_{i}, \beta_{i}$, and $\gamma_{i}$ are scalars representing the contribution of the basis functions. The right-hand side terms $\mathbf{f}$ and $q$ can be expressed in terms of the basis functions for $\mathbf{v}$ and $T$ by

$$
\mathbf{f}=\sum_{i=1}^{k} \phi_{i} \mathbf{v}_{i}, \quad q=\sum_{i=1}^{l} \psi_{i} T_{i}
$$

where $\phi_{i}$ and $\psi_{i}$ are scalars representing the contribution of the basis functions. Using these bases, the problem in (5.32) can be written in terms of matrices and vectors. The elements of the vectors represent the contributions of each basis function, i.e.,

$$
\mathrm{v}=\left\{\alpha_{i}\right\}, \quad \mathrm{T}=\left\{\beta_{i}\right\}, \quad \mathrm{p}=\left\{\gamma_{i}\right\}, \quad \mathrm{f}=\left\{\phi_{i}\right\}, \quad \mathrm{q}=\left\{\psi_{i}\right\} \quad \text { (5.60a,b,c,d,e) }
$$

The elements of the matrices $\mathrm{A}_{v}, \mathrm{~A}_{T}, \mathrm{~B}_{v}, \mathrm{~B}_{T}$ and $\mathrm{C}$ hold the values resulting from evaluating the corresponding bilinear forms for all combinations of the basis func- 
tions;

$$
\begin{aligned}
\mathrm{A}_{v} & =\left[\begin{array}{ccc}
a_{v}\left(\mathbf{v}_{1}, \mathbf{v}_{1}\right) & \cdots & a_{v}\left(\mathbf{v}_{1}, \mathbf{v}_{k}\right) \\
\vdots & \ddots & \vdots \\
a_{v}\left(\mathbf{v}_{k}, \mathbf{v}_{1}\right) & \cdots & a_{v}\left(\mathbf{v}_{k}, \mathbf{v}_{k}\right)
\end{array}\right], \quad \mathrm{A}_{T}=\left[\begin{array}{ccc}
a_{T}\left(T_{1}, T_{1}\right) & \cdots & a_{T}\left(T_{1}, T_{l}\right) \\
\vdots & \ddots & \vdots \\
a_{T}\left(T_{l}, T_{1}\right) & \cdots & a_{T}\left(T_{l}, T_{l}\right)
\end{array}\right], \\
\mathrm{B}_{v} & {\left[\begin{array}{ccc}
b_{v}\left(\mathbf{v}_{1}, p_{1}\right) & \cdots & b_{v}\left(\mathbf{v}_{1}, p_{m}\right) \\
\vdots & \ddots & \vdots \\
b_{v}\left(\mathbf{v}_{k}, p_{1}\right) & \cdots & b_{v}\left(\mathbf{v}_{k}, p_{m}\right)
\end{array}\right], \quad \mathrm{B}_{T}=\left[\begin{array}{ccc}
b_{T}\left(T_{1}, p_{1}\right) & \cdots & b_{T}\left(T_{1}, p_{m}\right) \\
\vdots & \ddots & \vdots \\
b_{T}\left(T_{l}, p_{1}\right) & \cdots & b_{T}\left(T_{l}, p_{m}\right)
\end{array}\right], } \\
\mathrm{C}=\left[\begin{array}{ccc}
c\left(p_{1}, p_{1}\right) & \cdots & c\left(p_{1}, p_{m}\right) \\
\vdots & \ddots & \vdots \\
c\left(p_{m}, p_{1}\right) & \cdots & c\left(p_{m}, p_{m}\right)
\end{array}\right] &
\end{aligned}
$$

The matrix form of problem (5.39) now takes the form

$$
\left[\begin{array}{ccc}
\mathrm{A}_{v} & 0 & \mathrm{~B}_{v}^{H} \\
0 & \mathrm{~A}_{T} & \mathrm{~B}_{T}^{H} \\
\mathrm{~B}_{v} & \mathrm{~B}_{T} & \mathrm{C}
\end{array}\right]\left\{\begin{array}{c}
\mathrm{v} \\
\mathrm{T} \\
\mathrm{p}
\end{array}\right\}=\left\{\begin{array}{l}
\mathrm{f} \\
\mathrm{q} \\
0
\end{array}\right\}
$$

To improve legibility, the following compound matrices, vectors and functions are defined

$$
\mathrm{A}=\left[\begin{array}{cc}
\mathrm{A}_{v} & 0 \\
0 & \mathrm{~A}_{T}
\end{array}\right], \quad \mathrm{B}=\left[\begin{array}{ll}
\mathrm{B}_{v} & \mathrm{~B}_{T}
\end{array}\right], \quad \mathrm{w}=\{\mathrm{v}, \mathrm{T}\}, \quad \mathbf{w}=(\mathbf{v}, T) \quad(5.63 \mathrm{a}, \mathrm{b}, \mathrm{c}, \mathrm{d})
$$

\subsubsection{The inf-sup condition and the singular value decomposition}

In section 5.2.6 it is stated that the inf-sup condition is satisfied if there is a $\beta_{0}$ so that $\beta_{h} \geq \beta_{0}$ for all $h$, where $\beta_{h}$ is defined by inequality (5.44). Restated in terms of the compound vector $\mathbf{w}$, the inequality takes the form ${ }^{13}$ :

$$
\inf _{q_{h} \in\left(\operatorname{ker} B^{H}\right)^{\perp}} \sup _{\mathbf{w} \in X_{h} \times Y_{h}} \frac{\left|b\left(\mathbf{w}, q_{h}\right)\right|}{\|\mathbf{w}\|_{X \times Y}\left\|q_{h}\right\|_{Z}} \geq \beta_{h}
$$

After squaring both numerator and denominator, this inequality can also be written in terms of the matrices and vectors defined above as:

$$
\inf _{\mathrm{p} \in\left(\operatorname{ker} \mathrm{B}^{H}\right)^{\perp}} \sup _{w \in \mathbb{C}^{n k} \times \mathbb{C}^{C}} \frac{\left|\mathrm{p}^{H} \mathrm{Bw}\right|^{2}}{\left|\mathrm{w}^{H} \mathrm{Mw}\right|\left|\mathrm{p}^{H} \mathrm{~Np}\right|} \geq \beta_{h}
$$

\footnotetext{
${ }^{13}$ Note that with respect to (5.44), the quotient norm is replaced by \|\|$_{Z}$, and $q_{h}$ is sought in the orthogonal complement of $\operatorname{ker} B$, thus yielding the same value for $\beta_{h}$.
} 
where the elements of $\mathrm{M}$ and $\mathrm{N}$ represent the values of the inner product corresponding to the space $H^{1}$ and $L^{2}$ for pairs of the basis functions spanning $X_{h} \times Y_{h}$ and $Z_{h}$, respectively, so

$$
\mathrm{m}_{i j}=\langle\mathbf{w}, \mathbf{w}\rangle_{H^{1}}, \quad \mathrm{n}_{i j}=\left\langle p_{i}, p_{j}\right\rangle_{L^{2}}
$$

The value for $\beta_{h}$ in inequality (5.65) corresponds to a singular value of the matrix $\mathrm{B}$ if $\mathrm{M}=\mathrm{N}=I[61]$. For $\mathrm{M} \neq I$ and/or $\mathrm{N} \neq I$ (which is true for the case at hand), the value for $\beta_{h}$ corresponds to a generalized singular value of the matrix B (see [61]). Instead of calculating the generalized singular values directly, it is convenient to switch to a different basis for $\mathrm{v}, \mathrm{T}$ and $\mathrm{p}$, so that algorithms for the standard singular value decomposition can be used. Inequality (5.65) can be brought in such a form by applying the following coordinate transformation:

$$
\hat{w}=\hat{M} w
$$$$
\hat{p}=\hat{N} p
$$

where $\hat{\mathrm{M}}$ and $\hat{\mathrm{N}}$ must satisfy

$$
\hat{\mathrm{M}}^{H} \hat{\mathrm{M}}=\mathrm{M} \quad \hat{\mathrm{N}}^{H} \hat{\mathrm{N}}=\mathrm{N}
$$

Since $M$ and $N$ are positive definite by definition, they can be obtained by performing a Cholesky decomposition ${ }^{14}$. Substitution of the new coordinates in inequality (5.65) yields

$$
\inf _{\hat{\mathbf{p}} \in\left(\operatorname{ker} \hat{\mathrm{B}}^{H}\right)^{\perp}} \sup _{\hat{\mathbf{w}} \in \mathbb{C}^{n k} \times \mathbb{C}^{l}} \frac{\left|\hat{\mathrm{p}}^{H} \hat{\mathrm{B}} \hat{\mathrm{w}}\right|}{\left|\hat{\mathrm{w}}^{H} \hat{\mathrm{w}}\right|\left|\hat{\mathrm{p}}^{H} \hat{\mathrm{p}}\right|} \geq \beta_{h}, \quad \text { where } \quad \hat{\mathrm{B}}=\hat{\mathrm{N}}^{-H} \mathrm{~B} \hat{\mathrm{M}}^{-1}
$$

After this transformation, the value $\beta_{h}$ corresponds to a singular value of $\hat{B}$. The singular values of this matrix are obtained by performing the standard singular value decomposition given by

$$
\mathrm{U} \Sigma \mathrm{V}^{H}=\hat{\mathrm{B}}
$$

where the colons of matrices $\mathrm{U}$ and $\mathrm{V}$ are the left and right hand singular vectors, and the diagonal elements of the pseudo-diagonal matrix $\Sigma$ are the singular values (usually listed in order of decreasing magnitude). Note that the matrix norm $\|\hat{B}\|$ corresponds to the highest singular value, which converges to $\|b\|$ for $h$ goes to zero $^{15}$. Furthermore, if $\hat{p}$ in inequality (5.69) is sought in $\mathbb{C}^{k}$ instead of $\left(\operatorname{ker} \hat{B}^{H}\right)^{\perp}$, the value of $\beta_{h}$ corresponds to the smallest singular value of $\hat{B}$. The singular values (and vectors) of $\hat{B}$ can alternatively be determined by solving the following associated generalized eigenvalue problems (see [61]):

$$
\mathrm{BM}^{H} \mathrm{~B}^{H} \mathrm{U}=\mathrm{NU} \Sigma^{2}, \quad \mathrm{~B}^{H} \mathrm{~N}^{H} \mathrm{BV}=\mathrm{MV} \Sigma^{2}
$$

\footnotetext{
${ }^{14}$ Other decompositions that satisfy (5.68) can be obtained and may be used as well.

${ }^{15}$ The norm on $b\left(\right.$, ) is defined as $\|b\|=\sup _{p \in Z \mathbf{v} \in X} \frac{|b(v, p)|}{|\mathbf{v}|_{X}|p|_{Z}}$.
} 


\subsubsection{The inf-sup test}

The numerical inf-sup test consists of calculating $\beta_{h}$ for a family of meshes obtained by consecutive mesh refinements. The result can be used to predict whether the value of $\beta_{h}$ will remain bounded above zero as $h \downarrow 0$. If $\beta_{h}$ is non-zero and does not vanish to zero as $h \downarrow 0$, the FE method is said to pass the numerical inf-sup test. Naturally, the outcome of the numerical inf-sup test must not be confused with a definitive analytical proof that the inf-sup condition is met for a certain FE method; The asymptotical behavior of $\beta_{h}$ is predicted based on just a few values of $h$. In addition, a method that satisfies the numerical inf-sup test for a certain family of meshes and boundary conditions might still not satisfy the inf-sup condition for other meshes and boundary conditions. So, proving robustness of an FE method would require performing the inf-sup test for all possible meshes and boundary conditions. In practice, conclusions are usually drawn based on the results of the numerical inf-sup test for a regular and a distorted mesh on a square geometry satisfying certain 'simple' boundary conditions (for instance Dirichlet boundary conditions).

Obviously, it can be argued that such results cannot be considered conclusive. Nevertheless, the numerical inf-sup test can be useful to asses whether a new FE method will fail the inf-sup condition, or if it is likely to satisfy it. In this thesis, the test serves precisely that purpose. An introduction to the numerical inf-sup test can be found for instance in $[110,111]$.

Apart from the convergence behavior of $\beta_{h}$, the value of the following ratio is also of interest when the accuracy of an FE method is determined:

$$
\psi=\frac{\beta_{h}}{\|\hat{B}\|}
$$

Recall that for small values of $\omega$, the matrix norm on $\hat{C}$ is small compared to that of $\hat{A}$. In that case, the condition of the the matrix $\hat{B}$ will have an significant impact on the condition of the total system of equations in (5.62) describing the FE problem. Since, $\psi$ is the ratio between the inf-sup value corresponding with the lowest (or second lowest) singular value and the sup-sup value corresponding with the highest singular value, it is inversely proportional to the condition of matrix $\hat{B}$. In other words, if $\omega$ is small, the ratio gives an indication of the condition of the system of discrete LNSF equations. If the value of $\psi$ is much smaller than unity, large inaccuracies in the FE solution can be expected for systems with few degrees of freedom (even if the inf-sup condition is satisfied - implying $\beta_{h}$ is bounded from below - and the FE method under consideration has optimal convergence). Naturally, if the inf-sup condition is not satisfied and $\beta_{h}$ vanishes when $h$ goes to zero, $\psi$ will also vanish (since the value of $\|\hat{B}\|$ converges to the constant $\|b\|$ for $h \downarrow 0$.). 
In the presentation of the test results in the next subsections, the ratio $\psi$ will be plotted instead of the inf-sup value $\beta_{h}$. This way, both the phenomena of locking and ill-conditioning of the FE system due to other causes can be monitored.

\section{Smallest non-zero singular values}

Above it was explained that the value of $\beta_{h}$ can be associated with a singular value of the matrix $\hat{B}$. This implies that the values of $\beta_{h}$ for a family of meshes can be determined numerically by performing a singular value decomposition on $\hat{B}$ for each $h$. What remains, is to determine which singular value corresponds with $\beta_{h}$. The matrices $\mathrm{U}$ and $\mathrm{V}$ defined by the decomposition in equation (5.70) serve as an orthonormal basis for $X_{h} \times Y_{h}$ and $Z_{h}$. So, for each $h$, the original system of equations in (5.32) can be transformed to a basis in which $\hat{B}$ takes a pseudo-diagonal form. The new coordinates $\bar{w}$ and $\bar{p}$, and right-hand side term $\bar{f}$ and the matrices $\bar{A}$ and $\bar{C}$ in these new coordinates are defined, respectively, as:

$$
\begin{array}{crr}
\bar{w}=\mathrm{V}^{H} \hat{\mathrm{M}} \mathrm{w}, & \overline{\mathrm{p}}=\mathrm{U}^{H} \hat{\mathrm{N}} \mathrm{p}, \quad \overline{\mathrm{f}}=\mathrm{V}^{H} \hat{\mathrm{Mf}} \\
\overline{\mathrm{A}}=\mathrm{V}^{H} \hat{\mathrm{M}}^{-H} \mathrm{~A} \hat{M}^{-1} \mathrm{~V}, \quad \overline{\mathrm{C}}=\mathrm{U}^{H} \hat{\mathrm{N}}^{-H} \mathrm{CN}^{-1} \mathrm{U}
\end{array}
$$

The singular values and the corresponding coordinates are divided into three sets, so the system in (5.32) takes the form:

$$
\left[\begin{array}{ccccccc}
\overline{\mathrm{A}}_{11} & \overline{\mathrm{A}}_{12} & \overline{\mathrm{A}}_{13} & \overline{\mathrm{A}}_{14} & \Sigma_{1} & 0 & 0 \\
\overline{\mathrm{A}}_{21} & \overline{\mathrm{A}}_{22} & \overline{\mathrm{A}}_{23} & \overline{\mathrm{A}}_{24} & 0 & \Sigma_{2} & 0 \\
\overline{\mathrm{A}}_{31} & \overline{\mathrm{A}}_{32} & \overline{\mathrm{A}}_{33} & \overline{\mathrm{A}}_{34} & 0 & 0 & \Sigma_{3} \\
\overline{\mathrm{A}}_{41} & \overline{\mathrm{A}}_{42} & \overline{\mathrm{A}}_{43} & \overline{\mathrm{A}}_{44} & 0 & 0 & 0 \\
\Sigma_{1} & 0 & 0 & 0 & \overline{\mathrm{C}}_{11} & \overline{\mathrm{C}}_{12} & \overline{\mathrm{C}}_{13} \\
0 & \Sigma_{2} & 0 & 0 & \overline{\mathrm{C}}_{21} & \overline{\mathrm{C}}_{22} & \overline{\mathrm{C}}_{23} \\
0 & 0 & \Sigma_{3} & 0 & \overline{\mathrm{C}}_{31} & \overline{\mathrm{C}}_{32} & \overline{\mathrm{C}}_{33}
\end{array}\right]\left\{\begin{array}{c}
\overline{\mathrm{w}}_{1} \\
\overline{\mathrm{w}}_{2} \\
\overline{\mathrm{w}}_{3} \\
\overline{\mathrm{w}}_{4} \\
\overline{\mathrm{p}}_{1} \\
\overline{\mathrm{p}}_{2} \\
\overline{\mathrm{p}}_{3}
\end{array}\right\}=\left\{\begin{array}{c}
\overline{\mathrm{f}}_{1} \\
\overline{\mathrm{f}}_{2} \\
\overline{\mathrm{f}}_{3} \\
\overline{\mathrm{f}}_{4} \\
0 \\
0 \\
0
\end{array}\right\}
$$

The singular values in $\Sigma_{1}, \Sigma_{2}$ and $\Sigma_{3}$ are stored in the vectors $\sigma_{1}, \sigma_{2}, \sigma_{3}$ in increasing order. The separation of the singular values into three sets is such, that $\sigma_{3}$ contains all zero singular values, $\sigma_{2}$ contains singular values that vanish as $h$ goes to zero, and $\sigma_{1}$ contains the remaining 'stable part'. The singular values in $\sigma_{1}$ and $\sigma_{2}$ are called stable and unstable, respectively. The rate of growth of the dimension of the vectors $\sigma_{1}, \sigma_{2}$, and $\sigma_{3}$ as $h$ goes to zero determines whether the FE method is stable or not.

The dimension of $\sigma_{3}$ is denoted by $m$, where $m=\operatorname{dim}\left(\operatorname{ker} B_{h}^{H}\right)$. The dimension of the null space of the continuous operator is denoted by $n=\operatorname{dim}\left(\operatorname{ker} B^{H}\right)$. For the LNSF equations $n$ equals either zero (at zero frequency) or one (for non-zero 
frequencies). If $n=1$ and the element in $\operatorname{ker} B^{H}$ can be associated with an element in $\operatorname{ker} B_{h}^{H}$, the value of $\beta_{h}$ corresponds to the second smallest singular value, otherwise (for non-zero frequency) $\beta_{h}$ corresponds to the smallest singular value. Depending on the FE spaces that are used, the dimension of $\operatorname{ker} B_{h}^{H}$ can be bigger than that of $\operatorname{ker} B^{H}$ (i.e., $m>n$ ). In that case, the method suffers from spurious modes, and the value of $\beta_{h}$ corresponds to an element of $\sigma_{3}$, i.e., $\beta_{h}=0$ for all $h$.

The dimension of $\sigma_{2}$ is denoted by $k$. If $m=n$ (no spurious modes) and $k>0$, the value of $\beta_{h}$ corresponds to the smallest singular value in $\Sigma_{2}$ and vanishes as $h$ goes to zero. In that case, convergence of the FE solution is compromised and the FE method is likely to suffer from locking to some degree. Lastly, if $m=n$ (no spurious modes) and $k=0$ (no locking), the value of $\beta_{h}$ corresponds to the smallest singular value in $\Sigma_{3}$ and remains bounded above zero for all $h$. In that case, the element is stable.

If the FE method suffers from spurious modes $(m>n)$, obtaining a solution might still be useful despite the fact that the $\beta_{h}$ is zero and the inf-sup test fails according to the above definitions. In that case, it is more useful to relate $\beta_{h}$ to the smallest non-zero singular value, which can be an element of $\Sigma_{2}$ or $\Sigma_{3}$. By doing this, the stability of the solution without the influence of spurious modes can still be assessed by observing the value $\beta_{h}$ as $h$ goes to zero. Redefining $\beta_{h}$ in this way corresponds to replacing the quotient norm \|\|$_{Z / \mathrm{ker} B^{H}}$ in inequality (5.44) with the quotient norm \|\|$_{Z / k e r B_{h}^{H}}$. This definition of $\beta_{h}$ is used from this point on. Comparing the dimension of $\Sigma_{3}$ with that of $\operatorname{ker} B_{h}^{H}$ will still allow to determine whether $m>n$, indicating that the FE method suffers from spurious modes.

\subsubsection{Influence of $\mathrm{B}_{v}$ and $\mathrm{B}_{T}$}

As explained above, the dimension of the matrices $\Sigma_{1}, \Sigma_{2}$, and $\Sigma_{3}$ containing the generalized singular values of $B$ indicates whether an FE method might suffer from spurious modes or locking. Not surprisingly, the dimensions of these matrices are dependent on the properties of both $\mathrm{B}_{v}$ and $\mathrm{B}_{T}$. The properties of these sub matrices are influenced by the FE spaces that are used to approximate $\mathbf{v}, T$ and $p$. To investigate the individual influence of these matrices, a similar diagonalization as proposed above for $\mathrm{B}$ is carried out for $\mathrm{B}_{v}$ and $\mathrm{B}_{T}$ individually. First, the coordinate transformation as proposed in (5.67) is applied so a standard singular value decomposition can be used. Subsequently, a singular value decomposition is performed on $\mathrm{B}_{v}$ and $\mathrm{B}_{T}$ independently. The matrices holding the singular vectors can be used to perform a second coordinate transformation as proposed in $(5.73 \mathrm{a}, \mathrm{b}, \mathrm{c})$ and $(5.74 \mathrm{a}, \mathrm{b})$. Note that the second coordinate transformation affects either $\mathrm{A}_{v}, \mathrm{~B}_{v}, \mathrm{w}_{v}$ and $\mathrm{f}_{v}$ or $\mathrm{A}_{T}, \mathrm{~B}_{T}, \mathrm{w}_{T}$ and $\mathrm{f}_{T}$ depending on which set of singular values is used. The following two equivalent systems of equations are thus 
obtained:

$$
\left[\begin{array}{ccc}
\overline{\mathrm{A}}_{v} & 0 & \Sigma_{1} \\
0 & \hat{\mathrm{A}}_{T} & \hat{\mathrm{B}}_{T} \\
\Sigma_{v} & \hat{\mathrm{B}}_{T} & \overline{\mathrm{C}}
\end{array}\right]\left\{\begin{array}{c}
\overline{\mathrm{w}}_{v} \\
\hat{\mathrm{w}}_{T} \\
\overline{\mathrm{p}}
\end{array}\right\}=\left\{\begin{array}{c}
\overline{\mathrm{f}}_{v} \\
\hat{\mathrm{f}}_{T} \\
0
\end{array}\right\}, \quad\left[\begin{array}{ccc}
\hat{\mathrm{A}}_{v} & 0 & \hat{\mathrm{B}}_{v} \\
0 & \overline{\mathrm{A}}_{T} & \Sigma_{2} \\
\hat{\mathrm{B}}_{v} & \Sigma_{T} & \overline{\mathrm{C}}
\end{array}\right]\left\{\begin{array}{c}
\hat{\mathrm{w}}_{v} \\
\overline{\mathrm{w}}_{T} \\
\overline{\mathrm{p}}
\end{array}\right\}=\left\{\begin{array}{c}
\hat{\mathrm{f}}_{v} \\
\overline{\mathrm{f}}_{T} \\
0
\end{array}\right\}
$$

Note that diagonalizing $B_{v}$ in this way, does not necessarily diagonalizes $B_{T}$ and vice versa. This implies that a right singular vector (describing pressure) corresponding to a singular value of $\hat{\mathrm{B}}_{v}$ can be non-orthogonal to multiple right singular vectors corresponding to singular values of $\hat{\mathrm{B}}_{T}$. This coupling between $\hat{\mathrm{B}}_{v}$ and $\hat{\mathrm{B}}_{T}$ through their right singular vectors determines how the different stable, unstable and zero singular values of the individual matrices contribute to the singular values of $\hat{B}$. The following inequality is useful when trying to determine the nature of the singular values $\sigma$ of the matrix $\hat{\mathrm{B}}$ based on the singular values $\sigma_{v}$ and $\sigma_{T}$ of the matrices $\hat{\mathrm{B}}_{v}$ and $\hat{\mathrm{B}}_{T}$

$$
\min \left(\sigma_{i}\right) \geq \min \left(\sigma_{v i}, \sigma_{T i}\right)
$$

This inequality implies that the singular values obtained by concatenating two matrices are at least of the same magnitude as the singular values of the individual matrices.

Both $\Sigma_{v}$ and $\Sigma_{T}$ can be split into sets of stable, unstable and zero singular values as is done in equation (5.75). Using inequality (5.77) it can for instance be concluded that if either $\sigma_{v}$ or $\sigma_{T}$ consist of stable singular values only, $\sigma$ will consist of stable singular values only. Note, however, that the properties of the operators $B_{v}$ and $B_{T}$ describing the continuous problem, suggest that this situation will not occur. On the other hand, if for instance $\sigma_{v}$ consists of stable and zero singular values, and $\sigma_{T}$ consists of stable and unstable singular values, $\sigma$ can consist of any mix of stable, unstable and zero singular values. The coupling through the right-hand singular vectors of $\hat{\mathrm{B}}_{v}$ and $\hat{\mathrm{B}}_{T}$ (which is governed by their orthogonality) determines the types of singular values observed in the compound matrix.

\subsubsection{Stable FE methods}

If the weak form of the LNSF equations and the Stokes equations are compared, it is easily observed that the inf-sup condition for $B_{v h}$ in the context of the LNSF equations is equal to the inf-sup condition in the context of the Stokes problem. From the literature on FE methods for the Stokes equations, it is known for a large number of FE methods whether spurious modes or locking occurs (see for instance $[60,61,62]$ ). For these methods, the occurrence (and possibly the number) of stable, unstable and zero singular values for $\hat{B}_{v}$ can be predicted. If the FE 
spaces for $v$ and $p$ are such that the FE method does not lock, $\sigma_{\nu}$ consists of stable and zero singular values only. As mentioned previously, the number of zero singular values depends on the presence of spurious modes; if there are no spurious modes there is one zero singular value, otherwise, there are more. Note that the singular vector for pressure that can be associated with $\operatorname{ker} B_{v}^{H}$ is smooth (a constant), while the singular vectors for pressure associated with spurious modes are highly oscillatory. This difference influences how these pressure modes are coupled with the singular vectors for pressure corresponding to $\hat{B}_{T}$.

\section{Order of the trial functions}

Based on FE methods for the Stokes equations that are known to be stable, it can be concluded that the dimension of the FE space $\mathbf{v}_{h}$ must be taken large enough with respect to that of $p_{h}$ to ensure that spurious modes and unstable singular values of $\hat{\mathrm{B}}_{v}$ do not occur. The opposite holds true for $\hat{\mathrm{B}}_{T}$; if the dimension of the FE space $T_{h}$ is chosen to be large with respect to that of $p_{h}$, the FE operator $B_{T h}$ will not be closed in the limit that $h$ goes to zero (for an example see figure 5.10 in section 5.4.4). It turns out that the polynomials which are used to approximate $p$ have to be of higher order than those used to approximate $T$ in order to obtain a matrix $\hat{\mathrm{B}}_{T}$ with only stable singular values. This suggests that to obtain a matrix $\hat{\mathrm{B}}_{v}$ with only stable, and one zero singular value, and a matrix $\hat{\mathrm{B}}_{T}$ with only stable singular values it is required that $\operatorname{dim}(v)>\operatorname{dim}(p)>\operatorname{dim}(T)$. Under these conditions it follows from (5.77) that B will only have stable singular values and the FE method will thus be free from locking and spurious modes. However, looking at the error bounds in (5.42) and (5.53), it is clear that such an FE scheme yields optimal convergence, but would result in a low convergence speed since it is limited by the poor approximability of $T$. From the point of view of approximability, it is most advantageous to choose the FE spaces for $\mathbf{v}$ and $T$ of equal order, and to take the polynomials that span the FE space for $p$ one order lower than those for $\mathbf{v}$ and $T$.

\section{Inf-sup condition for the Stokes equations}

Fortunately, it is not necessary for $B_{T h}$ to be closed, to obtain an operator $B_{h}$ that is closed in the limit that $h$ goes to zero. If the solution spaces for $\mathbf{v}_{h}$ and $p_{h}$ of an FE method satisfy the inf-sup condition for the Stokes problem, all but one of the singular values of $B_{v h}$ are stable. The smallest singular value corresponds to $\operatorname{ker} B_{v h}^{H}$ and thus equals zero. As long as the corresponding right singular vector for $B_{v h}$ (being a constant pressure function) is non-orthogonal to at least one right singular vector for $B_{T h}$ corresponding to a stable singular value (for all $h$ ), the sin- 
gular values of $B_{h}$ are all non-zero and stable. In that case, $B_{T h}$ does not need to be closed for $B_{h}$ to be closed in the limit that $h$ goes to zero. In general this condition is met and it suffices to require that the solution spaces for $\mathbf{v}_{h}$ and $p_{h}$ of the FE method satisfy the inf-sup condition for the Stokes problem. Furthermore, if $\omega$ is zero or relatively small, $B_{T h}=0$ or its operator norm will be small compared to that of $B_{v h}$. In that case, the potentially stabilizing effects of $B_{T h}$ on any unstable part in $B_{h}$ due to $B_{v h}$ will vanish. This means that for small values of $\omega$ satisfaction of the inf-sup condition for $B_{v h}$ is not only sufficient but also necessary to ensure $B_{h}$ satisfies the inf-sup condition and a stable FE method is obtained.

It must thus be concluded that in order to obtain an FE method for the LNSF equations that is robust for $\omega \in[0, \infty)$, the solutions spaces for $\mathbf{v}_{h}$ and $p_{h}$ must be chosen so that the inf-sup condition for $B_{v h}$ is satisfied. Note that these are precisely the combinations of solution spaces for velocity and pressure that satisfy the inf-sup condition for the Stokes equation. The results presented in the next section support the predictions given above.

As mentioned above, in order to obtain an efficient FE method, the trial functions for $T_{h}$ must be of equal order to those for $v_{h}$. Such a combination of trial functions will yield an operator $B_{T h}$ that is not closed. Nevertheless, it must be stressed that the growth in the FE error of the temperature and pressure solution for vanishing $\omega$, which is observed for all conformal FE methods that are thus obtained, is not caused by the fact the $B_{T h}$ is not closed. It is the result of the fact that all singular values of $B_{T h}$ vanish as $\omega$ goes to zero. This causes the smallest singular value of $B_{h}$ to vanish, leading to ill-conditioning of the system of FE equations (which is responsible for the growth of the FE error for the temperature and pressure solution).

\subsection{Stability and convergence results}

\subsubsection{Test setup}

The numerical inf-sup test and convergence test are performed here for a simple 2D rectangular geometry being twice as long as high. The family of mesh patterns used in the tests are created by doubling the number of elements used in both directions by a factor of two for each mesh refinement (see figure 5.2).

\section{Boundary conditions}

In order to perform convergence studies, it is advantageous to have an exact solution available for the boundary conditions that are applied to the rectangle. To this end, the rectangular geometry is assumed to represent a small section of a $1 \mathrm{D}$ 
waveguide for which an exact solution was presented in chapter 3. The appropriate boundary conditions are thus as follows: No-slip isothermal boundary conditions are applied to two opposing sides of the square. At the other boundaries, velocity and temperature profiles corresponding to the first acoustic mode in a layer geometry as calculated with the techniques described in chapter 3 are prescribed. Using this setup, the velocity, temperature and pressure fields obtained by solving the FE system can be used to verify the convergence rate of different FE methods by a direct comparison with the exact solution.

\section{mesh patterns}

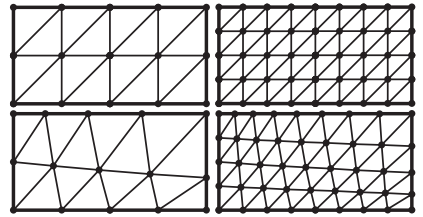

(a)

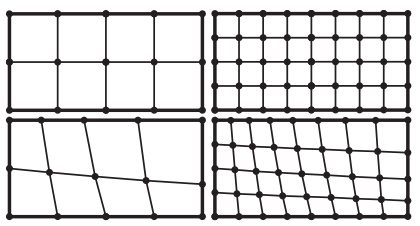

(d)

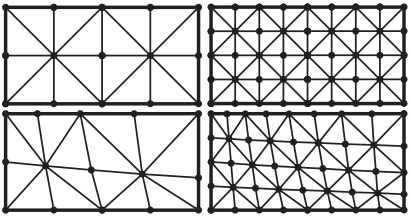

(b)

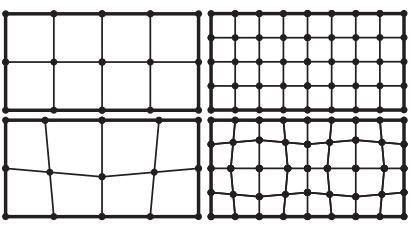

(e)

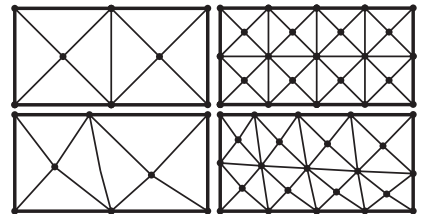

(c)

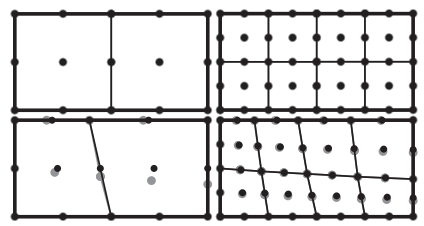

(f)

Figure 5.2: Different mesh patterns used in the inf-sup test and convergence studies. Besides a regular mesh (top), a distorted mesh is also shown (bottom). The distorted mesh of (a) (b)(c)(d) and (f) is obtained using a global mapping, while the distorted mesh in (e) is obtained by using a local mapping (and its mirror images) confined to repetitive groups of elements. The gray nodes in the bottom mesh of (f) are obtained after distorting the biquadratic elements of a quadrilateral mesh in the same way as done in (d). The black nodes are obtained after repositioning the midside and internal nodes to correspond with a bilinear mapping.

In order to detect possible adverse or beneficial effects due to structured ordering of elements, all mesh patterns depicted in figure 5.2 are considered on regular meshes and distorted meshes ${ }^{16}$. Note that two types of distortions are considered. The first type of distortion is achieved by using a mapping that globally distorts the

\footnotetext{
${ }^{16}$ In the presented results, the MINI and Crouzeix Raviart elements are only used in combination with the so-called Union Jack.
} 
meshes of different coarseness. The lengths of edge elements on the right and bottom are taken equal over the edges, while the element lengths for the top and right edges vary over the edges. The element lengths differ by a factor of two between the extremities. Examples of such a mesh distortion are given in the different subfigures in 5.2 except for figure 5.2(e). Note that even though the mappings that are used do not correspond to an affine transformation (some of the elements are warped) the resulting mesh family is still asymptotically affine. This means that the mapping between the shape of the reference elements and each element in the mesh ultimately corresponds to an affine transformation as the mesh is increasingly refined ${ }^{17}$.

New insights show that mesh families that are not asymptotically affine can lead to a lower order of convergence if quadrilaterals are used [112]. If bilinear mappings are used to transform (bi)linear shape functions on the reference element to their global shape, the accuracy of the best approximation of an arbitrary solution based on the resulting FE spaces might not converge with the expected order when the mesh is refined. In that case, an element that has optimal convergence might be falsely classified as not having optimal convergence. On the other hand, an element that is predicted to have optimal convergence, might show an order of convergence of a lower order if this phenomenon occurs. To investigate if the FE methods developed here for the LNSF equations also suffer from this phenomenon, the different quadrilateral elements are also tested using a second type of mesh distortion. In contrast to the first type, the mapping distorts the mesh locally. The mapping is not applied to the full meshes but is instead applied to the repetitive groups of elements that appear due to mesh refinement. An example of such a mesh distortion is illustrated by figure 5.2(e). The mesh family that is thus obtained is not asymptotically affine and can be used to asses the true rate of convergence of quadrilateral elements on arbitrary mesh patterns.

Note that for mixed elements involving (bi-)quadratic trial functions for the approximation of one of the variables, the midside nodes and internal nodes are repositioned after distorting the mesh to obtain elements that can be mapped to the corresponding reference elements by a (bi-)linear mapping (see figure 5.2(f). Using mixed elements that use (bi)-linear trial functions to approximate one or more of its variables in combination with a (bi-)quadratic geometrical mapping can lead to additional inaccuracies (resembling the case of superparametric elements for non-mixed methods). Note that in the cases presented in chapter 6 and 7, a (bi-)linear mapping is also used for all the elements in the interior of the domain. Nevertheless, if higher order trial functions are used for approximation of one or more of the variables, the midside nodes on the boundary of the domain

${ }^{17}$ For the meshes distortion considered, the elements will ultimately be shaped like rectangles. 
will not be centered between the element edges when the geometry of the domain involves curved boundaries.

\section{Stretched meshes}

The different combinations of element and mesh patterns (both regular and distorted) are also considered for stretched meshes. In general, the gradients of velocity and temperature of solutions to the LNSF equations will be much greater in directions normal to the fluid boundary than in directions tangential to it. If only the approximability of the mesh is taken into consideration, such solutions are most efficiently approximated if more elements are used in the direction normal to fluid boundaries than in direction tangential to it. In other words, an efficient FE method for the LNSF equations should allow the use of meshes that include heavily stretched elements. In the stretched meshes that are investigated, the length of the longer side of the rectangle is increased by a factor of ten.

\section{FE error and order of convergence}

Since the exact solution for the considered problem is known, the order of convergence of the different fields can be directly inferred by comparing the relative FE error on two consecutive mesh refinements. To improve legibility, the following error operator is defined for an exact solution $X$ and a corresponding FE approximation $X_{F E}$ :

$$
E(X)=\frac{\int_{\Omega}\left|X_{F E}-X\right| d \Omega}{\int_{\Omega}|X| d \Omega}
$$

The expression $E(X)$ thus represents the relative FE error, being defined as the quotient of the absolute difference between fields $X$ and $X_{F E}$ integrated over the domain, and the absolute value of the field $X$ itself integrated over the domain. For the proposed families of meshes, the order of convergence $r$ for the FE approximation of a field $X$ based on two consecutive meshes in a family of mesh refinements is now given by

$$
r=\log _{2}\left(\left.E(X)\right|_{i}-\left.E(X)\right|_{i+1}\right)
$$

where $\left.E(X)\right|_{i}$ and $\left.E(X)\right|_{i+1}$ indicate the error in the approximation for two consecutive mesh refinements.

\subsubsection{Element types}

The trial functions spanning the solutions space for $\mathbf{v}, T$ and $p$ can be chosen independently. In combination with variations in element shape, this offers a seemingly unlimited number of FE methods that can be used to discretize the LNSF 
equations. In the following, a combination of a certain element shape and solution spaces for the different variables is referred to as an element type or element for short. Below a number of element types are introduced for which the results of the inf-sup test and convergence studies are presented in this section.

\section{Element types for the Stokes equations}

- value of velocity $\quad \bigcirc$ value of pressure $\quad$ - - value of pressure derivatives

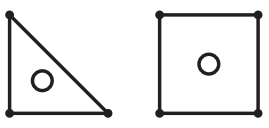

(a) $\mathrm{P} 1-\mathrm{P} 0 / \mathrm{Q} 1-\mathrm{Q} 0$
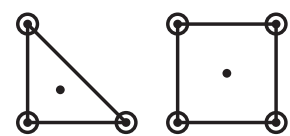

(d) MINI

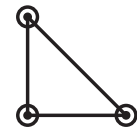

(b) $\mathrm{P} 1-\mathrm{P} 1 / \mathrm{Q} 1-\mathrm{Q} 1$

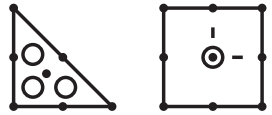

(e) Crouzeix Raviart
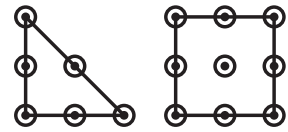

(c) $\mathrm{P} 2-\mathrm{P} 2 / \mathrm{Q} 2-\mathrm{Q} 2$

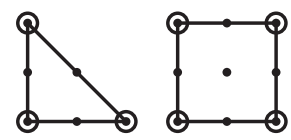

(e) Taylor Hood

Figure 5.3: Velocity and pressure degrees of freedom for six types of 2D element families for the Stokes equations. Elements from family (a), (b) and (c) do not satisfy the inf-sup criterium and suffer from locking and/or spurious modes. Elements from the families (d), (e) and (f) do satisfy the inf-sup conditions and lead to stable combinations of solution spaces for the Stokes equations.

In section 5.3.5 it is explained that the solution spaces for $\mathbf{v}_{h}$ and $p_{h}$ of elements for the LNSF equations which are robust in the frequency range $[0, \infty\rangle$ must satisfy the inf-sup condition for the Stokes equation. Robust elements for the LNSF equations can thus be obtained in a straightforward manner by extending an element that is stable for the Stokes equations to the LNSF equations by adding a solution space for temperature. FE methods for the Stokes equations have been in existence for some decades, and there is a vast amount of literature on stable (and unstable) conformal FE discretizations for the Stokes equations. Many 'simple' elements, such as those depicted in figure 5.3(a) and 5.3(b) which are obtained by taking equal velocity and pressure solution spaces do not satisfy the inf-sup criteria and suffer from locking and/or spurious modes. Other seemingly logical choices, for instance the element depicted in 5.3(c), which is obtained by taking the linear element in 5.3(b) and lowering the order of the pressure solution space (thus mimicking the order of the derivatives of velocity and pressure in the weak formulation) are also known to be unstable. 
Three types of element families that do satisfy the inf-sup condition for the Stokes equations are depicted in figure 5.3(d), 5.3(e) and 5.3(f). The elements in these families are thus promising candidates for obtaining stable elements for the LNSF equations by adding an appropriate solution space for temperature. The merits and disadvantages of each family is discussed briefly below. Possible choices for the temperature solution space that extend the applicability of these FE methods to the LNSF equations are also indicated per element family. Note that the elements for the LNSF equations that are thus obtained are given the same names as the element for the Stokes equation from which they were derived.

\section{Taylor Hood elements}

The first element family considered is the Taylor Hood family (see figure 5.3(f)). If the solution space for temperature is taken equal to that for the individual velocity components, the approximability of all fields is expected to be equal (in the $H^{1}$ norm) based on results from interpolation theory ${ }^{18}$. This implies that adding more degrees of freedom to the velocity or temperature solution space of each element would not increase convergence speed, since it is limited by the approximability that is lowest ${ }^{19}$. On the other hand, lowering the order of the functions spanning the pressure or temperature solution spaces still yields elements that pass the infsup test, but approximability of a variable for which the order was lowered will cause a reduction in convergence speed. In that sense the Taylor Hood elements with quadratic temperature fields are efficient elements. The family of Taylor Hood elements can easily be extended to 3D. As an example, the velocity and pressure nodes for three standard 3D elements are given in figure 5.4.

- value of velocity

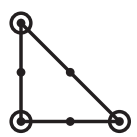

(b) $2 \mathrm{D}$ elements
○ value of pressure
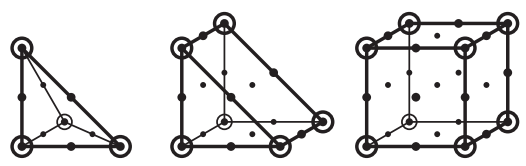

(b) $3 \mathrm{D}$ elements

Figure 5.4: Velocity and pressure degrees of freedom for 1D, 2D and 3D elements of the Taylor Hood family.

The large variety of 1D, 2D and 3D shapes that are available (lines, triangle,

\footnotetext{
${ }^{18}$ As already mentioned, according to new insights, this does not always hold true for mesh families with quadrilateral elements that are not asymptotically affine [112].

${ }^{19}$ Increasing the amount of pressure degrees of freedom, for instance by adding a bubble degree of freedom, leads to an unstable element [110].
} 
quadrilateral, brick, tetrahedron, prism) can be easily coupled. This is especially advantageous if a mesh of 2D or 3D LNSF elements are to be coupled to structural or thermal FE elements at a boundary of the fluid domain. In those cases, the different models can be coupled directly without the need for a coupling element.

\section{Crouzeix Raviart elements}

An alternative family of elements satisfying the inf-sup condition are the Crouzeix Raviart elements (see figure 5.3(e)). If the trial functions for temperature are taken to be equal to those for the velocity components (or if quadratic trial functions are used for temperature), the approximability of all variables is equal making it an efficient element in terms of degrees of freedom vs. convergence speed. Characteristic to Crouzeix Raviart elements is that the functions in the solution space for pressure are discontinuous over the element boundaries. Moreover, the pressure fields on different element do not share degrees of freedom. This makes it possible to eliminate the pressure degrees of freedom on element level, which increases its performance in terms of efficiency even more. To the author's knowledge, available extensions to 3D elements are currently limited to tetrahedra [61]. The Crouzeix Raviart elements are somewhat more cumbersome to implement than the elements of the Taylor Hood family. In addition, the discontinuous pressure makes a coupling between LNSF elements and structural elements less straightforward.

\section{MINI elements}

As a complement to these FE formulations for which the functions spanning the different solution spaces are not equal for all variables, the so-called MINI element is considered here as well (see figure 5.3(d)). The element is obtained by adding socalled bubble functions to the elements in figure 5.3(b). Bubble functions are socalled nodeless degrees of freedom with smooth trial/test functions that are zero on the element boundaries. For triangular elements (and tetrahedral elements), a 'standard' bubble function (see figure 5.5(a)) is added separately to the solution space of both velocity components. For quadrilateral elements, a 'standard' bubble function (see figure 5.5(a)) is added separately to the solution space of both velocity components, and one of two bubble functions of second $\operatorname{order}^{20}$ (see figure 5.5(c) and 5.5(d)), which share the same degree of freedom, is added to the

\footnotetext{
${ }^{20}$ Note that the second order bubble functions may be defined in different ways. The second order bubble functions depicted in figure 5.5(c) and 5.5(d) are obtained by taking the bubble functions as defined in [113] and subtracting the 'simple' bubble function 5.5(b). The solution space spanned by the different sets of bubble functions is identical.
} 
solution spaces of each velocity component.

The MINI element for triangular and tetrahedral elements was first proposed for the Stokes equation by Arnold et al. [113]. The MINI element for quadrilateral elements was proposed by Bai [114]. An enhanced version of these MINI elements that can be used in the context of the FLNS equations was proposed by Malinen et al. [24]. The enhancement consists of adding a solution space for temperature equal to that of the solution space for pressure. Malinen et al. suggest that an extension of the quadrilateral MINI element to the $3 \mathrm{D}$ case is possible. The resulting brick element has trilinear shape functions for velocity, temperature and pressure, and four additional bubble degrees of freedom for velocity; A 'standard' tetrahedral bubble is added separately to the solution space of each velocity component, and one of three bubble functions of second order, which share the same degree of freedom, is added to the solution spaces of each velocity component. To the author's knowledge, the stability of this brick MINI element for the Stokes equation (and its counterpart for the LNSF equation) has not yet been proven. Nevertheless, preliminary results show that the numerical inf-sup test for regular meshes is passed and the expected order of convergence is obtained. Note that the degrees of freedom associated with the bubble functions can be condensed out of the system prior to solving it. In practice, this means that MINI elements provide an equal order approximation for mixed methods ${ }^{21}$.

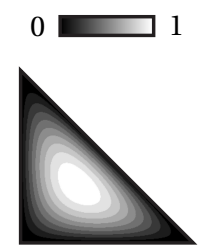

(a)

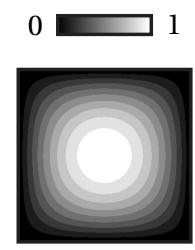

(b)

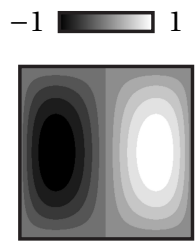

(c)

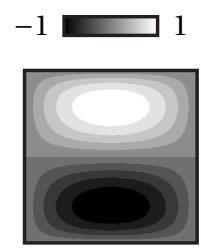

(d)

Figure 5.5: Bubble functions for triangle and quadrilateral elements; standard bubble functions (a)(b), and second order bubble functions (c)(d)

\subsubsection{Example: stable vs. unstable LNSF element}

The results of the numerical inf-sup test are given below for a stable and an unstable LNSF element. The presented case is an example of the numerous results for different element types, mesh patterns and values for $k / s$ and $s$ that were considered. In the next sections, the different trends are indicated that can be observed

\footnotetext{
${ }^{21}$ Extensions of the MINI family to quadratic or higher order shape functions are also possible [112].
} 
in the results of various other cases that were considered.

In figure 5.6, the ratio $\psi$, defined in equation (5.72) as the ratio between the inf-sup and the sup-sup value is plotted as a function of the number of elements $n$ used in the thickness direction of the waveguide section (see figure 5.2)

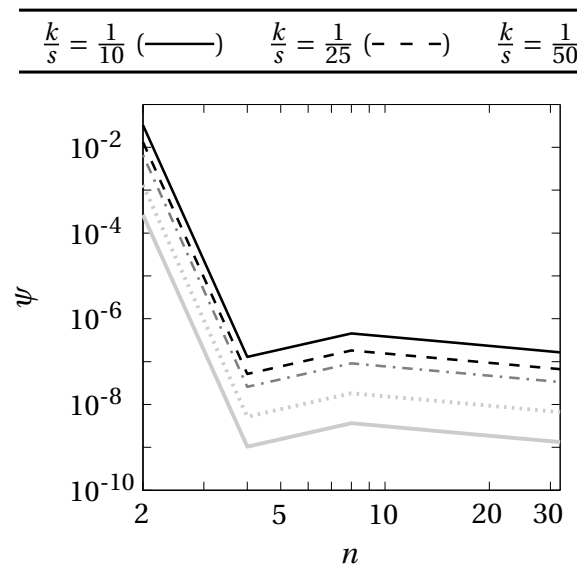

(a) Enhanced P1-P0 element

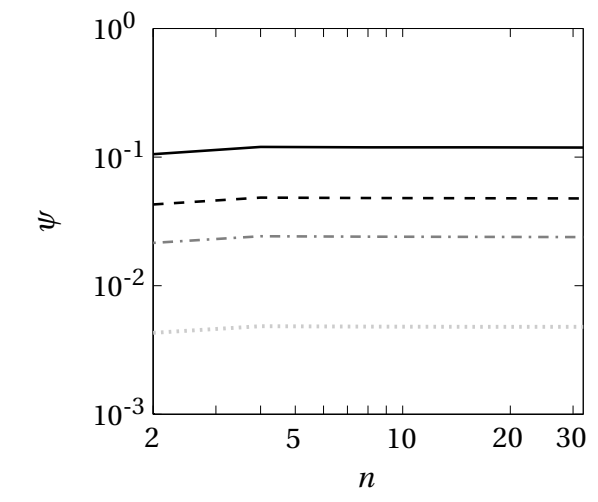

(b) Enhanced MINI element

Figure 5.6: The ratio $\psi$ between the inf-sup and sup-sup value as a function of the number of elements in thickness direction of the waveguide section, for $\tilde{s}=5$ and different values of $k / s$.

In figure 5.6(a), results are shown for the P1-P0 element (see figure 5.3), which have linear trial functions for velocity and temperature, and a constant value for pressure. In figure 5.6(b), results are shown for the MINI element (see figure 5.3), which have linear trial functions for velocity, temperature and pressure, and an enrichment of the velocity solution space with bubble functions. In both cases, triangular elements are used on a stripped distorted mesh (see figure 5.2). Note that the P1-P0 element suffers from spurious modes, and $\psi$ corresponds to the lowest non-zero singular value of the matrix $\hat{B}$ that is calculated in the inf-sup test.

The values for $\psi$ for the P1-P0 element do not seem to converge to a constant value as the mesh is refined, while the value for the MINI element remains virtually unchanged upon mesh refinement. Note that the P1-P0 element suffers from two global spurious modes. The plotted results for $\psi$ correspond to the first non-zero singular value. Based on these results, it can be concluded that the P1-P0 element does not pass the (numerical) inf-sup test and is expected to exhibit unstable convergence behavior. This is supported by the convergence results in figure 5.7(a), which correspond to the same family of meshes used in the numerical inf-sup test. 


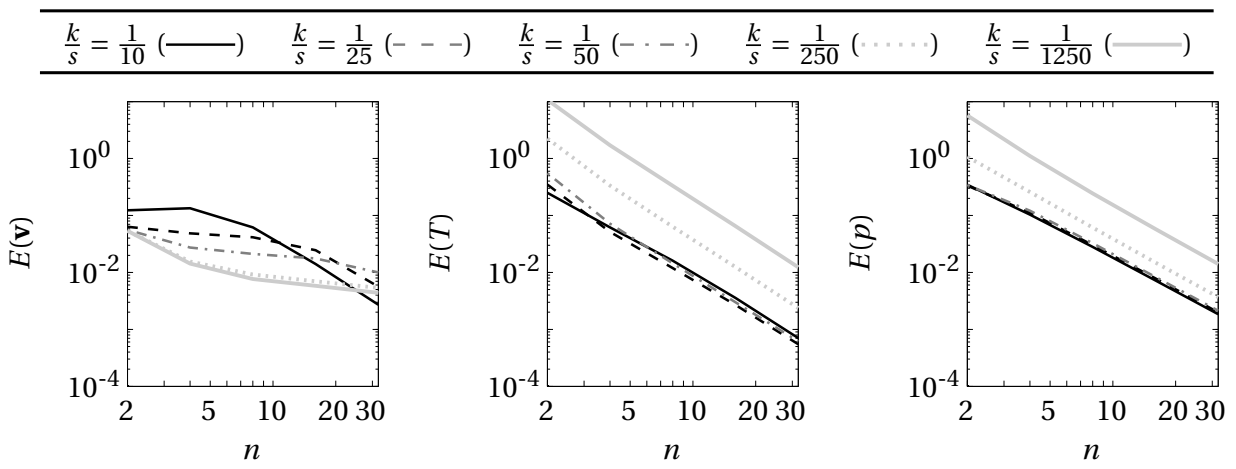

(a) Enhanced P1-P0 element
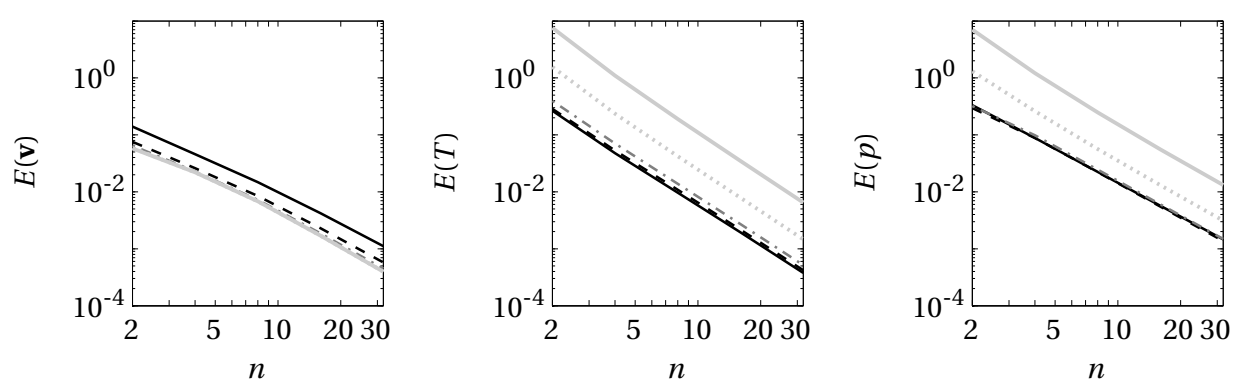

(b) Enhanced MINI element

Figure 5.7: Convergence behavior of relative error in the FE approximation of velocity, temperature and pressure for $\tilde{s}=5$ and different values of $\mathrm{k} / \mathrm{s}$.

First the convergence results for velocity are considered for the P1-P0 element. For small values of $k / s$ (implying small values of $\omega$ ) the convergence rate is not constant for subsequent mesh refinements. The element does converge within the considered range of $h$, but the convergence rate decreases and is not optimal as $h$ goes to zero. For bigger values of $k / s$ (implying bigger values of $\omega$ ) the rate of convergence is initially lower, however, as the number of degrees of freedom increases and $h$ goes to zero, eventually optimal convergence is observed. The behavior corresponds with a locking phenomenon; the range of $h$ where optimal convergence is observed is postponed, and the extent of the postponement depends on $k / s$ (or $\omega$ ). In section 2.5.2 it is shown that the FE approximations for the LNSF equations are expected to behave similarly to FE approximation for linear elasticity involving nearly incompressible materials. From the literature it is known that the P1-P0 element fails the inf-sup test in the case of linear elasticity involving (nearly) incompressible materials (see for instance [111]). Furthermore, the extent to which 
the region where convergence is optimal is postponed depends on the ratio between bulk and shear modulus of the elastic material. In the analogy, between the LNSF equations and the equations for linear elasticity, $k / s$ takes a similar role as the ratio between the bulk and shear modulus. This is easily observed by rewriting (2.28) in terms of the wave numbers $k$ and $s$. The condition for near incompressible behavior derived in section 2.5.2 becomes

$$
\frac{k^{2}}{s^{2}} \ll 1
$$

This condition for nearly incompressible behavior corresponds well with the dependency of the convergence behavior on $k / s$ as described above. Note that the condition implies that for most practical cases, the LNSF equations describe nearly incompressible material behavior. This is of great significance for the selection of suitable element types for the LNSF equations; it underlines the earlier claim that useful element types for the LNSF equations should have a combination of velocity and pressure solution spaces that satisfy the inf-sup test for the Stokes equations (since the Stokes equations are obtained if the equations for linear elasticity are considered for the limit case of full incompressibility). Note that despite the suboptimal convergence of $\mathbf{v}$, the convergence of $T$ and $p$ are optimal for the entire range of $h$ that is considered. Nevertheless, the errors in the approximation of different fields are coupled for mixed methods ${ }^{22}$, and the convergence rates for $T$ and $p$ will ultimately be affected if the convergence rate for $\mathbf{v}$ does not recover quick enough.

The MINI element is known to be stable for the case of linear elasticity and the Stokes equation, and is therefore also expected to be stable for the LNSF equations. The results presented in 5.6(b) suggest that the element satisfies the inf-sup condition for the LNSF equations, which implies stable convergence behavior. The convergence results in figure 5.7(b) which are based on the same set of meshes confirm that the MINI element is indeed stable for the considered case. The solutions of $\mathbf{v}, T$ and $p$ exhibit convergence rates close to optimal convergence over the entire range of $h$.

Another interesting feature of the convergence plots in both 5.7(a) and 5.7(b) is that below $k / s \approx 1 / 50$ the relative FE error or $T$ and $p$ rises with a decrease in $k / s$. This behavior which is related to the condition of the matrix $\hat{B}$ is further elaborated on below.

\footnotetext{
${ }^{22}$ This follows from the error bounds given in section 5.2.6. See also [61, 112].
} 


\subsubsection{Numerical inf-sup test}

The form in which the results of the numerical inf-sup test in figure 5.6 are presented is used in most articles on the numerical inf-sup test. Nevertheless, if results are to be presented for large numbers of cases (for instance for several values of $k / s, \tilde{s})$ the form is not efficient. As an alternative, the values of $\psi$ in figure 5.8 are plotted as a function $k / s$ for different values of $n$.

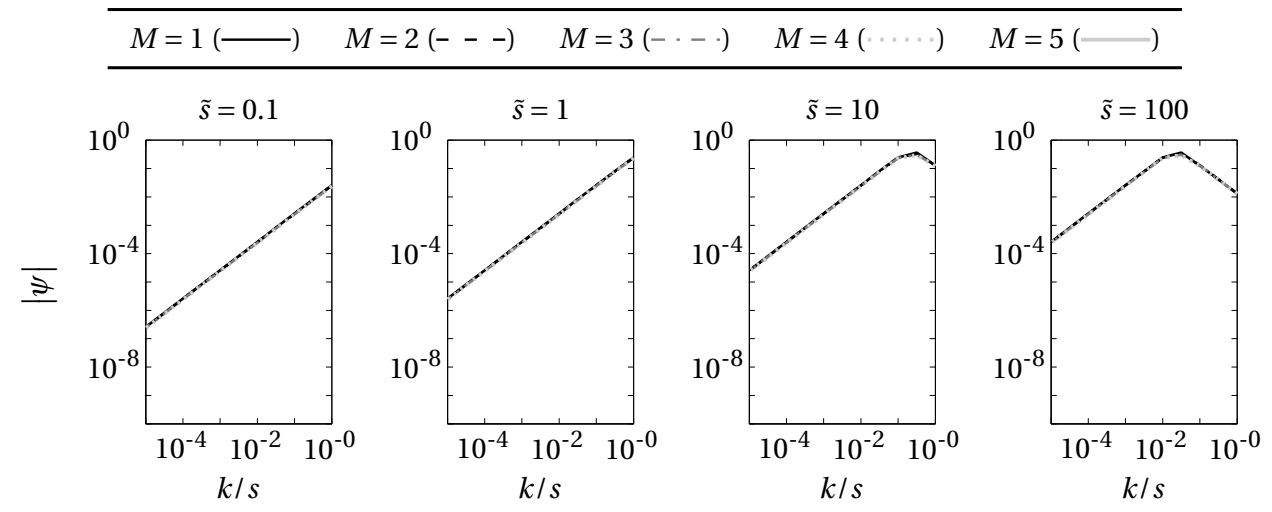

(a) Triangular MINI elements, distorted mesh
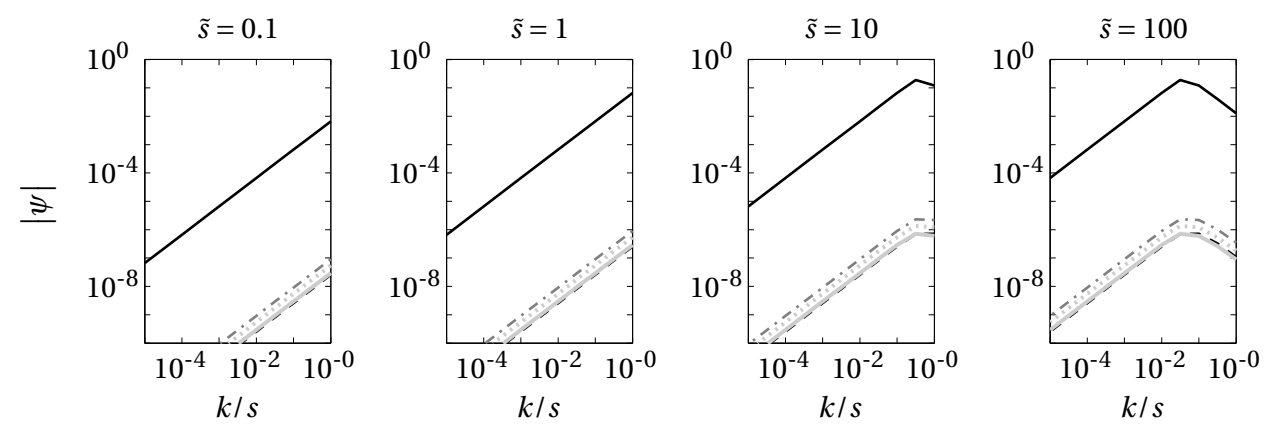

(b) Triangular P1-P0 elements, distorted mesh

Figure 5.8: Ratio between the inf-sup and sup-sup as a function of $k / s$ for different subsequent mesh refinements ( $M$ indicates the mesh number, with $M=1$ being the coarsest mesh) and different values of $\tilde{s}$.

Using this presentation, an element passes the numerical inf-sup test for a certain value of $k / s$, if the lines for different values of $n$ coincide at that location. This behavior corresponds with the results for the MINI element in figure 5.8(a) for the entire range of $k / s$ and $\tilde{s}$ that is considered. If the distance between lines on a logarithmic scale are equally spaced for increasing $n$, the value of $\psi$ is not bounded 
from below, and the element does not pass the inf-sup test. This behavior corresponds with the results for the P1-P0 element in figure 5.8(b) for the entire range of $k / s$ and $\tilde{s}$ that is considered (based on the last two mesh refinements).

All results of the inf-sup test for the MINI and Crouzeix Raviart elements are practically identical to those in figure 5.8. The curves for $\psi$ seem unaffected if the mesh is distorted or if the mesh pattern is changed. Stretching the elements by a factor of 10 in the direction of the waveguide also leaves the curves virtually unchanged.

The biggest differences are found for the Taylor Hood elements. For elements in this family the behavior for high $k / s$ is somewhat different. This is illustrated by figure 5.9 in which the results of the inf-sup test are plotted as a function of $k / s$ for $\tilde{s}=100$ for the triangular MINI element and the triangular Taylor Hood element.

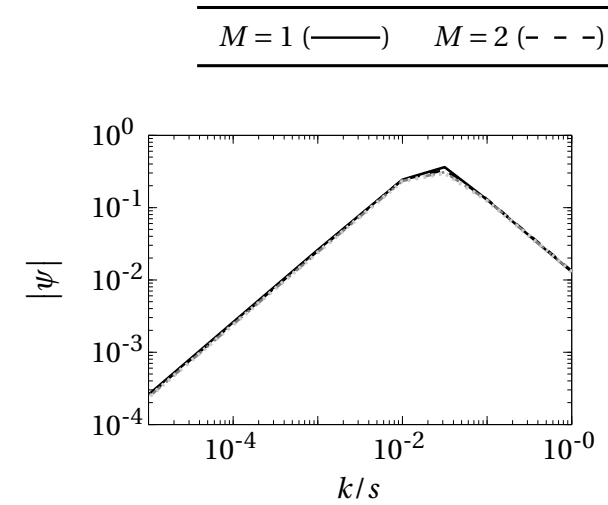

(a) Triangular MINI elements, distorted mesh

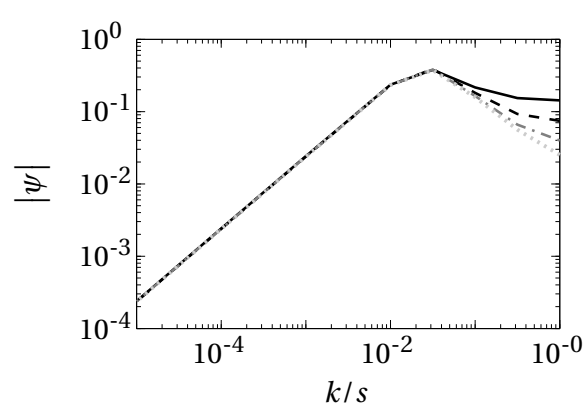

(b) Triangular Taylor Hood elements, distorted mesh

Figure 5.9: Ratio between the inf-sup and sup-sup as a function of $k / s$ for different subsequent mesh refinements ( $M$ indicates the mesh number, with 1 being the coarsest mesh) and $\tilde{s}=100$.

\section{Influence of $\mathrm{B}_{v}$ and $\mathrm{B}_{T}$}

The shape of the curves in 5.9(a) and 5.9(b) and the differences between them can be explained by looking at the influence of the corresponding singular values of $\hat{\mathrm{B}_{v}}$ and $\hat{\mathrm{B}_{T}}$ on the singular values of $\hat{\mathrm{B}}$. The sup-sup and inf-sup value corresponding with the biggest and smallest singular values of these matrices are plotted as a function of $n$, being the number of elements in thickness direction of the waveguide in figure 5.10 for a number of values of $k / s$ and $\tilde{s}=100$. 


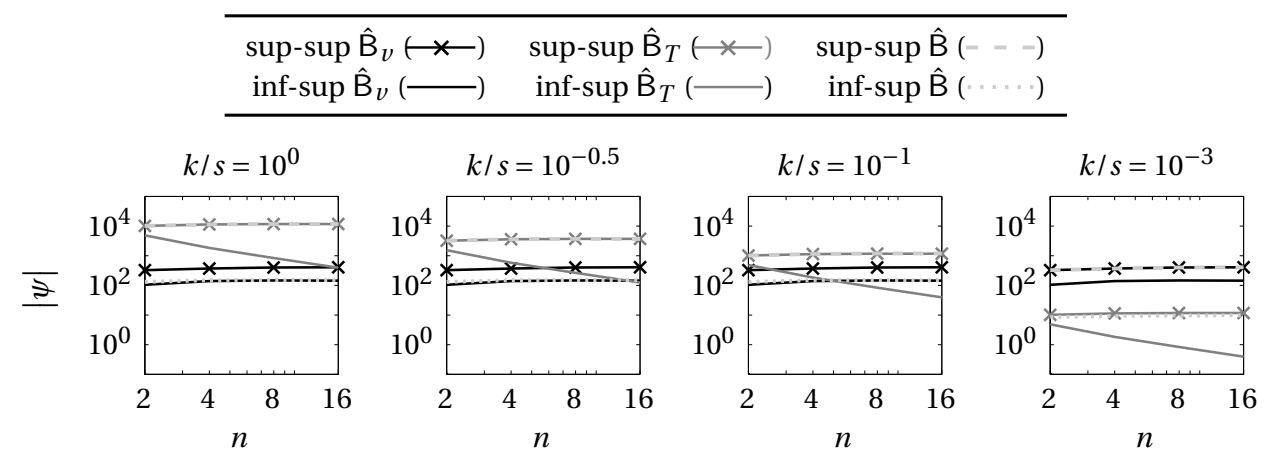

(a) Triangular MINI elements, distorted mesh
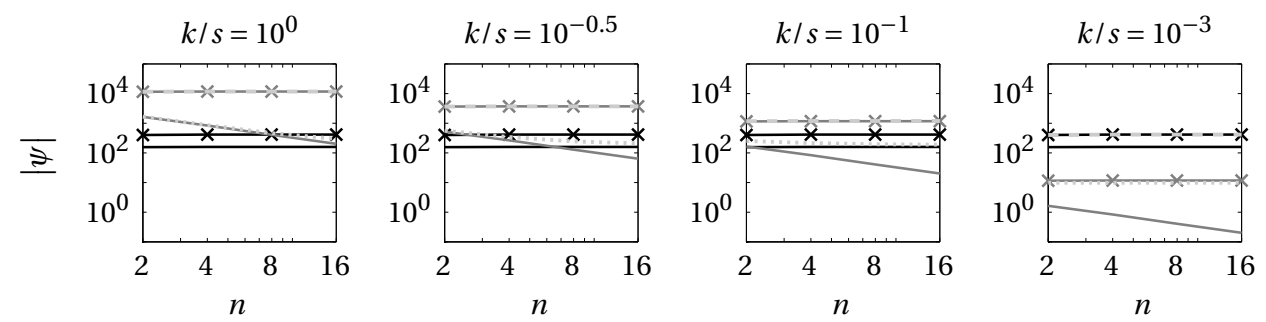

(b) Triangular Taylor Hood elements, distorted mesh

Figure 5.10: Biggest singular value (sup-sup) and smallest singular value (inf-sup) of $\hat{\mathrm{B}}_{v}, \hat{\mathrm{B}}_{T}$ and $\hat{\mathrm{B}}$, as a function of the number of elements $n$ in thickness direction of the waveguide for various values of $k / s$ and $\tilde{s}=100$.

For $k / s<10^{-2}$ the value of $\psi$ in both 5.9(a) and 5.9(b) decreases as $k / s$ goes to zero. For this range of $k / s$, the highest singular value of $\mathrm{B}$ appears to be dominated by the highest singular value of $B_{v}$, while the lowest singular value of $B$ appears to be dominated by the lowest singular value of $B_{T}$. This is illustrated by the plotted results in 5.9 for $k / s=10^{-3}$. Since all singular values of $B_{T}$ decrease with $\omega$ and thus with $k / s$, and all singular values of $\mathrm{B}_{v}$ are independent of $\omega$ and thus independent of $k / s$, the ratio between the smallest and biggest singular value of $\mathrm{B}$ vanishes as $k / s$ goes to zero.

For $k / s>10^{-1}$ the reverse occurs; the value of $\psi$ in 5.9(a) decreases at a constant rate as $k / s$ increases. For the MINI element, the highest singular value of $\mathrm{B}$ appears to be dominated by the highest singular value of $B_{T}$, while the lowest singular value of $\mathrm{B}$ appears to be dominated by the lowest singular value of $\mathrm{B}_{v}$. This is illustrated by the plotted results in 5.9(a) for $k / s=10^{0}, k / s=10^{-0.5}$ and $k / s=10^{-1}$. Since all singular values of $\mathrm{B}_{T}$ increase with $\omega$ and thus with $k / s$, and all singular values of $\mathrm{B}_{v}$ are independent of $\omega$ and thus independent of $k / s$, the ratio between 
the smallest and biggest singular value of $\mathrm{B}$ grows as $k / s$ increases. Note that results in 5.8 show that the transition in the dependency of $\psi$ on $k / s$ is dependent on $\tilde{s}$.

\section{Taylor Hood Elements}

For the Taylor Hood element, it appears that the smallest singular value of $B$ is still dominated by the smallest singular value of $\mathrm{B}_{T}$ if $n$ is small. However, as $n$ increases and the value of the smallest singular value of $B_{T}$ drops below that of the smallest singular value of $B_{v}$, the smallest singular value of $B$ is now determined by that of $\mathrm{B}_{v}$ due to relation (5.77). This is illustrated by the plotted results in 5.9(b) for $k / s=10^{0}$. The described behavior implies that initially the ratio between smallest and biggest singular value of $\mathrm{B}$ will decrease with $n$, but will eventually converge to a constant value. It can be seen from the plots in 5.9(b) that the number of mesh refinements needed before $\psi$ reaches a stable value depends on the values of $k / s$ (and thus $\omega$ ).

Note that this behavior should not be confused with the phenomenon of locking, in which case the value of $\psi$ would not stabilize and vanish as $n$ increases. In fact, the net effect on the rate of convergence is not expected to be negative. In the region where $\psi$ is decreasing, the value of $\psi$ is higher for Taylor Hood elements than the value of $\psi$ for other elements. Since $\psi$ is a measure for the condition of the system, the height of the relative FE error for Taylor Hood elements is expected to be smaller for high values of $k / s$. In practice the rate of convergence does not seem to be affected (see the results in appendix I).

Just as for the elements in the MINI and Crouzeix Raviart family, the $\psi$-curves for elements in the Taylor Hood family seem unaffected by changes in mesh pattern, mesh distortion and stretching of the mesh. The difference between elements in the Taylor Hood family and the MINI and Crouzeix Raviart family are observed for both triangles and quadrilaterals, and all considered mesh patterns.

Note that if quadrilateral Taylor Hood elements are considered with linear trial functions for temperature on a regular, distorted and/or stretched mesh, the resulting $\psi$-curves are virtually identical to the cases where quadratic trial functions are used.

Summarizing, the results for the considered cases suggest that the $\psi$-curves depicted in figure 5.9(a) are representative for all elements having a solution space for $\mathbf{v}_{h}$ and $p_{h}$ that satisfies the inf-sup condition for the Stokes equations. Changing the element type, mesh pattern, order of the trial functions for temperature or stretching the elements by a factor of 10 only results in minute changes. 


\subsubsection{Convergence results}

The convergence data corresponding to the cases for which the inf-sup test was performed is presented in appendix I in condensed form. Instead of plotting the relative error for different meshes as a function of $k / s$, the rate of convergence is given for the beginning, middle, and end of the range considered for $k / s$. Results are presented for different elements, mesh patters, distorted and undistorted meshes and stretched meshes. The following trends are observed from the results ${ }^{23}$ :

- For values of $k / s$ and $\tilde{s}$ that yield $\tilde{k}<1$, a stable convergence rate is observed after 2 mesh refinements for all element types and mesh patterns both on distorted/non-distorted meshes that are not stretched. For stretched meshes the same is true for $\tilde{k}<1 / 10$. In all other cases, convergence will not set in until four or more elements per wavelength are available to describe the oscillations in the direction of the waveguide.

- The relative error in the FE solution for $T$ and $p$ is higher on distorted meshes than on non-distorted meshes for smaller values of $k / s$.

- The order of convergence for $p$ is one or two orders higher than expected based on results from interpolation theory for smaller values of $k / s$. This is true for all considered cases except triangular and quadrilateral MINI elements and quadrilateral elements with the order of the trial functions for temperature lower than those for the velocity.

- For $T$ similar unexpected high values of the order of convergence are observed, but only on distorted meshes.

- For higher values of $k / s$ and lower values of $\tilde{s}$, the order of convergence is as expected for $\mathbf{v}, T$ and $p$, except for quadrilateral Crouzeix Raviart and Taylor Hood elements and triangular Taylor Hood elements on a striped mesh, which all have a convergence rate for $\mathbf{v}$ and $T$ that is one order higher than expected. In these cases, the order of convergence in the $L^{2}$ norm is the same as the order of convergence in the $H^{1}$ norm (while for other cases, the order of convergence in the $L^{2}$ norm is one order higher as predicted by interpolation theory).

- For asymptotic non-affine mesh families with quadrilateral elements the order of convergence drops one order for $\mathbf{v}$ and $T$ for the MINI element and

\footnotetext{
${ }^{23}$ The effects of the first item that is described overshadows all other listed trends when $\tilde{k}>1$ or $\tilde{k}>1 / 10$ for non-stretched and stretched elements, respectively.
} 
one or two orders for $\mathbf{v}$ and $T$ for the Taylor Hood and Crouzeix Raviart element. The order of convergence for $p$ is not affected ${ }^{24}$.

Note that the MINI and Taylor Hood element also have optimal convergence on quadrilateral mesh families that are asymptotically non-affine (the occurrences of super-convergence described above are no longer observed). On such mesh families, the Crouzeix Raviart element no longer has optimal convergence for $\mathbf{v}$. The conclusions based on the convergence results can thus be summarized as: all considered elements that are robust according to the numerical inf-sup test have optimal convergence or convergence of a higher order, except for Crouzeix Raviart elements on asymptotic non-affine mesh families. In this case, the lack of optimal convergence can most likely be attributed to the limited approximability offered by a bilinear mapping of (bi)linear trial functions for pressure on distorted elements (see [112]).

\subsubsection{Example of convergence results}

A number of the phenomena that are described above are illustrated by the results in figure 5.11. Here, the relative error of $\mathbf{v}, T$ and $p$ are plotted as a function of $k / s$ for subsequent mesh refinements and $\tilde{s}=1$.

On the non-distorted mesh (figure 5.11(a)), the errors do not increase when $k / s$ becomes small, but remain constant (the non-constant error in the pressure for $M=4$ is due to the large condition of the numerical system and limited machine precision). For the distorted mesh (figure 5.11(b)), the relative FE error of $T$ and $p$ are virtually the same as the non-distorted mesh for $k / s>10^{-2}$, but rise as $k / s$ decreases for $k / s<10^{-2}$. Note that this dependency on the mesh is not observed for the velocity. For both $T$ and $p$ the convergence rate is of higher order in the region where the FE error is dependent on the mesh. Note, however, that for $T$ the increase in convergence order is not observed for the non-distorted mesh, while for $p$ the increase is observed for both meshes. Note that these observations correspond with the results in [27]. In this reference convergence results in the $L^{2}$ norm are presented for non-distorted meshes. An unexpected high convergence order is reported for $p$, but not for $T$. Taking the difference norm that is used, and the range of $k / s$ for which results are presented into account, these findings correspond with the results in figure 5.11(a).

An important feature of the differences in FE error and convergence rate is that the value of $k / s$ for which the differences are observed, shifts to smaller values

\footnotetext{
${ }^{24}$ It must be noted that the observed order of convergence for $p$ does not concur with the results in [112], which predict a drop in the order of convergence for both $\mathbf{v}$ and $p$ on asymptotically non-affine meshes in the case of the Stokes equations.
} 


\begin{tabular}{llll}
\hline$M=1(-)$ & $M=2(---)$ & $M=3(-\cdot-)$ & $M=4(\cdots \cdots)$ \\
\hline
\end{tabular}
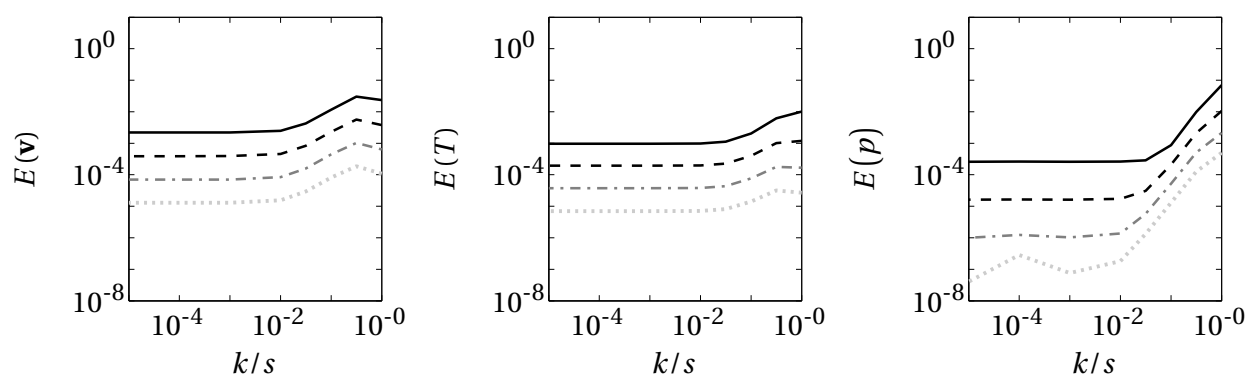

(a) Triangular Taylor Hood element, non-distorted crossed mesh
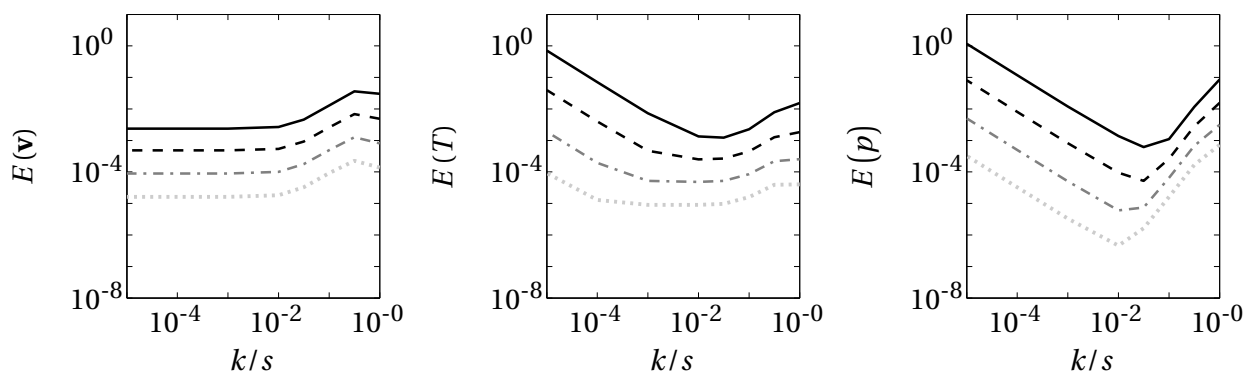

(b) Triangular Taylor Hood element, distorted crossed mesh

Figure 5.11: Relative FE error as a function of $k / s$ for different subsequent mesh refinements ( $M$ indicates the mesh number, with $M=1$ being the coarsest mesh) and $\tilde{s}=1$.

for increasing numbers of mesh refinements. This suggests that ultimately, the solution will converge with the predicted order independent of the value of $\mathrm{k} / \mathrm{s}$ if enough mesh refinements are made.

Note that the increase in FE errors can be related to the fact that the matrix $\hat{\mathrm{B}}$ becomes increasingly ill-conditioned as $\omega$ and thus $k / s$ vanish. The behavior is predicted by the error bounds in section 5.2.6. The rise in the error of $T$ and $p$ for decreasing $k / s$ (and thus $\omega$ ) follows from the error bound in (5.42). The error bound predicts a (possible) increase in the error of $\mathbf{v}, T$ and $p$ as the infsup value becomes smaller, while the results in section 5.4.4 show that the inf-sup value vanishes as $k / s$ goes to zero. The fact that the solution of $\mathbf{v}$ is not affected by ill-conditioning of the matrix B was already predicted by the error bound in (5.49) (which bounds the error of $\mathbf{v}$ independent of $k / s$ - and thus $\omega$ ). 


\subsubsection{Velocity-temperature based elements}

The results presented in the previous sections are limited to mixed FE methods. In this context, 'mixed' indicates that a mixture of primal variables ( $\mathbf{v}$ and $T$ ) and a derived or dual variable $(p)$ are used in the description of the material behavior. As an alternative to the presented mixed methods, a number of primal FE methods based on a description in terms of velocity and temperature are also developed.

The FE methods used to produce the result presented below are equivalent to the primal (v/T-based) FE method described by Joly ${ }^{25}$ [48]. Such methods can be seen as an extension of primal (v-based) FE methods for the Stokes equations, or equations for linear elasticity to the case of the LNSF equations. From the literature, it is known that primal FE methods for the Stokes equations generally suffer from locking. This becomes apparent if pressure is reintroduced as an (auxiliary) variable after the equations are already in weak form and discretized using FE techniques. In this way, a mixed method is obtained with velocity and pressure degrees of freedom. Note that the solution space for pressure that is thus obtained cannot be chosen freely. Instead, it is implied by the solution space that is used for velocity. The resulting combination of solution spaces for velocity and pressure does not automatically satisfy the inf-sup condition [115]. In fact, in most cases it does not. For instance if linear shape functions are used for a velocity only FE approximation of the Stokes equations, the inf-sup condition is not satisfied for the associated mixed problem, suggesting that such an FE method is numerically unstable. In practice, such elements are indeed known to suffer from locking ${ }^{26}$. The tendency of primal methods for the Stokes problem to be susceptible to locking transcends to FE methods for the LNSF equations in the case of velocity/temperature-based methods. The drawback of using such methods is illustrated below with two examples.

\section{Waveguide}

The setup for the first example is identical to that used for the numerical inf-sup test and convergence studies presented above. A small piece of waveguide for which the exact solution is known is modeled with FE methods as described at the beginning of section 5.4 .

\footnotetext{
${ }^{25}$ The referenced work also describes a $(\mathbf{v}, T)$-based FE method, however, it is not specified what kind of trial functions are used.

${ }^{26}$ Note that the order of discretization and eliminating pressure from the set of equations is important. As mentioned before, the family of Crouzeix Raviart elements are known to be stable, while pressure can be condensed out of the algebraic system yielding a description in terms of velocity (and temperature) only. Eliminating the pressure from a discrete system of equations is not the same as discretizing a system of equations from which the pressure is eliminated.
} 
In figure 5.12, the convergence results for different primal (v/ $T$-based) FE methods are given. The primal methods that are used have linear or quadratic trial functions for both velocity and temperature, and are indicated by $P 1 / Q 1$ and $P 2 / Q 2$ for triangular/quadrilateral elements, respectively.

The presented results are obtained for $\tilde{s}=10$ and $\tilde{k}=0.01$ corresponding to a

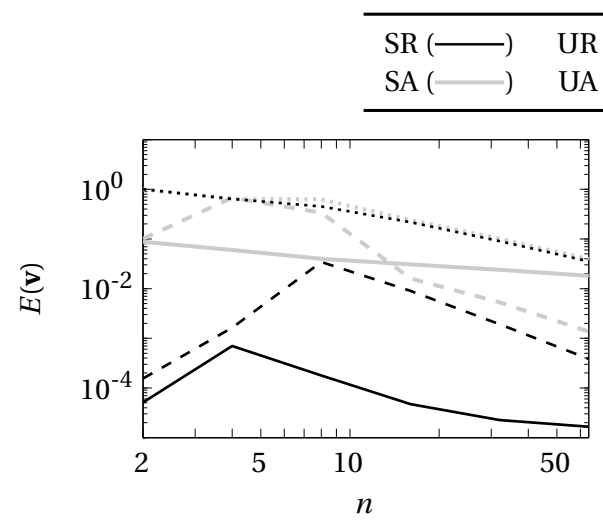

(a) P1 elements (triangular, linear)

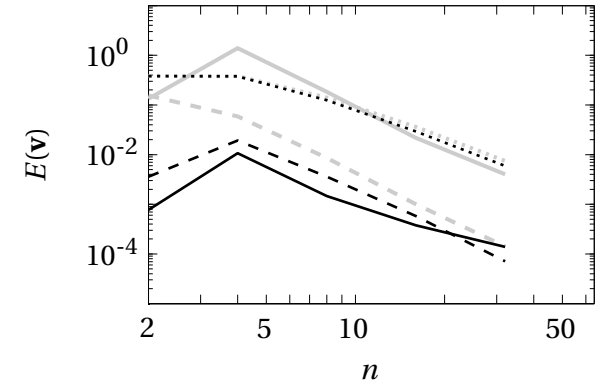

(b) P2 elements (triangular, quadratic)

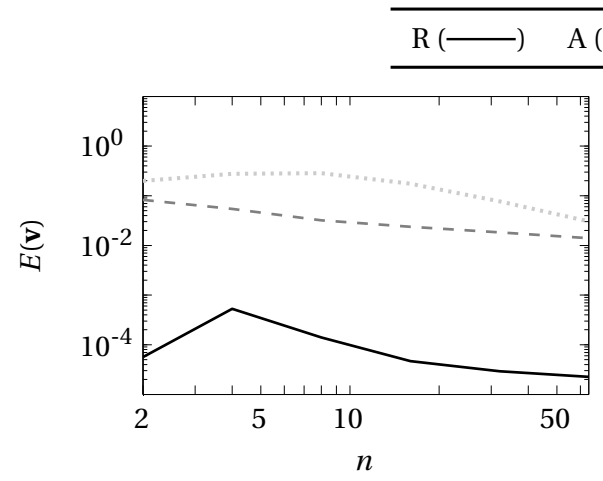

(c) Q1 elements (quadrilateral, linear) (d) Q2 elements (quadrilateral, quadratic)

Figure 5.12: Convergence behavior of primal FE methods for element types P1, P2, Q1 and Q2 and different mesh patterns. The letters $S, U$, and $C$ indicate the mesh pattern for triangular meshes: $S$ stripped mesh (figure 5.2(a)), $U$ union-jack mesh (figure 5.2(b)), and $C$ crossed mesh (figure 5.2(c)). The letters $R, A$ and $N$ indicate the type of mesh distortion: $R$ regular (undistorted) mesh, $A$ asymptotically affine mesh (figure 5.2(d)), and $N$ asymptotically non-affine mesh (figure 5.2(e)). All solutions are obtained for $\tilde{s}=10$ and $\tilde{k}=0.01$. 
frequency of $1223 \mathrm{~Hz}$, which is well within the range where element types that are susceptible to locking due to nearly incompressible behavior will suffer from numerical instabilities. Triangular elements are considered on both undistorted and distorted meshes for three types of mesh patterns: striped (figure 5.2(a)), unionjack (figure 5.2(b)), and crossed (figure 5.2(c)) . Quadrilateral elements are considered for the following three types of meshes: undistorted, distorted asymptotically affine meshes (figure 5.2(e)), and asymptotically non-affine meshes (figure 5.2(e)).

From the different plots in figure 5.12 it can be concluded that the $P 1, Q 1$ and $Q 2$ elements are not robust. Optimal convergence for the $P 1$ element is not observed for undistorted and distorted meshes having a striped mesh pattern. The Q1 element appears to be stable only on meshes including distorted elements. The Q2 element fails on meshes with distorted elements but has optimal convergence on undistorted meshes and meshes that become less distorted with mesh refinements. Only the P2 element seems to have optimal convergence on all considered meshes for the considered range, except on undistorted striped meshes. The trend in the convergence rate observed for undistorted striped meshes suggests that the order of convergence will become even lower for systems having more degrees of freedom. Nevertheless, based on the results in 5.12(b) the P2 element is expected to perform well on unstructured meshes.

Note that the Q1 element does not seem to have optimal convergence on regular and asymptotically affine meshes, while it does have optimal convergence for asymptotically non-affine meshes. This peculiar (perhaps counterintuitive) behavior is not uncommon in the case of (near) incompressibility. Such behavior is for instance also encountered for the $Q 1-P 0$ (bilinear velocity-constant pressure) element in the context of the Stokes equations. This element seems to perform well for arbitrary meshes if the element shapes are distorted and for meshes consisting of element groups for which the elements are distorted, but suffers from a certain degree of locking (not complete) on regular (undistorted) meshes [61].

\section{Stepped cavity}

To illustrate the practical consequences of the locking phenomena observed for P1, P2 and Q2 elements on certain mesh patterns, an air-filled cavity having a 2D geometry as depicted in figure 5.13 and 5.14 is considered. The geometry consists of two coupled layers of unequal thickness. The thick layer is closed off at one end, and the system is excited by a unit normal force at the open end of the thin layer. Adiabatic boundary conditions are assumed at all boundaries of the domain. The FE solution for velocity is depicted in figure 5.13 and 5.14 for several meshes that are obtained by subsequent mesh refinements.

The results in 5.13(a) illustrate the locking effect that is encountered when us- 

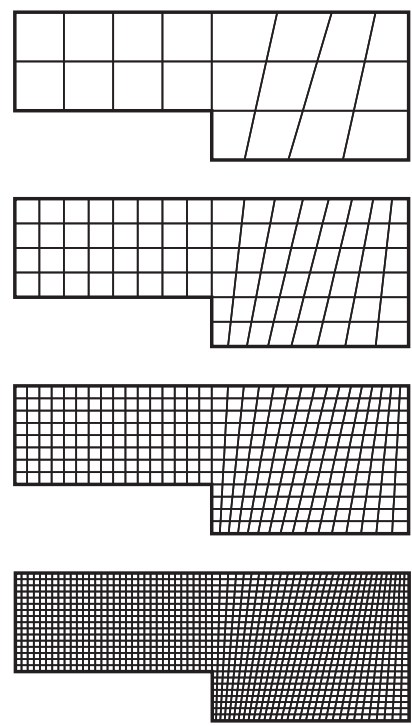

(a) Mesh patterns
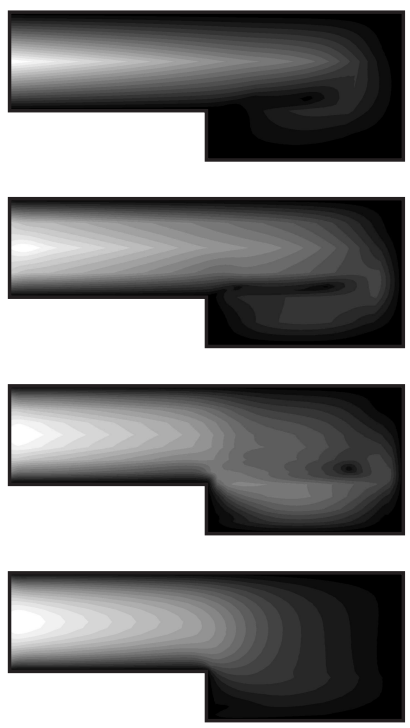

(b) Velocity solution, $|\mathbf{v}|$, for $(\mathbf{v}, T)$-based method with quadratic elements (Q2)
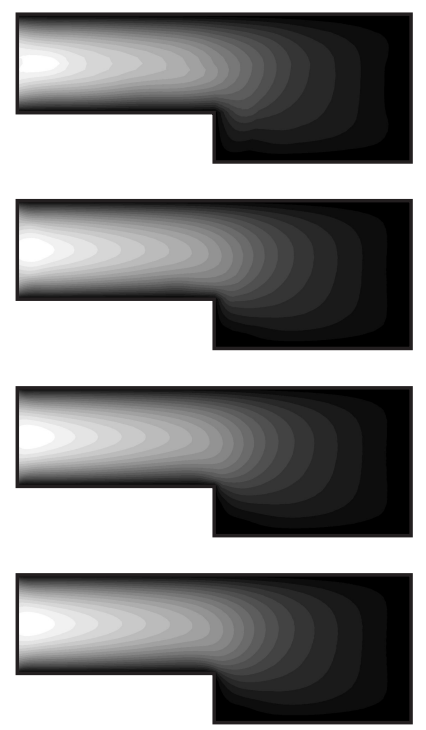

(c) Velocity solution, $|\mathbf{v}|$, for mixed method with Taylor Hood elements

Figure 5.13: $(\mathbf{v}, T)$-based and $(\mathbf{v}, T, p)$-based FE approximations of velocity on various quadrilateral meshes for $\tilde{s}=10$ and $\tilde{k}=0.01$ (for the thin layer).

ing a primal method with quadrilateral elements having quadratic shape functions (Q2 elements). The accuracy of the solution is clearly not solely determined by the approximability of the solution for the given meshes. A striking artefact is the area of lower velocity that seems to protrude from where the boundary of the thin layer meets the thick layer. This behavior is not observed in figure 5.13(a) that shows the velocity results for the stable Taylor Hood element (which involves an algebraic system of less degrees of freedom). The apparent sudden convergence of the solution for the Q2 element between the two finest meshes is due to the fact that the elements become increasingly less distorted as the mesh is refined. This causes a change in convergence speed with mesh refinements since the results in figure 5.12 suggest that the Q2 element has optimal convergence behavior on regular meshes. Nevertheless, it is clear from the results in figure 5.13, that the accuracy of the velocity solution for Q2 elements on arbitrary quadrilateral meshes (including significantly distorted elements) cannot be inferred solely from the level of mesh refinement. In other words, elements for primal FE methods like the Q2 element should be used with caution.

A similar conclusion can be drawn based on the results presented in figure 

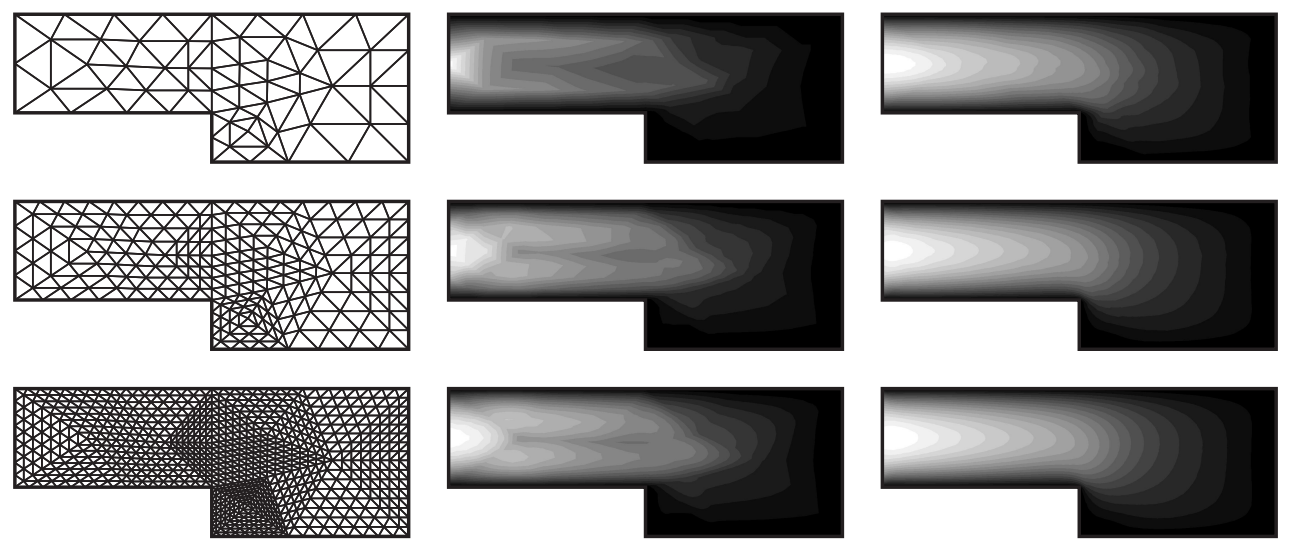

(a) Mesh patterns

(b) Velocity solution, $|\mathbf{v}|$, for P1 elements

(c) Velocity solution, $|\mathbf{v}|$, for P2 elements

Figure 5.14: $(\mathbf{v}, T)$-based FE approximations of velocity on various triangular meshes using $\mathrm{P} 1$ and $\mathrm{P} 2$ elements for $\tilde{s}=10$ and $\tilde{k}=0.01$ (for the thin layer).

5.14. Here, the velocity results for two primal FE methods are shown for a family of triangular meshes. The results of the P1 element in figure 5.14(b) show a typical case of locking. The solution hardly seems to change as the mesh is repeatedly refined, and the accuracy of the solution clearly cannot be predicted based on the level of mesh refinement. Closer inspection of the meshes reveals that they consist of groups of elements which have a striped mesh pattern corresponding with figure 5.2(a). The boundaries of these groups remain intact as the mesh is refined and can clearly be observed in the velocity solution for the P1 element. These observations together with the results in 5.12(a) indicate that use of the P1 element, which might seems attractive in terms of efficiency, should be avoided.

The situation is somewhat different when the P2 element is considered. The corresponding results in figure 5.14(b) concur with the fact that optimal convergence is observed for the Q2 element in figure 5.12(b) for all considered mesh patterns, except undistorted striped meshes. Figure 5.14(b) suggests that the element does not suffer from locking on arbitrary meshes that might be encountered in practical cases. In this case, patches of elements in figure 5.14(b) that have a striped mesh pattern do not seem to influence the solution adversely. To the author's knowledge, no proof for the stability of the P2 element (or its equivalent for the Stokes equations or the equations for linear elasticity) exist. Together with the fact that the convergence rate seems to decline with mesh refinement on undistorted meshes having a striped mesh pattern, it must be concluded that these elements can be useful for unstructured meshes, but should be used with 
some caution.

\subsection{Conclusions}

The main results of the investigation after the numerical stability of the different FE methods presented in this chapter are given below. The first four remarks concern mixed FE methods, while the last two remarks concern primal FE methods.

- The results of the numerical inf-sup test suggest that all considered elements for the LNSF equations that are based on elements satisfying the inf-sup condition for the Stokes equations are robust for all $\omega$; altering the element shape, mesh pattern, order of the trial functions for temperature or stretching the elements by a factor of ten only results in minute changes of the observed inf-sup values.

- The presented convergence results concur with the results of the numerical inf-sup test; all elements that are robust according to the numerical inf-sup test have optimal convergence or convergence of a higher order ${ }^{27}$.

- Failure to pass the inf-sup test does not necessarily result in poor convergence $^{28}$. For large values of $k / s(k / s \gtrsim 1)$ convergence is optimal independent of the outcome of the inf-sup test ${ }^{29}$.

- The FE error in pressure and temperature of all considered elements grows as $k / s$ goes to zero. This concurs with the results of the numerical inf-sup test. The rise in the FE error is due to an ill-conditioning of the system that is unrelated to the locking phenomenon observed for nearly incompressible material behavior.

- Several examples of primal (v/T-based) FE methods, such as the P1,Q1 and Q2 elements, do not appear to be robust. The presented examples show that their use should be avoided.

\footnotetext{
${ }^{27}$ In the case of the Crouzeix Raviart elements on asymptotic non-affine mesh families, the low convergence rate can be attributed to the limited approximability offered by a bilinear mapping of (bi)linear trial functions for pressure on distorted elements (see [112]). Convergence is however still optimal.

${ }^{28}$ With respect to this behavior, the results obtained with FE methods for the LNSF equations are similar to those obtained for the equivalent FE method for the Stokes equations.

${ }^{29}$ This is analogue to the results obtained with FE methods for linear elasticity in the case of compressible material behavior.
} 
- The P2 element (another examples of a primal FE method), seems to have optimal convergence on arbitrary meshes. Nevertheless, the order of convergence that is observed for regular (undistorted) meshes with a stripped mesh pattern is slightly lower than optimal. The presented results suggest that these elements can be useful in the case of unstructured meshes, but should be used with some caution. 


\section{Chapter 6}

\section{Performance}

The LRF solutions provide a simple and efficient way to model viscothermal wave propagation in waveguides. Beltman [5, 10,36] and Tijdeman [35] point out that for wave propagation in many gases and liquids, the need for a full Navier Stokes model is very limited. This view is supported for instance by the results plotted in figure 6.6 portraying the relative error of the LRF approximation of the propagation constant for an air slit and a cylindrical air-filled tube compared to the exact Kirchhoff solutions. The work of Beltman and Tijdeman shows that LRF models for simple geometries are much simpler to solve than the full LNSF model and in general only fail in extreme cases.

\section{LRF assumptions and criteria}

In chapter 4 three criteria are given that are necessary for the assumptions leading the LRF approximation to hold. The criteria are given in terms of the dimensionless wave numbers $\tilde{k}, \tilde{s}$ and $\tilde{s}_{t}$. The first two criteria in (4.10), can be denoted as $\tilde{k} / \tilde{s} \ll 1$ and $\tilde{k} / \tilde{s}_{t} \ll 1$ (or alternatively in dimensional wave numbers as $k / s \ll 1$ and $k / s_{t} \ll 1$, respectively). These conditions are requirements for the viscous and thermal boundary layer to be small compared to the acoustic wavelength. It was already mentioned in chapter 4 that these quotients are only dependent on frequency and material properties and not on geometry. As an example, the value of these quotients as a function of frequency is given in figure 6.1(b) for air under standard atmospheric conditions (see appendix B). From these plots, it is clear that the first two criteria in (4.10) are not satisfied only in the case of very high frequencies. Beltman [10] states that for many fluids, the value of $k / s$ and $k / s_{t}$ will only approach unity under conditions involving high frequencies or low temperature or pressure. Under such conditions, the assumptions required for continuum mechanics are almost always violated and the full model also yields inaccurate re- 


\begin{tabular}{lll}
$s(-)$ & $s_{t}(---)$ & $k(\cdots \cdots)$ \\
\hline
\end{tabular}

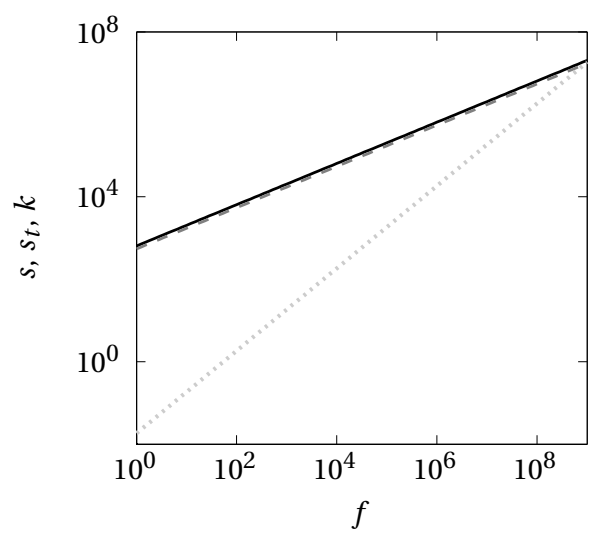

(a) Dimensional wave numbers
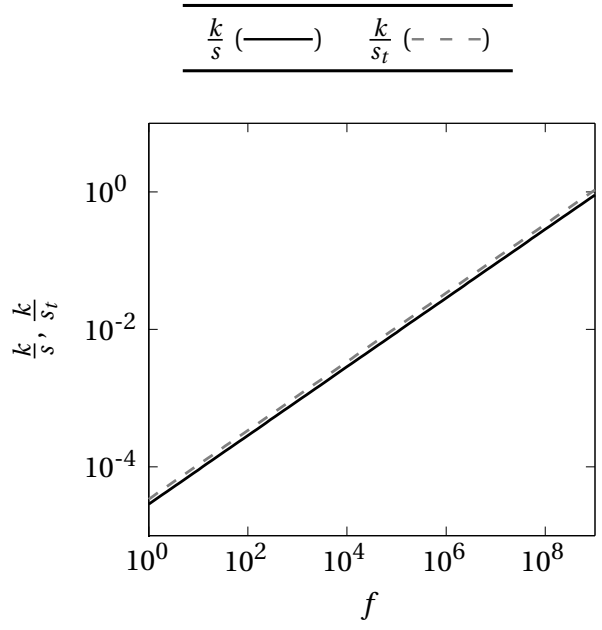

(b) Ratios of wave numbers

Figure 6.1: Dimensional wave numbers for air under standard atmospheric conditions (see appendix B for properties).

sults.

The third criterion in (4.10), which can be denoted as $\tilde{k}<1$, must ensure that the pressure can be assumed constant in directions other than the propagation direction. In practice, this assumption is valid for cases involving straight (long) waveguides if the excitation of higher order modes at the waveguide entrances is small. The results in figure 6.6 show that even if $\tilde{k} \sim 1$ or $\tilde{k}>1$ the LRF model still predicts the propagation constant of the first propagating wave very well as long as $k / s \ll 1$. Summarizing, it can be said that based on accuracy (and efficiency) results found in the literature, the use of LRF models is almost always preferred over full LNSF models.

\section{Coupled LRF models}

A serious shortcoming of the studies on the accuracy of LRF models encountered in the literature is that they generally only focus on examples involving very simple geometries and no-slip isothermal boundary conditions. While various studies $[10,35]$ point out that accuracy is guaranteed for LRF models of infinite waveguides of constant cross section (and constant curvature) having no-slip velocity and isothermal temperature boundary conditions, the same cannot automatically be said for cases involving more complex geometries or other boundary conditions. An example of a case where the LRF criteria are met but LRF models yield 
results of insufficient accuracy is given in section 7.2.

More complex LRF models are generally obtained by coupling multiple LRF models of sections of infinite waveguides. In this way, systems involving for instance junctions of waveguides, or changes in boundary conditions, cross section, or curvature, can be modeled in a straightforward way with the techniques described in section 4.3. When considering such coupled LRF models, it is important to realize that the sub models are based on an approximation of the first acoustic mode in infinite waveguides having constant boundary conditions, cross section ${ }^{1}$ and curvature in propagation direction ${ }^{2}$. Naturally, the solution for such coupled LRF models cannot represent the local velocity, temperature and pressure fields accurately. If the LRF approximation of these fields is locally inaccurate, the higher order acoustic, vorticity and thermal modes contribute significantly to these fields. The knowledge on how neglecting these modes affects the global properties of the solution if multiple waveguides are coupled is limited. This implies that the usefulness/necesity of other, less efficient viscothermal models should not automatically be ruled out in the case of more general geometries or boundary conditions.

\section{Literature}

Unfortunately, the literature on the accuracy of coupled LRF models for more complex geometries and/or boundary conditions is not systematic. In virtually all instances, the accuracy of coupled LRF models is assessed by comparing calculated data with measurement data instead of with results obtained with models based on the full LNSF equations. This makes it difficult to systematically investigate the influence of certain parameters (such as geometry, and material parameters) on the accuracy of coupled LRF models. It also leaves the causes for possible differences in calculated and measured data open to speculation.

In most studies dealing with coupled viscothermal waveguide models, it is simply assumed that only the effect of higher order acoustic modes can become significant, and that the effects of neglecting these modes can always be accounted for by applying a correction technique used in inviscid adiabatic acoustics, namely, the use of length corrections. The fact that these length corrections are derived for the inviscid adiabatic case and do not take viscothermal effects into account, and the possible effects of neglecting thermal and vorticity modes are usually not ad-

\footnotetext{
${ }^{1}$ The cross section is constant in $p d$-direction with respect to the $c d$-coordinates, but may vary in Cartesian coordinates.

${ }^{2}$ This was demonstrated for two specific cases in section 3.5.2.
} 
dressed $^{3}$.

Exceptions where full LNSF models are compared with LRF results for systems of more complex geometry, are found in the recent work of Kampinga [1] and Christens et al. [26]. Kampinga uses the FE models that are presented in chapter 5 to verify the LRF solution and the solution of a new method called the Sequential Linear Navier Stokes Model. Comparisons are made for a number of ad hoc cases (including the case study presented in section 7.2). Christens et al. compare FE models of the LNSF equations that are very similar to those presented in chapter 5 with results of the viscothermal BE models presented in $[49,50]$ and LRF models for a case involving three coupled circular cylindrical tubes. In both instances, the error made by using LRF approximations compared to full LNSF models is not analyzed, and the presented cases are ad hoc. To the author's knowledge, a systematic study on the accuracy of coupled LRF models for more complex geometries is not available in the literature. An account of the accuracy of LRF models in the case of adiabatic boundary conditions (instead of isothermal boundary conditions) was also not encountered in the literature.

\section{Outline}

The results presented in this chapter are an attempt to extend the knowledge on the accuracy of LRF models compared to solutions of the full set of LNSF equations in a more systematic way. In the next sections, the relative accuracy of different LRF solutions are explored for a variety of 'less standard' geometries and adiabatic boundary conditions for a range of values of $k$ and $s$. The 'exact' or reference solutions are obtained by employing the Kirchhoff models presented in chapter 3 , and the robust FE models presented in chapter 5 for which a predefined accuracy can always be achieved by consecutive mesh refinements.

First, the validity of the criteria that are necessary for the assumptions that lead to LRF models to hold is considered for general geometries and boundary conditions, and a generic test case is introduced. In section 6.2 the accuracy of LRF models of straight infinite waveguides having constant cross section and boundary conditions is investigated for isothermal and adiabatic boundary conditions. Sections 6.3, 6.4, 6.5 and 6.6 deal with waveguide bifurcation, and changes in thermal boundary conditions, cross section and curvature respectively. In the last section a scattering problem including higher order modes based on the exact Kirchhoff solutions is considered.

\footnotetext{
${ }^{3}$ An exception is the work of Mechel $[75,76]$ who investigates the coupling of multiple waveguides, using a description in terms of approximated higher order acoustic modes that account for viscothermal effects. However, the thermal and vorticity modes are not considered in the coupling, and errors made by using an LRF or inviscid adiabatic acoustic approximation are not considered.
} 


\subsection{LRF assumptions and relative accuracy}

Under the LRF assumptions discussed above and in section 4.1.2, an order of magnitude analysis shows that a number of terms in the full LNSF equations are expected to be relatively small and can be neglected, thus leading to the LRF approximation. Checking the validity of the LRF assumptions directly, however, cannot be done a priori. Therefore, the LRF assumptions are in practice usually replaced by the related criteria posed in (4.10), which can be verified a priori. Nevertheless, satisfying these criteria does not always imply that the different terms neglected in the LRF models are small. If the gradients involved in the neglected terms are large, the original assumptions are violated and the neglected terms might have a significant magnitude compared to terms that are retained. The following conditions can have a negative influence on the validity of the LRF assumptions:

- Non-isothermal boundary conditions

- Changes in boundary conditions along the direction of propagation

- Changes in waveguide cross section along the direction of propagation

- Changes in waveguide curvature along the direction of propagation

- Inlet effects ${ }^{4}$ at the waveguide entrances

In the next section the influence of the different conditions on the relative accuracy of LRF models is investigated. In that context, it is important to define when an LRF model can be considered accurate.

\subsubsection{Relative accuracy of LRF models}

When arbitrary geometries and boundary conditions are considered, the LRF assumptions are not guaranteed to hold under the criteria given in 4.1.2 for the entire fluid domain. Nevertheless, locally violating the assumptions does not necessarily imply that the resulting approximation is useless. The properties of the global solution that are of interest may remain relatively unaffected by locally violating the assumptions that are deemed necessary for the approximation. In many cases, the exact velocity, temperature and pressure fields in a waveguide system are not of particular importance. The properties that are usually of interest take the form of 'transfer functions' between one or more quantities at the entrances of a waveguide system. For instance, the averaged amplitude of pressure or outward velocity at one entrance as a function of the pressure or outward velocity at another

\footnotetext{
${ }^{4}$ Inlet effects at waveguide entrances can also be thought of as sudden changes in the boundary conditions or the geometry of the waveguide in the direction of propagation.
} 
entrance, or the averaged pressure at an entrance as a function of the averaged inward velocity at that entrance. Note that in many cases, the exact velocity, temperature, and pressure profiles at the entrances are not even of interest, and the averaged values over the cross-sectional surface of the entrance suffice. If one is indeed only interested in the averaged values of certain quantities at the waveguide entrances, it is usually sufficient that the model can accurately predict the amplitudes of in and outgoing propagating waves at each entrance of the waveguide system.

\subsubsection{Scattering calculation}

In this chapter, scattering calculations are used as a generic test case to investigate how changes in boundary conditions and geometry in the direction of propagation influence the relative accuracy of LRF models. An abstract representation of a model used for scattering calculations is given in figure 6.2. The model consists of two coupled waveguides of infinite length, indicated by $A$ and $B$ having arbitrary, constant cross section and boundary conditions in $p d$-direction. The waveguides may be coupled directly or via a waveguide system having arbitrary geometry and boundary conditions.

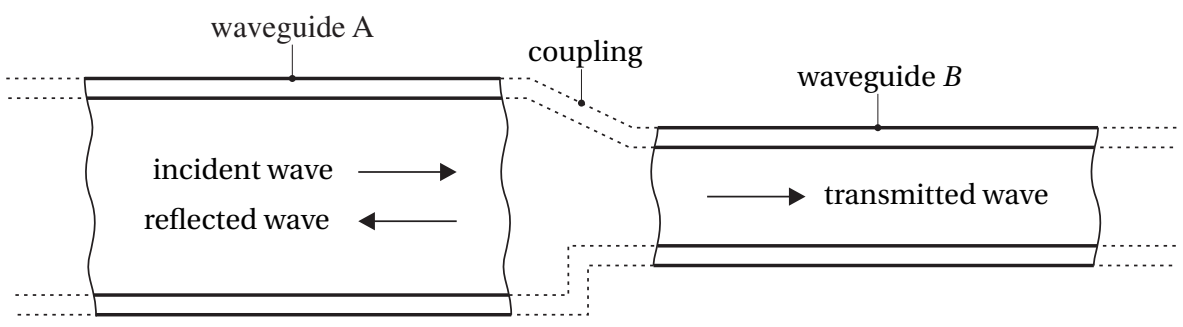

Figure 6.2: An abstract scattering problem; incident, transmitted and reflected waves in two arbitrary, coupled waveguides of infinite length.

Based on the theory in chapter 3 both infinite waveguide sections will support an infinite number of acoustic, thermal and vorticity modes. These modes represent acoustic, thermal and vorticity waves that are supported by the waveguide. The direction of the waves is determined by the attenuation of their amplitude (and not necessarily by the direction in which the wave tops are traveling) as defined in chapter 3 . This implies that waves will always damp out as they travel through the waveguide. It is assumed that all waves in each infinite waveguide section will damp out relatively quickly compared to waves corresponding to the 
first acoustic mode ${ }^{5}$. Furthermore, it is assumed that no waves are traveling towards the junctions, except for one incident wave in waveguide $A$, which corresponds to the first acoustic mode impinging on the junction. Consequently, at a certain distance from the junction, the incident, transmitted and reflected waves which correspond to the first acoustic mode in each infinite waveguide section dominate the velocity, temperature and pressure field.

\section{Accuracy of LRF models}

In the resulting model, the velocity, temperature and pressure field in parts of the infinite waveguides well away from any change in cross section or boundary condition can be accurately modeled if the propagation constants of these propagating waves and their complex amplitudes at a certain location in the waveguides are known with sufficient accuracy. So, under the above assumptions, the accuracy of an LRF model can be defined by the error that is made in the approximation of the propagation constant and the approximation of the complex amplitudes of the backward and forward traveling waves in both infinite waveguides. The error in the LRF approximation of the propagation constant is studied in section 6.2. In the sections thereafter, the error in the complex amplitudes is studied for different cases. The generic scattering problem describing these cases is introduced below. In section 6.7 a scattering problem that includes multiple acoustic and vorticity modes in both waveguide sections is considered. The modes used in this model are obtained with the techniques presented in chapter 3 . The coupling of the different modes in the waveguide sections is described in section 3.6. The resulting model is used to study the influence of using higher order acoustic modes and vorticity modes on the complex amplitude of the first acoustic modes.

\subsubsection{Generic problem}

The objective of the scattering calculation is to obtain the complex amplitude of the transmitted and reflected waves corresponding to the first acoustic modes that result from an incident acoustic wave of known amplitude impinging on the waveguide junction as depicted in figure 6.2. As these propagating waves damp out and slow down due to viscothermal effects, the complex amplitude of the incident, transmitted, and reflected wave depends on the position in the waveguide. The errors in the approximation of the amplitudes of the different waves are calculated at the location in the infinite waveguide sections $A$ and $B$ close to the junction. For the cases studied in this chapter these locations are indicated in figure 6.3 by

\footnotetext{
${ }^{5}$ Note that for the case in section 6.4, this assumption does not hold and the effects of the first thermal mode are also taken into account.
} 
dashed lines, and in figure 6.4 and 6.5 by the origin of the coordinates $x_{A}$ and $x_{B}$. For the cases depicted in figure $6.3 \mathrm{~b}, 6.3 \mathrm{c}$, and $6.3 \mathrm{~d}$ this means all wave amplitudes are taken at the same location, while for the cases depicted in figure 6.3a, the location where the amplitude of the transmitted wave is taken differs from where the amplitude of the incident and reflected waves are taken.

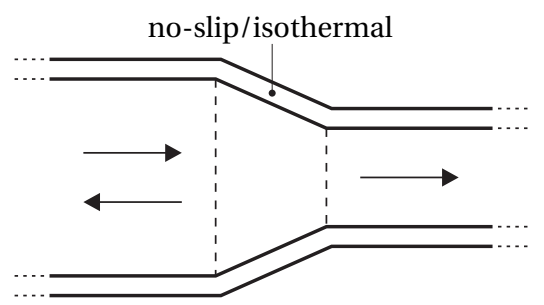

(a)

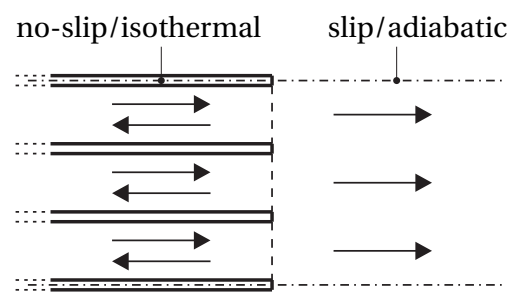

(c)

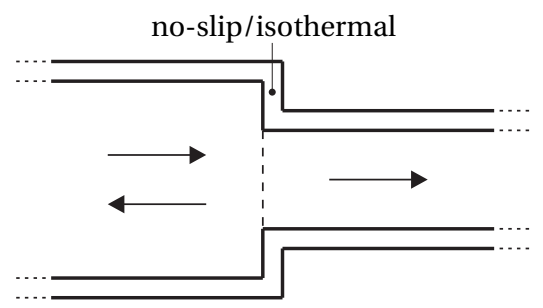

(b)

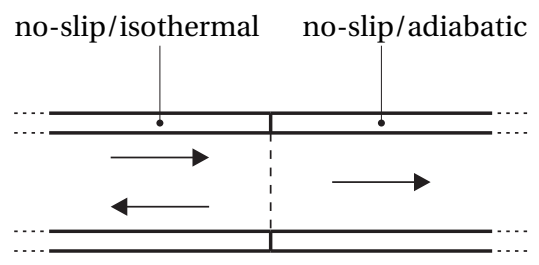

(d)

Figure 6.3: Scattering problems for changes in waveguide cross section or boundary conditions: Gradual change in waveguide cross section (a), sudden change in waveguide cross section (b), bifurcation of a waveguide (c), and a sudden change in thermal boundary conditions (d).

Only a finite section of the infinite waveguides are modeled, yielding a finite waveguide system with two entrances. For typical scattering calculations, the boundary conditions at the entrance supporting the incident wave must be such that the first acoustic wave traveling inwards has a known non-zero amplitude, while all other waves traveling towards the junction in both waveguide sections have zero amplitude. Below it is described how this is accomplished for both the approximate LRF models and the FE models that are used as a reference solution.

\section{LRF model}

The LRF model used for the infinite waveguide sections and the method to couple these models are described in section 4.3. Waveguide sections of non-constant cross sections are approximated with multiple coupled waveguide models as de- 


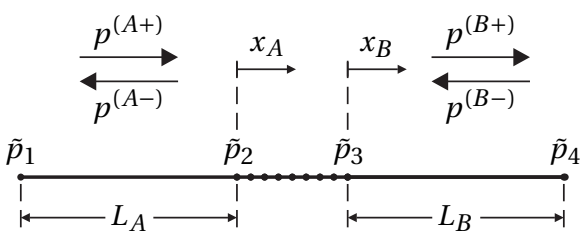

(a)

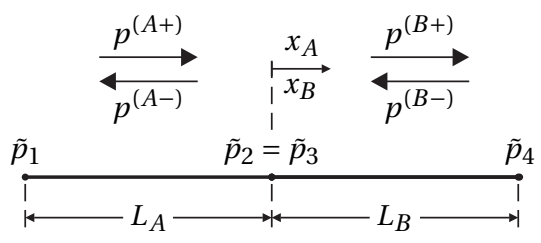

(b)

Figure 6.4: LRF models for scattering problems: Gradual change in waveguide cross section - multiple coupled LRF models (a), sudden change in waveguide cross section or boundary conditions - two coupled LRF models (b). At $p_{1}$ a unit pressure is prescribed, and at $p_{4}$ the characteristic impedance of the corresponding waveguide is prescribed.

scribed in section 4.2.3. The number of waveguides used in the approximation is such that further increase does not significantly change the calculated error.

In practice, for the LRF models, a non-zero pressure is prescribed at the entrance with the incident wave, while an impedance condition matching the characteristic impedance of the waveguide is prescribed at the other entrance (thus ensuring this entrance only supports outgoing waves). The complex amplitude of the resulting backward and forward traveling waves in the infinite waveguide sections can be determined based on the pressure at the entrances of the sections of the infinite waveguide, as was done in section 4.3. Rewriting equation (4.54), so that the complex amplitudes are calculated at the waveguide entrances yields:

$$
\begin{array}{ll}
\left\{\begin{array}{l}
\tilde{p}_{1} \\
\tilde{p}_{2}
\end{array}\right\}=[V]\left\{\begin{array}{l}
p^{(A+)} \\
p^{(A-)}
\end{array}\right\}, & \text { with }[V]=\left[\begin{array}{cc}
e^{i k \Gamma_{A} L_{A}} & e^{-i k \Gamma_{A} L_{A}} \\
1 & 1
\end{array}\right] \\
\left\{\begin{array}{l}
\tilde{p}_{3} \\
\tilde{p}_{4}
\end{array}\right\}=[V]\left\{\begin{array}{l}
p^{(B+)} \\
p^{(B-)}
\end{array}\right\}, & \text { with }[V]=\left[\begin{array}{cc}
1 & 1 \\
e^{-i k \Gamma_{B} L_{B}} & e^{i k \Gamma_{B} L_{B}}
\end{array}\right]
\end{array}
$$

where $\tilde{p}_{1}, \tilde{p}_{2}, \tilde{p}_{3}$ and $\tilde{p}_{4}$ are the pressures at the entrances of the sections of the infinite waveguide (see figure 6.4), $p^{(A+)}, p^{(A-)}, p^{(B+)}$ and $p^{(B-)}$ are the complex amplitudes of the acoustic waves traveling in positive and negative $x$-direction in each section at $x_{A}=0$ and $x_{B}=0$, respectively, and $\Gamma_{A}$ and $\Gamma_{B}$ are the LRF approximation of the propagation constant corresponding to these waves. Solving the systems in (6.1) yields the complex amplitude of the incident, transmitted, and reflected acoustic wave. The resulting amplitudes are scaled so that the incident wave has unit amplitude and zero phase at the end of the section of the infinite waveguide close to the junction. 


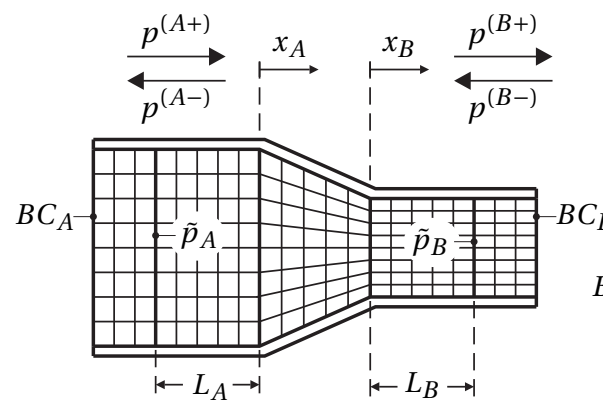

(a)

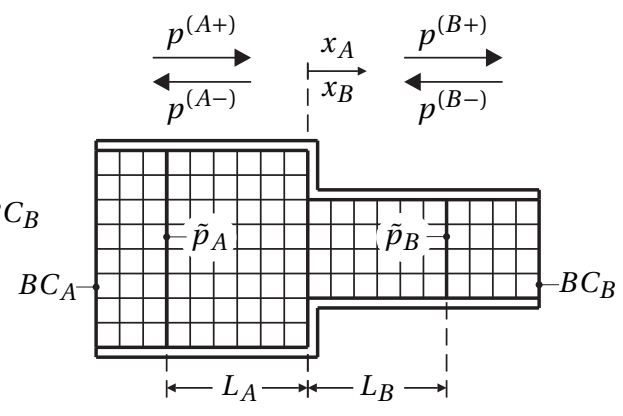

(b)

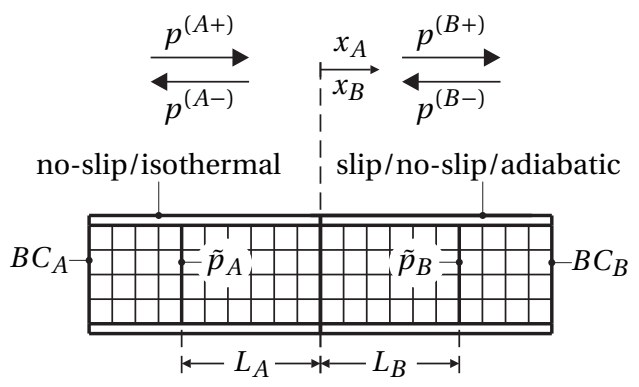

(c)

Figure 6.5: FE models for scattering problems: Gradual change in waveguide cross section (a), sudden change in waveguide cross section (b), and sudden change in boundary conditions (c). The velocity and temperature profile corresponding to the first acoustic mode is prescribed at $B C_{A}$, and the stress vector and heat flux expressed in terms of the velocity and temperature profile for an outward traveling acoustic wave ${ }^{7}$ is prescribed at $B C_{B}$.

\section{FE models}

For the FE models, the velocity and temperature profiles corresponding to the first acoustic mode are prescribed at the entrance where the incident wave originates. At the other entrance an impedance boundary condition is prescribed; Based on the mode shapes and propagation factor of an outward traveling acoustic wave, the stress vector and normal heat flux are expressed in terms of velocity and temperature, and subsequently prescribed as boundary conditions. In both instances, the propagating acoustic mode is determined with the techniques in chapter 3 . The following equations can be used to determine the complex amplitude of the

\footnotetext{
${ }^{7}$ Note that these expressions depend on the boundary conditions applied at the waveguide surface.
} 
backward and forward traveling waves:

$$
\begin{aligned}
& \left\{\begin{array}{c}
\tilde{p}_{A} \\
\frac{\partial \tilde{p}_{A}}{\partial x}
\end{array}\right\}=[V]\left\{\begin{array}{l}
p^{(A+)} \\
p^{(A-)}
\end{array}\right\}, \quad \text { with } \quad[V]=\left[\begin{array}{cc}
e^{\left(i k \Gamma_{A} L_{A}\right)} & e^{\left(-i k \Gamma_{A} L_{A}\right)} \\
-i k \Gamma_{A} e^{\left(i k \Gamma_{A} L_{A}\right)} & i k \Gamma_{A} e^{\left(-i k \Gamma_{A} L_{A}\right)}
\end{array}\right] \\
& \left\{\begin{array}{c}
\tilde{p}_{B} \\
\frac{\partial \tilde{p}_{B}}{\partial x}
\end{array}\right\}=[V]\left\{\begin{array}{l}
p^{(B+)} \\
p^{(B-)}
\end{array}\right\}, \quad \text { with } \quad[V]=\left[\begin{array}{cc}
e^{\left(-i k \Gamma_{B} L_{B}\right)} & e^{\left(i k \Gamma_{B} L_{B}\right)} \\
-i k \Gamma_{B} e^{\left(-i k \Gamma_{B} L_{B}\right)} & i k \Gamma_{B} e^{\left(i k \Gamma L_{B}\right)}
\end{array}\right]
\end{aligned}
$$

where $\tilde{p}_{A}$ and $\tilde{p}_{B}$ are the (dimensionless) pressure of the FE solution averaged over the waveguide cross section ${ }^{8}$ at distance $L_{A}$ and $L_{B}$ from the junction (see figure 6.5), and $\Gamma_{A}$ and $\Gamma_{B}$ are the propagation constant for the first acoustic modes determined with the techniques in chapter 3. Note that $L_{A}$ and $L_{B}$ must be taken large enough to ensure that influence of the higher order acoustic, thermal and vorticity modes on the pressure and its derivative can be neglected at the location where these quantities are used in the calculation of the amplitudes of the inward and outward traveling waves. Note that the length of the section of the infinite waveguide that is modeled is larger than $L_{A}$ and $L_{B}$. This ensures that the influence of inward traveling waves caused by slight inaccuracies induced by prescribing the boundary conditions ${ }^{9}$ are small at the location where the pressure and its derivative are taken, and thus can be neglected as well.

Again, once the complex amplitudes of the incident, transmitted, and reflected wave are determined by solving the system in (6.2), the results can be scaled so that the incident wave has unit amplitude and zero phase at the end of the section of the infinite waveguide close to the junction.

\section{Accuracy}

Using this setup, an appropriate measure for the accuracy of the proposed LRF models is the magnitude of the error in the approximation of the complex amplitude of the transmitted wave $p^{(B+)}$ and the reflected wave $p^{(A-)}$ compared to that obtained with an FE calculation. Note that the thus obtained errors do not include errors due to the LRF approximation of wave propagation in the sections of the infinite waveguide. For the cases in figure $6.3 \mathrm{~b}, 6.3 \mathrm{c}$, and $6.3 \mathrm{~d}$, the observed errors are caused solely by the 'jump' in waveguide cross section or boundary condition, while for the cases in figure 6.3a, the error due the LRF approximation of wave propagation in the 'tapered' section is included.

\footnotetext{
${ }^{8}$ The amplitude of the modes determined with the techniques in chapter 3 are all normalized by taking the averaged dimensionless pressure over the waveguide surface equal to unity.

${ }^{9}$ Inaccuracies can be the results of, for instance, numerical integration and the fact that the domain includes corners.
} 
In the next sections the relative error of the complex amplitudes of the transmitted and reflected wave of the LRF approximation compared to the exact solution is presented for a number of LRF models. To ensure that the accuracy of FE solutions, which are used to obtain the 'exact' or reference solution, does not dominate these errors, a second FE calculation on a coarser mesh is performed to verify if mesh size influences the errors significantly. For all data presented in the next section, the influence of the mesh size on the relative error is small compared to the value of the error itself. As a consequence of eliminating inaccurate FE results, some of the plots in the presented figures are discontinued above or below certain parameter values.

\subsubsection{Relative errors}

The next subsection contains a large number of figures depicting relative errors of LRF approximations of, for instance, the real and imaginary part of the propagation constant and the absolute value of the complex amplitude of propagating waves. To keep the labels and captions of these plots legible these relative errors are expressed as operators on the quantity of interest. From hereon, the operators $E_{R}, E_{I}$, and $E_{A}$ represent the relative error in the real, imaginary and absolute value of the LRF approximation of a certain quantity compared to the corresponding exact solution. For completeness, the difference in phase (which is not a relative error) is assigned to the operator $E_{P}$. The error operators are defined as follows for a quantity $X$ and its (LRF) approximation $X_{L}$ :

$$
\begin{array}{ll}
E_{R}(X)=\frac{\Re\left(X_{L}\right)-\Re(X)}{\Re(X)} & E_{I}(X)=\frac{\Im\left(X_{L}\right)-\Im(X)}{\Im(X)} \\
E_{A}(X)=\frac{\left|X_{L}\right|-|X|}{|X|} & E_{P}(X)=\angle X_{L}-\angle X
\end{array}
$$

Additionally, an error operator for the relative error of a quantity $X$ and a corresponding approximation $X_{L}$ is also defined;

$$
E(X)=\frac{X_{L}-X}{X}
$$

This operator is used in section 6.7 to represent relative errors in the approximation of a solution $X_{L}$ obtained by mode matching (as described in section 3.6) instead of an LRF solution.

\subsection{Propagation constant}

For straight infinite waveguides having constant cross section and boundary conditions, the difference between the exact solution of the first acoustic mode and 
the LRF approximation is governed by the error in the propagation constant and the error in the velocity and pressure profiles. The latter errors are not considered here, the former errors are investigated for a 1D layer and prismatic circular cylindrical tube having no-slip and isothermal boundary conditions, and a 1D layer having no-slip adiabatic boundary conditions. The presented results are the relative errors in the LRF approximation of the propagation constant compared to the exact solution. Besides the accuracy of the LRF approximation for various conditions, the results also reveal trends in how the errors change with the parameters $\tilde{k}, \tilde{s}$ and $k / s$ which were previously not observed. In addition, the relative errors in the LRF approximation of the complex amplitude of a propagating acoustic wave after traveling the length of a number of waveguide thicknesses is presented for each case.

\subsubsection{Isothermal no-slip boundary conditions}

The relative error in the LRF approximation of the real and imaginary part of propagation constant compared to the exact solution is given in figure 6.6 for an infinite prismatic circular tube and a $1 \mathrm{D}$ layer, both having no-slip isothermal boundary conditions. The relative error is plotted as a function of $\tilde{k}$ for various values of $k / s$. The ratio $k / s$ is lowered incrementally by a factor of two. To improve intelligibility, points on the $k / s$ lines having a value of $\tilde{s}$ equal to $0.01,0.1,1,10$ and 100 are connected by thin, dotted, black lines. Note that the trends in the plots for the prismatic tube and the 1D layer are very similar and all the following comments apply to both cases.

As expected from results found in the literature [35, 10,36], the lines for different values of $k / s$ demonstrate that the LRF approximation is more accurate as the value of $k / s$ becomes bigger. The relative difference in the real part of the propagation constant (governing phase speed) and the relative difference in the imaginary part (governing attenuation) are both constant for constant $k / s$ and $s<1$. For $\tilde{k}>1$ frequencies above the so-called cut-on frequency are encountered. In this frequency range, wave propagation in $c d$-direction is possible and not all higher order acoustic modes will die out rapidly. A common misconception is that the LRF approximation fails in that range of the reduced frequency. Figure 6.6 demonstrates that for $\tilde{k}>1$, LRF models still approximate the first mode found by solving Kirchhoff's dispersion equation very well if $k / s \ll 1$. Nevertheless, practical use of LRF models in this range of the reduced frequency is limited. Boundary conditions at the waveguide openings can easily excite propagating higher order acoustic modes. Secondly, the criteria $\tilde{k}>1, k / s \ll 1$ imply relatively high values of the shear and thermal wave number, suggesting that the use of other approximating models (for instance based on the assumptions for inviscid adiabatic acoustics or 
boundary layer admittance) are more attractive.

An important feature observed for both waveguides is that the error decreases monotonously for $k / s<1 / \pi$. For $\tilde{s}<1$ the error in both the real and imaginary part decreases quadratically with $k / s$. For $\tilde{s}>1$, the error in the real part decreases cubically, while the error in the imaginary part decreases only linearly with $k / s$.

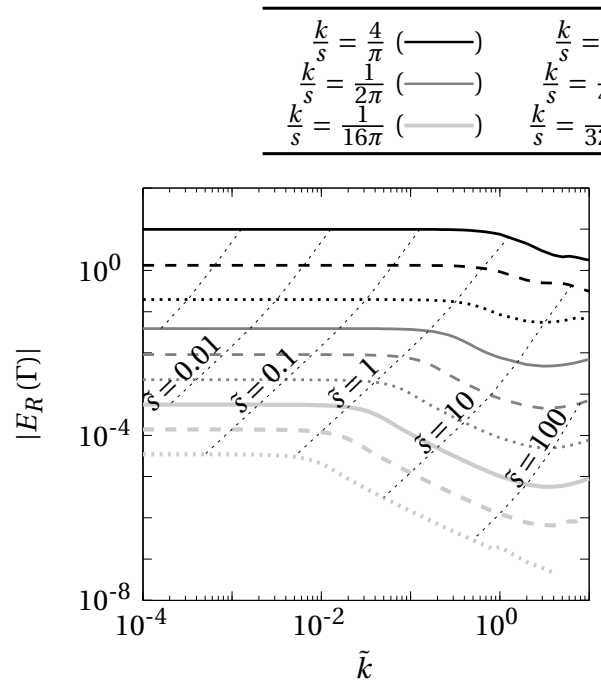

(a) Prismatic tube, real part

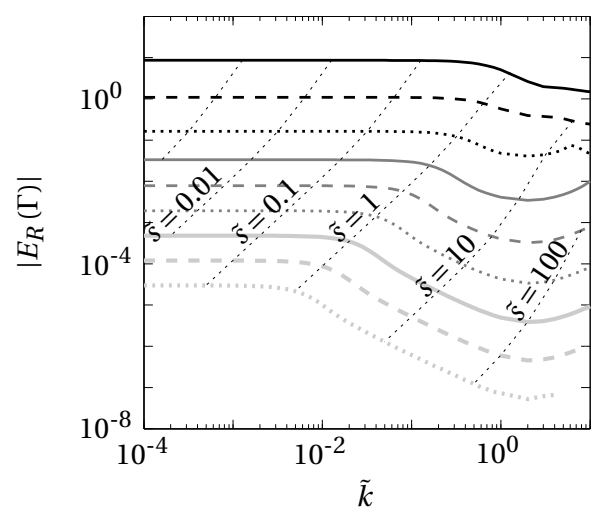

(c) Plane layer, real part

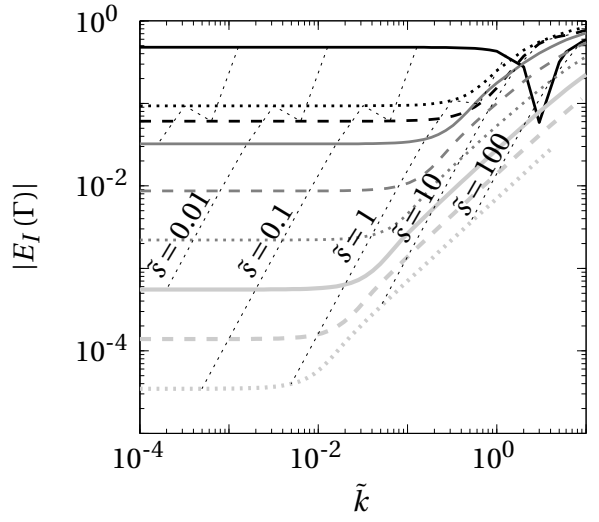

(b) Prismatic tube, imaginary part

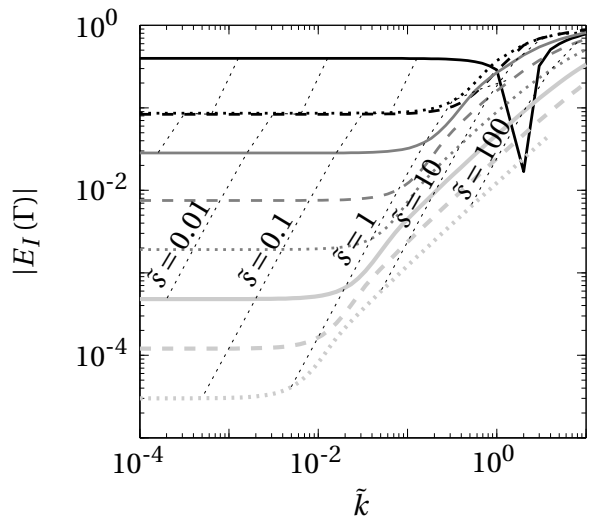

(d) Plane layer, imaginary part

Figure 6.6: Relative error in the LRF approximation of the propagation constant $\left(\Gamma_{L}\right)$ compared to the Kirchhoff solution $(\Gamma)$ for a prismatic cylindrical tube and a plain layer with fixed isothermal walls. 


\subsubsection{Adiabatic no-slip boundary conditions}

A presentation of the relative errors in the LRF approximation of the propagation constant (similar to figure 6.6) is given for no-slip adiabatic boundary conditions in figure 6.7. Again, the relative error is plotted as a function of $\tilde{k}$ for various values of $k / s$ and points of constant values of $\tilde{s}$ are connected by thin, dotted, black lines.

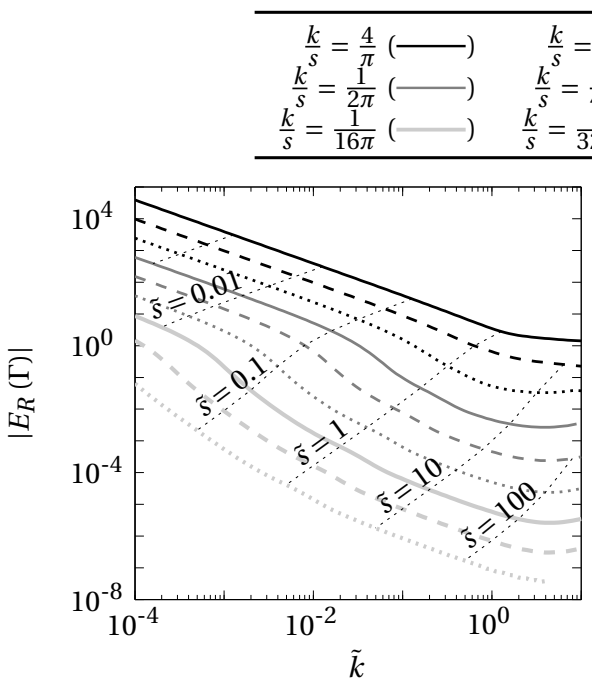

(a) Real part

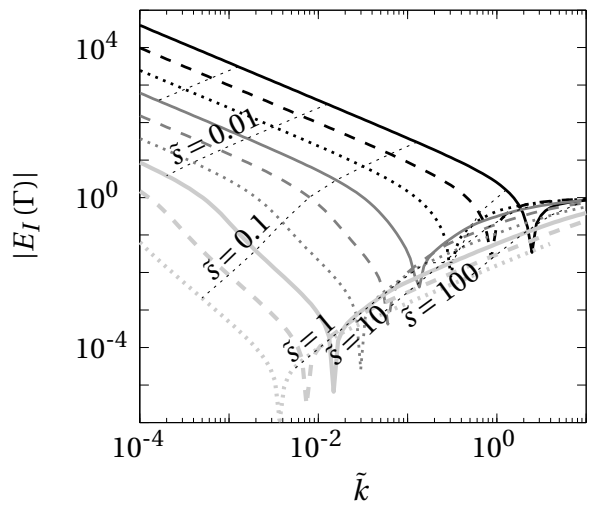

(b) Imaginary part

Figure 6.7: Error in the LRF approximation of the propagation constant $\left(\Gamma_{L}\right)$ compared to the Kirchhoff solution $(\Gamma)$ for a plain layer with fixed adiabatic walls.

When the results in figure 6.7 are compared to those in figure 6.6(c) and 6.6(d), the behavior is found to be very similar for $\tilde{s}>1$. On the other hand, for $\tilde{s}<1$, the results are, perhaps counterintuitively, radically different. The relative error of both the real and imaginary part grows linearly as $\tilde{k}$ decreases, reaching values well above unity. As a consequence, even for $k / s \ll 1$ the relative error in the approximation of the propagation constant is not necessarily small. In fact, it can be made arbitrarily big, by decreasing $\tilde{k}$. The causes for the observed differences between isothermal and adiabatic boundary conditions are discussed in appendix K. 


\section{Practical relevance}

It can be inferred from results in figure 6.7 that the error decreases as the value of $\tilde{s}^{2} / \tilde{k}$ rises $^{10}$. It is observed that the relative error will only be significant if the value of $\tilde{s}^{2} / \tilde{k}$ is not too large. For instance, a value of $\tilde{s}^{2} / \tilde{k}>15$ leads to a relative error smaller than $1 \%$. The value of $\tilde{s}^{2} / \tilde{k}$ can also be expressed in terms of the characteristic length $l$ using the equations in (2.34). For air under standard atmospheric conditions this yields:

$$
l=\left(\frac{\mu}{\rho_{0} c_{0}}\right) \frac{\tilde{s}^{2}}{\tilde{k}}=4.44 \cdot 10^{-8} \frac{\tilde{s}^{2}}{\tilde{k}}
$$

This implies that values of $\tilde{s}^{2} / \tilde{k}$ that lead to significant errors are only encountered for waveguides with very small cross-sectional dimensions. In fact, relative errors larger than $1 \%$ will only be encountered for cases where $l$ (and thus the dimension of the waveguide) is so small that the assumptions required for continuum mechanics ${ }^{11}$ will not hold.

\subsubsection{Error in complex amplitude}

Next, it is investigated how the errors in the approximation of the propagation constant influence the complex amplitude of propagating waves. Figure 6.8 shows the relative error in amplitude and phase of an acoustic wave after traveling eight layer thicknesses in a 2D layer with no-slip isothermal and adiabatic boundary conditions. The error is plotted as a function of $\tilde{k}$ for different values of $k / s$. Again, the points on the $k / s$ lines that have a constant value of $\tilde{s}$ are connected by thin, dotted, black lines. Note that the length of eight wavelengths is arbitrary. Since the errors in the approximation of the complex amplitude vary linearly with the length of the waveguide, changing lengths will merely shift all curves up or down the $y$-axis.

From figure 6.8, it is clear that the error in the approximation of the complex amplitude decreases with $k / s$ as is to be expected. Naturally, the errors for $\tilde{s}>1$ are very similar for the isothermal and adiabatic case, since the propagation constant which governs the errors in figure 6.8 are also very similar. For $\tilde{s}<1$ the errors differ for the isothermal and adiabatic case. Note that in contrast to the errors in the propagation constant, the errors in the complex amplitude stabilize for smaller values of $\tilde{k}$ and ultimately also assume a constant value for constant $k / s$. Despite the differences that are observed, the LRF models offer a very high level of accuracy

\footnotetext{
${ }^{10}$ Recall that $k / s=\tilde{k} / \tilde{s}$

${ }^{11}$ A requirement for the use of models based on continuum mechanics is that the length scales of the considered fluid domain are larger than $10^{-7}$ (following [10]).
} 
for both the isothermal and adiabatic case for most practically relevant values of $\tilde{s}$ and $k / s$.

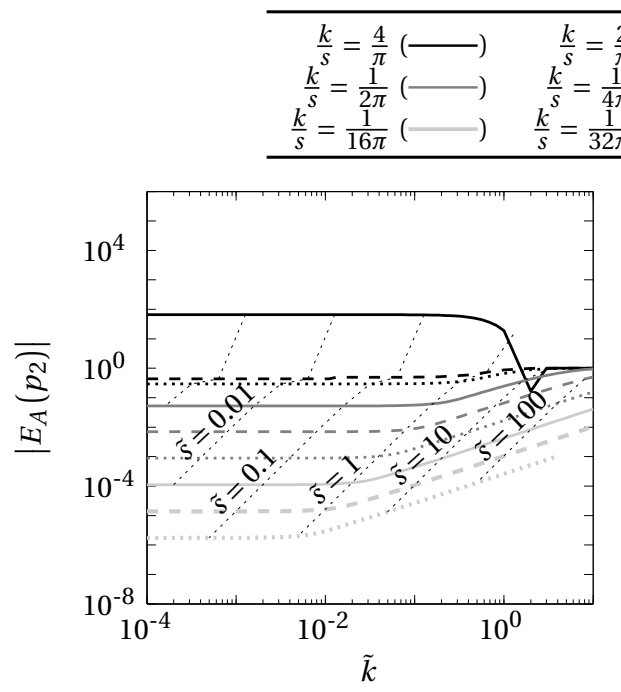

(a) Isothermal walls, error in amplitude

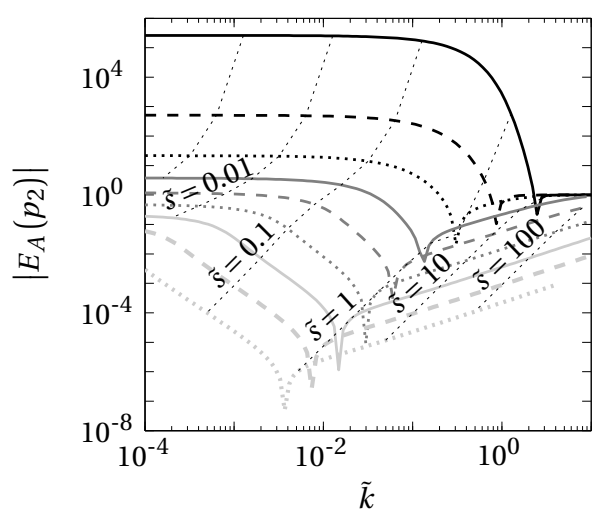

(c) Adiabatic walls, error in amplitude

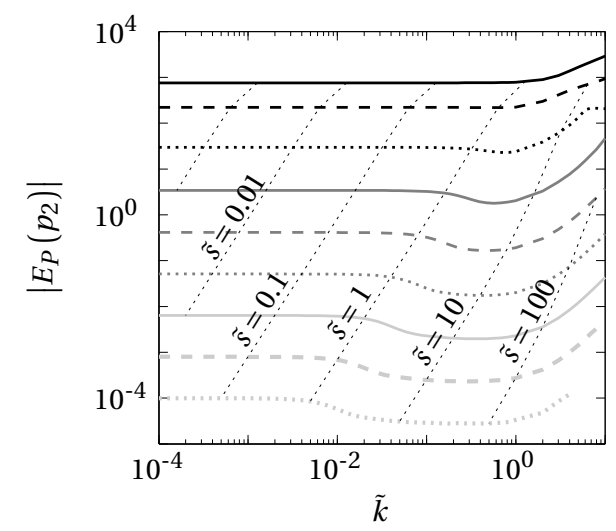

(b) Isothermal walls, error in phase

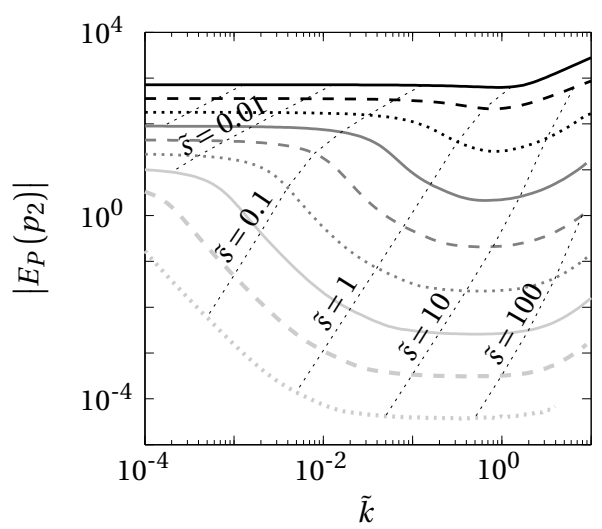

(d) Adiabatic walls, error in phase

Figure 6.8: Error in the LRF approximation of the amplitude and complex phase of a wave after traveling eight thicknesses in a plain layer with fixed isothermal walls (a) (b), and fixed adiabatic walls (c)(d). 


\subsection{Bifurcation of waveguides}

The previous section looked at errors in the LRF approximation of wave propagation in infinite straight waveguides having constant cross section and boundary conditions. In this section and the next, additional errors due to abrupt changes in boundary conditions are investigated. The present section deals with the case of waveguide bifurcation; a system is considered where a relatively wide waveguide is connected to many narrow neighboring waveguides of identical cross section (e.g., rectangular, triangular or hexagonal). A cutout of a $2 \mathrm{D}$ version of such a system, where a wide layer bifurcates into many narrow layers, is sketched in figure 6.9 (a).

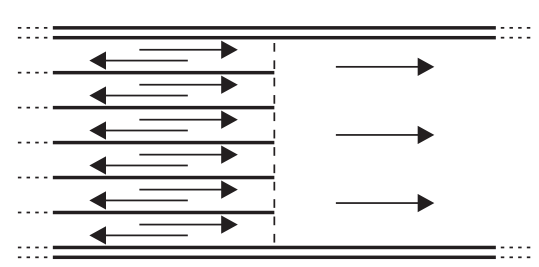

(a)

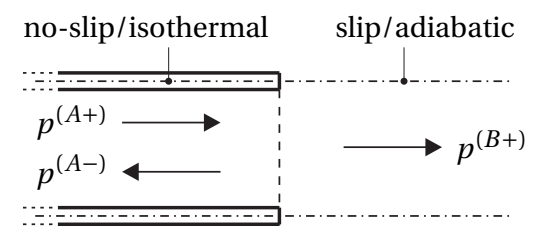

(b)

Figure 6.9: Bifurcation of a wide waveguide into multiple narrow waveguides having isothermal no-slip boundary conditions (a). Abstract representation of a scattering problem involving a waveguide system based on a recurring subsystem of one narrow waveguide.(b).

No-slip isothermal boundary conditions are assumed at all waveguide surfaces. The wide waveguide is assumed to be wide enough to allow the use of inviscid adiabatic acoustics, while the narrow waveguides have cross-sectional dimensions that require a model that takes viscothermal effects into account. In addition, the frontal surface of the boundaries of the narrow waveguide at the junctions is assumed to be relatively small compared to the cross-sectional surface of the wide waveguide. Under these conditions, the behavior of the total system can now be efficiently modeled by modeling only one narrow waveguide, having noslip isothermal boundary conditions, which is coupled to a waveguide of identical cross section, having slip-adiabatic boundary conditions. In other words, the boundaries of the narrow waveguide can be extended into the wide waveguide, and act as symmetry planes. The resulting subsystem for a single narrow waveguide is sketched in 6.9(b). This section investigates the scattering problem that results if an incident wave is impinging on the surface where the boundary conditions change. 
Figure 6.10 depicts the velocity and temperature field for various values of the shear wave number. The results, which are obtained with the FE models presented in chapter 5 , give an impression of the errors that are locally made by using a coupled LRF model. In contrast to fields for the coupled LRF model, which exhibit discontinuities at the interface, the velocity and temperature profiles over the waveguide cross section change gradually over a distance in the order of the viscous and thermal wave length for $\tilde{s}>1$, and a distance in the order of the waveguide width for $\tilde{s}<1$.
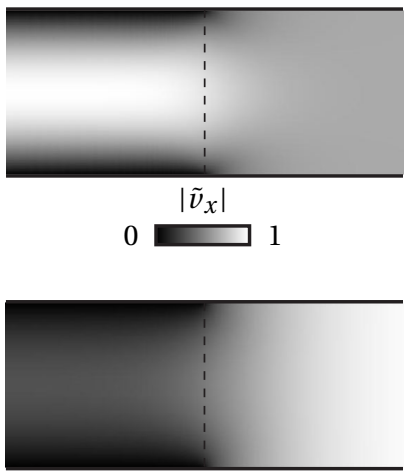

0 0.25

(a) $\tilde{s}=1, \tilde{k}=0.001$

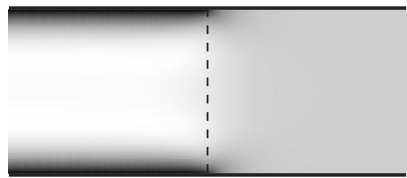

0
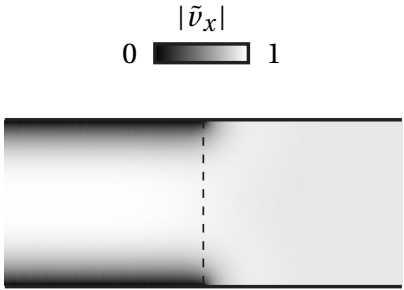

0

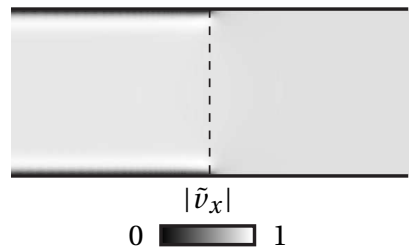

0

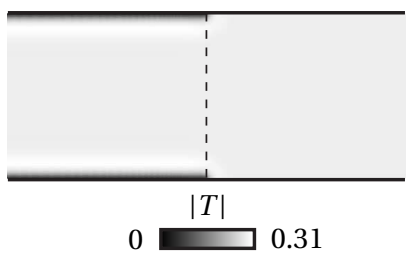

(c) $\tilde{s}=20, \tilde{k}=0.001$

Figure 6.10: Velocity and temperature fields of FE model of the scattering problem corresponding to waveguide bifurcation for various values of $\tilde{s}$.

The resulting errors in the LRF approximation of the magnitude and phase of the complex amplitude of the transmitted and reflected acoustic waves are plotted in figure 6.11 as a function of $\tilde{k}$. The different lines correspond with constant values of $k / s$. Points on the curves that have a constant value of $\tilde{s}$ are connected by thin, dotted, black lines. Note that only the errors due to the LRF approximation are considered here. The errors due to the assumptions that are necessary to arrive at the scattering problem depicted in figure 6.9(b) may be larger than the errors presented in figure 6.11 .

The most important feature of the results in figure 6.11 is that all errors in the LRF approximation of the complex amplitude of the transmitted wave decrease linearly (or faster) with $k / s$ (for $k / s<1 / \pi$ ). It can be concluded that LRF models involving sudden changes in boundary conditions as proposed in this section are very accurate for $k / s \ll 1$ and $\tilde{k}<1$, and become more accurate as $k / s$ decreases. In most practical cases, the errors due to neglecting the frontal surface of 
\begin{tabular}{lllll}
\hline$\frac{k}{s}=\frac{1}{\pi}(\longrightarrow)$ & $\frac{k}{s}=\frac{1}{2 \pi}(\cdots \cdots)$ & $\frac{k}{s}=\frac{1}{4 \pi}(\longrightarrow)$ & $\frac{k}{s}=\frac{1}{8 \pi}(\cdots \cdots)$ & $\frac{k}{s}=\frac{1}{16 \pi}(\longrightarrow)$ \\
\hline
\end{tabular}

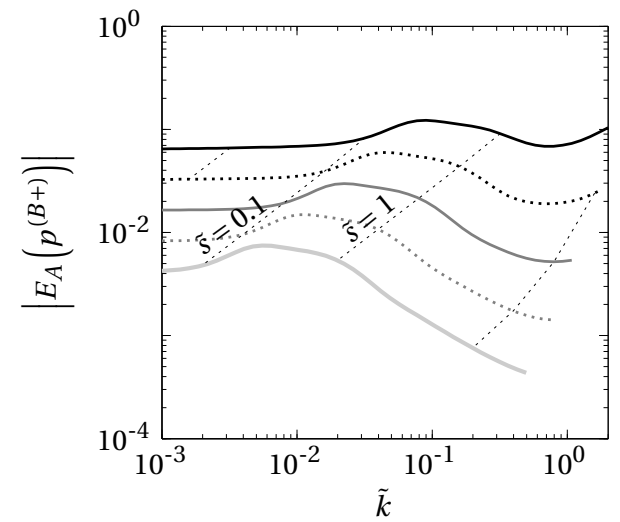

(a) Transmitted wave, error in amplitude

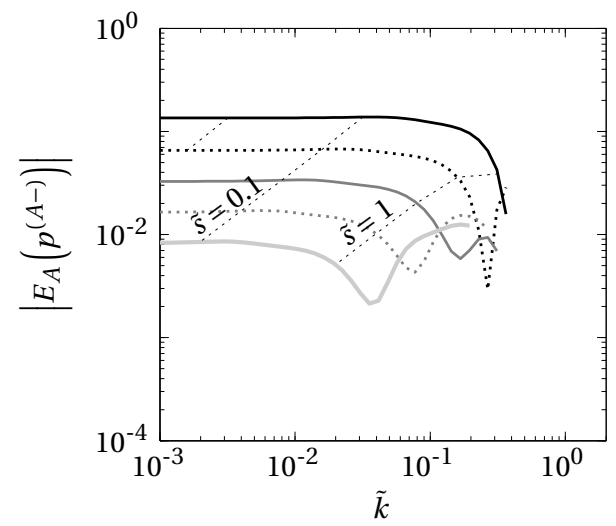

(c) Reflected wave, error in amplitude

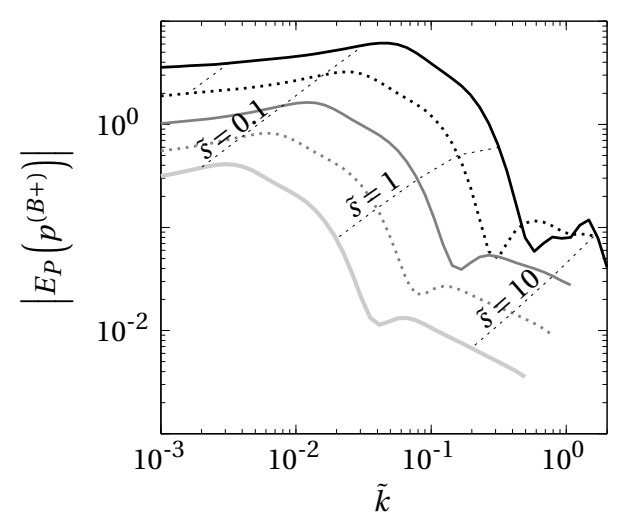

(b) Transmitted wave, error in phase

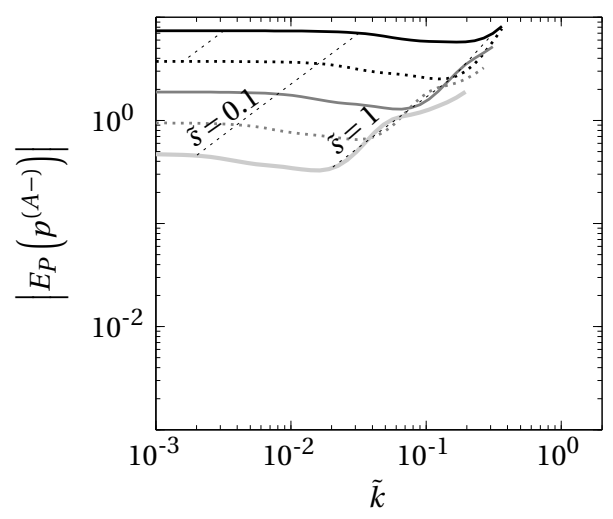

(d) Reflected wave, error in phase

Figure 6.11: Relative error in the LRF approximation of the amplitude and phase of the transmitted and reflected wave as a function of $\tilde{k}$ for various values of $k / s$.

the boundaries of the narrow waveguides at their opening and neglecting the influence of the boundaries of the wide waveguide probably outweigh the errors due to using an LRF approximation. Note that as $\tilde{k}$ increases, the error due to using an LRF approximation grows, and higher order modes have to be taken into account (or suitable length/end corrections have to be used) to arrive at accurate results. 


\subsection{Change in thermal boundary conditions}

In the previous section a simultaneous change in both traction and thermal boundary conditions was examined. In this section the effects of a sudden change in thermal boundary conditions are considered, while for the traction boundary conditions a no-slip condition is assumed throughout the entire waveguide. Again, two infinite straight waveguides of equal constant cross section are coupled. One waveguide has isothermal boundary conditions, while an adiabatic boundary condition is assumed for the other waveguide. Again, an incident acoustic propagating wave is impinging on the surface of the junction. The resulting scattering problem using $1 \mathrm{D}$ layers as waveguides is depicted in figure 6.12.

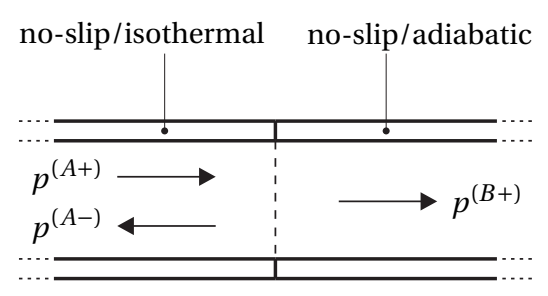

Figure 6.12: Abstract representation of a scattering problem involving a waveguide system with an abrupt change in thermal boundary conditions.

The resulting temperature fields very closely resemble those depicted in figure 6.10. The velocity profiles in both waveguide sections resemble the profiles observed in the left part of these plots very closely, and appear not to be affected by the sudden change in thermal boundary conditions. This agrees with the fact that the velocity fields for the first acoustic mode of a waveguide are virtually equal for isothermal and adiabatic boundary conditions. Nevertheless, due to the difference in characteristic impedance of waves influenced by viscothermal effects and free waves, part of the incident wave is reflected. The magnitude and phase of the complex amplitude of the transmitted and reflected waves are plotted in figure 6.13 as a function of $\tilde{k}$ for the corresponding FE and LRF model. Again, different lines indicate constant values of $k / s$ for the LRF model, while the corresponding results of the FE model are marked by crosses and pluses. Points on the lines for the LRF results that have a constant value of $\tilde{s}$ are connected by thin, dotted, black lines.

For $\tilde{s}>1$ the difference in impedance is small and the amplitude of the reflected wave, plotted in figure 6.13, is also small. For $\tilde{s}<1$ the difference in impedance is significant and consequently, so is the amplitude of the reflected wave and the impact on the amplitude of the transmitted wave. For $\tilde{s}>1$ the FE results seem to converge to the LRF results as $\tilde{s}$ increases, i.e., both models predict that 


\begin{tabular}{llllll}
\hline LRF & $\frac{k}{s}=\frac{1}{\pi}(\longrightarrow)$ & $\frac{k}{s}=\frac{1}{2 \pi}(\cdots \cdots)$ & $\frac{k}{s}=\frac{1}{4 \pi}(\longrightarrow)$ & $\frac{k}{s}=\frac{1}{8 \pi}(\cdots \cdots)$ & $\frac{k}{s}=\frac{1}{16 \pi}(\longrightarrow)$ \\
FEM & $\frac{k}{s}=\frac{1}{\pi}(+++)$ & $\frac{k}{s}=\frac{1}{2 \pi}(\times \times \times)$ & $\frac{k}{s}=\frac{1}{4 \pi}(+++)$ & $\frac{k}{s}=\frac{1}{8 \pi}(\times \times \times)$ & $\frac{k}{s}=\frac{1}{16 \pi}(+++)$ \\
\hline
\end{tabular}

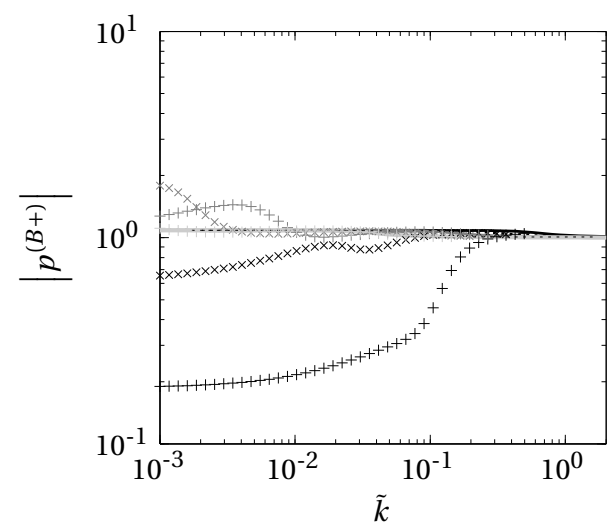

(a) Transmitted wave, amplitude

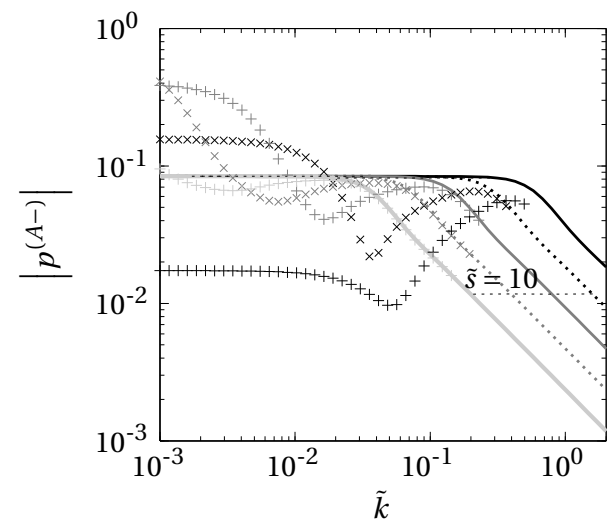

(c) Reflected wave, amplitude

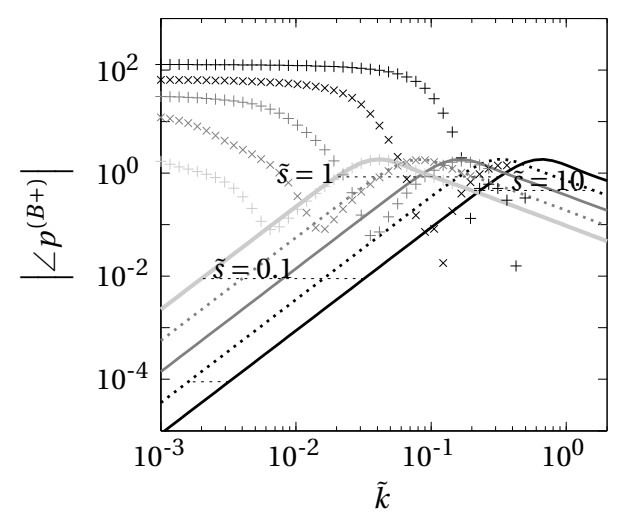

(b) Transmitted wave, phase

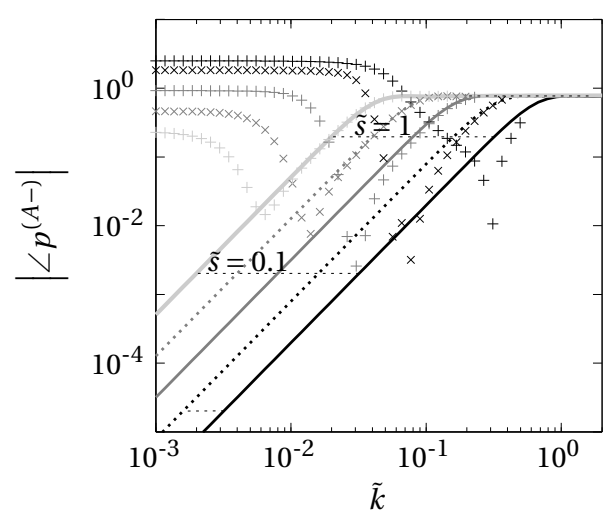

(d) Reflected wave, phase

Figure 6.13: Amplitude and phase of the transmitted and reflected wave as a function of $\tilde{k}$ for both the LRF and FE solution and various values of $k / s$.

the influence of the difference in impedance is small. However, for $\tilde{s}<1$, the results predicted by the LRF model are very different from those predicted by the FE model. Differences in the amplitude in the order of unity and differences in the angle in the order of 180 degrees are observed in figure 6.13. The large difference are reflected by the relative errors presented in figure 6.14.

The most important feature of these results is that the error decreases with 


\begin{tabular}{lllll}
\hline$\frac{k}{s}=\frac{1}{\pi}(\longrightarrow)$ & $\frac{k}{s}=\frac{1}{2 \pi}(\cdots \cdots)$ & $\frac{k}{s}=\frac{1}{4 \pi}(\longrightarrow)$ & $\frac{k}{s}=\frac{1}{8 \pi}(\cdots \cdots)$ & $\frac{k}{s}=\frac{1}{16 \pi}(\square)$ \\
\hline
\end{tabular}

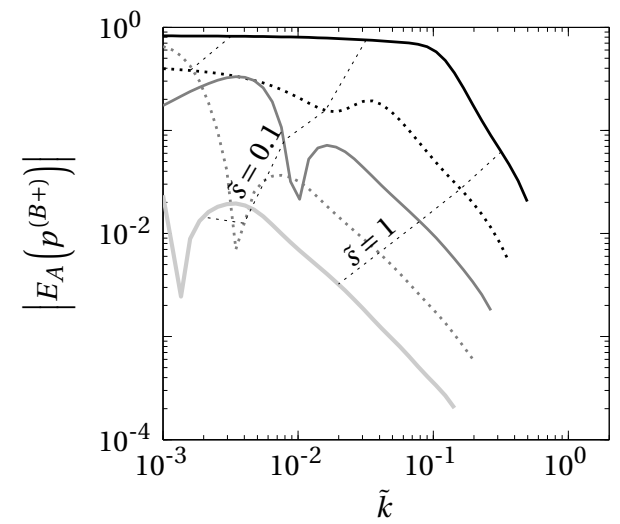

(a) Transmitted wave, error in amplitude

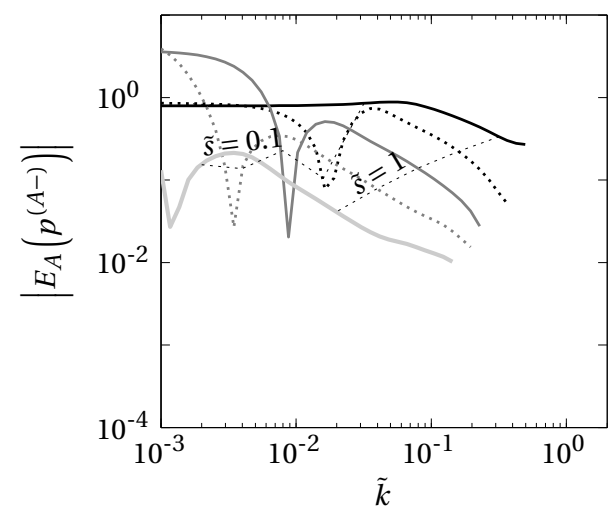

(c) Reflected wave, error in amplitude

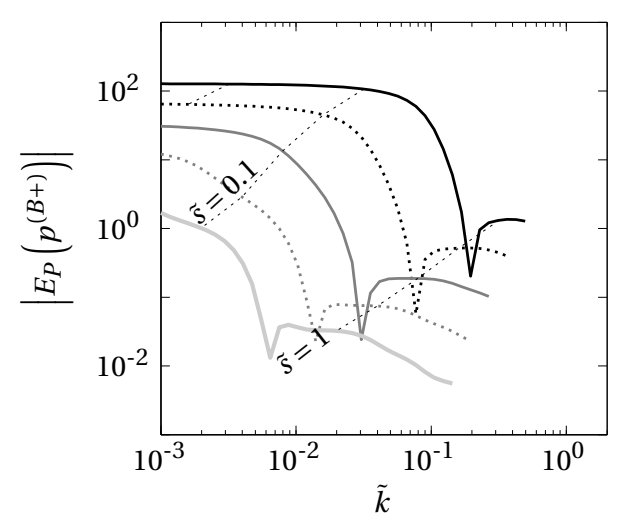

(b) Transmitted wave, error in phase

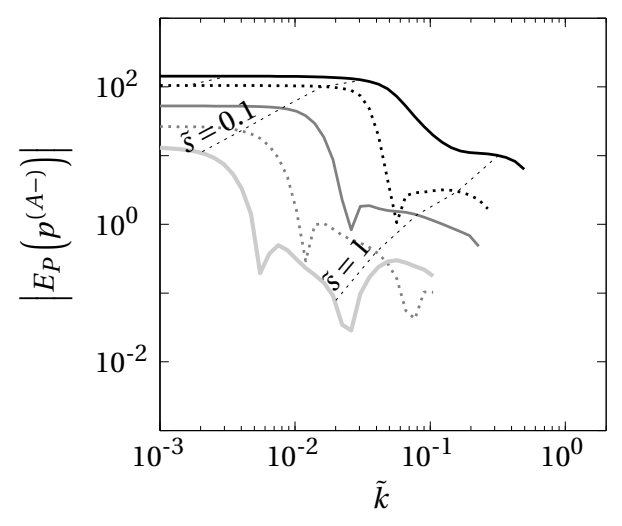

(d) Reflected wave, error in phase

Figure 6.14: Error in the LRF approximation of the amplitude and phase of the transmitted and reflected wave as a function of $\tilde{k}$ for various values of $k / s$.

$k / s$ for $\tilde{s}>1$. The large errors for $\tilde{s}<1$ can be explained in part by the fact that the propagation constant is not well approximated by the LRF model for adiabatic boundary conditions, as was pointed out in section 6.2. Nevertheless, the observed errors are dominated by the fact that for no-slip adiabatic boundary conditions, the first thermal mode increasingly resembles the first acoustic mode as $\tilde{s}$ decreases. This causes the LRF model, which only accounts for the effects of the first acoustic mode, to become inaccurate. A discussion on this phenomenon is given in appendix K.2. 
Based on the results in figure 6.14, it can be concluded that the errors will only become significant for values of $\tilde{s}$ and $k / s$ for which the assumptions required for continuum mechanics do not hold (analogue to the results for the LRF approximation of the propagation constant).

\subsection{Change in cross-sectional geometry}

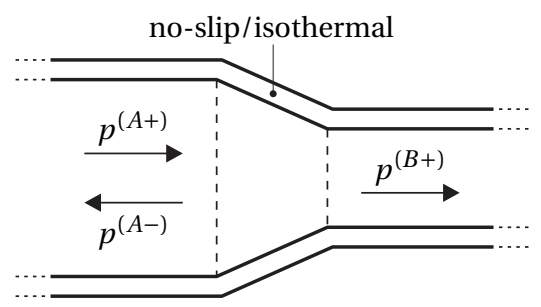

(a)

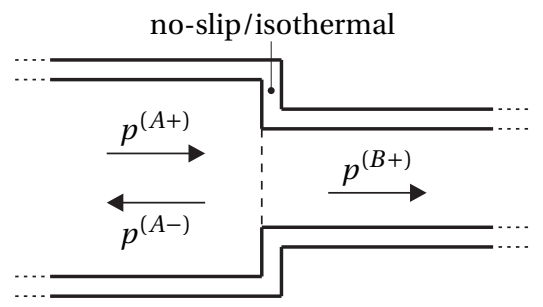

(b)

Figure 6.15: Abstract representation of scattering problems involving a waveguide system with a gradual (a), and abrupt (b) change in cross-sectional geometry.

Besides changes in thermal- or traction boundary conditions which are discussed in the previous two sections, another source of inaccuracies in coupled LRF models are changes in the cross-sectional geometry of a waveguide. The errors due to such changes are investigated in this section. Both the influence of the magnitude of the change and the rate at which the changes occur are considered.

Recall that the value of the ratio $k / s$ is not dependent on geometry, implying that the ratio has a constant value throughout the waveguide. On the other hand, the dimensionless acoustic wave number $\tilde{k}$ and dimensionless shear wave number $\tilde{s}$, which are also used as characteristic parameter, are dependent on the waveguide cross section, and therefore, will not be constant throughout a waveguide with changing cross section. In all cases, the values for $\tilde{k}$ and $\tilde{s}$ are based on the characteristic length that corresponds to the widest part of the waveguide.

Again, the scattering problem described in 6.1.3 and the corresponding LRF and FE models are used to investigate the accuracy of an approximate LRF solution compared to a reference FE solution. Two infinite waveguide sections of different cross section are coupled, either directly 6.15(b), or by a third transitional waveguide 6.15(a). For the cases where the change in cross-sectional geometry is sudden, the relative errors that are presented do not include the errors due to wave propagation in the infinite waveguide sections (just as for the cases in the previous sections). For the cases where the change in cross-sectional geometry is 


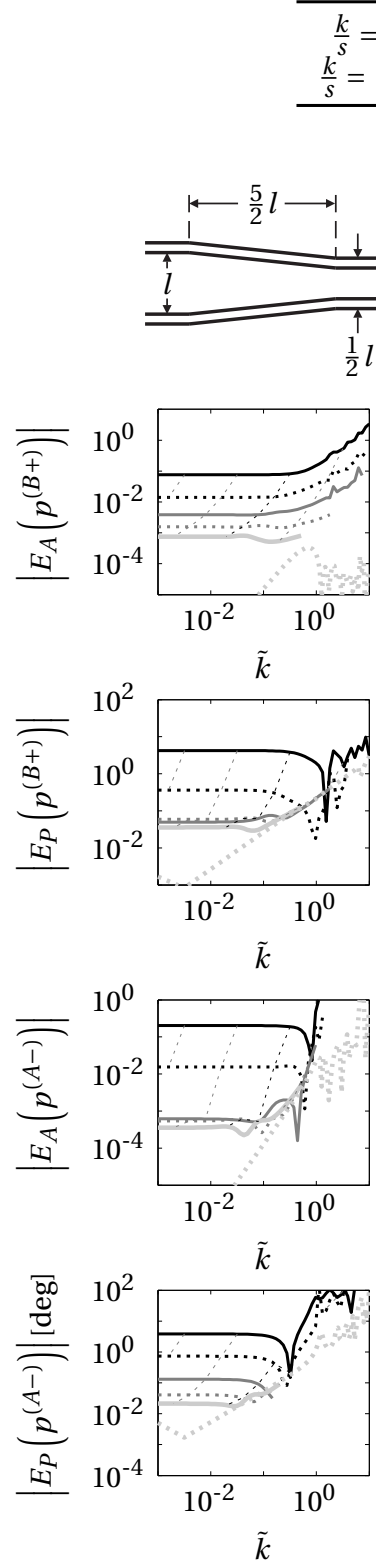

(a)
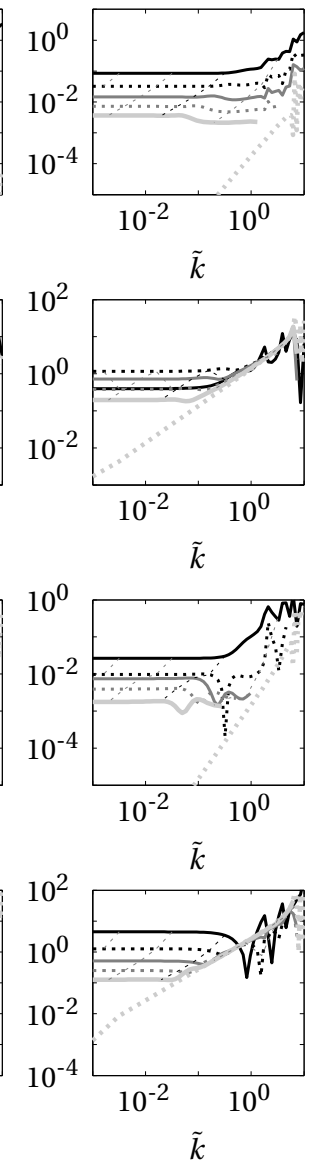

(b)
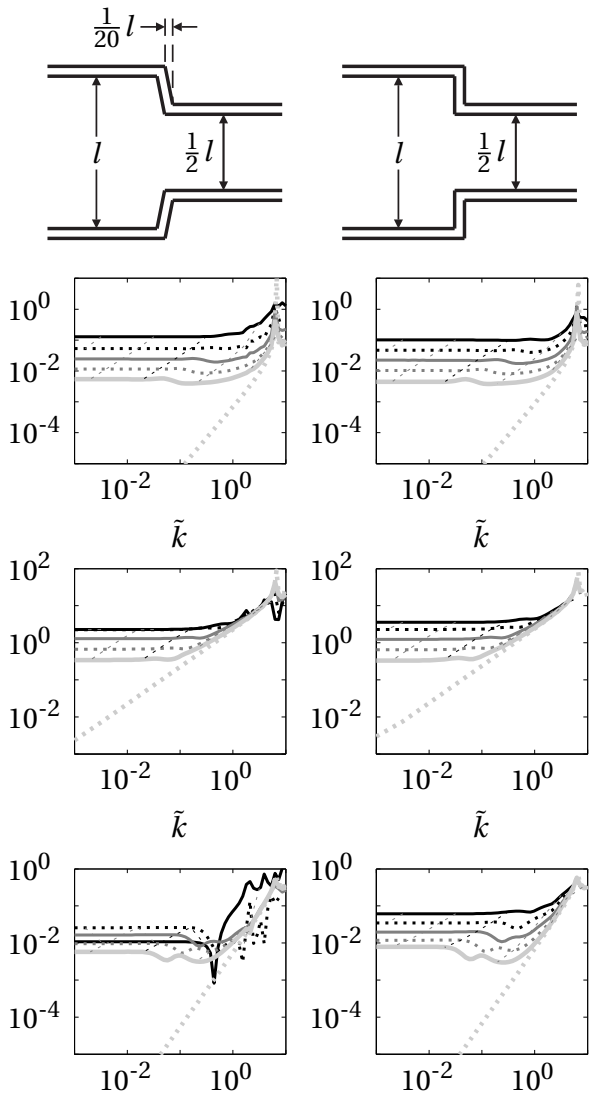

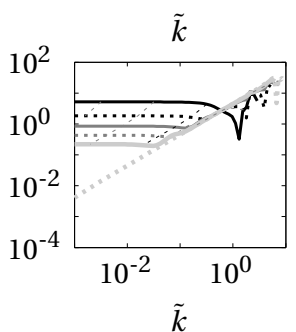

(c)

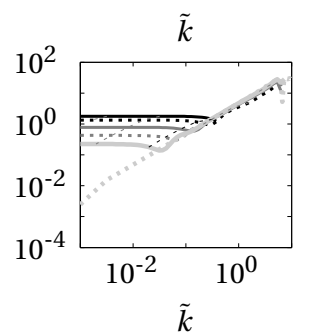

(d)

Figure 6.16: Relative error in the LRF approximation of the amplitude and the phase of the transmitted and reflected waves for different waveguide junctions and various values of $k / s$. The thin dashed lines $(---)$ connect data for which $\tilde{s}$ is constant $(\tilde{s}=0.01,0.1$, 1 , and 10) with the black line indicating $\tilde{s}=1$. 


\begin{tabular}{rrr}
\hline$\frac{k}{s}=\frac{1}{\pi}(\longrightarrow)$ & $\frac{k}{s}=\frac{1}{4 \pi}(\longrightarrow)$ & $\frac{k}{s}=\frac{1}{16 \pi}(\longrightarrow)$ \\
$\frac{k}{s}=\frac{1}{2 \pi}(\cdots \cdots)$ & $\frac{k}{s}=\frac{1}{8 \pi}(\cdots \cdots)$ & $\frac{k}{s}=0(\cdots \cdots)$ \\
\hline
\end{tabular}
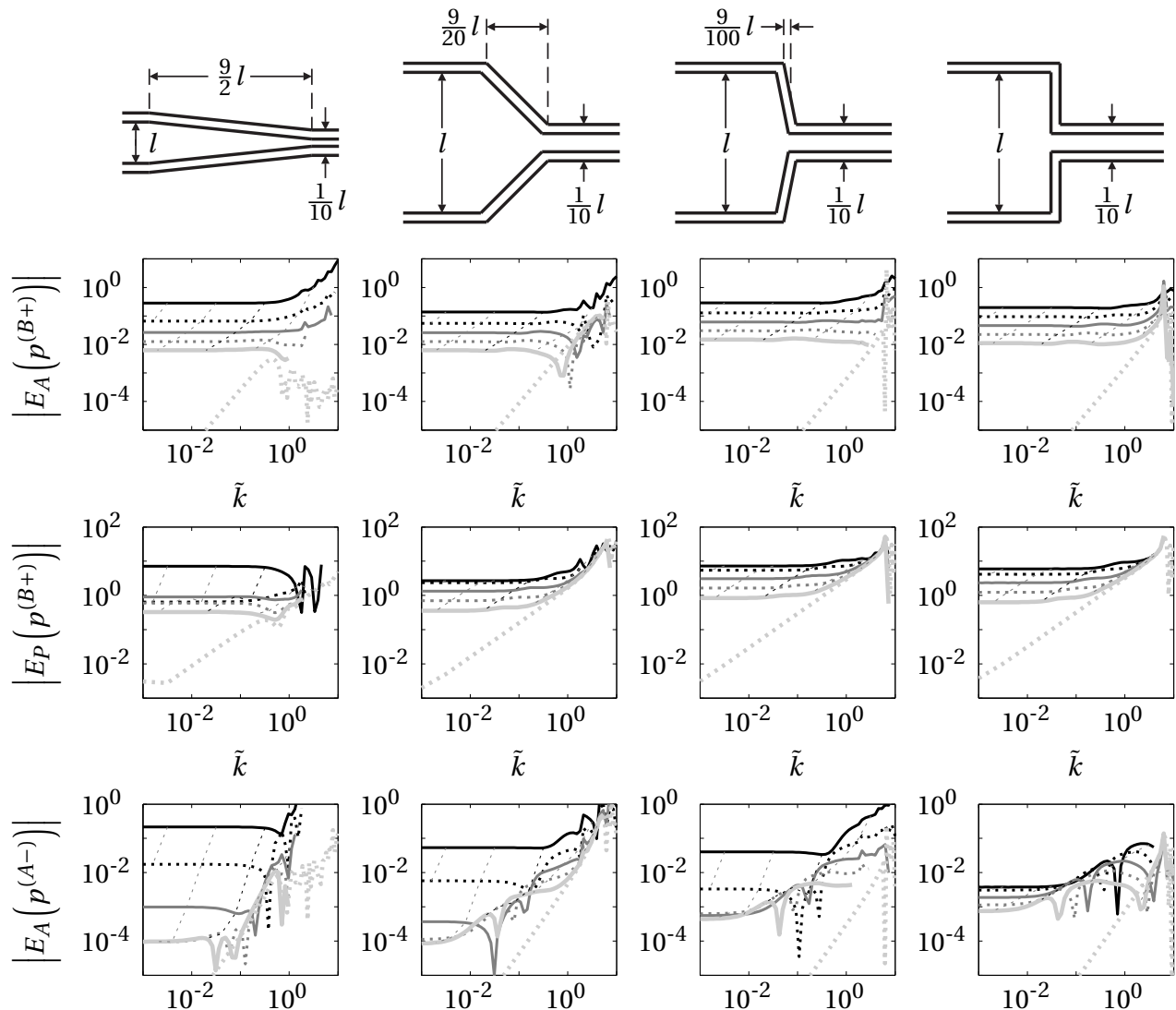

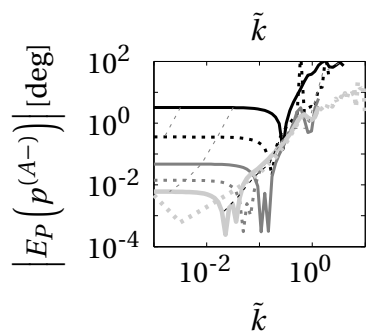

(a)

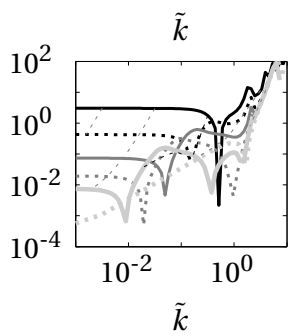

(b)

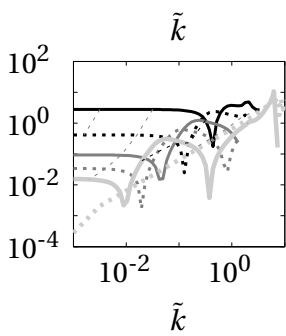

(c)

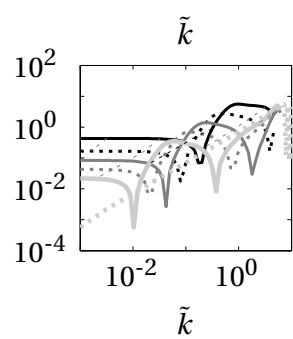

(d)

Figure 6.17: Relative error in the LRF approximation of the amplitude and the phase of the transmitted and reflected waves for different waveguide junctions and various values of $k / s$. The thin dashed lines (- - ) connect data for which $\tilde{s}$ is constant $(\tilde{s}=0.01,0.1$, 1 , and 10 ) with the black line indicating $\tilde{s}=1$. 
gradual, the errors do include the errors due to wave propagation in the transition waveguide. Naturally, these errors are expected to become larger as the transition is more gradual, and the waveguide section with the transition is longer. Note that the number of waveguide sections that are used in the LRF two-port approximation to represent the transitional part of the system is such, that the observed errors do not change significantly when more waveguide sections are used. In figure 6.16 and 6.17 the relative errors are given for a change in layer thickness of a factor two and ten, respectively. Different rates of changes in layer thickness are considered for both cases. Again, points on the lines for constant values of $k / s$ for which $\tilde{s} \in\{0.01,0.1,1,10,100\}$ are connected by thin, dotted lines (the black line indicates $\tilde{s}=1)$.

\section{Dominant causes for errors}

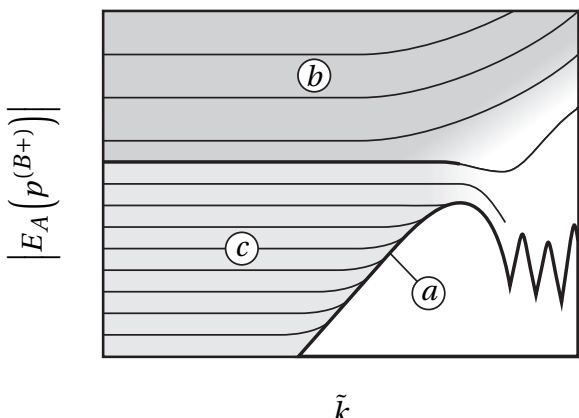

(a) Schematic representation of the relative errors plotted in figure 6.16(a) and 6.16(a)

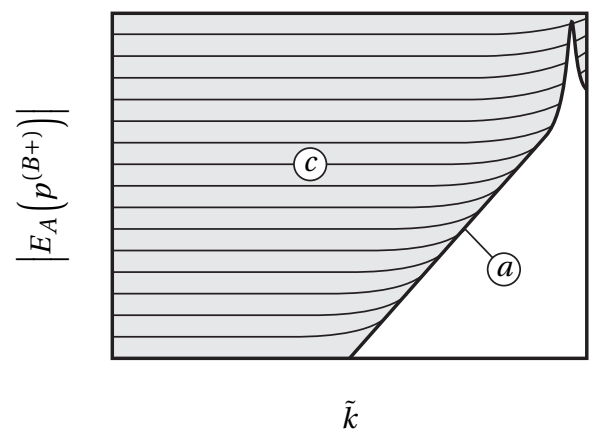

(b) Schematic representation of the relative errors plotted in figure 6.17(d) and $6.17(\mathrm{~d})$

Figure 6.18: Schematic representation of the trends in the relative error for constant values of $k / s$ as a function of $\tilde{k}$. The error for $k / s=0$ is dominated by omission of the higher order acoustic modes (a). The error in the area indicated by (b) is dominated by the error in the LRF approximation of the first mode. The error in the area indicated by (c) is dominated by the omission of both vorticity and higher order acoustic modes.

Certain trends can be recognized in the plots of the relative error presented in figure 6.16 and 6.17. To identify the different trends more easily, a schematic representation of the relative error in the amplitude of the transmitted wave for two cases is given in figure 6.18. One of the most obvious trends is that the relative errors decrease or remain unchanged as the value of $k / s$ is lowered. For small values of $\tilde{k}$ the error is constant with $\tilde{k}$. For higher values of $\tilde{k}$ the error for most values of $k / s$ coincides with the error for $k / s=0$. The case $k / s=0$ corresponds 
with inviscid adiabatic acoustics and the presented error is based on an inviscid adiabatic FE and two-port model. In that case, the observed error can only be caused by the omission of higher order modes. The results suggest that the error in the LRF model is dominated by omission of the higher order acoustic modes when the error for $k / s \neq 0$ coincides with the error for $k / s=0$.

For cases where the change in cross section is gradual (as, for instance, in figure 6.16(a) and 6.17(a)), the area where the relative error is constant with $k / s$ can be split into two areas based on the spacing between subsequent lines of constant $k / s$. This split into two regions having big and small spacing between lines is indicated in figure 6.18(a). For cases where the change in cross section is sudden (as, for instance, in figure 6.16(d) and 6.17(d)), the spacing between subsequent lines of constant $k / s$ appears to be equal to the smaller spacing independent of the value of $k / s$. For cases with a sudden change in geometry, the relative error does not include errors due to using an LRF approximation of the propagation constant for modeling wave propagation of the first acoustic mode. And since the error is not dominated solely by the omission of the higher order acoustic modes, it is most likely that the error is dominated by the omission of vorticity and thermal modes. In section 6.7 it is shown that the thermal modes do not influence the error, and the omission of the vorticity modes indeed have a large influence on the error in these regions. It turns out that the errors of this magnitude result if the influence of either the viscosity or higher order acoustic modes is not accounted for. Summarizing, the following conclusions can be drawn based on the results presented above:

- In general, the relative errors due to changes in cross section decrease as $k / s$ goes to zero. Nevertheless, the relative error is bounded from below by the error obtained for the inviscid adiabatic case. For cases where the errors coincide with the line for $k / s=0$ (indicated by (a) in figure 6.18), the observed error is due to omission of the higher order acoustic modes.

- For gradual changes in geometry, and larger values of $k / s$ (area indicated by (b) in figure 6.18(a)), the relative error due to changes in cross section is dominated by using an LRF approximation of the propagation constant to model wave propagation of the first acoustic mode.

- For sudden changes in geometry or gradual changes in geometry and smaller values of $k / s$ (area indicated by (c) in figure 6.18), the relative error due to changes in cross section is dominated by the omission of both vorticity and higher order acoustic modes.

- Apart from cases where the error is dominated by omission of the higher 
order acoustic modes, the observed errors are small for $k / s \ll 1$. Note, however, the errors can add up if multiple waveguides of different cross section are coupled. This can reduce the range of $k / s$ where LRF models are guaranteed to produce results of a certain accuracy.

\subsection{Change in curvature}
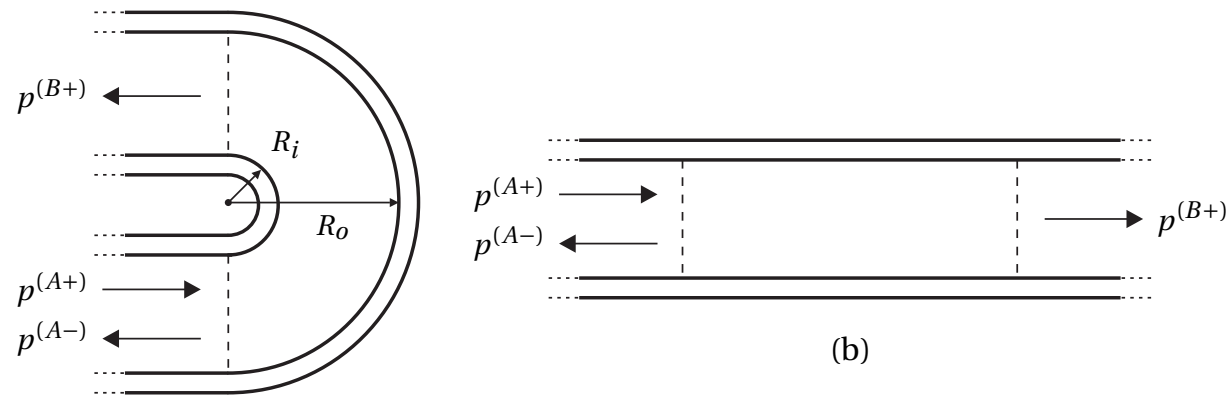

(b)

(a)

Figure 6.19: Abstract representation of a scattering problem for a waveguide system involving a curved section (a), and a waveguide system of equivalent length involving only straight waveguides (b).

For curved waveguides, the operator $\Delta_{c d}$ in equation (4.11b) cannot be written as a scalar operator. As a result, the term $\Delta_{c d} \mathbf{v}_{p d}$ not only depends on the velocity in $p d$-direction but also introduces terms that depend on $\mathbf{v}_{c d}$. These additional terms have to be discarded in order to follow the solution strategy presented in section 4.1.3 leading to the solution presented in section 4.2.2. Neglecting these terms, however, can lead to small but significant errors.

A closer look at the different terms in the full LNSF equations shows that due to the approximation the accuracy of the LRF is not only determined by the magnitude of the ratios $k / s$ and $k / s_{t}$, but also by the distance of the curved waveguide with respect to the origin. In the case of a layer with a constant curvature, the neglected terms become significant as curvature increases. Figure 6.20 illustrates the effect of the (additional) approximation on the accuracy of the propagation constant obtained with the LRF model compared to the exact solution obtained with the techniques in chapter 3. It is clear that for the considered values of $k / s$ and $\tilde{s}$ the relative errors in the propagation constant for larger curvatures are considerably bigger than those observed for the LRF model of an equivalent straight waveguide section in figure 6.6(c) and 6.6(d). Another difference between the er- 


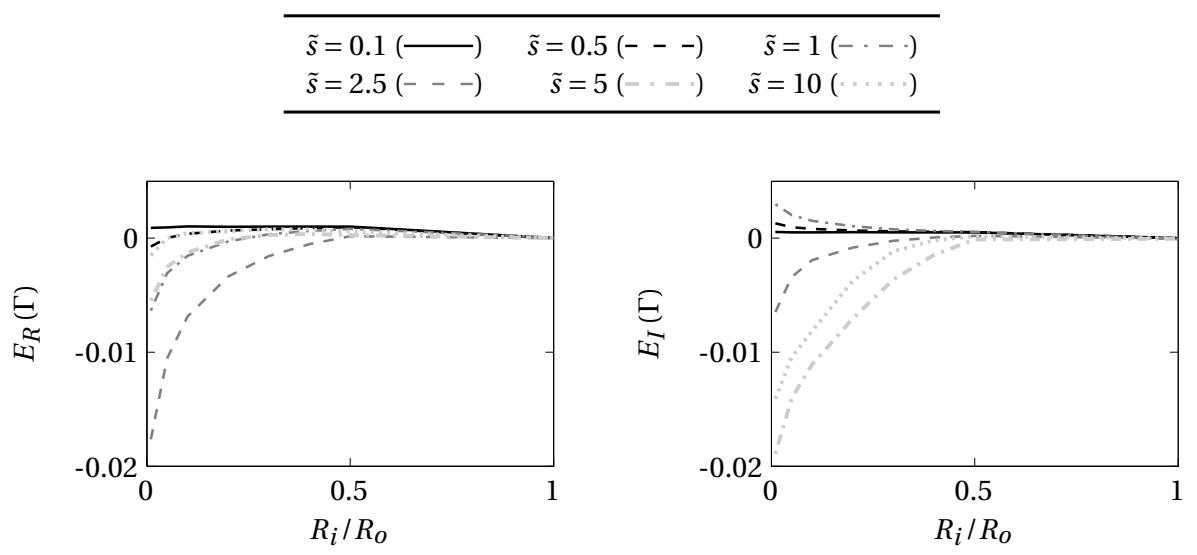

Figure 6.20: Relative error in real and imaginary part of the propagation constant $\Gamma$ for a curved layer as function of $R_{i} / R_{O}$, the ratio between inner and outer layer radius, for $k / s=10^{-3}$ and different shear wave numbers.

rors for straight and curved waveguides is that the errors for curved waveguides will not become increasingly smaller as $k / s$ decreases.

\section{Scattering problem}

In section 4.2.2 differences in the propagation constant for a straight and curved waveguide are investigated. For higher curvatures, the differences are significant. The propagation constant is related to the characteristic impedance of a waveguide, which describes how the fluid in the waveguides impedes enforced motion. From waveguide theory, it is known that differences in impedance (for instance due to a change in medium or a waveguide bifurcation) cause propagating waves to be scattered. When straight and curved waveguides are coupled, the differences in propagation constant imply a difference in impedance. As a result, a wave impinging on the interface of a coupled straight and curved waveguide section is scattered.

In figure 6.19(a) a setup is depicted with two infinite straight waveguide sections coupled by a curved waveguide section. The most efficient way to model viscothermal wave propagation in such a setup is by modeling the curved section with a straight waveguide of equivalent length. For the equivalent model in 6.19(b), the arc length of a curve passing through the center of the cross section of the curved waveguide is taken as the equivalent length.

The generic scattering problem described in 6.1.3 and the corresponding LRF and FE models are used to investigate the accuracy of the equivalent LRF solution compared to a reference FE solution. Note that the equivalent (straight) LRF model 
does not yield a reflected wave. Therefore, only the relative errors in the complex amplitude of the transmitted wave are presented in figure 6.21. Besides the errors of the equivalent LRF model compared to the FE model for the curved geometry, the errors of the equivalent LRF model compared to an the exact solution for a straight waveguide of equivalent length are also given.

The errors in figure 6.21 which are obtained by comparing the LRF model with the curved FE model are considered first. In general, the relative errors decrease as $k / s$ goes to zero, but appear to be bounded from below by the errors observed for the inviscid adiabatic case $(k / s=0)$, just as was observed for the cases involving changes in waveguide cross section described in the previous section. It is known from the literature for the inviscid adiabatic case that the observed errors for this case are due to the omission of higher order acoustic modes [90, 91]. The results in figure 6.21 suggest that for larger values of $\tilde{k}$ and smaller values of $k / s$ the observed relative errors are also dominated by omission of the higher order acoustic modes. Naturally, the errors obtained by comparing the straight LRF solution with the straight exact solution do not suffer from such errors. In the following, the errors in the area above the line for $k / s=0$ are commented upon.

If the results for a waveguide section with small curvature are considered (figure 6.21(a)), the relative error of the LRF model compared to the curved FE model, and the relative error of the LRF model compared to the straight exact solution are nearly the same. In this case, the error due to using an LRF approximation of the propagation constant for a straight waveguide to model wave propagation of the first acoustic mode is dominating the error in the amplitude of the transmitted wave. As the curvature of the curved section becomes larger, the distance traveled in the curved waveguide section becomes smaller and these errors decrease. Nevertheless, as the curvature becomes larger (ultimately resulting in the tightly curved waveguide presented in figure 6.21(f)) the relative error in the approximation of the amplitude of the transmitted wave based on the curved FE model increases (for most values of $k / s$ ) and becomes bigger than the error based on the straight exact model. In addition, the decrease in the error as $k / s$ goes to zero is much slower than for waveguides of smaller curvature.

Possible causes for the errors observed for tighter waveguides that are unrelated to neglecting higher order acoustic modes are: using the LRF approximation for a straight waveguide instead of a curved waveguide, disregarding terms involving velocity in $c d$-direction (which becomes significant as can be seen in the FE results presented in figure 6.21), and omission of vorticity modes. Again, neglecting the thermal modes is not expected to contribute significantly to the observed error based on the results in section 6.7. It is not investigated further which of these sources of errors contributes most to the observed errors. Summarizing, the 

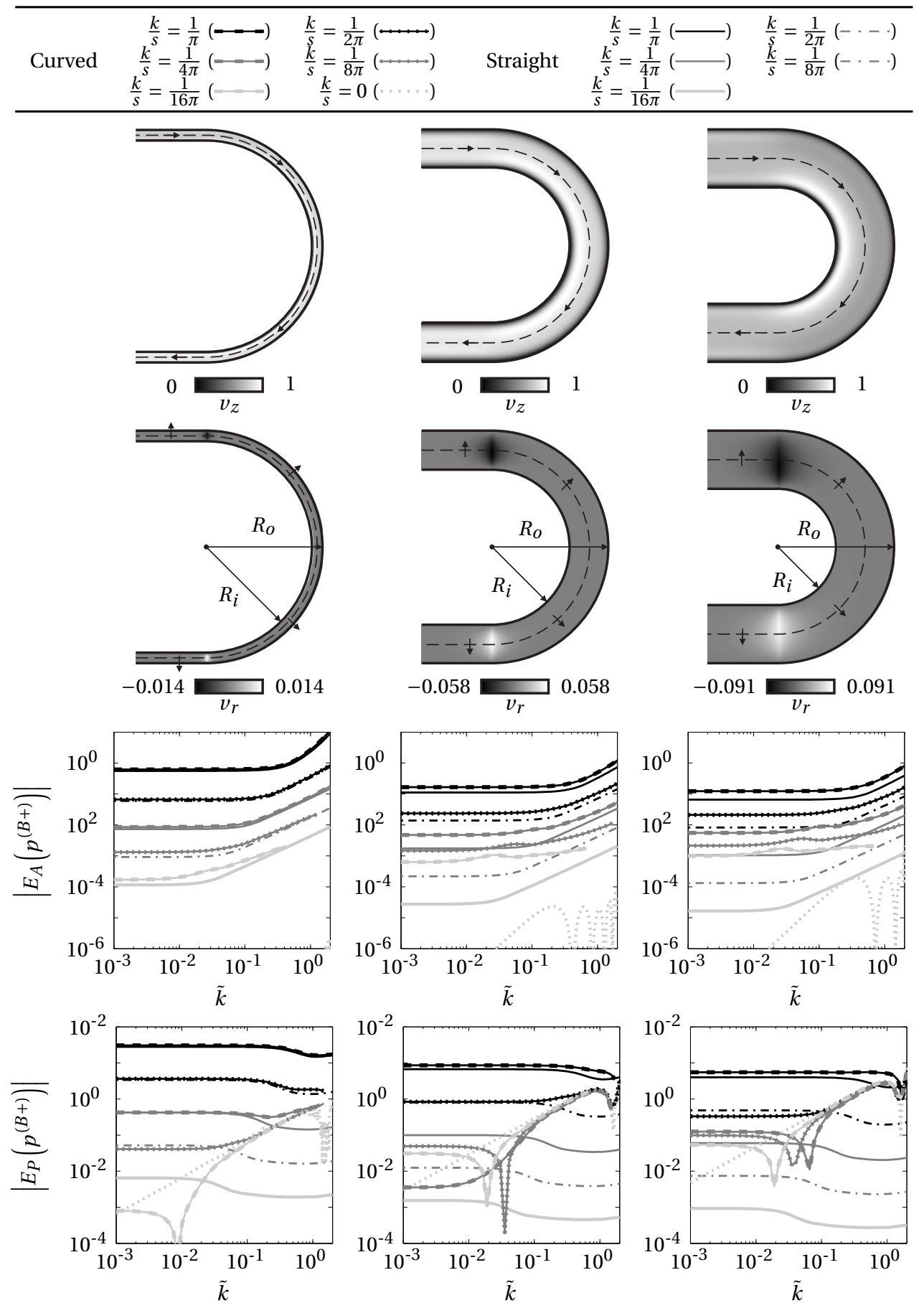

(a) $R_{o} / R_{i}=1.1$

(b) $R_{o} / R_{i}=1.5$

(c) $R_{o} / R_{i}=2$

Figure 6.21: Relative error in the absolute value and phase of the transmitted wave in a curved layer and straight layer of equivalent length. 


\begin{tabular}{|c|c|c|c|c|c|}
\hline Curved & $\begin{array}{r}\frac{k}{s}=\frac{1}{\pi}(\longrightarrow) \\
\frac{k}{s}=\frac{1}{4 \pi}(-(-) \\
\underline{k}=1\end{array}$ & $\begin{array}{l}\frac{k}{s}=\frac{1}{2 \pi}(\longrightarrow) \\
\frac{k}{s}=\frac{1}{8 \pi}(\longrightarrow) \\
\underline{k}=0\end{array}$ & Straight & $\begin{array}{r}\frac{k}{s}=\frac{1}{\pi}(\square) \\
\frac{k}{s}=\frac{1}{4 \pi}(\square) \\
k=1\end{array}$ & $\begin{array}{l}\frac{k}{s}=\frac{1}{2 \pi}(-\cdot \cdot) \\
\frac{k}{s}=\frac{1}{8 \pi}(-\cdot-\cdot)\end{array}$ \\
\hline & $\frac{k}{s}=\frac{1}{16 \pi}(---\cdots)$ & $\frac{k}{s}=0(\cdots)$ & & $\frac{\kappa}{s}=\frac{1}{16 \pi}(\Longrightarrow)$ & \\
\hline
\end{tabular}

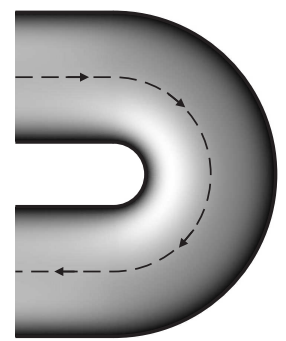

0

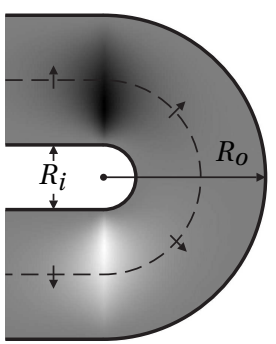

$-0.18$

0.18

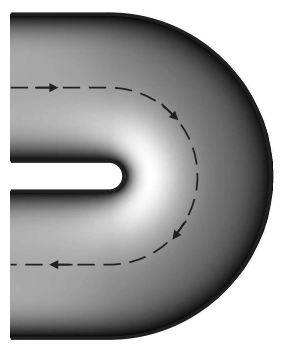

0
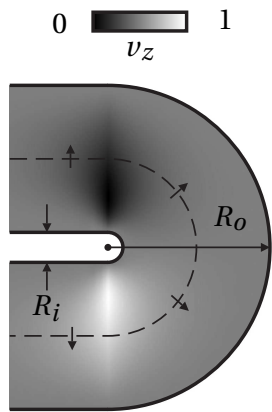

$-0.24$

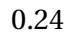

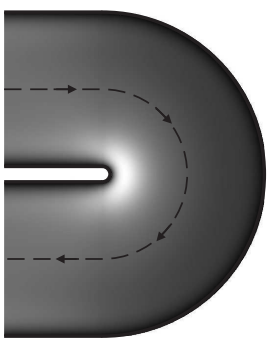

$0 \underset{v_{z}}{ } 1$

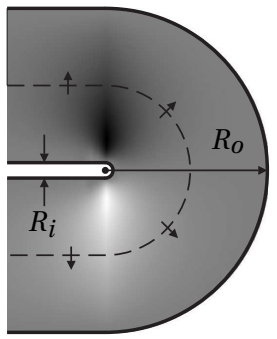

$-0.26 \underset{v_{r}}{\longmapsto} 0.26$
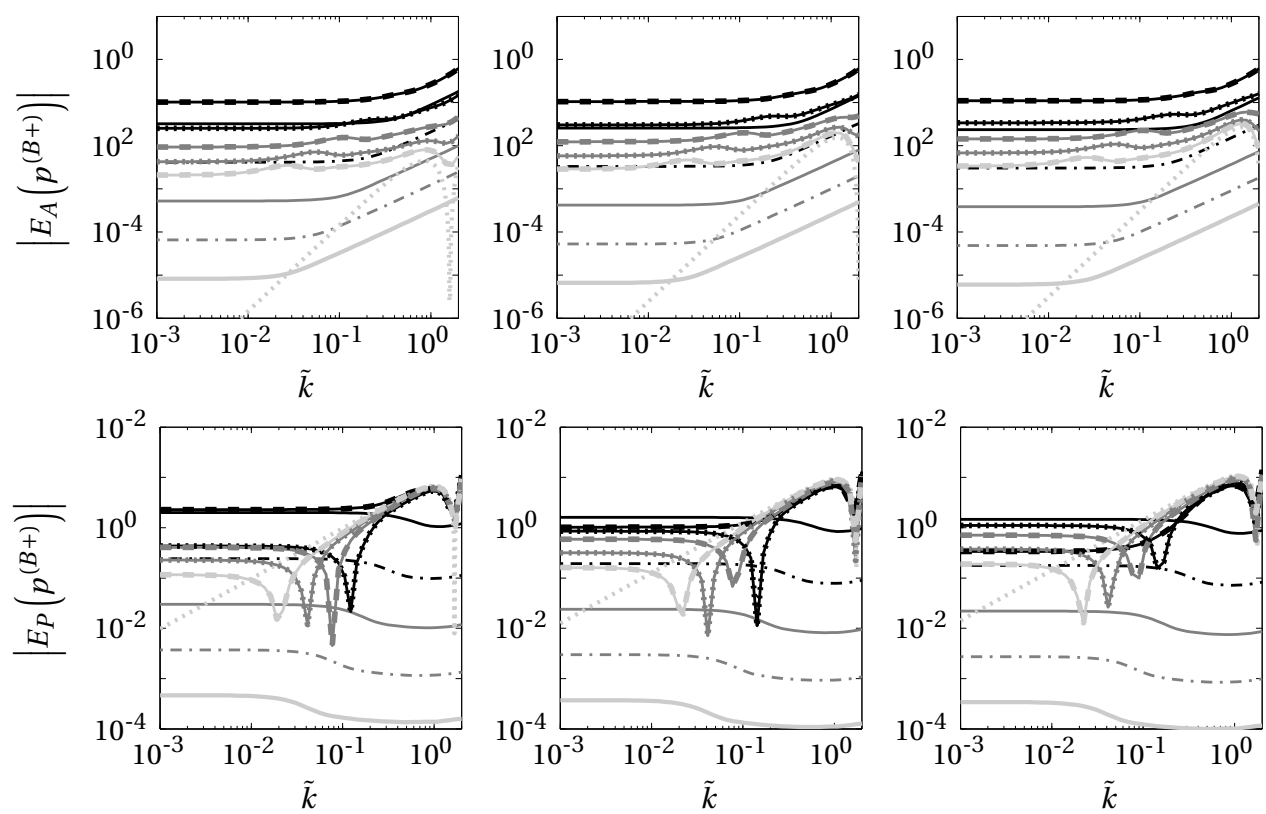

(d) $R_{o} / R_{i}=5$

(e) $R_{o} / R_{i}=11$

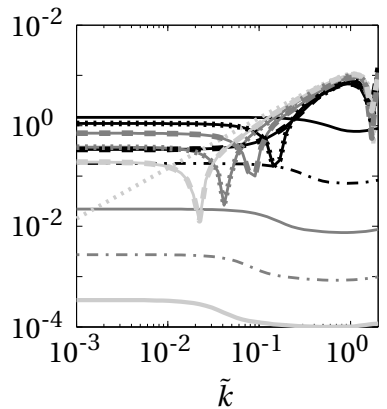

(f) $R_{o} / R_{i}=21$

Figure 6.21: Continuation: Additionally, FE results for axial velocity $\left(v_{z}\right)$ and tangential velocity $\left(v_{r}\right)$ are plotted for $\tilde{s}=10$ and $\tilde{k}=0.01$. 
following conclusions can be drawn based on the results presented above:

- In general, the relative errors due to changes in cross section decrease as $k / s$ goes to zero. Nevertheless, the relative error is bounded from below by the error obtained for the inviscid adiabatic case. For the case where the errors coincide with the line for $k / s=0$, the observed error is due to omission of the higher order acoustic modes.

- For small curvatures, the relative error due to changes in cross section is dominated by using an LRF approximation of the propagation constant to model wave propagation of the first acoustic mode. The fact that the LRF approximation for a straight waveguide is used has no significant influence on the error in this case.

- For large curvatures, the relative errors are dominated by: using the LRF approximation for a straight waveguide instead of a curved waveguide, disregarding terms involving velocity in $c d$-direction, and omission of vorticity and higher order acoustic modes.

- Apart from cases where the error is dominated by omission of the higher order acoustic modes, the observed errors are small for $k / s \ll 1$. Note, however, that for waveguides including many coupled curved and straight sections, the errors add up, reducing the range of $k / s$ where LRF models are guaranteed to produce results of a certain accuracy.

\subsection{Scattering calculation using mode matching}

In section 6.5 the effects on the accuracy of LRF models for straight waveguides with a sudden change in cross section are discussed. It is stated there that if the observed error in the LRF approximation is not dominated solely by the omission of higher order acoustic modes, it is dominated by the omission of both vorticity and higher order acoustic modes. This claim is investigated here by considering a similar scattering problem that includes higher order acoustic modes and vorticity modes. Furthermore, the influence of thermal modes on the solution of scattering problems is discussed.

Instead of using the models described in section 6.1.3, the method of mode matching described in section 3.6 is used to model the scattering of an acoustic wave impinging on a waveguide junction of two layers of different thickness (see figure 6.22). The ratio between the thicknesses is five in all considered cases. The waveguides are assumed to be infinite, and apart from the impinging wave, all waves are traveling outward from the waveguide junction. Recall that the direction 
of the different waves is determined by the direction in which a wave attenuates and not by the direction in which the wave tops travel.

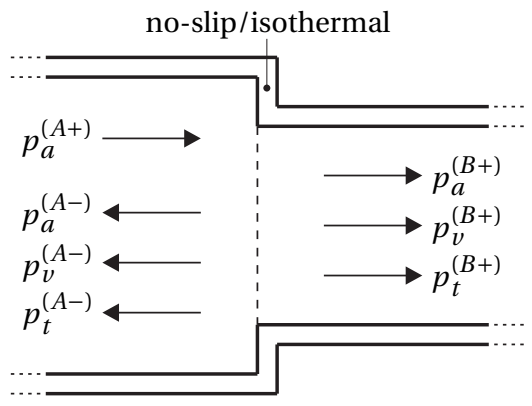

Figure 6.22: Abstract representation of a scattering problem involving acoustic, vorticity and thermal modes.

\subsubsection{Orthogonality}

Before discussing the results of mode matching for specific cases, the orthogonality of the different modes found in the individual waveguides is investigated first for different values of $\tilde{k}$ and $\tilde{s}$. The orthogonality between modes is an indication of how strong modes are coupled. Such information can serve as an indication of how disregarding certain modes in a mode-matching scheme might influence the contribution of other modes to the reconstructed solution. The following measure for the orthogonality of two modes is used:

$$
\Phi_{i j}=\frac{\left|\left\langle\mathbf{v}_{i}, \mathbf{v}_{j}\right\rangle+\left\langle T_{i}, T_{j}\right\rangle\right|}{\sqrt{\left|\mathbf{v}_{\mathbf{i}}\right|+\left|T_{i}\right|} \sqrt{\left|\mathbf{v}_{\mathbf{j}}\right|+\left|T_{j}\right|}}
$$

where $\mathbf{v}_{i}$ and $T_{i}$ are the velocity and temperature profiles over the waveguide cross section (padded with zeros outside the waveguide geometry), respectively, and the inner product $\langle$,$\rangle (and its associated norm) are as defined in 5.9. Two modes are$ perfectly correlated if $\Phi=1$, while two modes are orthogonal when $\Phi=0$. The orthogonality results for the first five acoustic, vorticity and thermal modes is depicted in figure 6.23.

An important observation is that the thermal modes are only coupled strongly with the first acoustic mode for $\tilde{k}<1$ (for $\tilde{k}>1$ the coupling between higher order acoustic modes and thermal modes becomes significant). This can also be deducted from a direct observation of the velocity and temperature profiles (see figures 3.7, 3.8 and 3.9). The thermal modes have relatively high values for (dimensionless) temperature compared to (dimensionless) velocity, while all other 
waveguide A $(\tilde{s}=1, \tilde{k}=0.01) \quad$ waveguide $\mathrm{B}(\tilde{s}=5, \tilde{k}=0.05)$

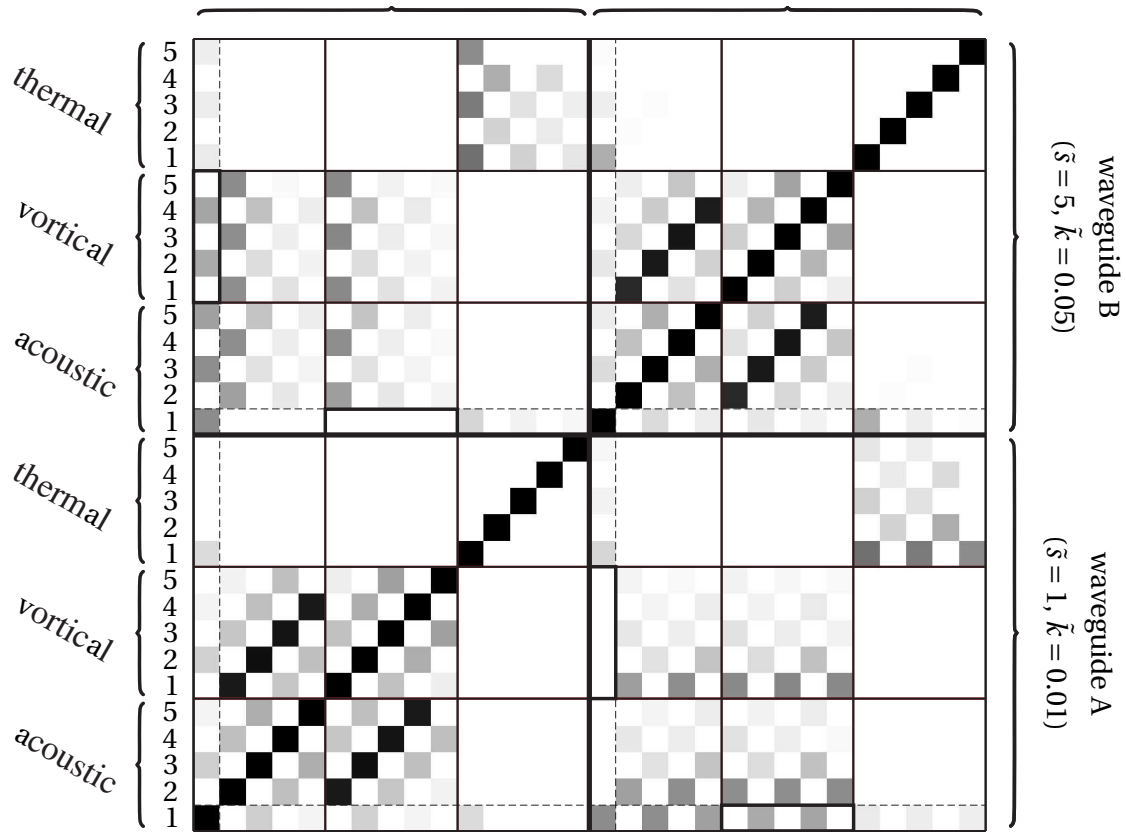

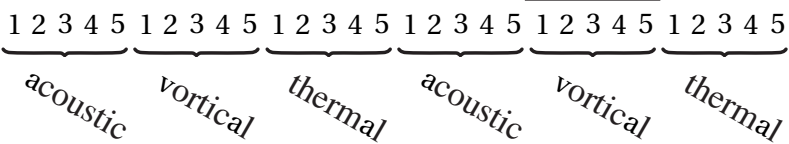
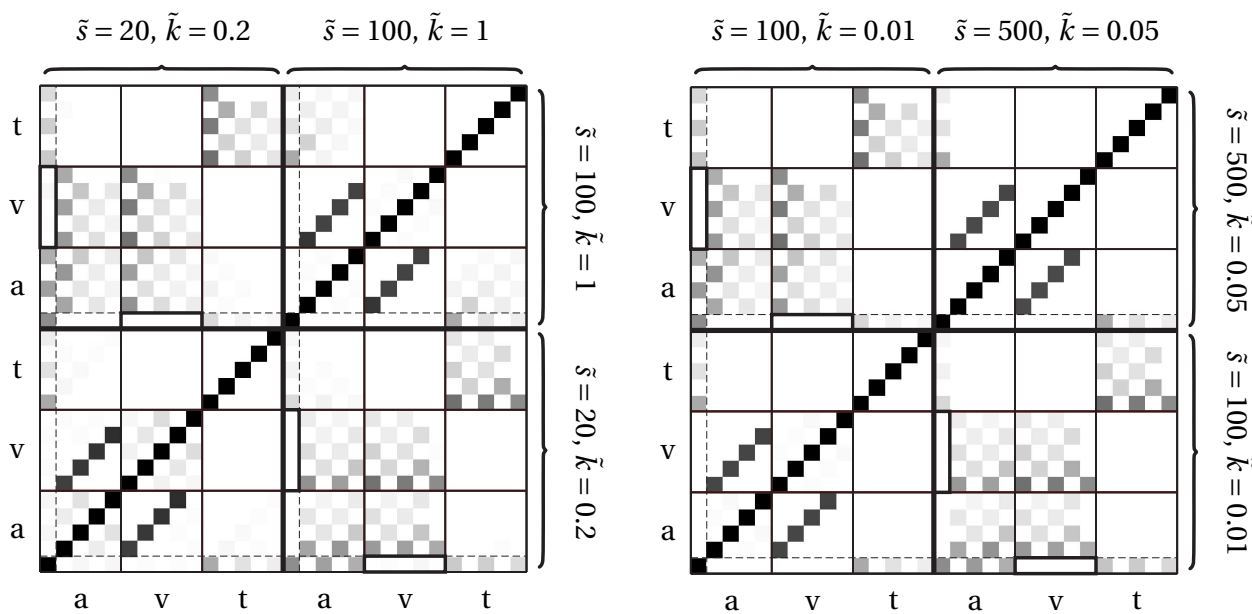

Figure 6.23: Orthogonality of the first 5 acoustic (a), vorticity ( $v$ ) and thermal (t) modes in two coupled waveguides of different cross section. White indicates fully orthogonal functions $(\Phi=0)$, black indicates fully identical functions $(\Phi=1)$. 
modes (except the first acoustic one) have high (dimensionless) velocities with respect to (dimensionless) temperatures. Since the thermal modes are only strongly coupled to the first acoustic modes, excluding them will have little effect on a reconstruction of the sound field. If desired, continuity of temperature can always be enforced by solving the contribution of the thermal modes after the contributions of all other modes are already determined.

The acoustic modes and vorticity modes are strongly coupled. This suggests that both higher order acoustic modes and vorticity modes are required to obtain an accurate value of the contribution of the first acoustic mode. However, upon closer inspection, the higher order vorticity modes appear to be strongly coupled to the first acoustic modes only if the shear wave number is low $(\tilde{s}<10)$. Consequently, for $\tilde{s}>10$ the coupling between the first acoustic mode and vorticity modes is indirect. Based on these observations, it is expected that for $\tilde{s}<10$ the impact of neglecting vorticity modes on the accuracy of the amplitudes of the reflected and transmitted wave corresponding to the first acoustic mode will be significant. For $\tilde{s}>10$ the impact is expected to be much lower.

\subsubsection{Influence of vorticity modes}

The influence of neglecting the vorticity modes as described above is confirmed by the results in figure 6.24. Here, the relative error in the complex amplitude of the transmitted and reflected wave obtained by mode matching is presented for different values of $\tilde{s}$ and $\tilde{k}$ (values are given for the narrow waveguide). The reference solution for the amplitudes of the reflected and transmitted wave is obtained with an FE model as described in section 6.1.3. The error is plotted as a function of the number of acoustic modes $n$ used for mode matching, and is given for the case where both acoustic and vorticity modes are used, and the case where only acoustic modes are used in the reconstruction. The error in the amplitudes when the system is modeled as an acoustic two-port (see section 6.5) is also indicated.

The results in figure 6.24(a) show that for $\tilde{s}=1$ neglecting the vorticity modes halts convergence to the correct solution after only a couple of modes are taken into account, while the model including vorticity modes continues to converge. On the other hand, for the results in figure 6.24(b) and 6.24(c), $\tilde{s}>10$ and neglecting the vorticity modes does not halt convergence in the considered range of $n$. The results in figure 6.24(a) also confirm the claim made in section 6.5, that for sudden changes in waveguide cross section the error in the LRF approximation of the amplitude of the reflected and transmitted waves are not dominated solely by the omission of the higher order acoustic modes.

In figure 6.25 an example of the reconstructed velocity field is given for the case in figure 6.24(a) when the mode-matching technique is used with and with- 
out vorticity modes and when different numbers of acoustic modes are taken into account. The relative errors of the solutions reconstructed with mode matching compared to the results of a (reference) FE model are also depicted.

Note that errors are only significant very close to the waveguide junction if both acoustic and vorticity modes are used in the reconstruction. This is equally true for the case where 5 acoustic modes and 30 acoustic modes are used. The area where the errors are significant extends much further when vorticity modes are neglected. This implies a much bigger area where the assumption that the first acoustic mode dominates the velocity does not hold. Significant errors can be expected if a second waveguide junction is located within this area and vorticity modes are not taken into account. This can become important, for instance, if the behavior of a system of multiple coupled waveguides is approximated with a two-port system of coupled LRF models.

\begin{tabular}{lll}
\hline$p_{a+\nu}^{(B+)}(\longrightarrow)$ & $p_{a}^{(B+)}(---)$ & $p^{(B+)}(\cdots \cdots)$ \\
$p_{a+\nu}^{(A-)}(\longrightarrow)$ & $p_{a}^{(A-)}\left(-{ }^{(A-)}\right.$ & $p^{(A-)}(\cdots \cdots)$ \\
\hline
\end{tabular}

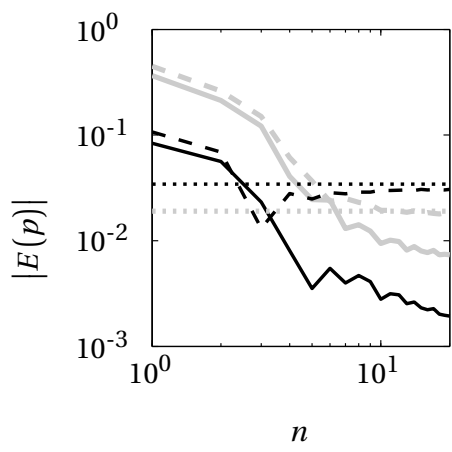

(a) $\tilde{s}=1, \tilde{k}=0.01$

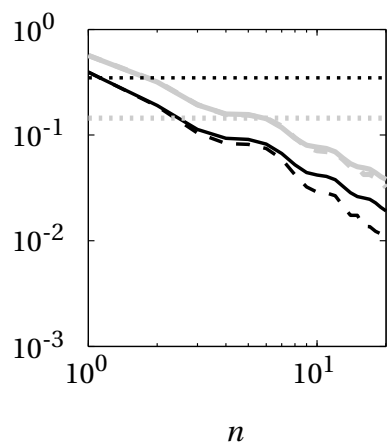

(b) $\tilde{s}=20, \tilde{k}=0.2$

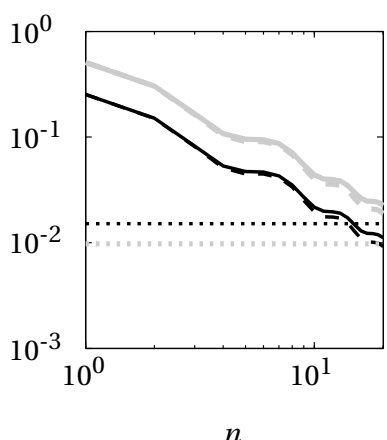

(c) $\tilde{s}=100, \tilde{k}=0.01$

Figure 6.24: Relative error in the complex amplitude of the transmitted and reflected wave obtained by mode matching for different values of $\tilde{s}$ and $\tilde{k}$ (values are given for the narrow waveguide). The error is plotted as a function of the number of acoustic modes used for mode matching. For $p_{a+v}^{(B+)}$ and $p_{a+v}^{(A-)}$ both acoustic and vorticity modes are used, while for $p_{a}^{(B+)}$ and $p_{a}^{(A-)}$ only acoustic modes are used. The lines for $p^{(B+)}$ and $p^{(A-)}$ correspond to the error when the system is modeled as an acoustic two-port. 

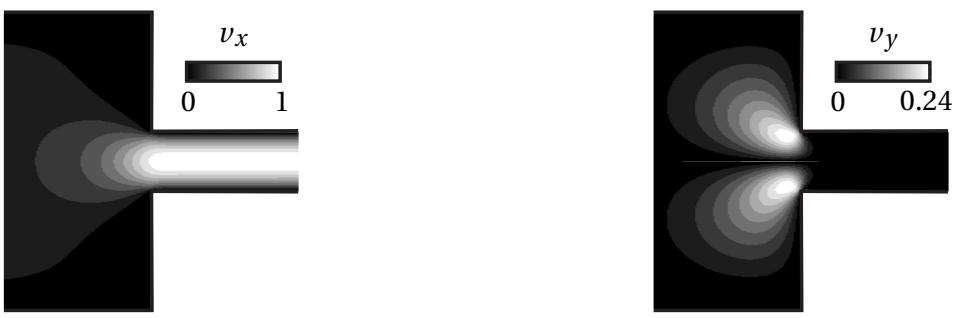

(a) FE solution
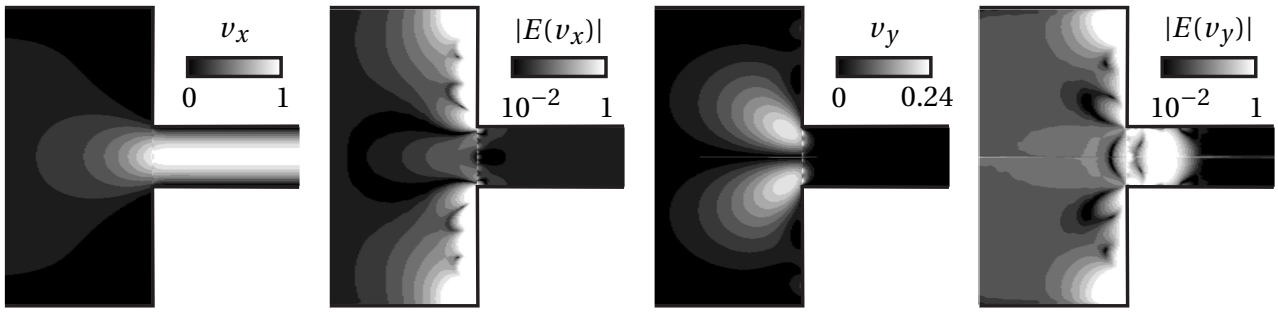

(b) Mode-matching solution using 5 acoustic and 5 vorticity modes
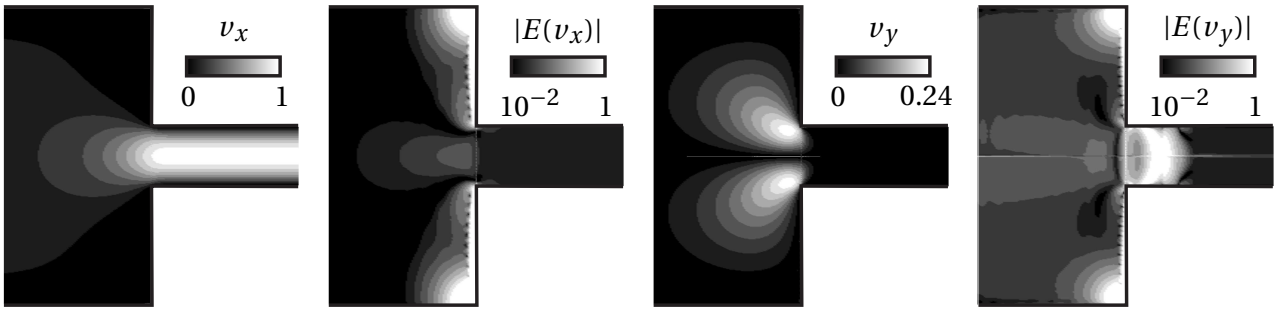

(c) Mode-matching solution using 30 acoustic and 30 vorticity modes
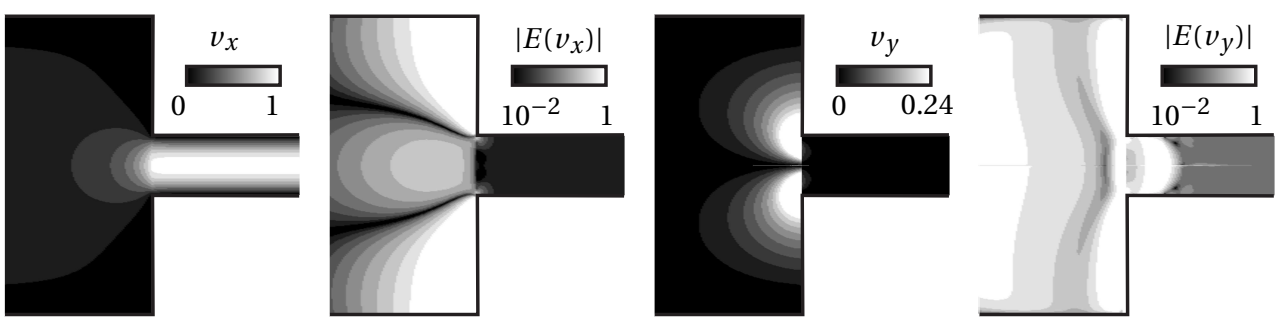

(d) Mode-matching solution using 30 acoustic modes and no vorticity modes

Figure 6.25: Junction of two waveguides; Velocity fields calculated with FE and modematching method, and the relative error of the mode-matching solution compared to the FE solution on a logarithmic scale. Results for a varying number of included acoustic and thermal modes are presented for $\tilde{s}=1$, and $\tilde{k}=0.01$. 


\subsection{Conclusions}

The main conclusions of the presented parameter studies on the accuracy of LRF models presented in this chapter are:

- The a priori criteria $k / s \ll 1, k / s_{t} \ll 1$ and $\tilde{k}<1$ are not sufficient to ensure an accurate approximation of the propagation constant for LRF models involving no-slip ${ }^{12} /$ adiabatic boundary conditions in cases where $\tilde{s}<1$. However, in the range of values for $\tilde{s}, \tilde{s}_{t}$ and $\tilde{k}$ where the assumptions for continuum mechanics hold, the observed errors are small.

- In general, the relative errors due to changes in waveguide cross section or waveguide curvature decrease as $k / s$ goes to zero. Nevertheless, the relative error is bounded from below by the error obtained for the inviscid adiabatic case. For the case where the errors coincide with the errors for $k / s=0$ (the inviscid adiabatic case), the observed errors are due to omission of the higher order acoustic modes.

- For larger values of $k / s$ and gradual changes in waveguide cross section or small changes in waveguide curvature, the relative error due to changes in cross section/curvature is dominated by using an (equivalent) LRF approximation of the propagation constant to model wave propagation of the first acoustic mode.

- For sudden changes in waveguide cross section or gradual changes in cross section and smaller values of $k / s$, the relative error due to changes in cross section is dominated by the omission of both vorticity and higher order acoustic modes.

- For all considered cases, the observed errors due to changes in waveguide cross section and curvature are small for $k / s \ll 1$, except for cases where the error is dominated by omission of the higher order acoustic modes. Note, however, the errors can add up if many waveguides of different cross section or curvature are coupled. This can reduce the range of $k / s$ where LRF models are guaranteed to produce results of a certain accuracy.

Furthermore, based on the results in appendix K.2, it can be concluded that for no-slip/adiabatic boundary conditions, both the propagation constant and the velocity/temperature profiles of the first thermal mode will closely resemble

\footnotetext{
${ }^{12}$ For slip/adiabatic boundary conditions the criteria are sufficient. In that case, satisfying the a priori criteria reduces LRF models to the equations for 1D or 2D inviscid adiabatic acoustic wave propagation.
} 
those of the first acoustic mode if $s \ll 1$ and $k / s \ll 1$. The resemblance is closer if $s \ll 1$ and $k / s \ll 1$ are smaller. If the resemblance is strong, the first thermal mode will act as an additional propagating mode. This causes significant errors in the predicted amplitudes of waves corresponding to the first acoustic modes if waveguides with no-slip/adiabatic boundary conditions are coupled to waveguides having no-slip/isothermal boundary conditions. Nevertheless, based on the results in section 6.4 it can be concluded that coupling waveguides having no-slip/isothermal boundary conditions with waveguides having slip-adiabatic boundary conditions ${ }^{13}$ only causes minor errors in the range of values for $\tilde{s}, \tilde{s}_{t}$ and $\tilde{k}$ where the assumptions for continuum mechanics hold.

\footnotetext{
${ }^{13}$ In the case of slip/adiabatic boundary conditions, the propagation constant of the first acoustic mode equals unity ${ }^{12}$, corresponding with a truly propagating wave, while the propagation constant of the first thermal mode corresponds to a heavily damped wave.
} 


\section{Chapter 7}

\section{Engineering applications}

Two engineering applications involving passive noise reduction using resonators are presented in this chapter. The first case describes the use of side resonators for noise reduction of an axial in-duct fan ${ }^{1}$. The second case describes the use of resonators for the absorption of aircraft cabin noise.

\subsection{Passive reduction of fan noise}

One of the main noise sources in high performance computers is the cooling fans. Tonal noise at the rotational frequency of the fan, the so-called Blade Passing Frequency (BPF) and its higher harmonics are important contributors to the sound pressure level of fan noise, and are often a cause of annoyance. A possible solution to reduce the sound level at specific frequencies in a ducted system is the application of so-called side resonators. A schematic representation of a setup for noise reduction of an axial in-duct fan with side resonators is given in figure 7.1.

In a broad sense, a side resonator can be defined as an air-filled cavity of arbitrary geometry, connected to the circumference of a duct system. When a side resonator is inserted into a duct system, traveling waves will experience a sudden change in impedance at the location of the side resonator. Changes in impedance cause incident waves to be scattered at the location where the changes occur, just as is observed for impedance changes due to a change in media (see figure 7.2(a)).

\footnotetext{
${ }^{1}$ Note that the research presented in the first section was initially aimed at noise reduction of cooling fans in personal computers. Although still relevant for high performance systems, the introduction of multicore technology, and variable processor clocking has lessened the need for extensive cooling in most personal computers. The dramatic noise levels of a few years back have dropped to acceptable levels. Nevertheless, the presented noise reduction principles can be applied to reduce the noise of other machinery involving ducted fans.
} 


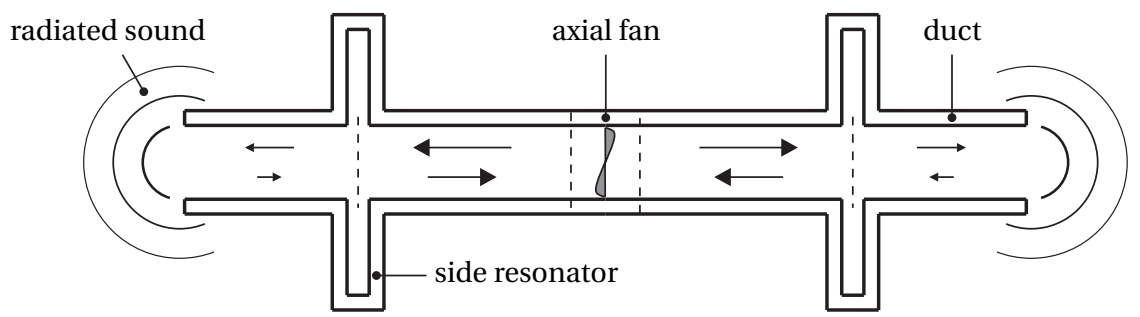

Figure 7.1: Schematic representation of a setup for noise reduction of an axial in-duct fan with side resonators.

The dimensions of the side resonator relative to the dimensions of the duct determine how much of the incident wave is reflected and how much is transmitted for a given frequency. It must be noted, however, that a partial reflection of noise back to the source does not necessarily imply a reduction of the sound level of the transmitted sound waves. The effect of inserting side resonators into a duct system is also heavily influenced by the type of noise source, the geometry of the duct system and the location of the side resonators in the duct system.

\subsubsection{Influence of source impedance and termination impedance}

\section{source impedance}

To illustrate the effects of the source impedance, consider the simple setup in figure 7.2(a). A resonator is connected to a duct with a noise source generating sound waves of constant amplitude at one end, while the duct extends infinitely to the other end ${ }^{2}$. Depending on the impedance of the source, waves that are reflected by the side resonator $\left(p_{r}\right)$ can be absorbed, or can be reflected once more in the direction of the resonator. In the former case, the impedance at the source is such that it matches the impedance of the duct. This representation is adequate, for instance, if the duct at the source side is terminated by a (perfect) sound absorber (see figure 7.2(b)). On the other hand, if the impedance of the source is very high, a resonance effect between source and resonator can occur for certain frequencies, causing the sound levels that are observed on the other side of the side resonator to rise. For other frequencies, the re-reflected wave can also (partially) cancel the waves emitted by the source thus lowering the sound level observed past the resonators.

If the considered source is an axial in-duct fan, the source impedance will not

\footnotetext{
${ }^{2}$ An equivalent setup is obtained if the infinite end is cut off and replaced by a perfectly acoustic absorbing material.
} 


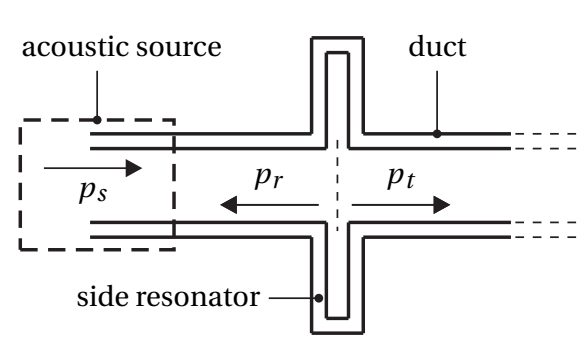

(a)

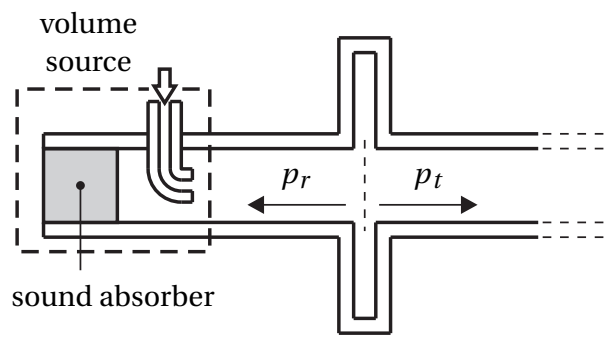

(b)

Figure 7.2: Schematic representation of an (infinite) duct with an acoustic resonator and sound source (a), an infinite duct with resonator and a sound source having a matched impedance (b). The source wave, reflected wave and transmitted wave are indicated by $p_{s}, p_{r}$ and $p_{t}$.

match the impedance of the duct; part of the sound waves that are traveling towards the fan will be reflected, and a part will be transmitted through the fan. This distribution between reflected and transmitted wave is frequency dependent. An efficient way to model these properties for waves approaching from both sides of the fan is by using a scattering-matrix approach as described in section 4.3.

\section{Termination impedance}

In the cases considered above, the duct extends infinitely and waves are not reflected once they have passed the resonator. However, for practical problems, the ducting system has a termination with a specific impedance. Depending on the termination impedance, frequency, length of the duct section and the equivalent impedance of source and resonator, resonance or anti-resonance can also occur between the resonator and duct termination. In short, the effect of inserting side resonators into a system is influenced by many parameters such as frequency, geometry of the duct system, resonator geometry and location, and the characteristics of the noise source.

In order to optimize the dimensions of a setup such as depicted in figure 7.1, accurate modeling of the source characteristics of the axial fan is important. Subsections 7.1.3, 7.1.4, and 7.1.5 are devoted to the fan model that is used to optimize the resonator design. The subsections describe, respectively, the fan model, the setup build to determine the different frequency dependent coefficients that govern the source characteristic of the fan, and measurement results used for verifying the accuracy of the model and the coefficients that were obtained experimentally. First, the influence of mean flow on wave propagation in the main duct is discussed. 


\subsubsection{Wave propagation in the presence of mean flow}

The LRF models for viscothermal wave propagation in ducts given in 4 are valid for quiescent fluids. A simple modification allows the model to be used in the case of steady uniform fluid flow, also known as plug flow. The modification to account for a plug flow of velocity $v_{0}$ consists of replacing the wave number $k$ by an adjusted wave number defined for waves traveling in positive and negative $x$ direction respectively as

$$
k_{(+)}=\frac{\omega}{c_{0}+v_{0}}, \quad k_{(-)}=\frac{\omega}{c_{0}-v_{0}}
$$

Note that this substitution of $k$ is carried out for all models of duct sections supporting mean flow described in this section. This is also true for all cases where equations in chapter 4 are referenced. In those cases, the equations are used with the adjusted wave numbers without restating them.

The assumption of plug flow is valid when the airflow velocities are relatively small (low Mach numbers) and wave propagation is inviscid and adiabatic by approximation [116]. It was verified with the models including mean flow presented in section 3.4, that the assumption of plug flow is indeed valid for the flow speeds and shear wave numbers in the duct encountered in the present study ${ }^{3}$.

\subsubsection{Fan model}

Following Larentjev, the axial fan is modeled as an acoustic two-port. A schematic representation of such a two-port model, based on wave amplitudes, is given in figure 7.3.

The degrees of freedom for this two-port model are: the amplitudes of the incident waves $p^{(1-)}$ and $p^{(2-)}$, the amplitudes of the sums of the resulting reflected and transmitted waves $p^{(1+)}$ and $p^{(2+)}$, and the amplitudes of the source waves $p^{(1 s)}$ and $p^{(2 s)}$. The source waves are the outward traveling waves observed when no sound is reflected back to the fan from either side. When the source waves are paired to form a vector as is done in figure 7.3(b) the result is referred to as the source strength vector. The relation between the different waves in figure 7.3 is given by

$$
\left\{\begin{array}{l}
p^{(1+)} \\
p^{(2+)}
\end{array}\right\}=[\mathbf{S}]\left\{\begin{array}{l}
p^{(1-)} \\
p^{(2-)}
\end{array}\right\}+\left\{\begin{array}{l}
p^{(1 s)} \\
p^{(2 s)}
\end{array}\right\}
$$

\footnotetext{
${ }^{3}$ If viscous effects due to main flow are large or if wave propagation is viscothermal in nature, the models including mean flow presented in section 3.4 can be used. Other examples of more complete viscothermal acoustic models that account for the presence of main flow for arbitrary velocity profiles can be found for instance in $[39,58,59]$.
} 


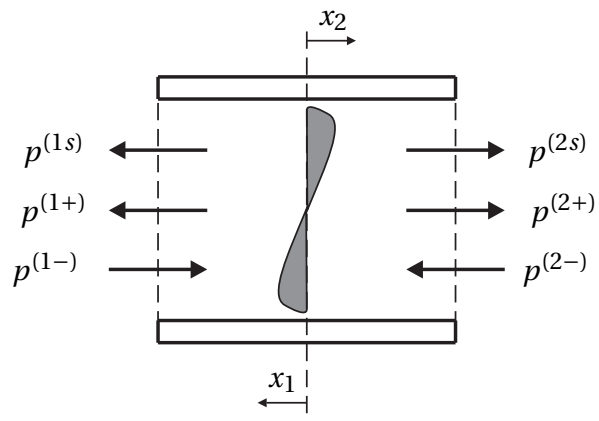

(a)

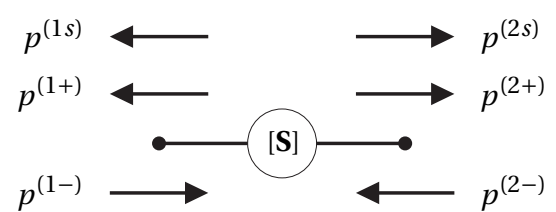

(b)

Figure 7.3: Schematic representation of wave propagation in a duct section housing an axial fan (a). Corresponding two-port model of the axial in-duct fan based on the scattering-matrix approach (b).

where $\mathbf{S}$ is the so-called scattering matrix of size $2 \times 2$. To be able to use this twoport model for the fan in combination with the two-port models for the various geometries derived in section 4.3, it is rewritten in terms of the mobility-matrix approach (having pressure and volume flows as degrees of freedom). This is done easiest by splitting the fan model into an active and passive model as is indicated in figure 7.4.

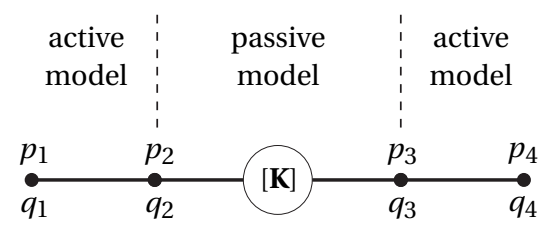

Figure 7.4: Axial in-duct fan represented by an active and passive model based on the mobility matrix approach. The mobility matrix $\mathbf{K}$ of the passive model is given in equation 4.67.

\section{Passive mode}

The passive model only describes the relation between incident sound waves and resulting outward traveling sound waves. When the source strength vector is disregarded, equation (7.2) relates the amplitudes of these waves through the scattering matrix. The equation is rewritten to yield an expression for the volume flows on both sides of the fan in terms of the pressure on both sides of the fan (as was done 
in section 4.3.4). The resulting equation ${ }^{4}$ is

$$
\left\{\begin{array}{l}
q_{2} \\
q_{3}
\end{array}\right\}=\frac{\gamma}{\rho_{0} c_{0}}[\mathbf{K}]\left\{\begin{array}{l}
p_{2} \\
p_{3}
\end{array}\right\}
$$

where the matrix $\mathbf{K}$ is as defined in equation (4.67).

\section{Active model}

The active model describes the outward traveling sound waves $p^{(1 s)}$ and $p^{(2 s)}$. In order to represent the 'active behavior' of the fan in both directions, the model for the passive behavior is sandwiched between two duct sections in which a sound wave is induced in a single direction (see figure 7.4). Each active model involves two volume sources that add and extract fluid from the system at a certain phase difference. As a consequence waves will travel in both directions from all locations of volume injection. This situation is portrayed in figure 7.5.

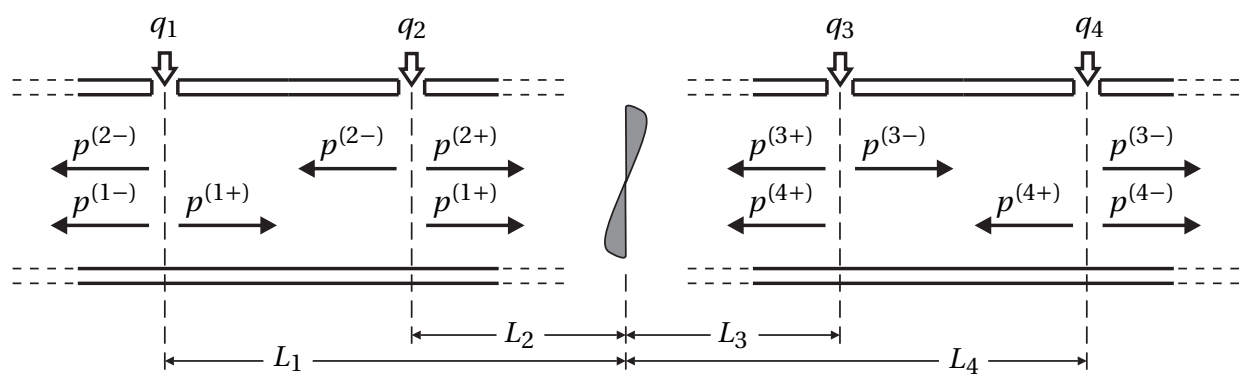

Figure 7.5: Schematic representation of sound waves in the active models.

The amplitude and the phase of the sources $q_{1}$ and $q_{2}$ can be chosen in such a way that the generated waves cancel each other out at one side of the corresponding active element (on the left), while a wave of a desired amplitude and phase travels outward from the other end. Similarly $q_{3}$ and $q_{4}$ can be used to control the amplitudes of the waves traveling outward for the other active element (on the right).

If a wave is to be generated in a duct section of constant cross section, a standard two-port model of that duct section (as presented in section 4.3.2) can serve as active element. The volume flows $q_{1}$ through $q_{4}$ required to generate sound waves of amplitude $p^{(s-)}$ and $p^{(s+)}$ and in the negative and positive $x$-direction

\footnotetext{
${ }^{4}$ The same equation in dimensionless form is given in (4.67).
} 
(obtained after extensive use of equation 4.53) are defined, respectively, by

$$
\begin{aligned}
& \left\{\begin{array}{l}
q_{1} \\
q_{2}
\end{array}\right\}=-\frac{2}{\Gamma n} \frac{\gamma}{\rho_{0} c_{0}} S\left[\begin{array}{cc}
e^{\left(-i k_{(-)} \Gamma L_{1}\right)} & e^{\left(-i k_{(-)} \Gamma L_{2}\right)} \\
-e^{\left(i k_{(+)} \Gamma L_{1}\right)} & -e^{\left(i k_{(+)} \Gamma L_{2}\right)}
\end{array}\right]^{-1}\left\{\begin{array}{c}
p^{s-} \\
0
\end{array}\right\} \\
& \left\{\begin{array}{l}
q_{3} \\
q_{4}
\end{array}\right\}=-\frac{2}{\Gamma n} \frac{\gamma}{\rho_{0} c_{0}} S\left[\begin{array}{cc}
e^{\left(i k_{(-)} \Gamma L_{3}\right)} & e^{\left(i k_{(-)} \Gamma L_{3}\right)} \\
-e^{\left(-i k_{(+)} \Gamma L_{4}\right)} & -e^{\left(-i k_{(+)} \Gamma L_{4}\right)}
\end{array}\right]^{-1}\left\{\begin{array}{c}
0 \\
p^{s+}
\end{array}\right\}
\end{aligned}
$$

with $L_{1}$ through $L_{4}$ the distances of the volume sources to the center of the fan, and $\Gamma$ the propagation constant for the duct section housing the in-duct fan. Using the above definition, the amplitude of the resulting source waves is calculated at the center of the fan. The calculated volume flows $q_{1}$ through $q_{4}$ can be applied as forcing terms in the model of the two-port network involving the fan.

Note that the lengths of the passive and active model defined by $L_{1}$ through $L_{4}$ can be chosen arbitrarily. The chosen lengths determine how much of the duct length is included in the fan model. Consequently, the lengths influence the values of $q_{1}$ through $q_{4}$ and the values of the coefficients in the scattering matrix. The value of the measured scattering matrix coefficients in section 7.1.5 are valid for $L_{2}=L_{3}=0$. In that case, the passive model represents scattering of waves at a plane through the center of the fan and wave propagation in the fan is modeled using a two-port model for duct sections.

\subsubsection{Determining the scattering matrix and source strength vector}

The different methods available to determine the frequency-dependent parameters in the scattering matrix and the source strength vector are described by Lavrentjev et al. [117]. A distinction can be made between techniques with and without external sources. It is argued by Lavrentjev et al. that the use of external sources yields the best results when the correlation between the source strength components is unknown. In this case there is no prior knowledge about the source strength components and the external source method is adopted.

\section{External source method}

The measurement data needed to determine the coefficients of the scattering matrix and the source strength vector are the wave amplitudes of the waves traveling backward and forward in the duct on both sides of the fan for multiple load cases. A schematic overview of the setup that was used to collect the required data is given in figure 7.6.

Note that the open duct terminations of the setup must be located in different rooms. In this way, the sound field observed by the reference microphones are 


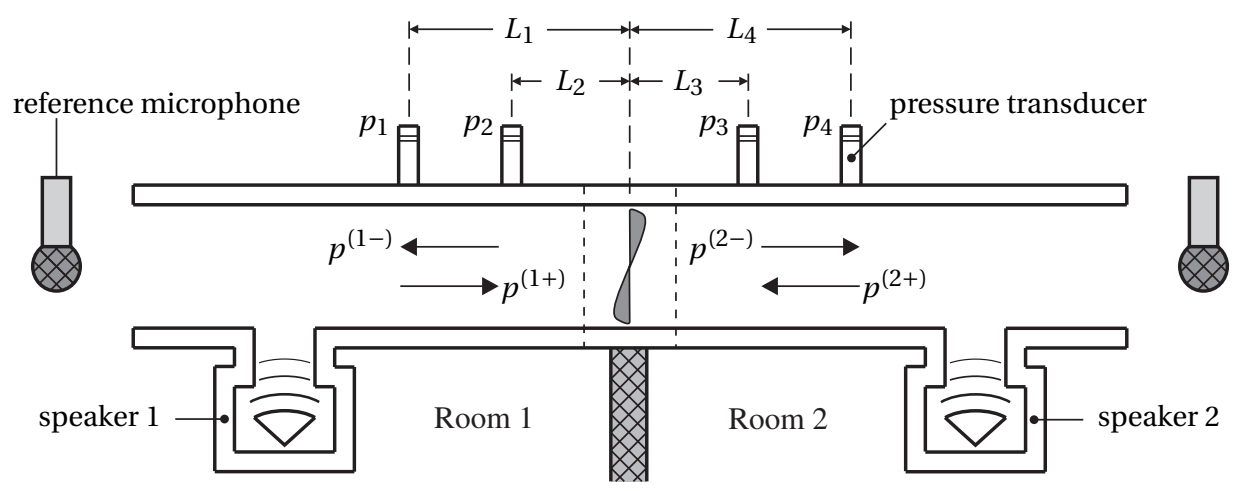

Figure 7.6: Schematic representation of the setup for determining the coefficients of the scattering matrix and the source strength vector.

only correlated to the sound radiated from one of the duct terminations. The amplitudes of the backward and forward traveling waves can be calculated based on the signals from the pressure transducers located on either side of the fan by

$$
\begin{aligned}
\left\{\begin{array}{l}
p^{(1-)} \\
p^{(1+)}
\end{array}\right\}=[\mathbf{V}]^{-1}\left\{\begin{array}{l}
p_{1} \\
p_{2}
\end{array}\right\}, \quad \text { with }[\mathbf{V}]=\left[\begin{array}{ll}
e^{i k_{(-)} \Gamma L_{1}} & e^{-i k_{(+)} \Gamma L_{1}} \\
e^{i k_{(-)} \Gamma L_{2}} & e^{-i k_{(+)} \Gamma L_{2}}
\end{array}\right] \\
\left\{\begin{array}{l}
p^{(2-)} \\
p^{(2+)}
\end{array}\right\}=[\mathbf{W}]^{-1}\left\{\begin{array}{l}
p_{3} \\
p_{4}
\end{array}\right\}, \quad \text { with }[\mathbf{W}]=\left[\begin{array}{lll}
e^{i k_{(-)} \Gamma L_{3}} & e^{-i k_{(+)} \Gamma L_{3}} \\
e^{i k_{(-)} \Gamma L_{4}} & e^{-i k_{(+)} \Gamma L_{4}}
\end{array}\right]
\end{aligned}
$$

An external noise source is used to generate a sound field in the duct system of much higher amplitude than the field generated by the fan. Hence, the source strength vector in equation (7.2) can be neglected in the description on the sound field. Note that only one of the speakers is active in each measurement. Switching to the other speaker is a quick way to change the acoustic load on the system. Another way to change the acoustic load is to replace one of the duct segments with a longer or shorter version. After the amplitude of the backward and forward traveling sound waves is measured for multiple acoustical load cases, the following system emerges:

$$
[\mathbf{M}]=\left[\begin{array}{ll}
S_{11} & S_{12} \\
S_{21} & S_{22}
\end{array}\right][\mathbf{N}]
$$

With $\mathbf{M}$ and $\mathbf{N}$ matrices that contain the measured wave amplitudes for the different load cases:

$$
[\mathbf{M}]=\left[\begin{array}{lll}
p_{1}^{(1+)} & \cdots & p_{n}^{(1+)} \\
p_{1}^{(2+)} & \cdots & p_{n}^{(2+)}
\end{array}\right], \quad[\mathbf{N}]=\left[\begin{array}{ccc}
p_{1}^{(1-)} & \cdots & p_{n}^{(1-)} \\
p_{1}^{(2-)} & \cdots & p_{n}^{(2-)}
\end{array}\right]
$$


where $n$ indicates the number of load cases. For $n=2$, pre-multiplying the equation with $\mathbf{N}^{-1}$ yields the scattering matrix. For cases where $n \neq 2$, matrix $\mathbf{M}$ has to be pre-multiplied with a pseudo inverse of $\mathbf{N}$ to obtain the scattering matrix. If the Moore-Penrose pseudo inverse is used, the calculated scattering matrix will be a minimum least square fit for the supplied loading cases. In such cases, the exact scattering matrix may be dependent on the acoustic boundary condition that is applied to the system. A measure for this dependency is given by:

$$
\epsilon=\frac{\operatorname{det}\left(\left|\mathbf{M N}^{H}\right|^{2}\right)}{\operatorname{det}\left(\mathbf{M M}^{H}\right) \operatorname{det}\left(\mathbf{N N}^{H}\right)}
$$

This 'linearity coefficient' ranges between one and zero. If it equals unity, the sets of amplitudes form a perfect linear system and the scattering matrix is not dependent on the acoustic load that is applied. A value of $\epsilon$ unequal to 1 means the set of amplitudes does not form a linear system. This indicates that the calculated scattering matrix is dependent on the applied acoustic load or that measurement noise/errors prevent an accurate calculation of the scattering matrix. Note that instead of using actual pressure signals, transfer functions between the pressure signals and the signal to drive the active speaker are used to calculate the amplitudes of the backward and forward traveling waves occupying the matrices $\mathbf{M}$ and $\mathbf{N}$. This suppresses the influence of flow noise and fan noise that is not masked by the external noise source.

The source strength vector of the fan is determined in a second experiment in which the external noise source is switched off and the fan itself serves as noise source. Now that the coefficients of the scattering matrix $\mathbf{S}$ are known, equation (7.2) can be rewritten as follows to yield the source strength vector:

$$
\left\{\begin{array}{l}
p^{(1 s)} \\
p^{(2 s)}
\end{array}\right\}=\left\{\begin{array}{l}
p^{(1+)} \\
p^{(2+)}
\end{array}\right\}-[\mathbf{S}]\left\{\begin{array}{l}
p^{(1-)} \\
p^{(2-)}
\end{array}\right\}
$$

Substitution of the measured wave amplitudes on both sides of the fan yields the source strength vector. Again, instead of using actual pressure sensor data, transfer functions are used to determine the amplitude of the backward and forward traveling wave. In this case, a signal that is driving the sound source is not available. The sound radiated from the duct openings is correlated to the fan noise and is relatively free from flow noise. Therefore, microphones positioned close to the duct openings (outside of the airflow) are used as an alternative to obtain a reference signal. 


\subsubsection{Measured source characteristics}

The experimental setup depicted in figure 7.6 was manufactured to determine the scattering matrix and source strength vector used in the model described above. The modular design of the setup allows it to be modified easily, so it can be used for verification of the presented theory as well (see the next section). The coefficients of the scattering matrix and source strength vector were determined for the axial cooling fan depicted in figure 7.7 at different operating voltages.

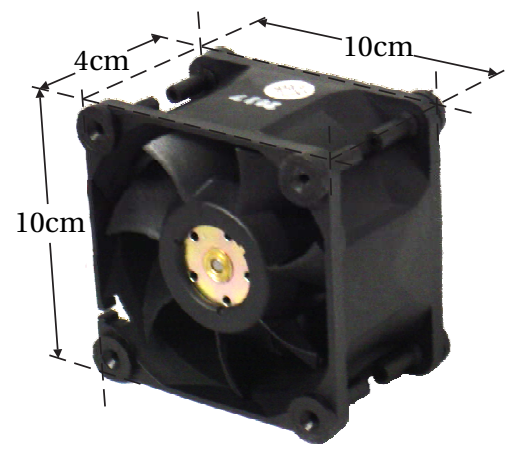

Figure 7.7: Dimensions of the axial in duct fan.

\section{Pressure transducers}

The methods to derive the coefficients of the scattering matrix and source strength vector that are described above are derived from or analogue to the so-called $2 p$ method or two microphone method. This is the standard method to obtain the ratio of the amplitudes of the backward and forward traveling wave based on the transfer function between the signals of two pressure sensors. The accuracy of the obtained ratio of amplitudes is sensitive to possible phase offsets of the pressure sensors that are used. The pressure transducers that are used in all measurements described below ${ }^{5}$ are very lightly-damped dynamic systems with essentially no phase shift. A drawback of these sensors, however, is their relatively high noise floor. In a number of cases, the pressure level of the signal that is to be measured is similar (and in some cases lower) than the noise floor. It is indicated below when the values of the different coefficients become unreliable due to this issue. Note that the noise floor of the sensors increases significantly below $300 \mathrm{~Hz}$ which explains the deviations in that region.

\footnotetext{
${ }^{5}$ The pressure transducers used are $1 / 4$ inch Kulite microphones.
} 


\section{Scattering matrix coefficients}

An example of the measured scattering matrix parameters and source strength components of the fan running at $12 \mathrm{~V}$, the maximum operating voltage of the fan, is plotted in figure 7.8. It follows from the dimensions of the test setup that the (1D) LRF model used is valid for frequencies below the cut-on frequency of the tube at $3300 \mathrm{~Hz}$. For higher frequencies the wave propagation in the duct cannot be considered 1D and the model can no longer describe the sound field in the duct. This is observed in the plots of the parameters in the scattering matrix in figure 7.8. For the present case, the frequency range of interest is well below the cut-on frequency. The methods in [118] can be used if source characteristics of the fan are needed for frequencies above the cut-on frequency. The coefficients of the

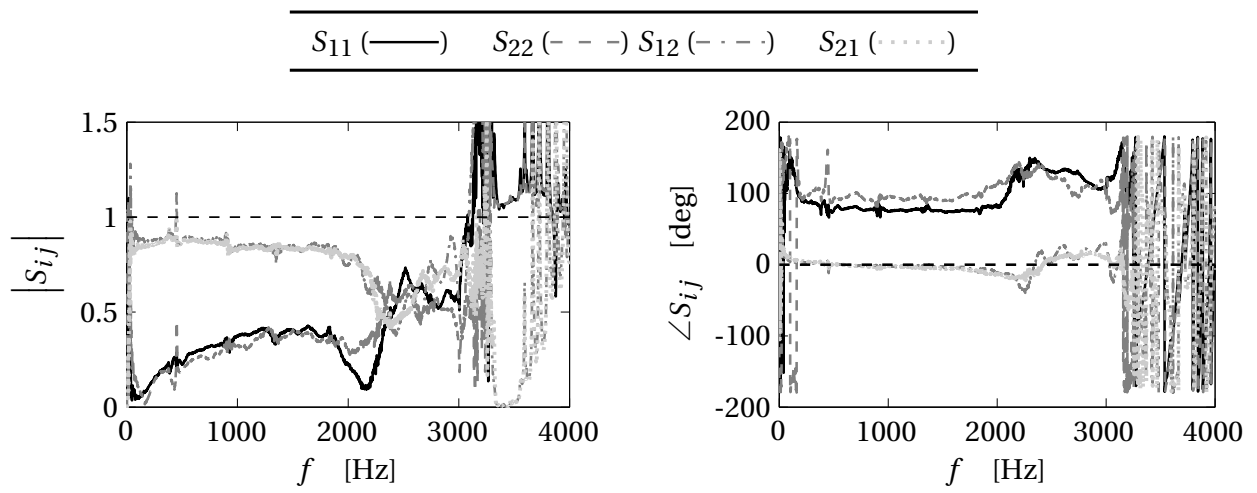

Figure 7.8: Measured scattering matrix coefficients at an operating voltage of $12 \mathrm{~V}$.

scattering matrix for other operating voltages in the range $0-12 \mathrm{~V}$ were found to be virtually identical for all considered voltages. Since the operating speed is directly related to the operating voltage, the scattering matrix coefficients in figure 7.8 are also virtually independent of fan speed.

The presented results are calculated with equation 7.8 from the measurement data of the four different load cases depicted in figure 7.9(a). For two cases the setup in figure 7.6 is used alternatively using one of the two speakers as a noise source. For the other two cases, the T-junction including the non-active noise source and the duct piece leading to the duct termination are removed from the duct system. The linearity coefficient in equation (7.9) was calculated for the resulting overdetermined set of equations to determine to what extent the coefficients in the scattering matrix are dependent on the loading conditions. The linearity coefficient $\epsilon$ is plotted in figure 7.9(b) as a function of frequency for different operating voltages of the fan. 


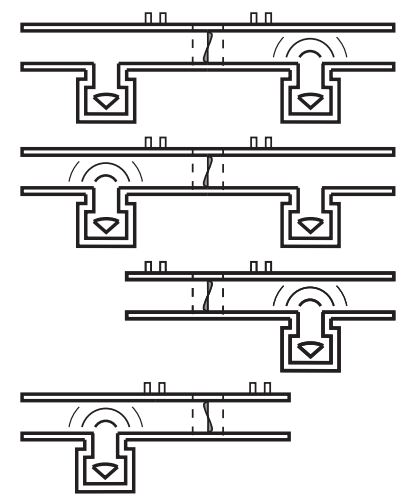

(a) load cases

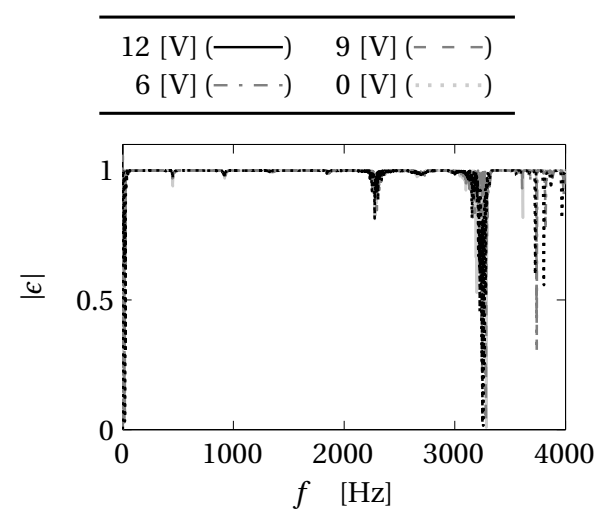

(b) linearity coefficient

Figure 7.9: Four different load cases used to determine the scattering matrix coefficients and the corresponding linearity coefficient.

The value of the linearity coefficient suggests that for most frequencies the coefficients of the scattering matrix hardly depend on the acoustic load. Besides the dip observed around $2300 \mathrm{~Hz}$, the value of $\epsilon$ only deviates significantly from unity at frequencies above the cut-on frequency. Virtually equivalent results were obtained for $\epsilon$ at other operating voltages. The results suggest that the values of the scattering matrix coefficients in figure 7.9 can be used for the model aimed at optimizing a side-resonator design presented in section 7.1.7. However, an inspection of the signal levels of the pressure sensors compared to the level of the noise source reveals that the signal-to-noise ratio around certain frequencies is very poor. These frequencies correspond with resonance frequencies of the duct. The poor signal-to-noise ratio is indicated by a poor coherence between the pressure signals and the reference signal (the signal used to drive the active speaker). Figure 7.10(a) plots the 'combined coherence', which is the product of the coherence between the reference signal and the individual pressure signals.

The frequencies where the 'combined coherence' is very low correspond with the frequencies where the linearity coefficient deviates from unity. In these regions, the measured values for the scattering matrix are expected to be less accurate. Because of the relatively smooth character of the coefficients of the scattering matrix in the areas where coherence is good, the values of these coefficients are extrapolated to the areas where the combined coherence is low when the data is used in the optimization of the side-resonator design in section 7.1.7. 


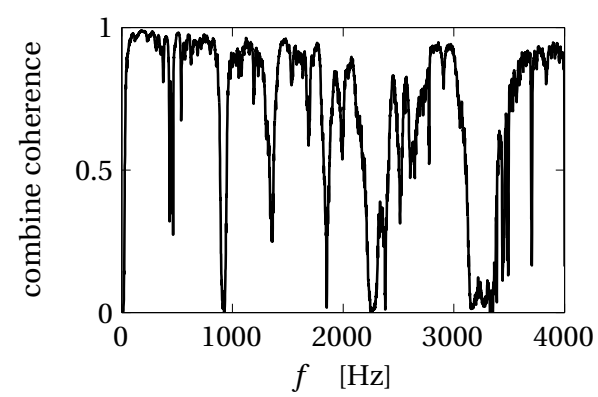

(a) scattering matrix measurement

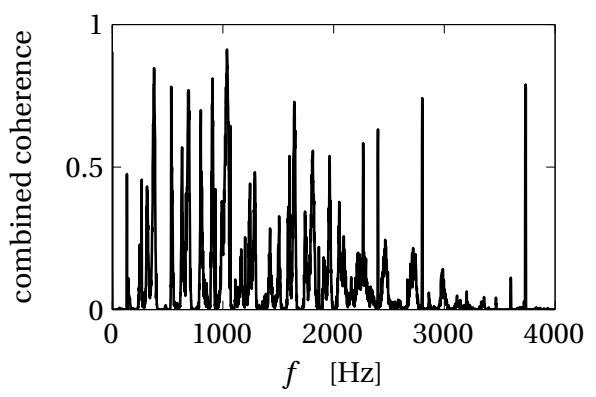

(b) source strength vector measurement

Figure 7.10: Product of coherence between reference signal and individual pressure signals.

\section{Source strength vector coefficients}

The source strength vector is determined by a separate measurement, using the setup in figure 7.6 without speakers and T-junctions. As an example, the measured amplitudes and phase difference of the coefficients for the source strength vector are plotted in figure 7.11 for an operating voltage of $12 \mathrm{~V}$.
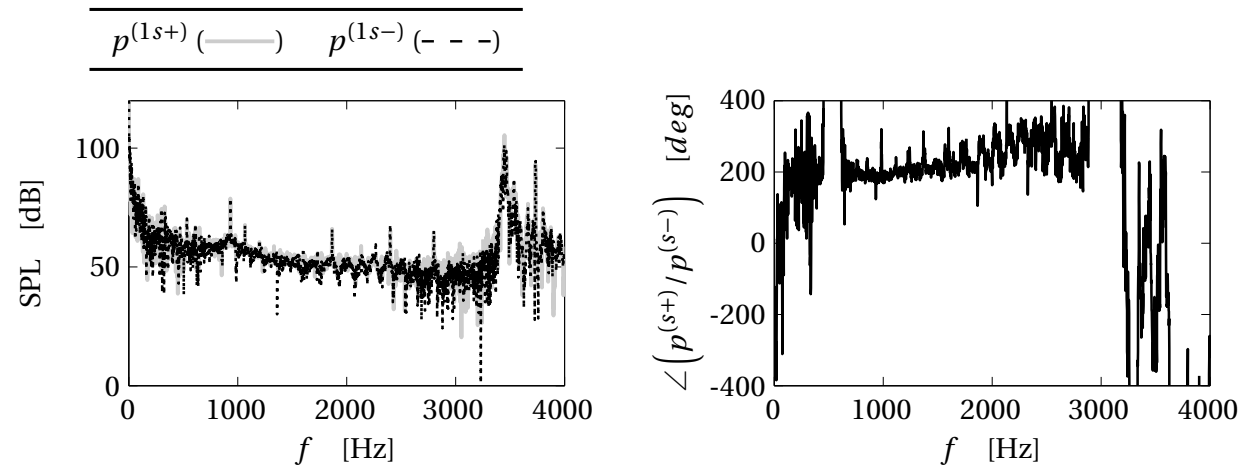

Figure 7.11: Sound pressure level (SPL) and phase difference of the measured coefficients of the source strength vector at an operating voltage of $12 \mathrm{~V}$.

The BPF and its higher harmonics found at 934, 1868 and $2801 \mathrm{~Hz}$ can be identified easily, having a sound level that is 10 to $15 \mathrm{~dB}$ higher than the observed broadband noise. Other peaks related to multiples of the BPF divided by seven (the number of blades on the fan) can also be identified. In the literature axial fans are sometimes modeled as ideal dipoles [119]. The phase difference between the source strength signals in figure 7.11 varies somewhat with frequency, but has a 
value close to 200 degrees on average. The sound power of both source strength components also matches fairly well. The assumption that the active source characteristic of a fan can be modeled as a dipole therefore seems to be reasonable. Nevertheless, the actual source data is already available and will be used throughout this section.

Due to the fact that the sound field generated by the fan is of much lower amplitude than the field generated by the speakers used to determine the scattering matric coefficients, the 'combined coherence' of the pressure signals is significantly reduced, compared to the measurement results presented for the scattering matrix coefficients. The values of the 'combined coherence' in figure 7.10 suggest that the accuracy of the measured source strength vector is expected to be low, except at a great number of peaks. Not surprisingly, the coherence is relatively good at the frequencies where the sound source strength has a peak. Since the primary goal of using side resonators is reducing the amplitudes of these peaks, accurate source strength data is only required to be accurate at the corresponding frequencies when optimizing the resonator design presented in section 7.1.7. Note that the steep increase in the source strength below $300 \mathrm{~Hz}$ is not related to sound produced by the fan, but is due to the sudden rise in the noise floor of the pressure transducers.

\section{Plug flow velocity}

A method to obtain the plug flow velocity from acoustic measurement data is presented in [120]. An analogue method used in the present study is described shortly. The axial fan is placed in a duct with two pairs of pressure transducers on one side of the fan. The pressure measurements that are obtained can be used to obtain the backward and forward traveling waves at the same location for each pair of transducers. The plug flow velocity must be such that the calculated amplitudes of these waves are identical for both transducer pairs. Since the plug flow is independent of frequency, there are as many independent equations to determine the plug flow as there are measured frequencies. A least square fit of the plug flow velocity provides an accurate approximation [120]. The observed flow at the maximum operator voltage of $12 \mathrm{~V}$ is $7.5 \mathrm{~m} / \mathrm{s}$. In the range of $6-12 \mathrm{~V}$, the flow velocity is found to change linearly with the voltage.

\subsubsection{Experimental validation}

To validate the theory of the passive and active fan elements, the gathered values for the coefficients of the scattering matrix and source strength vector are used to model the sound field in the duct for a setup with different acoustic loads than 
used above. The new loads are obtained by removing the speakers and T-junction and shortening the ducts on both sides of the fan. The calculated and measured pressure signal for one of the transducers and the transfer functions between pressure signals are depicted in figure 7.12.

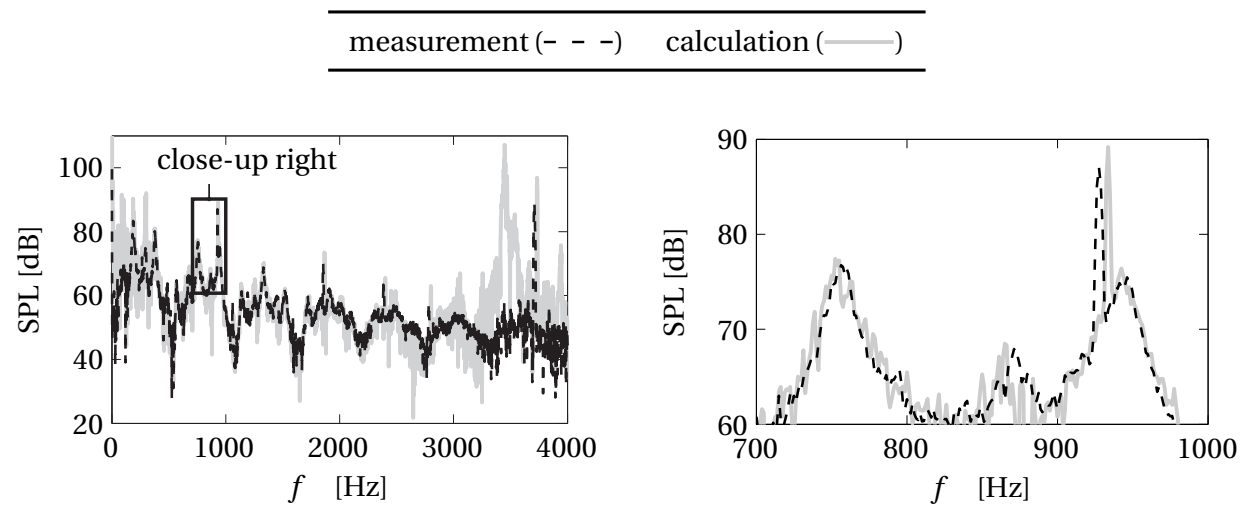

(a) Signal of a pressure transducers
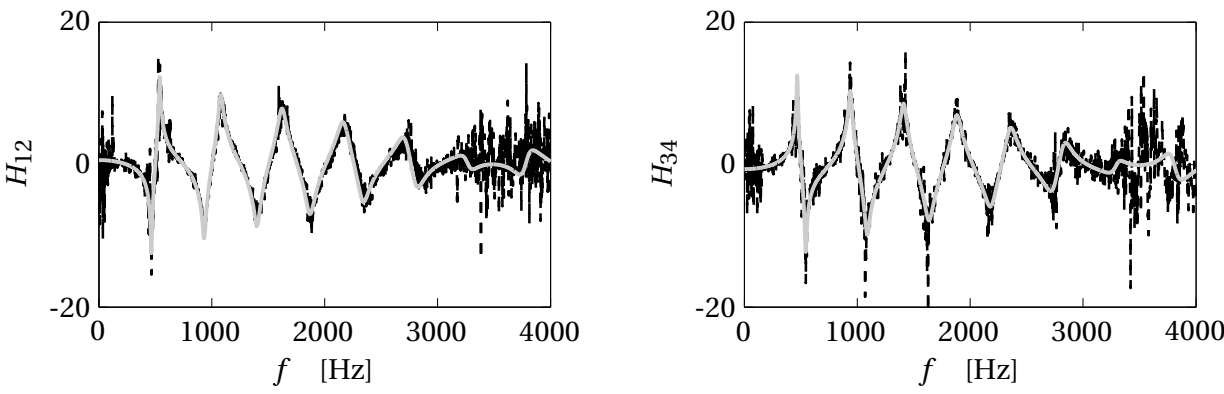

(b) Transfer functions between pressure transducer signals

Figure 7.12: Calculated and measured pressure signals and transfer functions between pressure signals.

The measured and calculated pressure signals, presented in figure 7.12, show good correspondence between 300 and $2500 \mathrm{~Hz}$. Note that the strength of the sensor signals diminishes with increasing frequencies as a result of the influence of the duct system. This causes the sensor signal to gradually sink into the noise floor, resulting in larger deviations between predicted and measured values for higher frequencies.

The close-up in 7.12(a) on the other hand shows a fairly tight match for the lower frequency range. Note that the location of the peak at $934 \mathrm{~Hz}$ is slightly shifted between measurement and prediction due to minor fluctuations in fan 
speed that are always observed for constant operating voltages. As a result, the predicted amplitude of the peaks is also lower. A closer look shows that the difference between the height of the peak and the level of broadband noise immediately beside the peak is almost identical for measurement and prediction. In fact, when the operating voltage is actively changed, the peaks related to the speed of the fan shift along the wider peaks and valleys observed in the broadband noise. These wider peaks and valleys result from resonances and anti-resonances in the duct system. The main objective of the optimization of a side-resonator design for a speed-controlled fan (as presented in the next section) is lowering the wider peaks observed in the broadband noise that are caused by the duct system. Based on the presented results, it can be concluded that the developed model and the obtained values for the various coefficients are accurate enough to be used in the optimization of a resonator design for frequencies between 300 and $2500 \mathrm{~Hz}$.

\subsubsection{Resonator design}

\section{Broadband fan noise reduction}

The resonator designs presented below are the result of an optimization aimed at broadband noise reduction of a speed-controlled fan. If broadband noise reduction is achieved, the fan can be operated at lower voltages, thus saving power and lowering the overall noise level, while the extremes in the noise spectrum remain suppressed. Another advantage of a design aimed at broadband noise reduction is that its performance is not sensitive to unintended changes in fan speed, for instance due to changes in back-pressure (by re-arranging or placing new components in the air flow) or wear of the fan bearings over time.

As mentioned previously, side resonators generally have a relatively narrow frequency band in which they are most effective. This size of the frequency range can be manipulated somewhat by the choice of the general resonator shape and by altering the dimensions to increase or decrease the influence of viscothermal effects. In general, increasing the effective frequency range in this way will lower the level of noise reduction that is achieved. A more effective way to increase the frequency range is the use of multiple resonators of different dimensions (see for instance [19]). This approach is taken in the present study.

\section{Resonator setup}

In order to achieve noise reduction using side resonators, the fan needs to be placed in a duct with a certain length. The duct length is preferably kept to a minimum to facilitate a compact design of the resonator setup. However, there are certain minimum distances needed between different acoustical elements for the 
sound waves to become planar again after a local disturbance found at the junction between duct and resonator and in the area close to the fan. If elements are placed too close to each other, the 1D model that is used for the optimization will become inaccurate. It was decided to build two demonstrators: a long version $(20 \mathrm{~cm})$ using four resonators and a shorter more compact version $(12 \mathrm{~cm})$ using three resonators. The 1D model for the setup with the longer duct is expected to offer better accuracy than the model for the smaller duct having only small distances between the fan and the individual resonators.

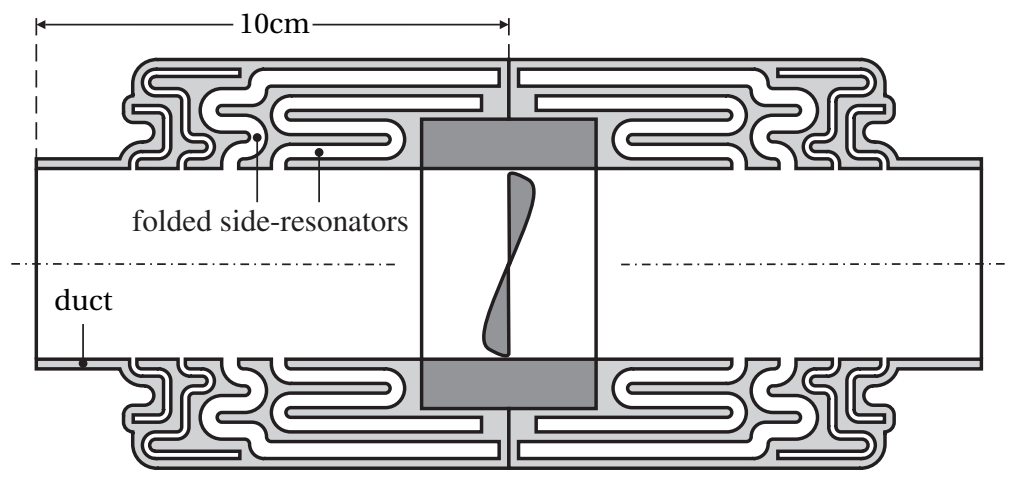

Figure 7.13: Schematic representation of a resonator setup using multiple folded side resonators.

The emitted noise spectra were calculated for six operation voltages between 6 and $12 \mathrm{~V}$ for a setup where the fan is mounted in the center of a 12-centimeter duct and a 20-centimeter duct. For these two cases, respectively three and four frequency bands were selected where noise reduction is most needed based on the emitted noise spectra. The different side resonators are targeted primarily at noise reduction in these frequency bands. In order to reduce the noise level around the lowest targeted frequency of $750 \mathrm{~Hz}$ a resonator length of approximately $12 \mathrm{~cm}$ is needed in case resonators in the form of air slits are used. To facilitate a compact design, the air layers are 'folded' alongside the direction of the main duct a number of times. The layout of a such a folded resonator design is sketched in figure 7.13. An optimization routine was developed to determine the lengths and thicknesses of the different side-resonator sections. The final resonator designs for the 3-resonator and 4-resonator setup are depicted in appendix L. The large amount of parameters in the optimization of the resonator design requires the use of an efficient viscothermal model. The approximations that are made to arrive at an efficient LRF model are discussed shortly. 


\section{Coupled LRF model}

The curvature of the sections of cylindrical air layers only has a small influence on the acoustic behavior of the system, since the inner and outer radius of the boundary surfaces only differ slightly. These sections can be modeled more efficiently by straight layers. The same reasoning is applied to model the doubly curved layers at the waveguide folds. It is assumed that these sections can be accurately modeled by (singly) curved air layers as described in section 4.2.2. The parameter study in section 6.6 suggests that the resulting curved layer geometries can be modeled efficiently, and with good accuracy, by representing them as straight layers of equivalent length if $k / s$ and $\tilde{k}$ are small. For the present case, the highest target frequency (for the 4-resonator design) is around $3300 \mathrm{~Hz}$. In combination with the smallest resonator thickness of 1.5 in the final design, the largest values for $\tilde{k}$ and $k / s$ are $9.2 \cdot 10^{-4}$ and $1.6 \cdot 10^{-5}$ respectively. Based on the results in section 6.6 , it is concluded that the curved sections at the folds can be modeled as straight air layers of equivalent length without significant loss of accuracy.

It was found that neglecting the effects of expansion of the sound waves in the folded sections due to wave propagation in radial direction has a significant influence on the acoustic behavior of the resonators. In [121] folded resonators are investigated for the inviscid adiabatic case, which support these findings. The model for a circular air layer in section 4.3.3 can be used to model the expansion effect accurately. Therefore, the side resonators are modeled as multiple coupled circular and cylindrical air layers. The LRF models for the resonator are coupled to the LRF model for the duct carrying a mean flow and the active and passive fan model described in 7.1.3. The lengths of the resonator sections that are connected to the main tube are adjusted with the length correction for a slit in a baffle [70]. The resulting LRF model for the 3-resonator setup is given in figure 7.14.

\subsubsection{Results}

Finally, the results of the measurements from which noise reduction and effectiveness of both demonstrators can be determined are presented. First, the results of the sound power measurements that were carried out are discussed. Thereafter, the results of measurements carried out with a binaural measurement system are given.

\section{Sound power measurement}

The sound power measurements were carried out in an anechoic room in one of Intel's research labs. The setup that was used complies with ISO 7779. The demonstrator is placed in the center of a half dome with a radius of $1 \mathrm{~m}$ (see figure 7.15(a)). 

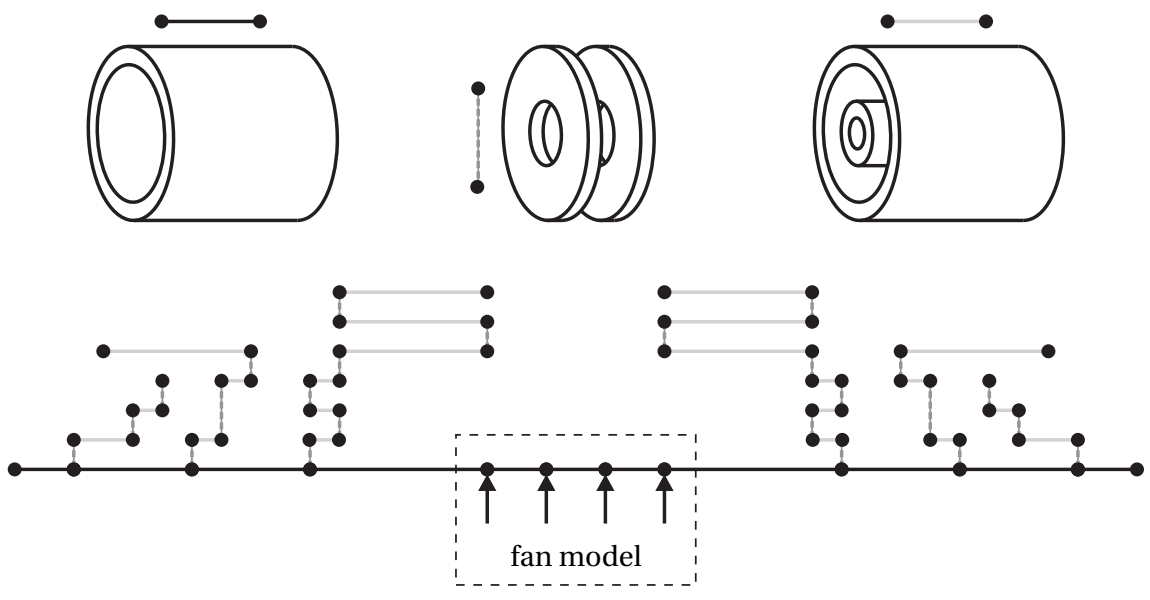

Figure 7.14: Schematic representation of the LRF model for the 3- resonator setup.

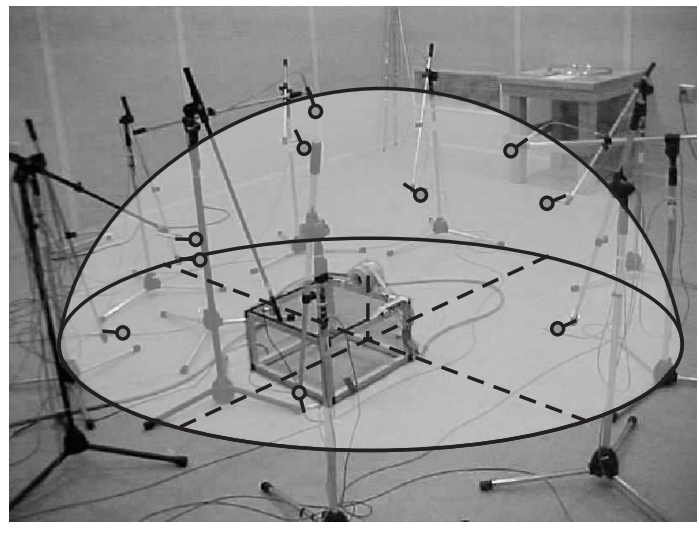

(a) ISO 7779 compliant setup
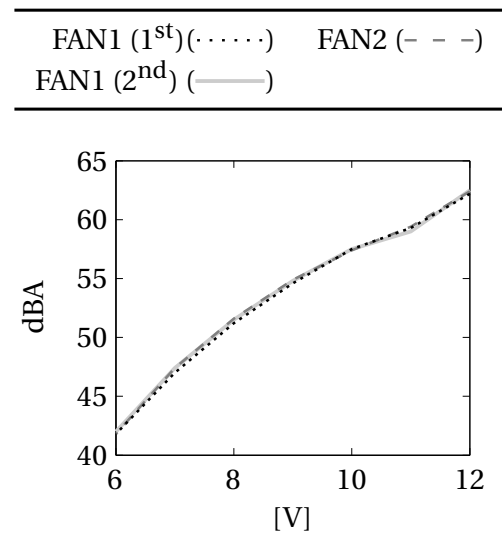

(b) Sound power of the fan (two measurements) and of a second fan of the same type and make

Figure 7.15: Sound power measurement setup and measurement results for the fan.

Ten microphones are positioned on the surface of the dome in predefined locations. Based on their signals, the total sound power transmitted through the surface of the dome and the transmitted power per square meter is calculated. The measured 3rd octave band sound spectra are subjected to A-weighting before they are used in the calculation. The final output of the sound power measurement is a single value in $\mathrm{dB}(\mathrm{A})$, reflecting the sound power emitted by the test object over all 
audible frequencies compensated for the frequency-dependent sensitivity of the human ear.

Various measurements of the cooling fans under consideration were taken to determine the variation in sound power between measurements and between fans of the same type and make. The observed variation in the sound power is never greater than $0.5 \mathrm{~dB}(\mathrm{~A})$. To investigate the effect of a duct without resonators (having the same length as a resonator setup) on the sound power emitted by a fan, special duct pieces were manufactured. The measured sound power of the demonstrator and duct without resonators are plotted as a function of the applied voltage for the (short) 3-resonator setup and (long) 4-resonator setup in figure 7.16.

\begin{tabular}{rr}
\hline $\begin{array}{r}\text { resonator setup with foil }(- \\
\text { duct, no resonators }(\cdots \cdots)\end{array}$ & resonator setup without foil $\left(-{ }^{--}\right)$ \\
fan only $(-)$ \\
\hline
\end{tabular}

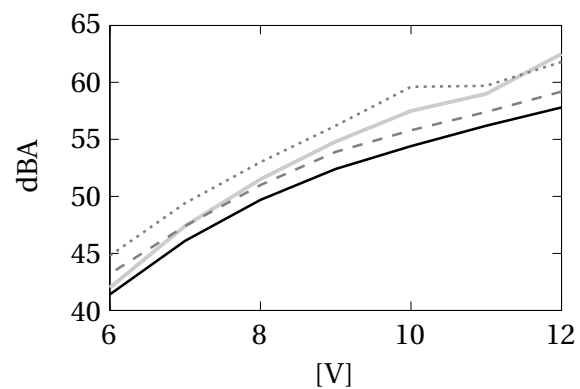

(a) 3-resonator setup

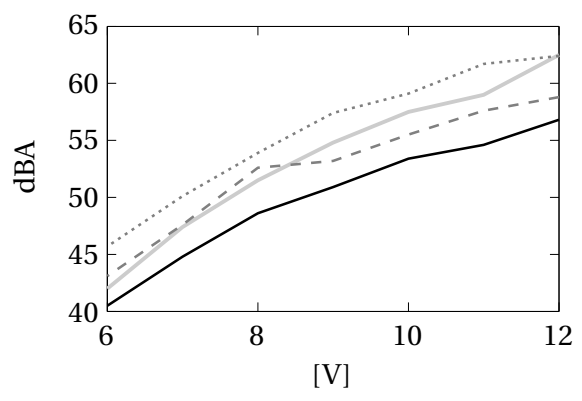

(b) 4-resonator setup

Figure 7.16: Sound power measurement results of the 3-resonator and 4-resonator setup.

Closer inspection of the noise spectrum pointed out that there is an increase in flow noise when resonators are introduced in the duct. The most likely cause of this increase is the fact that the airflow becomes turbulent when it passes the resonator openings. This problem was (partially) circumvented by placing a thin foil (Saran Wrap) over the resonator entrances. The foil is light and weak enough for the sound field to pass through it virtually unaltered, yet it prevents flow separation of the air flow passing the resonator opening. In this way, the flow noise created by the resonator openings is reduced without changing the acoustical properties of the resonators. The results of the demonstrators with foil-covered resonator entrances are also included in figure 7.16.

A reduction of approximately $3 \mathrm{~dB}(\mathrm{~A})$, and $5.5 \mathrm{~dB}(\mathrm{~A})$ is achieved at higher voltages for the 3-resonator and 4-resonator setup, respectively. For lower voltages the demonstrators become less efficient $(1 \mathrm{~dB}(\mathrm{~A})$, and $2.5 \mathrm{~dB}(\mathrm{~A})$ for the 3-resonator 
and 4-resonator setup, respectively). Furthermore, it is observed that placing the fan in a duct without resonators leads to an increase in emitted sound power. With respect to a ducted fan the 3 -resonator setup reduces the fan noise by $3.5-5 \mathrm{~dB}(\mathrm{~A})$ over the entire voltage range while the 4-resonator setup reduces the fan noise by $5-7 \mathrm{~dB}(\mathrm{~A})$.

\section{Binaural measurements}

In addition to sound power measurements the so-called binaural HEAD measurement system was used to determine the effectiveness of the demonstrators. The HEAD measurement system consists of a human head shaped dummy with microphones mounted in the ears, a playback unit that allows realistic play-back of the recorded sounds, and special software tools that help analyze the recorded sound files. An important quantity that defines the 'quality' of a sound that has both broadband and tonal aspects in its spectrum is the so-called tonality. Tonality is a measure that reflects to what extent a sound is perceived as tonal. Tests carried out with various test panels showed that sound that is more tonal is more annoying than broadband sound [122, 123]. These findings support the noise reduction strategy that was applied, namely reducing the highest peaks in the frequency spectrum. The tonality of the different resonator setups is calculated with the software tools of the HEAD measurement system. The results for the measurements of the cooling fan are plotted in figure 7.17(a). For this particular test, the dummy head was placed at the ISO 7779 seated operator position, using a square desk (ca. $80 \times 80 \mathrm{~cm}$ ). The test object was placed $20 \mathrm{~cm}$ above the desk's surface.

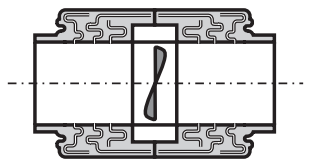

(a)

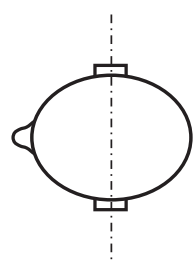

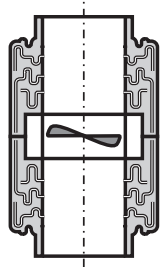

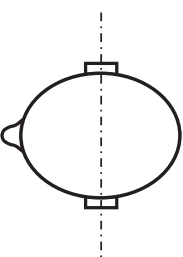

(b)

Figure 7.17: Orientation of the demonstrator with respect to the HEAD system; Dummy facing the inlet of the demonstrator (a) and dummy facing the side of the demonstrator (b).

The orientation of the object with respect to the dummy head is also important for this type of test. The results of this test can be influenced substantially by the orientation of the object in case it creates a directional sound field. All mea- 
surements are carried out for two orientations of the test object; in one case the inlet opening of the fan is facing the dummy head (this orientation is indicated by 'frontal', see figure 7.17(a)). In the second case the fan axis is parallel to an axis through the ears of the dummy head (this orientation is indicated by 'side', see figure $7.17(\mathrm{~b})$ ).

The measured tonality of the 3-resonator and 4-resonator setup as a function of the operating voltage is plotted in figure 7.18(a) and 7.18(b), respectively. The tonality of both demonstrators was measured with and without the foil that reduces flow noise. The results of both demonstrators show a considerable drop in tonality. This concurs with the adopted noise reduction strategy which was aimed at reducing the peaks in sound power at the BPF and higher harmonics. The foil that was added to reduce the (broadband) flow noise over the resonator openings has little influence on the tonality.

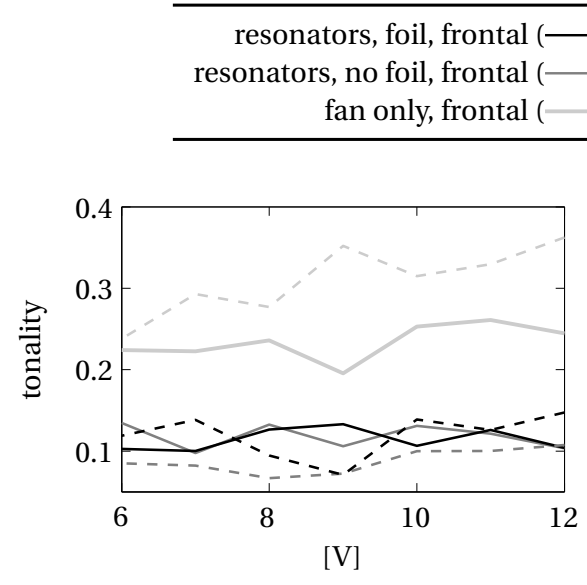

(a) 3-resonator setup

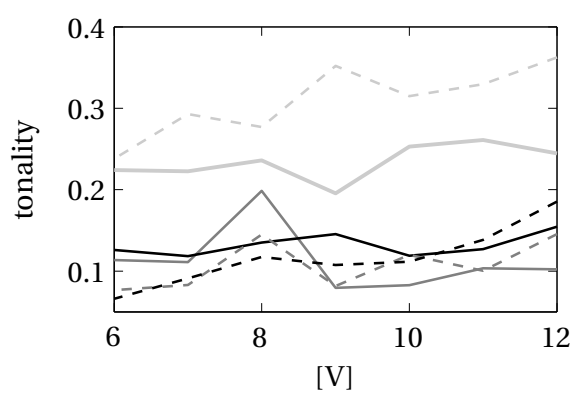

(b) 4-resonator setup

Figure 7.18: Tonality as a function of operating voltage of the 3-resonator and 4-resonator setup for different orientations.

For a fan without resonators or duct, a tonality level between 0.2 and 0.35 is found. When the dummy is facing the duct opening, flow noise is more pronounced especially at higher frequencies. In that case, the tonal content of the information is lower, which is reflected by the tonality results. The tonality is reduced to a level between 0.07 and 0.14 for the 3-port setup, and a level between 0.07 and 0.20 for the 4 -port setup. At these levels, the tonal content appears to be less dependent on the orientation of the dummy. Results of subjective listening tests carried out with a number of test panels imply that this reduction in tonality contributes considerably to the sound quality of the emitted noise especially at 
higher noise levels (or in this case higher voltages) [122, 123].

\subsection{Sound absorption of aircraft cabin noise}

Cabin noise is an important topic of research in the aircraft industry. The acoustic pressure waves emitted by the propelling system are high enough to find their way into the cabin despite a wall of thermal insulation that blocks much of the sound. In addition, the turbulent boundary layer around the fuselage of an aircraft causes structural vibrations that are observed as an excessive source of noise in the interior of the aircraft. Flow noise typically has a broadband characteristic and has a pronounced presence at frequencies below $2000 \mathrm{~Hz}$.

One of the possibilities to reduce the sound pressure levels in the cabin is the placement of acoustic resonators in the trim panels lining the inner surface of the cabin. In the work of Hannink such a solution is proposed [124]. The acoustic resonators that are considered are so-called tube resonators which are known to reduce the sound within a fairly narrow frequency band [19]. To overcome this limitation, Hannink proposes the use of multiple resonators of different dimension targeting multiple frequencies. If the dimensions are properly chosen, broadband absorption over a relatively wide frequency range can be achieved. Unfortunately, the frequency bands where the resonators have a significant impact overlap. As a result, the different resonators influence each other, and the absorption behavior of a surface having multiple resonators of different dimensions is complex. An optimization algorithm is used to tune the resonator dimensions in order to obtain broadband absorption over a desired frequency range. The influence of viscosity and thermal conductivity have a pronounced effect on the acoustic behavior of tube resonators and must be modeled accurately to predict the sound absorbing capabilities of a resonator design. To simplify matters Hannink uses a setup with a so-called impedance tube (see figure 7.19) and only considers frequencies well below the cut-on frequency ${ }^{6}$ so efficient $1 \mathrm{D}$ LRF models can be used to describe the viscothermal wave propagation in the resonators.

\section{Coupled LRF model}

Due to their efficiency, using coupled LRF models is an attractive means of modeling a multiple resonator system if a large number of resonator dimension needs to be optimized simultaneously. The demonstrator setup described below was already presented by Hannink in [124], and results from an optimization for broad-

\footnotetext{
${ }^{6}$ Above the cut-on frequency, higher order acoustic waves change from evanescent to propagating waves.
} 
band noise reduction between $1000-2000 \mathrm{~Hz}$. It is concluded by Hannink that there are significant discrepancies between the performance of the LRF model and measured performance. From the results presented by Hannink, it can also be concluded that the accuracy of the LRF model limits the accuracy of the optimization. Therefore, the demonstrator setup presented in [124] serves as an example of a practical situation where LRF models fail to produce results of the desired accuracy.

In this section, it is investigated whether the discrepancies between the results of the LRF model and measurements are caused by the approximations required to obtain LRF models. In this context, the full FE model is used as a reference model since it does not involve any of these approximations. The setup is also modeled with the FE-BLA method described in appendix J, which is a more efficient alternative to using an FE model based on the full LNSF equations. First the demonstrator setup is described in more detail. The results obtained for the LRF model, the FE model, and the FE-BLA model are compared with measurement results in section 7.2 .3

\subsubsection{Demonstrator setup}

As proof of concept, a number of resonator designs were created by Hannink to demonstrate that broadband sound absorption with multiple resonators can be achieved in a setup using an impedance tube. A schematic representation of the setup is given in figure 7.19.

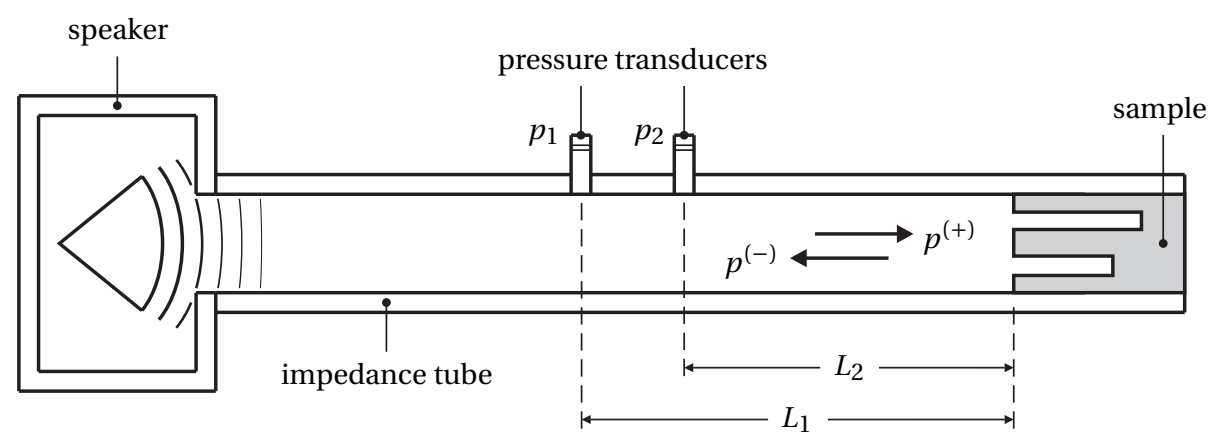

Figure 7.19: Impedance tube setup for measuring the acoustic absorption coefficient.

A speaker on one end of the impedance tube generates sound waves that travel through the tube and impinge on the sample of which the acoustic absorption characteristics are to be determined. Two pressure transducers are flush mounted in the tube to measure the amplitude of the resulting sound field. The impedance 
tube functions as a waveguide and can be modeled efficiently as an acoustic twoport network as described in section 4.3. Based on signals of the pressure transducer the amplitudes of the waves traveling to and from the sample, indicated by $p^{(+)}$and $p^{(-)}$, are determined by:

$$
\left\{\begin{array}{l}
p^{(+)} \\
p^{(-)}
\end{array}\right\}=\left[\begin{array}{ll}
e^{-i k \Gamma_{A} L_{1}} & e^{i k \Gamma_{A} L_{1}} \\
e^{-i k \Gamma_{A} L_{2}} & e^{i k \Gamma_{A} L_{2}}
\end{array}\right]^{-1}\left\{\begin{array}{l}
p_{1} \\
p_{2}
\end{array}\right\}=
$$

Based on the complex amplitudes of the backward and forward traveling wave, the so-called absorption coefficient is defined as

$$
\alpha=1-\left|\frac{p^{(-)}}{p^{(+)}}\right|^{2}
$$

If the absorption coefficient equals zero, acoustic waves are completely reflected $\left(\left|p^{(-)}\right|=\left|p^{(+)}\right|\right)$. On the other hand, if the absorption coefficient equals unity, the incident acoustic wave is completely absorbed by the sample $\left(\left|p^{(-)}\right|=0\right)$.

\section{Sample design}

A sample design including 20 circular cylindrical tube resonators of different length and radius was obtained by an optimization of the dimensions aimed at achieving full absorption between $1000-2000 \mathrm{~Hz}$. The geometry of the sample and a schematic representation of the location of the different resonators is given in figure 7.20. After the sample was manufactured, the measurements of the true dimensions of the sample were taken. These dimensions are listed in table 7.1.

\begin{tabular}{lrrrrrrrrrrr}
\hline $\begin{array}{l}\text { Resonator } \\
\text { number }\end{array}$ & 1 & 2 & 3 & 4 & 5 & 6 & 7 & 8 & 9 & 10 \\
\hline $\begin{array}{l}\text { Length [mm] } \\
\text { Radius [mm] }\end{array}$ & 43.6 & 63.6 & 45.4 & 46.8 & 48.6 & 50.5 & 40.6 & 52.1 & 54.4 & 56.7 \\
\hline \hline $\begin{array}{l}\text { Resonator } \\
\text { number }\end{array}$ & 11 & 12 & 13 & 14 & 15 & 16 & 17 & 18 & 19 & 20 \\
\hline $\begin{array}{l}\text { Length [mm] } \\
\text { Radius [mm] }\end{array}$ & 59.0 & 61.7 & 64.7 & 66.9 & 69.8 & 72.9 & 76.1 & 79.8 & 36.8 & 82.3 \\
\hline
\end{tabular}

Table 7.1: Resonator dimensions. Resonator number corresponds with the number in figure 7.20 (c) 


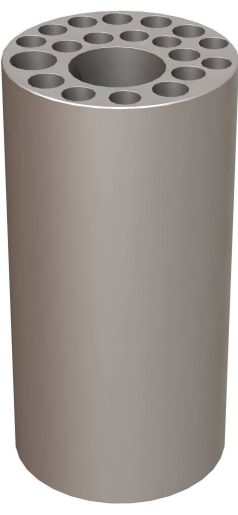

(a)

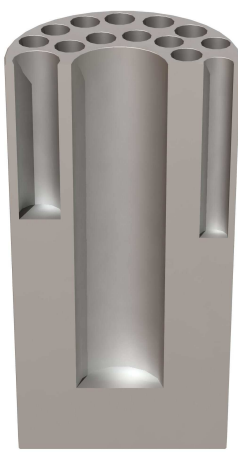

(b)

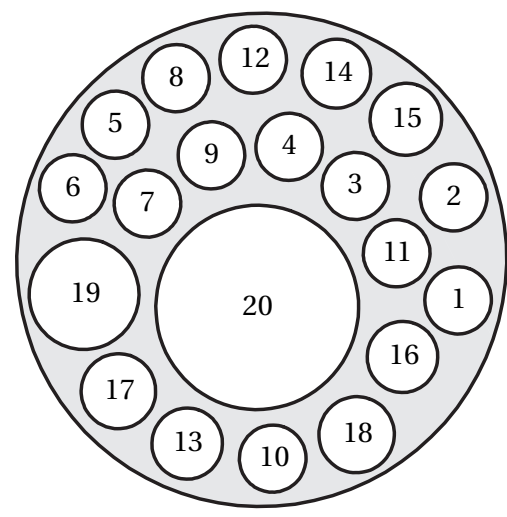

(c)

Figure 7.20: Geometry of the sample (a) (b) and a schematic representation of the location of the different resonators (c).

\subsubsection{LRF, FE, and FE-BLA model}

Below the LRF, FE, and FE-BLA models that are used to predict the absorption coefficient are briefly introduced.

\section{Two-port LRF model}

A schematic representation of the two-port LRF network that is used is given in figure 7.21. A fluid flow of zero is prescribed at all openings of the two-port network, except at $p_{1}$, where a unit pressure is described. Based on the pressure at $p_{1}$ and $p_{2}$, equations (7.11) and (7.12) can be used to determine the absorption coefficient. Note that the lengths of the resonator were adjusted with the length correction for a circular tube centrally located in another circular tube [70].

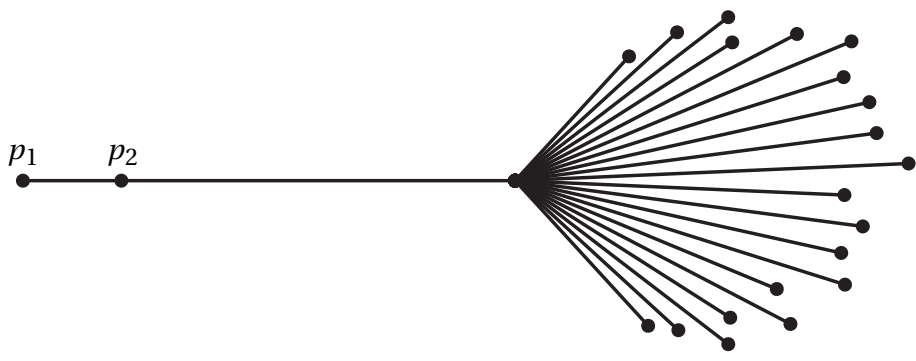

Figure 7.21: Schematic representation of the two-port LRF model. 


\section{FE model}

The air volume inside the resonators and a section of the impedance tube is modeled with the FE methods described in chapter 5 . The elements that are used are from the Taylor Hood family. The corresponding FE mesh is depicted in figure 7.22. To accurately capture the viscothermal effects in the boundary layers, the surface of the tube resonators and the interface between the sample and the fluid in the impedance tube are refined. Note that the mesh is not refined at the bottom of the resonators and inner surface of the impedance tube. Refining the mesh along these surfaces results in a significantly larger numerical problem, while the resulting solutions do not lead to significant improvements in the accuracy of the predicted values of the absorption coefficient. At these surfaces symmetry boundary conditions are applied; a zero normal velocity, traction force, and heat flux are assumed. At the other surfaces of the sample, no-slip/isothermal boundary conditions are applied. A unit normal force, zero traction, and zero heat flux are applied at the entrance of the section of the impedance tube.

From the solution of the FE model, the averaged pressure over the impedance tube cross section is obtained at the same locations as the pressure transducers. Subsequently, equations (7.11) and (7.12) are used to determine the absorption coefficient.

\section{BLA model}

The boundary layer approach as described in appendix $\mathrm{J}$ is used with quadratic (Lagrangian) elements. Since the influence of viscous and thermal effects in the boundary layers are already accounted for by the FE-BLA method, the mesh refinements at the surface of the resonators and at the interface of the sample and the fluid in the impedance tube are no longer necessary to obtain accurate results. Besides the absence of mesh refinements at the boundaries, the mesh used for the FE-BLA model is very similar to that depicted in 7.22.

A normal velocity of zero is applied everywhere on the boundaries, except at the entrance of the section of the impedance tube where a unit normal force is prescribed. Again, the averaged pressure over the impedance tube cross section is obtained at the locations of the pressure transducers, and equations (7.11) and (7.12) are used to determine the absorption coefficient.

\subsubsection{Results}

The values for the absorption coefficient obtained with the different models and by performing measurements are presented in figure 7.23. Here the absorption coefficient is plotted as a function of frequency in the range $900-2100 \mathrm{~Hz}$. Note 


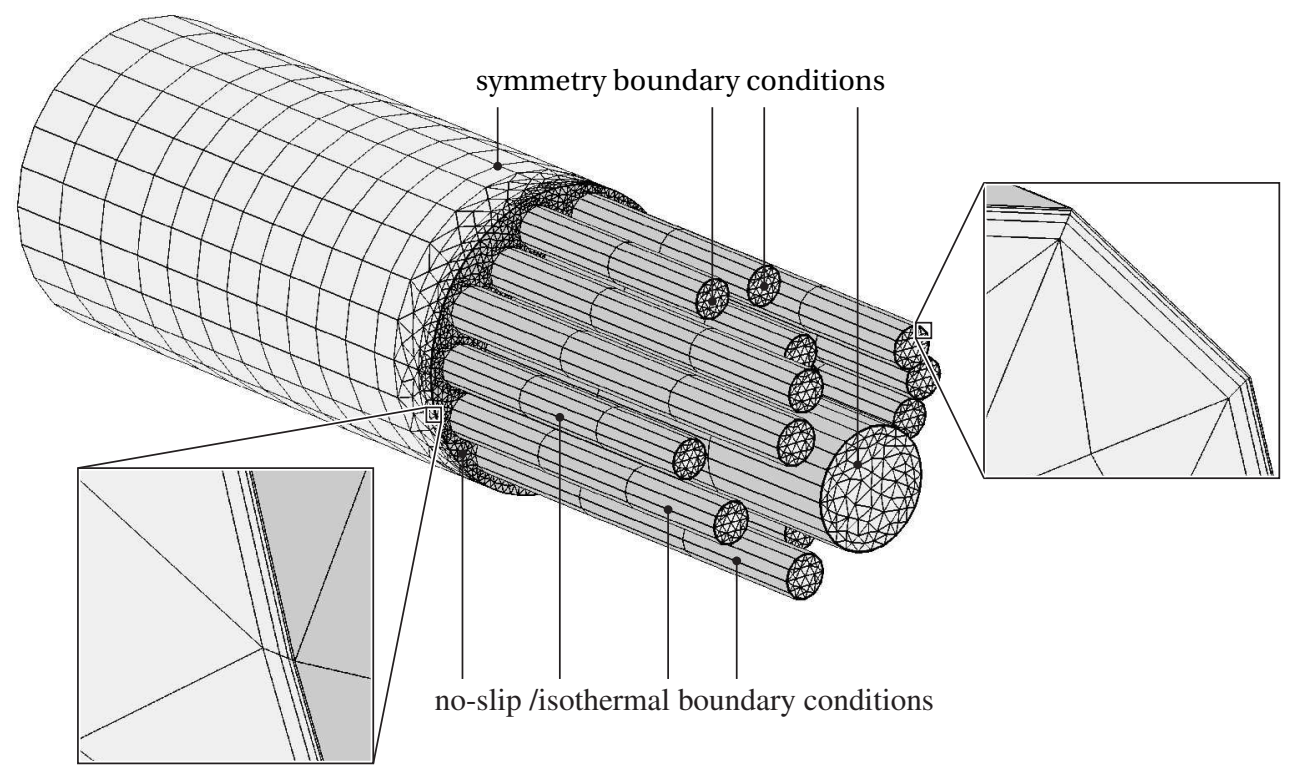

Figure 7.22: Mesh used for the FE model of the sample. Two close-ups show the mesh refinement intended to capture the viscous and thermal boundary layer at the resonator surface and at the interface of the sample and the fluid in the impedance tube.

that the absorption coefficient for the LRF model presented in figure 7.23 deviates from unity for certain frequencies between $1000-2000 \mathrm{~Hz}$. These deviations are not due to a poor optimization, but are caused by deviations between the dimensions for the optimized LRF model and the actual dimension of the manufactured sample. Naturally, the dimensions of the manufactured model are used when the LRF model (and the other two models) are compared with measurement data.

The general trend of all models and the measured results are similar; the absorption coefficient is close to unity between $1000-2000 \mathrm{~Hz}$ and quickly falls to zero outside that range. Nevertheless, a significant difference can be observed at various frequencies. It is clear that the results of the LRF model resemble the measurement data least of all. Especially at frequencies below $1800 \mathrm{~Hz}$, the LRF model predicts absorption coefficients that are very close to unity, while the measurement data shows considerable drops in the absorption coefficients for several frequencies. In that respect, the results for the FE models follow the patterns of peaks and valleys observed for the measurement data much closer. Only above $1800 \mathrm{~Hz}$ is the trend in the results of the FE data significantly different from those observed for the measurement data. Note that the results for the FE and FE-BLA model are very similar. This concurs with the notion that FE-BLA models are expected to of- 


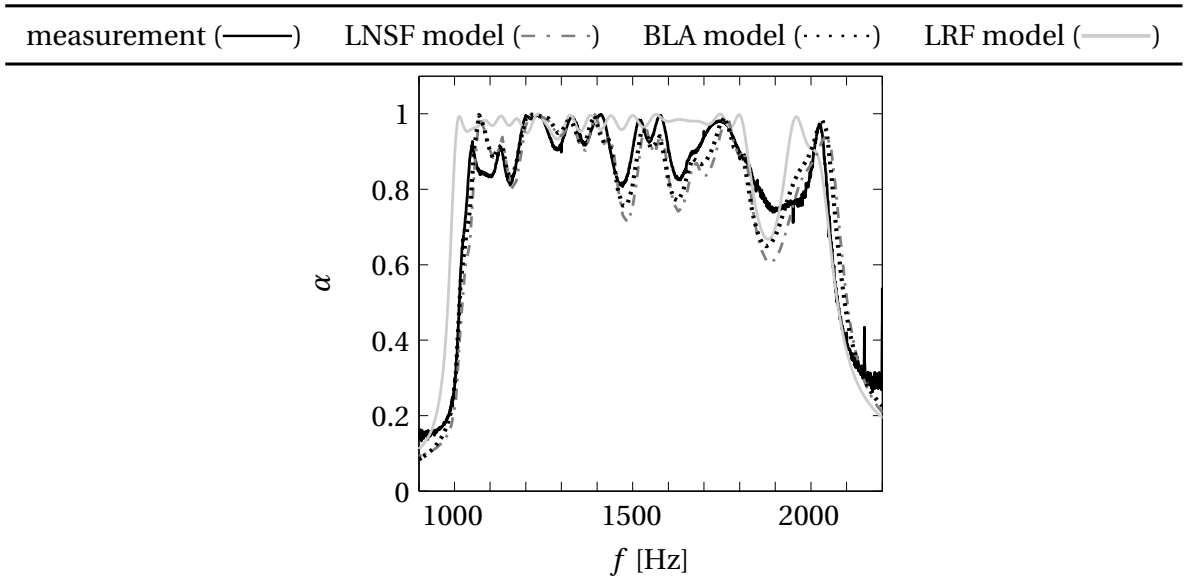

Figure 7.23: Measured and calculated absorption coefficient for the sample shown in figure 7.20 .

fer a good approximation of the solutions for the full LNSF equations if the viscous and thermal boundary layers are well separated and the domain boundaries can be considered as locally flat. For the present case, the dimensionless shear wave number in the narrowest resonators at $900 \mathrm{~Hz}$ is 65 , and the radii of curvature of the boundary are small compared to the shear- and thermal wave lengths, implying that the use of the FE-BLA model is appropriate.

To give an indication of the efficiency of the different models, table 7.2 shows the degrees of freedom involved in the resulting numerical systems and the averaged computation time required to obtain a solution for a single frequency.

\begin{tabular}{lcc}
\hline Model & number of DOFs & time $[\mathrm{s}]$ \\
\hline FE (LNSF) & $532 \cdot 10^{3}$ & 1802 \\
FE-BLA & $96 \cdot 10^{3}$ & 22 \\
LRF & 22 & 0.011 \\
\hline
\end{tabular}

Table 7.2: Efficiency of the LRF, FE and FE-BLA model in terms of computation time and degrees of freedom. The given computation time is the averaged time required for computation at a single frequency.

Based on the presented results, it can be concluded that the FE-BLA model is the most suitable model in terms of accuracy and efficiency to be used for optimization of the dimensions of the resonator design. The LRF model does not provide the desired accuracy, and the FE model based on the full LNSF equations, 
which is very computationally intensive, does not provide an increase in accuracy compared to the FE-BLA model. 


\section{Chapter 8}

\section{Conclusions and discussion}

In the course of this study various models for viscothermal wave propagation were developed, and the accuracy of new and existing models was investigated. The presented models are aimed at predicting wave propagation in fluids when excited at a certain frequency for cases where viscous and thermal effects cannot be neglected. Viscothermal effects can become important when the dimensions of the fluid domain become small compared to the so-called viscous and thermal boundary layers, or when a fluid system is excited at a frequency near resonance.

The toolkit of models for viscothermal wave propagation was extended with several FE methods for the equations governing viscothermal wave propagation. A distinction can be made between so-called mixed methods, which are based on velocity, temperature and pressure variables, and so-called primal methods, which are based on velocity and temperature variables only. The convergence behavior of both types of methods was instigated. In addition, the numerical stability and robustness of various mixed methods was assessed with the so-called numerical inf-sup test.

In addition, the toolkit of models for viscothermal wave propagation was extended with several new approximate and exact models. New approximate models, belonging to the class of so-called Low Reduced Frequency models (or LRF models), were developed for curved and tapered waveguides. The range of available 'exact' models was extended to include a solution for curved layers. Furthermore, a new model that is a hybrid between an FE method and an analytical solution was developed for straight, curved, twisted, and coiled waveguides of arbitrary cross section (including the effects of mean flow).

Lastly, a parameter study on the accuracy of coupled LRF models was carried out. The effects of changes in boundary conditions, waveguide cross section and curvature were investigated in a systematical fashion. 
The main conclusions of the study on convergence and numerical stability of the different $\mathrm{FE}$ methods are given below, and the outcomes of the parameter study on the accuracy of coupled LRF models are presented. Thereafter, some of the results presented in this thesis are discussed briefly.

\subsection{Conclusions}

The main conclusions of the work presented in this thesis are summarized below:

\section{Mixed FE methods (Chapter 5)}

- Triangular and quadrilateral Taylor Hood, Crouzeix Raviart and MINI elements which are numerically stable for the Stokes equations are robust for all frequencies when extended to the LNSF equations by choosing the trial functions for temperature equal to the trial functions for velocity.

- Failure of FE methods for the LNSF equation to pass the inf-sup test does not necessarily result in poor convergence. This behavior is similar to that of equivalent FE methods for the Stokes equations. For high frequencies (corresponding to $k / s \gtrsim 1$ ) convergence of FE methods for the LNSF equations is optimal, independent of the outcome of the inf-sup test. This behavior is similar to that of equivalent FE methods for linear elasticity in the case of compressible material behavior.

\section{Primal FE methods (Chapter 5)}

- Several examples of primal (v/T-based) FE methods, resulting in the P1, Q1 and Q2 elements, do not appear to be robust. The presented examples show that their use should be avoided.

- The P2 element appears to have optimal convergence on unstructured meshes. The presented results suggest that these elements can be useful, but should be used with caution, since convergence was not optimal for one of the considered structured meshes.

\section{LRF performance (Chapter 6)}

The first three remarks apply to situations where 1D layer geometries with changes in cross section and curvature are modeled using multiple coupled LRF models for straight waveguides. 
- The relative errors due to changes in waveguide cross section and waveguide curvature are relatively small when $\tilde{k} \ll 1$ and $k / s \ll 1$.

- The relative errors due to changes in waveguide cross section and waveguide curvature decrease as $k / s$ goes to zero. The error is bounded from below by the error obtained for the inviscid adiabatic case. For $s>10$ the observed error is similar to that for the inviscid adiabatic case, and it is dominated by the omission of the higher order acoustic modes.

- The relative errors due to changes in waveguide cross section are dominated by the omission of both vorticity and higher order acoustic modes if viscothermal effects are pronounced in (some part of) the waveguide $(\tilde{s}<5)$. However, for $k / s \ll 1$ these errors are relatively small.

- The a priori criteria $k / s \ll 1, k / s_{t} \ll 1$ and $\tilde{k}<1$ are not sufficient to ensure accurate approximation of the propagation constant for LRF models involving no-slip/adiabatic boundary conditions in cases where $\tilde{s}<1$. However, for most fluids the errors are minor in the range of values for $\tilde{s}, \tilde{s}_{t}$ and $\tilde{k}$ where the assumptions for continuum mechanics hold.

- For no-slip/adiabatic boundary conditions, the propagation constant and the velocity/temperature profiles of the first thermal mode will closely resemble those of the first acoustic mode if $\tilde{s} \ll 1$ and $k / s \ll 1$. Using (coupled) LRF models, this can cause significant errors in the predicted amplitudes of propagating acoustic waves due to inlet effects. However, for most fluids the errors are minor in the range of values for $\tilde{s}, \tilde{s}_{t}$ and $\tilde{k}$ where the assumptions for continuum mechanics hold.

\subsection{Discussion}

A concise discussion on some of the results gathered in this thesis is given below:

\section{Primal FE methods}

The numerically robust FE methods that were presented can serve as a benchmark tool for the accuracy of approximate models. In that sense, they provide a valuable extension of the toolkit of models for viscothermal wave propagation. However, the efficiency of the resulting FE methods in terms of computation time and memory use is very poor compared to the alternative models, especially in 3D. In this context the presented primal methods seem attractive because one of the variables is eliminated before discretization, resulting in systems of fewer degrees of 
freedom. However, these FE methods are not guaranteed to have optimal convergence for all meshes. In particular, primal FE methods with linear trial functions suffer from severe locking. On the other hand, eliminating the pressure degrees of freedom on element level from a mesh of Crouzeix Raviart elements leads to a system that has an equivalent number of degrees of freedom as obtained for a primal FE method using quadratic trial functions. This implies that there is no need for primal FE methods with quadratic trial functions (for which numerical stability is questionable) since robust FE methods are available offering the same efficiency.

\section{Ill-conditioning for small frequencies}

The FE error in pressure and temperature of all considered elements grows as the frequency (or $k / s$ ) goes to zero. This concurs with the results of the numerical inf-sup test. The rise in the FE error is due to an ill-conditioning of the system that is unrelated to the locking phenomenon observed for nearly incompressible material behavior. It is caused by the fact that the solution for pressure will only be unique up to a constant in the limit of zero frequency. The large errors due to the ill-conditioning are accompanied by an order of convergence of pressure and temperature that is above optimal for some of the considered cases. The order of convergence is eventually expected to drop so optimal convergence is obtained if the mesh is continuously refined.

\section{Temperature trial functions}

Based on the results for mixed FE methods, it is expected that any numerically stable FE element for the Stokes equation is robust for all frequencies when extended to the LNSF equations by choosing arbitrary trial functions for temperature (assuming the functions are conformal and offer approximability). From the presented inf-sup and convergence results, it was observed that in the case of the Taylor Hood element, the choice of trial functions for temperature has no effect on stability of the FE elements for the LNSF equations when it is of equal or higher order than the trial functions. Choosing a lower order trial function for temperature than for velocity does not seem to affect the order of convergence of velocity for the number of mesh refinements that were considered (lower convergence rates are expected eventually if the mesh is continuously refined). The order of convergence for pressure reduces in some the evaluated conditions, but convergence still remains optimal for the number of mesh refinements that were considered (lower convergence rates are expected eventually if the mesh is continuously refined). 


\section{Extension of LRF models}

The toolkit of models for viscothermal wave propagation was extended with LRF models for curved and tapered waveguides. The model for tapered geometries accurately captures the spreading/contracting of the wave as it travels through a tapered waveguide. This so-called geometric spreading is not captured when a tapered waveguide is modeled by coupling multiple straight layers of increasing thickness. The value of the tapered LRF model is limited, however, since it is unclear how a tapered and straight waveguide can be coupled to yield an accurate description of the local impedance changes experienced by traveling waves.

Another addition to the toolkit of models for viscothermal wave propagation are LRF models for curved waveguides. The LRF models yield a fairly accurate description of viscothermal wave propagation through curved tubes. The error in the approximation of the propagation constant increases as the waveguide is curved tighter and experiences a maximum for shear wave numbers between $5<\tilde{s}<10$. Nevertheless, the errors remain relatively small ${ }^{1}$ for all curvatures and values of $\tilde{s}$.

\section{LRF performance}

In chapter 6 the errors caused by modeling geometries with changes in waveguide cross section and curvature with multiple coupled LRF models for straight waveguides were investigated. The observed errors for all considered changes in waveguide cross section and curvature are small for $k / s \ll 1$, except in cases where the error is dominated by omission of the higher order acoustic modes $(\tilde{k} \sim 1)$. Nevertheless, the range of $k / s$ where LRF models are guaranteed to produce results of a certain accuracy is reduced compared to the situation where LRF models are used to describe geometries without changes in cross section of curvature. The additional errors can add up (or cancel out) if multiple LRF models of different cross section or curvature are coupled. The accuracy of multiple coupled LRF models is thus not automatically guaranteed for $k / s \ll 1$ and $\tilde{k} \sim 1$. This is illustrated by the application in section 7.2. A point of further research might be a parameter study on the errors of LRF models for geometries having waveguide junctions involving several waveguides of different dimensions. Results from such a study could ultimately lead to a practical set of engineering rules, providing an estimation of the accuracy of multiple coupled LRF models.

\footnotetext{
${ }^{1}$ Relative errors with a maximum of $2 \%$ in the real and imaginary part of the propagation constant are observed.
} 


\section{Extension of Kirchhof models}

Another addition to the toolkit of models for viscothermal wave propagation is an exact solution for curved layers. It is made plausible that exact solutions only exist for three types of layers: flat layers, cylindrical layers and spherical layers ${ }^{2}$. Furthermore, a hybrid FE-Kirchhoff solution is developed for 1D waveguides. Such solutions exist for coordinate systems that allow a separation of coordinates so that the solution in $p d$-direction is known analytically, while the waveguide cross section and curvature remain constant in $p d$-direction. The FE techniques presented in chapter 5 can be used to discretize the remaining equations for the solution in $c d$-direction. A modification allows the resulting system of equations to be written as an eigenvalue problem that yields the different modes and corresponding propagation constants. This is an efficient method for calculating the different modes for straight, curved, coiled and twisted waveguides of arbitrary constant cross section. The different terms accounting for convection of mass, impulse and energy can be easily added to account for the effects of a mean flow. Solutions for coupled waveguides of different cross section and curvature can be obtained by using the modes in the mode-matching scheme presented in chapter 3.

\footnotetext{
${ }^{2}$ In this context, an annular tube can be seen as a curved layer, and a closed tube can be seen as a curved layer with zero inner radius (having continuity boundary conditions at the center).
} 


\section{Nomenclature}

\section{Roman}

A

Velocity profile inhomogeneous equation, homogeneous boundary conditions

$A_{b} \quad$ Velocity profile homogeneous equation, inhomogeneous boundary conditions

b Specific body force vector

$B$ Averaged velocity profile inhomogeneous equation, ho$\left[\mathrm{Nkg}^{-1}\right]$ mogeneous boundary conditions

$B_{b} \quad$ Averaged velocity profile homogeneous equation, inhomogeneous boundary conditions

$c$ Speed of sound

$c_{0}$ Undisturbed (adiabatic) speed of sound Temperature profile inhomogeneous equation, homogeneous boundary conditions

$C_{b} \quad$ Temperature profile homogeneous equation, inhomogeneous boundary conditions Specific heat at constant pressure Specific heat at constant volume $\left[\mathrm{Jkg}^{-1} \mathrm{~K}^{-1}\right]$ Averaged temperature profile inhomogeneous equation, homogeneous boundary conditions

$D_{b}$ Averaged temperature profile homogeneous equation, inhomogeneous boundary conditions

$e$

$\mathbf{e}_{n}$

$f$

$h$

$H_{n}^{1}$ $H_{n}^{2}$ Specific internal energy Unit normal vector Frequency Measure for size of the elements in a mesh Hankel function of the first kind of order $n$ Hankel function of the second kind of order $n$ $\left[\mathrm{ms}^{-1}\right]$ $\left[\mathrm{ms}^{-1}\right]$ 


\begin{tabular}{|c|c|c|}
\hline$i=\sqrt{-1}$ & Imaginary unit & {$[-]$} \\
\hline$J_{n}$ & Bessel function of the first kind of order $n$ & {$[-]$} \\
\hline$k$ & Acoustic wave number & {$\left[\mathrm{m}^{-1}\right]$} \\
\hline$\tilde{k}$ & Dimensionless acoustic wave number & {$[-]$} \\
\hline $\mathbf{K}$ & Mobility matrix & {$[-]$} \\
\hline$l$ & Characteristic length scale & [m] \\
\hline$L$ & Resonator length & [m] \\
\hline$m$ & Mass & [kg] \\
\hline$n$ & Polytropic coefficient/Dimensionality of FE problem & {$[-]$} \\
\hline$p$ & Pressure & {$[\mathrm{Pa}]$} \\
\hline$p_{0}$ & Mean pressure & [Pa] \\
\hline $\bar{p}$ & Pressure variation & {$[\mathrm{Pa}]$} \\
\hline$\tilde{p}$ & Dimensionless pressure variation & {$[-]$} \\
\hline$p^{(+)}$ & $\begin{array}{l}\text { Complex amplitude of wave traveling in positive } \\
x \text {-direction }\end{array}$ & {$[-]$} \\
\hline$p^{(-)}$ & $\begin{array}{l}\text { Complex amplitude of wave traveling in negative } \\
x \text {-direction }\end{array}$ & {$[-]$} \\
\hline $\operatorname{Pr}$ & Prandtl number & {$[-]$} \\
\hline$q$ & Pressure weighting function & [-] \\
\hline $\mathbf{q}$ & Heat flux vector & {$\left[\mathrm{Wm}^{-2}\right]$} \\
\hline$q_{n}$ & Normal heat flux & {$\left[\mathrm{Wm}^{-2}\right]$} \\
\hline$\tilde{q}$ & Normalized $^{3}$ volume flow rate & {$\left[\mathrm{m}^{2}\right]$} \\
\hline$r$ & Coordinate in radial direction & [m] \\
\hline$r_{e}$ & Rate of specific energy supply & {$\left[\mathrm{Wkg}^{-1}\right]$} \\
\hline$R_{0}$ & Specific gas constant & {$\left[\mathrm{Jkg}^{-1} \mathrm{~K}^{-1}\right]$} \\
\hline$s$ & Shear wave number & {$\left[\mathrm{m}^{-1}\right]$} \\
\hline$\tilde{s}$ & Dimensionless shear wave number & {$[-]$} \\
\hline$s_{t}$ & Thermal wave number & {$\left[\mathrm{m}^{-1}\right]$} \\
\hline$\tilde{s}_{t}$ & Dimensionless thermal wave number & {$[-]$} \\
\hline \multirow[t]{2}{*}{$S$} & Cross-sectional surface area & {$\left[\mathrm{m}^{2} / \mathrm{m}\right]$} \\
\hline & Chapter 5: Temperature weighting function & {$[-]$} \\
\hline$\partial S$ & Boundary of cross-sectional area & {$[\mathrm{m} /-]$} \\
\hline $\mathbf{S}$ & Scattering matrix & {$[-]$} \\
\hline$t$ & Time & [s] \\
\hline$T$ & Temperature & {$[\mathrm{K}]$} \\
\hline$T_{0}$ & Mean temperature & {$[\mathrm{K}]$} \\
\hline $\bar{T}$ & Temperature variation & {$[\mathrm{K}]$} \\
\hline$\tilde{T}$ & Dimensionless temperature variation & \\
\hline
\end{tabular}

\footnotetext{
${ }^{3}$ The volume flow rate is normalized with respect to the speed of sound $c_{0}$.
} 
$\mathbf{u} \quad$ Velocity weighting function vector

$v \quad$ Fluid velocity

$[-]$

$\tilde{v} \quad$ Dimensionless fluid velocity

$\left[\mathrm{ms}^{-1}\right]$

Fluid velocity vector

$\mathbf{v}$

Dimensionless fluid velocity vector

$\left[\mathrm{ms}^{-1}\right]$

$\tilde{\mathbf{v}}$

Cartesian coordinates

[-]

$x, y, z$

Trial and test spaces for velocity, temperature and pres-

$X, Y, Z$ sure

$Y_{n}$

Bessel function of the second kind of order $n$

\section{Greek}

$\begin{array}{ll}\gamma & \text { Ratio of specific heats } \\ \Gamma & \text { Propagation coefficient } \\ \eta & \text { Bulk viscosity } \\ \theta & \text { Coordinate in tangential direction } \\ \kappa & \text { Thermal conductivity } \\ \lambda & \text { Second coefficient of viscosity } \\ \mu & \text { Dynamic (shear) viscosity } \\ \xi & \text { Viscosity ratio } \\ \rho & \text { Density } \\ \rho_{0} & \text { Mean density } \\ \sigma & \text { Stress tensor } \\ \tau & \text { Viscous stress tensor } \\ \Phi & \text { Viscous dissipation function } \\ \omega & \text { Angular frequency }\end{array}$

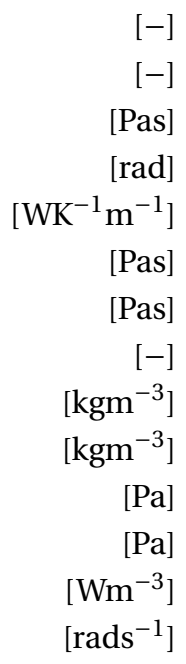

\section{Miscellaneous}

$\Re$

$p d$

$c d$

ker

Im

inf

sup
Squeeze term

Propagation direction

Cross-sectional direction

Kernel or null space

Image or range

Infimum or greatest lower bound

Supremum or least upper bound 


\section{Abbreviations}

$\mathrm{BE}$

Boundary element

BEM

Boundary element method

BLA

Boundary layer approximation

BPF

Blade passing frequency

DOF

Degree(s) of freedom

FE

Finite element

FEM

Finite element method

IL

Insertion loss

LNSF

Linearized Navier-Stokes-Fourier

LRF

Low reduced frequency

NSF

Navier-Stokes-Fourier

SLNS

Sequential linearized Navier-Stokes

SPL

Sound pressure level

\section{Operators}

$\begin{array}{ll}\partial / \partial t & \text { Partial derivative with respect to time } \\ D / D t & \text { Total derivative with respect to time } \\ \nabla & \text { Gradient operator } \\ \nabla_{x} & \text { Gradient operator in direction } x \\ \Delta & \text { Laplace operator } \\ \langle,\rangle & \text { Inner product } \\ R() & \text { Real part } \\ \Im() & \text { Imaginary part } \\ L & \text { Angle in radians or degrees } \\ \| I & \text { Absolute value } \\ \|\| & \text { Norm } \\ T & \text { Transpose } \\ H & \text { Hermitian transpose } \\ E_{(}() & \text {Error operator } \\ E_{R}() & \text { Error operator measuring difference in real part } \\ E_{I}() & \text { Error operator measuring difference in imaginary part } \\ E_{A}() & \text { Error operator measuring difference in magnitude } \\ E_{P}() & \text { Error operator measuring difference in phase } \\ d(,) & \text { Magnitude of the approximation error } \\ {[L]} & \text { Boundary condition operator }\end{array}$




\section{Appendix A}

\section{Derivation of the basic equations}

In the literature, the construction of models describing the dynamic behavior of fluids and their solutions are often obtained without a clear statement of the underlying assumptions. For instance, the term 'Navier-Stokes equations' is used (and misused) to identify a large number of different descriptions of fluid dynamics. In most cases it refers to the incompressible form of the momentum equation including the constitutive equation for incompressible Newtonian fluids. Sometimes the equations are already partly linearized, often without full or accurate description of all the assumptions made.

This appendix is intended to give a concise derivation of the equations governing dynamic behavior of Newtonian fluids that adhere to Fourier's law of heat conduction, which are referred to as Navier-Stokes-Fourier fluids (or NSF fluids) from this point onwards. The description includes derivation of the equations stating the conservation of mass, momentum and energy, the appropriate constitutive relations and the assumptions needed to arrive at the set of equations that describes the dynamic behavior of all NSF fluids. Additional assumptions that are needed in order to apply this set of equations to describe acoustic viscothermal wave propagation in ideal (or perfect) gases are given in chapter 2. References $[125,126,127,128,129]$ were used (not exclusively) in the derivation presented in this appendix.

\section{A.1 Continuum hypothesis}

The basic equations of continuum mechanics are the conservation laws for mass, momentum and energy and some form of the second law of thermodynamics. These equations hold for all materials, e.g. solids, fluids and rigid bodies under the assumptions of the so-called continuum hypothesis: 
All macroscopic length and time scales are considerably larger than the largest molecular length and time scales

By adhering to these assumptions, a medium is considered a continuum. In other words; matter is taken to occupy every point of the space of interest, regardless of how closely the material is examined. These assumptions permit the use of (thermodynamic) quantities such as fluid velocities, density, pressure, and temperature, which can be taken as fields, i.e., piecewise continuous functions of space and time. However, if the spatial and temporal gradients are too large, local thermodynamical equilibrium (LTE) cannot be maintained over the macroscopic imposed time scale and the behavior of the medium can no longer be accurately described by the different thermodynamic quantities. Temperature for instance is, by definition, proportional to the average kinetic and vibrational energy of moving particles in an equilibrated neighborhood. If the macroscopic length scales considered are smaller than the length scale of an equilibrated neighborhood, or the macroscopic time scale considered is smaller than the time it takes the molecules in this neighborhood to reach LTE, the very concept of temperature breaks down, and the temperature becomes undefined. In other words, if the assumptions of the continuum hypothesis are violated, one must resort to statistical mechanics (or kinetic theory) to obtain a valid model.

\section{A.2 Conservation laws}

The conservation law of an extensive property $N$, such as mass, momentum or energy, in integral conservation form is found by applying Reynolds transport theorem.

$$
\int_{V} \frac{\partial \rho N_{v}}{\partial t} d V+\int_{S} \rho N_{v}\left(\mathbf{v}_{b} \cdot \mathbf{n}\right) d S+\int_{S} \rho N_{v}\left(\mathbf{v}_{r} \cdot \mathbf{n}\right) d S=\frac{D N}{D t}
$$

with $N_{v}$ the extensive property $N$ per unit mass, $\rho$ the density, $\frac{D}{D t}$ the total derivative (also known as substantive or material derivative) and $\frac{\partial}{\partial t}$ the (partial) time derivative. The control surface $S$ bounds the (arbitrary, finite, moving) control volume $V$ and the vector $\mathbf{n}$ is the outward pointing normal on $S$. The velocity of the control surface is represented by $\mathbf{v}_{b}$ and the velocity of the medium relative to the control surface is $\mathbf{v}_{r}$. (The velocity of the medium $\mathbf{v}$ is thus defined as $\mathbf{v}=\mathbf{v}_{b}+\mathbf{v}_{r}$.)

Reynolds transport theorem states that for a system bounded by an arbitrary control volume $V$ the rate of change of an extensive property $N$ is equal to the time rate of change of $N$ within the control volume and the net rate of flux of the property $\mathrm{N}$ through the corresponding control surface. 
In Eularian coordinates, the first surface integral on the left-hand side of equation (A.1) vanishes since the control volume is chosen to be fixed in space $\left(\mathbf{v}_{b}=0\right.$ and $\mathbf{v}_{r}=\mathbf{v}$ ). In Lagrangian coordinates the second surface integral vanishes since the control volume is chosen to follow the flow of the medium $\left(\mathbf{v}_{b}=\mathbf{v}\right.$ and $\left.\mathbf{v}_{r}=0\right)$. This evaluation demonstrates that the equations stating conservation of an extensive property can be obtained without choosing a specific coordinate system ${ }^{1}$. However, the choice of a coordinate system will prove to be very important when boundary conditions are concerned.

\section{A.2.1 Conservation of mass}

If equation (A.1) is considered with respect to mass, the specific property $N_{v}$ equals unity and the substantive derivative (the rate of change of mass) equals zero since no mass can be generated or destroyed.

$$
\int_{V} \frac{\partial \rho}{\partial t} d V+\int_{S} \rho(\mathbf{v} \cdot \mathbf{n}) d S=0
$$

The above equation simply states that the time rate of change of mass in $V$, represented by the first term, combined with the flux of mass flowing out of $V$ though $S$, represented by the second term, equals zero.

\section{A.2.2 Conservation of momentum}

If equation (A.1) is considered with respect to momentum, the substantive derivative (the rate of change of momentum) expresses force, and the specific property $N_{\nu}$ is found to be the velocity of the medium $\mathbf{v}$. The following vector equation is obtained:

$$
\int_{V} \frac{\partial \rho \mathbf{v}}{\partial t} d V+\int_{S} \rho \mathbf{v}(\mathbf{v} \cdot \mathbf{n}) d S=\int_{S} \sigma \cdot \mathbf{n} d S+\int_{V} \rho \mathbf{b} d V
$$

with $\sigma$ the stress tensor and $\mathbf{b}$ the body force density. The first term in the lefthand side of equation (A.3) represents the time rate of change of momentum of mass in $V$. The second term represents the momentum of the mass flowing out of $V$ through $S$ per unit time. The first term on the right-hand side represents the stress acting on $S$ and the second term the volumetric forces that are exerted on $V$.

\footnotetext{
${ }^{1}$ The dynamic principles described by the resulting equations are not only coordinate independent but also frame indifferent [125].
} 


\section{A.2.3 Conservation of energy}

If equation (A.1) is considered with respect to energy, $N_{\nu}$ is found to be the specific energy $E$ and the substantive derivative (the rate of change of energy) expresses power.

$$
\int_{V} \frac{\partial \rho E}{\partial t} d V+\int_{S} \rho E(\mathbf{v} \cdot \mathbf{n}) d S=\int_{S}(\sigma \cdot \mathbf{v}) \cdot \mathbf{n} d S-\int_{S} \mathbf{q} \cdot \mathbf{n} d S+\int_{V} \rho r_{e} d V+\int_{V} \rho \mathbf{b} \cdot \mathbf{v} d V
$$

with $q$ the heat flux vector and $r_{e}$ the rate of specific energy supply. The specific energy $E$ is defined as the sum of specific internal energy $(e)$ and specific kinetic energy $\left(\frac{1}{2}|\mathbf{v} \cdot \mathbf{v}|\right)$.

The first left-hand side term of equation (A.4) represents the time rate of change of the sum of the internal and kinetic energy in $V$. The second term represents the internal and kinetic energy flow out of $V$ through $S$ per unit time. The first righthand side term expresses the work done per unit time by the stress acting on $S$, and the second term expresses the heat flux through $S$. The third term represents the volumetric energy supply to $V$ per unit time, and the fourth term represents the work done per unit time by body forces exerted on $V$.

\section{A.3 Balance equations}

The conservation laws given in equation (A.2) through (A.4) are in integral form which is the so-called 'weak' representation. A restriction placed on the validity of the integral form is that the different integrands must be integrable on arbitrary $V$ (and corresponding $S$ ). This restriction is 'weaker' than the restrictions imposed on the partial differential form ${ }^{2}$ (or local form) of the equations which is given below.

$$
\begin{aligned}
\frac{\partial \rho}{\partial t}+\nabla \cdot \rho \mathbf{v} & =0 \\
\frac{\partial \rho \mathbf{v}}{\partial t}+\nabla \cdot \rho \mathbf{v} \mathbf{v} & =\nabla \cdot \sigma+\rho \mathbf{b} \\
\frac{\partial \rho E}{\partial t}+\nabla \cdot \rho E \mathbf{v} & =\nabla \cdot(\sigma \cdot \mathbf{v})-\nabla \cdot \mathbf{q}+\rho r_{e}+\rho \mathbf{b} \cdot \mathbf{v}
\end{aligned}
$$

\footnotetext{
${ }^{2}$ The partial differential form is obtained by applying Gauss theorem to the surface integrals in equation (A.2) through (A.4) and collecting all terms in each equation under one integral. Since the control volume $V$ is arbitrary, these integrals can only equal zero if the integrand is zero for all points in $V$, yielding the PDE conservation form.
} 
These balance equations or continuity equations ${ }^{3}$ are the so-called 'strong' representation of the conservation laws. A restriction to the validity of this form is that the different space and time derivatives must exist. This is a 'stronger' restriction in the sense that it limits the use of the partial differential equations to solutions for which the different variables are continuous. It implies equations (A.5) only govern the smooth part of the flow. Modeling discontinuous behavior in the medium, for instance shock waves, requires (some form of) the weak form of the balance equations, or the inclusion of the so-called jump shock conditions ${ }^{4}$.

Equation (A.5b) is often denoted in a slightly different form. Rewriting the terms in the left hand with the chain rule and applying equation (A.5a) yields

$$
\rho\left[\frac{\partial \mathbf{v}}{\partial t}+(\mathbf{v} \cdot \nabla) \mathbf{v}\right]=\nabla \cdot \sigma+\rho \mathbf{b}
$$

The right-hand side now represents the rate of change in momentum due to the rate of change in velocity. Notice that the rate of change in momentum due to the rate of change in density is zero because no mass is generated or removed from the system, which is already expressed by the equation for conservation of mass. A second form of the energy equation can be derived by rewriting the terms in the left-hand side of equation (A.5c) with the chain rule and applying equation (A.5a). Hereafter the total energy $E$ is substituted by $E=e+\frac{1}{2}|\mathbf{v} \cdot \mathbf{v}|$ and the term associated with kinetic energy is substituted by the dot product of the velocity vector with equation (A.6). Rearranging the different terms yields:

$$
\rho\left[\frac{\partial e}{\partial t}+(\mathbf{v} \cdot \nabla) e\right]=\sigma:(\nabla \mathbf{v})^{T}-\nabla \cdot \mathbf{q}+\rho r_{e}
$$

This result is known as the so-called thermal energy equation. The first term on the right-hand side is the part of the work done by the stress which changes the internal or thermal energy. The rest of the stress and the body forces simply modulate the kinetic energy. Such changes in kinetic energy are already accounted for by the momentum equation.

\section{A.4 Constitutive Relations}

All materials are expected to satisfy the fundamental conservation laws derived above. However, the dramatic difference in the behavior of solids, fluids and viscoelastic materials is due to the difference in the way they resist deformation. To

\footnotetext{
${ }^{3}$ The term 'continuity equation' is often used for equation (A.5a), the partial differential equation that describes conservation of mass. In a broader definition, the name continuity equation is used for any differential equation that describes the conservative transport of some kind of quantity.

${ }^{4}$ In fluid dynamics, these conditions are also known as Rankine-Hugonoit jump conditions [130].
} 
complete the description of any material, the so-called constitutive relations or constitutive laws for that material have to be stated. These relations express the dependence of the stress tensor $\sigma$, the heat flux vector $q$ and the internal energy $e$ on the different field quantities such as density, temperature and velocity. The constitutive relations are a mathematical expression for the response of a material when taken out of equilibrium.

\section{A.4.1 Constitutive relations for Navier-Stokes-Fourier fluids}

The constitutive relations for a Navier-Stokes-Fourier fluid are listed below. The coefficients used in the description of the material behavior are expected to meet certain requirements; the coefficient must be chosen in such a way that fluid friction always opposes shear gradients and dilatation in a flow and that heat flows from hot to cold. These requirements are consequences of the need to satisfy the second law of thermodynamics.

The heat flux vector is written as a function of the temperature.

$$
\mathbf{q}=-\kappa \nabla T
$$

with $\kappa$ the coefficient of thermal conductivity. This expression is known as Fourier's law. The second law of thermodynamics demands that $k \geq 0$.

A second constitutive relation expresses the stress as a function of the rate of strains and pressure $p$.

$$
\sigma=-p \mathbf{I}+\tau
$$

with $\tau$ the viscous stress tensor defined as

$$
\tau=\lambda(\nabla \cdot \mathbf{v}) \mathbf{I}+\mu\left(\nabla \mathbf{v}+(\nabla \mathbf{v})^{T}\right)
$$

where $\mu$ is the first coefficient of viscosity or dynamic (shear) viscosity and $\lambda$ the second coefficient of viscosity. Note that in this definition the viscous stress tensor only depends on the symmetric part of the (rate of) deformation tensor. This is a good approximation if stresses resulting from rigid body rotations are assumed to be negligible. In that case, the equations for conservation of angular momentum (not included in this derivation) decouple from the other conservation equations $[128,129]$ and can be omitted. Since the viscous stress tensor is a symmetric second order tensor, its trace is invariant. The mean normal viscous stress $\tau_{m}$ is based on this invariant.

$$
\tau_{m}=\frac{1}{3} \operatorname{tr}(\tau)=\left(\lambda+\frac{2}{3} \mu\right) \nabla \cdot \mathbf{v}=\eta \nabla \cdot \mathbf{v}
$$

where $\eta$ is the coefficient of bulk viscosity. This is the coefficient that describes dilatational viscosity. As mentioned above, viscous friction must oppose (rate of) 
deformation (both from shear and dilatation), therefore the parameters $\mu$ and $\eta$ must satisfy:

$$
\mu \geq 0, \quad \eta \geq 0 \quad \text { (A.12a,b) }
$$

\section{A.4.2 Material parameters in the literature}

The values for the coefficient of thermal conductivity and the first coefficient of viscosity are readily available for many gases and fluids (see [131, 132]). On the other hand, consensus on values for the second coefficient of viscosity or coefficient of bulk viscosity is rarely encountered in the literature even for common fluids like air or water ${ }^{5}$. This is mainly due to the fact that there are no measurement setups in existence that are capable of measuring the bulk viscosity coefficient directly [134]. Instead, the bulk viscosity coefficient is estimated for instance by performing acoustic absorption measurements aimed at determining the absorption coefficient for free sound waves. Using this approach, the value of the bulk viscosity coefficient is inferred by using an (approximate) expression for the absorption coefficient that depends on the bulk viscosity coefficient. For instance, Pierce [28] concluded from absorption data for air that $\eta=0.60 \mu$ which concurs with an expression for the absorption coefficient for air in the high frequency limit at standard atmospheric conditions [134]. However, using this approach, the inferred or 'measured' bulk viscosity coefficient differs from the bulk viscosity coefficient as defined in the framework of continuum mechanics. This is due to the fact that the absorption coefficient (and thus the inferred bulk viscosity coefficient) is also related to relaxation processes that are caused by slight departures from the continuum hypothesis (see section A.1). For changes in pressure, density or temperature, the fluid does not instantly reach a new equilibrium, but only after a certain relaxation time. A consequence of the 'lag in equilibrium' that is of particular interest to acoustics, is that changes in pressure lag changes in density by a small amount, which results in attenuation of sound waves. These losses that are the result of (slightly) violating the continuum hypothesis thus effect the absorption coefficient and can be accounted for in the Navier-Stokes equation by adjusting the bulk viscosity coefficient accordingly. Note that the effects of relaxation depend on the relaxation time which depends on the state and molecular make-up of the fluid. As a consequence, the bulk viscosity coefficient (and the related second coefficient of viscosity) for air is considered to be dependent on temperature, humidity and (in some cases) frequency (see [135]). Not surprisingly, the reported values for the bulk viscosity coefficient and the second coefficient of viscosity vary

\footnotetext{
${ }^{5}$ Only for (dilute) monatomic gases, theory and experiments consistently predict that the bulk viscosity coefficient equals zero (see [133]).
} 
substantially in different sources ${ }^{6}$. For an overview of the literature on the bulk viscosity coefficient see for instance [133].

\section{A.5 Governing equations for Navier-Stokes-Fourier fluids}

The equations governing the behavior of Navier-Stokes-Fourier fluids are obtained by inserting the, material specific, constitutive equations (A.8) and (A.9) in balance equations (A.6) and (A.7) which express the fundamental conservation principles. The equations are stated once more using the total derivative

$$
\begin{aligned}
\frac{D \rho}{D t}+\rho \nabla \cdot \mathbf{v} & =0 \\
\rho \frac{D \mathbf{v}}{D t} & =\nabla \cdot \sigma+\rho \mathbf{b} \\
\rho \frac{D e}{D t} & =-p \nabla \cdot \mathbf{v}+\Phi+\kappa \Delta T+\rho r_{e}
\end{aligned}
$$

with $\Phi$ the viscous dissipation function defined as

$$
\Phi=\tau:(\nabla \mathbf{v})^{T}
$$

and the total derivative defined as

$$
\frac{D}{D t}=\frac{\partial}{\partial t}+(\mathbf{v} \cdot \nabla)
$$

Equations (A.13) are so-called field equations and can be used to describe the behavior of all Navier-Stokes-Fourier fluids. A count of equations and variables shows that all that remains is to choose two suitable equations of state to complete the system. In section 2.2.1 the appropriate equations of state for an ideal gas are substituted and the equations are linearized. The resulting set of equations is the basis of the different models for acoustic viscothermal wave propagation in ideal gases that are presented in this thesis.

\footnotetext{
${ }^{6}$ Tisza reported values of the second coefficient of viscosity for air in the same order as the values of the first coefficient of viscosity (see [135]). Marcy [136] and Ash [135] reported values of the second coefficient of viscosity for air that are 2000 to 20000 times higher.
} 


\section{Appendix B}

\section{Properties of air}

The values for the various properties of air under atmospheric conditions that are used throughout this thesis are listed in the table below. Note that several of the properties are related. Only seven of the properties can be determined independently. The second half of the table contains 'derived' properties.

\begin{tabular}{lcl}
\hline Property & Value & unit \\
\hline$C_{p}$ & 1004 & {$\left[\mathrm{Jkg}^{-1} \mathrm{~K}^{-1}\right]$} \\
$C_{v}$ & 717 & {$\left[\mathrm{Jkg}^{-1} \mathrm{~K}^{-1}\right]$} \\
$\rho_{0}$ & 1.2 & {$\left[\mathrm{kgm}^{-3}\right]$} \\
$\kappa$ & $25.6 \cdot 10^{-3}$ & {$\left[\mathrm{Wm}^{-1} \mathrm{~K}^{-1}\right]$} \\
$\mu$ & $18.2 \cdot 10^{-6}$ & {$\left[\mathrm{Nsm}^{-2}\right]$} \\
$\lambda$ & $-12.1 \cdot 10^{-6}$ & {$\left[\mathrm{Nsm}^{-2}\right]$} \\
$T_{0}$ & 290 & {$[\mathrm{~K}]$} \\
\hline$R_{0}$ & 287 & {$\left[\mathrm{Jkg}^{-1} \mathrm{~K}^{-1}\right]$} \\
$p_{0}$ & $99.9 \cdot 10^{3}$ & {$\left[\mathrm{Nm}^{-2}\right]$} \\
$c_{0}$ & 341 & {$\left[\mathrm{~ms}^{-1}\right]$} \\
\hline
\end{tabular}

Table B.1: Air properties under atmospheric conditions 


\section{Appendix C}

\section{LNSF equations for arbitrary fluids}

In chapter 2 the LNSF equations, which govern viscothermal wave propagation, were introduced and linearized. Prior to linearization, the equations of state for an ideal gas were substituted. Below, it is discussed to what extend the various models and solution techniques that are presented in this thesis are applicable to arbitrary fluids.

For an ideal or perfect gas, the relatively simple but approximate equations of state can be derived from kinetic theory. The model of an ideal gas follows from a statistical treatment of particles which exchange kinetic and vibrational energy in perfect elastic collisions. Potential energy due to intermolecular forces is neglected in this model. The magnitude of intermolecular forces decreases with the distance between molecules, so the ideal gas approximation becomes better as the average distance between particles becomes larger ${ }^{1}$. Consequently, the approximation becomes less appropriate if density is high and intermolecular forces become significant.

For liquids and for gases under high pressure/low temperature conditions, the ideal gas approximation is inappropriate and simple but accurate equations of state that are based on physical principles are often not available. In contrast, a large number of empirical equations of state for pure gases and liquids under various conditions can be found in the literature accompanied by a large variety of mixing rules for fluid mixtures [137, 138, 139].

Depending on the equations of state that are used, the form of the energy equation derived in section 2.2.2, and equation (2.7) can differ from that of an ideal gas. In acoustics, only small perturbations of the state variables are considered, al-

\footnotetext{
${ }^{1}$ Note that if the distance between particles in a diluted gas becomes too big, the fluid is no longer in local thermodynamic equilibrium and the assumptions needed for continuum mechanics break down.
} 
lowing linearization of the equations ${ }^{2}$ (see section 2.3). In general, the linearized equations of state of an arbitrary fluid that complete equations (2.1) can be cast in a form that yields pressure changes in terms of density and temperature changes and changes in internal energy in terms of temperature and pressure changes. This means that the form of the linearized thermal equation of state, and the energy equation that is derived in the next section, can be used unaltered for different equations of state. As a result, the form of the linearized equations derived in chapter 2 is appropriate to describe viscothermal acoustic wave propagation in arbitrary NSF fluids. Since the forms of the equations remain unchanged, the different solution techniques described in chapter 3 through 5 can be used unaltered. Naturally, the coefficients and material parameters involved in the different terms resulting from the equations of state that are used may vary depending on the material under consideration. In addition, the amplitude of the density, pressure and temperature variations for which the linearized model provides results of reasonable accuracy is dependent on the different coefficients in the equations of state that are used. In extreme cases (for instance if the fluid is in a state close to a phase change), the requirements proposed in section 2.3 (specifically equation (2.14)) may not be stringent enough to justify linearization of the equations of state.

\footnotetext{
${ }^{2}$ Note that equations of state are known to be non-analytic at phase boundaries making linearization impossible for these states [137].
} 


\section{Appendix D}

\section{Coordinate systems}

The scale factors for arbitrary orthogonal coordinates, and the general solution to the homogeneous scalar Helmholtz equation are given below for Cartesian, circular cylindrical and spherical coordinate systems.

\section{Scale factors}

The scale factors used in the definition of the gradient and Laplace operator for an orthogonal coordinate system with coordinates $\xi_{1}, \xi_{2}$, and $\xi_{3}$ is defined as

$$
h_{i}=\sqrt{g_{i i}} \quad \text { with } \quad g_{i j}=\sum_{k} \frac{\xi_{k}}{x_{i}} \frac{\xi_{k}}{x_{j}} \quad \text { where } \quad i, j, k=1,2,3
$$

with $g_{i j}$ the metric tensor of the coordinate system and $x_{1}, x_{2}$, and $x_{3}$ Cartesian coordinates.

\section{Helmholtz equation}

The homogeneous scalar Helmholtz equation is given by:

$$
\Delta \psi+k \psi=0
$$

\section{Cartesian coordinate systems}

coordinates: $x, y, z, \quad$ scale factors: $\quad h_{x}=1, \quad h_{y}=1, \quad h_{z}=1$

$$
\begin{aligned}
\psi(x, y, z) & =\psi_{x}(x) \psi_{y}(y) \psi_{z}(z), \quad k^{2}=k_{x}^{2}+k_{y}^{2}+k_{z}^{2} \\
\psi_{x}(x) & =C_{x 1} e^{i k_{x} x}+C_{x 2} e^{-i k_{x} x} \\
\psi_{y}(y) & =C_{y 1} e^{i k_{y} y}+C_{y 2} e^{-i k_{y} y} \\
\psi_{z}(z) & =C_{z 1} e^{i k_{z} z}+C_{z 3} e^{-i k_{z} z}
\end{aligned}
$$




\section{Circular cylindrical coordinate systems}

coordinates: $\quad r, \theta, z, \quad$ scale factors: $\quad h_{r}=1, \quad h_{\theta}=r, \quad h_{z}=1$

$$
\begin{gathered}
x=r \cos \theta, \quad y=r \sin \theta, \quad z=z \\
\psi(r, \theta, z)=\psi_{r}(r) \psi_{\theta}(\theta) \psi_{z}(z), \quad k^{2}=k_{r}^{2}+k_{z}^{2} \\
\psi_{r}(r)=C_{r 1} H_{m}^{(1)}\left(k_{r} r\right)+C_{r 2} H_{m}^{(2)}\left(k_{r} r\right) \\
\psi_{\theta}(\theta)=C_{\theta 1} e^{i k_{\theta} \theta}+C_{\theta 1} e^{-i k_{\theta} \theta} \\
\psi_{z}(z)=C_{z 1} e^{i k_{z} z}+C_{z 2} e^{-i k_{z} z}
\end{gathered}
$$

\section{Spherical coordinate systems}

coordinates: $\quad r, \theta, \phi, \quad$ scale factors: $\quad h_{r}=1, \quad h_{\theta}=r \sin (\phi), \quad h_{\phi}=r$

$$
\begin{aligned}
& x=r \cos \theta \sin \phi, \quad y=r \sin \theta \sin \phi, \quad z=r \cos \phi \\
\psi(r, \theta, z)= & \psi_{r}(r) \psi_{\theta}(\theta) \psi_{z}(z) \\
\psi_{r}(r)= & C_{r 1} h_{m}^{(1)}(k r)+C_{r 2} h_{m}^{(2)}(k r) \\
\psi_{\theta}(\theta)= & C_{\theta 1} e^{i k_{\theta} \theta}+C_{\theta 1} e^{-i k_{\theta} \theta} \\
\psi_{\phi}(\phi)= & C_{\phi} P_{k_{\phi}}^{k_{\theta}}(\cos (\phi))
\end{aligned}
$$




\section{Appendix E}

\section{Geometry specific LRF solutions}

For straight waveguides having simple cross-sectional geometries, the analytical expressions for the velocity profile $A$ and its averaged value $B$ are given in section E.1 for no-slip/isothermal boundary conditions. A general method to obtain semi-analytical solutions for other simple geometries and boundary conditions is given in section E.2. As an example, the method is applied to the case of curved waveguides with rectangular cross section.

The temperature profile $C$ and its averaged value $D$ can also be obtained using these expressions and this method, by substituting the shear wave number $s$ with the thermal wave number $s_{t}$. The expressions for $A_{b}, B_{b}, C_{b}$ and $D_{b}$ corresponding to the velocity and temperature profiles and their averaged values for inhomogeneous boundary conditions are defined by

$$
A_{b}=1-A, \quad B_{b}=1-B, \quad C_{b}=1-C, \quad D_{b}=1-D \quad \text { (E.1a,b,c,d) }
$$

\section{E.1 Analytical solutions}

All presented expressions correspond with the case of no-slip/isothermal boundary conditions. Expressions for more general velocity and temperature boundary conditions for these geometries are presented in $[66,20]$. Note that the shear wave number in the presented expressions is dimensional. To obtain the corresponding dimensionless forms, the shear or thermal wave numbers must be substituted by $\tilde{s} / l$ or $\tilde{s}_{t} / l$, respectively, where $l$ is the characteristic length. Although no analytical solution is available for the case of a rectangular cross section, an efficient semianalytical solution that is much more efficient than the method described in E.2) is available. It is also given here for completeness. 


\section{Layer}

$$
\begin{array}{cc}
x \uparrow \ldots l & x \in[-L / 2, L / 2] \\
A=1-\frac{\cos (\sqrt{-i} s x)}{\cos (\sqrt{-i}(s L / 2))}, & B=1-\frac{\tan (\sqrt{-i}(s L / 2))}{\sqrt{-i}(s L / 2)}
\end{array}
$$

\section{Circle}

$$
\underset{\substack{l=R \\+}}{i=[0, R]}
$$

$$
A=1-\frac{J_{0}(\sqrt{-i} s r)}{J_{0}(\sqrt{-i} s R)}, \quad B=-\frac{J_{2}(\sqrt{-i} s R)}{J_{0}(\sqrt{-i} s R)}
$$

\section{Annulus}

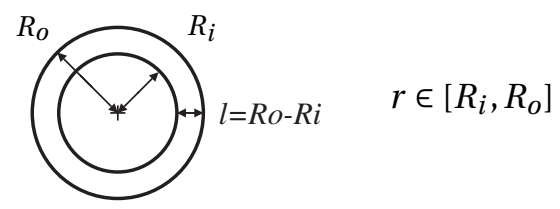

$$
\begin{gathered}
c_{1}=\frac{H_{0}^{(2)}\left(\sqrt{-1} R_{o}\right)}{H_{0}^{(2)}\left(\sqrt{-1} R_{i}\right)}, \quad c_{2}=\frac{H_{0}^{(1)}\left(\sqrt{-1} R_{i}\right)}{H_{0}^{(1)}\left(\sqrt{-1} R_{o}\right)}, \quad \alpha=\frac{1-c_{1}}{1-c_{1} c_{2}}, \quad \beta=\frac{1-c_{2}}{1-c_{1} c_{2}} \\
A=1-\alpha \frac{H_{0}^{(1)}(\sqrt{-1} s r)}{H_{0}^{(1)}\left(\sqrt{-1} s R_{0}\right)}-\beta \frac{H_{0}^{(2)}(\sqrt{-1} s r)}{H_{0}^{(2)}\left(\sqrt{-1} s R_{i}\right)} \\
B=\frac{R_{i}^{2}}{R_{0}^{2}-R_{0}^{2}}\left[\alpha \frac{H_{2}^{(1)}\left(\sqrt{-1} s R_{i}\right)}{H_{0}^{(1)}\left(\sqrt{-1} s R_{o}\right)}+\beta \frac{H_{2}^{(2)}\left(\sqrt{-1} s R_{i}\right)}{H_{0}^{(2)}\left(\sqrt{-1} s R_{i}\right)}\right]-\frac{R_{o}^{2}}{R_{0}^{2}-R_{0}^{2}}\left[\alpha \frac{H_{2}^{(1)}\left(\sqrt{-1} s R_{o}\right)}{H_{0}^{(1)}\left(\sqrt{-1} s R_{o}\right)}+\beta \frac{H_{2}^{(2)}\left(\sqrt{-1} s R_{o}\right)}{H_{0}^{(2)}\left(\sqrt{-1} s R_{i}\right)}\right]
\end{gathered}
$$




\section{Triangle}

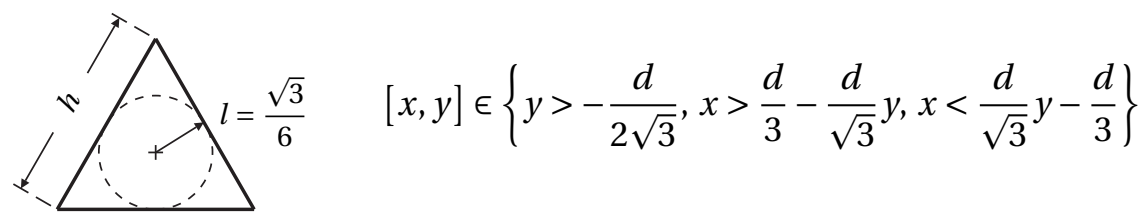

$$
\begin{aligned}
\alpha & =\left(\frac{1}{3} y+\frac{\sqrt{3}}{3} x\right) \sqrt{-i} s, \quad \beta=\left(\frac{1}{3} y-\frac{\sqrt{3}}{3} x\right) \sqrt{-i} s, \quad \gamma=\frac{2}{3} y \sqrt{-i} s \\
c_{1} & =\frac{\cos \left(\frac{1}{3} \sqrt{-1} s\right)}{\sin (\sqrt{-1} s)}, \quad c_{2}=\frac{\sin \left(\frac{1}{3} \sqrt{-1} s\right)}{\sin (\sqrt{-1} s)} \\
A & =1-[\sin (\alpha)+\sin (\beta)-\sin (\gamma)] c_{1}-[\cos (\alpha)+\cos (\beta)+\cos (\gamma)] c_{2} \\
B & =1-3 \frac{\tan (\sqrt{-1} s)-(\sqrt{-1} s)}{-1 s^{2} \tan (\sqrt{-1} s)}
\end{aligned}
$$

\section{Rectangle}

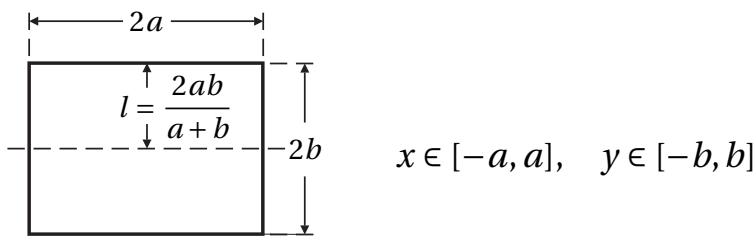

$$
\begin{aligned}
& \alpha_{n}=\sqrt{i s^{2}-\left(\frac{\gamma_{n}}{a}\right)^{2}}, \quad \beta_{n}=\sqrt{i s^{2}-\left(\frac{\gamma_{n}}{b}\right)^{2}}, \quad \gamma_{n}=\left(n+\frac{1}{2}\right) \pi \\
& A=-i s^{2} \sum_{n=0}^{\infty} \frac{(-1)^{n}}{\gamma_{n}}\left[\frac{\cos \left(\frac{\gamma_{n} x}{a}\right)}{\alpha_{n}^{2}}\left(1-\frac{\cos \left(\alpha_{n} y\right)}{\cos \left(\alpha_{n} b\right)}\right)+\frac{\cos \left(\frac{\gamma_{n} x}{b}\right)}{\beta_{n}^{2}}\left(1-\frac{\cos \left(\beta_{n} x\right)}{\cos \left(\beta_{n} a\right)}\right)\right] \\
& B=-i s^{2} \sum_{n=0}^{\infty}\left[\alpha_{n}^{-2} \gamma^{-2}\left(1-\frac{\tan \left(\alpha_{n} b\right)}{\alpha_{n} b}\right)+\beta_{n}^{-2} \gamma^{-2}\left(1-\frac{\tan \left(\beta_{n} a\right)}{\beta_{n} a}\right)\right]
\end{aligned}
$$




\section{Tapered Layer}

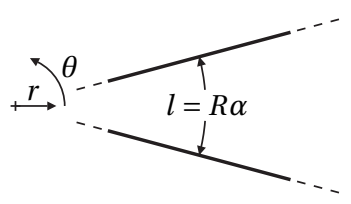

$$
A=1-\frac{\cos (\sqrt{-i} s r \alpha)}{\cos (\sqrt{-i}(\operatorname{sr} \alpha / 2))}
$$

$\theta \in[-\alpha / 2, \alpha / 2]$

where $\alpha$ is the opening angle

$$
B=1-\frac{\tan (\sqrt{-i}(s r \alpha / 2))}{\sqrt{-i}(s r \alpha / 2)}
$$

\section{E.2 Semi-analytical solutions}

For certain simple waveguide geometries and homogeneous boundary conditions, equations (4.11b) and (4.11d) can be efficiently solved by expanding the velocity or temperature profile $f\left(\mathbf{x}_{c d}\right)$ in a series of functions $f_{m, n}\left(\mathbf{x}_{c d}\right)$ that satisfy the boundary conditions as

$$
f\left(\mathbf{x}_{c d}\right)=\sum_{m, n} A_{m, n} f_{m, n}\left(\mathbf{x}_{c d}\right)
$$

with $A_{m, n}$ participation factors that have to be chosen so the inhomogeneous equation is satisfied. The expansion is substituted in the differential equation, multiplied by a weighing function and integrated over the cross section. If the functions in the expansion are also used as weighing functions, the following system of equations is obtained.

$$
\int_{S}\left(\Delta_{c d}+i \lambda^{2}\right) \sum_{m, n}\left[A_{m, n} f_{m, n}\right] f_{q, r} d S=\int_{S} \lambda^{2} g(p) f_{q, r} d S
$$

with $f_{q, r}$ weighing functions and $\lambda$ and $g(p)$ representing, respectively, the wave number and pressure dependent term in equation (4.11b) and (4.11d). The resulting system of size $m n \times q r$ can be solved for the participation factors $A_{m, n}$. If the set of functions used for the expansion is orthogonal and complete, convergence (in the mean) is guaranteed and the approximation error is controlled by the number of functions included in the series (see [89]). In addition, the equations in the system of equation (E.4) decouple and obtaining an expression for the individual participation values is straightforward. The eigenfunctions of the Laplace operator satisfying the boundary conditions form a convenient orthogonal complete set to expand the inhomogeneous Helmholtz equation. Substitution in equation (E.4) 
yields the following expression for the participation factors

$$
A_{m, n}=\frac{\lambda^{2} \int_{S} g(p) f_{m, n} d S}{\left(\lambda_{m, n}^{2}+i \lambda^{2}\right) \int_{S} f_{m, n}^{2} d S}
$$

with $\lambda_{m, n}^{2}$ the eigenvalue corresponding to the eigenfunction $f_{m, n}$. For waveguide geometries, described by coordinate surfaces of a coordinate system in which the scalar Helmholtz equation is separable, expressions for the eigenfunctions of the Laplace operator are readily available (see [73]). In general, finding the eigenvalues for arbitrary boundary conditions (that are constant over the boundaries) requires calculating the (first countable infinite number of) roots of a transcendental equation for each $c d$-direction. Depending on the geometry, the transcendental equations for the different equations may be coupled requiring them to be solved simultaneously (this is the case when the Jacobian of the coordinate system is dependent on more than one coordinate, an example being cylindrical-elliptical coordinate systems).

Note that for small wave numbers only a limited amount of functions is needed for an accurate approximation. However, for increasing wave numbers the number of required functions rises considerably ${ }^{1}$. It is pointed out in section 4.2.1, that for higher shear wave numbers a good approximation of the propagation constant can be obtained efficiently by evaluating equivalent cross sections of much simpler geometrical shape. Depending on the geometry and boundary conditions, obtaining the eigenfunctions and eigenvalues, and calculating the integrals in equation (E.5) can be time consuming. Fortunately, the integrals are independent of the shear wave number (and thus frequency) and once they are known for a certain geometry, evaluation of the participation factors for different values of the shear wave number is very efficient. Using numerical schemes such as FEM or BEM to obtain a solution may be more efficient if results are only required for a few values of the shear wave number. A variational approach (based on Ritz-functions) can be an efficient alternative for cross sections defined by arbitrary polygons (see [38]).

\footnotetext{
${ }^{1}$ The number of eigenfunctions required to stay below a certain maximum error rises approximately quadratic with the wave number for $2 \mathrm{D}$ cross sections
} 


\section{E.3 Example curved tube of rectangular cross section}

A semi-analytical solution for the velocity profile in a curved waveguide of rectangular cross section can be obtained by expanded velocity in a series of functions that satisfy the boundary conditions according to equation (E.3). The pressure dependent function $g(p)$ in equation (E.4) equals $p / r$ in case the velocity profile is reconstructed (and $p$ in case the temperature profile is reconstructed). Suitable functions for the expansion are the analytical expressions for the eigenfunctions and eigenvalues of the Laplace operator. For the present case in circular cylindrical coordinates, eigenfunctions can be constructed from a radial dependent part and the tangential dependent part which can be determined independently. Using such a separation, the eigenfunctions and corresponding eigenvalues can be written as

$$
f_{m, n}(r, z)=f_{m}(r) f_{n}(z), \quad \quad \lambda_{m, n}=\sqrt{\lambda_{m}^{2}+\lambda_{n}^{2}}
$$

The (radial) $r$-dependent part of the eigenfunctions of the Laplacian are found by solving the following (transcendental) equation for eigenvalues $\lambda_{m}$

$$
\mid\left[\begin{array}{ll}
\left.L_{i} J_{1}\left(\lambda_{m} r\right)\right|_{r=R_{i}} & \left.L_{i} Y_{1}\left(\lambda_{m} r\right)\right|_{r=R_{i}} \\
\left.L_{o} J_{1}\left(\lambda_{m} r\right)\right|_{r=R_{o}} & \left.L_{o} Y_{1}\left(\lambda_{m} r\right)\right|_{r=R_{o}}
\end{array}||=0\right.
$$

where $R_{i}$ and $R_{o}$ are the inner and outer radius corresponding to the waveguide boundaries, and $L_{i}$ and $L_{o}$ are operators expressing the boundary conditions at each boundary (see section 2.7). The corresponding eigenfunctions are described by

$$
f_{m}(r)=\left.J_{1}\left(\lambda_{m} r\right) L_{i} Y_{1}\left(\lambda_{m} r\right)\right|_{r=R_{i}}-\left.Y_{1}\left(\lambda_{m} r\right) L_{i} J_{1}\left(\lambda_{m} R_{i}\right)\right|_{r=R_{i}}=0
$$

The expressions for the eigenfunctions and eigenvalues of the Laplacian in tangential direction are found by solving the following (transcendental) equation for the eigenfunctions $\lambda_{n}$

$$
\left|\left[\begin{array}{cc}
\left.L_{i} \cos \left(\lambda_{n} z\right)\right|_{z=-w} & \left.L_{i} \sin \left(\lambda_{n} r\right)\right|_{z=-w} \\
\left.L_{o} \cos \left(\lambda_{n} z\right)\right|_{z=w} & \left.L_{o} \sin \left(\lambda_{n} z\right)\right|_{z=w}
\end{array}\right]\right|=0
$$

where the corresponding eigenfunctions are described by

$$
f_{n}(z)=\left.\cos _{n}\left(\lambda_{n} z\right) L_{i} \sin \left(\lambda_{n} w\right)\right|_{z=R_{i}}-\left.\sin \left(\lambda_{n} z\right) L_{i} \cos \left(\lambda_{n} w\right)\right|_{z=R_{i}}=0
$$

Substitution of the radial and tangential dependent part in E.6a,b yield the desired eigenfunctions and eigenvalues. The participation factors required to reconstruct the velocity and temperature profile with equation (E.3) can be obtained by substituting the eigenfunctions and eigenvalues in equation (E.5). 


\section{Appendix F}

\section{$1 D$ waveguides of non-constant cross section}

A discussion on various aspects of modeling waveguides of non-constant cross section is given below.

\section{F.1 One-parameter waves}

The horn equation given in (4.49) only accounts for $p d$-velocities, while it does not involve $c d$-velocities. Similarly, equation (4.16) only accounts for the impulse balance in $p d$-direction, while it does not involve the impulse balance in $c d$-direction. For the inviscid adiabatic case, certain combinations of waveguide and coordinate systems allow solutions that exhibit velocities in $p d$-direction only. Such solutions are governed by a single spatial parameter and are known as one-parameter waves [72]. In all other cases, the horn equation describes the acoustic behavior of the waveguide by approximation (one-parameter approximation). Putland [71] demonstrated that there are only three types of true one-parameter waves: plane waves, cylindrical waves and spherical waves, which occur in straight, tapered and conical waveguides, respectively. Note that for all these waveguide geometries, the rate of change of the cross sections is constant with the $p d$-coordinates. Geometries for which the rate of change is not constant are said to be flaring. Oneparameter approximations of flaring waveguide geometries become less accurate as the variations in the rate of change of the cross section become larger. The approximation becomes better as the acoustic wavelength becomes bigger $(\tilde{k}$ becomes smaller). The coordinates system in which a flaring waveguide is described influences the accuracy of one-parameter approximation. Keefe [140] demonstrated that for an arbitrarily varying cross section, the use of the horn equation in 
an appropriate curvilinear coordinate system provides an approximation of higher accuracy than in a Cartesian, conical or spherical coordinate system. Alfredson [141] described a flaring waveguide in terms of multiple waveguides of constant cross section. By including higher order modes in these sections, the effects of velocities in $c d$-direction are accounted for in the approximation, thereby improving its accuracy. The error that is made can be controlled by the number of sections used to describe the waveguide geometry and the number of higher order modes that is taken into account. In the work of Nederveen and Dalmont [142], a method is proposed to derive a correction factor that accounts for changes of the pressure gradient in $c d$-directions in rapidly flaring horns (for $\tilde{k}<1$ ). The effects of viscosity on the accuracy of one-parameter LRF approximations are investigated in chapter 6.

\section{F.2 Numerical solutions for tapered 1D waveguides}

A numerical solution to equation (4.16) can be obtained by rewriting the equation as a system of two ordinary first order differential equations. The resulting system can easily be solved numerically with a so-called step-integration-algorithm (see [143] for an example for the inviscid adiabatic case). Using this approach, surfaces of constant pressure are not necessarily plain, but are determined by the coordinates system. A drawback of this method is that both boundary conditions have to be applied at one waveguide entrance.

Another approach that is related to the two-port approach described in section 4.2.3, is presented by Kulik [144] ${ }^{1}$. He considered an equivalent two-port network using a transfer matrix approach in the limit of an infinite number of two-ports. It is demonstrated that the resulting system can be rewritten as a single acoustic twoport, where the matrix coefficients involve an integral of the position dependent propagation constant over the length of the conical section. Although the transfer matrix is derived analytically, the solutions to the integrals have to be obtained with numerical integration schemes for arbitrary duct shapes. If these integrals are solved numerically with the midpoint rule, the result is identical to that obtained by using a finite number of coupled two-ports if the number of integration steps equals the number of two-ports.

The two-port approach described in section 4.2.3 and the approach suggested by Kulik are most attractive for modeling waveguides of varying cross section. Both approaches allow the boundary conditions to be applied at a single waveguide en-

\footnotetext{
${ }^{1}$ Kulik uses the propagation constant defined by Kirchhoff which is only valid for $\tilde{s} \gg 1$. Straightforward substitution of the propagation constant in equation (4.23) extends the domain of validity to all values of $\tilde{s}$.
} 
trance, or at both waveguide entrances. The approaches offer easy integration of the waveguide in two-port networks of more waveguides, thus offering more flexibility than the approach based on a step-integration-algorithm. In addition, they are more efficient in case the waveguide includes prismatic sections.

\section{F.3 Boundary layer approximation}

An alternative to solving equation (4.16) for the viscothermal case is adopting a boundary layer approximation. An equation similar to the horn equation is derived in $[28,140]$ by applying a variational approach to solve the Helmholtz equation (for the inviscid adiabatic case). In both derivations, the change in phase speed of traveling waves due to the change in viscothermal effects with the axial coordinate are determined by applying a boundary layer approximation. The viscothermal effects are represented by an admittance-like boundary condition. Keefe [140] generalized the method proposed by Pierce [28] to yield approximate solutions for arbitrary axisymmetric orthogonal coordinate systems. Another approach for arbitrary axisymmetric geometries that yields similar equations and uses an admittance-like boundary condition to represent viscothermal effects is presented in [145].

All these models involve an equivalent admittance-like boundary condition that represents the viscothermal effects. Because of the boundary layer approximation that is used to obtain an expression for this boundary condition, a necessary assumption is that the viscous and thermal boundary layers are small compared to the waveguide radius throughout the waveguide. 


\section{Appendix G}

\section{Non-orthogonality of mode shapes}

In the scattering problem investigated in chapter 6 , there are three main sources of non-orthogonality that can make the system of equations in (3.67) ill-conditioned for most practical choices of weighing functions.

The first source of non-orthogonality is due to the influence of viscous effects on the velocity and stress profiles. The acoustic and vorticity modes become a strongly non-orthogonal set of functions as the shear wave number decreases (see figure 6.23 in chapter 6 ).

The second source of non-orthogonality is due to the fact that the mode shapes that are considered are a combination of the velocity and temperature profiles and the profiles for the stresses and normal heat flux. These combinations of profiles result in a set of functions that becomes more non-orthogonal as more modes are added. This occurs even in the case of the inviscid adiabatic limit where profiles for the individual quantities form an orthogonal set when considered individually. In addition, some combinations of acoustic and vorticity modes are strongly non-orthogonal (see figure 6.23 in chapter 6). Mechel [76] argues that because of this non-orthogonality, the use of vorticity modes in mode-matching schemes should be limited to cases where shear waves are enforced as boundary conditions. He does not include the vorticity modes when he applies mode matching to solve scattering problems such as presented in chapter 6 . However, in chapter 6 it is demonstrated that in order for the mode-matching method to converge for $\tilde{s}<10$, the vorticity modes have to be taken into account because of their strong non-orthogonality with respect to the first acoustic mode.

The third source of non-orthogonality is the discontinuity in waveguide cross section. The profiles for velocity, temperature, stress and normal heat flux in waveguides of different cross section form non-orthogonal sets of functions. The level of non-orthogonality is significantly influenced by the extent to which the cross 
sections differ and the alignment of the waveguide sections. Note that the first two sources can be important even if the waveguides that are coupled have an identical cross section. 


\section{Appendix H}

\section{Mathematical backgrounds}

\section{H.1 Null spaces, image and closed range theorem}

Two subspaces of $V$ and $Q$ that are important in the study of existence and uniqueness are the so-called null spaces or kernels of the operators $B$ and $B^{T}$, denoted by $\operatorname{ker} B$ and $\operatorname{ker} B^{T}$, respectively. The subspaces can be defined using bilinear forms or their associated operators as

$$
\begin{array}{rrr}
\operatorname{ker} B=\{v \in V \mid B v=0\} & =\{v \in V \mid b(v, q)=0, & \forall q \in Q\} \\
\operatorname{ker} B^{T}=\left\{q \in Q \mid B^{T} q=0\right\} & =\{q \in Q \mid b(v, q)=0, & \forall v \in V\}
\end{array}
$$

Two subspaces of $V^{\prime}$ and $Q^{\prime}$ that are important are the so-called range or image of the operators $B$ and $B^{T}$ denoted by $\operatorname{Im} B$ and $\operatorname{Im} B^{T}$, respectively. The subspaces are defined as

$$
\begin{array}{rr}
\operatorname{Im} B=\left\{q \in Q^{\prime} \mid q=B v,\right. & \forall v \in V\} \\
\operatorname{Im} B^{T}=\left\{v \in V^{\prime} \mid v=B^{T} q,\right. & \forall q \in Q\}
\end{array}
$$

Since $\operatorname{ker} B$ and $\operatorname{ker} B^{T}$ are subspaces of $V$ and $Q$, their so-called orthogonal complement in these spaces can also be defined:

$$
\begin{aligned}
(\operatorname{ker} B)^{\perp} & =\left\{v \in V \mid\left\langle v, v_{0}\right\rangle=0,\right. & \left.\forall \nu_{0} \in \operatorname{ker} B\right\} \\
\left(\operatorname{ker} B^{T}\right)^{\perp} & =\left\{q \in Q \mid\left\langle q, q_{0}\right\rangle=0,\right. & \left.\forall q_{0} \in \operatorname{ker} B^{T}\right\}
\end{aligned}
$$

The so-called close range theorem now asserts that the following statements are equivalent:

- $\operatorname{Im} B$ is closed in $Q^{\prime}$ 
- $\operatorname{Im} B^{T}$ is closed in $V^{\prime}$

- $\operatorname{Im} B=\left(\operatorname{ker} B^{T}\right)^{\perp}$

- $\operatorname{Im} B^{T}=(\operatorname{ker} B)^{\perp}$

- $\exists v_{g} \in V$ and $k_{0}>0$ for all $g \in Q^{\prime}$, such that $B v_{g}=g$ and $\left\|v_{g}\right\|_{V} \leq 1 / k_{0}\left\|B v_{g}\right\|_{Q^{\prime}}$

- $\exists q_{f} \in Q$ and $k_{0}>0$ for all $f \in V^{\prime}$, such that $B^{T} g_{f}=f$ and $\left\|q_{f}\right\|_{Q} \leq 1 / k_{0}\left\|B^{T} q_{f}\right\|_{V^{\prime}}$

The theorem implies that an operator with a closed range shares the algebraic properties of a matrix (an operator in finite-dimensional space) which has a closed range by definition. The last two statements in the above list imply that if $\operatorname{Im} B$ is closed in $Q^{\prime}$, the operator $B$ is invertible from $\operatorname{Im} B$ on the orthogonal complement of $\operatorname{ker} B$.

\section{H.2 Requirements for existence and uniqueness for sesquilin- ear forms}

In section 5.2.3, the weak formulation in equation (5.4) was given in a form very similar to equation (5.23) presented in section 5.2.1 for which existence and uniqueness results were presented by Brezzi and Fortin [61]. One of the main differences between the two sets of equations is that the problem described by (5.23) involves bilinear forms, while the problem in (5.32) involves sesquilinear forms. The requirements for the existence and uniqueness of solutions have to be altered slightly to account for this change, while the proof remains virtually unchanged. The key changes in the requirements revolve around the fact that the proof for existence and uniqueness in [61] hinges on the condition that $|c(p, q)| /\|q\|_{Z}$ is uniformly bounded (amongst others in terms of $\mathbf{f}, g$ and $\|a\|)$ for all $(p, q) \in Z$. The requirements for this condition to be met are discussed below for the bilinear and sesquilinear case.

\section{The bilinear case}

In the case of bilinear forms, Brezzi and Fortin demonstrated that this condition holds when $C$ is symmetric, and both $a($, ) and $c($, ) are positive semi-definite (the proof also holds if they are both negative semi-definite). The proof uses the fact that if $C$ is symmetric and positive or negative semi-definite, the following inequality holds

$$
|c(p, q)|^{2} \leq|c(p, p) c(q, q)|, \quad \forall p, q \in Z
$$


Using this inequality and the fact that $c($, ) is a bounded form, the following inequality is obtained that can be used to bound $|c(p, q)| /\|q\|_{Z}$ in terms of $|c(p, p)|$ and $\|c\|$ :

$$
\frac{|c(p, q)|^{2}}{\|q\|_{Z}^{2}} \leq \frac{|c(p, p) c(q, q)|}{\|q\|_{Z}^{2}} \leq|c(p, p)|\|c\|, \quad \forall p, q \in Z
$$

It then remains to prove that $|c(p, p)|$ is uniformly bounded. The requirement that both $a($, ) and $c($, ) are positive semi-definite implies

$$
|c(p, p)| \leq|a(p, p)+c(p, p)|
$$

which is crucial in this proof.

\section{The sesquilinear case}

If $c($, ) is a sesquilinear form, it is useful to split it into its Hermitian and skewHermitian part, using equation (5.28). The Pythagorean theorem yields:

$$
|c(p, q)|^{2}=\left|c_{h}(p, q)\right|^{2}+\left|c_{s}(p, q)\right|^{2}
$$

If both $c_{h}\left(\right.$, ) and $c_{s}($,$) are either positive or negative semi-definite, inequality (H.7)$ yields the following for the right-hand side terms:

$$
\begin{array}{ll}
\left|c_{h}(p, q)\right|^{2} \leq\left|c_{h}(p, p)\right|\left|c_{h}(q, q)\right|, & \forall p, q \in Z \\
\left|c_{s}(p, q)\right|^{2} \leq\left|c_{s}(p, p)\right|\left|c_{s}(q, q)\right|, & \forall p, q \in Z
\end{array}
$$

Combining these results and the fact that $c_{h}\left(\right.$, ) and $c_{s}($, ) are bounded (since $c($,$) is$ bounded) yields the equivalent of (H.8) for sesquilinear forms:

$$
\frac{|c(p, q)|^{2}}{\|q\|_{Z}^{2}} \leq\left(\left|c_{h}(p, p)\right|+\left|c_{s}(p, p)\right|\right)\|c\|, \quad \forall p, q \in Z
$$

So, if $c($, ) is bounded and has positive or negative semi-definite Hermitian and skew-Hermitian parts, $|c(p, q)| /\|q\|_{Z}$ is uniformly bounded if $|c(p, p)|$ is uniformly bounded. In the case of bilinear forms, the requirement that both $a($, ) and $c($, ) are positive or negative semi-definite ensures that inequality (H.9) holds, which is used to prove that $|c(p, p)|$ is uniformly bounded. For sesquilinear forms inequality (H.9) holds, if this requirement is replaced by demanding that the values of $a((\mathbf{v}, T),(\mathbf{v}, T))$ and $c(p, p)$ lie in the same quadrant for all $(\mathbf{v}, T, p) \in X \times Y \times Z$. This implies that $a_{h}\left(\right.$, ) and $c_{h}($, ) are both either positive semi-definite or negative semi-definite, and $a_{s}\left(\right.$, ) and $c_{s}($, ) are both either positive semi-definite or negative semi-definite. Note that these requirements include the requirements needed for inequality (H.12) to hold and are thus sufficient. 
Appendix I

Convergence results 


\begin{tabular}{|c|c|c|c|c|c|c|c|c|c|c|c|c|c|c|c|c|}
\hline $\begin{array}{l}\text { Element } \\
\text { family }\end{array}$ & $\begin{array}{l}\text { Mesh } \\
\text { type }\end{array}$ & & & $E_{H^{2}}(\tilde{\mathbf{v}})$ & & & & & $\mathrm{E}_{H^{2}}(\tilde{T})$ & & & & & $E_{L^{2}}(\tilde{p})$ & & \\
\hline$s$ & & 0.1 & 1 & 10 & 31 & 100 & 0.1 & 1 & 10 & 31 & 100 & 0.1 & 1 & 10 & 31 & 100 \\
\hline MINI & $3 \mathrm{UR}_{4}$ & $1,1, r$ & $1,1, r$ & $1,1, r$ & $1,1, r$ & $0,0, r$ & $1,1, r$ & $1,1, r$ & $1,1, r$ & $1,1, r$ & $0,0, r$ & $2,2, d$ & $2,2, d$ & $2,2, d$ & $2,2,0$ & $1,1,0$ \\
\hline MINI & $3 \mathrm{UD}_{4}$ & $1,1, r$ & $1,1, r$ & $1,1, r$ & $1,1, r$ & $0,0, r$ & $2,1, r$ & $2,1, r$ & $2,1, r$ & $2,1, r$ & $0,0, r$ & $2,2, d$ & $2,2, d$ & $2,2, d$ & $2,2,0$ & $0,1,0$ \\
\hline $\mathrm{CR}$ & $3 \mathrm{UR}_{4}$ & $2,2,2$ & $2,2,2$ & $2,2,2$ & $2,2,0$ & $1,1, r$ & $2,2,2$ & $2,2,2$ & $2,2,2$ & $2,2,1$ & $1,1, r$ & $4,4, d$ & $4,4, d$ & $4,4,2$ & $4,4,0$ & $2,2,0$ \\
\hline $\mathrm{CR}$ & $3 \mathrm{UD}_{4}$ & $2,2,2$ & $2,2,2$ & $2,2,2$ & $2,2,0$ & $1,1, r$ & $4,2,2$ & $4,2,2$ & $4,2,2$ & $4,2,1$ & $1,1,2$ & $4,4, d$ & $4,4, d$ & $4,4,2$ & $4,4,0$ & $0,2,0$ \\
\hline $\mathrm{TH}$ & $3 \mathrm{UR}_{5}$ & $2,2,2$ & $2,2,2$ & $2,2,2$ & $2,2,0$ & $1,1, r$ & $2,2,2$ & $2,2,2$ & $2,2,2$ & $2,2,1$ & $1,1, r$ & $4, d, 2$ & $4,4,2$ & $4,4,2$ & $4,4,0$ & $2,2,0$ \\
\hline $\mathrm{TH}$ & $3 \mathrm{UD}_{5}$ & $2,2,2$ & $2,2,2$ & $2,2,2$ & $2,2,0$ & $1,1, r$ & $4,2,2$ & $4,2,2$ & $4,2,2$ & $4,2,1$ & $2,2, r$ & $4, d, 2$ & $4,4,2$ & $4,4,2$ & $4,4,0$ & $0,2,0$ \\
\hline $\mathrm{TH}$ & $3 \mathrm{CR}_{4}$ & $2,2,2$ & $2,2,2$ & $2,2,2$ & $2,2,0$ & $1,1, d$ & $2,2,2$ & $2,2,2$ & $2,2,2$ & $2,2,0$ & $1,1,1$ & $4, d, 2$ & $4,4,2$ & $4,4,2$ & $4,4,0$ & $2,2,0$ \\
\hline $\mathrm{TH}$ & $3 \mathrm{CD}_{4}$ & $2,2,2$ & $2,2,2$ & $2,2,2$ & $2,2,0$ & $1,1, d$ & $4,2,2$ & $4,2,2$ & $4,2,2$ & $4,2,0$ & $1,1, d$ & $4, d, 2$ & $4,4,2$ & $4,4,2$ & $4,4,0$ & $0,2,0$ \\
\hline $\mathrm{TH}$ & $3 \mathrm{SR}_{4}$ & $3,3,3$ & $3,3,3$ & $3,3,3$ & $3,3,0$ & $1,1, r$ & $3,3,3$ & $3,3,3$ & $3,3,3$ & $3,3,1$ & $1,1, r$ & $4, d, 2$ & $4,4,2$ & $4,4,2$ & $4,4,0$ & $2,2,0$ \\
\hline $\mathrm{TH}$ & $3 \mathrm{SD}_{4}$ & $3,3,3$ & $3,3,3$ & $3,3,3$ & $3,3,0$ & $1,1, r$ & $4, d, 3$ & $4, d, 3$ & $4,3,3$ & $4,3,1$ & $1,1, r$ & $4, d, 2$ & $4,4,2$ & $4,4,2$ & $4,4,0$ & $0,2,0$ \\
\hline MINI & $4 R_{4}$ & $2,2,2$ & $2,2,2$ & $2,2,2$ & $1,1,1$ & $0,0, r$ & $2,2,2$ & $2,2,2$ & $2,2,2$ & $1,1,1$ & $0,0, r$ & $2,2, d$ & $2,2, d$ & $2,2,2$ & $2,2,0$ & $1,1,0$ \\
\hline MINI & $4 \mathrm{D}_{4}$ & $2,2,2$ & $2,2,2$ & $2,2,2$ & $1,1,1$ & $0,0, r$ & $2,2,2$ & $2,2,2$ & $2,2,2$ & $1,1,1$ & $0,0, r$ & $2,2, d$ & $2,2, d$ & $2,2,2$ & $2,2,0$ & $0,1, d$ \\
\hline $\mathrm{CR}$ & $4 R_{5}$ & $3,3,3$ & $3,3,3$ & $3,3,3$ & $3,3,0$ & $2,2,0$ & $3,3,3$ & $3,3,3$ & $3,3,3$ & $3,3,0$ & $2,2,0$ & $4,4,2$ & $4,4,2$ & $4,4,2$ & $4,4,0$ & $3,3,0$ \\
\hline CR & $4 \mathrm{D}_{5}$ & $3,3,3$ & $3,3,3$ & $3,3,3$ & $3,3,0$ & $2,2,0$ & $4,4,3$ & $4,4,3$ & $4, d, 3$ & $4,3,0$ & $3,2,0$ & $4,4,2$ & $4,4,2$ & $4,4,2$ & $4,4,0$ & $0,2,0$ \\
\hline $\mathrm{TH}$ & $4 R_{5}$ & $3,3,3$ & $3,3,3$ & $3,3,3$ & $3,3,0$ & $2,2,0$ & $3,3,3$ & $3,3,3$ & $3,3,3$ & $3,3,0$ & $2,2,0$ & $4,4,3$ & $4,4,2$ & $4,4,2$ & $4,4,0$ & $4,4,0$ \\
\hline $\mathrm{TH}$ & $4 \mathrm{D}_{5}$ & $3,3,3$ & $3,3,3$ & $3,3,3$ & $3,3,0$ & $2,2,0$ & $4, d, 3$ & $4,4,3$ & $4,3, r$ & $4,3,0$ & $2,2,0$ & $4, d, 2$ & $4,4,2$ & $4,4,2$ & $4,4,0$ & $0,2,0$ \\
\hline TH-LT & $4 \mathrm{R}_{5}$ & $3,3,3$ & $3,3,3$ & $3,3,2$ & $3,3,0$ & $2,2,0$ & $2,2,2$ & $2,2,2$ & $2,2,2$ & $2,2,0$ & $1,1,0$ & $2,2, r$ & $2,2,2$ & $2,2,2$ & $2,2,0$ & $2,2,0$ \\
\hline TH-LT & $4 \mathrm{D}_{5}$ & $3,3,3$ & $3,3,3$ & $3,3,2$ & $3,3,0$ & $2,2,0$ & $2,2,2$ & $4,2,2$ & $4,2,2$ & $4,1,0$ & $0,0, r$ & $1, r, 2$ & $4,2,2$ & $4,2,2$ & $4,2,0$ & $0,2,0$ \\
\hline
\end{tabular}

Table I.1: Order of convergence for different element families and mesh types for non-stretched elements. MINI, CR and TH indicate elements of the MINI family, the Crouzeix Raviart family, and Taylor Hood family, respectively (see figure 5.3). The suffix LT indicates that a linear field is taken for the temperature. The numbers and letters of the mesh type indicate: (3) triangular elements, (4) quadrilateral elements, $(\mathrm{U})(\mathrm{C})(\mathrm{S})$ Union-jack, Crossed, and Striped mesh pattern, respectively (see figure 5.2), $(R)(D)$ Regular and Distorted mesh patterns, respectively (see figure 5.2). The subscript indicates the number of meshes used to determine the order of convergence. Black entries indicate a stable convergence rate, light gray entries indicate the convergence rate is still changing with mesh refinements. Dark gray entries indicate convergence is halted due to the limited machine precision. The first, second and third values listed, indicate the order of convergence around $k / s=10^{-5}, k / s=10^{-2.5}$ and $k / s=10^{-0}$, respectively. Alternatively, the letters $r$ and $d$ indicate that the rate of convergence is rising or descending with $k / s$ around these values. 


\begin{tabular}{|c|c|c|c|c|c|c|c|c|c|c|c|c|c|c|c|c|}
\hline $\begin{array}{l}\text { Element } \\
\text { family }\end{array}$ & $\begin{array}{l}\text { Mesh } \\
\text { type }\end{array}$ & & & $E_{H^{2}}(\tilde{\mathbf{v}})$ & & & & & $E_{H^{2}}(\tilde{T}$ & & & & & $E_{L^{2}}(\tilde{p})$ & & \\
\hline$s$ & & 0.1 & 1 & 10 & 31 & 100 & 0.1 & 1 & 10 & 31 & 100 & 0.1 & 1 & 10 & 31 & 100 \\
\hline MINI & $3 \mathrm{UR}_{4}$ & $1,1, r$ & $1,1, r$ & $1,1, r$ & $0,0, r$ & $0,0, r$ & $1,1, r$ & $1,1, r$ & $1,1, r$ & $0,0, r$ & $0,0, r$ & $2,2, d$ & $2,2, d$ & $2,2,0$ & $2,2,0$ & $1,1,0$ \\
\hline MINI & $3 \mathrm{UD}_{4}$ & $1,1, r$ & $1,1, r$ & $1,1, r$ & $0,0, r$ & $0,0, r$ & $2,1, r$ & $2,1, r$ & $2,1, r$ & $2,0, r$ & $0,0, r$ & $2,2, d$ & $2,2, d$ & $2,2,0$ & $0,2,0$ & $0,1,0$ \\
\hline CR & $3 \mathrm{UR}_{4}$ & $2,2,2$ & $2,2,2$ & $2,2,2$ & $2,2,2$ & $1,1,0$ & $2,2,2$ & $2,2,2$ & $2,2,2$ & $2,2,2$ & $1,1,0$ & $4,2,2$ & $4, d, 2$ & $4,4,0$ & $4,4,0$ & $2,2,0$ \\
\hline CR & $3 \mathrm{UD}_{4}$ & $2,2,2$ & $2,2,2$ & $2,2,2$ & $2,2,0$ & $1,1,0$ & $4,2,2$ & $3,2,2$ & $3,2,2$ & $3,2,0$ & $1,1,0$ & $4,2, d$ & $4, d, 2$ & $4,3,0$ & $3,3,0$ & $0,2,0$ \\
\hline TH & $3 \mathrm{UR}_{4}$ & $2,2,2$ & $2,2,2$ & $2,2,2$ & $2,2,0$ & $1,1,0$ & $2,2,2$ & $2,2,2$ & $2,2,2$ & $2,2,0$ & $1,1,0$ & $4,2,2$ & $4, d, 2$ & $4, d, 0$ & $4,3,0$ & $2,2,0$ \\
\hline $\mathrm{TH}$ & $3 \mathrm{UD}_{4}$ & $2,2,2$ & $2,2,2$ & $2,2,0$ & $1,1,0$ & $1,1,0$ & $4,2,2$ & $3,2,2$ & $3,2,2$ & $2,2,0$ & $1,1,0$ & $4,2,2$ & $4,2,2$ & $3,3,0$ & $3,3,0$ & $0,2,0$ \\
\hline $\mathrm{TH}$ & $3 \mathrm{CR}_{4}$ & $2,2,2$ & $2,2,2$ & $2,2, d$ & $2,2,0$ & $1,1,0$ & $2,2,2$ & $2,2,2$ & $2,2, d$ & $2,2,0$ & $1,1,0$ & $4,2,2$ & $4, d, 2$ & $4, d, 1$ & $4,4,0$ & $2,2,0$ \\
\hline $\mathrm{TH}$ & $3 \mathrm{CD}_{4}$ & $2,2,2$ & $2,2,2$ & $2,2, d$ & $2,2,0$ & $1,1,0$ & $4,2,2$ & $4,2,2$ & $4,2,2$ & $3,2,0$ & $1,1,0$ & $4,2,2$ & $4, d, 2$ & $4,3,0$ & $3,3,0$ & $0,2,0$ \\
\hline $\mathrm{TH}$ & $3 \mathrm{SR}_{4}$ & $2,2,2$ & $3,3,3$ & $2,2, d$ & $2,2,0$ & $1,1,0$ & $2,2,2$ & $3,3,3$ & $2,2, d$ & $2,2,1$ & $1,1,0$ & $4,2,2$ & $4, d, 2$ & $4, d, 0$ & $4,3,0$ & $2,2,0$ \\
\hline TH & $3 \mathrm{SD}_{4}$ & $2,2,2$ & $3,3,3$ & $2,2, d$ & $2,2,0$ & $1,1,0$ & $4,2,2$ & $4,3,3$ & 4,2, & $3,2,0$ & $1,1,0$ & $4,2,2$ & $4, d, 2$ & $4, d, 0$ & $3,3,0$ & $0,2,0$ \\
\hline MINI & $4 \mathrm{R}_{4}$ & $2,2, r$ & $2,2, r$ & $1,1,2$ & $1,1,0$ & $0,0, r$ & $2,2, r$ & $2,2, r$ & 2,2, & $1,1, d$ & $0,0, r$ & $2,2, d$ & $2,2, d$ & $2,2,0$ & $2,2,0$ & $1, d, 0$ \\
\hline MINI & $4 \mathrm{D}_{4}$ & $2,2, r$ & $2,2, r$ & $1,1,2$ & $1,1,0$ & $0,0, r$ & $2,2, r$ & $2,2, r$ & $2,2, r$ & $1,1,0$ & $0,0, r$ & $2,2, d$ & $2,2, d$ & $2,2,0$ & $0,2,0$ & $0,1,0$ \\
\hline CR & $4 \mathrm{R}_{4}$ & $3,3, d$ & $3,3, d$ & $3,3,0$ & $3,3,0$ & $1,1,0$ & $3,3,3$ & $3,3,3$ & $3,3,0$ & $3,3,0$ & $1,1,0$ & $4,2,2$ & $4,2,2$ & $4, d, 0$ & $3,3,0$ & $2,2,0$ \\
\hline CR & $4 \mathrm{D}_{4}$ & $3,3, d$ & $3,3, d$ & $3,3, d$ & $3,3,0$ & $1,2,0$ & $4,3,3$ & $4,2,2$ & $4,3,2$ & $3,3,0$ & $1,2,0$ & $4,2,2$ & $4, d, 2$ & $4, d, 0$ & $3,3,0$ & $0,3,0$ \\
\hline $\mathrm{TH}$ & $4 R_{5}$ & $3,3,3$ & $3,3,3$ & $3,3,1$ & $3,3,0$ & $1,1,0$ & $3,3,3$ & $3,3,3$ & $3,3,1$ & $3,3,0$ & $1,1,0$ & $4,2, d$ & $4,2, d$ & $4, d, 0$ & $4, d, 0$ & $2,2,0$ \\
\hline $\mathrm{TH}$ & $4 \mathrm{D}_{5}$ & $3,3,3$ & $3,3,3$ & $3, d, 0$ & $3, d, 0$ & $1,2,0$ & $4,2,3$ & $4,2,3$ & $4,3,1$ & $3,3,0$ & $1,2,0$ & $4,2, d$ & $4,2, d$ & $4, d, 0$ & $3,3,0$ & $0,2,0$ \\
\hline TH-LT & $4 R_{5}$ & $3,3,3$ & $3,3,3$ & $3, d, 0$ & $3, d, 0$ & $1,1,0$ & $2,2, r$ & $2,2, r$ & $2,2,2$ & $1,1,0$ & $0,0, r$ & $2,2, d$ & $2,2, d$ & $2,2,0$ & $2,2,0$ & $2, d, 0$ \\
\hline TH-LT & $4 \mathrm{D}_{5}$ & $3,3,3$ & $3,3,3$ & $3, d, 0$ & $3, d, 0$ & $1,1,0$ & $2,2, r$ & $2,2, r$ & $2,2,2$ & $2,1,0$ & $0,0, r$ & $4,2,2$ & $3,2,2$ & $4,2,0$ & $3,2,0$ & $0,2,0$ \\
\hline
\end{tabular}

Table 1.2: Order of convergence for different element families and mesh types for stretched elements. MINI, CR and TH indicate elements of the MINI family, the Crouzeix Raviart family, and Taylor Hood family, respectively (see figure 5.3). The suffix LT indicates that a linear field is taken for the temperature. The numbers and letters of the mesh type indicate: (3) triangular elements, (4) quadrilateral elements, $(U)(C)(S)$ Union-jack, Crossed, and Striped mesh pattern, respectively (see figure 5.2), (R)(D) Regular and Distorted mesh patterns, respectively (see figure 5.2). The subscript indicates the number of meshes used to determine the order of convergence. Black entries indicate a stable convergence rate, light gray entries indicate the convergence rate is still changing with mesh refinements. Dark gray entries indicate convergence is halted due to the limited machine precision. The first, second and third values listed, indicate the order of convergence around $k / s=10^{-5}, k / s=10^{-2.5}$ and $k / s=10^{-0}$, respectively. Alternatively, the letters $r$ and $d$ indicate that the rate of convergence is rising or descending with $k / s$ around these values. 


\section{Appendix J}

\section{FE boundary layer approximation}

In this appendix the finite element formulation, including boundary conditions that account for viscothermal boundary effects as proposed by Bossart et al. [47], is presented with small alterations that make this method even more efficient. The finite element formulation used is a straightforward finite element discretization of the Helmholtz equation for pressure by using a Galerkin approach. In regions of the domain boundary where velocity, impedance or admittance boundary conditions are prescribed, the boundary conditions are adjusted to account for viscothermal effects. This is accomplished by rewriting velocity or impedance boundary conditions as (equivalent) prescribed admittance boundary conditions, and subsequently adding terms that represent the admittance of the thermal and viscous boundary layers as proposed by Cremer [32].

\section{J.1 FEM Helmholtz equation for pressure}

The Helmholtz equation for inviscid adiabatic acoustic pressure fluctuations was already presented in section 2.5.1 as a limiting case of the more general theory for viscothermal wave propagation. The equation is stated here once more for convenience

$$
\Delta \bar{p}+k^{2} \bar{p}=0
$$

Transforming the equation into weak form for a domain $\Omega$ yields

$$
\left\langle\nabla p, \nabla p_{w}\right\rangle+k_{a}^{2}\left\langle p, p_{w}\right\rangle=\left\langle f, p_{w}\right\rangle_{S}
$$

where the forcing term $f$ can be expressed as the pressure gradient in the direction of the boundary or, using Euler's equation ${ }^{1}$, in terms of fluid velocity in the

\footnotetext{
${ }^{1}$ In the absence of viscosity and body forces, equation (2.16b) is known as Euler's equation.
} 
direction of the boundary

$$
f=\nabla p \cdot \mathbf{n}=-i \omega \rho_{0} v_{n}
$$

with $\mathbf{n}$ and $v_{n}$ the unit vector and fluid velocity normal to the domain boundary $S$. The inner products $\langle$,$\rangle and \langle,\rangle_{S}$ are sesquilinear forms over the domain $\Omega$ and domain boundary $S$, respectively defined as

$$
\langle a, b\rangle=\int_{\Omega} a \bar{b} d \Omega, \quad\langle a, b\rangle_{S}=\int_{S} a \bar{b} d S
$$

All that remains it to generate FE approximation spaces in the usual way by defining elements and trial and test functions.

\section{J.2 Boundary layer theory}

Cremer [32] was the first to apply boundary layer theory to acoustics and derived expressions for an (equivalent) admittance that accounts for the thermal and viscous boundary layers at a rigid isothermal wall. An essentially identical derivation can be found in the work of Pierce [28]. In both publications, the Laplacian in direction tangential to the boundary that arises in the contribution of the viscous effects is eliminated by substituting

$$
\frac{\partial^{2} p}{\partial x_{t}^{2}}=k_{t}^{2} p=k^{2} \sin ^{2}(\theta) p
$$

where $x_{t}$ is the coordinate direction tangential to the boundary, and $\theta$ is the assumed angle of an incident wave. Bossart et al. [47] derive the admittance in a similar way, but leave the tangential part of the Laplacian in the expressions. Instead, they propose to perform an inviscid adiabatic acoustic FEM calculation to determine the value of this unknown term (it is suggested to use a boundary layer admittance corresponding to a wave inciding at 45 degrees for this preliminary calculation). On the one hand, this solution allows the boundary layer approach to be used on FE grids of arbitrary shape in a straightforward way, since it does not require a (local or global) coordinate direction that is tangential to the element boundary. On the other hand, the solution requires solving the FE element system twice (once with an estimated expression and once with a corrected expression for the boundary admittance ${ }^{2}$ ). Fortunately, a simple and efficient alternative exists that allows the correct admittance to be applied at once. The proposed solution is presented here for the first time.

\footnotetext{
${ }^{2}$ Bossart et al. do not discuss the need for additional iterations to improve accuracy.
} 
Following Bossart et al. ${ }^{3}$ the expression for the boundary layer admittance in terms of the viscous and thermal wave numbers $s$ and $s_{t}$ is

$$
\frac{v_{n}}{p}=i \frac{k}{\rho_{0} c_{0}}\left(\frac{k_{t}^{2}}{k^{2}} \frac{1}{\sqrt{i} s}+(\gamma-1) \frac{1}{\sqrt{i} s_{t}}\right)
$$

where $k_{t}$ is the wave number tangential to the boundary. Note that the thermal wave number used here (as in most texts on acoustic boundary layer theory) is the approximate thermal wave number defined in equation (3.46). In order to apply the admittance as a right-hand side in equation (J.2) the equation is rewritten to yield velocity in terms of pressure

$$
v_{n}=i \frac{k}{\rho_{0} c_{0}}\left(\frac{\partial^{2} p}{\partial x_{t}^{2}} \frac{1}{\sqrt{i} s k^{2}}+(\gamma-1) \frac{1}{\sqrt{i} s_{t}} p\right)
$$

Note that the Laplacian in tangential direction is re-introduced. Substitution of this expression in equation (J.3) yields a right-hand side $f$ in terms of $p$ and its second derivative in normal direction

$$
f=-k^{2}\left(\frac{\partial^{2} p}{\partial x_{t}^{2}} \frac{1}{\sqrt{i} s k_{0}^{2}}+(\gamma-1) \frac{1}{\sqrt{i} s_{t}} p\right)
$$

If second order (Lagrangian) elements are used, discretization of $f$ (including the second order derivative) is straightforward and the resulting terms can be included in the system matrix. This alteration allows the correct viscothermal boundary effects to be imposed without the need for an additional calculation to determine the (approximate) equivalent admittance.

The equations above are valid for rigid, isothermal walls. A prescribed velocity can be taken into account by adding it to the right-hand side of equation (J.7) and continuing the derivation. A prescribed admittance can be taken into account by adding it to equation (J.6) and continuing the derivation. An adiabatic wall can easily be modeled by discarding the second term between the brackets in equation (J.8).

Note that effects of bulk viscosity, relaxation and heat conduction in $p d$-direction can all be incorporated in the wave number $k$ if desired as is done in the work of Bossart et al.

\footnotetext{
${ }^{3}$ The expression in [47] contains two typographical errors which are corrected in the present expression.
} 


\section{Appendix K}

\section{LRF performance}

\section{K.1 No-slip isothermal/adiabatic boundary conditions}

\section{Adiabatic boundary conditions}

In section 6.2.2 the relative error in the LRF approximation of the propagation constant was investigated for a straight $1 \mathrm{D}$ layer with no-slip adiabatic boundary conditions. The presented results show that the LRF approximation is clearly unreliable or even worthless in a range of parameters where the a priori criteria in (4.10) hold $\left(k / s \ll 1, k / s_{t} \ll 1\right.$ and $\left.\tilde{k}<1\right)$. The conclusion must be that the a priori criteria do not ensure that LRF assumptions are met. More specifically, the assumption that the temperature gradient in $p d$-direction is small compared to the temperature gradient in $c d$-direction is not valid for $\tilde{s}<1$. In fact, under the assumption that $p$ is constant over the cross section, it follows from equation (2.32c) governing the temperature, that $T$ will also be constant over the cross section if adiabatic boundary conditions are applied. As a consequence, the gradient in $c d$ direction is zero (for the first acoustic mode), thus reducing equation (2.32c) to a one dimensional inhomogeneous Helmholtz equation for $T$ as a function of the $p d$-coordinate.

If the wavelength of the waves described by this Helmholtz equation, being the thermal wavelength, is small compared to the wavelength of the acoustic wave, the influence of heat transport in $p d$-direction is small, and temperature will rise and fall with the changes in pressure as the acoustic wave passes. For $\tilde{s} \gg 1$, the propagation constant has a value close to unity, and the actual wave number of the acoustic wave, given by $k \Gamma$ is very well approximated by the acoustic wave number for free waves, being $k$. In that case, conduction of heat in $p d$-direction will be small if $k / s \ll 1$. On the other hand, if $\tilde{s}<1$, viscous effects slow down the acoustic wave. In that case, both the real and imaginary part of $\Gamma$ can be much bigger 
than unity, making the real and imaginary parts of actual acoustic wave number $k \Gamma$ much bigger than $k$. Ultimately, the increasingly smaller wavelength of the acoustical wave becomes of the same order of magnitude as the wavelength of the thermal wave, resulting in significant transport of heat in $p d$-direction as a result of the passing wave. Since the LRF approximations include the assumption of zero heat flow in $p d$-direction, this effect is not described by LRF models, and the error of the LRF approximation grows rapidly.

\section{Isothermal boundary conditions}

The question arises why this dramatic growth of the error of the LRF approximation does not occur for no-slip isothermal boundary conditions. The answer resides in the fact that for isothermal boundary conditions, a significant temperature gradient in $c d$-direction is present due to heat conduction between fluid and boundary for $\tilde{s}<1$. For $\tilde{s}<1$ the magnitude of the temperature gradient in $c d$ direction scales with $\tilde{s}$, and it turns out that the gradient in $p d$-direction which is governed by the actual wave number $k \gamma$ does not decrease faster with $\tilde{s}$ than the gradient in $c d$-direction. Consequently, for $k / s \ll 1$, the original LRF assumption requiring that the gradient in $p d$-direction is small compared to that in $c d$ direction, is satisfied for $\tilde{s}<1$ in the case of isothermal boundary conditions. For $\tilde{s}>1$, the gradient in $c d$-direction becomes increasingly smaller in the center of the waveguide, however, since the actual wave number $k \Gamma$ is very close to the wave number of a free acoustic wave $k$, the requirement that $k / s \ll 1$ is enough to ensure that the gradient (and resulting heat flow) in $p d$-direction can be neglected ${ }^{1}$.

\section{K.2 Changes in thermal boundary conditions}

In section 6.4 the accuracy of a coupled LRF model for a waveguide having a sudden change in thermal boundary conditions was investigated. It was observed that the errors in the LRF approximation of the resulting scattering problem can become large for $\tilde{s}<1$. The observed errors can be explained in part by the fact that the propagation constant is not well approximated by the LRF model for adiabatic boundary conditions, as was pointed out in section 6.2. Since the propagation constant governs the characteristic impedance, significant errors for $\tilde{s}$ are expected. What is surprising, is that the FE results for the amplitude of the transmitted and reflected wave in figure 6.14 suggest that a significant amount of en-

\footnotetext{
${ }^{1}$ Note that the term is neglected irrespective of the relative difference between the magnitudes of the gradient in $c d$ - and $p d$-directions.
} 
ergy is lost at the junction ${ }^{2}$. The question arises how the energy is dissipated. It turns out that for no-slip adiabatic boundary conditions, the first thermal mode increasingly resembles the first acoustic mode as $\tilde{s}$ decreases. The dimensionless velocity, temperature and pressure profiles corresponding to the first acoustic and first thermal mode are depicted in figure K.1. Only the absolute values of the profiles are plotted here, since the differences in the angle of the profiles between the two modes is several orders lower.

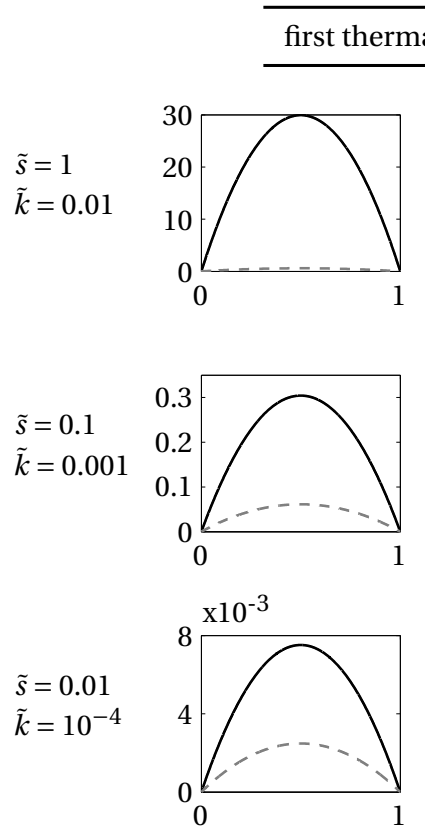

(a) $\tilde{v}_{x}$
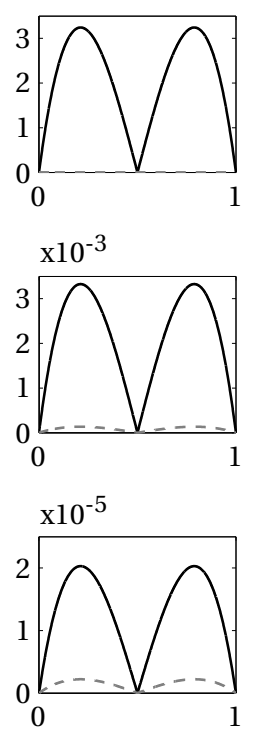

(b) $\tilde{v}_{y}$
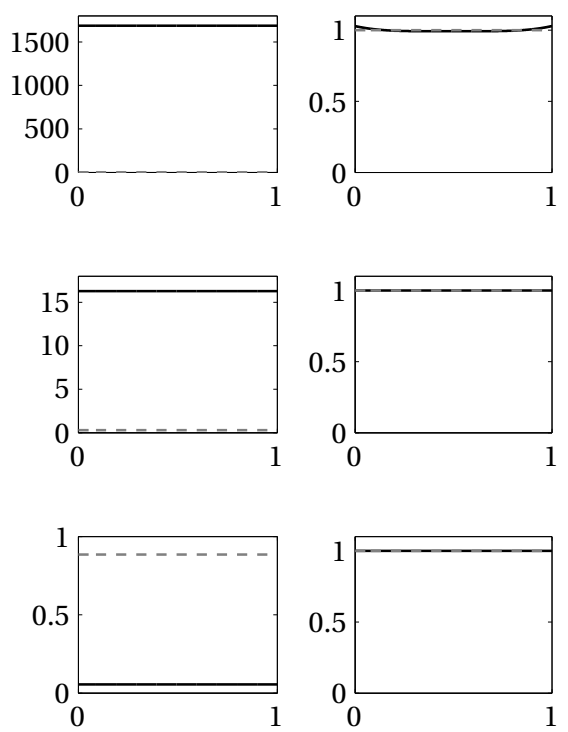

(c) $\tilde{T}$

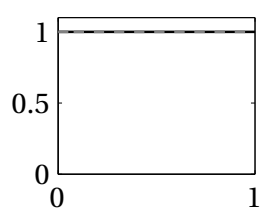

(d) $\tilde{p}$

Figure K.1: Absolute value of the velocity, temperature and pressure profiles for the first acoustic and first thermal mode.

Note that for $\tilde{s}=1$, the magnitude of the temperature for the thermal mode is a few orders higher than that of the velocities, while this ceases to be true for lower values of $\tilde{s}$. For the acoustic mode, the (dimensionless) magnitude of the temperature and velocity is similar for $\tilde{s}>1$, while the magnitude of temperature becomes increasingly bigger compared to the velocities for lower values of $\tilde{s}$. It appears that for $\tilde{s}<1$ the behavior of the thermal and acoustic modes gradually becomes more

\footnotetext{
${ }^{2}$ The energy content of the incident, reflected and transmitted wave is related to their amplitude, and the combined amplitude of the transmitted and reflected wave is significantly smaller than that of the incident wave.
} 
alike and even appears to switch role if $\tilde{s}$ is chosen small enough. Recall that as $\tilde{s}$ decreases, the real and imaginary parts of the propagation constant gradually become equal in magnitude. This implies that the propagating wave corresponding to the first acoustic mode is very dissipative. It is found that in the case of adiabatic boundary conditions, the propagation constant of the first thermal mode equals $i \sqrt{i} s_{t}$ for large values of $\tilde{s}_{t}$ and approaches values equal to twice the propagation constant of the first acoustic mode as $\tilde{s}_{t}$ decreases. This means that the waves corresponding to the first thermal mode are also very dissipative, but their wavelength and rate of attenuation is of the same order as that of acoustic waves. Summarizing, as $\tilde{s}$ decreases both the velocity and temperature profiles and the propagation constant become similar for the first acoustic and the first thermal mode. Note that for a given $\tilde{s}$, the resemblance becomes stronger as $k / s$ increases. Considering the similarities in profiles and propagation constant, it is not surprising that if the scattering problem is carried out with a thermal wave corresponding to the first thermal mode as a second transmitted wave, the contribution of the thermal wave to the outgoing sound field is significant. And since the amplitude of the incident wave is now 'divided' between two 'propagating' waves, the contribution of the first acoustic mode is reduced or is amplified (depending on the whether the two type of modes cancel or enforce each other at the location of the junction). The effects of the first thermal mode are accounted for by the FEM model, which explains the variations in the complex amplitude of the transmitted wave for low values of $\tilde{s}$. Since the LRF model does not account for the effects of the first thermal mode, the LRF result will deviate strongly.

Note that for lower values of $\tilde{s}$, the contribution of the first thermal mode can interfere when reconstructing the complex amplitude of the first acoustic mode from the FE results. Since the wavelength and rate of attenuation of the first acoustic and first thermal mode only differ by a factor of (approximately) two for $\tilde{s} \ll 1$, the distance between the location where the pressure is used to calculate the backward and forward traveling waves and the location where the boundary conditions change must be relatively big (resulting in large FE models). As an alternative, equation (6.2b) can be modified to account for two outward traveling waves corresponding to the first acoustic and thermal mode (instead of a backward and forward traveling wave both based on the first acoustic mode). The results of both reconstruction methods converge to one another if $L_{A}$ is increased for the method without the thermal mode and is kept constant for the method with the thermal mode. 


\section{Appendix L}

\section{Resonator design}

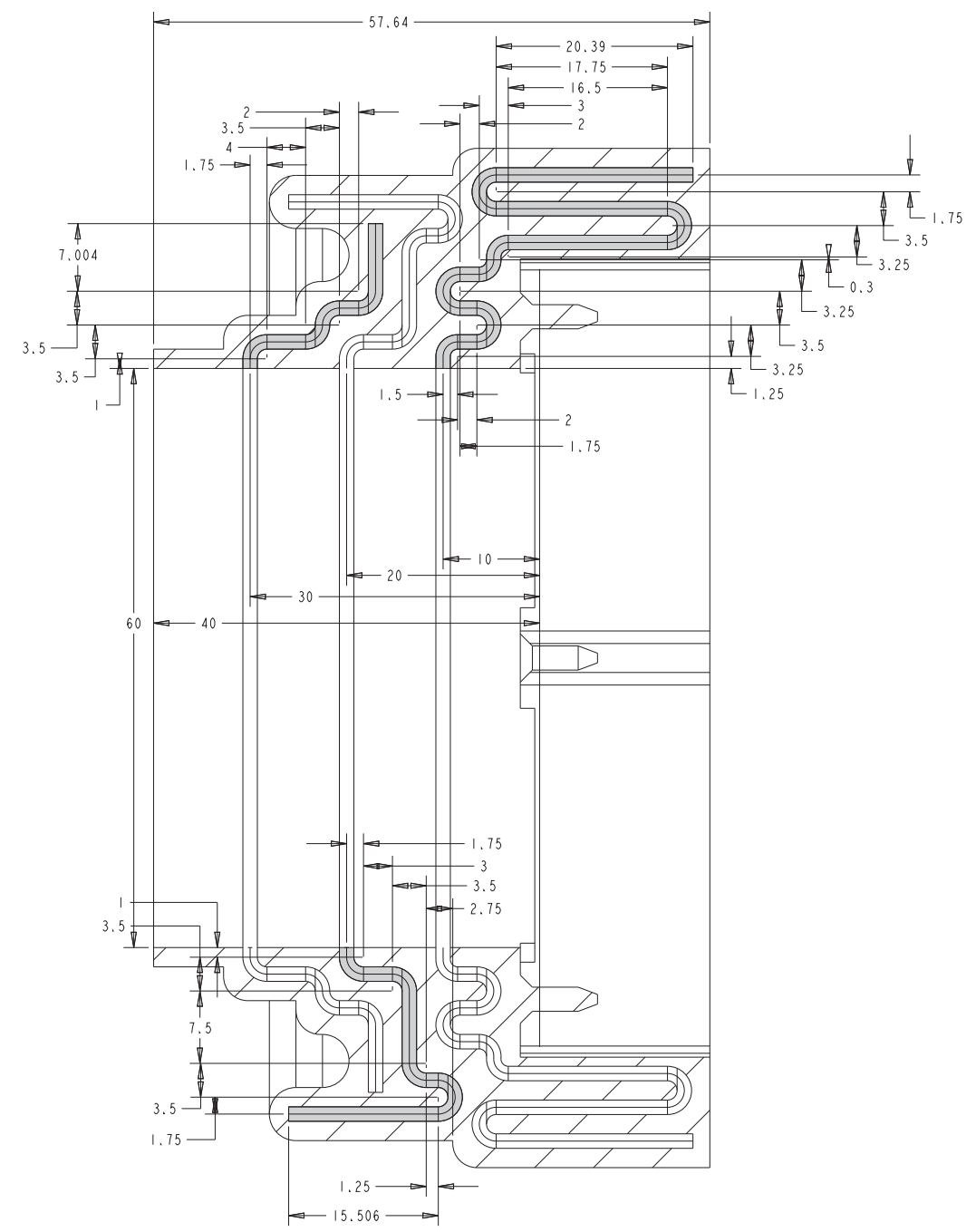




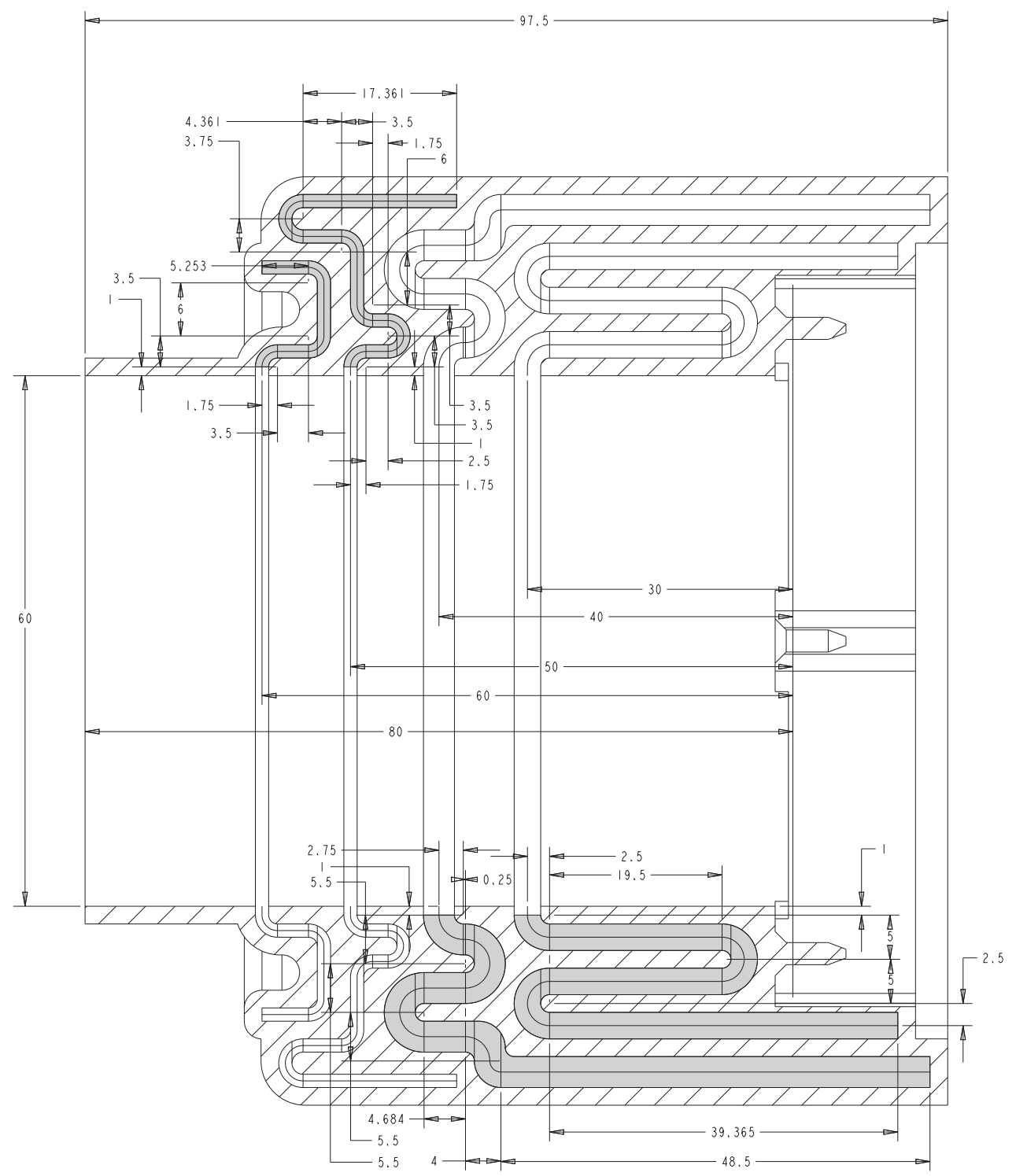




\section{Bibliography}

[1] W. Kampinga, Viscothermal acoustics using finite elements. Analysis tools for engineers. PhD thesis, University of Twente, Enschede, Netherlands, 2010.

[2] T. Veijola and M. Turowski, "Compact damping models for laterally moving microstructures with gas-rarefaction effects," Journal of Microelectromechanical Systems, vol. 10, pp. 263-273, 2001.

[3] T. Veijola, "A two-port model for wave propagation along a long circular microchannel," Microfluidics and Nanofluidics, vol. 3, pp. 359-368, 2007.

[4] T. Veijola, "Compact models for squeezed-film dampers with inertial and rarefied gas effects," Journal of Micromechanics and Microengineering, vol. 14, pp. 11091118, 2004.

[5] W. Beltman, "Viscothermal wave propagation including acousto-elastic interaction, Part II: Applications,” Journal of Sound and Vibration, vol. 227, pp. 587-609, 1999.

[6] G. Plantier and M. Bruneau, "Heat conduction effects on the acoustic response of a membrane separated by a very thin air film from a backing electrode," Journal Acoustique, vol. 3, pp. 243-250, 1990.

[7] M. Bruneau, A. Bruneau, and P. Hamery, "An improved approach to modelling the behaviour of thin fluid films trapped between a vibrating membrane and a backing wall surrounded by a reservoir at the periphery," Acustica, vol. 1, pp. 227-234, 1993.

[8] C. Karra and M. Ben Tahar, "Boundary element analysis of vibro-acoustic interaction between vibrating membrane and thin fluid layer," Flow, Turbulence and Combustion, vol. 61, pp. 179-187, 1999.

[9] H. Wijshoff, Structure- and fluid-dynamics in piezo inkjet printheads. $\mathrm{PhD}$ thesis, University of Twente, Enschede, Netherlands, 2007.

[10] W. Beltman, Viscothermal wave propagation including acousto-elastic interaction. PhD thesis, University of Twente, Enschede, The Netherlands, 1998.

[11] M. Moser, "Damping of structure born sound by the viscosity of a layer between two plates," Acustica, vol. 46, pp. 210-217, 1980. 
[12] L. Chow and R. Pinnington, "Practical industrial method of increasing structural damping in machinery, i: squeeze film damping with air," Journal of Sound and Vibration, vol. 118, pp. 123-139, 1987.

[13] L. Chow and R. Pinnington, "Practical industrial method of increasing structural damping in machinery, ii: squeeze film damping with liquids," Journal of Sound and Vibration, vol. 128, pp. 333-347, 1989.

[14] T. Onsay, "Effects of layer thickness on the vibration response of a plate-fluid layer system," Journal of Sound and Vibration, vol. 163, pp. 231-259, 1993.

[15] C. Karra and M. Ben Tahar, "Simulation of viscothermal losses on the acoustic behaviour of a thin fluid layer enclosed between two oscillating plates," Building Acoustics, vol. 13, pp. 113-126, 2006.

[16] T. Basten, P. van der Hoogt, R. Spiering, and H. Tijdeman, "On the acousto-elastic behaviour of double-wall panels with a viscothermal air layer," Journal of Sound and Vibration, vol. 243, pp. 699-719, 2001.

[17] T. Basten, Noise reduction by visco-thermal acousto-elastic interaction in double wall panels. PhD thesis, University of Twente, Enschede, Netherlands, 2001.

[18] F. White, Viscous Fluid Flow. McGraw-Hill, $3^{\text {rd }}$ ed., 2006.

[19] F. van der Eerden, Noise reduction with coupled prismatic tubes. $\mathrm{PhD}$ thesis, Universiteit Twente, Enschede, The Netherlands, 2000.

[20] M. Hannink, Acoustic resonators for the reduction of sound radiation and transmission. PhD thesis, University of Twente, Enschede, The Netherlands, 2007.

[21] M. Nijhof, Y. Wijnant, and A. de Boer, "Elliptical side resonators for broadband noise reduction: theory and experiments," in ICSV13, (Vienna, Austria), 2006.

[22] M. Nijhof, W. Beltman, Y. Wijnant, and A. de Boer, "Optimizing circular sideresonators to reduce computer fan noise," in NOISE-CON2004, (Baltimore, Maryland, U.S.A), 2004.

[23] M. Nijhof, W. Beltman, Y. Wijnant, and A. de Boer, "Reduction of fan noise by means of (circular) side-resonators; theory and experiment," in ISMA2004, (Leuven, Belgium), 2004.

[24] M. Malinen, M. Lyly, P. Råback, A. Kärkkäinen, and L. Kärkkäinen, "A finite element method for the modeling of thermo-viscous effects in acoustics," in ECCOMAS2004, (Jyväskylä, Finland), 2004.

[25] M. Nijhof, Y. Wijnant, and A. de Boer, "An acoustic finite element including viscothermal effects," in ICSV14, (Cairns, Australia), 2007.

[26] R. Christensen, P. Juhl, and V. C. Henriquez, "Modeling of acoustic losses in air due to heat conduction and viscosity,” in EURONOISE2008, (Paris, France), 2008.

[27] R. Kampinga, "Performance of several viscothermal acoustic finite elements," Acta acustica united with acustica, vol. 95, 2009. 
[28] A. Pierce, Acoustics, An Introduction to its Physical Principles and Applications. McGraw-Hill, 1994 edition ed., 1981.

[29] J. W. S. Rayleigh, Theory of Sound, Volume II. Dover, New York, $2^{\text {nd }}$ ed., 1896.

[30] G. Stokes, Mathematical and Physical Papers, Volume I. Cambridge: University Press, $1^{\text {st }}$ ed., 1880.

[31] G. Kirchhoff, "Ueber den Einfluss der Wärmeleitung in einem Gase auf die Schallbewegung," Annalen der Physik und Chemie, vol. 210, no. 6, pp. 177-193, 1868.

[32] L. Cremer, "On the acoustic boundary layer outside a rigid wall," Archiv Der Elektrischen Und Ubertragung, vol. 2, pp. 236-239, 1948.

[33] R. Beatty, "Boundary layer attenuation of higher order modes in rectangular and circular tubes," The Journal Of The Acoustical Society Of America, vol. 22, pp. 850854, 1950.

[34] C. Zwikker and C. Kosten, Sound Absorbing Materials. Elsevier, $5^{\text {th }}$ ed., 1949.

[35] H. Tijdeman, "On the propagation of sound waves in cylindrical tubes," Journal of Sound and Vibration, vol. 39, pp. 1-33, 1975.

[36] W. Beltman, "Viscothermal wave propagation including acousto-elastic interaction, Part I: Theory,” Journal of Sound and Vibration, vol. 227, pp. 555-586, 1999.

[37] W. Beltman, P. van der Hoogt, R. Spiering, and H. Tijdeman, "Implementation and experimental validation of a new viscothermal acoustic finite element for acoustoelastic problems," Journal of Sound and Vibration, vol. 216, pp. 159-185, 1998.

[38] A. Cummings, "Sound propagaton in narrow tubes of arbitrary cross section," Journal of Sound and Vibration, vol. 162, pp. 27-42, 1993.

[39] R. Astley and A. Cummings, "Wave propagation in catalytic converters: Formulation of the problem and finite element to solution scheme," Journal of Sound and Vibration, vol. 188, pp. 635-657, 1995.

[40] F. Shields, K. Lee, and W. Wiley, "Numerical solution for sound velocity and absorption in cylindrical tubes," Journal of the Acoustical Society of America, vol. 37, pp. 724-729, 1965.

[41] H. Scarton, Waves and stability in viscous and inviscid compressible liquids contained in rigid and elastic tubes by the method of eigenvalleys. $\mathrm{PhD}$ thesis, CarnegieMellon University, Pittsburgh., U.S.A., 1970.

[42] H. Scarton and W. Rouleau, "Axisymmetric waves in compressible Newtonian liquids contained in rigid tubes: Steady-periodic mode shapes and dispersion by the method of eigenvalleys," Journal of Fluid Mechanics, vol. 58, pp. 595-621, 1973.

[43] M. Bruneau, P. Herzog, J. Kergomard, and J. Polack, "General formulation of the dispersion equation in bounded visco-thermal fluid, and application to some simple geometries," Wave Motion 11, pp. 441-451, 1989. 
[44] P. Liang and H. Scarton, "Attenuation of higher order circumferential thermoacoustic waves in viscous fluid lines," Journal of Sound and Vibration, vol. 193, pp. 10991113, 1996.

[45] E. Dokumaci, "Prediction of the effects of entropy fluctuations on sound radiation from vibrating bodies using an integral equation approach," Journal of Sound and Vibration, vol. 186, pp. 805-819, 1995.

[46] C. Karra and M. Ben Tahar, "Effects of entropic wave in vibroacoustic problem using boundary element analysis," Flow, Turbulence and Combustion, vol. 74, pp. 49-66, 2005.

[47] R. Bossart, N. Joly, and M. Bruneau, "Hybrid numerical and analytical solutions for acoustic boundary problems in thermo-viscous fluids," Journal of Sound and Vibration, vol. 263, pp. 69-84, 2003.

[48] N. Joly, "Finite element modeling of thermoviscous acoustics in closed cavities," in EURONOISE2008, (Paris, France), 2008.

[49] V. Cutanda and P. Juhl, "Calculation of visco-thermal losses in thin fluid layers using BEM,” in ICSV9, (Orlando, Florida, U.S.A.), 2002.

[50] V. Cutanda, Numerical transducer modeling. PhD thesis, Technical University of Denmark, Ørsted, Denmark, 2001.

[51] S. Makarov and M.Ochmann, "Nonlinear and thermoviscous phenomena in acoustics, Part I,” Acustica - acta acustica, vol. 82, pp. 579-606, 1996.

[52] S. Makarov and M.Ochmann, "Nonlinear and thermoviscous phenomena in acoustics, Part II," Acustica - acta acustica, vol. 83, pp. 197-222, 1997.

[53] M. Ochmann and S. Makarov, "Nonlinear and thermoviscous phenomena in acoustics, Part III," Acustica - acta acustica, vol. 83, pp. 827-846, 1997.

[54] W. Chester, "Resonant oscillations in closed tubes," Journal of Fluid Mechanics, vol. 18, pp. 44-64, 1964.

[55] M. Mortell, “The evolution of nonlinear standing waves in bounded media," Journal of Applied Mathematics and Physics (ZAMP), vol. 28, pp. 33-46, 1977.

[56] B. Seymour and M. Mortell, "Resonant acoustic oscillations with damping: Small rate theory," Journal of Applied Mathematics and Physics (ZAMP), vol. 58, pp. 353373, 1973.

[57] K. Peat, "Evaluation of four-pole parameters for ducts with flow by the finite element method," Journal of Sound and Vibration, vol. 84, pp. 389-395, 1982.

[58] K. Peat and R. Kirbya, "Acoustic wave motion along a narrow cylindrical duct in the presence of an axial mean flow and temperature gradient," Journal of the Acoustical Society of America, vol. 107, p. 1859Ü1867, 2000. 
[59] E. Dokumaci, "An approximate dispersion equation for sound waves in a narrow pipe with ambient gradients," Journal of Sound and Vibration, vol. 240, pp. 637-646, 2001.

[60] M. Gunzburger, Finite element methods for viscous incompressible flows, A guide to Theory, Practice and Algorithms. Academic Press, $1^{\text {st }}$ ed., 1989.

[61] F. Brezzi and M. Fortin, Mixed and hybrid finite element methods. Springer, $1^{\text {st }}$ ed., 1991.

[62] K. Bathe, Finite element procedures in engineering analysis. Prentice-Hall, $1^{\text {st }}$ ed., 1982.

[63] C. Truesdell, "Precise theory of the absorption and dispersion of forced plane infinitisimal waves according to the Navier-Stokes equations," Journal of Mathematics and Mechanics, vol. 2, pp. 643-741, 1953.

[64] K. Rathnam and M. Oberai, "Acoustic wave propagation in cylindrical tubes containting slighly rarefied gas," Journal of Sound and Vibration, vol. 60, pp. 379-388, 1978.

[65] K. Rathnam and M. Oberai, "Influence of velocity slip and temperature jump in rarefied gas acoustic oscillation in cylindrical tubes," Journal of Sound and Vibration, vol. 103, pp. 448-452, 1985.

[66] V. Kozlov and A. Fedorov, "Acoustic properties of rarefied gases inside pores of simple geometries," Acoustical Society of America, vol. 117, p. 3402Ü3412, 2005.

[67] M. Stinson, "Propagation of sound and the assignment of shape factors in model porous materials having simple pore geometries," Journal of the Acoustical Society of America, vol. 91, pp. 685-695, 1992.

[68] M. Stinson, "The propagation of plane sound waves in narrow and wide circular tubes, and generalization to uniform tubes of arbitrary cross sectional shape," Journal of the Acoustical Society of America, vol. 89, pp. 550-558, 1991.

[69] H. Roh, W. Arnott, and J. Sabatier, "Measurement and calculation of acoustic propagation constants in arrays of small air-filled rectangular tubes," Journal of the Acoustical Society of America, vol. 89, pp. 2617-2624, 1991.

[70] D. Bies and C. Hansen, Engineering noise control. E\&FN SPON, $1^{\text {st }}$ ed., 1996.

[71] G. Putland, "Every one-parameter acoustic field obeys Webster's horn equation," Journal of the Audio Engineering Society, vol. 41, pp. 435-450, 1993.

[72] P. Morse and K. Ingard, Theoretical acoustics. McGraw-Hill, $1^{\text {st }}$ ed., 1968.

[73] P. Moon and D. Spencer, Field Theory Handbook. Springler, $2^{\text {nd }}$ ed., 1971.

[74] P. Morse and H. Feshbach, Methods of theoretical physics. McGraw-Hill, $1^{\text {st }}$ ed., 1953.

[75] F. Mechel, "Revision der Kirchhoff-Rayleigh-Theorie zur Schallausbreitung in thermo-viskoser Luft. teil i: Grundgleichungen und Kapillaren-Moden," Acta Acustica United With Acustica, vol. 93, pp. 507-534, 2007. 
[76] F. Mechel, "Revision der Kirchhoff-Rayleigh-Theorie zur Schallausbreitung in thermo-viskoser Luft. teil ii: Feldberechnungen in Flach-Kapillaren," Acta Acustica United With Acustica, vol. 93, pp. 675-705, 2007.

[77] F. Mechel, Formulas of acoustics. Springer, $1^{\text {st }}$ ed., 2002.

[78] L. Cheng, R. White, and K. Grosh, "Three-dimensional viscous finite element formulation for acoustic fluid-structure interaction," Computer Methods in Applied Mechanics and Engineering, vol. 197, pp. 4160-4172, 2008.

[79] E. Kita and N. Kamiya, "Trefftz method: An overview," Advances in Engineering Software, vol. 24, pp. 3-12, 1995.

[80] E. Trefftz, "Ein Gegenstück zum Ritzschen Verfahren," in Proceedings of the 2nd international congress on applied mechanics, (Zürich, Switzerland), 1926.

[81] A. Zielinski, "On trial functions applied in the generalized Trefftz method," Advances in Engineering Software, vol. 24, pp. 147-155, 1995.

[82] F. Tisseur and K. Meerbergen, "The quadratic eigenvalue problem," SIAM review, vol. 43, pp. 235-286, 2001.

[83] Q. Yu and G. Hu, "Development of a helical coordinate system and its application to analysis of polymer flow in screw extruders part i. the balance equations in a helical coordinate system," Journal of Non-Newtonian Fluid Mechanics, vol. 69, pp. 155$167,1997$.

[84] L. Campos and P. Serrao, "On helicoidal rectangular coordinates for the acoustics of bent and twisted tubes,” Wave motion, vol. 38, pp. 53-66, 2003.

[85] H. Hudde and U. Letens, "Scattering matrix of a discontinuity with a nonrigid wall in a lossless circular duct," Journal of the Acoustical Society of America, vol. 78, pp. 1826-1837, 1985.

[86] R. Kirby, "Transmission loss predictions for dissipative silencers of arbitrary cross section in the presence of mean flow," Journal of the Acoustical Society of America, vol. 114, pp. 200-209, 2003.

[87] R. Astley, "Fe mode-matching schemes for the exterior Helmholtz problem and their relationship to the fe-dtn approach," Communications in Numerical Methods in Engineering, vol. 12, pp. 257-267, 1996.

[88] R. Mittra and S. Lee, Analytical techniques in the theory of guided waves. The MacMillan Company, $1^{\text {st }}$ ed., 1971.

[89] G. Arfken, Mathematical Methods for Physicists. FL: Academic Press, $3^{\text {rd }}$ ed., 1985.

[90] W. Rostafinski, "Analysis of propagation of waves of acoustic frequencies in curved ducts," Journal of the Acoustical Society of America, vol. 56, pp. 11-15, 1974.

[91] W. Rostafinski, "Acoustic systems containing curved duct sections," Journal of the Acoustical Society of America, vol. 60, pp. 23-28, 1976. 
[92] E. Eisner, "Complete solutions of the Webster horn equation," Journal of the Acoustical Society of America, vol. 41, pp. 1126-1146, 1967.

[93] S. Rienstra, "Webster's horn equation revisited," Society for Industrial and Applied Mathematics, vol. 65, pp. 1981-2004, 2005.

[94] E. Dokumaci, "An exact transfer matrix formulation of plane sound wave transmission in inhomogeneous ducts," Journal of Sound and Vibration, vol. 217, pp. 869882, 1998.

[95] R. Glav, P. Regaud, and M. Äbom, "A general formalism for analyzing acoustic 2-port networks," Journal of Sound and Vibration, vol. 202, pp. 739-747, 1997.

[96] F. Brown, Engineering system dynamics. CRC Press, $2^{\text {st }}$ ed., 2007.

[97] M. Munjal, Acoustics of Ducts and Mufflers. Wiley, $1^{\text {st }}$ ed., 1987.

[98] W. Eversman, "A systematic procedure for the analysis of multiply branched acoustic transmission lines," Transactions of the American Society of Mechanical Engineers Journal of Vibrations, Acoustics, Stress and Reliability in Design, vol. 109, pp. 168177, 1987.

[99] A. Frid, "Fluid vibration in piping systems-a structural mechanics approach, I: theory," Journal of Sound and Vibration, vol. 133, pp. 423-438, 1989.

[100] S. Brenner, The mathematical theory of finite element methods. Springer, $3^{\text {st }}$ ed., 2008.

[101] H. Elmar, D. Silverster, and A. Wathem, Finite elements and fast iterative solvers. Oxford University Press, $1^{\text {st }}$ ed., 2005.

[102] V. Girault and P. Raviart, Finite element methods for Navier-Stokes equations. Springer, $1^{\text {st }}$ ed., 1986.

[103] D. Boffi, F. Brezzi, L. Demkowicz, R. Durán, R. Falk, and M. Fortin, Mixed finite elements, compatibility conditions, and applications. Springer, $1^{\text {st }}$ ed., 2008.

[104] A. Ern and J. Guermond, Theory and practice of finite elements. Springer, $1^{\text {st }}$ ed., 2004.

[105] O. Zienkiewicz and R. Taylor, The Finite Element Method, Volume I: The basis. Butterworth-Heinemann, $5^{\text {th }}$ ed., 2000.

[106] T. Hughes, The finite element method, linear static and dynamic element analysis. Dover publications, inc., $1^{\text {st }}$ ed., 2000.

[107] P. Ciarlet and P. Raviart, "Interpolation theory over curved elements, with applications to finite element methods," Computer Methods in Applied Mechanics and Engineering, vol. 1, pp. 217-249, 1972.

[108] I. Babuska and M. Suri, "On locking and robustness in the finite element method," Society for Industrial and Applied Mathematics, vol. 29, pp. 1261-1293, 1992. 
[109] O. Ladyzhenskaya, The mathematical theory of viscous incompressible flow. Gordon and Breach, $1^{\text {st }}$ ed., 1969.

[110] D. Chapelle and K. Bathe, "The inf-sup test," Computers \& Structures, vol. 47, pp. 537-545, 1993.

[111] K. Bathe, "The inf-sup condition and its evaluation for mixed finite element methods," Computers \& Structures, vol. 79, pp. 243-252, 2001.

[112] D. Arnold, D. Boffi, R. Falk, and L. Gastaldi, "Finite element approximation on quadrilateral meshes," Communications In Numerical Methods In Engineering, vol. 17, pp. 805-812, 2001.

[113] D. Arnold, F. Brezzi, and M. Fortin, "A stable finite element for the Stokes equations," Estratto da Calcolo, vol. 21, pp. 337-344, 1984.

[114] W. Bai, "The quadrilateral 'MINI' finite element for the Stokes problem," Computational Methods in Applied Mechanical Engineering, vol. 143, pp. 41-47, 1997.

[115] P. Bochev and M. Gunzburger, Least-squares Finite Element Methods. Springer, 2009.

[116] E. Dokumaci, "On the propagation of plane sound waves in ducts carrying an incompressible axial mean flow having an arbitrary velocity profile," Journal of Sound and Vibration, vol. 249, pp. 824-827, 2002.

[117] J. Lavrentjev, M. Äbom, and H. Bodén, "A measurement method for determining the source data of acoustic two-port sources," Journal of Sound and Vibration, vol. 183, pp. 517-531, 1995.

[118] J. Lavrentjev and M. Äbom, "Characterization of fluid machines as acoustic multiport sources," Journal of Sound and Vibration, vol. 197, pp. 1-16, 1996.

[119] M. Äbom and H. Bodén, "A note on the aeroacoustic source character of in-duct axial fans," Journal of Sound and Vibration, vol. 186, pp. 589-598, 1995.

[120] M. Äbom and H. Bodén, "Error analysis of two-microphone measurements in ducts with flow," Journal of the Acoustical Society of America, vol. 83, pp. 2429-2438, 1988.

[121] R. Glav, P. Regaud, and M. Äbom, "Study of a folded resonator including the effects of higher order modes," Journal of Sound and Vibration, vol. 273, pp. 777-792, 2004.

[122] R. Dohertya, E. Salskov, P. Corriveau, D. Gabel, P. Sorenson, and W. Beltman, "Human annoyance levels to pc sounds in the home background noise environment," in INTER-NOISE2006, (Honolulu, Hawaii, USA), 2006.

[123] W. Beltman, R. Doherty, E.Salskov, P. Corriveau, D. Gabel, and E. Baugh, "Applications of psychoacoustics to information technology products," in EURONOISE2008, (Paris, France), 2008.

[124] M. Hannink, Y. Wijnant, and A. de Boer, "Optimised sound absorbing trim panels for the reduction of aircraft cabin noise," in ICSV11, (St. Petersburg, Russia), 2004.

[125] C. Wang and C. Truesdell, Introduction to Rational Elasticity. Noordhof International Publishing, $1^{\text {st }}$ ed., 1973. 
[126] M. Cramer, “Navier Stokes equations.” http://www.esm.vt.edu/ macramer/, 2003.

[127] A. Spencer, Continuum Mechanics. Longman Group Limited, $1^{\text {st }}$ ed., 1980.

[128] G. Batchelor, An introduction to fluid dynamics. Cambridge University Press, 1967.

[129] L. Landau and E. Lifshitz, Fluid mechanics. Pergamon Press, $1^{\text {st }}$ ed., 1987.

[130] M. Salas, "The curious events leading to the theory of shock waves," in Proc. 17th Shock Interaction Symposium, (Rome, Italy), 2006.

[131] T. C. and J. F. A.L. Yarin, Springer handbook of experimental fluid mechanics. Springer, $1^{\text {st }}$ ed., 2007.

[132] N. Vargaftik, Handbook of physical properties of liquids and gases : pure substances and mixtures. Springer, $2^{\text {st }}$ ed., 1983.

[133] R. Graves and B. Argrow, "Bulk viscosity: Past to present," Journal of Thermophysics and Heat Transfer, vol. 13, pp. 337-342, 1999.

[134] D. Blackstock, Fundamentals of Physical Acoustics. Springer, 2000.

[135] R. Ash, "Second coefficient of viscosity in air," Progress report 0325544, NASA, Langley Research Center, Hampton, Virginia 23665, 1991.

[136] S. Marcy, "Evaluating the second coefficient of viscosity from sound dispersion or absorption data," AIAA Journal, vol. 28, pp. 171-173, 1990.

[137] J. Sengers, Equations of state for fluids and fluid mixtures. CBa $536.7 \mathrm{e} 044,1^{\text {st }}$ ed., 2002.

[138] B. Poling, The properties of gases and liquids. CBa 541.11 p065, $1^{\text {st }}$ ed., 2000.

[139] K. Chao and R. Robinson, Equations of State in Engineering and Research. CBa 541.121 e034, $1^{\text {st }}$ ed., 2000.

[140] D. Keefe, "Acoustic propagation in flaring, axisymmetric horns: II. numerical results, wkb theory, and viscothermal effects," Acta Acustica United with Acustica, vol. 85, pp. 285-293, 1999.

[141] R. Alfredson, "The propagation of sound in a circular duct of continuously varying cross-sectional area," Journal of Sound and Vibration, vol. 23, pp. 433-442, 1972.

[142] C. Nederveen and J. Dalmont, "Corrections to the plane-wave approximation in rapidly flaring horns," Acta Acustica United with Acustica, vol. 94, pp. 461-473, 2008.

[143] A. Cummings, "Acoustics of a wine bottle," Journal of Sound and Vibration, vol. 31, pp. 331-343, 1973.

[144] Y. Kulik, "Transfer matrix of conical waveguides with any geometric parameter for increased precision in computer modeling," Journal of the Acoustical Society of America, vol. 122, p. 179Ü184, 2007.

[145] T. Hélie, "Unidimensional models of acoustic propagation in axisymmetric waveguides," Journal of the Acoustical Society of America, vol. 114, pp. 2633-2647, 2003. 


\section{Nawoord}

Klaar! Meer dan zes jaar onderweg en nu is het eindelijk af. Dat was niet gelukt zonder de hulp van een hele hoop mensen. De laatste pagina's van dit boekje wil ik dan ook graag gebruiken om aantal hiervan te bedanken.

Allereerst wil ik André bedanken. Zonder jouw uitnodiging om te gaan promoveren, je begeleiding, vertrouwen op de goede afloop en je aanmoedigingen om het toch eindelijk eens af te maken had dit boekje er niet gelegen. Ysbrand, bedankt voor het enthousiasme en de opbouwende kritiek die je me als dagelijks begeleider keer op keer hebt geven. We waren het soms eens en vaak ook niet. Door al ons discussiëren is het boekje stukken beter geworden. Ook wil ik graag Intel bedanken voor het mogelijk maken van dit project, en in het speciaal Marco Beltman; het was een prachtige ervaring om een paar maanden bij Intel in de keuken te kunnen kijken. Ik denk er nog graag en vaak aan terug.

Op het inhoudelijk vlak ben ik ook dank verschuldigd aan de overige leden van mijn leescommissie. Ruud, bedankt voor je immer nauwkeurige en kritische kijk op de zaken. Zonder jouw input was het misschien een nog dikker boekje geworden. Ronald, we zaten lange tijd in (bijna) hetzelfde schuitje. Bedankt voor het sparren. Jelmer, strikt genomen las je niet mee, maar je dacht altijd mee. Je brede interesse voor wat dan ook was erg aanstekelijk. Ik houd jou dan ook mede verantwoordelijk voor de dikte van dit boekje.

Bert, bedankt voor de assistentie bij het op- en aftuigen van de diverse meetopstellingen (Zelfs het slaan van een gat in de akoestische kamer was je niet te gek!). Debbie, gelukkig was je direct aan de overkant van de gang te vinden. Bedankt voor werkelijk van alles en nog wat (variërend van het invullen van idiote formulieren, het regelen van vliegtuigtickets tot broodnodige pep-talks)! Ook een woord van dank voor de twee mannen die tijdens hun afstuderen nuttig werk voor me hebben verricht: Jeroen en Koen, het was een genoegen met jullie samen te werken.

Verder zijn er een hoop mensen die wellicht geen directe bijdrage aan de totstandkoming van dit boekje hebben geleverd, maar wel mijn promotie tijd tot een prachttijd hebben gemaakt. Door mijn ietwat lange verblijf aan de UT heb ik vele 
nieuwe collega's mogen begroeten. Ik zou dan ook graag willen zeggen: oude AIO ploeg, nieuwe AIO ploeg, mannen van TW, bedankt voor alle lunchwandelingen, conferenties, filmpjes, biertjes, koffiepauzes en lab/bankhang-sessies (met gesponsorde speculaas!). Of course a special thanks to all the roommates I have had over the last couple of years; you have been excellent company.

Voor de broodnodige afleiding wil ik ook een aantal niet vakgroep genoten bedanken waaronder vele Harrambee-ers en UTV-ers, studentenwintersporters, centerparkers en geo-(nacht)cashers. In het speciaal bedank ik Paul, Chris en Rien voor de ontelbare biertjes, maaltijden en potjes pool die we hebben gedronken, gegeten en gespeeld de afgelopen paar jaar.

Ma, Lodewijk, Karine en Marieke, bedankt voor jullie blijvende interesse voor wat ik aan het uitspoken was en alle aanmoedigingen om het af te maken. (Marieke, natuurlijk ook bedankt voor de omslag.) Als laatste wil ik Carina bedanken voor het geduld (wanneer het werken aan het boekje toch telkens weer wat langer bleek te duren) en vier hele mooie jaren!

Marten Nijhof

Enschede, november 2010 



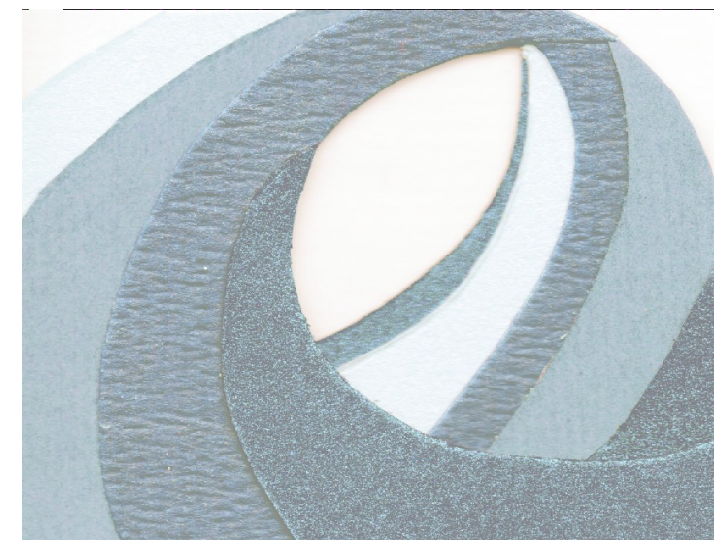

UNIVERSITY OF TWENTE. 